Supporting Information

\title{
Novel Benzo[a]quinolizidine Analogs Induce Cancer Cell Death through Paraptosis and Apoptosis
}

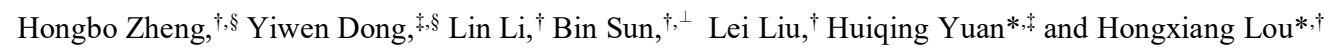

†Department of Natural Products Chemistry, Key Laboratory of Chemical Biology of Ministry of Education, School of Pharmaceutical Sciences, Shandong University, Jinan, China.

*Department of Biochemistry and Molecular Biology, School of Medicine, Shandong University, Jinan, China.

${ }^{\perp}$ National Glycoengineering Research Center, Shandong University, Jinan, China.

\section{Table of contents}

1. Preparation of tetrhydroisoquinolines, methyl vinyl ketone and reaction optimization

2. Configuration determination, and MTT results of benzo $[a]$ quinolizidine derivatives

3. Analytical data for these derivatives

4. Optimization of the Z-VAD-fmk and Ac-DEVD-CHO assays

5. Westernblot analysis of IkB-a and p-P65 reponse to CHX and ActD

6. CI values calculated by CompuSyn software

7. Weight of individual excised tumor

8. Figures of HR-ESI-MS, IR, ${ }^{1} \mathrm{H},{ }^{13} \mathrm{C}$ and $2 \mathrm{D}$ NMR spectra for derivatives 


\section{Preparation of tetrhydroisoquinolines, vinyl ketone and reaction optimization.}

Tetrahydroisoquinoline are commercial available and other tetrahydroisoquinolines with alkoxyl or benzyloxy group(s) are prepared with phenethylamines by using using a Pictet-Spengler reaction as described in text and ref.1. Next demethylation, N-Boc protection and O-Bn protection was performed acoording to ref.2-4 and give the intermediates SA1-SA14 all shown in scheme S1.

Scheme S1. Preparation of tetrhydroisoquinolines. ${ }^{\mathrm{a}}$

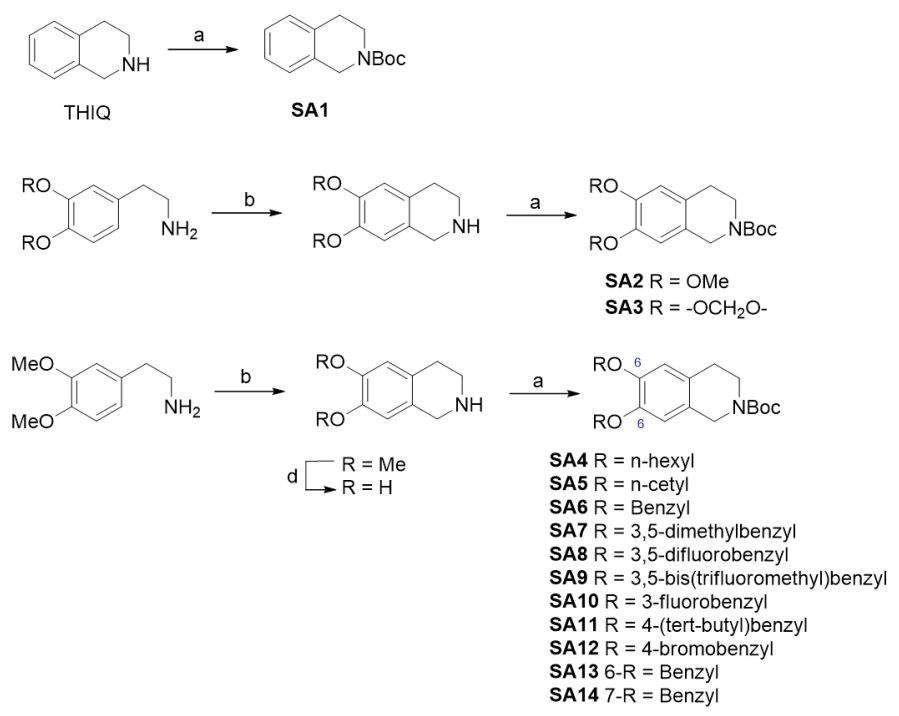

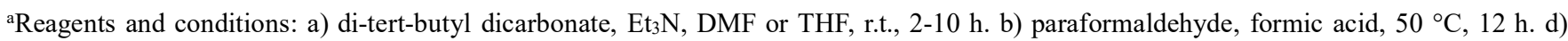
$\mathrm{HBr}\left(48 \%\right.$ in $\left.\mathrm{H}_{2} \mathrm{O}\right)$, reflux, 5 h. d) $\mathrm{RBr}, \mathrm{K}_{2} \mathrm{CO}_{3}$, DMF, r.t., 5-12 h.

Methyl vinyl ketones SB1-SB13 was prepared by Claisen-Schmidt reaction or Witting reaction which could found in ref.5-6, and the rest two commercial available materials was also shown in scheme S2.

Scheme S2. Preparation of tetrhydroisoquinolines. ${ }^{\mathrm{a}}$

${ }^{1}$ Okano, K.; Tokuyama, H.; Fukuyama, T. Total synthesis of (+)-yatakemycin. J. Am. Chem. Soc. 2006, 3, 296-309.

${ }^{2}$ Dalence-Guzmán, M. F.; Berglund, M.; Skogvall, S.; Sterner, O. SAR studies of capsazepinoid bronchodilators. Part 1: The importance of the catechol moiety and aspects of the B-ring structure. Bioorg. Med. Chem. 2008, 16, 2499-2512.

${ }^{3}$ Berglund, M.; Dalence, M. F. SAR studies of capsazepinoid bronchodilators. Part 2: Chlorination and catechol replacement in the A-ring. Bioorg. Med. Chem. 2008, 16, 2513-2528.

${ }^{4}$ Li, Y.; Zhou, Y.; Qi, B.; Gong, T.; Sun, X.; Fu, Y.; Zhang, Z. (2014). Brain-specific delivery of dopamine mediated by N,N-dimethyl amino group for the treatment of Parkinson's disease. Mol. Pharm. 2014, 11, 3174-3185.

${ }^{5}$ Weber, W. M.; Hunsaker, L. A.; Abcouwer, S. F.; Deck, L. M.; Vander Jagt, D. L. Anti-oxidant activities of curcumin and related enones. Bioorg. Med. Chem. 2005, 13, 3811-3820.

${ }^{6}$ Wube, A. A.; Hüfner, A.; Thomaschitz, C.; Blunder, M.; Kollroser, M.; Bauer, R.; Bucar, F. Design, synthesis and antimycobacterial activities of 1-Methyl-2-alkenyl-4(1H)-quinolones.. Bioorg. Med. Chem. 2011, 19, 567-579. 


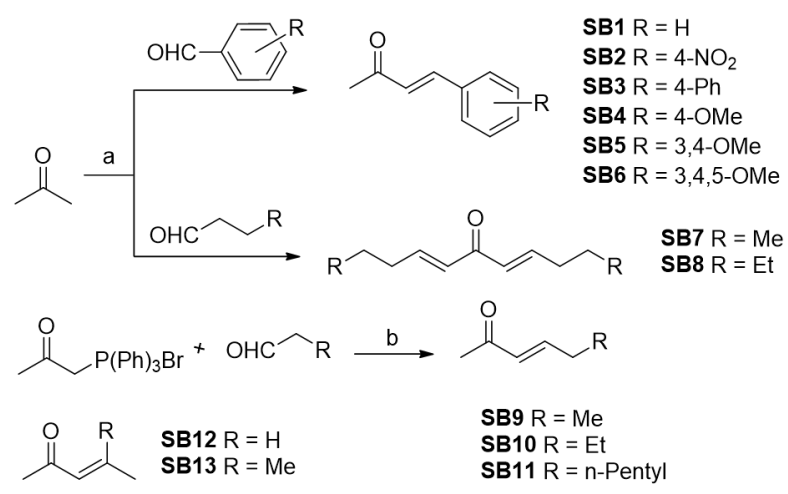

aReagents and conditions: a) $10 \% \mathrm{NaOH}$, EtOH, r.t., 3 h. b) $\mathrm{CH}_{2} \mathrm{Cl}_{2}$, r.t., 24 h.

Condition for the Synthesis of 6,7-dihydrobenzo[a]quinolizidine derivatives which not discribed in text were shown in scheme S3.

Palladium/C catalyst was employed for deprotection and compound SD4,SE4a-SE4b were obtained.

Scheme S3 Preparation of tetrhydroisoquinolines. ${ }^{\mathrm{a}}$

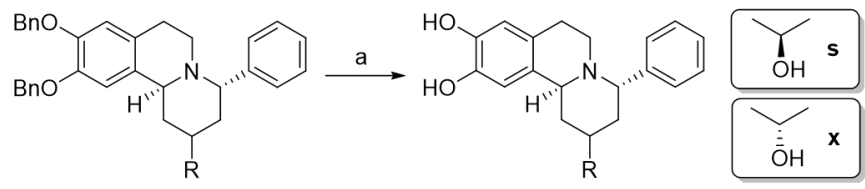

SD4, SE4a-SE4b

aReagents and conditions: a) $\mathrm{Pd} / \mathrm{C}, \mathrm{MeOH}$, r.t. 12 h. b) $\mathrm{CH}_{2} \mathrm{Cl}_{2}$, r.t., 24 h.

Coupling of N-Boc ethoxy tetrahydroisoquinoline SC1 with trimethylsilyl enol ether SC2 was examined for reaction optimization shown in table $\mathrm{S} 1$.

Table S1. Model reaction optimization. ${ }^{\mathrm{a}}$

\begin{tabular}{|c|c|c|c|c|}
\hline & & & & \\
\hline Entry & Lewis acid & eq & $\mathrm{t}(\mathrm{h})$ & Yiled $(\%)^{b}$ \\
\hline 1 & TMSOTf & 0.3 & 2 & $23 \%$ \\
\hline 2 & $\mathrm{TfOH}$ & 0.3 & 2 & $<5 \%$ \\
\hline 3 & $\mathrm{BF} \cdot \mathrm{EtO}_{2}$ & 0.3 & 2 & $21 \%$ \\
\hline 4 & $\operatorname{In}(\mathrm{OTf})_{3}$ & 0.3 & 2 & $46 \%$ \\
\hline 5 & $\mathrm{Sc}(\mathrm{OTf})_{3}$ & 0.3 & 2 & $61 \%$ \\
\hline 6 & $\mathrm{Ga}(\mathrm{OTf})_{3}$ & 0.3 & 2 & $57 \%$ \\
\hline 7 & $\mathrm{Bi}(\mathrm{OTf}) 3$ & 0.3 & 2 & $64 \%$ \\
\hline 8 & $\mathrm{Yb}(\mathrm{OTf})_{3}$ & 0.3 & 2 & $82 \%$ \\
\hline 9 & $\mathrm{Yb}(\mathrm{OTf})_{3}$ & 1.0 & 2 & $76 \%$ \\
\hline 10 & $\mathrm{Yb}(\mathrm{OTf})_{3}$ & 0.3 & 24 & $71 \%$ \\
\hline
\end{tabular}

${ }^{\mathrm{a} C}$ Conditions: SC1 $(0.1 \mathrm{mmol})$, SC2 $(0.2 \mathrm{mmol})$, Lewis acid $(0.03 \mathrm{mmol})$ in $1.0 \mathrm{~mL} \mathrm{CH}_{2} \mathrm{Cl}_{2}$ at room temperature for $2 \mathrm{~h}$ or $24 \mathrm{~h}$. ${ }^{\mathrm{b}}$ Isolated yield. 


\section{Benzo $[a]$ quinolizidine derivatives, configuration determination and their MTT results}

Relative configuration of compound 1, 4, 16a, 11b, 18 was determined by NOESY spectrum and shown in Fig. S1.
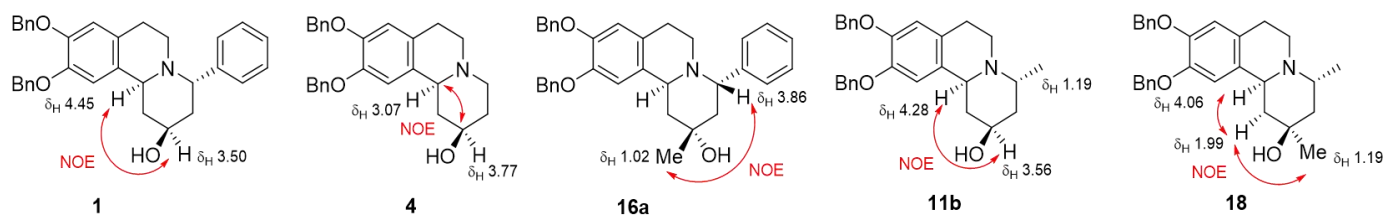

Figure S1. NOESY $(\mathrm{H} \leftrightarrow \mathrm{H})$ correlations for compound 1, 4, 16a, 11b, 18 .

Benzo $[a]$ quinolizidine derivatives and their MTT results which not shown in the text and now exhibited in and table S2-S4.

Table S2. Derivatives with alkoxy moieties and benzyloxy group. ${ }^{a}$

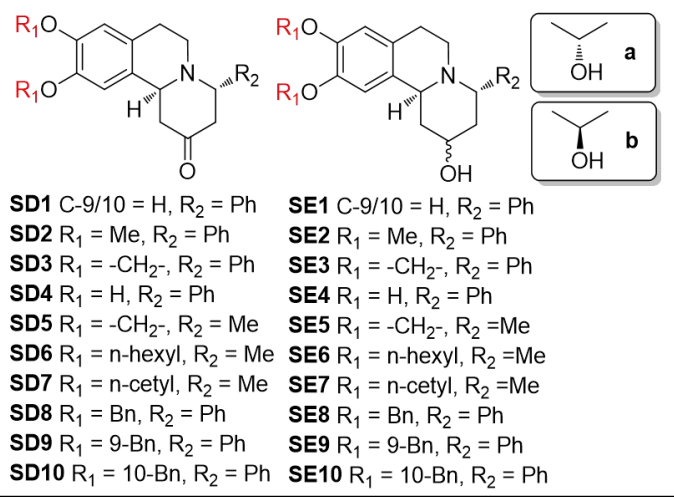

\begin{tabular}{cccc}
\hline Compound $^{\mathrm{b}}$ & MDA-MB-231 & Hela & K562 \\
\hline 2(SE9b) & $13.3 \pm 1.52$ & $8.55 \pm 0.42$ & $16.3 \pm 0.99$ \\
3(SE10b) & $12.6 \pm 0.78$ & $5.87 \pm 0.30$ & - $^{\mathrm{b}}$ \\
\hline
\end{tabular}

${ }^{\mathrm{a} C}$ Compounds SD1-SE10 was all tested inactive $\left(\mathrm{IC}_{50}>20 \mu \mathrm{M}\right)$ except compound 2-3. ${ }^{\mathrm{b}}$ Not active $\left(\mathrm{IC}_{50}>20 \mu \mathrm{M}\right)$.

Table S3. Derivatives with 4-substitutes and position $2 .^{\mathrm{a}}$<smiles>[R2]C1CC(=O)C[C@H]2c3cc(OCc4ccccc4)c(OC)cc3CCN12</smiles>

SF1 $R_{1}=M e, R_{2}=M e$

$S F 2 R_{1}=H, R_{2}=4$-nitrobenzen

SF3 $R_{1}=H, R_{2}=4$-methoxylbenzene

SF4 $R_{1}=H, R_{2}=3$,4-dimethoxylbenzene

SF5 $R_{1}=H, R_{2}=3,4,5$-trimethoxylbenzene

SF6 $R_{1}=H, R_{2}=4$-phenylbenzene

SF7 R $R_{1}=H, R_{2}=E t$

SF8 $R_{1}=H, R_{2}=n-P r$

SF9 $R_{1}=H, R_{2}=n-H e x y l$

$34 \mathrm{R}_{1}=\mathrm{H}, \mathrm{R}_{2}=\mathrm{Me}$

$36 \mathrm{R}_{1}=\mathrm{H}, \mathrm{R}_{2}=\mathrm{C}-3$

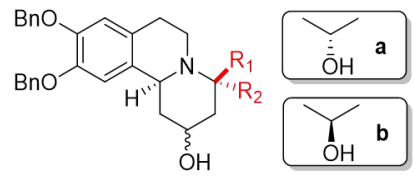

$4^{\mathrm{b}} \mathrm{R}_{1}=\mathrm{H}, \mathrm{R}_{2}=\mathrm{H}$

$5^{\mathrm{b}} \mathrm{R}_{1}=\mathrm{Me}, \mathrm{R}_{2}=\mathrm{Me}$

$6 \mathrm{R}_{1}=\mathrm{H}, \mathrm{R}_{2}=4$-nitrobenzene

$7 R_{1}=H, R_{2}=4$-methoxylbenzene

$8 \mathrm{R}_{1}=\mathrm{H}, \mathrm{R}_{2}=3$,4-dimethoxylbenzene

$9 R_{1}=H, R_{2}=3,4,5$-trimethoxylbenzene

$10 R_{1}=H, R_{2}=4$-phenylbenzene

$11 \mathrm{R}_{1}=\mathrm{H}, \mathrm{R}_{2}=\mathrm{Me}$

$12 \mathrm{R}_{1}=\mathrm{H}, \mathrm{R}_{2}=\mathrm{Et}$

$13 \mathrm{R}_{1}=\mathrm{H}, \mathrm{R}_{2}=\mathrm{n}-\mathrm{Pr}$

$14 R_{1}=H, R_{2}=n-H e x y l$

\begin{tabular}{cccccr}
\hline Compound & MDA-MB-231 & Hela & K562 & PC3 & DU145 \\
\hline $\mathbf{4}$ & $11.8 \pm 0.65$ & $10.6 \pm 0.74$ & $23.2 \pm 1.78$ & c & $21.3 \pm 0.91$ \\
$\mathbf{7 b}$ & $7.26 \pm 1.14$ & $12.5 \pm 0.87$ & $13.0 \pm 0.91$ & & \\
$\mathbf{9 b}$ & $-\mathrm{d}(25.4)$ & $-(32.3)$ & - & & \\
$\mathbf{1 4 a}$ & $7.35 \pm 0.80$ & $6.87 \pm 0.52$ & $9.83 \pm 0.48$ & & \\
$\mathbf{1 4 b}$ & $4.61 \pm 0.48$ & $2.18 \pm 0.33$ & $5.09 \pm 0.26$ & & \\
$\mathbf{1 6 a}$ & $-(29.8)$ & - & $-(37.4)$ & & \\
$\mathbf{1 7 b}$ & $-(34.68)$ & $-(36.2)$ & - & &
\end{tabular}




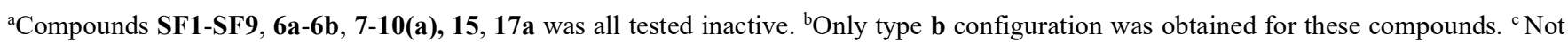
tested. ${ }^{\mathrm{N}}$ ot active $\left(\mathrm{IC}_{50}>20 \mu \mathrm{M}\right)$.

Table S4. Derivatives with various benzyl groups. ${ }^{a}$<smiles>[R7]C=Cc1ccc(COc2cc3c(cc2OCc2cc[R7]([R])c2)[C@H]2CC(=O)C[C@H]([R2])N2CC3)cc1</smiles>

SG1 $R_{1}=3,5-F, R_{2}=3$,4-dimethoxylbenzene SG2 $R_{1}=3,5-M e, R_{2}=M e$ SG $3 R_{1}=3,5-F, R_{2}=M e$, SG4 $R_{1}=3,5-C_{3}, R_{2}=M e$ SG5 $R_{1}=4-B r, R_{2}=M e$ SG6 $R_{1}=4-\mathrm{tBu}, \mathrm{R}_{2}=\mathrm{Me}$ $35 R_{1}=3-F, R_{2}=M e$

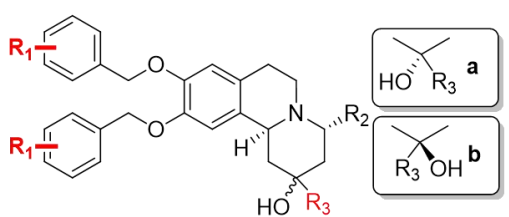

$20 \mathrm{R}_{1}=3,5-\mathrm{Me}, \mathrm{R}_{2}=3,4$-dimethoxylbenzene, $\mathrm{R}_{3}=\mathrm{H}$

$21 R_{1}=3,5-F, R_{2}=3$,4-dimethoxylbenzene, $R_{3}=H$

$22 \mathrm{R}_{1}=3,5-\mathrm{Me}, \mathrm{R}_{2}=\mathrm{Me}, \mathrm{R}_{3}=\mathrm{H}$

$23 R_{1}=3,5-F, R_{2}=M e, R_{3}=H$

$24 \mathrm{R}_{1}=3,5-\mathrm{CF}_{3}, \mathrm{R}_{2}=\mathrm{Me}, \mathrm{R}_{3}=\mathrm{H}$

$25 R_{1}=3-F, R_{2}=M e, R_{3}=H$

$26 \mathrm{R}_{1}=4-\mathrm{Br}, \mathrm{R}_{2}=\mathrm{Me}, \mathrm{R}_{3}=\mathrm{H}$

$27 R_{1}=4-t B u, R_{2}=M e, R_{3}=H$

$28^{\mathrm{a}} \mathrm{R}_{1}=3,5-\mathrm{Me}, \mathrm{R}_{2}=\mathrm{Me}, \mathrm{R}_{3}=\mathrm{Me}$

29 ${ }^{\mathrm{a}} \mathrm{R}_{1}=3,5-\mathrm{F}, \mathrm{R}_{2}=\mathrm{Me}, \mathrm{R}_{3}=\mathrm{Me}$

$30^{\mathrm{a}} \mathrm{R}_{1}=3,5-\mathrm{CF}_{3}, \mathrm{R}_{2}=\mathrm{Me}, \mathrm{R}_{3}=\mathrm{Me}$

$31^{\mathrm{a}} \mathrm{R}_{1}=3-\mathrm{F}, \mathrm{R}_{2}=\mathrm{Me}, \mathrm{R}_{3}=\mathrm{Me}$

$32^{\mathrm{a}} \mathrm{R}_{1}=4-\mathrm{Br}, \mathrm{R}_{2}=\mathrm{Me}, \mathrm{R}_{3}=\mathrm{Me}$

$33^{\mathrm{a}} \mathrm{R}_{1}=4-\mathrm{tBu}, \mathrm{R}_{2}=\mathrm{Me}, \mathrm{R}_{3}=\mathrm{Me}$

\begin{tabular}{cccc}
\hline Compound & MDA-MB-231 & Hela & K562 \\
\hline SG4 & $12.2 \pm 0.54$ & $7.31 \pm 0.17$ & $20.0 \pm 1.32$ \\
SG5 & $14.1 \pm 0.61$ & $6.95 \pm 0.72$ & $13.1 \pm 0.86$ \\
SG6 & $13.6 \pm 0.96$ & $13.8 \pm 0.88$ & $6.71 \pm 0.17$ \\
35 & $1.33 \pm 0.28$ & $7.24 \pm 0.67$ & $15.1 \pm 1.44$ \\
\hline
\end{tabular}

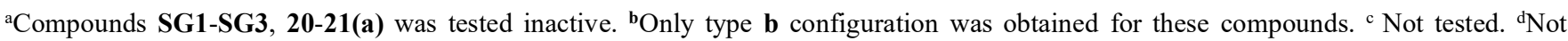
active $\left(\mathrm{IC}_{50}>20 \mu \mathrm{M}\right)$.

Table S5. Octahydroazocino[2,1-a]isoquinoline derivative. ${ }^{\mathrm{a}}$

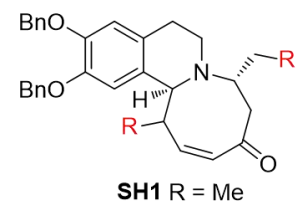

$\mathrm{SH} 1 \mathrm{R}=\mathrm{Me}$
$\mathrm{SH} 2 \mathrm{R}=\mathrm{Et}$

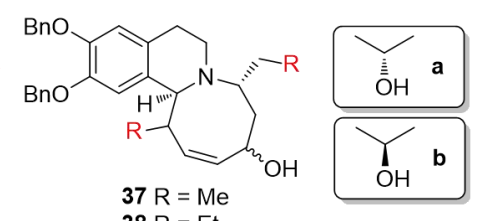

$38 \mathrm{R}=\mathrm{Et}$

\begin{tabular}{cccc}
\hline Compound & MDA-MB-231 & Hela & K562 \\
\hline 37a & $12.9 \pm 1.03$ & $9.11 \pm 0.59$ & $13.0 \pm 0.50$ \\
37b & $8.34 \pm 0.44$ & $9.99 \pm 0.25$ & $11.7 \pm 0.87$ \\
\hline
\end{tabular}

${ }^{a}$ Compounds SH-1, SH-2, 38a was tested inactive.

Isolation of compound $\mathbf{2 2 b}$ was shown in Fig. S2. Compound 22b-1 and 22b-2 exhibits equal cytotoxicity against PC3 cell line, 0.72 and $0.91 \mu \mathrm{M}$, respectively.

Column: Chiralpak AS-H $(250 \mathrm{~mm} \times 4.6 \mathrm{~mm} \times 5 \mu \mathrm{m}) ; \quad$ Mobile phase: Hexane-Isopropanol $(85: 15, \mathrm{v} / \mathrm{v})$;

Wavelength: $223 \mathrm{~nm}$;

Rate: $1 \mathrm{~mL} / \mathrm{min}$; 
Temperature: $25^{\circ} \mathrm{C}$;

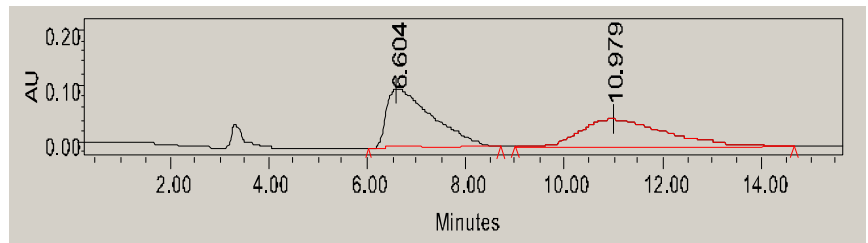

\begin{tabular}{|c|c|c|c|c|c|}
\hline & Name & $\begin{array}{c}\text { Retention } \\
\text { Time }\end{array}$ & Area & \% Area & Height \\
\hline 1 & $\mathbf{2 2 b - 1}$ & 6.604 & 6795705 & 52.69 & 104571 \\
\hline 2 & $\mathbf{2 2 b - 2}$ & 10.979 & 6101968 & 47.31 & 46876 \\
\hline
\end{tabular}

22b (before islation)

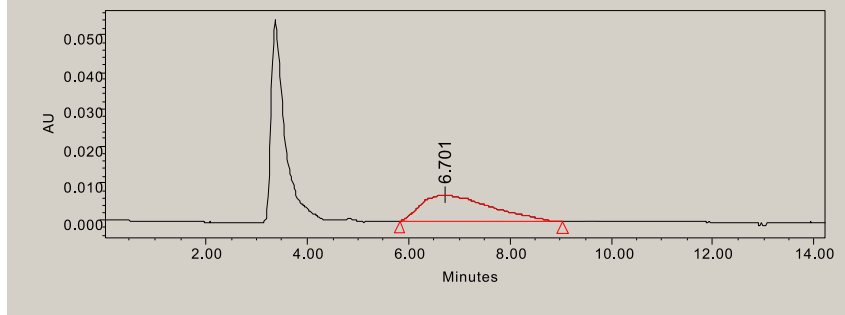

22b-1 (after islation)

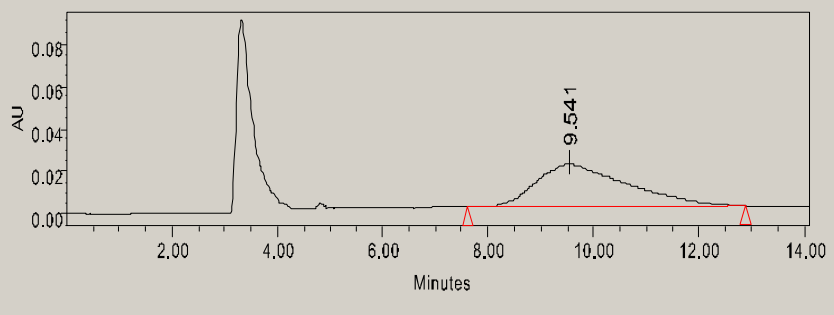

22b-2 (after islation)

Figure S2. HPLC analysis for racemic 22b and the two enantiomers 22b-1, 22b-2.

\section{Analytical data for these derivatives}

Spectra of HPLC assays of several compounds was provided in Fig. S3-S11.

Column: Eclipse XDB-C18 $(150 \mathrm{~mm} \times 4.6 \mathrm{~mm} \times 5 \mu \mathrm{m})$;

Wavelength: $210 \mathrm{~nm}$;

Rate: $0.8 \mathrm{~mL} / \mathrm{min}$;

Temperature: $25^{\circ} \mathrm{C}$;

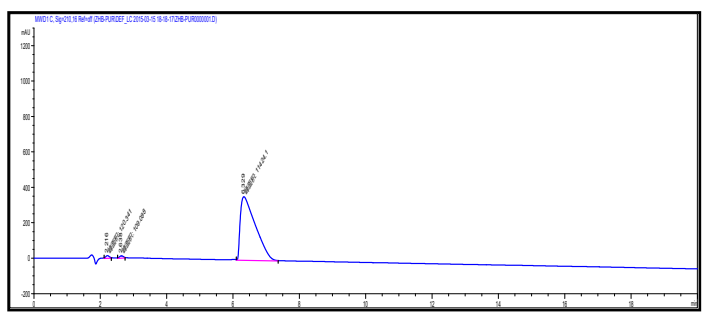

Figure S3. HPLC analysis of 1, 98.4\%. Mobile phase: Methanol-Water( $0.5 \%$ Triethylamine) $(65: 35, \mathrm{v} / \mathrm{v})$.

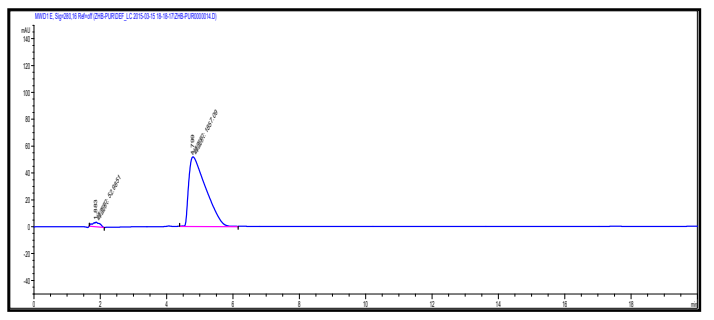

Figure S4. HPLC analysis of 11b, 97.2\%. Mobile phase: Methanol-Water( $0.5 \%$ Triethylamine) $(65: 35, \mathrm{v} / \mathrm{v})$. 


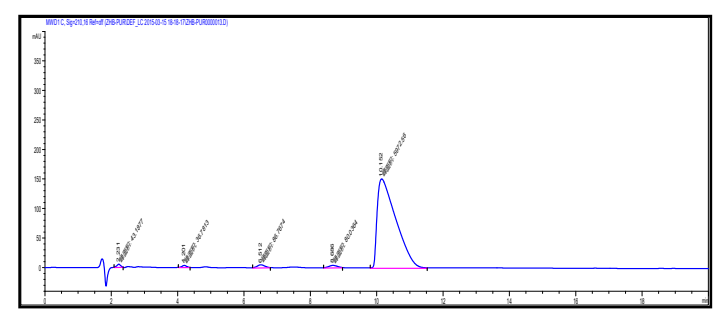

Figure S5. HPLC analysis of 20b, 96.0\%. Mobile phase: Methanol-Water( $0.5 \%$ Triethylamine) (75:25, v/v).

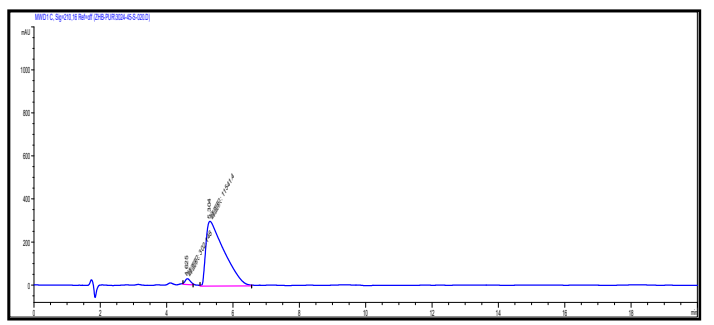

Figure S6. HPLC analysis of 22a, 97.5\%. Mobile phase: Methanol-Water( $0.5 \%$ Triethylamine) (75:25, v/v).

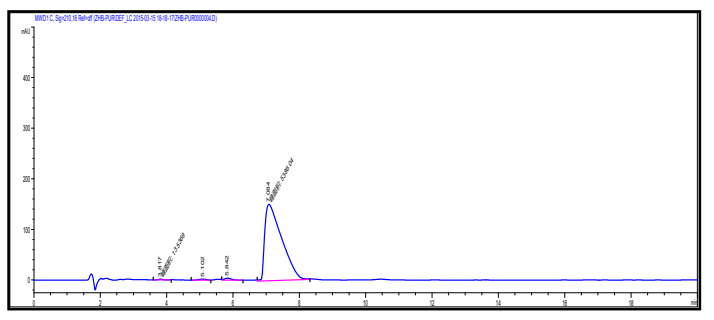

Figure S7. HPLC analysis of 22b, 98.1\%. Mobile phase: Methanol-Water( $0.5 \%$ Triethylamine) $(65: 25, \mathrm{v} / \mathrm{v})$.

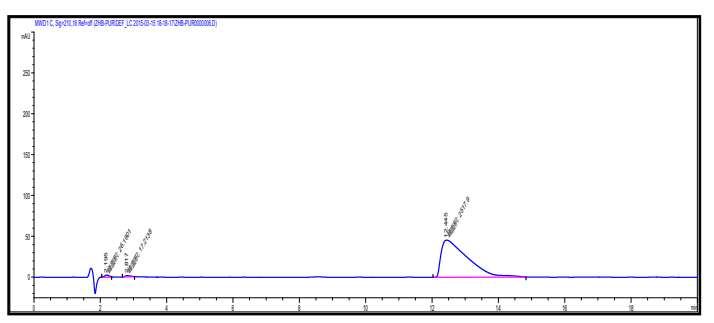

Figure S8. HPLC analysis of 24a, 98.2\%. Mobile phase: Methanol-Water( $0.5 \%$ Triethylamine) $(75: 25, \mathrm{v} / \mathrm{v})$;

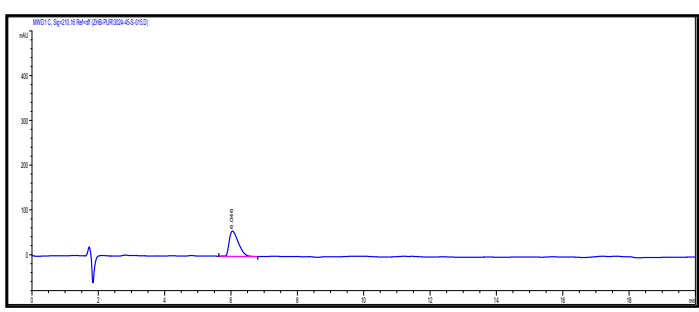

Figure S9. HPLC analysis of 24b, 100\%. Mobile phase: Methanol-Water( $0.5 \%$ Triethylamine) $(75: 25, \mathrm{v} / \mathrm{v})$.

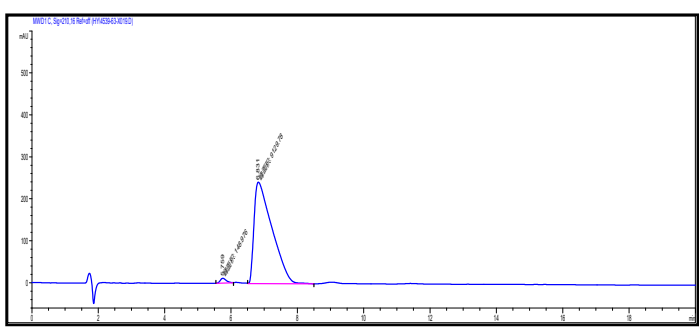

Figure S10. HPLC analysis of 31, 98.4\%. Mobile phase: Methanol-Water( $0.5 \%$ Triethylamine) $(65: 35, \mathrm{v} / \mathrm{v})$; 


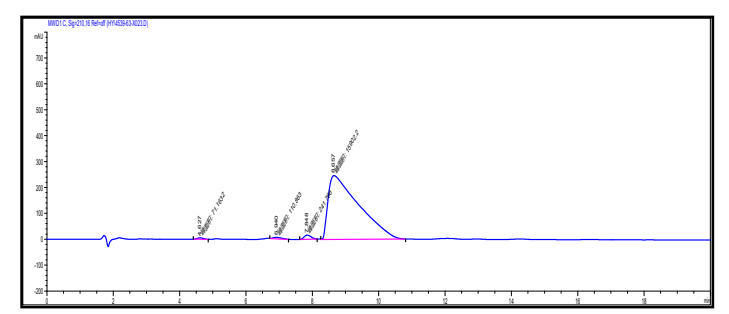

Figure S11. HPLC analysis of 33, 97.4\%. Mobile phase: Methanol-Water(0.5\% Triethylamine) (75:25, v/v);

Analytical data for products.

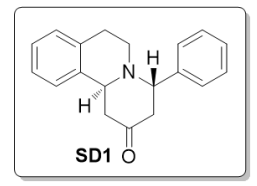

trans-4-Phenyl-3,4,6,7-tetrahydro-1H-pyrido[2,1-a]isoquinolin-2(11bH)-one (SD1). Colorless oil. ${ }^{1} \mathrm{H}$ NMR (400 $\left.\mathrm{MHz}, \mathrm{CDCl}_{3}\right) \delta$ 7.46-7.29 (m, 4H), 7.29-7.23 (m, 1H), 7.20-7.06 (m, 3H), 7.07-6.93 (m, 1H), $4.45(\mathrm{t}, J=5.0 \mathrm{~Hz}$, $1 \mathrm{H}), 4.17(\mathrm{dd}, J=9.7,4.0 \mathrm{~Hz}, 1 \mathrm{H}), 3.36-3.13(\mathrm{~m}, 1 \mathrm{H}), 3.11-2.59(\mathrm{~m}, 7 \mathrm{H}) .{ }^{13} \mathrm{C} \mathrm{NMR}\left(101 \mathrm{MHz}, \mathrm{CDCl}_{3}\right) \delta 209.0$, 139.5, 136.9, 134.1, 129.1, 128.4, 128.2, 127.6, 126.5, 126.2, 126.1, 63.9, 54.5, 46.9, 46.3, 43.8, 29.1. ESI-MS m/z $278[\mathrm{M}+\mathrm{H}]^{+}$, HR-ESI-MS: m/z $278.1538[\mathrm{M}+\mathrm{H}]^{+}$, (calcd for $\left.\mathrm{C}_{19} \mathrm{H}_{20} \mathrm{NO}^{+}, 278.1539\right)$.

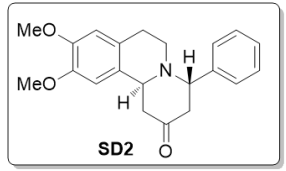

trans-9,10-Dimethoxy-4-phenyl-3,4,6,7-tetrahydro-1H-pyrido[2,1-a]isoquinolin-2(11bH)-one (SD2). White solid, mp 101-102 ${ }^{\circ} \mathrm{C} .{ }^{1} \mathrm{H}$ NMR (600 MHz, $\left.\mathrm{CDCl}_{3}\right) \delta$ 7.41-7.32 (m, 4H), 7.31-7.28 (m, 1H), $6.62(\mathrm{~s}, 1 \mathrm{H}), 6.49$ (s, $1 \mathrm{H}), 4.48(\mathrm{~s}, 1 \mathrm{H}), 4.11(\mathrm{~d}, J=9.7 \mathrm{~Hz}, 1 \mathrm{H}), 3.87(\mathrm{~s}, 3 \mathrm{H}), 3.82(\mathrm{~s}, 3 \mathrm{H}), 3.34-3.24(\mathrm{~m}, 1 \mathrm{H}), 3.02-2.83(\mathrm{~m}, 5 \mathrm{H})$, 2.82-2.70 (m, 2H). ${ }^{13} \mathrm{C}$ NMR (151 MHz, $\left.\mathrm{CDCl}_{3}\right) \delta$ 209.2, 147.7, 147.4, 139.5, 128.7, 128.4, 128.2, 127.6, 126.1, 111.5, 108.9, 64.0, 55.9, 55.9, 54.2, 467.0, 46.6, 43.6, 28.7. ESI-MS m/z $338[\mathrm{M}+\mathrm{H}]^{+}$, HR-ESI-MS: m/z 338.1754 [M + H] $]^{+}$( calcd for $\left.\mathrm{C}_{21} \mathrm{H}_{24} \mathrm{NO}_{3}{ }^{+}, 338.1751\right)$.

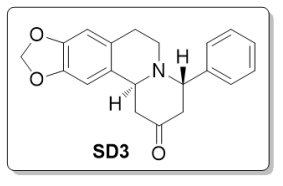

trans-4-Phenyl-3,4,6,7-tetrahydro- $1 H$-[1,3]dioxolo[4,5-g]pyrido[2,1-a]isoquinolin-2(12bH)-one (SD3). Light yellow crystal, mp 156-158 ${ }^{\circ} \mathrm{C} .{ }^{1} \mathrm{H}$ NMR $\left(400 \mathrm{MHz}, \mathrm{CDCl}_{3}\right) \delta 7.46-7.27(\mathrm{~m}, 5 \mathrm{H}), 6.58(\mathrm{~s}, 1 \mathrm{H}), 6.47(\mathrm{~s}, 1 \mathrm{H}), 5.89$ $(\mathrm{dd}, J=4.2,1.3 \mathrm{~Hz}, 2 \mathrm{H}), 4.44(\mathrm{t}, J=5.1 \mathrm{~Hz}, 1 \mathrm{H}), 4.08(\mathrm{dd}, J=9.2,4.2 \mathrm{~Hz}, 1 \mathrm{H}), 3.30-3.16(\mathrm{~m}, 1 \mathrm{H}), 2.97-2.61(\mathrm{~m}$, 7H). ${ }^{13} \mathrm{C}$ NMR $\left(101 \mathrm{MHz}, \mathrm{CDCl}_{3}\right) \delta$ 209.0, 146.5, 146.3, 139.7, 129.9, 128.6, 128.3, 127.8, 127.5, 108.8, 106.3, 101.0, 63.9, 54.9, 47.1, 46.6, 44.0, 29.2. ESI-MS m/z $344[\mathrm{M}+\mathrm{N} a]^{+}$, HR-ESI-MS: m/z $322.1440[\mathrm{M}+\mathrm{H}]^{+}$, (calcd for $\left.\mathrm{C}_{20} \mathrm{H}_{20} \mathrm{NO}_{3}{ }^{+}, 322.1438\right)$.

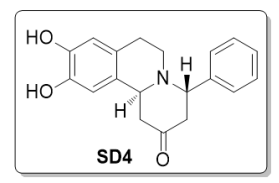

trans-9,10-Dihydroxy-4-phenyl-3,4,6,7-tetrahydro-1 $H$-pyrido[2,1- $a]$ isoquinolin-2(11bH)-one (SD4). Yellow oil. ${ }^{1} \mathrm{H}$ NMR (400 MHz, Acetone) $\delta 7.74(\mathrm{~s}, 2 \mathrm{H}), 7.43-7.37$ (m, 2H), 7.37-7.30 (m, 2H), 7.30-7.21 (m, 1H), 6.57 (s, 1H), $6.52(\mathrm{~s}, 1 \mathrm{H}), 4.52-4.40(\mathrm{~m}, 1 \mathrm{H}), 4.02(\mathrm{dd}, J=9.5,3.9 \mathrm{~Hz}, 1 \mathrm{H}), 3.41-3.17(\mathrm{~m}, 1 \mathrm{H}), 2.88-2.50(\mathrm{~m}, 7 \mathrm{H}) .{ }^{13} \mathrm{C}$ NMR (101 MHz, Acetone) $\delta$ 207.3, 143.8, 143.3, 140.9, 128.4, 128.1, 128.0, 127.1, 125.4, 115.1, 112.8, 63.4, 54.5, 46.6, 45.8, 43.2,28.1. ESI-MS m/z $310[\mathrm{M}+\mathrm{H}]^{+}$, HR-ESI-MS: m/z $310.1444[\mathrm{M}+\mathrm{H}]^{+}$, (calcd for $\mathrm{C}_{19} \mathrm{H}_{20} \mathrm{NO}_{3}{ }^{+}, 310.1438$ ).

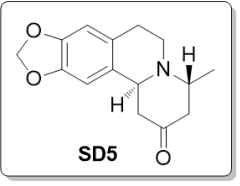

trans-4-Methyl-3,4,6,7-tetrahydro-1 $H$-[1,3]dioxolo[4,5-g]pyrido[2,1-a]isoquinolin-2(12bH)-one (SD5). White solid, mp 131-133 ${ }^{\circ} \mathrm{C} .{ }^{1} \mathrm{H}$ NMR $\left(400 \mathrm{MHz}, \mathrm{CDCl}_{3}\right) \delta 6.59(\mathrm{~s}, 1 \mathrm{H}), 6.52(\mathrm{~s}, 1 \mathrm{H}), 5.91(\mathrm{~s}, 2 \mathrm{H}), 4.13(\mathrm{dd}, J=11.1,3.3$ $\mathrm{Hz}, 1 \mathrm{H}), 3.63-3.50(\mathrm{~m}, 1 \mathrm{H}), 3.17-3.07(\mathrm{~m}, 1 \mathrm{H}), 2.98-2.90(\mathrm{~m}, 1 \mathrm{H}), 2.88-2.78(\mathrm{~m}, 3 \mathrm{H}), 2.65$ (ddd, $J=14.1,3.5,2.1$ $\mathrm{Hz}, 1 \mathrm{H}), 2.50(\mathrm{dd}, J=13.8,11.4 \mathrm{~Hz}, 1 \mathrm{H}), 2.24(\mathrm{dt}, J=13.6,2.0 \mathrm{~Hz}, 1 \mathrm{H}), 1.13(\mathrm{~d}, J=7.0 \mathrm{~Hz}, 3 \mathrm{H}) .{ }^{13} \mathrm{C} \mathrm{NMR}\left(101 \mathrm{MHz}, \mathrm{CDCl}_{3}\right) \delta 209.2$, 146.5, 146.3, 130.4, 127.5, 108.8, 105.9, 101.0, 57.6, 54.5, 47.5, 47.2, 46.4, 30.1, 15.2. ESI-MS m/z 260 [M + H] $]^{+}$HR-ESI-MS: m/z $260.1283[\mathrm{M}+\mathrm{H}]^{+}$, (calcd for $\left.\mathrm{C}_{15} \mathrm{H}_{18} \mathrm{NO}_{3}{ }^{+}, 260.1281\right)$.

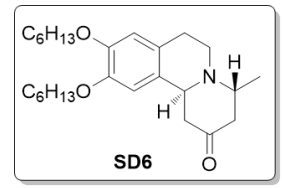

trans-9,10-Bis(hexyloxy)-4-methyl-3,4,6,7-tetrahydro- $1 H$-pyrido[2,1-a]isoquinolin-2(11b $H$ )-one

Yellow powder, mp 76-78 ${ }^{\circ} \mathrm{C} .{ }^{1} \mathrm{H}$ NMR $\left(400 \mathrm{MHz}, \mathrm{CDCl}_{3}\right) \delta 6.54(\mathrm{~s}, 1 \mathrm{H}), 6.48(\mathrm{~s}, 1 \mathrm{H}), 4.12-4.01(\mathrm{~m}, 1 \mathrm{H})$, $3.95-3.76(\mathrm{~m}, 4 \mathrm{H}), 3.66-3.32(\mathrm{~m}, 1 \mathrm{H}), 3.15-2.96(\mathrm{~m}, 1 \mathrm{H}), 2.95-2.66(\mathrm{~m}, 4 \mathrm{H}), 2.65-2.54(\mathrm{~m}, 1 \mathrm{H}), 2.44(\mathrm{dd}, J=$ 
13.6, $11.5 \mathrm{~Hz}, 1 \mathrm{H}), 2.22-2.10(\mathrm{~m}, 1 \mathrm{H}), 1.78-1.66(\mathrm{~m}, 4 \mathrm{H}), 1.46-1.34(\mathrm{~m}, 4 \mathrm{H}), 1.31-1.20(\mathrm{~m}, 8 \mathrm{H}), 1.05(\mathrm{~d}, J=6.8 \mathrm{~Hz}, 3 \mathrm{H}), 0.83(\mathrm{t}, J=6.2$ $\mathrm{Hz}, 6 \mathrm{H}) .{ }^{13} \mathrm{C} \mathrm{NMR}\left(101 \mathrm{MHz}, \mathrm{CDCl}_{3}\right) \delta 209.6,148.2,147.8,129.6,126.5,114.2,111.7,69.8,69.5,57.8,54.1,47.6,47.4,46.5,31.8,29.7$, 29.5, 29.5, 25.9, 25.9, 22.8, 15.4, 14.2. ESI-MS m/z $416[\mathrm{M}+\mathrm{H}]^{+}$, HR-ESI-MS: m/z $416.3159\left[\mathrm{M}+\mathrm{H}^{+},\left(\right.\right.$calcd for $\mathrm{C}_{26} \mathrm{H}_{42} \mathrm{NO}_{3}{ }^{+}$, 416.3159).

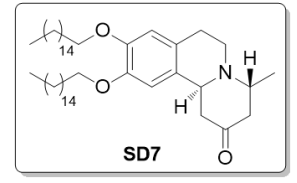

trans-9,10-Bis(hexadecyloxy)-4-methyl-3,4,6,7-tetrahydro-1H-pyrido[2,1-a]isoquinolin-2(11bH)-one (SD7). white solid, mp 80-81 ${ }^{\circ} \mathrm{C} .{ }^{1} \mathrm{H}$ NMR $\left(400 \mathrm{MHz}, \mathrm{CDCl}_{3}\right) \delta 6.54(\mathrm{~s}, 1 \mathrm{H}), 6.47$ (s, 1H), 4.14-3.99 (m, 1H), 3.97-3.79 $(\mathrm{m}, 4 \mathrm{H}), 3.59-3.44(\mathrm{~m}, 1 \mathrm{H}), 3.11-2.96(\mathrm{~m}, 1 \mathrm{H}), 2.92-2.67(\mathrm{~m}, 4 \mathrm{H}), 2.66-2.57(\mathrm{~m}, 1 \mathrm{H}), 2.51-2.37(\mathrm{~m}, 1 \mathrm{H})$, 2.24-2.12 (m, 1H), 1.79-1.62 (m, 4H), 1.46-1.33 (m, 4H), 1.29-1.14 (m, 48H), $1.06(\mathrm{~d}, J=6.8 \mathrm{~Hz}, 3 \mathrm{H}), 0.81(\mathrm{t}, J=6.7 \mathrm{~Hz}, 6 \mathrm{H}) .{ }^{13} \mathrm{C} \mathrm{NMR}$ $\left(101 \mathrm{MHz}, \mathrm{CDCl}_{3}\right) \delta 209.4,148.0,147.6,129.4,126.4,114.1,111.6,69.6,69.4,57.6,54.0,47.5,47.2,46.3,31.9,29.7,29.7,29.7,29.5$,

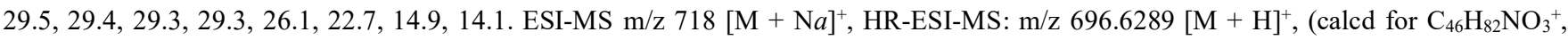
696.6289).

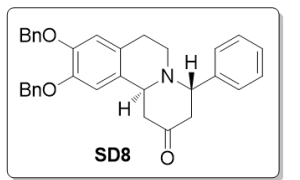

trans-9,10-Bis(benzyloxy)-4-phenyl-3,4,6,7-tetrahydro-1 $H$-pyrido[2,1-a]isoquinolin-2(11bH)-one

(SD8). Colorless crystal, mp 146-148 ${ }^{\circ} \mathrm{C} .{ }^{1} \mathrm{H}$ NMR $\left(600 \mathrm{MHz}, \mathrm{CDCl}_{3}\right) \delta$ 7.46-7.41 (m, 4H), 7.39-7.27 (m, 11H), 6.69 (s, $1 \mathrm{H}), 6.58(\mathrm{~s}, 1 \mathrm{H}), 5.13(\mathrm{~s}, 2 \mathrm{H}), 5.07(\mathrm{~s}, 2 \mathrm{H}), 4.44(\mathrm{t}, J=4.9 \mathrm{~Hz}, 1 \mathrm{H}), 4.06(\mathrm{dd}, J=9.5,4.0 \mathrm{~Hz}, 1 \mathrm{H}), 3.29-3.16(\mathrm{~m}$, $1 \mathrm{H}), 2.93-2.85(\mathrm{~m}, 3 \mathrm{H}), 2.85-2.78(\mathrm{~m}, 2 \mathrm{H}), 2.71-2.58(\mathrm{~m}, 2 \mathrm{H}) .{ }^{13} \mathrm{C} \mathrm{NMR}\left(101 \mathrm{MHz}, \mathrm{CDCl}_{3}\right) \delta 208.9,147.9,147.4,139.5,137.3,137.3$, 129.7, 128.5, 128.4, 128.1, 127.8, 127.8, 127.5, 127.4, 127.3, 127.2, 115.4, 113.3, 71.7, 71.4, 63.9, 54.2, 47.0, 46.4, 43.8, 28.6. ESI-MS m/z $490[\mathrm{M}+\mathrm{H}]^{+}$, HR-ESI-MS: $\mathrm{m} / \mathrm{z} 490.2377[\mathrm{M}+\mathrm{H}]^{+}$, (calcd for $\left.\mathrm{C}_{33} \mathrm{H}_{32} \mathrm{NO}_{3}{ }^{+}, 490.2375\right)$.

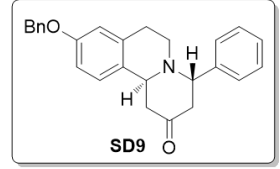

trans-9-(Benzyloxy)-4-phenyl-3,4,6,7-tetrahydro-1H-pyrido[2,1-a]isoquinolin-2(11bH)-one（SD9). White crystyl, mp $129-131^{\circ} \mathrm{C} .{ }^{1} \mathrm{H}$ NMR $\left(400 \mathrm{MHz}, \mathrm{CDCl}_{3}\right) \delta 7.48-7.25(\mathrm{~m}, 10 \mathrm{H}), 6.95(\mathrm{~d}, J=8.6 \mathrm{~Hz}, 1 \mathrm{H}), 6.84-6.70(\mathrm{~m}$, 2H), $5.04(\mathrm{~s}, 2 \mathrm{H}), 4.43(\mathrm{t}, J=5.1 \mathrm{~Hz}, 1 \mathrm{H}), 4.17(\mathrm{dd}, J=9.2,4.2 \mathrm{~Hz}, 1 \mathrm{H}), 3.37-3.21(\mathrm{~m}, 1 \mathrm{H}), 3.12-2.93(\mathrm{~m}, 1 \mathrm{H})$, 2.93-2.68 (m, 6H). ${ }^{13} \mathrm{C}$ NMR (101 MHz, $\left.\mathrm{CDCl}_{3}\right) \delta 209.0,157.3,139.6,137.1,135.5,129.4,128.6,128.4,128.1,128.0,127.6,127.4,127.2$, 114.8, 113.2, 70.0, 63.6, 54.4, 46.9, 46.4, 44.1, 29.1. ESI-MS m/z $384[\mathrm{M}+\mathrm{H}]^{+}$, HR-ESI-MS: m/z $384.1969[\mathrm{M}+\mathrm{H}]^{+}$, (calcd for $\left.\mathrm{C}_{26} \mathrm{H}_{26} \mathrm{NO}_{2}^{+}, 384.1958\right)$.

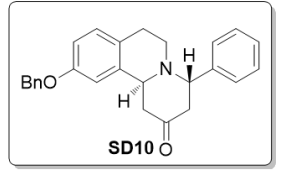

trans-10-(Benzyloxy)-4-phenyl-3,4,6,7-tetrahydro-1H-pyrido[2,1-a]isoquinolin-2(11bH)-one (SD10). White powder, $146-148^{\circ} \mathrm{C} .{ }^{1} \mathrm{H}$ NMR $\left(400 \mathrm{MHz}, \mathrm{CDCl}_{3}\right) \delta 7.63-7.18(\mathrm{~m}, 10 \mathrm{H}), 7.05(\mathrm{~d}, J=8.4 \mathrm{~Hz}, 1 \mathrm{H}), 6.81(\mathrm{dd}, J=8.4$, $2.3 \mathrm{~Hz}, 1 \mathrm{H}), 6.64(\mathrm{~d}, J=1.5 \mathrm{~Hz}, 1 \mathrm{H}), 5.00(\mathrm{~s}, 2 \mathrm{H}), 4.54-4.33(\mathrm{~m}, 1 \mathrm{H}), 4.15(\mathrm{~d}, J=3.1 \mathrm{~Hz}, 1 \mathrm{H}), 3.36-3.17(\mathrm{~m}, 1 \mathrm{H})$, 3.14-2.42 (m, 7H). ${ }^{13} \mathrm{C}$ NMR (101 MHz, $\left.\mathrm{CDCl}_{3}\right) \delta 209.0,157.3,139.6,138.1,137.2,130.2,128.8,128.6,128.3,128.1,127.8,127.6,126.6$, 114.1, 112.3, 70.3, 64.0, 54.9, 47.3, 46.5, 44.0, 28.4. ESI-MS m/z $384[\mathrm{M}+\mathrm{H}]^{+}$, HR-ESI-MS: m/z $384.1966[\mathrm{M}+\mathrm{H}]^{+}$, (calcd for $\left.\mathrm{C}_{26} \mathrm{H}_{26} \mathrm{NO}_{2}{ }^{+}, 384.1958\right)$.

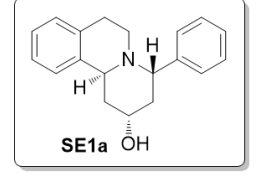

4 $\alpha$-Phenyl-2,3,4,6,7,11b $\alpha$-hexahydro-1 $H$-pyrido[2,1- $a$ ]isoquinolin-2 $\alpha$-ol (SE1a). White solid, mp $126-127{ }^{\circ} \mathrm{C} .{ }^{1} \mathrm{H}$ NMR $\left(400 \mathrm{MHz}, \mathrm{CDCl}_{3}\right) \delta 7.56(\mathrm{~d}, J=7.8 \mathrm{~Hz}, 2 \mathrm{H}), 7.34(\mathrm{t}, J=7.5 \mathrm{~Hz}, 2 \mathrm{H}), 7.24(\mathrm{~d}, J=7.3 \mathrm{~Hz}, 1 \mathrm{H}), 7.21-7.10$ (m, 3H), 7.13-7.06 (m, 1H), $4.26(\mathrm{t}, J=4.3 \mathrm{~Hz}, 1 \mathrm{H}), 4.06$ (ddd, $J=18.8,9.4,3.9 \mathrm{~Hz}, 2 \mathrm{H}), 3.41-3.26$ (m, 1H), 3.10-2.95 (m, 1H), 2.95-2.81 (m, 2H), $2.39(\mathrm{dt}, J=13.2,3.3 \mathrm{~Hz}, 1 \mathrm{H}), 2.07(\mathrm{dt}, J=12.6,3.3 \mathrm{~Hz}, 1 \mathrm{H}), 2.03-1.87(\mathrm{~m}, 2 \mathrm{H}) .{ }^{13} \mathrm{C} \mathrm{NMR}(101 \mathrm{MHz}$, $\left.\mathrm{CDCl}_{3}\right) \delta 142.0,138.7,134.9,129.4,128.6,127.5,126.9,126.8,126.4,126.0, \quad 66.5,61.2,53.6,46.3,37.8,34.4,29.2$. ESI-MS m/z 280 $[\mathrm{M}+\mathrm{H}]^{+}$, HR-ESI-MS: m/z $280.1695[\mathrm{M}+\mathrm{H}]^{+}$, (calcd for $\left.\mathrm{C}_{19} \mathrm{H}_{22} \mathrm{NO}^{+}, 280.1696\right)$.

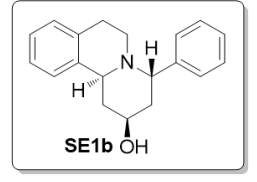

4 $\alpha$-Phenyl-2,3,4,6,7,11b $\alpha$-hexahydro-1 $H$-pyrido[2,1-a]isoquinolin-2 $\beta$-ol (SE1b). White powder, mp $149-151{ }^{\circ} \mathrm{C}$. ${ }^{1} \mathrm{H}$ NMR $\left(400 \mathrm{MHz}, \mathrm{CDCl}_{3}\right) \delta$ 7.48-7.16 (m, 8H), $7.11(\mathrm{~d}, J=6.8 \mathrm{~Hz}, 1 \mathrm{H}), 4.59(\mathrm{~s}, 1 \mathrm{H}), 3.86-3.52(\mathrm{~m}, 2 \mathrm{H})$, $3.18-2.67(\mathrm{~m}, 4 \mathrm{H}), 2.44-2.24(\mathrm{~m}, 1 \mathrm{H}), 2.13-1.88(\mathrm{~m}, 2 \mathrm{H}), 1.69(\mathrm{q}, J=11.5 \mathrm{~Hz}, 1 \mathrm{H}) .{ }^{13} \mathrm{C} \mathrm{NMR}\left(101 \mathrm{MHz}, \mathrm{CDCl}_{3}\right) \delta$ 
143.4, 1364, 135.4, 129.7, 128.9, 127.6, 127.6, 126.8, 126.6, 125.7, 65.5, 58.2, 57.5, 46.7, 45.3, 37.1, 23.2. ESI-MS m/z 280 [M + H] ${ }^{+}$, HR-ESI-MS: m/z $280.1696[\mathrm{M}+\mathrm{H}]^{+}$, (calcd for $\left.\mathrm{C}_{19} \mathrm{H}_{22} \mathrm{NO}^{+}, 280.1696\right)$.

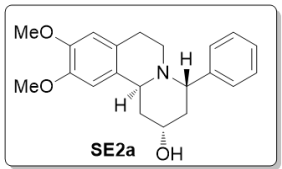

9,10-Dimethoxy-4 $\alpha$-phenyl-2,3,4,6,7,11b $\alpha$-hexahydro-1 $H$-pyrido[2,1- $a]$ isoquinolin-2 $\alpha$-ol(SE2a). Yellow oil. ${ }^{1} \mathrm{H}$ NMR $\left(400 \mathrm{MHz}, \mathrm{CDCl}_{3}\right) \delta 7.56(\mathrm{~d}, J=7.7 \mathrm{~Hz}, 2 \mathrm{H}), 7.34(\mathrm{t}, J=7.5 \mathrm{~Hz}, 2 \mathrm{H}), 7.30-7.20(\mathrm{~m}, 1 \mathrm{H}), 6.63(\mathrm{~s}, 1 \mathrm{H})$, $6.58(\mathrm{~s}, 1 \mathrm{H}), 4.25(\mathrm{t}, J=4.2 \mathrm{~Hz}, 1 \mathrm{H}), 4.09-3.96(\mathrm{~m}, 2 \mathrm{H}), 3.87(\mathrm{~s}, 3 \mathrm{H}), 3.82(\mathrm{~s}, 3 \mathrm{H}), 3.38-3.21(\mathrm{~m}, 1 \mathrm{H}), 2.99-2.78$ $(\mathrm{m}, 3 \mathrm{H}), 2.48-2.28(\mathrm{~m}, 1 \mathrm{H}), 2.14-2.02(\mathrm{~m}, 1 \mathrm{H}), 2.02-1.83(\mathrm{~m}, 2 \mathrm{H}) .{ }^{13} \mathrm{C}$ NMR $\left(101 \mathrm{MHz}, \mathrm{CDCl}_{3}\right) \delta 147.6,147.4,141.9,130.3,128.4$, 127.3, 126.7, 126.6, 111.8, 109.8, 66.2, 61.0, 56.0, 55.9, 53.1, 46.2, 37.7, 34.4, 28.6, 28.4. ESI-MS m/z 340 [M + H] , HR-ESI-MS: m/z $340.1910[\mathrm{M}+\mathrm{H}]^{+}$, (calcd for $\left.\mathrm{C}_{21} \mathrm{H}_{26} \mathrm{NO}_{3}{ }^{+}, 340.1907\right)$.

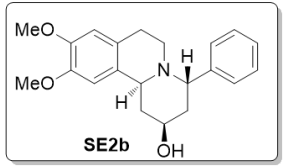

9,10-Dimethoxy-4 $\alpha$-phenyl-2,3,4,6,7,11b $\alpha$-hexahydro-1H-pyrido[2,1-a]isoquinolin-2 $\beta$-ol (SE2b). Yellow crystal, mp 191-193 ${ }^{\circ} \mathrm{C} .{ }^{1} \mathrm{H}$ NMR $\left(400 \mathrm{MHz}, \mathrm{CDCl}_{3}\right) \delta$ 7.50-7.20 (m, 5H), $6.85(\mathrm{~s}, 1 \mathrm{H}), 6.59(\mathrm{~s}, 1 \mathrm{H}), 4.51(\mathrm{~s}, 1 \mathrm{H})$, $3.86(\mathrm{~s}, 6 \mathrm{H}), 3.79-3.64(\mathrm{~m}, 2 \mathrm{H}), 3.03-2.81(\mathrm{~m}, 3 \mathrm{H}), 2.81-2.64(\mathrm{~m}, 1 \mathrm{H}), 2.25(\mathrm{~d}, J=16.0 \mathrm{~Hz}, 1 \mathrm{H}), 2.19$ (brs, $0 \mathrm{H})$, $2.04(\mathrm{td}, J=12.7,4.9 \mathrm{~Hz}, 2 \mathrm{H}), 1.96(\mathrm{~d}, J=10.6 \mathrm{~Hz}, 1 \mathrm{H}), 1.67(\mathrm{q}, J=11.5 \mathrm{~Hz}, 1 \mathrm{H}) .{ }^{13} \mathrm{C} \mathrm{NMR}\left(101 \mathrm{MHz}, \mathrm{CDCl}_{3}\right) \delta 147.8,147.7,143.3$, 128.6, 128.0, 127.5, 127.4, 112.2, 109.0, 65.2, 57.7, 57.2, 56.3, 55.9, 46.6, 45.1, 37.1, 22.6. ESI-MS m/z 340 [M + H] $]^{+}$HR-ESI-MS: m/z $340.1911[\mathrm{M}+\mathrm{H}]^{+}$, (calcd for $\left.\mathrm{C}_{21} \mathrm{H}_{26} \mathrm{NO}_{3}{ }^{+}, 340.1907\right)$.

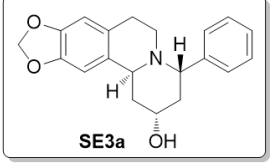

4 $\alpha$-Phenyl-2,3,4,6,7,12b $\alpha$-hexahydro-1 $H$-[1,3]dioxolo[4,5-g]pyrido[2,1-a]isoquinolin-2 $\alpha$-ol (SE3a). Whitel solid, mp 177-178 ${ }^{\circ} \mathrm{C} .{ }^{1} \mathrm{H}$ NMR $\left(400 \mathrm{MHz}, \mathrm{CDCl}_{3}\right) \delta$ 7.67-7.46 (m, 2H), 7.44-7.28 (m, 2H), 7.28-7.19 (m, 1H), $6.60(\mathrm{~s}, 1 \mathrm{H}), 6.56(\mathrm{~s}, 1 \mathrm{H}), 5.90(\mathrm{~s}, 2 \mathrm{H}), 4.25(\mathrm{~s}, 1 \mathrm{H}), 4.13-3.83(\mathrm{~m}, 1 \mathrm{H}), 3.32-3.18(\mathrm{~m}, 1 \mathrm{H}), 3.01-2.64(\mathrm{~m}, 3 \mathrm{H})$, 2.43-2.29 (m, 1H), 2.10-1.84 (m, 3H). ${ }^{13} \mathrm{C}$ NMR (101 MHz, $\left.\mathrm{CDCl}_{3}\right) \delta 146.0,145.9,141.8,131.3,128.5,127.7,127.3,126.7,108.7,106.6$,

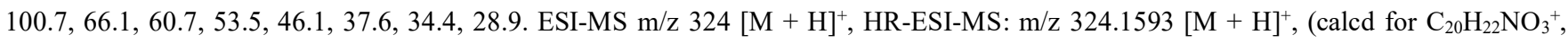
324.1594).

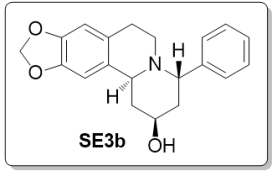

4 $\alpha$-Phenyl-2,3,4,6,7,12b $\alpha$-hexahydro-1 $H$-[1,3]dioxolo[4,5-g]pyrido[2,1-a]isoquinolin-2 $\beta$-ol (SE3b). White powder, mp 190-192 ${ }^{\circ} \mathrm{C} .{ }^{1} \mathrm{H}$ NMR (400 MHz, DMSO) $\delta$ 7.52-7.28 (m, 4H), 7.29-7.18 (m, 1H), $6.92(\mathrm{~s}, 1 \mathrm{H}), 6.64$ (s, 1H), $5.96(\mathrm{~d}, J=3.3 \mathrm{~Hz}, 2 \mathrm{H}), 4.63(\mathrm{~d}, J=4.5 \mathrm{~Hz}, 1 \mathrm{H}), 4.32(\mathrm{~s}, 1 \mathrm{H}), 3.55(\mathrm{dd}, J=11.4,2.4 \mathrm{~Hz}, 1 \mathrm{H}), 3.47-3.36$ $(\mathrm{m}, 1 \mathrm{H}), 2.89-2.56(\mathrm{~m}, 4 \mathrm{H}), 2.26-2.11(\mathrm{~m}, 1 \mathrm{H}), 1.82-1.64(\mathrm{~m}, 2 \mathrm{H}), 1.40(\mathrm{dd}, J=23.5,12.0 \mathrm{~Hz}, 1 \mathrm{H}) .{ }^{13} \mathrm{C} \mathrm{NMR}(101 \mathrm{MHz}, \mathrm{DMSO}) \delta 146.4$, 145.8, 144.2, 129.9, 128.8, 128.2, 127.6, 127.5, 109.3, 106.0, 100.9, 63.8, 57.7, 57.1, 46.4, 45.6, 37.6, 23.0. ESI-MS m/z 324 [M + H] $]^{+}$ HR-ESI-MS: m/z $324.1594[\mathrm{M}+\mathrm{H}]^{+}$, (calcd for $\left.\mathrm{C}_{20} \mathrm{H}_{22} \mathrm{NO}_{3}{ }^{+}, 324.1594\right)$.

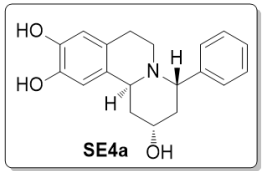

4 $\alpha$-Phenyl-2,3,4,6,7,11b $\alpha$-hexahydro-1 $H$-pyrido[2,1- $a$ ]isoquinoline-2 $\alpha, 9,10$-triol (SE4a). Orange oil. ${ }^{1} \mathrm{H}$ NMR (400 MHz, MeOD) $\delta 7.59-7.39(\mathrm{~m}, 2 \mathrm{H}), 7.36-7.29(\mathrm{~m}, 2 \mathrm{H}), 7.26-7.21(\mathrm{~m}, 1 \mathrm{H}), 6.52(\mathrm{~s}, 1 \mathrm{H}), 6.50(\mathrm{~s}, 1 \mathrm{H}), 4.27$ (t, $J$ $=4.1 \mathrm{~Hz}, 1 \mathrm{H}), 4.08-3.94(\mathrm{~m}, 2 \mathrm{H}), 3.26-3.12(\mathrm{~m}, 1 \mathrm{H}), 2.80-2.71(\mathrm{~m}, 3 \mathrm{H}), 2.32(\mathrm{dt}, J=10.0,3.3 \mathrm{~Hz}, 1 \mathrm{H}), 2.08(\mathrm{dt}, J$ $=13.1,3.2 \mathrm{~Hz}, 1 \mathrm{H}), 1.98-1.88(\mathrm{~m}, 1 \mathrm{H}), 1.87-1.75(\mathrm{~m}, 1 \mathrm{H}) .{ }^{13} \mathrm{C} \mathrm{NMR}(101 \mathrm{MHz}, \mathrm{MeOD}) \delta 145.0,144.6,142.2,129.5,129.4,128.8,128.0$, 126.1, 116.1, 114.2, 66.1, 63.2, 54.9, 48.0, 38.7, 35.8, 29.1. ESI-MS m/z $312[\mathrm{M}+\mathrm{H}]^{+}$, HR-ESI-MS: m/z 312.1595[M + H $]^{+}$, (calcd for $\left.\mathrm{C}_{19} \mathrm{H}_{22} \mathrm{NO}_{3}^{+}, 312.1594\right)$.

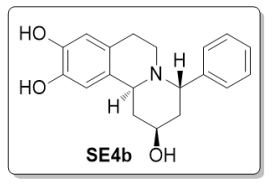

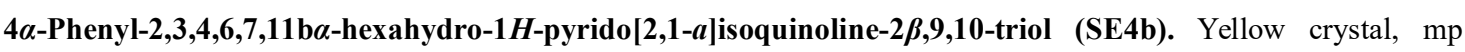
157-159 ${ }^{\circ} \mathrm{C} .{ }^{1} \mathrm{H}$ NMR $(400 \mathrm{MHz}, \mathrm{MeOD}) \delta 7.56-7.28(\mathrm{~m}, 5 \mathrm{H}), 6.85(\mathrm{~s}, 1 \mathrm{H}), 6.59(\mathrm{~s}, 1 \mathrm{H}), 4.58(\mathrm{~s}, 1 \mathrm{H}), 3.89(\mathrm{~d}, J=$ $11.9 \mathrm{~Hz}, 1 \mathrm{H}), 3.82-3.67(\mathrm{~m}, 1 \mathrm{H}), 3.15-2.97(\mathrm{~m}, 1 \mathrm{H}), 2.97-2.82(\mathrm{~m}, 2 \mathrm{H}), 2.82-2.73(\mathrm{~m}, 1 \mathrm{H}), 2.41-2.26(\mathrm{~m}, 1 \mathrm{H})$, 2.10-1.92 (m, 2H), $1.74(\mathrm{dd}, J=23.7,11.9 \mathrm{~Hz}, 1 \mathrm{H}) .{ }^{13} \mathrm{C}$ NMR (101 MHz, MeOD) $\delta$ 145.6, 145.6, 142.0, 130.0, 129.2, 128.6, 125.8, 125.6, 116.9, 113.3, 64.9, 59.6, 59.0, 47.9, 44.8, 37.1, 22.9. ESI-MS m/z $312[\mathrm{M}+\mathrm{H}]^{+}$, HR-ESI-MS: m/z $312.1596[\mathrm{M}+\mathrm{H}]^{+}$, (calcd for $\left.\mathrm{C}_{19} \mathrm{H}_{22} \mathrm{NO}_{3}{ }^{+}, 312.1594\right)$ 


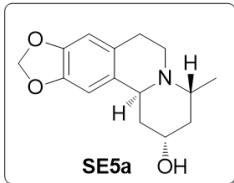

4 $\alpha$-Methyl-2,3,4,6,7,12b $\alpha$-hexahydro-1 $H$-[1,3]dioxolo[4,5-g]pyrido[2,1-a]isoquinolin-2 $\alpha$-ol (SE5a). White solid, mp 155-157 ${ }^{\circ} \mathrm{C} .{ }^{1} \mathrm{H}$ NMR $\left(400 \mathrm{MHz}, \mathrm{CDCl}_{3}\right) \delta 6.56(\mathrm{~s}, 1 \mathrm{H}), 6.48(\mathrm{~s}, 1 \mathrm{H}), 5.82(\mathrm{~s}, 2 \mathrm{H}), 4.07-3.89$ (m, $\left.1 \mathrm{H}\right), 3.70(\mathrm{~d}, J$ $=10.5 \mathrm{~Hz}, 1 \mathrm{H}), 3.44-3.13(\mathrm{~m}, 1 \mathrm{H}), 2.95-2.68(\mathrm{~m}, 2 \mathrm{H}), 2.67-2.51(\mathrm{~m}, 2 \mathrm{H}), 2.26(\mathrm{~m}, 1 \mathrm{H}), 1.85-1.57(\mathrm{~m}, 2 \mathrm{H}), 1.35$ $(\mathrm{dd}, J=23.1,11.5 \mathrm{~Hz}, 1 \mathrm{H}), 1.06(\mathrm{~d}, J=7.0 \mathrm{~Hz}, 2 \mathrm{H}) .{ }^{13} \mathrm{C} \mathrm{NMR}\left(101 \mathrm{MHz}, \mathrm{CDCl}_{3}\right) \delta 145.9,145.8,131.3,127.7,108.5,105.7,100.7,65.8$, 55.6, 52.6, 47.9, 41.4, 39.3, 30.1, 12.7. ESI-MS m/z $262[\mathrm{M}+\mathrm{H}]^{+}$, HR-ESI-MS: m/z 262.1438 [M + $]^{+},\left(\right.$calcd for $\mathrm{C}_{15} \mathrm{H}_{20} \mathrm{NO}_{3}{ }^{+}$, 262.1438).

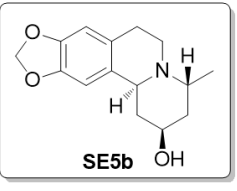

4 $\alpha$-Methyl-2,3,4,6,7,12b $\alpha$-hexahydro-1 $H$-[1,3]dioxolo[4,5-g]pyrido[2,1-a]isoquinolin-2 $\beta$-ol(SE5b). Colorless crystal, mp 134-136 ${ }^{\circ} \mathrm{C} .{ }^{1} \mathrm{H}$ NMR $\left(400 \mathrm{MHz}, \mathrm{CDCl}_{3}\right) \delta 6.76(\mathrm{~s}, 1 \mathrm{H}), 6.55(\mathrm{~s}, 1 \mathrm{H}), 5.90(\mathrm{~s}, 2 \mathrm{H}), 4.31(\mathrm{~s}, 1 \mathrm{H})$, 3.90-3.70 (m, 1H), 3.42-3.23 (m, 1H), 3.13-2.79 (m, 3H), 2.56-2.42 (m, 1H), 2.42-2.31 (m, 1H), 2.00-1.90 (m, $2 \mathrm{H}), 1.51-1.37(\mathrm{~m}, 1 \mathrm{H}), 1.22(\mathrm{~d}, J=6.5 \mathrm{~Hz}, 3 \mathrm{H}) .{ }^{13} \mathrm{C} \mathrm{NMR}\left(101 \mathrm{MHz}, \mathrm{CDCl}_{3}\right) \delta 146.5,146.1,129.9,128.3,109.0,105.8,100.9,65.6,55.9$, 48.5, 47.1, 42.2, 38.2, 25.0, 18.8. ESI-MS m/z $262[\mathrm{M}+\mathrm{H}]^{+}$, HR-ESI-MS: m/z $262.1439[\mathrm{M}+\mathrm{H}]^{+}$, (calcd for $\mathrm{C}_{15} \mathrm{H}_{20} \mathrm{NO}_{3}{ }^{+}, 262.1438$ ).

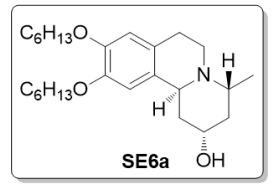

9,10-Bis(hexyloxy)-4 $\alpha$-methyl-2,3,4,6,7,11b $\alpha$-hexahydro-1 $H$-pyrido[2,1- $a]$ isoquinolin-2 $\alpha$-ol (SE6a). Yellow solid, mp 74-76 ${ }^{\circ} \mathrm{C} .{ }^{1} \mathrm{H}$ NMR $\left(400 \mathrm{MHz}, \mathrm{CDCl}_{3}\right) \delta 6.68(\mathrm{~s}, 1 \mathrm{H}), 6.58(\mathrm{~s}, 1 \mathrm{H}), 4.19-4.02(\mathrm{~m}, 1 \mathrm{H}), 4.00-3.88(\mathrm{~m}, 4 \mathrm{H})$, $3.84-3.69(\mathrm{~m}, 1 \mathrm{H}), 3.38-3.27(\mathrm{~m}, 1 \mathrm{H}), 3.12-2.82(\mathrm{~m}, 2 \mathrm{H}), 2.79-2.55(\mathrm{~m}, 2 \mathrm{H}), 2.40(\mathrm{~d}, J=12.1 \mathrm{~Hz}, 1 \mathrm{H}), 1.90-1.68$ $(\mathrm{m}, 7 \mathrm{H}), 1.53-1.41(\mathrm{~m}, 5 \mathrm{H}), 1.38-1.30(\mathrm{~m}, 8 \mathrm{H}), 1.12(\mathrm{~d}, J=7.0 \mathrm{~Hz}, 3 \mathrm{H}), 0.95-0.87(\mathrm{~m}, 6 \mathrm{H}) .{ }^{13} \mathrm{C} \mathrm{NMR}\left(101 \mathrm{MHz}, \mathrm{CDCl}_{3}\right) \delta 147.8,147.5$, 130.6, 127.1, 114.2, 112.0, 69.9, 69.4, 66.1, 55.8, 52.3, 48.4, 41.6, 39.8, 31.8, 31.8, 29.8, 29.6, 29.5, 25.9, 22.8, 22.8, $14.2,14.2,12.7$. ESI-MS m/z $418[\mathrm{M}+\mathrm{H}]^{+}$, HR-ESI-MS: m/z $418.3312[\mathrm{M}+\mathrm{H}]^{+}$, (calcd for $\left.\mathrm{C}_{26} \mathrm{H}_{44} \mathrm{NO}_{3}{ }^{+}, 418.3315\right)$.

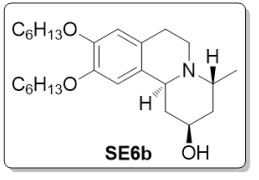

9,10-Bis(hexyloxy)-4 $\alpha$-methyl-2,3,4,6,7,11b $\alpha$-hexahydro- $1 H$-pyrido[2,1-a] isoquinolin-2 $\beta$-ol (SE6b). Yellow oil. ${ }^{1} \mathrm{H}$ NMR $\left(400 \mathrm{MHz}, \mathrm{CDCl}_{3}\right) \delta 6.77(\mathrm{~s}, 1 \mathrm{H}), 6.57(\mathrm{~s}, 1 \mathrm{H}), 4.30(\mathrm{~s}, 1 \mathrm{H}), 3.99-3.89(\mathrm{~m}, 4 \mathrm{H}), 3.86-3.66(\mathrm{~m}, 1 \mathrm{H}), 3.34$ (dd, $J=12.5,4.5 \mathrm{~Hz}, 1 \mathrm{H}), 3.09-2.78(\mathrm{~m}, 3 \mathrm{H}), 2.50-2.24(\mathrm{~m}, 3 \mathrm{H}), 2.02-1.87$ (m, 2H), 1.83-1.74 (m, 4H), 1.51-1.40 $(\mathrm{m}, 5 \mathrm{H}), 1.37-1.29(\mathrm{~m}, 8 \mathrm{H}), 1.20(\mathrm{~d}, J=6.4 \mathrm{~Hz}, 2 \mathrm{H}), 0.90(\mathrm{t}, J=6.8 \mathrm{~Hz}, 6 \mathrm{H}) .{ }^{13} \mathrm{C} \mathrm{NMR}\left(101 \mathrm{MHz}, \mathrm{CDCl}_{3}\right) \delta 148.0,147.7,129.1,127.7$, $114.4,112.3,70.1,69.3,65.5,55.6,48.3,47.2,42.5,38.0,31.8,31.8,29.6,29.5,29.5,25.9,24.3,22.8,18.9,14.2$. ESI-MS m/z 418 [M + $\mathrm{H}]^{+}$, HR-ESI-MS: m/z $418.3312[\mathrm{M}+\mathrm{H}]^{+}$, (calcd for $\left.\mathrm{C}_{26} \mathrm{H}_{44} \mathrm{NO}_{3}{ }^{+}, 418.3316\right)$.

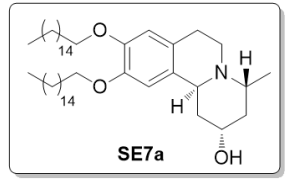
White powder, mp $74-76{ }^{\circ} \mathrm{C} .{ }^{1} \mathrm{H}$ NMR $\left(400 \mathrm{MHz}, \mathrm{CDCl}_{3}\right) \delta 6.68(\mathrm{~s}, 1 \mathrm{H}), 6.58(\mathrm{~s}, 1 \mathrm{H}), 4.16-4.02(\mathrm{~m}, 1 \mathrm{H})$, 4.02-3.85 (m, 4H), $3.78(\mathrm{~d}, J=11.0 \mathrm{~Hz}, 1 \mathrm{H}), 3.42-3.26(\mathrm{~m}, 1 \mathrm{H}), 3.05-2.80(\mathrm{~m}, 2 \mathrm{H}), 2.84-2.55(\mathrm{~m}, 2 \mathrm{H})$, 2.49-2.32 (m, 1H), 1.91-1.71 (m, 6H), 1.54-1.41 (m, 5H), 1.36-1.20 (m, 48H), $1.12(\mathrm{~d}, J=6.8 \mathrm{~Hz}, 4 \mathrm{H}), 0.89(\mathrm{t}, J=6.7 \mathrm{~Hz}, 6 \mathrm{H}) .{ }^{13} \mathrm{C} \mathrm{NMR}$ $\left(101 \mathrm{MHz}, \mathrm{CDCl}_{3}\right) \delta 147.9,147.6,130.7,127.2,114.3,112.1,70.0,69.5,66.2,55.8,52.4,48.4,41.6,39.9,32.1,29.9,29.9,29.9,29.7$, 29.7, 29.6, 29.6, 26.3, 22.9, 14.3, 12.7. ESI-MS m/z $698[\mathrm{M}+\mathrm{Na}]^{+}$, HR-ESI-MS: m/z 698.6448 $[\mathrm{M}+\mathrm{H}]^{+},\left(\right.$calcd for $\mathrm{C}_{46} \mathrm{H}_{84} \mathrm{NO}_{3}{ }^{+}$, 698.6446).

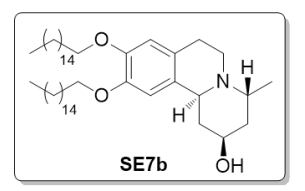

9,10-Bis(hexadecyloxy)-4 $\alpha$-methyl-2,3,4,6,7,11b $\alpha$-hexahydro-1 $H$-pyrido[2,1-a]isoquinolin-2 $\beta$-ol

(SE7b). White solid, mp 79-81 ${ }^{\circ} \mathrm{C} .{ }^{1} \mathrm{H}$ NMR $\left(400 \mathrm{MHz}, \mathrm{CDCl}_{3}\right) \delta 6.70(\mathrm{~s}, 1 \mathrm{H}), 6.50(\mathrm{~s}, 1 \mathrm{H}), 4.24(\mathrm{~s}, 1 \mathrm{H}), 3.93-3.80(\mathrm{~m}$, $4 \mathrm{H}), 3.77-3.64(\mathrm{~m}, 1 \mathrm{H}), 3.27(\mathrm{dd}, J=12.4,4.5 \mathrm{~Hz}, 1 \mathrm{H}), 3.04-2.71(\mathrm{~m}, 3 \mathrm{H}), 2.46-2.24(\mathrm{~m}, 2 \mathrm{H}), 1.93-1.79(\mathrm{~m}$, $2 \mathrm{H}), 1.76-1.64(\mathrm{~m}, 4 \mathrm{H}), 1.45-1.33(\mathrm{~m}, 5 \mathrm{H}), 1.26-1.08(\mathrm{~m}, 51 \mathrm{H}), 0.81(\mathrm{t}, J=6.7 \mathrm{~Hz}, 6 \mathrm{H}) .{ }^{13} \mathrm{C} \mathrm{NMR}\left(101 \mathrm{MHz}, \mathrm{CDCl}_{3}\right) \delta 147.9,147.6$, 129.0, 127.6, 114.4, 112.2, 70.0, 69.2, 65.6, 55.3, 48.2, 47.1, 42.3, 37.9, 31.9, 29.7, 29.9, 29.5, 29.5, 29.4, 26.1, 24.3, $22.7,18.7,14.1$. ESI-MS m/z $698[\mathrm{M}+\mathrm{N} a]^{+}$, HR-ESI-MS: m/z $698.6450[\mathrm{M}+\mathrm{H}]^{+}$, (calcd for $\left.\mathrm{C}_{46} \mathrm{H}_{84} \mathrm{NO}_{3}{ }^{+}, 698.6446\right)$.

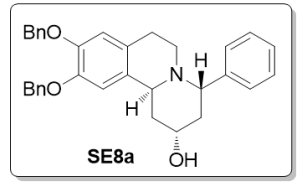

9,10-Bis(benzyloxy)-4 $\alpha$-(4-nitrophenyl)-2,3,4,6,7,11b $\alpha$-hexahydro-1 $H$-pyrido[2,1- $a]$ isoquinolin-2 $\alpha$-ol (SE8a). 
Yellow oil. ${ }^{1} \mathrm{H}$ NMR (400 MHz, $\left.\mathrm{CDCl}_{3}\right) \delta 7.60-7.21(\mathrm{~m}, 15 \mathrm{H}), 6.73(\mathrm{~s}, 1 \mathrm{H}), 6.68(\mathrm{~s}, 1 \mathrm{H}), 5.31-4.98(\mathrm{~m}, 4 \mathrm{H}), 4.22(\mathrm{t}, J=4.2 \mathrm{~Hz}, 1 \mathrm{H}), 4.00$ $(\mathrm{dd}, J=8.4,3.9 \mathrm{~Hz}, 2 \mathrm{H}), 3.36-3.10(\mathrm{~m}, 1 \mathrm{H}), 2.92-2.68(\mathrm{~m}, 3 \mathrm{H}), 2.44-2.18(\mathrm{~m}, 1 \mathrm{H}), 2.05-1.77(\mathrm{~m}, 3 \mathrm{H}) .{ }^{13} \mathrm{C} \mathrm{NMR}\left(101 \mathrm{MHz}, \mathrm{CDCl}_{3}\right) \delta$ 147.7, 147.1, 142.0, 137.5, 137.5, 131.1, 128.5, 128.5, 127.8, 127.4, 127.4, 127.3, 126.7, 115.4, 114.0, 71.8, 71.4, 66.0, 60.4, 53.2, 46.3, 37.4, 34.8, 28.1.ESI-MS m/z $492[\mathrm{M}+\mathrm{H}]^{+}$, HR-ESI-MS: m/z 492.2531 [M + H] $]^{+}$, (calcd for $\left.\mathrm{C}_{33} \mathrm{H}_{34} \mathrm{NO}_{3}{ }^{+}, 492.2533\right)$.

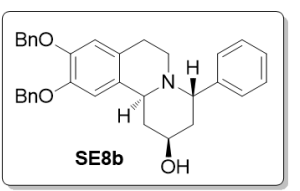

9,10-Bis(benzyloxy)-4 $\alpha$-(4-nitrophenyl)-2,3,4,6,7,11b $\alpha$-hexahydro-1 $H$-pyrido[2,1-a]isoquinolin-2 $\beta$-ol (SE8b, 1). Pale yellow crystal, mp 146-148 ${ }^{\circ} \mathrm{C} .{ }^{1} \mathrm{H}$ NMR $\left(400 \mathrm{MHz}, \mathrm{CDCl}_{3}\right) \delta 7.55-7.44(\mathrm{~m}, 4 \mathrm{H}), 7.44-7.25(\mathrm{~m}, 12 \mathrm{H})$, $6.89(\mathrm{~s}, 1 \mathrm{H}), 6.69(\mathrm{~s}, 1 \mathrm{H}), 5.33-5.06(\mathrm{~m}, 4 \mathrm{H}), 4.46(\mathrm{~s}, 1 \mathrm{H}), 3.62(\mathrm{dd}, J=11.5,2.4 \mathrm{~Hz}, 1 \mathrm{H}), 3.56-3.42(\mathrm{~m}, 1 \mathrm{H})$, 3.01-2.73 (m, 3H), $2.57(\mathrm{dd}, J=12.9,2.5 \mathrm{~Hz}, 1 \mathrm{H}), 2.21(\mathrm{dd}, J=15.7,4.7 \mathrm{~Hz}, 1 \mathrm{H}), 1.98(\mathrm{td}, J=12.2,4.8 \mathrm{~Hz}, 1 \mathrm{H}), 1.94-1.87(\mathrm{~m}, 1 \mathrm{H}), 1.63$ $(\mathrm{dd}, J=23.0,11.6 \mathrm{~Hz}, 2 \mathrm{H}) .{ }^{13} \mathrm{C}$ NMR $\left(101 \mathrm{MHz}, \mathrm{CDCl}_{3}\right) \delta 147.9,147.2,143.3,137.7,137.4,128.7,128.6,128.5,128.3,127.8,127.7$, 127.5, 127.5, 127.4, 115.5, 113.6, 71.9, 71.3, 65.1, 57.6, 57.1, 46.5, 44.8, 37.2, 22.5. ESI-MS m/z 492 [M + H] $]^{+}$HR-ESI-MS: m/z $492.2532[\mathrm{M}+\mathrm{H}]^{+}$, (calcd for $\left.\mathrm{C}_{33} \mathrm{H}_{34} \mathrm{NO}_{3}{ }^{+}, 492.2533\right)$.

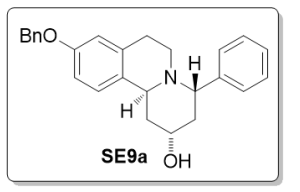

9-(Benzyloxy)-4 $\alpha$-phenyl-2,3,4,6,7,11b $\alpha$-hexahydro-1 $H$-pyrido[2,1-a]isoquinolin-2 $\alpha$-ol (SE9a). White solid, mp 125-127 ${ }^{\circ} \mathrm{C} .{ }^{1} \mathrm{H}$ NMR $\left(400 \mathrm{MHz}, \mathrm{CDCl}_{3}\right) \delta 7.59(\mathrm{~d}, J=7.6 \mathrm{~Hz}, 2 \mathrm{H}), 7.55-7.33(\mathrm{~m}, 7 \mathrm{H}), 7.33-7.27(\mathrm{~m}, 1 \mathrm{H})$, $7.05(\mathrm{~d}, J=8.6 \mathrm{~Hz}, 1 \mathrm{H}), 6.92-6.72(\mathrm{~m}, 2 \mathrm{H}), 5.09(\mathrm{~s}, 2 \mathrm{H}), 4.36-4.22(\mathrm{~m}, 1 \mathrm{H}), 4.22-3.96(\mathrm{~m}, 2 \mathrm{H}), 3.41-3.24(\mathrm{~m}$, $1 \mathrm{H}), 3.10-2.79(\mathrm{~m}, 3 \mathrm{H}), 2.55-2.31(\mathrm{~m}, 1 \mathrm{H}), 2.13-1.88(\mathrm{~m}, 3 \mathrm{H}) .{ }^{13} \mathrm{C} \mathrm{NMR}\left(101 \mathrm{MHz}, \mathrm{CDCl}_{3}\right) \delta 157.3,142.0,137.4,136.1,131.1,128.7$, 128.6, 128.1, 127.8, 127.6, 127.5, 126.8, 114.9, 113.2, 70.2, 66.3, 60.8, 53.2, 46.2, 37.7, 34.8, 29.2. ESI-MS m/z 408 [M + Na $]^{+}$, HR-ESI-MS: m/z $386.2125[\mathrm{M}+\mathrm{H}]^{+}$, (calcd for $\left.\mathrm{C}_{26} \mathrm{H}_{28} \mathrm{NO}_{2}{ }^{+}, 386.2115\right)$.

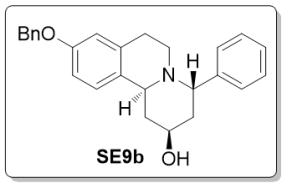

9-(Benzyloxy)-4 $\alpha$-phenyl-2,3,4,6,7,11b $\alpha$-hexahydro-1H-pyrido[2,1-a]isoquinolin-2 $\beta$-ol (SE9b, 2). Colorless crystal, mp 198-200 ${ }^{\circ} \mathrm{C} .{ }^{1} \mathrm{H}$ NMR (400 MHz, DMSO) $\delta 7.54-7.19(\mathrm{~m}, 11 \mathrm{H}), 6.87(\mathrm{dd}, J=8.4,2.2 \mathrm{~Hz}, 1 \mathrm{H}), 6.75$ $(\mathrm{d}, J=2.0 \mathrm{~Hz}, 1 \mathrm{H}), 5.06(\mathrm{~s}, 2 \mathrm{H}), 4.65(\mathrm{~d}, J=4.0 \mathrm{~Hz}, 1 \mathrm{H}), 4.36(\mathrm{~s}, 1 \mathrm{H}), 3.55(\mathrm{~d}, J=10.5 \mathrm{~Hz}, 1 \mathrm{H}), 3.47-3.37(\mathrm{~m}$, $1 \mathrm{H}), 2.92-2.61(\mathrm{~m}, 4 \mathrm{H}), 2.24(\mathrm{~d}, J=12.5 \mathrm{~Hz}, 1 \mathrm{H}), 1.86-1.62(\mathrm{~m}, 2 \mathrm{H}), 1.42(\mathrm{dd}, J=22.8,11.3 \mathrm{~Hz}, 1 \mathrm{H}) .{ }^{13} \mathrm{C} \mathrm{NMR}(101 \mathrm{MHz}, \mathrm{DMSO}) \delta$ 157.0, 144.5, 137.8, 136.8, 130.1, 129.2, 128.9, 128.2, 128.1, 127.6, 127.5, 127.0, 115.3, 113.7, 69.6, 63.7, 57.4, 57.1, 55.4, 46.4, 45.7, 37.3, 23.1. ESI-MS m/z $386[\mathrm{M}+\mathrm{H}]^{+}$, HR-ESI-MS: m/z $386.2125[\mathrm{M}+\mathrm{H}]^{+}$, (calcd for $\left.\mathrm{C}_{26} \mathrm{H}_{28} \mathrm{NO}_{2}{ }^{+}, 386.2115\right)$.

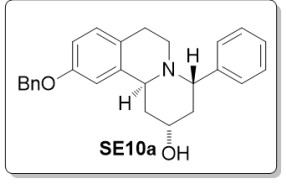

10-(Benzyloxy)-4 $\alpha$-phenyl-2,3,4,6,7,11b $\alpha$-hexahydro-1H-pyrido[2,1- $a]$ isoquinolin-2 $\alpha$-ol $\quad$ (SE10a). Orange solid, mp 47-49 ${ }^{\circ} \mathrm{C} .{ }^{1} \mathrm{H}$ NMR $\left(400 \mathrm{MHz}, \mathrm{CDCl}_{3}\right) \delta 7.55(\mathrm{~d}, J=7.7 \mathrm{~Hz}, 2 \mathrm{H}), 7.47-7.16(\mathrm{~m}, 8 \mathrm{H}), 7.06(\mathrm{~d}, J=8.4$ $\mathrm{Hz}, 1 \mathrm{H}), 6.83(\mathrm{dd}, J=8.4,2.4 \mathrm{~Hz}, 1 \mathrm{H}), 6.72(\mathrm{~d}, J=2.3 \mathrm{~Hz}, 1 \mathrm{H}), 5.03(\mathrm{~s}, 2 \mathrm{H}), 4.25(\mathrm{t}, J=4.5 \mathrm{~Hz}, 1 \mathrm{H}), 4.16-3.97$ (m, 2H), 3.36-3.19 (m, 1H), 2.98-2.73 (m, 3H), 2.35 (dt, $J=13.1,3.6 \mathrm{~Hz}, 1 \mathrm{H}), 2.11-1.85(\mathrm{~m}, 3 \mathrm{H}) .{ }^{13} \mathrm{C} \mathrm{NMR}\left(101 \mathrm{MHz}, \mathrm{CDCl}_{3}\right) \delta 156.9$, 141.8, 139.5, 137.2, 130.1, 128.6, 128.5, 127.9, 127.5, 127.3, 127.0, 126.7, 113.4, 112.7, 70.1, 66.1, 60.7, 53.7, 46.4, 37.4, 34.6, 27.9. ESI-MS m/z $386[\mathrm{M}+\mathrm{H}]^{+}$, HR-ESI-MS: m/z $386.2125[\mathrm{M}+\mathrm{H}]^{+}$, (calcd for $\left.\mathrm{C}_{26} \mathrm{H}_{28} \mathrm{NO}_{2}{ }^{+}, 386.2115\right)$.

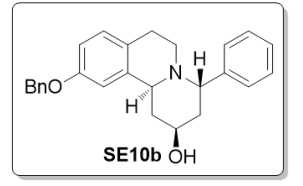

10-(Benzyloxy)-4 $\alpha$-phenyl-2,3,4,6,7,11b $\alpha$-hexahydro-1H-pyrido[2,1-a]isoquinolin-2 $\beta$-ol (SE10b, 3). White powder, mp 181-183 ${ }^{\circ} \mathrm{C} .{ }^{1} \mathrm{H}$ NMR $\left(400 \mathrm{MHz}, \mathrm{CDCl}_{3}\right) \delta 7.64-7.23(\mathrm{~m}, 10 \mathrm{H}), 7.13-6.94(\mathrm{~m}, 2 \mathrm{H}), 6.86(\mathrm{dd}, J=8.3$, $2.3 \mathrm{~Hz}, 1 \mathrm{H}), 5.09(\mathrm{~s}, 2 \mathrm{H}), 4.54(\mathrm{~s}, 1 \mathrm{H}), 3.81-3.54(\mathrm{~m}, 2 \mathrm{H}), 3.05-2.78(\mathrm{~m}, 3 \mathrm{H}), 2.74(\mathrm{dd}, J=12.8,2.9 \mathrm{~Hz}, 1 \mathrm{H})$, 2.41-2.25 (m, 1H), 2.09-1.92 (m, 2H), $1.68(\mathrm{dd}, J=23.1,11.5 \mathrm{~Hz}, 1 \mathrm{H}) .{ }^{13} \mathrm{C} \mathrm{NMR}\left(101 \mathrm{MHz}, \mathrm{CDCl}_{3}\right) \delta 157.5,143.2,137.3,137.2,130.3$, 128.6, 128.6, 128.0, 127.5, 127.5, 127.4, 112.9, 112.3, 70.2, 65.2, 58.2, 57.3, 46.6, 44.9, 37.0, 22.1. ESI-MS m/z $386[\mathrm{M}+\mathrm{H}]^{+}$, HR-ESI-MS: m/z $386.2126[\mathrm{M}+\mathrm{H}]^{+}$, (calcd for $\mathrm{C}_{26} \mathrm{H}_{28} \mathrm{NO}_{2}{ }^{+}, 386.2115$ ).

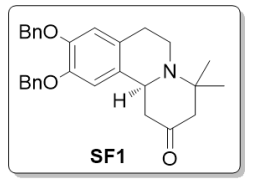

9,10-Bis(benzyloxy)-4,4-dimethyl-3,4,6,7-tetrahydro-1 $H$-pyrido[2,1-a]isoquinolin-2(11bH)-one (SF1). White solid, mp 104-106 ${ }^{\circ} \mathrm{C} .{ }^{1} \mathrm{H}$ NMR (400 MHz, $\left.\mathrm{CDCl}_{3}\right) \delta 7.52-7.39(\mathrm{~m}, 4 \mathrm{H}), 7.41-7.27(\mathrm{~m}, 6 \mathrm{H}), 6.69(\mathrm{~s}, 1 \mathrm{H}), 6.62(\mathrm{~s}$, $1 \mathrm{H}), 5.12(\mathrm{~d}, J=12.1 \mathrm{~Hz}, 4 \mathrm{H}), 4.09(\mathrm{dd}, J=11.6,3.0 \mathrm{~Hz}, 1 \mathrm{H}), 3.28-3.12(\mathrm{~m}, 1 \mathrm{H}), 2.95-2.81(\mathrm{~m}, 1 \mathrm{H}), 2.76-2.51(\mathrm{~m}$, 
4H), $2.42(\mathrm{dd}, J=13.5,12.2 \mathrm{~Hz}, 1 \mathrm{H}), 2.23(\mathrm{dd}, J=13.5,2.0 \mathrm{~Hz}, 1 \mathrm{H}), 1.35(\mathrm{~s}, 3 \mathrm{H}), 1.07(\mathrm{~s}, 3 \mathrm{H}) .{ }^{13} \mathrm{C} \mathrm{NMR}\left(101 \mathrm{MHz}^{\mathrm{C}} \mathrm{CDCl}_{3}\right) \delta 209.3$, 148.0, 147.4, 137.5, 137.5, 130.8, 128.7, 128.0, 128.0, 127.6, 127.5, 115.2, 113.3, 71.9, 71.5, 58.0, 56.3, 54.1, 47.6, 41.5, 30.4, 30.3, 19.9. ESI-MS m/z $442[\mathrm{M}+\mathrm{H}]^{+}$, HR-ESI-MS: m/z $442.2376[\mathrm{M}+\mathrm{H}]^{+}$, (calcd for $\left.\mathrm{C}_{29} \mathrm{H}_{32} \mathrm{NO}_{3}{ }^{+}, 442.2377\right)$.

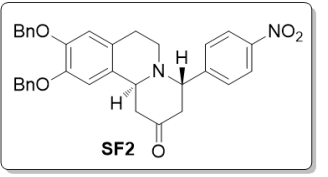

trans-9,10-Bis(benzyloxy)-4-(4-nitrophenyl)-3,4,6,7-tetrahydro-1 $H$-pyrido[2,1-a]isoquinolin-2(11bH)-one (SF2). Yellow oil. ${ }^{1} \mathrm{H}$ NMR (400 MHz, $\left.\mathrm{CDCl}_{3}\right) \delta 8.19(\mathrm{~d}, J=8.8 \mathrm{~Hz}, 2 \mathrm{H}), 7.59$ (d, $\left.J=8.6 \mathrm{~Hz}, 2 \mathrm{H}\right), 7.54-7.27$ $(\mathrm{m}, 10 \mathrm{H}), 6.71(\mathrm{~s}, 1 \mathrm{H}), 6.53(\mathrm{~s}, 1 \mathrm{H}), 5.13(\mathrm{~s}, 2 \mathrm{H}), 5.07(\mathrm{~s}, 2 \mathrm{H}), 4.52(\mathrm{t}, J=5.0 \mathrm{~Hz}, 1 \mathrm{H}), 4.01(\mathrm{dd}, J=9.8,3.6$ $\mathrm{Hz}, 1 \mathrm{H}), 3.40-3.19(\mathrm{~m}, 1 \mathrm{H}), 3.01-2.77(\mathrm{~m}, 5 \mathrm{H}), 2.72(\mathrm{dd}, J=14.8,10.0 \mathrm{~Hz}, 1 \mathrm{H}), 2.59(\mathrm{dd}, J=14.8,3.7 \mathrm{~Hz}, 1 \mathrm{H}) .{ }^{13} \mathrm{C} \mathrm{NMR}(101 \mathrm{MHz}$, $\left.\mathrm{CDCl}_{3}\right) \delta 207.6,148.1,147.5,147.3,137.2,137.1,128.9,128.8,128.5,128.5,127.8,127.8,127.3,127.3,126.7,123.7,115.3,113.3,71.6$, 71.4, 63.6, 54.8, 46.5, 45.9, 42.6, 28.6. ESI-MS m/z $535[\mathrm{M}+\mathrm{H}]^{+}$, HR-ESI-MS: m/z 535.2223 $\left[\mathrm{M}+\mathrm{H}^{+}\right.$, (calcd for $\mathrm{C}_{33} \mathrm{H}_{31} \mathrm{~N}_{2} \mathrm{O}_{5}{ }^{+}$, $535.2227)$.

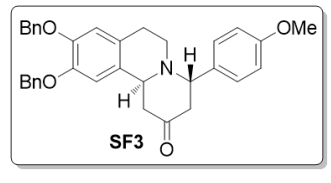

trans-9,10-Bis(benzyloxy)-4-(4-methoxyphenyl)-3,4,6,7-tetrahydro-1 $H$-pyrido[2,1-a] isoquinolin-2(11bH)one (SF3). Light yellow oil. ${ }^{1} \mathrm{H}$ NMR (400 MHz, $\left.\mathrm{CDCl}_{3}\right) \delta$ 7.47-7.40 (m, 4H), 7.40-7.29 (m, 6H), 7.27-7.24 $(\mathrm{m}, 2 \mathrm{H}), 6.87(\mathrm{~d}, J=8.7 \mathrm{~Hz}, 2 \mathrm{H}), 6.69(\mathrm{~s}, 1 \mathrm{H}), 6.59(\mathrm{~s}, 1 \mathrm{H}), 5.10(\mathrm{~d}, J=20.2 \mathrm{~Hz}, 4 \mathrm{H}), 4.41(\mathrm{~s}, 1 \mathrm{H}), 4.18-3.94$ (m, 1H), 3.30-3.16 (m, 1H), 2.94-2.76 (m, 5H), 2.74-2.57 (m, 2H). ${ }^{13} \mathrm{C}$ NMR (101 MHz, $\left.\mathrm{CDCl}_{3}\right) \delta 209.0,159.0,147.9,147.5,137.3$, 137.2, 129.5, 129.3, 128.6, 128.5, 127.8, 127.8, 127.4, 127.3, 127.1, 115.3, 113.8, 113.2, 77.4, 77.0, 76.7, 71.7, 71.4, 63.3, 55.3, 54.1, 46.9, 46.3, 44.0, 28.5. ESI-MS m/z $520[\mathrm{M}+\mathrm{H}]^{+}$, HR-ESI-MS: m/z 520.2481 [M + H] $]^{+}$, (calcd for $\left.\mathrm{C}_{34} \mathrm{H}_{34} \mathrm{NO}_{4}{ }^{+}, 520.2482\right)$.

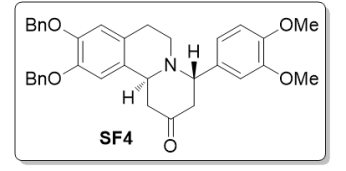

trans-9,10-Bis(benzyloxy)-4-(3,4-dimethoxyphenyl)-3,4,6,7-tetrahydro-1 $H$-pyrido[2,1-a]isoquinolin-2(11 bH)-one (SF4). Pale yellow crystal, mp 59-61 ${ }^{\circ} \mathrm{C} .{ }^{1} \mathrm{H}$ NMR $\left(400 \mathrm{MHz}, \mathrm{CDCl}_{3}\right) \delta$ 7.53-7.40 (m, 4H), $7.40-7.26(\mathrm{~m}, 6 \mathrm{H}), 6.93(\mathrm{~s}, 1 \mathrm{H}), 6.87(\mathrm{dd}, J=8.3,1.5 \mathrm{~Hz}, 1 \mathrm{H}), 6.82(\mathrm{~d}, J=8.3 \mathrm{~Hz}, 1 \mathrm{H}), 6.71(\mathrm{~s}, 1 \mathrm{H}), 6.59$ (s, $1 \mathrm{H}), 5.13(\mathrm{~s}, 3 \mathrm{H}), 5.08(\mathrm{~s}, 3 \mathrm{H}), 4.38(\mathrm{t}, J=4.9 \mathrm{~Hz}, 1 \mathrm{H}), 4.08(\mathrm{t}, J=6.8 \mathrm{~Hz}, 1 \mathrm{H}), 3.87(\mathrm{~s}, 6 \mathrm{H}), 3.33-3.13(\mathrm{~m}, 1 \mathrm{H}), 3.01-2.75(\mathrm{~m}, 5 \mathrm{H}), 2.67$ $(\mathrm{d}, J=6.8 \mathrm{~Hz}, 2 \mathrm{H}) .{ }^{13} \mathrm{C} \mathrm{NMR}\left(101 \mathrm{MHz}, \mathrm{CDCl}_{3}\right) \delta 209.0,149.0,148.4,147.9,147.4,137.4,137.3,132.4,129.8,128.5,127.8,127.8$, $127.4,127.3,127.2,120.4,115.4,113.3,111.5,110.8,71.7,71.4,63.6,56.0,55.9,54.4,46.8,46.2,43.9,28.6$. ESI-MS m/z 550 [M + H] $]^{+}$ HR-ESI-MS: $\mathrm{m} / \mathrm{z} 550.2586[\mathrm{M}+\mathrm{H}]^{+}$, (calcd for $\left.\mathrm{C}_{35} \mathrm{H}_{36} \mathrm{NO}_{5}{ }^{+}, 550.2588\right)$.

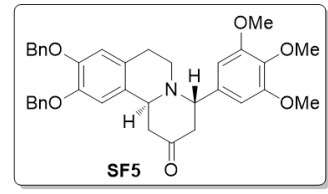

trans-9,10-Bis(benzyloxy)-4-(3,4,5-trimethoxyphenyl)-3,4,6,7-tetrahydro-1 $H$-pyrido[2,1- $a$ ] isoquinolin-2( 11bH)-one (SF5). White solid, mp 141-143 ${ }^{\circ} \mathrm{C} .{ }^{1} \mathrm{H}$ NMR (400 MHz, $\left.\mathrm{CDCl}_{3}\right) \delta 7.50-7.41$ (m, 4H), 7.41-7.28 $(\mathrm{m}, 6 \mathrm{H}), 6.71(\mathrm{~s}, 1 \mathrm{H}), 6.64-6.53(\mathrm{~m}, 3 \mathrm{H}), 5.11(\mathrm{~d}, J=17.4 \mathrm{~Hz}, 4 \mathrm{H}), 4.34(\mathrm{t}, J=5.1 \mathrm{~Hz}, 1 \mathrm{H}), 4.14(\mathrm{t}, J=6.7$ $\mathrm{Hz}, 1 \mathrm{H}), 3.89-3.81(\mathrm{~m}, 9 \mathrm{H}), 3.34-3.18(\mathrm{~m}, 1 \mathrm{H}), 2.98-2.89(\mathrm{~m}, 1 \mathrm{H}), 2.89-2.75(\mathrm{~m}, 4 \mathrm{H}), 2.73-2.64(\mathrm{~m}, 2 \mathrm{H}) .{ }^{13} \mathrm{C} \mathrm{NMR}\left(101 \mathrm{MHz}, \mathrm{CDCl}_{3}\right) \delta$ 209.1, 153.4, 148.2, 147.6, 137.5, 137.4, 135.9, 129.8, 128.7, 128.7, 128.0, 128.0, 127.6, 127.5, 127.4, 115.6, 113.6, 105.5, 71.9, 71.6, 64.1, 61.0, 56.4, 55.0, 47.0, 46.3, 44.3, 28.6. ESI-MS m/z $580[\mathrm{M}+\mathrm{H}]^{+}$, HR-ESI-MS: m/z 580.2692 [M + $]^{+},\left(\right.$calcd for $\mathrm{C}_{36} \mathrm{H}_{38} \mathrm{NO}_{6}{ }^{+}$, $580.2694)$.

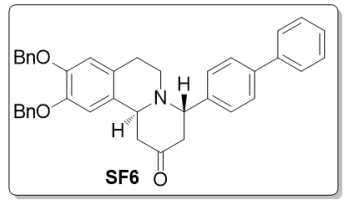

trans-4-([1,1'-Biphenyl]-4-yl)-9,10-bis(benzyloxy)-3,4,6,7-tetrahydro-1H-pyrido[2,1-a]isoquinolin-2(11b H)-one (SF6). Yellow crystal, mp 149-151 ${ }^{\circ} \mathrm{C} .{ }^{1} \mathrm{H}$ NMR (400 MHz, $\left.\mathrm{CDCl}_{3}\right) \delta 7.53-7.45(\mathrm{~m}, 4 \mathrm{H}), 7.41-7.19$ $(\mathrm{m}, 15 \mathrm{H}), 6.62(\mathrm{~s}, 1 \mathrm{H}), 6.50(\mathrm{~s}, 1 \mathrm{H}), 5.04(\mathrm{~s}, 2 \mathrm{H}), 4.98(\mathrm{~s}, 2 \mathrm{H}), 4.40(\mathrm{t}, J=4.8 \mathrm{~Hz}, 1 \mathrm{H}), 4.02(\mathrm{dd}, J=8.9,5.0$ $\mathrm{Hz}, 1 \mathrm{H}), 3.30-3.10(\mathrm{~m}, 1 \mathrm{H}), 2.96-2.67(\mathrm{~m}, 5 \mathrm{H}), 2.66-2.51(\mathrm{~m}, 2 \mathrm{H}) .{ }^{13} \mathrm{C}$ NMR $\left(101 \mathrm{MHz}, \mathrm{CDCl}_{3}\right) \delta 208.9,147.9,147.5,140.6,140.5$, 138.5, 137.4, 137.2, 129.7, 128.8, 128.6, 128.5, 127.8, 127.8, 127.4, 127.3, 127.2, 127.1, 127.1, 115.4, 113.3, 71.7, 71.4, 63.7, 54.2, 46.99, 46.4, 43.7, 28.7. ESI-MS m/z $566[\mathrm{M}+\mathrm{H}]^{+}$, HR-ESI-MS: m/z 566.2690 [M + Na $]^{+}$, (calcd for $\left.\mathrm{C}_{39} \mathrm{H}_{36} \mathrm{NO}_{3}{ }^{+}, 566.2690\right)$.

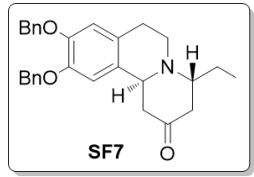
Colorless crystal, mp 104-106 ${ }^{\circ} \mathrm{C} .{ }^{1} \mathrm{H}$ NMR $\left(400 \mathrm{MHz}, \mathrm{CDCl}_{3}\right) \delta 7.64-7.40(\mathrm{~m}, 4 \mathrm{H}), 7.41-7.28(\mathrm{~m}, 6 \mathrm{H}), 6.71$ (s, 
$1 \mathrm{H}), 6.59(\mathrm{~s}, 1 \mathrm{H}), 5.13(\mathrm{~d}, J=11.9 \mathrm{~Hz}, 4 \mathrm{H}), 4.22-4.00(\mathrm{~m}, 1 \mathrm{H}), 3.18(\mathrm{dd}, J=11.5,6.7 \mathrm{~Hz}, 2 \mathrm{H}), 2.99-2.77(\mathrm{~m}, 3 \mathrm{H}), 2.72(\mathrm{dd}, J=13.6,6.2$ $\mathrm{Hz}, 1 \mathrm{H}), 2.57-2.38(\mathrm{~m}, 2 \mathrm{H}), 2.33(\mathrm{~d}, J=13.6 \mathrm{~Hz}, 1 \mathrm{H}), 1.65-1.53(\mathrm{~m}, 1 \mathrm{H}), 1.45-1.31(\mathrm{~m}, 1 \mathrm{H}), 0.93(\mathrm{t}, J=7.3 \mathrm{~Hz}, 4 \mathrm{H}) .{ }^{13} \mathrm{C} \mathrm{NMR}(101$ $\left.\mathrm{MHz}, \mathrm{CDCl}_{3}\right) \delta 209.3,147.9,147.3,137.4,137.3,130.3,128.5,127.8,127.8,127.4,127.3,127.1,115.3,113.2,71.6,71.4,64.9,54.5,46.6$, 46.5, 42.6, 29.7, 23.4, 11.3. ESI-MS m/z $442[\mathrm{M}+\mathrm{H}]^{+}$, HR-ESI-MS: m/z $442.2376[\mathrm{M}+\mathrm{H}]^{+}$, (calcd for $\left.\mathrm{C}_{29} \mathrm{H}_{32} \mathrm{NO}_{3}{ }^{+}, 441.2377\right)$.

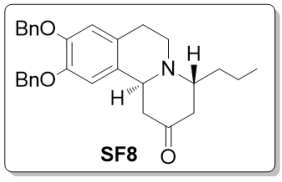

trans-9,10-Bis(benzyloxy)-4-propyl-3,4,6,7-tetrahydro-1 $H$-pyrido[2,1-a]isoquinolin-2(11b $H$ )-one

(SF8). White solid, mp $115-117^{\circ} \mathrm{C} .{ }^{1} \mathrm{H}$ NMR $\left(400 \mathrm{MHz}, \mathrm{CDCl}_{3}\right) \delta$ 7.53-7.41 (m, 4H), 7.41-7.28 (m, 6H), $6.71(\mathrm{~s}, 1 \mathrm{H})$, 6.59 (s, 1H), $5.12(\mathrm{~d}, J=11.2 \mathrm{~Hz}, 4 \mathrm{H}), 4.20-4.06(\mathrm{~m}, 1 \mathrm{H}), 3.29(\mathrm{dd}, J=12.3,6.1 \mathrm{~Hz}, 1 \mathrm{H}), 3.22-3.10(\mathrm{~m}, 1 \mathrm{H})$, $2.98-2.68(\mathrm{~m}, 4 \mathrm{H}), 2.58-2.38(\mathrm{~m}, 2 \mathrm{H}), 2.35-2.24(\mathrm{~m}, J=13.6 \mathrm{~Hz}, 1 \mathrm{H}), 1.57-1.50(\mathrm{~m}, 1 \mathrm{H}), 1.43-1.30(\mathrm{~m}, 3 \mathrm{H}), 0.91(\mathrm{t}, J=7.0 \mathrm{~Hz}, 3 \mathrm{H}) .{ }^{13} \mathrm{C}$ NMR $\left(101 \mathrm{MHz}, \mathrm{CDCl}_{3}\right) \delta 209.5,148.1,147.5,137.5,137.4,130.5,128.7,128.7,128.0,128.0,127.6,127.5,127.3,115.4,113.4,71.8$, 71.6, 63.1, 54.7, 46.7, 46.6, 43.2, 32.8, 29.9, 20.0, 14.1. ESI-MS m/z $456[\mathrm{M}+\mathrm{H}]^{+}$, HR-ESI-MS: m/z 456.2529 [M + H] $]^{+}$, (calcd for $\left.\mathrm{C}_{30} \mathrm{H}_{34} \mathrm{NO}_{3}{ }^{+}, 456.2533\right)$.

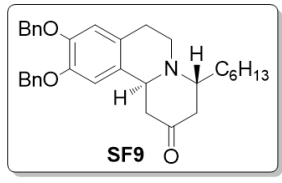

trans-9,10-Bis(benzyloxy)-4-hexyl-3,4,6,7-tetrahydro-1 $H$-pyrido[2,1-a]isoquinolin-2(11bH)-one

(SF9). Colorless crystal, mp 109-111 ${ }^{\circ} \mathrm{C} .{ }^{1} \mathrm{H}$ NMR $\left(400 \mathrm{MHz}, \mathrm{CDCl}_{3}\right) \delta 7.56-7.40(\mathrm{~m}, 4 \mathrm{H}), 7.42-7.27$ (m, 6H), 6.70 (s, 1H), $6.58(\mathrm{~s}, 1 \mathrm{H}), 5.13(\mathrm{~d}, J=11.7 \mathrm{~Hz}, 4 \mathrm{H}), 4.12(\mathrm{dd}, J=10.5,4.2 \mathrm{~Hz}, 1 \mathrm{H}), 3.38-3.20(\mathrm{~m}, 1 \mathrm{H}), 3.23-3.11(\mathrm{~m}$, $1 \mathrm{H}), 2.99-2.66(\mathrm{~m}, 4 \mathrm{H}), 2.57-2.40(\mathrm{~m}, 2 \mathrm{H}), 2.29(\mathrm{~d}, J=13.6 \mathrm{~Hz}, 1 \mathrm{H}), 1.59-1.48(\mathrm{~m}, 1 \mathrm{H}), 1.36-1.18(\mathrm{~m}, 9 \mathrm{H}), 0.87(\mathrm{t}, J=6.8 \mathrm{~Hz}, 3 \mathrm{H}) .{ }^{13} \mathrm{C}$ NMR $\left(101 \mathrm{MHz}, \mathrm{CDCl}_{3}\right) \delta{ }^{13} \mathrm{C}$ NMR $\left(101 \mathrm{MHz}, \mathrm{CDCl}_{3}\right) \delta 209.6,148.0,147.4,137.5,137.4,130.5,128.7,128.0,128.0,127.5,127.5$, 127.3, 115.4, 113.4, 71.8, 71.5, 63.4, 54.7, 46.7, 46.6, 43.5, 31.9, 30.7, 29.9, 29.4, 26.9, 22.8, 14.3. ESI-MS m/z 498 [M + H] ${ }^{+}$, HR-ESI-MS: $\mathrm{m} / \mathrm{z} 498.2999[\mathrm{M}+\mathrm{H}]^{+}$, (calcd for $\mathrm{C}_{33} \mathrm{H}_{39} \mathrm{NO}_{3}, 498.3003$ ).

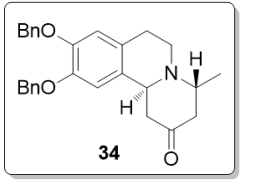

trans-9,10-Bis(benzyloxy)-4-methyl-3,4,6,7-tetrahydro-1H-pyrido[2,1-a]isoquinolin-2(11bH)-one (34). Pale yellow crystal, mp 107-108 ${ }^{\circ} \mathrm{C} .{ }^{1} \mathrm{H}$ NMR $\left(400 \mathrm{MHz}, \mathrm{CDCl}_{3}\right) \delta$ 7.51-7.40 (m, 4H), 7.40-7.27 (m, 6H), $6.71(\mathrm{~s}, 1 \mathrm{H})$, $6.62(\mathrm{~s}, 1 \mathrm{H}), 5.14(\mathrm{~s}, 2 \mathrm{H}), 5.14(\mathrm{~s}, 2 \mathrm{H}), 4.11(\mathrm{dd}, J=11.1,2.9 \mathrm{~Hz}, 1 \mathrm{H}), 3.66-3.42(\mathrm{~m}, 1 \mathrm{H}), 3.21-3.02(\mathrm{~m}, 1 \mathrm{H})$, $2.98-2.76(\mathrm{~m}, 4 \mathrm{H}), 2.60(\mathrm{~d}, J=13.1 \mathrm{~Hz}, 1 \mathrm{H}), 2.46(\mathrm{dd}, J=13.6,11.4 \mathrm{~Hz}, 1 \mathrm{H}), 2.24(\mathrm{~d}, J=13.6 \mathrm{~Hz}, 1 \mathrm{H}), 1.12(\mathrm{~d}, J=6.8 \mathrm{~Hz}, 3 \mathrm{H}) .{ }^{13} \mathrm{C}$ NMR $\left(101 \mathrm{MHz}, \mathrm{CDCl}_{3}\right) \delta$ 209.3, 148.1, 147.5, 137.5, 137.4, 130.3, 128.7, 128.7, 128.0, 128.0, 127.6, 127.5, 127.4, 115.5, 113.2, 71.9, 71.5, 57.7, 54.1, 47.6, 47.2, 46.5, 29.7, 15.0. ESI-MS m/z $428[\mathrm{M}+\mathrm{H}]^{+}, \mathrm{HR}-\mathrm{ESI}-\mathrm{MS}: \mathrm{m} / \mathrm{z} 428.2219\left[\mathrm{M}+\mathrm{H}^{+},\left(\right.\right.$calcd for $\mathrm{C}_{28} \mathrm{H}_{30} \mathrm{NO}_{3}{ }^{+}$, 428.2220).

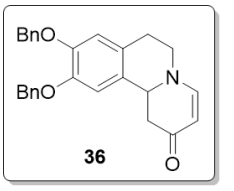

9,10-Bis(benzyloxy)-3,4,6,7-tetrahydro-1 $H$-pyrido[2,1-a]isoquinolin-2(11bH)-one (36). Light yellow crystal, mp 142-144 ${ }^{\circ} \mathrm{C} .{ }^{1} \mathrm{H}$ NMR $\left(600 \mathrm{MHz}, \mathrm{CDCl}_{3}\right) \delta$ 7.49-7.43 (m, 4H), 7.42-7.36 (m, 4H), 7.36-7.29 (m, 2H), 7.15 (d, $J=7.2$ $\mathrm{Hz}, 1 \mathrm{H}), 6.74(\mathrm{~s}, 1 \mathrm{H}), 6.70(\mathrm{~s}, 1 \mathrm{H}), 5.20-5.12(\mathrm{~m}, 4 \mathrm{H}), 5.06(\mathrm{~d}, J=7.3 \mathrm{~Hz}, 1 \mathrm{H}), 4.63(\mathrm{dd}, J=16.4,4.3 \mathrm{~Hz}, 1 \mathrm{H}), 3.58$ (ddd, $J=12.2,4.9,1.9 \mathrm{~Hz}, 1 \mathrm{H}), 3.39$ (td, $J=12.2,3.2 \mathrm{~Hz}, 1 \mathrm{H}), 3.09-3.01(\mathrm{~m}, 1 \mathrm{H}), 2.74-2.67(\mathrm{~m}, 2 \mathrm{H}), 2.47(\mathrm{t}, J=16.3 \mathrm{~Hz}, 1 \mathrm{H}) .{ }^{13} \mathrm{C} \mathrm{NMR}$ $\left(151 \mathrm{MHz}, \mathrm{CDCl}_{3}\right) \delta 192.8,154.2,148.0,148.0,137.1,136.9,128.6,128.6,128.0,127.9,127.5,127.3,127.3,126.3,114.9,112.1,98.4$, 71.4, 71.3, 56.3, 49.8, 44.1, 29.8. ESI-MS m/z 412 [M + H] $]^{+}$, HR-ESI-MS: m/z $412.1907[\mathrm{M}+\mathrm{H}]^{+}$, (calcd for $\mathrm{C}_{27} \mathrm{H}_{26} \mathrm{NO}_{3}{ }^{+}, 412.1907$ ).

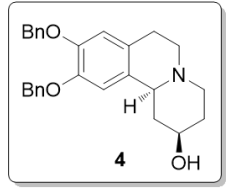

cis-9,10-Bis(benzyloxy)-2,3,4,6,7,11b-hexahydro-1 $H$-pyrido[2,1-a]isoquinolin-2-ol (4). Colorless crystal, mp 159-161 ${ }^{\circ} \mathrm{C} .{ }^{1} \mathrm{H}$ NMR $\left(400 \mathrm{MHz}, \mathrm{CDCl}_{3}\right) \delta$ 7.55-7.40 (m, 4H), 7.41-7.27 (m, 6H), $6.78(\mathrm{~s}, 1 \mathrm{H}), 6.69(\mathrm{~s}, 1 \mathrm{H}), 5.12(\mathrm{~d}$, $J=3.4 \mathrm{~Hz}, 4 \mathrm{H}), 3.86-3.68(\mathrm{~m}, 1 \mathrm{H}), 3.16-2.90(\mathrm{~m}, 4 \mathrm{H}), 2.67-2.55(\mathrm{~m}, 1 \mathrm{H}), 2.55-2.40(\mathrm{~m}, 2 \mathrm{H}), 2.34(\mathrm{td}, J=12.2,2.1$ $\mathrm{Hz}, 1 \mathrm{H}), 2.26-2.06(\mathrm{~m}, 1 \mathrm{H}), 1.98(\mathrm{dd}, J=12.0,1.7 \mathrm{~Hz}, 1 \mathrm{H}), 1.67$ (ddd, $J=24.0,12.2,4.2 \mathrm{~Hz}, 1 \mathrm{H}), 1.39(\mathrm{dd}, J=23.2,11.4 \mathrm{~Hz}, 1 \mathrm{H}) .{ }^{13} \mathrm{C}$ NMR $\left(101 \mathrm{MHz}, \mathrm{CDCl}_{3}\right) \delta 147.9,147.2,137.6,137.5,130.5,128.6,127.9,127.9,127.8,127.6,127.5,115.3,112.7,72.1,71.4,69.80$ 60.7, 54.5, 51.8, 40.6, 34.9, 29.3. ESI-MS m/z $416[\mathrm{M}+\mathrm{H}]^{+}$, HR-ESI-MS: m/z 416.2216 [M + H] $]^{+},\left(\right.$calcd for $\left.\mathrm{C}_{27} \mathrm{H}_{30} \mathrm{NO}_{3}{ }^{+}, 416.2220\right)$.

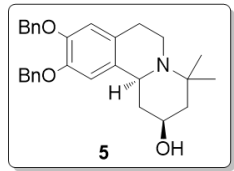

cis-9,10-Bis(benzyloxy)-4,4-dimethyl-2,3,4,6,7,11b-hexahydro-1H-pyrido[2,1-a]isoquinolin-2-ol (5). Brown oil. 
${ }^{1} \mathrm{H}$ NMR (400 MHz, $\left.\mathrm{CDCl}_{3}\right) \delta$ 7.42-7.31 (m, 4H), 7.31-7.19 (m, 6H), $6.70(\mathrm{~s}, 1 \mathrm{H}), 6.58(\mathrm{~s}, 1 \mathrm{H}), 5.02(\mathrm{~d}, J=7.7 \mathrm{~Hz}, 4 \mathrm{H}), 4.14(\mathrm{~s}, 1 \mathrm{H}), 3.95$ $(\mathrm{d}, J=11.6 \mathrm{~Hz}, 1 \mathrm{H}), 3.15-3.03(\mathrm{~m}, 1 \mathrm{H}), 2.92-2.70(\mathrm{~m}, 1 \mathrm{H}), 2.49(\mathrm{~d}, J=15.8 \mathrm{~Hz}, 1 \mathrm{H}), 2.31(\mathrm{td}, J=11.5,3.2 \mathrm{~Hz}, 1 \mathrm{H}), 2.09(\mathrm{dd}, J=13.9$, $2.3 \mathrm{~Hz}, 1 \mathrm{H}), 1.69(\mathrm{dd}, J=14.4,2.9 \mathrm{~Hz}, 1 \mathrm{H}), 1.61-1.50(\mathrm{~m}, 2 \mathrm{H}), 1.18(\mathrm{~s}, 3 \mathrm{H}), 1.12(\mathrm{~s}, 3 \mathrm{H}) .{ }^{13} \mathrm{C} \mathrm{NMR}\left(101 \mathrm{MHz}, \mathrm{CDCl}_{3}\right) \delta 147.5,147.2$, 137.8, 137.7, 132.7, 129.2, 128.6, 128.6, 127.9, 127.9, 127.7, 127.5, 115.4, 114.0, 72.2, 71.6, 66.4, 53.7, 50.9, 45.7, 42.7, 40.4, 31.7, 30.6, 19.3. ESI-MS m/z $444[\mathrm{M}+\mathrm{H}]^{+}$, HR-ESI-MS: $\mathrm{m} / \mathrm{z} 444.2531[\mathrm{M}+\mathrm{H}]^{+}$, (calcd for $\left.\mathrm{C}_{29} \mathrm{H}_{34} \mathrm{NO}_{3}{ }^{+}, 444.2533\right)$.

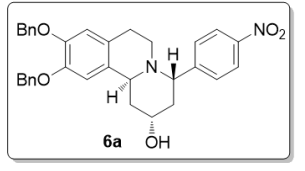

9,10-Bis(benzyloxy)-4 $\alpha$-(4-nitrophenyl)-2,3,4,6,7,11b $\alpha$-hexahydro- $1 H$-pyrido[2,1- $a$ ]isoquinolin-2 $\alpha$-ol

(6a). Yellow crystal, mp 60-62 ${ }^{\circ} \mathrm{C} .{ }^{1} \mathrm{H}$ NMR $\left(400 \mathrm{MHz}, \mathrm{CDCl}_{3}\right) \delta 8.18(\mathrm{~d}, J=8.7 \mathrm{~Hz}, 2 \mathrm{H}), 7.72(\mathrm{~d}, J=8.6 \mathrm{~Hz}, 2 \mathrm{H})$, $7.59-7.18(\mathrm{~m}, 10 \mathrm{H}), 6.73(\mathrm{~s}, 1 \mathrm{H}), 6.63(\mathrm{~s}, 1 \mathrm{H}), 5.35-4.92(\mathrm{~m}, 4 \mathrm{H}), 4.27(\mathrm{~s}, 1 \mathrm{H}), 3.99-3.71(\mathrm{~m}, 2 \mathrm{H}), 3.35-3.14(\mathrm{~m}$, 1H), 3.00-2.80 (m, 2H), 2.81-2.66 (m, 1H), 2.41-2.18 (m, 1H), 2.03-1.79 (m, 3H). ${ }^{13} \mathrm{C}$ NMR (101 MHz, $\left.\mathrm{CDCl}_{3}\right) \delta 150.0,147.9,147.2$, 146.9, 137.4, 137.4, 130.6, 128.5, 128.5, 128.1, 127.8, 127.4, 127.3, 123.7, 115.3, 114.0, 71.8, 71.4, 65.8, 60.4, 53.2, 46.0, 37.2, 34.0, 28.2. ESI-MS m/z $537[\mathrm{M}+\mathrm{H}]^{+}$, HR-ESI-MS: m/z $537.2381[\mathrm{M}+\mathrm{H}]^{+}$, (calcd for $\left.\mathrm{C}_{33} \mathrm{H}_{33} \mathrm{~N}_{2} \mathrm{O}_{5}{ }^{+}, 537.2383\right)$.

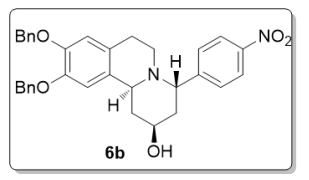

9,10-Bis(benzyloxy)-4 $\alpha$-(4-nitrophenyl)-2,3,4,6,7,11b $\alpha$-hexahydro-1H-pyrido[2,1-a]isoquinolin-2 $\beta$-ol (6b). Yellow crystal, mp $172-173{ }^{\circ} \mathrm{C} .{ }^{1} \mathrm{H}$ NMR $\left(400 \mathrm{MHz}, \mathrm{CDCl}_{3}\right) \delta 8.20(\mathrm{~d}, J=8.7 \mathrm{~Hz}, 2 \mathrm{H}), 7.76-7.17(\mathrm{~m}, 12 \mathrm{H}), 6.87$ (s, 1H), $6.69(\mathrm{~s}, 1 \mathrm{H}), 5.31-5.05(\mathrm{~m}, 4 \mathrm{H}), 4.45(\mathrm{~s}, 1 \mathrm{H}), 3.75(\mathrm{dd}, J=11.2,2.3 \mathrm{~Hz}, 1 \mathrm{H}), 3.57-3.39(\mathrm{~m}, 1 \mathrm{H}), 2.97$ (td, $J=13.4,5.2 \mathrm{~Hz}, 1 \mathrm{H}), 2.88-2.66(\mathrm{~m}, 2 \mathrm{H}), 2.60-2.50(\mathrm{~m}, 1 \mathrm{H}), 2.26(\mathrm{dd}, J=16.7,4.5 \mathrm{~Hz}, 1 \mathrm{H}), 2.01-1.84(\mathrm{~m}, 2 \mathrm{H}), 1.54(\mathrm{dd}, J=22.9,11.4$ $\mathrm{Hz}, 1 \mathrm{H}) .{ }^{13} \mathrm{C} \mathrm{NMR}\left(101 \mathrm{MHz}, \mathrm{CDCl}_{3}\right) \delta 151.2,148.0,147.6,137.6,137.3,128.6,128.3,128.2,127.9,127.8,127.5,127.4,124.0,115.4$, 113.6, 71.9, 71.3, 64.6, 57.5, 56.8, 46.6, 44.3, 37.1, 22.4. ESI-MS m/z $537[\mathrm{M}+\mathrm{H}]^{+}$, HR-ESI-MS: m/z $537.2380[\mathrm{M}+\mathrm{H}]^{+}$, (calcd for $\left.\mathrm{C}_{33} \mathrm{H}_{33} \mathrm{~N}_{2} \mathrm{O}_{5}{ }^{+}, 537.2383\right)$.

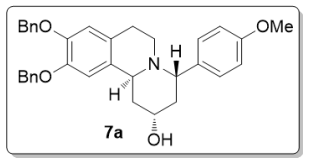

9,10-Bis(benzyloxy)-4 $\alpha$-(4-methoxyphenyl)-2,3,4,6,7,11b $\alpha$-hexahydro-1 $H$-pyrido[2,1- $a]$ isoquinolin-2 $\alpha$-ol (7a). Yellow oil. ${ }^{1} \mathrm{H}$ NMR $\left(400 \mathrm{MHz}, \mathrm{CDCl}_{3}\right) \delta 7.64-7.27(\mathrm{~m}, 12 \mathrm{H}), 6.88(\mathrm{~d}, J=8.7 \mathrm{~Hz}, 2 \mathrm{H}), 6.72(\mathrm{~s}, 1 \mathrm{H}), 6.67$ (s, 1H), 4.24-4.10 (m, 1H), 4.09-3.92 (m, 2H), $3.81(\mathrm{~s}, 3 \mathrm{H}), 3.36-3.12(\mathrm{~m}, 1 \mathrm{H}), 2.95-2.60$ (m, 3H), 2.35-2.16 (m, 1H), 2.02-1.74 (m, 3H). ${ }^{13} \mathrm{C}$ NMR $\left(101 \mathrm{MHz}, \mathrm{CDCl}_{3}\right) \delta 158.5,147.9,147.3,137.7,137.7,134.1,131.4,128.7,128.6,128.0,127.6$, 127.5, 115.6, 114.2, 113.9, 72.0, 71.6, 66.3, 60.0, 55.4, 53.3, 46.4, 37.6, 35.1, 28.3. ESI-MS m/z 522 [M + H], HR-ESI-MS: m/z 522.2637 $[\mathrm{M}+\mathrm{H}]^{+}$, (calcd for $\left.\mathrm{C}_{34} \mathrm{H}_{36} \mathrm{NO}_{4}{ }^{+}, 522.2639\right)$.

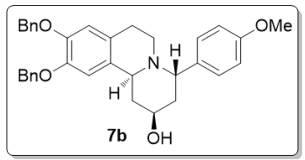

9,10-Bis(benzyloxy)-4 $\alpha$-(4-methoxyphenyl)-2,3,4,6,7,11b $\alpha$-hexahydro-1 $H$-pyrido[2,1-a]isoquinolin-2 $\beta$-ol (7b). Colorless needle crystal, $\mathrm{mp} 142-143{ }^{\circ} \mathrm{C} .{ }^{1} \mathrm{H}$ NMR (400 MHz, DMSO) $\delta$ 7.61-7.43 (m, 4H), 7.45-7.22 (m, $8 \mathrm{H}), 7.04(\mathrm{~s}, 1 \mathrm{H}), 6.89(\mathrm{~d}, J=8.3 \mathrm{~Hz}, 2 \mathrm{H}), 6.81(\mathrm{~s}, 1 \mathrm{H}), 5.10(\mathrm{~d}, J=12.8 \mathrm{~Hz}, 4 \mathrm{H}), 4.68-4.53(\mathrm{~m}, 1 \mathrm{H}), 4.31(\mathrm{~s}$, $1 \mathrm{H}), 3.74(\mathrm{~s}, 3 \mathrm{H}), 3.54-3.43(\mathrm{~m}, 1 \mathrm{H}), 2.91-2.56(\mathrm{~m}, 4 \mathrm{H}), 2.25-2.09(\mathrm{~m}, 1 \mathrm{H}), 1.83-1.61(\mathrm{~m}, 2 \mathrm{H}), 1.39(\mathrm{dd}, J=22.9,11.4 \mathrm{~Hz}, 1 \mathrm{H}) .{ }^{13} \mathrm{C} \mathrm{NMR}$ (101 MHz, DMSO) $\delta 158.7,147.5,147.2,138.0,137.9,136.4,129.5,128.8,128.8,128.6,128.5,128.2,128.2,128.0,115.5,114.2,113.4$, 71.4, 70.7, 63.9, 57.4, 56.3, 55.5, 46.4, 45.8, 37.4, 22.4. ESI-MS m/z $522[\mathrm{M}+\mathrm{H}]^{+}$, HR-ESI-MS: m/z $522.2637[\mathrm{M}+\mathrm{H}]^{+}$, (calcd for $\left.\mathrm{C}_{34} \mathrm{H}_{36} \mathrm{NO}_{4}{ }^{+}, 522.2639\right)$.

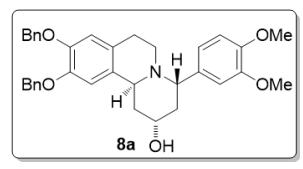

9,10-Bis(benzyloxy)-4 $\alpha$-(3,4-dimethoxyphenyl)-2,3,4,6,7,11b $\alpha$-hexahydro-1 $H$-pyrido[2,1- $a$ ]isoquinolin-2 $\alpha$-o I (8a). Yellow crystal, mp 71-73 ${ }^{\circ} \mathrm{C} .{ }^{1} \mathrm{H}$ NMR $\left(400 \mathrm{MHz}, \mathrm{CDCl}_{3}\right) \delta 7.60-7.42(\mathrm{~m}, 4 \mathrm{H}), 7.42-7.25$ (m, 6H), 7.10 (s, 1H), $7.02(\mathrm{~d}, J=8.2 \mathrm{~Hz}, 1 \mathrm{H}), 6.82(\mathrm{~d}, J=8.4 \mathrm{~Hz}, 1 \mathrm{H}), 6.70(\mathrm{t}, J=10.3 \mathrm{~Hz}, 2 \mathrm{H}), 5.20-4.94(\mathrm{~m}, 4 \mathrm{H}), 4.13(\mathrm{t}, J$ $=4.6 \mathrm{~Hz}, 1 \mathrm{H}), 4.08-3.95(\mathrm{~m}, 2 \mathrm{H}), 3.88(\mathrm{~s}, 3 \mathrm{H}), 3.86(\mathrm{~s}, 3 \mathrm{H}), 3.35-3.08(\mathrm{~m}, 1 \mathrm{H}), 2.91-2.70(\mathrm{~m}, 3 \mathrm{H}), 2.28-2.17(\mathrm{~m}, 1 \mathrm{H}), 2.06-1.94(\mathrm{~m}, 2 \mathrm{H})$, 1.91-1.82 (m, 1H). ${ }^{13} \mathrm{C}$ NMR (101 MHz, $\left.\mathrm{CDCl}_{3}\right) \delta 149.1,147.8,147.7,147.0,137.5,137.5,134.8,131.0,128.5,127.8,127.4,127.4,119.4$, 115.3, 114.0, 111.0, 110.8, 71.8, 71.4, 66.0, 59.3, 56.1, 55.9, 53.4, 46.3, 37.0, 35.6, 27.6. ESI-MS m/z 552 [M + H], HR-ESI-MS: m/z $552.2739[\mathrm{M}+\mathrm{H}]^{+},\left(\right.$calcd for $\left.\mathrm{C}_{35} \mathrm{H}_{38} \mathrm{NO}_{5}^{+}, 552.2744\right)$.

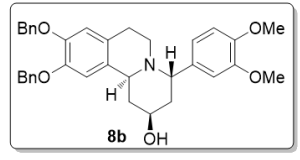


9,10-Bis(benzyloxy)-4 $\alpha$-(3,4-dimethoxyphenyl)-2,3,4,6,7,11b $\alpha$-hexahydro-1 $H$-pyrido[2,1-a]isoquinolin-2 $\beta$-ol (8b). Yellow oil. ${ }^{1} \mathrm{H}$ NMR (400 MHz, $\left.\mathrm{CDCl}_{3}\right) \delta$ 7.58-7.43 (m, 4H), 7.44-7.28 (m, 6H), $7.00(\mathrm{~s}, 1 \mathrm{H}), 6.93-6.83(\mathrm{~m}, 2 \mathrm{H}), 6.81(\mathrm{~d}, J=8.2 \mathrm{~Hz}, 1 \mathrm{H}), 6.70(\mathrm{~s}, 1 \mathrm{H})$, 5.39-5.05 (m, 4H), $4.45(\mathrm{~s}, 1 \mathrm{H}), 3.91(\mathrm{~s}, 3 \mathrm{H}), 3.88(\mathrm{~s}, 3 \mathrm{H}), 3.69-3.40(\mathrm{~m}, 2 \mathrm{H}), 3.04-2.72(\mathrm{~m}, 3 \mathrm{H}), 2.57(\mathrm{dd}, J=12.7,2.3 \mathrm{~Hz}, 1 \mathrm{H})$, $2.32-2.15(\mathrm{~m}, 1 \mathrm{H}), 2.02-1.85(\mathrm{~m}, 2 \mathrm{H}), 1.62(\mathrm{dd}, J=23.0,11.6 \mathrm{~Hz}, 1 \mathrm{H}) .{ }^{13} \mathrm{C} \mathrm{NMR}\left(101 \mathrm{MHz}, \mathrm{CDCl}_{3}\right) \delta 149.3,148.3,147.9,147.3,137.7$, $137.4,135.9,128.7,128.5,128.4,127.8,127.7,127.5,127.4,119.7,115.5,113.6,110.9,110.1,72.0,71.3,65.1,57.5,56.8,56.0,55.9,46.5$,

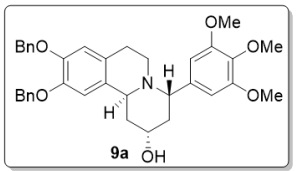
44.9, 37.1, 22.5. ESI-MS m/z $552[\mathrm{M}+\mathrm{H}]^{+}$, HR-ESI-MS: m/z 552.2740 $[\mathrm{M}+\mathrm{H}]^{+}$, (calcd for $\mathrm{C}_{35} \mathrm{H}_{38} \mathrm{NO}_{5}{ }^{+}$, $552.2744)$

9,10-bis(benzyloxy)-4 $\alpha$-(3,4,5-trimethoxyphenyl)-2,3,4,6,7,11b $\alpha$-hexahydro-1 $H$-pyrido[2,1- $a]$ isoquinolin-2 $\alpha$ -ol (9a). Light yellow oil. ' $\mathrm{H}$ NMR (400 MHz, $\left.\mathrm{CDCl}_{3}\right) \delta$ 7.54-7.42 (m, 4H), 7.42-7.28 (m, 6H), 6.80-6.66 (m, 4H), 5.25-5.04 (m, 4H), 4.11-3.97 (m, 3H), 3.87-3.80 (m, 9H), 3.27-3.16 (m, 1H), 2.90-2.75 (m, 2H), 2.75-2.60 (m, 1H), 2.21-2.10 (m, 1H), 2.05-1.98 (m, 2H), $1.91-1.83(\mathrm{~m}, 1 \mathrm{H}) .{ }^{13} \mathrm{C} \mathrm{NMR}\left(101 \mathrm{MHz}, \mathrm{CDCl}_{3}\right) \delta 153.5,147.9,147.1,138.4,137.7,137.6,137.0,131.0,128.7,128.0,128.0,127.9,127.6$,  $127.5,115.5,114.2,104.6,71.9,71.5,66.2,61.0,59.2,56.5,53.8,46.7,36.8,36.7,27.3$. ESI-MS m/z $582[\mathrm{M}+$ $\mathrm{H}]^{+}$, HR-ESI-MS: m/z $582.2850[\mathrm{M}+\mathrm{H}]^{+}$, (calcd for $\left.\mathrm{C}_{36} \mathrm{H}_{40} \mathrm{NO}_{6}{ }^{+}, 582.2850\right)$.

9,10-bis(benzyloxy)-4 $\alpha$-(3,4,5-trimethoxyphenyl)-2,3,4,6,7,11b $\alpha$-hexahydro- $1 H$-pyrido[2,1- $a]$ isoquinolin-2 $\beta$ ol (9b). Pale yellow crystal, mp 58-60 ${ }^{\circ} \mathrm{C} .{ }^{1} \mathrm{H}$ NMR $\left(400 \mathrm{MHz}, \mathrm{CDCl}_{3}\right) \delta$ 7.45-7.34 (m, 4H), 7.34-7.20 (m, 6H), $6.80(\mathrm{~s}, 1 \mathrm{H}), 6.62(\mathrm{~s}, 1 \mathrm{H})$, $6.54(\mathrm{~s}, 2 \mathrm{H}), 5.18-5.03(\mathrm{~m}, 4 \mathrm{H}), 4.37(\mathrm{~s}, 1 \mathrm{H}), 3.86-3.72(\mathrm{~m}, 9 \mathrm{H}), 3.55-3.27(\mathrm{~m}, 2 \mathrm{H}), 3.02-2.81(\mathrm{~m}, 2 \mathrm{H}), 2.81-2.66(\mathrm{~m}, 1 \mathrm{H}), 2.53-2.39(\mathrm{~m}$, 1H), 2.23-2.09 (m, 1H), 1.92-1.77 (m, 2H), 1.62-1.46 (m, 1H). $\left.{ }^{13} \mathrm{C} \mathrm{NMR} \mathrm{(101} \mathrm{MHz,} \mathrm{CDCl}_{3}\right) \delta 153.6,148.1,147.5,137.8,137.5,137.3$, 128.7, 128.0, 127.9, 127.7, 127.5, 115.7, 113.8, 104.5, 72.1, 71.5, 65.1, 61.0, 57.8, 56.4, 46.7, 45.0, 37.2, 22.8. ESI-MS m/z 582 [M + H] $]^{+}$ HR-ESI-MS: $\mathrm{m} / \mathrm{z} 582.2851[\mathrm{M}+\mathrm{H}]^{+}$, (calcd for $\left.\mathrm{C}_{36} \mathrm{H}_{40} \mathrm{NO}_{6}{ }^{+}, 582.2850\right)$

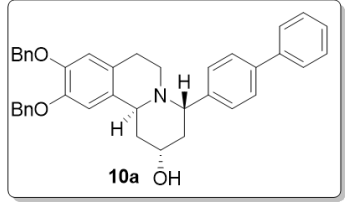

9,10-bis(benzyloxy)-4 $\alpha$-([1,1'-biphenyl]-4-yl)-2,3,4,6,7,11b $\alpha$-hexahydro-1H-pyrido[2,1- $a]$ isoquinolin-2 $\alpha$ ol (10a). Light yellow oil. ${ }^{1} \mathrm{H}$ NMR $\left(400 \mathrm{MHz}, \mathrm{CDCl}_{3}\right) \delta$ 7.75-7.54 (m, 6H), 7.54-7.21 (m, 13H), $6.75(\mathrm{~s}$, $1 \mathrm{H}), 6.70(\mathrm{~s}, 1 \mathrm{H}), 5.27-4.90(\mathrm{~m}, 4 \mathrm{H}), 4.27(\mathrm{~s}, 1 \mathrm{H}), 4.04(\mathrm{~d}, J=4.6 \mathrm{~Hz}, 2 \mathrm{H}), 3.39-3.19(\mathrm{~m}, 1 \mathrm{H}), 3.03-2.68(\mathrm{~m}$, $3 \mathrm{H}), 2.46-2.29(\mathrm{~m}, 1 \mathrm{H}), 2.07-1.82(\mathrm{~m}, 3 \mathrm{H}) .{ }^{13} \mathrm{C} \mathrm{NMR}\left(101 \mathrm{MHz}, \mathrm{CDCl}_{3}\right) \delta 147.8,147.2,141.1,140.9,139.6$, $137.5,137.5,131.2,128.8,128.5,128.5,127.8,127.8,127.4,127.4,127.2,127.2,127.1,115.4,114.0,71.8,71.4,66.1,60.4,53.2,46.3$, 37.5, 34.7, 28.3. ESI-MS m/z $568[\mathrm{M}+\mathrm{H}]^{+}$, HR-ESI-MS: m/z 568.2842 [M + H] $]^{+}$, (calcd for $\left.\mathrm{C}_{39} \mathrm{H}_{38} \mathrm{NO}_{3}{ }^{+}, 568.2846\right)$.

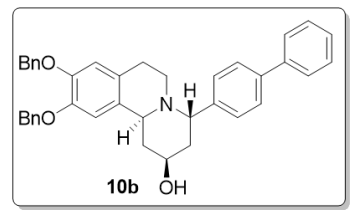

9,10-bis(benzyloxy)-4 $\alpha$-([1,1'-biphenyl]-4-yl)-2,3,4,6,7,11b $\alpha$-hexahydro-1 $H$-pyrido[2,1- $a]$ isoquinolin-2 $\beta$ -ol (10b). Colorless crystal, mp 213-214 ${ }^{\circ} \mathrm{C} .{ }^{1} \mathrm{H}$ NMR $\left(400 \mathrm{MHz}, \mathrm{CDCl}_{3}\right) \delta 7.66-7.56$ (m, 4H), 7.55-7.27 (m, $15 \mathrm{H}), 6.92(\mathrm{~s}, 1 \mathrm{H}), 6.72(\mathrm{~s}, 1 \mathrm{H}), 5.32-5.09(\mathrm{~m}, 4 \mathrm{H}), 4.49(\mathrm{~s}, 1 \mathrm{H}), 3.68(\mathrm{dd}, J=11.4,2.4 \mathrm{~Hz}, 1 \mathrm{H}), 3.61-3.42$ $(\mathrm{m}, 1 \mathrm{H}), 3.06-2.77(\mathrm{~m}, 3 \mathrm{H}), 2.60(\mathrm{dd}, J=12.6,2.1 \mathrm{~Hz}, 1 \mathrm{H}), 2.32-2.16(\mathrm{~m}, 1 \mathrm{H}), 2.05-1.91(\mathrm{~m}, 2 \mathrm{H}), 1.67(\mathrm{dd}$, $J=23.0,11.5 \mathrm{~Hz}, 1 \mathrm{H}) .{ }^{13} \mathrm{C} \mathrm{NMR}\left(101 \mathrm{MHz}, \mathrm{CDCl}_{3}\right) \delta 147.9,147.3,142.5,140.9,140.3,137.7,137.4,128.8,128.5,128.4,127.9,127.7$, 127.5, 127.4, 127.3, 127.2, 127.1, 115.5, 113.6, 72.0, 71.3, 65.1, 57.6, 56.8, 46.6, 44.8, 37.3, 22.6. ESI-MS m/z 568 [M + H] ${ }^{+}$, HR-ESI-MS: $\mathrm{m} / \mathrm{z} 568.2843[\mathrm{M}+\mathrm{H}]^{+}$, (calcd for $\left.\mathrm{C}_{39} \mathrm{H}_{38} \mathrm{NO}_{3}{ }^{+}, 568.2846\right)$.

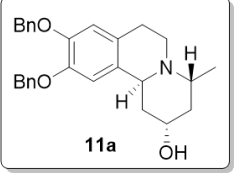

9,10-bis(benzyloxy)-4 $\alpha$-methyl-2,3,4,6,7,11b $\alpha$-hexahydro-1H-pyrido[2,1-a]isoquinolin-2 $\alpha$-ol (11a). Yellow oil. ${ }^{1} \mathrm{H}$ NMR $\left(400 \mathrm{MHz}, \mathrm{CDCl}_{3}\right) \delta$ 7.57-7.41 (m, 4H), 7.41-7.27 (m, 6H), $6.75(\mathrm{~s}, 1 \mathrm{H}), 6.67(\mathrm{~s}, 1 \mathrm{H}), 5.12(\mathrm{~s}, 4 \mathrm{H})$, 4.16-3.94 (m, 1H), $3.75(\mathrm{~d}, J=10.8 \mathrm{~Hz}, 1 \mathrm{H}), 3.42-3.22(\mathrm{~m}, 1 \mathrm{H}), 3.00-2.82(\mathrm{~m}, 2 \mathrm{H}), 2.79-2.55(\mathrm{~m}, 2 \mathrm{H}), 2.31(\mathrm{~d}, J=$ $12.1 \mathrm{~Hz}, 1 \mathrm{H}), 1.86(\mathrm{dd}, J=9.9,2.1 \mathrm{~Hz}, 1 \mathrm{H}), 1.75(\mathrm{td}, J=11.7,5.1 \mathrm{~Hz}, 1 \mathrm{H}), 1.38(\mathrm{dd}, J=23.2,11.6 \mathrm{~Hz}, 1 \mathrm{H}), 1.11(\mathrm{~d}, J=7.0 \mathrm{~Hz}, 3 \mathrm{H}) .{ }^{13} \mathrm{C}$ NMR $\left(101 \mathrm{MHz}, \mathrm{CDCl}_{3}\right) \delta 147.6$ 147.1, 137.5, 137.5, 131.3, 128.5, 127.9, 127.8, 127.7, 127.5, 127.3, 115.3, 113.4, 71.9, 71.4, 65.8, 55.6, 52.2, 48.1, 41.3, 39.5, 29.6, 12.6. ESI-MS m/z $430[\mathrm{M}+\mathrm{H}]^{+}$, HR-ESI-MS: $\mathrm{m} / \mathrm{z} 430.2375[\mathrm{M}+\mathrm{H}]^{+}$, (calcd for $\mathrm{C}_{28} \mathrm{H}_{32} \mathrm{NO}_{3}{ }^{+}, 430.2377$ ). 


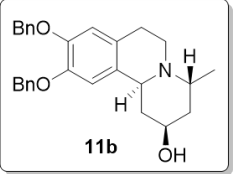

9,10-bis(benzyloxy)-4 $\alpha$-methyl-2,3,4,6,7,11b $\alpha$-hexahydro-1 $H$-pyrido[2,1-a]isoquinolin-2 $\beta$-ol (11b). Pale yellow crystal, mp 75-77 ${ }^{\circ} \mathrm{C} .{ }^{1} \mathrm{H}$ NMR $\left(400 \mathrm{MHz}, \mathrm{CDCl}_{3}\right) \delta 7.63-7.41(\mathrm{~m}, 4 \mathrm{H}), 7.41-7.27(\mathrm{~m}, 6 \mathrm{H}), 6.81(\mathrm{~s}, 1 \mathrm{H}), 6.67(\mathrm{~s}$, $1 \mathrm{H}), 5.28-5.03(\mathrm{~m}, 4 \mathrm{H}), 4.28(\mathrm{~s}, 1 \mathrm{H}), 3.70-3.52(\mathrm{~m}, 1 \mathrm{H}), 3.35(\mathrm{dd}, J=13.1,5.2 \mathrm{~Hz}, 1 \mathrm{H}), 3.03(\mathrm{td}, J=12.8,4.5 \mathrm{~Hz}$, 1H), 2.98-2.85 (m, 1H), 2.85-2.72 (m, 1H), 2.42 (dd, $J=16.3,4.0 \mathrm{~Hz}, 1 \mathrm{H}), 2.29$ (d, $J=13.0 \mathrm{~Hz}, 1 \mathrm{H}), 1.96-1.82(\mathrm{~m}, 2 \mathrm{H}), 1.47-1.35(\mathrm{~m}$, $1 \mathrm{H}), 1.20(\mathrm{~d}, J=6.4 \mathrm{~Hz}, 3 \mathrm{H}) .{ }^{13} \mathrm{C} \mathrm{NMR}\left(101 \mathrm{MHz}, \mathrm{CDCl}_{3}\right) \delta 147.7,147.2,137.6,137.4,129.6,128.5,128.5,128.3,127.8,127.7,127.5$, $127.4,115.4,113.5,71.9,71.3,65.3,55.5,48.0,46.9,42.1,37.8,24.0,18.8$. ESI-MS m/z $430[\mathrm{M}+\mathrm{H}]^{+}$, HR-ESI-MS: m/z 430.2372 [M + $\mathrm{H}]^{+}$, (calcd for $\mathrm{C}_{28} \mathrm{H}_{32} \mathrm{NO}_{3}{ }^{+}, 430.2377$ ).

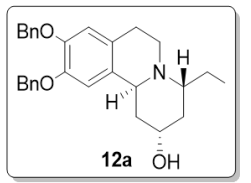

9,10-bis(benzyloxy)-4 $\alpha$-ethyl-2,3,4,6,7,11b $\alpha$-hexahydro-1 $H$-pyrido[2,1-a]isoquinolin-2 $\alpha$-ol (12a). Pale yellow crystal, mp 115-117 ${ }^{\circ} \mathrm{C} .{ }^{1} \mathrm{H}$ NMR $\left(400 \mathrm{MHz}, \mathrm{CDCl}_{3}\right) \delta 7.52-7.39(\mathrm{~m}, 4 \mathrm{H}), 7.42-7.28(\mathrm{~m}, 6 \mathrm{H}), 6.70(\mathrm{~s}, 1 \mathrm{H}), 6.67(\mathrm{~s}$, $1 \mathrm{H}), 5.12(\mathrm{~d}, J=3.9 \mathrm{~Hz}, 4 \mathrm{H}), 4.05-3.92(\mathrm{~m}, 1 \mathrm{H}), 3.78(\mathrm{~d}, J=10.3 \mathrm{~Hz}, 1 \mathrm{H}), 3.08-2.95(\mathrm{~m}, 1 \mathrm{H}), 2.97-2.87(\mathrm{~m}, 1 \mathrm{H})$, 2.89-2.77 (m, 1H), 2.78-2.64 (m, 2H), 2.18 (d, $J=13.8 \mathrm{~Hz}, 1 \mathrm{H}), 1.97$ (dd, $J=10.5,1.8 \mathrm{~Hz}, 1 \mathrm{H}), 1.68-1.57$ (m, 2H), $1.51-1.38$ (m, 2H), $0.94(\mathrm{t}, J=7.4 \mathrm{~Hz}, 3 \mathrm{H}) .{ }^{13} \mathrm{C} \mathrm{NMR}\left(101 \mathrm{MHz}, \mathrm{CDCl}_{3}\right) \delta 147.8,147.3,137.7,137.7,131.8,128.7,128.1,127.9,127.9,127.6,127.5,115.4$, 113.8, 72.1, 71.6, 66.3, 63.3, 52.9, 47.6, 40.8, 34.8, 30.1, 20.4, 12.1. ESI-MS m/z $466[\mathrm{M}+\mathrm{Na}]^{+}, \mathrm{HR}-\mathrm{ESI}-\mathrm{MS}: \mathrm{m} / \mathrm{z} 444.2530[\mathrm{M}+\mathrm{H}]^{+}$, (calcd for $\mathrm{C}_{29} \mathrm{H}_{34} \mathrm{NO}_{3}{ }^{+}, 444.2533$ ).

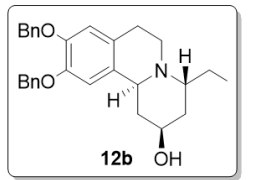

9,10-bis(benzyloxy)-4 $\alpha$-ethyl-2,3,4,6,7,11b $\alpha$-hexahydro-1 $H$-pyrido[2,1-a]isoquinolin-2 $\beta$-ol (12b). Light yellow oil. ${ }^{1} \mathrm{H}$ NMR (400 MHz, $\left.\mathrm{CDCl}_{3}\right) \delta$ 7.57-7.42 (m, 4H), 7.42-7.29 (m, 6H), $6.79(\mathrm{~s}, 1 \mathrm{H}), 6.67(\mathrm{~s}, 1 \mathrm{H}), 5.25-5.03$ (m, $4 \mathrm{H}), 4.30(\mathrm{~s}, 1 \mathrm{H}), 3.67-3.52(\mathrm{~m}, 1 \mathrm{H}), 3.35(\mathrm{dd}, J=13.3,4.0 \mathrm{~Hz}, 1 \mathrm{H}), 3.02(\mathrm{td}, J=12.6,4.6 \mathrm{~Hz}, 1 \mathrm{H}), 2.94-2.82(\mathrm{~m}$, $1 \mathrm{H}), 2.65-2.52(\mathrm{~m}, 1 \mathrm{H}), 2.52-2.40(\mathrm{~m}, 1 \mathrm{H}), 2.34-2.22(\mathrm{~m}, 1 \mathrm{H}), 1.90-1.81(\mathrm{~m}, 2 \mathrm{H}), 1.74-1.66(\mathrm{~m}, 1 \mathrm{H}), 1.65-1.55(\mathrm{~m}, 1 \mathrm{H}), 1.42(\mathrm{dd}, J=$ 21.5, $9.0 \mathrm{~Hz}, 1 \mathrm{H}), 0.92(\mathrm{t}, J=7.5 \mathrm{~Hz}, 3 \mathrm{H}) .{ }^{13} \mathrm{C} \mathrm{NMR}\left(101 \mathrm{MHz}, \mathrm{CDCl}_{3}\right) \delta$ 147.9, 147.3, 137.8, 137.6, 130.1, 128.7, 128.6, 128.5, 128.0, $127.9,127.7,127.6,127.5,115.5,113.8,72.1,71.5,65.9,55.7,54.1,46.8,37.8,37.5,24.7,24.5,10.1$. ESI-MS m/z $444[\mathrm{M}+\mathrm{H}]^{+}$, HR-ESI-MS: $\mathrm{m} / \mathrm{z} 444.2531[\mathrm{M}+\mathrm{H}]^{+}$, (calcd for $\left.\mathrm{C}_{29} \mathrm{H}_{34} \mathrm{NO}_{3}{ }^{+}, 444.2533\right)$.

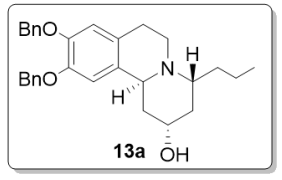

9,10-bis(benzyloxy)-4 $\alpha$-propyl-2,3,4,6,7,11b $\alpha$-hexahydro-1 $H$-pyrido[2,1-a]isoquinolin-2 $\alpha$-ol $\quad$ (13a). Pale yellow crystal, mp 128-130 ${ }^{\circ} \mathrm{C} .{ }^{1} \mathrm{H}$ NMR $\left(400 \mathrm{MHz}, \mathrm{CDCl}_{3}\right) \delta 7.50-7.42(\mathrm{~m}, 4 \mathrm{H}), 7.42-7.29(\mathrm{~m}, 6 \mathrm{H}), 6.71(\mathrm{~s}, 1 \mathrm{H})$, $6.68(\mathrm{~s}, 1 \mathrm{H}), 5.12(\mathrm{~d}, J=3.3 \mathrm{~Hz}, 4 \mathrm{H}), 4.05-3.93(\mathrm{~m}, 1 \mathrm{H}), 3.79(\mathrm{~d}, J=10.5 \mathrm{~Hz}, 1 \mathrm{H}), 3.09-2.95(\mathrm{~m}, 2 \mathrm{H}), 2.89-2.78$ (m, 1H), 2.78-2.65 (m, 2H), $2.17(\mathrm{~d}, J=12.2 \mathrm{~Hz}, 1 \mathrm{H}), 1.97-1.87(\mathrm{~m}, 1 \mathrm{H}), 1.63(\mathrm{dd}, J=12.0,5.2 \mathrm{~Hz}, 1 \mathrm{H}), 1.58-1.53(\mathrm{~m}, 1 \mathrm{H}), 1.49-1.33$ (m, 4H), $0.94(\mathrm{t}, J=7.1 \mathrm{~Hz}, 3 \mathrm{H}) .{ }^{13} \mathrm{C} \mathrm{NMR}\left(101 \mathrm{MHz}, \mathrm{CDCl}_{3}\right) \delta 147.7,147.3,137.7,137.6,131.8,128.6,128.5,128.0,127.9,127.9,127.6$, 127.5, 115.4, 113.8, 72.1, 71.5, 66.4, 61.3, 52.8, 47.5, 40.8, 35.3, 30.1, 30.0, 20.9, 14.4. ESI-MS m/z 458 [M + H], HR-ESI-MS: m/z $458.2684[\mathrm{M}+\mathrm{H}]^{+}$, (calcd for $\left.\mathrm{C}_{30} \mathrm{H}_{36} \mathrm{NO}_{3}{ }^{+}, 458.2690\right)$.

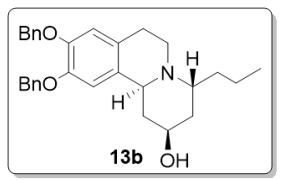

9,10-bis(benzyloxy)-4 $\alpha$-propyl-2,3,4,6,7,11b $\alpha$-hexahydro-1 $H$-pyrido[2,1-a]isoquinolin-2 $\beta$-ol (13b). Colorless oil. ${ }^{1} \mathrm{H}$ NMR (400 MHz, $\left.\mathrm{CDCl}_{3}\right) \delta 7.47(\mathrm{t}, J=7.3 \mathrm{~Hz}, 4 \mathrm{H}), 7.44-7.30(\mathrm{~m}, 6 \mathrm{H}), 6.81(\mathrm{~s}, 1 \mathrm{H}), 6.69$ (s, 1H), 5.22-5.05 $(\mathrm{m}, 4 \mathrm{H}), 4.35-4.23(\mathrm{~m}, 1 \mathrm{H}), 3.79-3.52(\mathrm{~m}, 1 \mathrm{H}), 3.46-3.22(\mathrm{~m}, 1 \mathrm{H}), 3.09-2.94(\mathrm{~m}, 1 \mathrm{H}), 2.94-2.83(\mathrm{~m}, 1 \mathrm{H})$, 2.77-2.59 (m, 1H), 2.58-2.40 (m, 1H), 2.40-2.22 (m, 1H), 1.95-1.89 (m, 1H), 1.89-1.82 (m, 1H), $1.7-1.50(\mathrm{~m}, 2 \mathrm{H}), 1.50-1.37(\mathrm{~m}, 2 \mathrm{H})$, $1.33-1.23(\mathrm{~m}, 1 \mathrm{H}), 0.95$ (t, $J=7.3 \mathrm{~Hz}, 3 \mathrm{H}) .{ }^{13} \mathrm{C} \mathrm{NMR}\left(101 \mathrm{MHz}, \mathrm{CDCl}_{3}\right) \delta 147.7,147.1,137.6,137.4,130.0,128.5,128.3,127.8,127.7$, 127.5, 127.4, 115.3, 113.6, 71.9, 71.3, 65.7, 55.2, 53.0, 46.6, 37.7, 34.0, 24.8, 19.0, 14.5. ESI-MS m/z 458 [M + H] $]^{+}$HR-ESI-MS: m/z $458.2685[\mathrm{M}+\mathrm{H}]^{+}$, (calcd for $\left.\mathrm{C}_{30} \mathrm{H}_{36} \mathrm{NO}_{3}{ }^{+}, 458.2690\right)$.

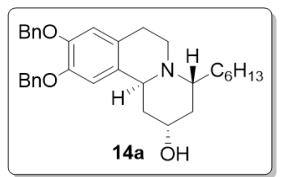

9,10-bis(benzyloxy)-4 $\alpha$-hexyl-2,3,4,6,7,11b $\alpha$-hexahydro-1 $H$-pyrido[2,1-a]isoquinolin-2 $\alpha$-ol (14a).

White solid, mp 107-109 ${ }^{\circ} \mathrm{C} .{ }^{1} \mathrm{H}$ NMR $\left(400 \mathrm{MHz}, \mathrm{CDCl}_{3}\right) \delta$ 7.51-7.41 (m, 4H), 7.41-7.28 (m, 6H), $6.70(\mathrm{~s}, 1 \mathrm{H})$, $6.67(\mathrm{~s}, 1 \mathrm{H}), 5.12(\mathrm{~d}, J=3.4 \mathrm{~Hz}, 4 \mathrm{H}), 4.09-3.88(\mathrm{~m}, 1 \mathrm{H}), 3.78(\mathrm{~d}, J=10.9 \mathrm{~Hz}, 1 \mathrm{H}), 3.11-2.95(\mathrm{~m}, 2 \mathrm{H}), 2.91-2.78$ 
(m, 1H), 2.78-2.64 (m, 2H), 2.31-2.10 (m, 1H), 1.96-1.80 (m, 1H), 1.65-1.55 (m, 2H), 1.48-1.38 (m, 2H), 1.37-1.23 (m, 9H), 0.90 (t, $J=$ $6.2 \mathrm{~Hz}, 3 \mathrm{H}) .{ }^{13} \mathrm{C} \mathrm{NMR}\left(101 \mathrm{MHz}, \mathrm{CDCl}_{3}\right) \delta$ 147.7, 147.3, 137.7, 137.6, 131.8, 128.6, 128.0, 127.9, 127.9, 127.6, 127.5, 115.4, 113.7, 72.0, 71.5, 66.4, 61.6, 52.8, 47.5, 40.8, 35.3, 32.0, 30.9, 29.6, 27.7, 27.7, 22.8, 14.3. ESI-MS m/z 522 [M + Na] ${ }^{+}$, HR-ESI-MS: m/z 500.3154[M $+\mathrm{H}]^{+},\left(\right.$calcd for $\left.\mathrm{C}_{33} \mathrm{H}_{42} \mathrm{NO}_{3}{ }^{+}, 500.3159\right)$.

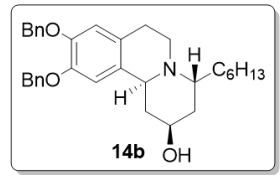

9,10-bis(benzyloxy)-4 $\alpha$-hexyl-2,3,4,6,7,11b $\alpha$-hexahydro-1 $H$-pyrido[2,1-a]isoquinolin-2 $\beta$-ol (14b).

Colorless oil. ${ }^{1} \mathrm{H}$ NMR $\left(400 \mathrm{MHz}, \mathrm{CDCl}_{3}\right) \delta$ 7.54-7.40 (m, 4H), 7.42-7.28 (m, 6H), $6.79(\mathrm{~s}, 1 \mathrm{H}), 6.67(\mathrm{~s}, 1 \mathrm{H})$, 5.26-4.99 (m, 4H), 4.37-4.20 (m, 1H), 3.76-3.42 (m, 1H), 3.44-3.22 (m, 1H), $3.00(\mathrm{td}, J=12.5,4.4 \mathrm{~Hz}, 1 \mathrm{H})$,

2.94-2.77 (m, 1H), $2.65(\mathrm{~d}, J=3.4 \mathrm{~Hz}, 1 \mathrm{H}), 2.56-2.35(\mathrm{~m}, 1 \mathrm{H}), 2.34-2.13(\mathrm{~m}, 1 \mathrm{H}), 1.95-1.78(\mathrm{~m}, 2 \mathrm{H}), 1.69-1.55(\mathrm{~m}, 2 \mathrm{H}), 1.48-1.36(\mathrm{~m}$, 2H), 1.33-1.24 (m, 7H), $0.88(\mathrm{t}, J=6.6 \mathrm{~Hz}, 3 \mathrm{H}) .{ }^{13} \mathrm{C} \mathrm{NMR}\left(101 \mathrm{MHz}, \mathrm{CDCl}_{3}\right) \delta$ 147.6, 147.1, 137.6, 137.4, 123.0, 128.5, 128.3, 127.8, $127.7,127.5,127.4,115.3,113.5,71.8,71.3,65.7,55.3,53.0,46.6,37.8,37.7,31.9,31.8,29.8,25.6,24.7,22.7,14.1$. ESI-MS m/z 500 [M $+\mathrm{H}]^{+}$, HR-ESI-MS: $\mathrm{m} / \mathrm{z} 500.3155[\mathrm{M}+\mathrm{H}]^{+}$, (calcd for $\left.\mathrm{C}_{33} \mathrm{H}_{42} \mathrm{NO}_{3}{ }^{+}, 500.3159\right)$.

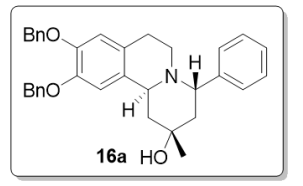

9,10-bis(benzyloxy)-2 $\beta$-methyl-4 $\alpha$-phenyl-2,3,4,6,7,11b $\alpha$-hexahydro-1 $H$-pyrido[2,1-a] isoquinolin-2 $\alpha$-ol (16a). Yellow oil. ${ }^{1} \mathrm{H}$ NMR $\left(400 \mathrm{MHz}, \mathrm{CDCl}_{3}\right) \delta$ 7.65-7.17 (m, 15H), $6.79(\mathrm{~s}, 1 \mathrm{H}), 6.69(\mathrm{~s}, 1 \mathrm{H}), 5.25-5.01(\mathrm{~m}, 4 \mathrm{H}), 4.48$ $(\mathrm{t}, J=5.6 \mathrm{~Hz}, 1 \mathrm{H}), 3.86(\mathrm{dd}, J=8.0,4.5 \mathrm{~Hz}, 1 \mathrm{H}), 3.05(\mathrm{dt}, J=11.9,4.5 \mathrm{~Hz}, 1 \mathrm{H}), 2.92-2.71(\mathrm{~m}, 2 \mathrm{H}), 2.45(\mathrm{dt}, J=$ 15.8, 4.2 Hz, 1H), $2.14(\mathrm{dd}, J=13.6,6.7 \mathrm{~Hz}, 1 \mathrm{H}), 2.06-1.96(\mathrm{~m}, 2 \mathrm{H}), 1.85(\mathrm{dd}, J=13.3,4.1 \mathrm{~Hz}, 1 \mathrm{H}), 1.02(\mathrm{~s}, 3 \mathrm{H}) .{ }^{13} \mathrm{C} \mathrm{NMR}(101 \mathrm{MHz}$, $\left.\mathrm{CDCl}_{3}\right) \delta 147.61,147.18,143.00,137.51,137.45,130.44,128.56,128.50,128.48,127.85,127.79,127.38,127.35,127.04,115.34,113.96$, 71.72, 71.39, 69.69, 58.95, 54.14, 46.10, 44.95, 41.32, 29.15, 25.35. ESI-MS m/z $506[\mathrm{M}+\mathrm{H}]^{+}, \mathrm{HR}-\mathrm{ESI}-\mathrm{MS}: \mathrm{m} / \mathrm{z} 506.2687[\mathrm{M}+\mathrm{H}]^{+}$, (calcd for $\mathrm{C}_{34} \mathrm{H}_{36} \mathrm{NO}_{3}{ }^{+}, 506.2690$ ).

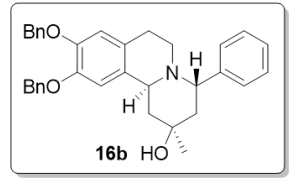

9,10-bis(benzyloxy)-2 $\alpha$-methyl-4 $\alpha$-phenyl-2,3,4,6,7,11b $\alpha$-hexahydro-1 $H$-pyrido[2,1- $a]$ isoquinolin-2 $\beta$-ol (16b). Yellow oil. ${ }^{1} \mathrm{H}$ NMR $\left(400 \mathrm{MHz}, \mathrm{CDCl}_{3}\right) \delta$ 7.59-7.24 (m, 15H), $6.85(\mathrm{~s}, 1 \mathrm{H}), 6.69(\mathrm{~s}, 1 \mathrm{H}), 5.29-5.07$ (m, $4 \mathrm{H}), 4.40(\mathrm{~d}, J=3.8 \mathrm{~Hz}, 1 \mathrm{H}), 3.93(\mathrm{t}, J=7.3 \mathrm{~Hz}, 1 \mathrm{H}), 3.05-2.80(\mathrm{~m}, 3 \mathrm{H}), 2.33(\mathrm{~d}, J=14.4 \mathrm{~Hz}, 1 \mathrm{H}), 2.25-2.13$ $(\mathrm{m}, 1 \mathrm{H}), 2.09(\mathrm{dd}, J=14.7,5.9 \mathrm{~Hz}, 1 \mathrm{H}), 1.69(\mathrm{~d}, J=7.7 \mathrm{~Hz}, 2 \mathrm{H}), 1.16(\mathrm{~s}, 3 \mathrm{H}) .{ }^{13} \mathrm{C} \mathrm{NMR}\left(101 \mathrm{MHz}, \mathrm{CDCl}_{3}\right) \delta$ $147.7,146.8,143.5,137.8,137.4,129.2,128.7,128.5,128.5,128.4,128.2,128.2,127.9,127.8,127.6,127.6,127.4,127.4,127.3,127.2$, 115.8, 113.9, 71.7, 71.3, 68.8, 55.8, 54.71, 47.6, 47.0, 39.5, 30.8, 23.0. ESI-MS m/z $506[\mathrm{M}+\mathrm{H}]^{+}, \mathrm{HR}-\mathrm{ESI}-\mathrm{MS}: \mathrm{m} / \mathrm{z} 506.2688[\mathrm{M}+\mathrm{H}]^{+}$, (calcd for $\mathrm{C}_{34} \mathrm{H}_{36} \mathrm{NO}_{3}{ }^{+}, 506.2690$ ).

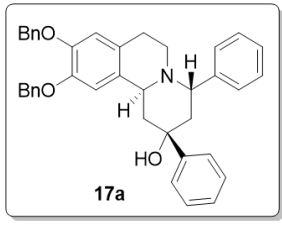

9,10-bis(benzyloxy)-2 $\beta, 4 \alpha$-diphenyl-2,3,4,6,7,11b $\alpha$-hexahydro- $1 H$-pyrido[2,1-a]isoquinolin-2 $\alpha$-ol

(17a). Yellow crystal, mp $153-154{ }^{\circ} \mathrm{C} .{ }^{1} \mathrm{H}$ NMR $\left(400 \mathrm{MHz}, \mathrm{CDCl}_{3}\right) \delta 7.58-7.22(\mathrm{~m}, 20 \mathrm{H}), 7.22-7.14(\mathrm{~m}, 1 \mathrm{H}), 6.84(\mathrm{~s}$, 1H), $6.72(\mathrm{~s}, 1 \mathrm{H}), 5.35-5.04(\mathrm{~m}, 4 \mathrm{H}), 4.58$ (d, $J=4.3 \mathrm{~Hz}, 1 \mathrm{H}), 4.14$ (dd, $J=11.3,2.5 \mathrm{~Hz}, 1 \mathrm{H}), 3.16-2.83(\mathrm{~m}, 3 \mathrm{H})$, $2.63(\mathrm{dd}, J=14.6,5.8 \mathrm{~Hz}, 1 \mathrm{H}), 2.50(\mathrm{~d}, J=14.5 \mathrm{~Hz}, 1 \mathrm{H}), 2.28-2.13(\mathrm{~m}, 1 \mathrm{H}), 2.13-1.99(\mathrm{~m}, 1 \mathrm{H}), 1.91-1.78(\mathrm{~m}$, 1H). ${ }^{13} \mathrm{C}$ NMR $\left(101 \mathrm{MHz}, \mathrm{CDCl}_{3}\right) \delta 147.6,147.2,143.0,137.5,137.4,130.4,128.6,128.5,128.5,127.8,127.8,127.4,127.4,127.1,115.3$, 113.9, 71.7, 71.4, 69.7, 58.9, 54.2, 46.1, 45.0, 41.3, 29.1, 25.3. ESI-MS m/z $568[\mathrm{M}+\mathrm{H}]^{+}$, HR-ESI-MS: m/z $568.2849[\mathrm{M}+\mathrm{H}]^{+}$, (calcd for $\left.\mathrm{C}_{39} \mathrm{H}_{38} \mathrm{NO}_{3}{ }^{+}, 568.2846\right)$.

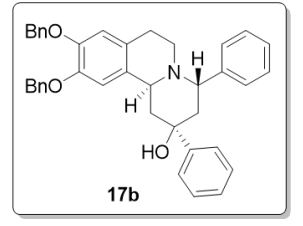

9,10-bis(benzyloxy)-2 $\alpha, 4 \alpha$-diphenyl-2,3,4,6,7,11b $\alpha$-hexahydro- $1 H$-pyrido[2,1-a]isoquinolin-2 $\beta$-ol

(17b). Yellow oil. ${ }^{1} \mathrm{H}$ NMR $\left(400 \mathrm{MHz}, \mathrm{CDCl}_{3}\right) \delta 7.70-7.09$ (m, 20H), $6.74(\mathrm{~s}, 1 \mathrm{H}), 6.71(\mathrm{~s}, 1 \mathrm{H}), 5.17$ (s, 2H), 5.09 (dd, $J$ $=23.8,12.0 \mathrm{~Hz}, 2 \mathrm{H}), 4.64(\mathrm{dd}, J=10.8,4.3 \mathrm{~Hz}, 1 \mathrm{H}), 4.26(\mathrm{t}, J=4.8 \mathrm{~Hz}, 1 \mathrm{H}), 3.37-3.25(\mathrm{~m}, 1 \mathrm{H}), 3.02-2.91(\mathrm{~m}$, $1 \mathrm{H}), 2.87-2.70(\mathrm{~m}, 2 \mathrm{H}), 2.54(\mathrm{dd}, J=14.5,6.5 \mathrm{~Hz}, 1 \mathrm{H}), 2.41-2.33(\mathrm{~m}, 1 \mathrm{H}), 2.24(\mathrm{dd}, J=13.6,11.1 \mathrm{~Hz}, 1 \mathrm{H})$, $2.04(\mathrm{dd}, J=13.6,2.5 \mathrm{~Hz}, 1 \mathrm{H}) .{ }^{13} \mathrm{C} \mathrm{NMR}\left(101 \mathrm{MHz}, \mathrm{CDCl}_{3}\right) \delta 148.0,147.7,147.4,143.0,137.5,137.4,131.5,128.7,128.5,128.4,128.3$, $127.7,127.7,127.7,127.5,127.3,127.3,127.2,126.9,126.8,124.6,115.3,113.9,73.6,71.7,71.5,60.5,51.1,45.9,41.7,40.4,28.1$. ESI-MS m/z $568[\mathrm{M}+\mathrm{H}]^{+}$, HR-ESI-MS: m/z $568.2850[\mathrm{M}+\mathrm{H}]^{+}$, (calcd for $\left.\mathrm{C}_{39} \mathrm{H}_{38} \mathrm{NO}_{3}{ }^{+}, 568.2846\right)$. 


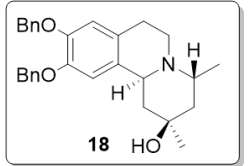

9,10-bis(benzyloxy)-2,4 $\alpha$-dimethyl-2,3,4,6,7,11b $\alpha$-hexahydro-1H-pyrido[2,1-a]isoquinolin-2 $\beta$-ol (18). Yellow crystal, mp 138-140 ${ }^{\circ} \mathrm{C} .{ }^{1} \mathrm{H}$ NMR $\left(400 \mathrm{MHz}, \mathrm{CDCl}_{3}\right) \delta 7.49-7.42(\mathrm{~m}, 4 \mathrm{H}), 7.40-7.28(\mathrm{~m}, 6 \mathrm{H}), 6.77(\mathrm{~s}, 1 \mathrm{H}), 6.67$ (s, $1 \mathrm{H}), 5.12(\mathrm{~d}, J=3.5 \mathrm{~Hz}, 4 \mathrm{H}), 4.06(\mathrm{~d}, J=10.4 \mathrm{~Hz}, 1 \mathrm{H}), 3.29-3.16(\mathrm{~m}, 1 \mathrm{H}), 3.04-2.85(\mathrm{~m}, 2 \mathrm{H}), 2.84-2.73(\mathrm{~m}, 1 \mathrm{H})$, 2.65-2.55 (m, 1H), 2.01-1.87 (m, 2H), $1.55(\mathrm{dd}, J=27.0,14.5 \mathrm{~Hz}, 2 \mathrm{H}), 1.28(\mathrm{~d}, J=6.7 \mathrm{~Hz}, 3 \mathrm{H}), 1.23(\mathrm{~s}, 2 \mathrm{H}) .{ }^{13} \mathrm{C} \mathrm{NMR}\left(101 \mathrm{MHz}, \mathrm{CDCl}_{3}\right)$ $\delta 147.6,147.3,137.8,137.7,132.1,128.6,128.4,127.9,127.9,127.8,127.7,127.6,115.6,113.8,72.1,71.6,70.5,54.6,50.1,48.4,45.3$, 43.1, 32.7, 29.4, 14.1. ESI-MS m/z $444[\mathrm{M}+\mathrm{H}]^{+}$, HR-ESI-MS: m/z $444.2530[\mathrm{M}+\mathrm{H}]^{+}$, (calcd for $\left.\mathrm{C}_{29} \mathrm{H}_{34} \mathrm{NO}_{3}{ }^{+}, 444.2533\right)$.

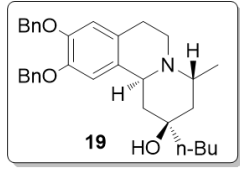

9,10-Bis(benzyloxy)-2-butyl-4 $\alpha$-methyl-2,3,4,6,7,11b $\alpha$-hexahydro-1 $H$-pyrido[2,1- $a]$ isoquinolin-2 $\beta$-ol

(19).

Yellow oil. ${ }^{1} \mathrm{H}$ NMR (400 MHz, Acetone) $\delta$ 7.55-7.43 (m, 4H), 7.43-7.22 (m, 6H), $6.85(\mathrm{~s}, 1 \mathrm{H}), 6.76(\mathrm{~s}, 1 \mathrm{H}), 5.10(\mathrm{~s}$, $4 \mathrm{H}), 3.23-3.12(\mathrm{~m}, 1 \mathrm{H}), 3.10(\mathrm{~s}, 1 \mathrm{H}), 2.89-2.78(\mathrm{~m}, 2 \mathrm{H}), 2.73-2.65(\mathrm{~m}, 1 \mathrm{H}), 2.65-2.48(\mathrm{~m}, 1 \mathrm{H}), 1.99(\mathrm{dt}, J=13.6$, $2.4 \mathrm{~Hz}, 1 \mathrm{H}), 1.80-1.67(\mathrm{~m}, 1 \mathrm{H}), 1.56(\mathrm{~d}, J=13.6 \mathrm{~Hz}, 1 \mathrm{H}), 1.45-1.28(\mathrm{~m}, 7 \mathrm{H}), 1.26(\mathrm{~d}, J=7.0 \mathrm{~Hz}, 3 \mathrm{H}), 0.91(\mathrm{t}, J=7.2 \mathrm{~Hz}, 3 \mathrm{H}) .{ }^{13} \mathrm{C} \mathrm{NMR}$ (101 MHz, Acetone) $\delta$ 147.3, 147.1, 138.0, 138.0, 132.9, 128.4, 128.3, 128.3, 127.6, 127.6, 127.5, 127.5, 115.0, 113.4, 71.2, 70.9, 70.8, $55.3,49.3,48.3,45.2,44.0,40.3,29.9,25.0,23.2,13.6,13.2$. ESI-MS m/z $486[\mathrm{M}+\mathrm{H}]^{+}$, HR-ESI-MS: m/z 486.2999 [M + Na $]^{+}$, (calcd for $\left.\mathrm{C}_{32} \mathrm{H}_{40} \mathrm{NO}_{3}{ }^{+}, 486.3003\right)$.

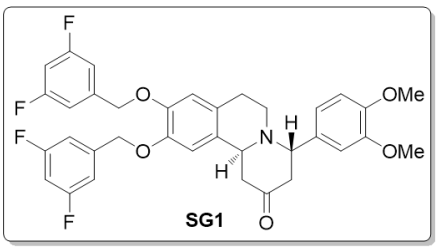

trans-9,10-Bis((3,5-difluorobenzyl)oxy)-4-(3,4-dimethoxyphenyl)-3,4,6,7-tetrahydro-1H-pyrido[2, 1-a] isoquinolin-2(11bH)-one (SG1). Yellow solid, mp 167-169 ${ }^{\circ} \mathrm{C} .{ }^{1} \mathrm{H}$ NMR $\left(400 \mathrm{MHz}, \mathrm{CDCl}_{3}\right) \delta$ 7.14-6.67 (m, 9H), $6.66(\mathrm{~s}, 1 \mathrm{H}), 6.56(\mathrm{~s}, 1 \mathrm{H}), 5.03(\mathrm{~s}, 4 \mathrm{H}), 4.37$ (t, $J=5.0 \mathrm{~Hz}, 1 \mathrm{H}), 4.15-4.03(\mathrm{~m}$, $1 \mathrm{H}), 3.87(\mathrm{~s}, 6 \mathrm{H}), 3.36-3.16(\mathrm{~m}, 1 \mathrm{H}), 3.00-2.72(\mathrm{~m}, 5 \mathrm{H}), 2.77-2.60(\mathrm{~m}, 2 \mathrm{H}) .{ }^{13} \mathrm{C} \mathrm{NMR}(101 \mathrm{MHz}$, $\left.\mathrm{CDCl}_{3}\right) \delta 209.0,164.7,164.7,164.6,164.6,162.2,162.1,162.1,149.2,148.7,147.4,147.0,141.5,141.4,141.3,141.3,141.2,132.6,130.5$, 128.0, 120.5, 115.4, 113.2, 111.6, 110.99, 110.0, 110.0, 109.9, 109.8, 109.8, 109.7, 103.7, 103.4, 103.4, 103.2, 103.2, 70.5, 70.3, 63.6, 56.2, 56.10, 54.7, 46.9, 46.4, 44.2, 28.6. ESI-MS m/z $622[\mathrm{M}+\mathrm{H}]^{+}$, HR-ESI-MS: m/z $622.2215[\mathrm{M}+\mathrm{H}]^{+}$, (calcd for $\left.\mathrm{C}_{35} \mathrm{H}_{32} \mathrm{~F}_{4} \mathrm{NO}_{5}{ }^{+}, 622.2211\right)$.

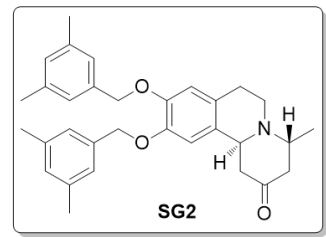

trans-9,10-Bis((3,5-dimethylbenzyl)oxy)-4-methyl-3,4,6,7-tetrahydro-1 $H$-pyrido[2,1-a]isoquinolin-2(11bH) -one (SG2). White solid, mp 133-135 ${ }^{\circ} \mathrm{C} .{ }^{1} \mathrm{H}$ NMR $\left(400 \mathrm{MHz}, \mathrm{CDCl}_{3}\right) \delta 7.07$ (d, J=6.1 Hz, 4H), $6.94(\mathrm{~s}, 2 \mathrm{H})$, $6.72(\mathrm{~s}, 1 \mathrm{H}), 6.64(\mathrm{~s}, 1 \mathrm{H}), 5.04(\mathrm{~d}, J=11.4 \mathrm{~Hz}, 4 \mathrm{H}), 4.18-4.08(\mathrm{~m}, 1 \mathrm{H}), 3.64-3.53(\mathrm{~m}, 1 \mathrm{H}), 3.16-3.05(\mathrm{~m}, 1 \mathrm{H})$, 2.99-2.76 (m, 4H), 2.67-2.56 (m, 1H), $2.48(\mathrm{dd}, J=13.9,11.3 \mathrm{~Hz}, 1 \mathrm{H}), 2.32(\mathrm{~s}, 12 \mathrm{H}), 2.26-2.22(\mathrm{~m}, 1 \mathrm{H}), 1.13$ $(\mathrm{d}, J=6.8 \mathrm{~Hz}, 3 \mathrm{H}) .{ }^{13} \mathrm{C}$ NMR $\left(101 \mathrm{MHz}, \mathrm{CDCl}_{3}\right) \delta 209.4,148.3,147.7,138.2,137.4,137.4,130.3,129.6,129.6,127.3,125.4,125.3,115.5$, 113.3, 72.0, 71.7, 57.8, 54.1, 47.6, 47.2, 46.5, 29.7, 21.5, 15.1. ESI-MS m/z 484 [M + H] $]^{+}$, HR-ESI-MS: m/z 484.2846 [M + H] , (calcd for $\left.\mathrm{C}_{32} \mathrm{H}_{38} \mathrm{NO}_{3}{ }^{+}, 483.2835\right)$

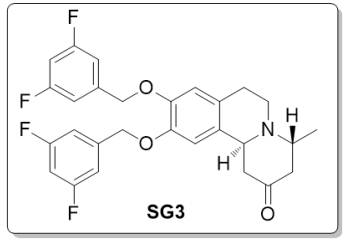

trans-9,10-Bis((3,5-difluorobenzyl)oxy)-4-methyl-3,4,6,7-tetrahydro-1H-pyrido[2,1-a]isoquinolin-2(11b H)-one (SG3). Yellow powder, mp 168-169 ${ }^{\circ} \mathrm{C} .{ }^{1} \mathrm{H}$ NMR $\left(400 \mathrm{MHz}, \mathrm{CDCl}_{3}\right) \delta 6.97(\mathrm{~d}, J=7.6 \mathrm{~Hz}, 4 \mathrm{H})$, 6.82-6.71 (m, 2H), $6.66(\mathrm{~s}, 1 \mathrm{H}), 6.60(\mathrm{~s}, 1 \mathrm{H}), 5.08(\mathrm{~d}, J=12.3 \mathrm{~Hz}, 4 \mathrm{H}), 4.16-4.06(\mathrm{~m}, 1 \mathrm{H}), 3.64-3.52(\mathrm{~m}$, $1 \mathrm{H}), 3.20-3.04(\mathrm{~m}, 1 \mathrm{H}), 3.02-2.74(\mathrm{~m}, 4 \mathrm{H}), 2.67-2.56(\mathrm{~m}, 1 \mathrm{H}), 2.49(\mathrm{dd}, J=13.7,11.4 \mathrm{~Hz}, 1 \mathrm{H}), 2.31-2.18$ $(\mathrm{m}, 1 \mathrm{H}), 1.13(\mathrm{~d}, J=6.8 \mathrm{~Hz}, 3 \mathrm{H}) .{ }^{13} \mathrm{C} \mathrm{NMR}\left(101 \mathrm{MHz}, \mathrm{CDCl}_{3}\right) \delta 164.7,164.6,162.2,162.1,147.4,147.0,141.5,141.4,141.3,141.2$, $131.0,128.1,115.3,112.9,110.0,110.0,110.0,109.9$, 109.8, 109.8, 109.8, 109.8, 103.7, 103.7, 103.4, 103.4, 103.2, 103.2, 100.2, 70.5,

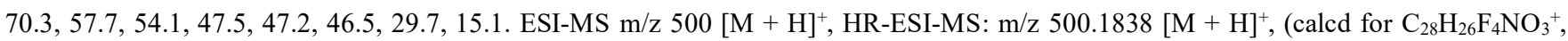
$500.1843)$

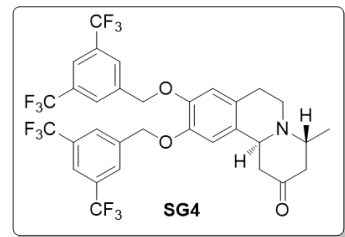

trans-9,10-Bis((3,5-bis(trifluoromethyl)benzyl)oxy)-4-methyl-3,4,6,7-tetrahydro-1H-pyrido[2,1-a]isoqui nolin-2(11bH)-one (SG4). White powder, mp 173-174 ${ }^{\circ} \mathrm{C} .{ }^{1} \mathrm{H}$ NMR $\left(400 \mathrm{MHz}, \mathrm{CDCl}_{3}\right) \delta 7.86(\mathrm{~d}, J=18.2$ $\mathrm{Hz}, 6 \mathrm{H}), 6.76(\mathrm{~s}, 1 \mathrm{H}), 6.70(\mathrm{~s}, 1 \mathrm{H}), 5.18(\mathrm{~d}, J=11.0 \mathrm{~Hz}, 4 \mathrm{H}), 4.17(\mathrm{dd}, J=10.9,2.8 \mathrm{~Hz}, 1 \mathrm{H}), 3.67-3.50$ (m, 
1H), 3.23-3.08 (m, 1H), 3.07-2.94 (m, 1H), 2.94-2.77 (m, 3H), 2.70-2.65 (m, 1H), 2.58-2.45 (m, 1H), 2.33-2.21 (m, $1 \mathrm{H}), 1.15(\mathrm{~d}, J=6.8$ $\mathrm{Hz}, 3 \mathrm{H}) .{ }^{13} \mathrm{C}$ NMR $\left(101 \mathrm{MHz}, \mathrm{CDCl}_{3}\right) \delta 208.9,147.4,147.0,139.8,139.7,132.7,132.3,132.0,131.7,128.8,127.4,124.7,122.2,122.2$, 122.1, 122.0, 119.3, 115.8, 113.3, 70.6, 70.3, 57.7, 54.1, 47.4, 47.2, 46.5, 29.7, 15.1. ESI-MS m/z 700 [M + H] $]^{+}$HR-ESI-MS: m/z $700.1721[\mathrm{M}+\mathrm{H}]^{+}$, (calcd for $\left.\mathrm{C}_{32} \mathrm{H}_{26} \mathrm{~F}_{12} \mathrm{NO}_{3}{ }^{+}, 700.1716\right)$.

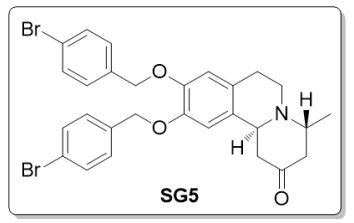

trans-9,10-Bis((4-bromobenzyl)oxy)-4-methyl-3,4,6,7-tetrahydro-1 $H$-pyrido[2,1-a]isoquinolin-2(11bH)one (SG5). Yellow oil. ${ }^{1} \mathrm{H}$ NMR $\left(400 \mathrm{MHz}, \mathrm{CDCl}_{3}\right) \delta 7.49(\mathrm{dd}, J=8.3,1.8 \mathrm{~Hz}, 4 \mathrm{H}), 7.29(\mathrm{dd}, J=8.6,4.1 \mathrm{~Hz}$, 4H), $6.66(\mathrm{~s}, 1 \mathrm{H}), 6.59(\mathrm{~s}, 1 \mathrm{H}), 5.04(\mathrm{~d}, J=11.2 \mathrm{~Hz}, 4 \mathrm{H}), 4.15-4.06(\mathrm{~m}, 1 \mathrm{H}), 3.69-3.45(\mathrm{~m}, 1 \mathrm{H}), 3.16-3.02$ (m, 1H), 3.01-2.69 (m, 4H), 2.66-2.55 (m, 1H), $2.47(\mathrm{dd}, J=13.7,11.4 \mathrm{~Hz}, 1 \mathrm{H}), 2.3-2.16(\mathrm{~m}, 1 \mathrm{H}), 1.12(\mathrm{~d}$, $J=6.8 \mathrm{~Hz}, 3 \mathrm{H}) .{ }^{13} \mathrm{C} \mathrm{NMR}\left(101 \mathrm{MHz}, \mathrm{CDCl}_{3}\right) \delta 209.0,147.5,147.0,136.2,136.2,131.6,130.5,129.0,128.9,127.6,121.8,121.8,115.3$, 112.9, 70.9, 70.6, 57.5, 53.9, 47.3, 47.0, 46.3, 29.5, 14.8. ESI-MS m/z $584[\mathrm{M}+\mathrm{H}]^{+}$, HR-ESI-MS: m/z $584.0436[\mathrm{M}+\mathrm{H}]^{+}$, (calcd for $\mathrm{C}_{28} \mathrm{H}_{28} \mathrm{Br}_{2} \mathrm{NO}_{3}{ }^{+}, 584.0430$ ).

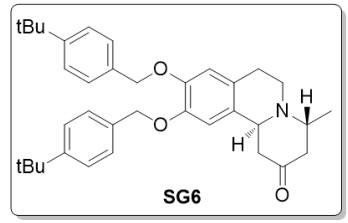

trans-9,10-Bis((4-(tert-butyl)benzyl)oxy)-4-methyl-3,4,6,7-tetrahydro-1H-pyrido[2,1- $a$ ]isoquinolin-2(11 bH)-one (SG6). Yellow crystal, mp 68-70 ${ }^{\circ} \mathrm{C} .{ }^{1} \mathrm{H}$ NMR $\left(400 \mathrm{MHz}, \mathrm{CDCl}_{3}\right) \delta$ 7.47-7.27 (m, 8H), $6.72(\mathrm{~s}, 1 \mathrm{H})$, $6.61(\mathrm{~s}, 1 \mathrm{H}), 5.09(\mathrm{~d}, J=11.5 \mathrm{~Hz}, 4 \mathrm{H}), 4.17-4.00(\mathrm{~m}, 1 \mathrm{H}), 3.64-3.50(\mathrm{~m}, 1 \mathrm{H}), 3.13-3.02(\mathrm{~m}, 1 \mathrm{H}), 2.99-2.73$ (m, 4H), 2.66-2.55 (m, 1H), $2.43(\mathrm{dd}, J=25.7,11.9 \mathrm{~Hz}, 1 \mathrm{H}), 2.23(\mathrm{~d}, J=13.4 \mathrm{~Hz}, 1 \mathrm{H}), 1.33(\mathrm{~d}, J=3.1 \mathrm{~Hz}$, $18 \mathrm{H}), 1.12(\mathrm{~d}, J=7.0 \mathrm{~Hz}, 3 \mathrm{H}) .{ }^{13} \mathrm{C}$ NMR $\left(101 \mathrm{MHz}, \mathrm{CDCl}_{3}\right) \delta 209.4,151.0,151.0,148.3,147.7,134.5,134.5,130.3,128.0,127.6,127.5$, 127.3, 125.9, 125.6, 125.6, 115.6, 113.5, 71.9, 71.5, 57.8, 54.1, 47.6, 47.2, 46.5, 34.8, 31.6, 31.6, 29.7, 15.0. ESI-MS m/z 540 [M + H] HR-ESI-MS: m/z $540.3472[\mathrm{M}+\mathrm{H}]^{+}$, (calcd for $\left.\mathrm{C}_{36} \mathrm{H}_{46} \mathrm{NO}_{3}{ }^{+}, 540.3472\right)$.

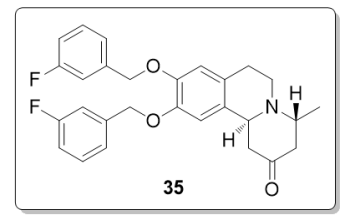

trans-9,10-Bis((3-fluorobenzyl)oxy)-4-methyl-3,4,6,7-tetrahydro- $1 H$-pyrido[2,1-a]isoquinolin-2(11bH)-o ne (35). White solid, mp 130-131 ${ }^{\circ} \mathrm{C} .{ }^{1} \mathrm{H}$ NMR $\left(400 \mathrm{MHz}, \mathrm{CDCl}_{3}\right) \delta$ 7.43-7.28 (m, 2H), 7.27-7.09 (m, 4H), $7.10-6.91(\mathrm{~m}, 2 \mathrm{H}), 6.68(\mathrm{~s}, 1 \mathrm{H}), 6.60(\mathrm{~s}, 1 \mathrm{H}), 5.11(\mathrm{~d}, J=11.0 \mathrm{~Hz}, 4 \mathrm{H}), 4.12(\mathrm{dd}, J=11.1,3.0 \mathrm{~Hz}, 1 \mathrm{H})$, $3.63-3.51(\mathrm{~m}, 1 \mathrm{H}), 3.18-3.03(\mathrm{~m}, 1 \mathrm{H}), 3.02-2.71(\mathrm{~m}, 4 \mathrm{H}), 2.69-2.56(\mathrm{~m}, 1 \mathrm{H}), 2.47(\mathrm{dd}, J=13.7,11.4 \mathrm{~Hz}$, $1 \mathrm{H}), 2.30-2.15(\mathrm{~m}, 1 \mathrm{H}), 1.12(\mathrm{~d}, J=7.0 \mathrm{~Hz}, 3 \mathrm{H}) .{ }^{13} \mathrm{C} \mathrm{NMR}\left(101 \mathrm{MHz}, \mathrm{CDCl}_{3}\right) \delta$ 209.2, 164.4, 162.0, 147.7, 147.2, 140.1, 140.0, 139.9, $130.7,130.3,130.2,127.7,122.8,122.8,122.8,122.7,115.4,115.1,115.0,114.9,114.8,114.4,114.4,114.2,114.2,113.0,71.0,70.7,57.7$, 54.1, 47.5, 47.2, 46.5, 29.7, 15.1. ESI-MS m/z $464[\mathrm{M}+\mathrm{H}]^{+}$, HR-ESI-MS: m/z $464.2030[\mathrm{M}+\mathrm{H}]^{+}$, (calcd for $\mathrm{C}_{28} \mathrm{H}_{27} \mathrm{~F}_{2} \mathrm{NO}_{3}, 464.2032$ ).

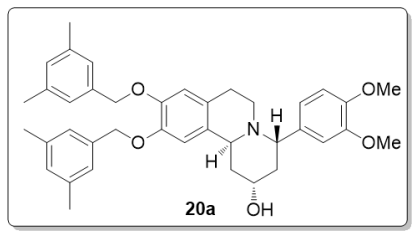

4 $\alpha$-(3,4-Dimethoxyphenyl)-9,10-bis((3,5-dimethylbenzyl)oxy)-2,3,4,6,7,11b $\alpha$-hexahydro- $1 H$-pyrido[ 2,1-a]isoquinolin-2 $\alpha$-ol (20a). Yellow oil. ${ }^{1} \mathrm{H}$ NMR $\left(400 \mathrm{MHz}, \mathrm{CDCl}_{3}\right) \delta 7.13-6.79(\mathrm{~m}, 8 \mathrm{H}), 6.78-6.71$ $(\mathrm{m}, 1 \mathrm{H}), 6.64(\mathrm{~s}, 2 \mathrm{H}), 5.07-4.82(\mathrm{~m}, 4 \mathrm{H}), 4.11-3.89(\mathrm{~m}, 3 \mathrm{H}), 3.84-3.74(\mathrm{~m}, 7 \mathrm{H}), 3.22-3.05(\mathrm{~m}, 1 \mathrm{H})$, 2.85-2.52 (m, 3H), 2.33-2.07 (m, 13H), 2.02-1.84 (m, 2H), 1.84-1.71 (m, 1H). ${ }^{13} \mathrm{C}$ NMR $(101 \mathrm{MHz}$, $\left.\mathrm{CDCl}_{3}\right) \delta 149.3,148.1,147.4,138.2,137.6,137.5,129.6,125.4,125.4,119.6,115.6,114.2,111.1,110.9,72.1,71.7,66.3,59.3,56.2,56.1$, 53.6, 46.6, 37.2, 36.0, 27.7, 21.5, 21.5. ESI-MS m/z $608[\mathrm{M}+\mathrm{H}]^{+}$, HR-ESI-MS: m/z $608.3372[\mathrm{M}+\mathrm{H}]^{+},\left(\right.$calcd for $\mathrm{C}_{39} \mathrm{H}_{46} \mathrm{NO}_{5}{ }^{+}$, $608.3371)$.

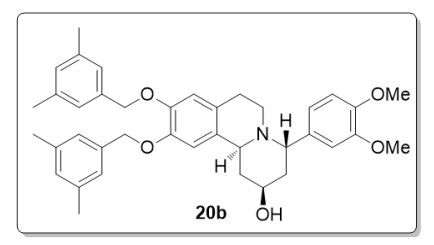

4 $\alpha$-(3,4-Dimethoxyphenyl)-9,10-bis((3,5-dimethylbenzyl)oxy)-2,3,4,6,7,11b $\alpha$-hexahydro- $1 H$-pyrido[

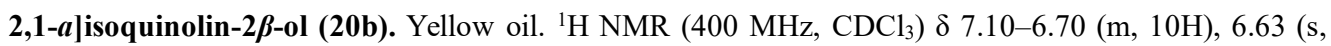
1H), 5.10-4.95 (m, 4H), 4.54-4.31 (m, 1H), 3.82 (d, $J=13.1 \mathrm{~Hz}, 7 \mathrm{H}), 3.57-3.41(\mathrm{~m}, 2 \mathrm{H}), 2.93-2.70$ (m, $3 \mathrm{H}), 2.52(\mathrm{~d}, J=11.7 \mathrm{~Hz}, 1 \mathrm{H}), 2.30-2.15(\mathrm{~m}, 14 \mathrm{H}), 2.01-1.89(\mathrm{~m}, 2 \mathrm{H}), 1.86(\mathrm{~d}, J=11.1 \mathrm{~Hz}, 1 \mathrm{H})$, $1.55-1.34(\mathrm{~m}, 1 \mathrm{H}) .{ }^{13} \mathrm{C}$ NMR $\left(101 \mathrm{MHz}, \mathrm{CDCl}_{3}\right) \delta 149.4,148.2,147.6,138.1,138.1,137.5,137.2,129.5,129.3,125.3,125.2,119.8,115.5$, 113.7, 110.9, 110.1, 72.3, 71.5, 65.0, 57.7, 57.1, 56.1, 55.9, 46.5, 44.6, 37.0, 22.5, 21.3, 21.3. ESI-MS m/z 608 [M + H] ${ }^{+}$, HR-ESI-MS: m/z $608.3373[\mathrm{M}+\mathrm{H}]^{+}$, (calcd for $\left.\mathrm{C}_{39} \mathrm{H}_{46} \mathrm{NO}_{5}{ }^{+}, 608.3371\right)$. 


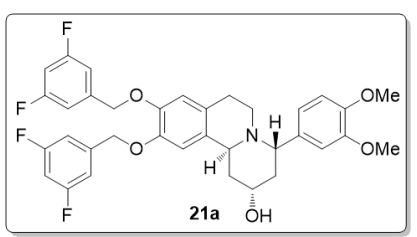

9,10-Bis((3,5-difluorobenzyl)oxy)-4 $\alpha$-(3,4-dimethoxyphenyl)-2,3,4,6,7,11b $\alpha$-hexahydro- $1 H$-pyrido[ 2,1-a]isoquinolin-2 $\alpha$-ol (21a). Yellow oil. ${ }^{1} \mathrm{H}$ NMR $\left(400 \mathrm{MHz}, \mathrm{CDCl}_{3}\right) \delta 7.10(\mathrm{~s}, 1 \mathrm{H}), 7.06-6.88(\mathrm{~m}$, $5 \mathrm{H}), 6.82(\mathrm{~d}, J=8.3 \mathrm{~Hz}, 1 \mathrm{H}), 6.79-6.69(\mathrm{~m}, 2 \mathrm{H}), 6.69-6.54(\mathrm{~m}, 2 \mathrm{H}), 5.17-4.99(\mathrm{~m}, 4 \mathrm{H}), 4.15(\mathrm{t}, J=4.4$ $\mathrm{Hz}, 1 \mathrm{H}), 4.11-3.97(\mathrm{~m}, 2 \mathrm{H}), 3.34-3.13(\mathrm{~m}, 1 \mathrm{H}), 2.93-2.60(\mathrm{~m}, 3 \mathrm{H}), 2.36-2.21(\mathrm{~m}, 1 \mathrm{H}), 2.04-1.80(\mathrm{~m}, 3 \mathrm{H}) .{ }^{13} \mathrm{C} \mathrm{NMR}\left(101 \mathrm{MHz}, \mathrm{CDCl}_{3}\right) \delta$ 164.6, 164.6, 164.5, 164.5, 162.2, 162.1, 162.0, 162.0, 149.3, 148.0, 147.2, 146.7, 141.6, 141.5, 141.4, 134.6, 131.9, 128.5, 119.5, 115.4, 113.9, 111.1, 111.0, 110.0, 109.9, 109.9, 109.9, 109.8, 109.8, 109.7, 109.7, 103.6, 103.3, 103.1, 70.6, 70.2, 66.2, 60.1, 56.2, 56.0, 53.3, 46.3, 37.4, 35.2, 28.2. ESI-MS m/z $624[\mathrm{M}+\mathrm{H}]^{+}$, HR-ESI-MS: $\mathrm{m} / \mathrm{z} 624.2366[\mathrm{M}+\mathrm{H}]^{+}$, (calcd for $\left.\mathrm{C}_{35} \mathrm{H}_{34} \mathrm{~F}_{4} \mathrm{NO}_{5}{ }^{+}, 624.2368\right)$.

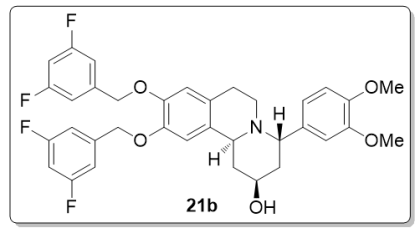

9,10-Bis((3,5-difluorobenzyl)oxy)-4 $\alpha$-(3,4-dimethoxyphenyl)-2,3,4,6,7,11b $\alpha$-hexahydro- $1 H$-pyrido[

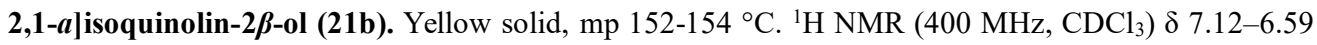
$(\mathrm{m}, 10 \mathrm{H}), 6.57(\mathrm{~s}, 1 \mathrm{H}), 5.03(\mathrm{~d}, J=7.6 \mathrm{~Hz}, 4 \mathrm{H}), 4.39(\mathrm{~s}, 1 \mathrm{H}), 3.90-3.71(\mathrm{~m}, 6 \mathrm{H}), 3.63-3.37(\mathrm{~m}, 2 \mathrm{H})$, 3.09-2.67 (m, 3H), 2.65-2.43 (m, 1H), 2.23-2.10 (m, 1H), 1.97-1.82 (m, 2H), 1.61-1.50 (m, 1H). ${ }^{13} \mathrm{C}$ NMR $\left(101 \mathrm{MHz}, \mathrm{CDCl}_{3}\right) \delta 164.7,164.7,164.6,164.6,162.2,162.2,162.1,162.1,149.6,148.5,147.5,147.1,141.6,141.5,141.5,141.5$, 141.4, 141.3, 135.9, 129.8, 129.3, 119.9, 115.7, 113.4, 111.10, 110.3, 110.2, 110.1, 110.0, 109.9, 109.8, 109.8, 103.7, 103.4, 103.2, 70.9, 70.2, 65.4, 57.7, 57.1, 56.2, 56.1, 46.6, 45.3, 37.1, 22.8. ESI-MS m/z $624[\mathrm{M}+\mathrm{H}]^{+}$, HR-ESI-MS: m/z $624.2367[\mathrm{M}+\mathrm{H}]^{+}$, (calcd for $\left.\mathrm{C}_{35} \mathrm{H}_{34} \mathrm{~F}_{4} \mathrm{NO}_{5}{ }^{+}, 624.2368\right)$.

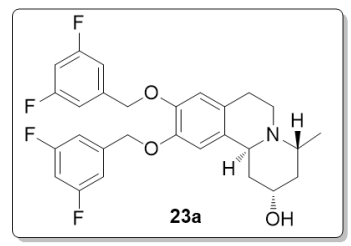

9,10-Bis((3,5-difluorobenzyl)oxy)-4 $\alpha$-methyl-2,3,4,6,7,11b $\alpha$-hexahydro-1H-pyrido[2,1-a]isoquinolin-2 $\alpha$ ol (23a). Pale yellow crystal, mp 145-146 ${ }^{\circ} \mathrm{C} .{ }^{1} \mathrm{H}$ NMR (400 MHz, $\left.\mathrm{CDCl}_{3}\right) \delta 7.0-6.91$ (m, 4H), 6.81-6.69 $(\mathrm{m}, 3 \mathrm{H}), 6.63(\mathrm{~s}, 1 \mathrm{H}), 5.14-5.00(\mathrm{~m}, 4 \mathrm{H}), 4.19-3.98(\mathrm{~m}, 1 \mathrm{H}), 3.76(\mathrm{~d}, J=10.5 \mathrm{~Hz}, 1 \mathrm{H}), 3.40-3.25(\mathrm{~m}, 1 \mathrm{H})$, $3.00-2.84(\mathrm{~m}, 2 \mathrm{H}), 2.78-2.58(\mathrm{~m}, 2 \mathrm{H}), 2.35-2.28(\mathrm{~m}, 1 \mathrm{H}), 1.92-1.82(\mathrm{~m}, 1 \mathrm{H}), 1.75(\mathrm{td}, J=11.8,5.2 \mathrm{~Hz}, 1 \mathrm{H})$, $1.40(\mathrm{dd}, J=23.1,11.5 \mathrm{~Hz}, 1 \mathrm{H}), 1.12(\mathrm{~d}, J=7.0 \mathrm{~Hz}, 3 \mathrm{H}) .{ }^{13} \mathrm{C} \mathrm{NMR}\left(101 \mathrm{MHz}, \mathrm{CDCl}_{3}\right) \delta 164.7,164.6,162.2,162.1,147.1,146.8,141.6$, 141.5, 132.2, 128.7, 115.3, 113.2, 110.1, 110.0, 109.9, 109.9, 109.8, 109.7, 103.6, 103.4, 103.3, 103.1, 70.7, 70.2, 66.0, 55.9, 52.3, 48.2, 41.6, 39.7, 29.9, 12.7. ESI-MS m/z $502[\mathrm{M}+\mathrm{H}]^{+}$, HR-ESI-MS: m/z 502.1995 [M + H] $]^{+}$, (calcd for $\mathrm{C}_{28} \mathrm{H}_{26} \mathrm{~F}_{4} \mathrm{NO}_{3}{ }^{+}, 502.2000$ ).

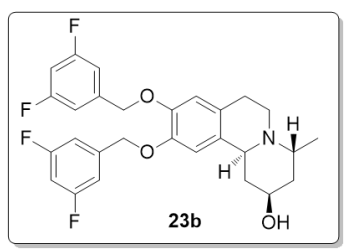

9,10-Bis((3,5-difluorobenzyl)oxy)-4 $\alpha$-methyl-2,3,4,6,7,11b $\alpha$-hexahydro-1 $H$-pyrido[2,1- $a]$ isoquinolin-2 $\beta$-o I (23b). Colorless needle crystal, mp 131-133 ${ }^{\circ} \mathrm{C} .{ }^{1} \mathrm{H}$ NMR $\left(400 \mathrm{MHz}, \mathrm{CDCl}_{3}\right) \delta 6.96-6.84(\mathrm{~m}, 4 \mathrm{H}), 6.74(\mathrm{~s}$, $1 \mathrm{H}), 6.70-6.61(\mathrm{~m}, 2 \mathrm{H}), 6.54(\mathrm{~s}, 1 \mathrm{H}), 4.99(\mathrm{~s}, 4 \mathrm{H}), 4.21(\mathrm{~s}, 1 \mathrm{H}), 3.71-3.62(\mathrm{~m}, 1 \mathrm{H}), 3.24(\mathrm{dd}, J=12.2,4.2 \mathrm{~Hz}$, $1 \mathrm{H}), 3.00-2.70(\mathrm{~m}, 3 \mathrm{H}), 2.44-2.31(\mathrm{~m}, 1 \mathrm{H}), 2.27-2.17$ (m, 1H), 2.08 (brs, 1H), 1.93-1.79 (m, 2H), 1.42-1.29 $(\mathrm{m}, 1 \mathrm{H}), 1.14(\mathrm{~d}, J=6.5 \mathrm{~Hz}, 3 \mathrm{H}) .{ }^{13} \mathrm{C} \mathrm{NMR}\left(101 \mathrm{MHz}, \mathrm{CDCl}_{3}\right) \delta 164.7,164.7,164.6,164.5,162.2,162.2,162.1,162.1,147.2,146.9$, 141.6, 141.5, 141.4, 141.4, 130.7, 129.2, 115.5, 113.3, 110.1, 110.1, 110.0, 109.9, 109.9, 109.9, 109.8, 109.7, 103.6, 103.4, 103.1, 70.8, 70.2, 65.7, 55.1, 48.8, 47.2, 42.0, 38.0, 24.7, 18.6. ESI-MS m/z $502[\mathrm{M}+\mathrm{H}]^{+}$, HR-ESI-MS: m/z $502.1996[\mathrm{M}+\mathrm{H}]^{+}$, (calcd for $\left.\mathrm{C}_{28} \mathrm{H}_{26} \mathrm{~F}_{4} \mathrm{NO}_{3}{ }^{+}, 502.2000\right)$.

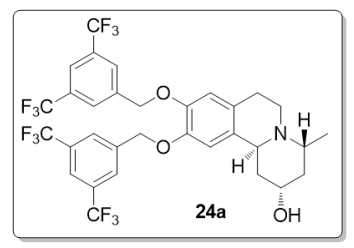

9,10-Bis((3,5-bis(trifluoromethyl)benzyl)oxy)-4 $\alpha$-methyl-2,3,4,6,7,11b $\alpha$-hexahydro-1 $H$-pyrido[2,1-a]iso quinolin-2 $\alpha$-ol (24a). Colorless crystal, mp $49-51{ }^{\circ} \mathrm{C} .{ }^{1} \mathrm{H}$ NMR $\left(400 \mathrm{MHz}, \mathrm{CDCl}_{3}\right) \delta 7.98-7.72$ (m, 6H), 6.82 (s, 1H), $6.72(\mathrm{~s}, 1 \mathrm{H}), 5.18(\mathrm{~s}, 4 \mathrm{H}), 4.17-3.98(\mathrm{~m}, 1 \mathrm{H}), 3.85-3.67(\mathrm{~m}, 1 \mathrm{H}), 3.39-3.28(\mathrm{~m}, 1 \mathrm{H}), 3.04-2.84(\mathrm{~m}$, 2H), 2.79-2.62 (m, 2H), 2.41-2.31 (m, 1H), 1.94-1.82 (m, 1H), $1.77(\mathrm{td}, J=11.7,5.1 \mathrm{~Hz}, 1 \mathrm{H}), 1.43(\mathrm{dd}, J=$ 23.2, $11.6 \mathrm{~Hz}, 1 \mathrm{H}), 1.14(\mathrm{~d}, J=7.0 \mathrm{~Hz}, 3 \mathrm{H}) .{ }^{13} \mathrm{C} \mathrm{NMR}\left(101 \mathrm{MHz}, \mathrm{CDCl}_{3}\right) \delta 147.1,146.7,140.0,139.9,132.9,132.6,132.3,132.3,132.0$, 132.0, 131.6, 131.6, 129.4, 127.4, 124.8, 122.2, 122.1, 122.0, 119.3, 115.7, 113.7, 70.8, 70.3, 66.0, 55.9, 52.35, 48.2, 41.6, 39.7, $29.9,12.7$. ESI-MS m/z $702[\mathrm{M}+\mathrm{H}]^{+}$, HR-ESI-MS: m/z $702.1874[\mathrm{M}+\mathrm{H}]^{+},\left(\right.$calcd for $\left.\mathrm{C}_{32} \mathrm{H}_{28} \mathrm{~F}_{12} \mathrm{NO}_{3}{ }^{+}, 702.1872\right)$. 


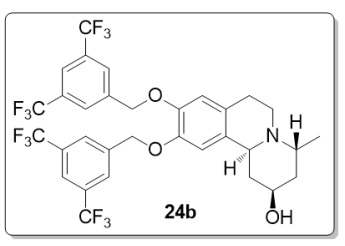

9,10-Bis((3,5-bis(trifluoromethyl)benzyl)oxy)-4 $\alpha$-methyl-2,3,4,6,7,11b $\alpha$-hexahydro-1 $H$-pyrido[2,1- $a$ ]isoq

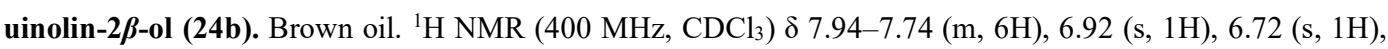
$5.19(\mathrm{~s}, 4 \mathrm{H}), 4.32(\mathrm{~s}, 1 \mathrm{H}), 3.86-3.69(\mathrm{~m}, 1 \mathrm{H}), 3.43-3.25(\mathrm{~m}, 1 \mathrm{H}), 3.14-2.81(\mathrm{~m}, 3 \mathrm{H}), 2.56-2.45(\mathrm{~m}, 1 \mathrm{H})$, 2.41-2.24 (m, 1H), 1.99-1.90 (m, 2H), $1.52-1.41(\mathrm{~m}, 1 \mathrm{H}), 1.24(\mathrm{~d}, J=6.4 \mathrm{~Hz}, 3 \mathrm{H}) .{ }^{13} \mathrm{C}$ NMR $(101 \mathrm{MHz}$, $\left.\mathrm{CDCl}_{3}\right) \delta 147.2,147.0,139.9,139.9,132.7,132.6,132.3,132.3,132.0,132.0,131.7,130.0,127.4,124.8,122.2,122.2,122.1,122.1,122.0$, $119.3,116.0,113.8,70.9,70.3,65.8,54.8,49.1,47.2,42.0,38.1,25.0,18.5$. ESI-MS m/z $702[\mathrm{M}+\mathrm{H}]^{+}$, HR-ESI-MS: m/z 702.1874 [M + $\mathrm{H}]^{+}$, (calcd for $\mathrm{C}_{32} \mathrm{H}_{28} \mathrm{~F}_{12} \mathrm{NO}_{3}{ }^{+}, 702.1872$ ).

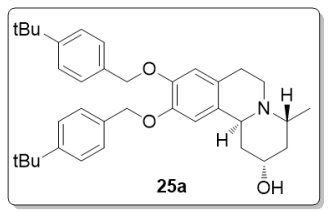

9,10-Bis((4-(tert-butyl)benzyl)oxy)-4 $\alpha$-methyl-2,3,4,6,7,11b $\alpha$-hexahydro-1 $H$-pyrido[2,1- $a]$ isoquinolin-2 $\alpha$ ol (25a). Yellow crystal, mp 59-61 ${ }^{\circ} \mathrm{C} .{ }^{1} \mathrm{H}$ NMR $\left(400 \mathrm{MHz}, \mathrm{CDCl}_{3}\right) \delta$ 7.54-7.27 (m, 8H), 6.75 (s, 1H), 6.68 (s, $1 \mathrm{H}), 5.08(\mathrm{~d}, J=2.9 \mathrm{~Hz}, 4 \mathrm{H}), 4.19-3.96(\mathrm{~m}, 1 \mathrm{H}), 3.91-3.68(\mathrm{~m}, 1 \mathrm{H}), 3.40-3.26(\mathrm{~m}, 1 \mathrm{H}), 2.94(\mathrm{~d}, J=5.9 \mathrm{~Hz}$, 2H), 2.79-2.54 (m, 2H), 2.37-2.26 (m, 1H), 1.94-1.82 (m, 1H), $1.76(\mathrm{td}, J=11.7,5.0 \mathrm{~Hz}, 1 \mathrm{H}), 1.42-1.27(\mathrm{~m}, 17 \mathrm{H}), 1.14(\mathrm{t}, J=11.2 \mathrm{~Hz}$, 3H). ${ }^{13} \mathrm{C} \mathrm{NMR}\left(101 \mathrm{MHz}, \mathrm{CDCl}_{3}\right) \delta 151.0,150.9,148.0,147.5,134.7,134.7,131.3,127.9,127.6,127.5,125.9,125.6,115.6,113.8,72.1$, 71.5, 66.1, 55.8, 52.5, 48.3, 41.5, 39.7, 34.8, 31.6, 29.7, 12.9. ESI-MS m/z $542[\mathrm{M}+\mathrm{H}]^{+}$, HR-ESI-MS: m/z 542.3624 [M + H] $]^{+}$, (calcd for $\left.\mathrm{C}_{36} \mathrm{H}_{48} \mathrm{NO}_{3}{ }^{+}, 542.3629\right)$.

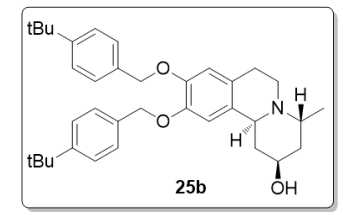

9,10-Bis((4-(tert-butyl)benzyl)oxy)-4 $\alpha$-methyl-2,3,4,6,7,11b $\alpha$-hexahydro-1H-pyrido[2,1-a]isoquinolin-2 $\beta$-o I (25b). Yellow solid, mp 72-74 ${ }^{\circ} \mathrm{C} .{ }^{1} \mathrm{H}$ NMR $\left(400 \mathrm{MHz}, \mathrm{CDCl}_{3}\right) \delta 7.48-7.19(\mathrm{~m}, 8 \mathrm{H}), 6.75(\mathrm{~s}, 1 \mathrm{H}), 6.60$ (s, 1H), $5.12-4.89(\mathrm{~m}, 4 \mathrm{H}), 4.26(\mathrm{~s}, 1 \mathrm{H}), 3.55(\mathrm{~s}, 1 \mathrm{H}), 3.29(\mathrm{dd}, J=13.0,5.2 \mathrm{~Hz}, 1 \mathrm{H}), 2.99(\mathrm{td}, J=12.7,4.4 \mathrm{~Hz}, 1 \mathrm{H})$, 2.95-2.70 (m, 2H), 2.46-2.34 (m, 1H), $2.25(\mathrm{~d}, J=13.0 \mathrm{~Hz}, 1 \mathrm{H}), 1.92-1.74(\mathrm{~m}, 2 \mathrm{H}), 1.45-1.34(\mathrm{~m}, 1 \mathrm{H})$, $1.32-1.21(\mathrm{~m}, 18 \mathrm{H}), 1.15(\mathrm{~d}, J=6.4 \mathrm{~Hz}, 3 \mathrm{H}) .{ }^{13} \mathrm{C} \mathrm{NMR}\left(101 \mathrm{MHz}, \mathrm{CDCl}_{3}\right) \delta 151.0,148.3,147.7,134.7,134.5,128.0,127.6,127.5,125.8$, 125.6, 125.6, 115.7, 113.8, 100.2, 72.1, 71.5, 65.4, 55.8, 48.6, 47.1, 42.0, 37.8, 34.8, 31.6, 31.6, 24.2, 18.7. ESI-MS m/z 542 [M + H] ${ }^{+}$, HR-ESI-MS: m/z $542.3625[\mathrm{M}+\mathrm{H}]^{+}$, (calcd for $\left.\mathrm{C}_{36} \mathrm{H}_{48} \mathrm{NO}_{3}{ }^{+}, 542.3629\right)$.

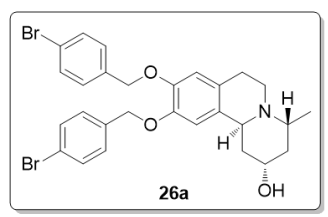

9,10-Bis((4-bromobenzyl)oxy)-4 $\alpha$-methyl-2,3,4,6,7,11b $\alpha$-hexahydro- $1 H$-pyrido[2,1- $\alpha]$ isoquinolin-2 $\alpha$-ol (26a). Pale yellow crystal, mp 158-160 ${ }^{\circ} \mathrm{C} .{ }^{1} \mathrm{H}$ NMR $\left(400 \mathrm{MHz}, \mathrm{CDCl}_{3}\right) \delta 7.48(\mathrm{~d}, J=8.2 \mathrm{~Hz}, 4 \mathrm{H}), 7.28(\mathrm{~d}, J$ $=9.2 \mathrm{~Hz}, 5 \mathrm{H}), 6.71(\mathrm{~s}, 1 \mathrm{H}), 6.63(\mathrm{~s}, 1 \mathrm{H}), 5.08-4.98(\mathrm{~m}, 4 \mathrm{H}), 4.14-4.00(\mathrm{~m}, 1 \mathrm{H}), 3.81-3.69(\mathrm{~m}, 1 \mathrm{H}), 3.37-3.28$ (m, 1H), 2.99-2.83 (m, 2H), 2.77-2.59 (m, 2H), 2.36-2.26 (m, 1H), 1.87 (d, J=10.3 Hz, 1H), $1.75(\mathrm{td}, J=11.6,5.0 \mathrm{~Hz}, 1 \mathrm{H}), 1.44-1.34(\mathrm{~m}$, $1 \mathrm{H}), 1.12(\mathrm{~d}, J=6.8 \mathrm{~Hz}, 3 \mathrm{H}) .{ }^{13} \mathrm{C} \mathrm{NMR}\left(101 \mathrm{MHz}, \mathrm{CDCl}_{3}\right) \delta 147.5,147.0,136.6,136.5,131.8,129.3,129.3,129.2,128.4,121.9,121.9$, 115.4, 113.6, 71.4, 70.8, 66.0, 55.8, 52.4, 48.2, 41.5, 39.7, 29.8, 12.8. ESI-MS m/z $586[\mathrm{M}+\mathrm{H}]^{+}$, HR-ESI-MS: m/z $586.0597[\mathrm{M}+\mathrm{H}]^{+}$, (calcd for $\mathrm{C}_{28} \mathrm{H}_{30} \mathrm{Br}_{2} \mathrm{NO}_{3}{ }^{+}, 586.0587$ ).

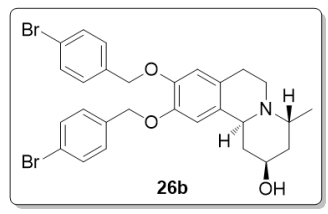

9,10-Bis((4-bromobenzyl)oxy)-4 $\alpha$-methyl-2,3,4,6,7,11b $\alpha$-hexahydro-1 $H$-pyrido[2,1- $a]$ isoquinolin-2 $\beta$-ol (26b). Yellow powder, mp 130-132 ${ }^{\circ} \mathrm{C} .{ }^{1} \mathrm{H}$ NMR $\left(400 \mathrm{MHz}, \mathrm{CDCl}_{3}\right) \delta$ 7.55-7.39 (m, 4H), 7.37-7.21 (m, 4H), $6.79(\mathrm{~s}, 1 \mathrm{H}), 6.62(\mathrm{~s}, 1 \mathrm{H}), 5.04(\mathrm{~d}, J=2.4 \mathrm{~Hz}, 4 \mathrm{H}), 4.30(\mathrm{~s}, 1 \mathrm{H}), 3.68(\mathrm{~s}, 1 \mathrm{H}), 3.34(\mathrm{dd}, J=12.5,4.7 \mathrm{~Hz}, 1 \mathrm{H})$, $3.11-2.77(\mathrm{~m}, 3 \mathrm{H}), 2.48-2.38(\mathrm{~m}, 1 \mathrm{H}), 2.35-2.27(\mathrm{~m}, 1 \mathrm{H}), 1.99-1.84(\mathrm{~m}, 2 \mathrm{H}), 1.50-1.39(\mathrm{~m}, 1 \mathrm{H}), 1.21(\mathrm{~d}, J=$ $6.4 \mathrm{~Hz}, 3 \mathrm{H}) .{ }^{13} \mathrm{C} \mathrm{NMR}\left(101 \mathrm{MHz}, \mathrm{CDCl}_{3}\right) \delta 147.7,147.2,136.6,136.5,131.8,131.8,123.0,129.4,129.3,129.1,128.7,122.0,121.9,115.6$, 113.7, 71.4, 70.8, 65.5, 55.4, 48.7, 47.1, 42.1, 37.8, 24.4, 18.7. ESI-MS m/z $586[\mathrm{M}+\mathrm{H}]^{+}$, HR-ESI-MS: m/z $586.0598[\mathrm{M}+\mathrm{H}]^{+}$, (calcd for $\left.\mathrm{C}_{28} \mathrm{H}_{30} \mathrm{Br}_{2} \mathrm{NO}_{3}{ }^{+}, 586.0587\right)$.

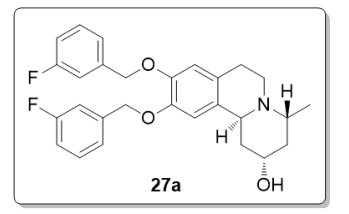

9,10-Bis((3-fluorobenzyl)oxy)-4 $\alpha$-methyl-2,3,4,6,7,11b $\alpha$-hexahydro- $1 H$-pyrido[2,1-a]isoquinolin-2 $\alpha$-ol (27a). White solid, mp $134-136{ }^{\circ} \mathrm{C} .{ }^{1} \mathrm{H}$ NMR $\left(400 \mathrm{MHz}, \mathrm{CDCl}_{3}\right) \delta 7.41-7.29$ (m, 2H), 7.24-7.12 (m, 4H), 7.07-6.93 (m, 2H), $6.74(\mathrm{~s}, 1 \mathrm{H}), 6.65(\mathrm{~s}, 1 \mathrm{H}), 5.14-5.06(\mathrm{~m}, 4 \mathrm{H}), 4.18-3.92(\mathrm{~m}, 1 \mathrm{H}), 3.78-3.68(\mathrm{~m}, 1 \mathrm{H}), 3.32$ 
(dd, $J=11.3,6.5 \mathrm{~Hz}, 1 \mathrm{H}), 3.00-2.86(\mathrm{~m}, 2 \mathrm{H}), 2.79-2.58(\mathrm{~m}, 2 \mathrm{H}), 2.38-2.23(\mathrm{~m}, 1 \mathrm{H}), 1.90-1.82(\mathrm{~m}, 1 \mathrm{H}), 1.75(\mathrm{td}, J=11.7,5.2 \mathrm{~Hz}, 1 \mathrm{H})$, $1.39(\mathrm{dd}, J=23.1,11.5 \mathrm{~Hz}, 1 \mathrm{H}), 1.11(\mathrm{~d}, J=7.0 \mathrm{~Hz}, 3 \mathrm{H}) .{ }^{13} \mathrm{C} \mathrm{NMR}\left(101 \mathrm{MHz}, \mathrm{CDCl}_{3}\right) \delta 164.4,162.0,147.4,147.0,140.3,140.2,140.1$, $131.8,130.3,130.2,128.4,122.9,122.9,122.8,122.8,115.3,115.0,115.0,114.8,114.7,114.5,114.4,114.3,114.2,113.3,71.2,70.7,66.0$, 55.8, 52.3, 48.2, 41.5, 39.7, 29.9, 12.7. ESI-MS m/z $466[\mathrm{M}+\mathrm{H}]^{+}$, HR-ESI-MS: m/z 466.2183 [M + $\mathrm{H}^{+},\left(\right.$calcd for $\mathrm{C}_{28} \mathrm{H}_{30} \mathrm{~F}_{2} \mathrm{NO}_{3}{ }^{+}$, 466.2188).

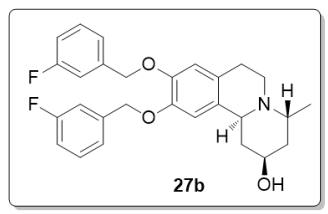

9,10-Bis((3-fluorobenzyl)oxy)-4 $\alpha$-methyl-2,3,4,6,7,11b $\alpha$-hexahydro-1 $H$-pyrido[2,1-a]isoquinolin-2 $\beta$-ol (27a). Yellow solid, mp 121-124 ${ }^{\circ} \mathrm{C} .{ }^{1} \mathrm{H}$ NMR (400 MHz, $\left.\mathrm{CDCl}_{3}\right) \delta 7.31-7.20(\mathrm{~m}, 2 \mathrm{H}), 7.16-7.04(\mathrm{~m}, 4 \mathrm{H}), 6.98-6.82$ $(\mathrm{m}, 2 \mathrm{H}), 6.74(\mathrm{~s}, 1 \mathrm{H}), 6.56(\mathrm{~s}, 1 \mathrm{H}), 5.09-4.92(\mathrm{~m}, 4 \mathrm{H}), 4.20(\mathrm{~s}, 1 \mathrm{H}), 3.67-3.50(\mathrm{~m}, 1 \mathrm{H}), 3.25(\mathrm{dd}, J=12.7,4.8$ $\mathrm{Hz}, 1 \mathrm{H}), 2.94(\mathrm{td}, J=12.6,4.2 \mathrm{~Hz}, 1 \mathrm{H}), 2.89-2.80(\mathrm{~m}, 1 \mathrm{H}), 2.75(\mathrm{~s}, 1 \mathrm{H}), 2.44-2.30(\mathrm{~m}, 1 \mathrm{H}), 2.22(\mathrm{~d}, J=13.1 \mathrm{~Hz}, 1 \mathrm{H}), 1.98(\mathrm{brs}, 1 \mathrm{H})$, $1.87-1.78(\mathrm{~m}, 2 \mathrm{H}), 1.40-1.28(\mathrm{~m}, 1 \mathrm{H}), 1.12(\mathrm{~d}, J=6.4 \mathrm{~Hz}, 3 \mathrm{H}) .{ }^{13} \mathrm{C} \mathrm{NMR}\left(101 \mathrm{MHz}, \mathrm{CDCl}_{3}\right) \delta 164.2,164.2,161.8,161.8,147.4,146.9$, 140.1 , 140.0, 139.9, 139.9, 130.1, 130.1, 130.0, 130.0, 128.7, 122.8, 122.8, 122.6, 122.6, 115.3, 114.8, 114.8, 114.6, 114.6, 114.4, 114.2, 114.1, 114.0, 113.3, 71.1, 70.5, 65.5, 55.2, 48.3, 46.9, 42.0, 37.8, 24.3, 18.6. ESI-MS m/z 466 [M + H] , HR-ESI-MS: m/z 466.2184 [M + $\mathrm{H}]^{+}$, (calcd for $\left.\mathrm{C}_{28} \mathrm{H}_{30} \mathrm{~F}_{2} \mathrm{NO}_{3}{ }^{+}, 466.2188\right)$.

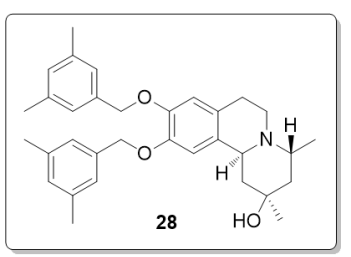

9,10-Bis((3,5-dimethylbenzyl)oxy)-2,4 $\alpha$-dimethyl-2,3,4,6,7,11b $\alpha$-hexahydro-1 $H$-pyrido[2,1- $\alpha$ ]isoquinolin-2 ק-ol (28). Yellow oil. ${ }^{1} \mathrm{H}$ NMR (400 MHz, $\left.\mathrm{CDCl}_{3}\right) \delta 7.09$ (s, 4H), 6.95 (s, 2H), 6.80 (s, 1H), 6.69 (s, 1H), 5.05 (s, 4H), $4.09(\mathrm{~d}, J=11.0 \mathrm{~Hz}, 1 \mathrm{H}), 3.28-3.16(\mathrm{~m}, 1 \mathrm{H}), 3.06-2.86(\mathrm{~m}, 2 \mathrm{H}), 2.86-2.75(\mathrm{~m}, 1 \mathrm{H}), 2.63(\mathrm{~d}, J=15.5 \mathrm{~Hz}$, $1 \mathrm{H}), 2.32(\mathrm{~s}, 12 \mathrm{H}), 2.06-1.91(\mathrm{~m}, 2 \mathrm{H}), 1.63-1.52(\mathrm{~m}, 2 \mathrm{H}), 1.29(\mathrm{~d}, J=6.8 \mathrm{~Hz}, 3 \mathrm{H}), 1.24(\mathrm{~s}, 3 \mathrm{H}) .{ }^{13} \mathrm{C} \mathrm{NMR}(101$ $\left.\mathrm{MHz}, \mathrm{CDCl}_{3}\right) \delta 147.8,147.5,138.1,138.1,137.6,137.6,132.0,129.5,129.5,128.4,125.6,125.3,115.6,113.9,72.3,71.7,70.5,54.5,50.1$, 48.4, 45.3, 43.1, 32.6, 29.4, 21.5, 14.1. ESI-MS m/z $500[\mathrm{M}+\mathrm{H}]^{+}$, HR-ESI-MS: m/z 500.3156 [M + $]^{+},\left(\right.$calcd for $\mathrm{C}_{33} \mathrm{H}_{42} \mathrm{NO}_{3}{ }^{+}$, 500.3159).

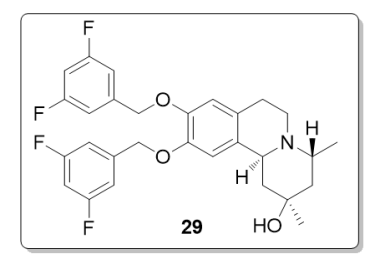

9,10-Bis((3,5-difluorobenzyl)oxy)-2,4 $\alpha$-dimethyl-2,3,4,6,7,11b $\alpha$-hexahydro-1H-pyrido[2,1-a]isoquinolin-2 ק-ol (29). Yellow solid, mp 104-106 ${ }^{\circ} \mathrm{C} .{ }^{1} \mathrm{H}$ NMR $\left(400 \mathrm{MHz}, \mathrm{CDCl}_{3}\right) \delta 7.00-6.76(\mathrm{~m}, 4 \mathrm{H}), 6.80-6.60$ (m, 3H), $6.54(\mathrm{~s}, 1 \mathrm{H}), 4.98(\mathrm{~d}, J=2.9 \mathrm{~Hz}, 4 \mathrm{H}), 3.98(\mathrm{~d}, J=10.9 \mathrm{~Hz}, 1 \mathrm{H}), 3.25-3.00(\mathrm{~m}, 1 \mathrm{H}), 2.96-2.75(\mathrm{~m}, 2 \mathrm{H})$, 2.76-2.62 (m, 1H), $2.53(\mathrm{~d}, J=15.9 \mathrm{~Hz}, 1 \mathrm{H}), 1.96-1.78(\mathrm{~m}, 2 \mathrm{H}), 1.51-1.40(\mathrm{~m}, 2 \mathrm{H}), 1.19$ (d, $J=7.0 \mathrm{~Hz}, 3 \mathrm{H})$, $1.16(\mathrm{~s}, 3 \mathrm{H}) .{ }^{13} \mathrm{C} \mathrm{NMR}\left(101 \mathrm{MHz}, \mathrm{CDCl}_{3}\right) \delta 164.5,164.3,162.0,161.9,146.6,146.4,141.5,141.4,141.3,132.5,128.8,115.1,113.2,110.0$, 109.9, 109.8, 109.7, 109.7, 109.6, 109.5, 103.4, 103.1, 102.9, 70.4, 70.3, 70.0, 54.5, 49.8, 48.1, 45.1, 42.8, 32.5, 29.3, 13.9. ESI-MS m/z $516[\mathrm{M}+\mathrm{H}]^{+}$, HR-ESI-MS: m/z 516.2151 [M+ H] $]^{+}$, (calcd for $\left.\mathrm{C}_{29} \mathrm{H}_{30} \mathrm{~F}_{4} \mathrm{NO}_{3}{ }^{+}, 516.2156\right)$.

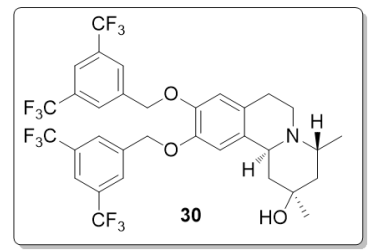

9,10-Bis((3,5-bis(trifluoromethyl)benzyl)oxy)-2,4 $\alpha$-dimethyl-2,3,4,6,7,11b $\alpha$-hexahydro-1 $H$-pyrido[2,1-a]

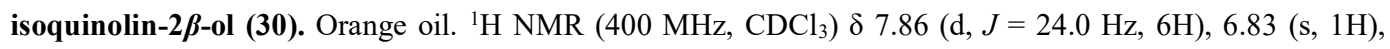
$6.71(\mathrm{~s}, 1 \mathrm{H}), 5.19(\mathrm{~s}, 4 \mathrm{H}), 4.17-3.95(\mathrm{~m}, 1 \mathrm{H}), 3.36-3.15(\mathrm{~m}, 1 \mathrm{H}), 3.10-2.88(\mathrm{~m}, 2 \mathrm{H}), 2.81(\mathrm{td}, J=10.9,3.7$ $\mathrm{Hz}, 1 \mathrm{H}), 2.72-2.61(\mathrm{~m}, 1 \mathrm{H}), 2.04-1.91(\mathrm{~m}, 2 \mathrm{H}), 1.61-1.49(\mathrm{~m}, 2 \mathrm{H}), 1.30(\mathrm{~d}, J=7.0 \mathrm{~Hz}, 3 \mathrm{H}), 1.26(\mathrm{~s}, 3 \mathrm{H})$. ${ }^{13} \mathrm{C}$ NMR $\left(101 \mathrm{MHz}, \mathrm{CDCl}_{3}\right) \delta 146.9,146.7,140.0,140.0,133.5,132.6,132.3,132.3,132.0,131.9,131.6,129.7,127.5,127.4,124.8$, 122.1, 119.4, 115.8, 113.8, 70.7, 70.5, 70.4, 54.8, 50.0, 48.3, 45.3, 43.0, 32.7, 29.6, 14.0. ESI-MS m/z 716 [M + H] $]^{+}$HR-ESI-MS: m/z $716.2038[\mathrm{M}+\mathrm{H}]^{+}$, (calcd for $\left.\mathrm{C}_{33} \mathrm{H}_{30} \mathrm{~F}_{12} \mathrm{NO}_{3}{ }^{+}, 716.2029\right)$.

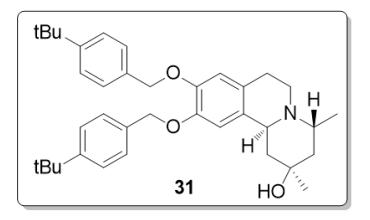

9,10-Bis((4-(tert-butyl)benzyl)oxy)-2,4 $\alpha$-dimethyl-2,3,4,6,7,11b $\alpha$-hexahydro-1 $H$-pyrido[2,1-a]isoquinolin -2ק-ol (31). Yellow oil. ${ }^{1} \mathrm{H}$ NMR (400 MHz, $\left.\mathrm{CDCl}_{3}\right) \delta 7.39$ (s, 8H), 6.78 (s, 1H), 6.69 (s, 1H), 5.20-5.00 (m, $4 \mathrm{H}), 4.07(\mathrm{~d}, J=10.9 \mathrm{~Hz}, 1 \mathrm{H}), 3.35-3.06(\mathrm{~m}, 1 \mathrm{H}), 3.03-2.84(\mathrm{~m}, 2 \mathrm{H}), 2.85-2.74(\mathrm{~m}, 1 \mathrm{H}), 2.68-2.54(\mathrm{~m}, 1 \mathrm{H})$, 2.03-1.86 (m, 2H), 1.60-1.46 (m, 2H), 1.38-1.31 (m, 18H), $1.28(\mathrm{~d}, J=6.8 \mathrm{~Hz}, 3 \mathrm{H}), 1.22(\mathrm{~s}, 3 \mathrm{H}) .{ }^{13} \mathrm{C}$ NMR $\left(101 \mathrm{MHz}, \mathrm{CDCl}_{3}\right) \delta 150.9$, 150.9, 147.7, 147.4, 134.8, 134.7, 131.9, 128.6, 127.6, 127.5, 125.5, 125.5, 115.7, 113.9, 72.0, 71.5, 70.5, 54.5, 50.1, 48.4, 45.2, 43.1, 34.7, 
32.6, 31.6, 29.3, 14.1. ESI-MS m/z $556[\mathrm{M}+\mathrm{H}]^{+}$, HR-ESI-MS: m/z $556.3785[\mathrm{M}+\mathrm{H}]^{+},\left(\right.$calcd for $\left.\mathrm{C}_{37} \mathrm{H}_{50} \mathrm{NO}_{3}{ }^{+}, 556.3785\right)$.

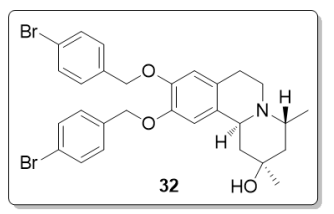

9,10-Bis((4-bromobenzyl)oxy)-2,4 $\alpha$-dimethyl-2,3,4,6,7,11b $\alpha$-hexahydro-1 $H$-pyrido[2,1-a]isoquinolin-2 $\beta$-ol (32). Orange oil. ${ }^{1} \mathrm{H}$ NMR (400 MHz, $\left.\mathrm{CDCl}_{3}\right) \delta 7.39(\mathrm{~d}, J=8.3 \mathrm{~Hz}, 4 \mathrm{H}), 7.20(\mathrm{~d}, 4 \mathrm{H}), 6.64(\mathrm{~s}, 1 \mathrm{H}), 6.54$ (s, $1 \mathrm{H}), 4.99-4.89(\mathrm{~m}, 4 \mathrm{H}), 3.97(\mathrm{~d}, J=10.1 \mathrm{~Hz}, 1 \mathrm{H}), 3.23-3.02(\mathrm{~m}, 1 \mathrm{H}), 2.97-2.74(\mathrm{~m}, 2 \mathrm{H}), 2.76-2.64(\mathrm{~m}, 1 \mathrm{H})$, $2.52(\mathrm{~d}, J=16.1 \mathrm{~Hz}, 1 \mathrm{H}), 1.95-1.76(\mathrm{~m}, 2 \mathrm{H}), 1.51-1.39(\mathrm{~m}, 2 \mathrm{H}), 1.19(\mathrm{~d}, J=6.8 \mathrm{~Hz}, 3 \mathrm{H}), 1.15(\mathrm{~s}, 3 \mathrm{H}) .{ }^{13} \mathrm{C}$ NMR $\left(101 \mathrm{MHz}, \mathrm{CDCl}_{3}\right) \delta 147.2,146.9,136.6,131.8,131.7,129.4,129.3,129.1,128.7,121.9,121.9,115.5,113.7,71.3,70.8,70.4,54.6$, 50.0, 48.4, 45.2, 43.0, 32.7, 29.4, 14.1. ESI-MS m/z $600[\mathrm{M}+\mathrm{H}]^{+}$, HR-ESI-MS: m/z 600.0750 [M $+\mathrm{H}^{+}$, $\left(\right.$calcd for $\mathrm{C}_{29} \mathrm{H}_{32} \mathrm{Br}_{2} \mathrm{NO}_{3}{ }^{+}$, $600.0743)$.

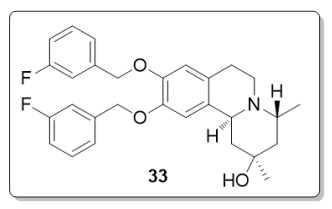

9,10-Bis((3,5-difluorobenzyl)oxy)-2,4 $\alpha$-dimethyl-2,3,4,6,7,11b $\alpha$-hexahydro-1H-pyrido[2,1-a]isoquinolin-2 ק-ol (33). Brown oil. ${ }^{1} \mathrm{H}$ NMR (400 MHz, $\left.\mathrm{CDCl}_{3}\right) \delta$ 7.30-7.20 (m, 2H), 7.17-7.05 (m, 4H), 7.00-6.83 (m, 2H), $6.67(\mathrm{~s}, 1 \mathrm{H}), 6.56(\mathrm{~s}, 1 \mathrm{H}), 5.02(\mathrm{~d}, J=3.1 \mathrm{~Hz}, 4 \mathrm{H}), 3.98(\mathrm{~d}, J=10.4 \mathrm{~Hz}, 1 \mathrm{H}), 3.20-3.02(\mathrm{~m}, 1 \mathrm{H}), 2.97-2.76(\mathrm{~m}$, $2 \mathrm{H}), 2.76-2.63(\mathrm{~m}, 1 \mathrm{H}), 2.61-2.43(\mathrm{~m}, 1 \mathrm{H}), 1.93-1.78(\mathrm{~m}, 2 \mathrm{H}), 1.56-1.39(\mathrm{~m}, 3 \mathrm{H}), 1.20(\mathrm{~d}, J=7.0 \mathrm{~Hz}, 3 \mathrm{H}), 1.15(\mathrm{~s}, 3 \mathrm{H}) .{ }^{13} \mathrm{C} \mathrm{NMR}(101$ $\left.\mathrm{MHz}, \mathrm{CDCl}_{3}\right) \delta 164.4,162.0,147.2,147.0,140.3,140.2,140.2,132.4,130.2,130.2,128.7,123.0,123.0,122.8,122.8,115.4,114.9,114.7$, 114.6, 114.4, 114.4, 114.2, 113.6, 71.2, 70.8, 70.5, 54.6, 50.0, 48.4, 45.3, 43.1, 32.7, 29.5, 14.1. ESI-MS m/z 480 [M + H] , HR-ESI-MS: $\mathrm{m} / \mathrm{z} 480.2343[\mathrm{M}+\mathrm{H}]^{+}$, (calcd for $\mathrm{C}_{29} \mathrm{H}_{32} \mathrm{~F}_{2} \mathrm{NO}_{3}{ }^{+}, 480.2345$ ).

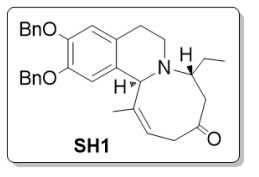

trans-2,3-Bis(benzyloxy)-8-ethyl-13-methyl-8,9,11,13a-tetrahydro-5H-azocino[2,1-a]isoquinolin-10(6H)-one (SH1). White solid, mp 129-131 ${ }^{\circ} \mathrm{C} .{ }^{1} \mathrm{H}$ NMR $\left(400 \mathrm{MHz}, \mathrm{CDCl}_{3}\right) \delta$ 7.54-7.41 (m, 4H), 7.41-7.29 (m, 6H), 6.71 (s, 1H), 6.62 (s, 1H), 5.32 (t, $J=6.9 \mathrm{~Hz}, 1 \mathrm{H}), 5.12$ (d, $J=11.6 \mathrm{~Hz}, 4 \mathrm{H}), 4.17$ (dd, $J=9.3,4.2 \mathrm{~Hz}, 1 \mathrm{H}), 3.62$ (t, $J=4.8$ $\mathrm{Hz}, 1 \mathrm{H}), 3.30-3.17$ (m, 1H), 2.99-2.82 (m, 2H), 2.82-2.69 (m, 1H), 2.69-2.57 (m, 2H), 2.50 (dt, J = 14.9, 4.5 Hz, 2H), 2.15-1.98 (m, 2H), $1.68(\mathrm{~s}, 3 \mathrm{H}), 0.96(\mathrm{t}, J=7.5 \mathrm{~Hz}, 3 \mathrm{H}) .{ }^{13} \mathrm{C}$ NMR $\left(101 \mathrm{MHz}, \mathrm{CDCl}_{3}\right) \delta 209.1,147.9,147.3,137.4,137.3,134.4,130.4,130.1,128.5,127.8$, 127.8, 127.4, 127.3, 115.4, 113.6, 71.7, 71.4, 66.3, 54.6, 45.8, 44.9, 41.3, 28.4, 21.3, 14.2, 14.0. ESI-MS m/z 482 [M + H] , HR-ESI-MS: $\mathrm{m} / \mathrm{z} 482.2687[\mathrm{M}+\mathrm{H}]^{+}$, (calcd for $\left.\mathrm{C}_{32} \mathrm{H}_{36} \mathrm{NO}_{3}{ }^{+}, 482.2690\right)$.

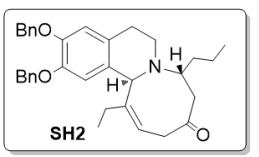

trans-2,3-Bis(benzyloxy)-13-ethyl-8-propyl-8,9,11,13a-tetrahydro-5 $H$-azocino[2,1-a]isoquinolin-10(6H)-one (SH2). White solid, mp 69-71 ${ }^{\circ} \mathrm{C} .{ }^{1} \mathrm{H}$ NMR $\left(400 \mathrm{MHz}, \mathrm{CDCl}_{3}\right) \delta 7.45(\mathrm{t}, J=8.0 \mathrm{~Hz}, 4 \mathrm{H}), 7.40-7.29(\mathrm{~m}, 6 \mathrm{H}), 6.72(\mathrm{~s}$, $1 \mathrm{H}), 6.57(\mathrm{~s}, 1 \mathrm{H}), 5.25$ (t, $J=7.0 \mathrm{~Hz}, 1 \mathrm{H}), 5.11(\mathrm{~d}, J=14.1 \mathrm{~Hz}, 4 \mathrm{H}), 4.10(\mathrm{dd}, J=10.7,3.6 \mathrm{~Hz}, 1 \mathrm{H}), 3.80(\mathrm{~s}, 1 \mathrm{H})$, $3.33-3.21(\mathrm{~m}, 1 \mathrm{H}), 3.10-2.96(\mathrm{~m}, 1 \mathrm{H}), 2.96-2.87(\mathrm{~m}, 1 \mathrm{H}), 2.76-2.66(\mathrm{~m}, 1 \mathrm{H}), 2.66-2.46(\mathrm{~m}, 3 \mathrm{H}), 2.38(\mathrm{dd}, J=15.0,3.5 \mathrm{~Hz}, 1 \mathrm{H})$, 2.23-2.08 (m, 2H), $2.03(\mathrm{dd}, J=14.6,7.4 \mathrm{~Hz}, 2 \mathrm{H}), 1.39(\mathrm{dd}, J=14.7,7.4 \mathrm{~Hz}, 2 \mathrm{H}), 0.95(\mathrm{t}, J=7.6 \mathrm{~Hz}, 3 \mathrm{H}), 0.90(\mathrm{t}, J=7.3 \mathrm{~Hz}, 3 \mathrm{H}) .{ }^{13} \mathrm{C}$ NMR $\left(101 \mathrm{MHz}, \mathrm{CDCl}_{3}\right) \delta 209.2,148.0,147.3,141.0,137.4,137.3,130.4,128.5,128.5,128.4,127.8,127.8,127.4,127.3,127.1,115.4$, 113.7, 71.7, 71.4, 64.9, 53.9, 45.5, 44.9, 40.3, 29.9, 29.4, 22.9, 21.8, 13.9, 13.6. ESI-MS m/z 510 [M + H] $]^{+}$HR-ESI-MS: m/z 510.3000 [M $+\mathrm{H}]^{+}$, (calcd for $\left.\mathrm{C}_{34} \mathrm{H}_{40} \mathrm{NO}_{3}{ }^{+}, 510.3003\right)$.

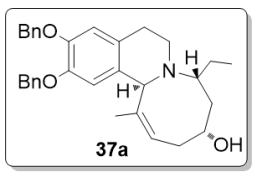

2,3-Bis(benzyloxy)-8 $\alpha$-ethyl-13-methyl-6,8,9,10,11,13a $\alpha$-hexahydro-5H-azocino[2,1- $a]$ isoquinolin-10 $\alpha$-ol (37a). Pale yellow oil. ${ }^{1} \mathrm{H}$ NMR (400 MHz, $\left.\mathrm{CDCl}_{3}\right) \delta$ 7.52-7.40 (m, 4H), 7.43-7.28 (m, 6H), $6.75(\mathrm{~s}, 1 \mathrm{H}), 6.69(\mathrm{~s}, 1 \mathrm{H})$, $5.45(\mathrm{t}, J=6.9 \mathrm{~Hz}, 1 \mathrm{H}), 5.23-5.01(\mathrm{~m}, 4 \mathrm{H}), 4.13-3.96(\mathrm{~m}, 2 \mathrm{H}), 3.42(\mathrm{~s}, 1 \mathrm{H}), 3.25-3.10(\mathrm{~m}, 1 \mathrm{H}), 2.89-2.74(\mathrm{~m}, 2 \mathrm{H})$, $2.68-2.55(\mathrm{~m}, 1 \mathrm{H}), 2.08-1.93(\mathrm{~m}, 4 \mathrm{H}), 1.65(\mathrm{~s}, 5 \mathrm{H}), 1.62-1.55(\mathrm{~m}, 2 \mathrm{H}), 0.96(\mathrm{t}, J=7.5 \mathrm{~Hz}, 3 \mathrm{H}) .{ }^{13} \mathrm{C} \mathrm{NMR}\left(101 \mathrm{MHz}^{-} \mathrm{CDCl}_{3}\right) \delta 147.7$, 147.0, 137.6, 137.5, 128.5, 127.8, 127.8, 127.4, 127.3, 115.4, 114.0, 71.8, 71.3, 66.1, 61.1, 53.4, 46.6, 36.2, 34.3, 26.7, $21.2,14.2,13.1$. ESI-MS m/z $484[\mathrm{M}+\mathrm{H}]^{+}$, HR-ESI-MS: m/z $484.2841[\mathrm{M}+\mathrm{H}]^{+}$, (calcd for $\left.\mathrm{C}_{32} \mathrm{H}_{38} \mathrm{NO}_{3}{ }^{+}, 484.2846\right)$.

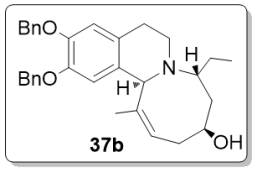

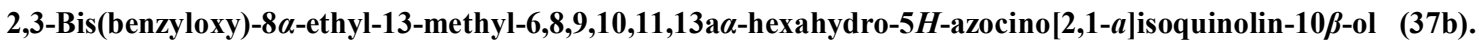
Colorless oil. ${ }^{1} \mathrm{H}$ NMR $\left(400 \mathrm{MHz}, \mathrm{CDCl}_{3}\right) \delta$ 7.62-7.41 (m, 4H), 7.43-7.27 (m, 6H), $6.83(\mathrm{~s}, 1 \mathrm{H}), 6.67(\mathrm{~s}, 1 \mathrm{H}), 5.38$ 
(t, $J=6.5 \mathrm{~Hz}, 1 \mathrm{H}), 5.30-4.99(\mathrm{~m}, 4 \mathrm{H}), 4.33(\mathrm{~s}, 1 \mathrm{H}), 3.48-3.33(\mathrm{~m}, 1 \mathrm{H}), 3.17-2.75(\mathrm{~m}, 4 \mathrm{H}), 2.46(\mathrm{dd}, J=12.8,3.2 \mathrm{~Hz}, 1 \mathrm{H}), 2.26(\mathrm{dd}, J=$ 16.7, $4.5 \mathrm{~Hz}, 1 \mathrm{H}), 2.08-1.98(\mathrm{~m}, 2 \mathrm{H}), 1.84-1.74(\mathrm{~m}, 1 \mathrm{H}), 1.65(\mathrm{~s}, 3 \mathrm{H}), 1.63-1.49(\mathrm{~m}, 2 \mathrm{H}), 0.95(\mathrm{t}, J=7.5 \mathrm{~Hz}, 3 \mathrm{H}) .{ }^{13} \mathrm{C} \mathrm{NMR}(101 \mathrm{MHz}$, $\left.\mathrm{CDCl}_{3}\right) \delta 147.7,147.0,137.7,137.4,135.4,129.7,128.6,128.5,127.8,127.7,127.5,127.4,115.4,113.5,71.9,71.3,65.1,60.1,57.0,46.6$, 40.5, 37.1, 22.5, 20.9, 14.0, 10.9. ESI-MS m/z $484[\mathrm{M}+\mathrm{H}]^{+}$, HR-ESI-MS: m/z $484.2842[\mathrm{M}+\mathrm{H}]^{+}$, (calcd for $\mathrm{C}_{32} \mathrm{H}_{38} \mathrm{NO}_{3}{ }^{+}, 484.2846$ ).

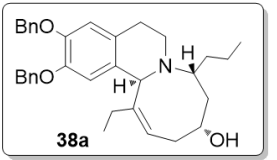

2,3-Bis(benzyloxy)-13-ethyl-8 $\alpha$-propyl-6,8,9,10,11,13a $\alpha$-hexahydro-5H-azocino[2,1-a]isoquinolin-10 $\alpha$-ol (38a). Yellow oil. ${ }^{1} \mathrm{H}$ NMR $\left(400 \mathrm{MHz}, \mathrm{CDCl}_{3}\right) \delta 7.56-7.40(\mathrm{~m}, 4 \mathrm{H}), 7.42-7.27(\mathrm{~m}, 6 \mathrm{H}), 6.69(\mathrm{~d}, J=3.1 \mathrm{~Hz}, 2 \mathrm{H}), 5.39(\mathrm{t}$, $J=7.0 \mathrm{~Hz}, 1 \mathrm{H}), 5.23-5.00(\mathrm{~m}, 4 \mathrm{H}), 4.18-3.96(\mathrm{~m}, 1 \mathrm{H}), 3.89(\mathrm{dd}, J=9.5,3.2 \mathrm{~Hz}, 1 \mathrm{H}), 3.66-3.43(\mathrm{~m}, 1 \mathrm{H})$, 3.37-3.06 (m, 1H), 2.89-2.61 (m, 3H), 2.29-2.13 (m, 1H), 2.13-1.97 (m, 4H), 1.97-1.85 (m, 1H), 1.85-1.71 (m, 1H), 1.74-1.56 (m,1H), $1.46-1.35(\mathrm{~m}, 2 \mathrm{H}), 0.96(\mathrm{t}, J=7.5 \mathrm{~Hz}, 3 \mathrm{H}), 0.92(\mathrm{t}, J=7.3 \mathrm{~Hz}, 3 \mathrm{H}) .{ }^{13} \mathrm{C} \mathrm{NMR}\left(101 \mathrm{MHz}, \mathrm{CDCl}_{3}\right) \delta 147.7,147.0,140.9,137.6,137.5$, 131.6, 128.5, 128.4, 128.0, 127.7, 127.7, 127.4, 127.3, 126.3, 115.3, 114.1, 71.9, 71.4, 66.3, 61.6, 53.0, 46.2, 37.3, 32.7, 30.0, 28.5, 23.1, 21.3, 14.0, 13.8. ESI-MS m/z $512[\mathrm{M}+\mathrm{H}]^{+}$, HR-ESI-MS: m/z $512.3153[\mathrm{M}+\mathrm{H}]^{+}$, (calcd for $\left.\mathrm{C}_{34} \mathrm{H}_{42} \mathrm{NO}_{3}{ }^{+}, 512.3159\right)$.

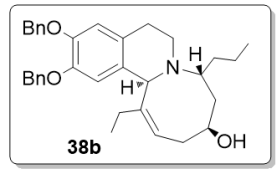

2,3-Bis(benzyloxy)-13-ethyl-8 $\alpha$-propyl-6,8,9,10,11,13a $\alpha$-hexahydro-5 $H$-azocino[2,1- $a$ ] isoquinolin-10 $\boldsymbol{\beta}$-ol (38b). Colorless oil. ${ }^{1} \mathrm{H}$ NMR $\left(400 \mathrm{MHz}, \mathrm{CDCl}_{3}\right) \delta$ 7.60-7.41 (m, 4H), 7.43-7.27 (m, 6H), $6.83(\mathrm{~s}, 1 \mathrm{H}), 6.68(\mathrm{~s}$, $1 \mathrm{H}), 5.37(\mathrm{t}, J=7.2 \mathrm{~Hz}, 1 \mathrm{H}), 5.26-5.03(\mathrm{~m}, 4 \mathrm{H}), 4.40-4.26(\mathrm{~m}, 1 \mathrm{H}), 3.46-3.25(\mathrm{~m}, 1 \mathrm{H}), 3.16(\mathrm{dd}, J=13.2,5.9 \mathrm{~Hz}$, $1 \mathrm{H}), 3.09-2.77(\mathrm{~m}, 3 \mathrm{H}), 2.46(\mathrm{dd}, J=12.8,2.6 \mathrm{~Hz}, 1 \mathrm{H}), 2.25(\mathrm{dd}, J=16.7,5.4 \mathrm{~Hz}, 1 \mathrm{H}), 2.21-2.09$ (m, 2H), 2.08-2.00 (m, 2H), $1.84-1.70$ $(\mathrm{m}, 2 \mathrm{H}), 1.55(\mathrm{dd}, J=22.9,11.4 \mathrm{~Hz}, 1 \mathrm{H}), 1.4-1.32(\mathrm{~m}, 2 \mathrm{H}), 1.04(\mathrm{t}, J=7.6 \mathrm{~Hz}, 3 \mathrm{H}), 0.91(\mathrm{t}, J=7.4 \mathrm{~Hz}, 4 \mathrm{H}) .{ }^{13} \mathrm{C} \mathrm{NMR}\left(101 \mathrm{MHz}, \mathrm{CDCl}_{3}\right)$ $\delta 148.0,147.3,142.1,137.9,137.6,129.3,128.9,128.7,128.0,127.9,127.7,127.6,115.7,113.7,72.1,71.5,65.4,60.5,57.2,46.9,41.7$, 37.3, 29.9, 23.2, 22.6, 19.8, 15.2, 14.1. ESI-MS m/z $512[\mathrm{M}+\mathrm{H}]^{+}$, HR-ESI-MS: m/z 512.3154[M + H] $]^{+}$, (calcd for $\left.\mathrm{C}_{34} \mathrm{H}_{42} \mathrm{NO}_{3}{ }^{+}, 512.3159\right)$.

\section{Optimization for the Z-VAD-fmk assays.}

MTT reslts of orthogonal experiments with different concentration of Z-VAD-fmk or Ac-DEVD-CHO and incubated time were shown as following. $10 \mu \mathrm{M}$ Z-VAD-fmk (or $50 \mu \mathrm{M}$ Ac-DEVD-CHO) pretreated and $24 \mathrm{~h}$ incubaied time is the most appropriate condition. Relative enzyme activity for caspase-3was also provided in the text. 

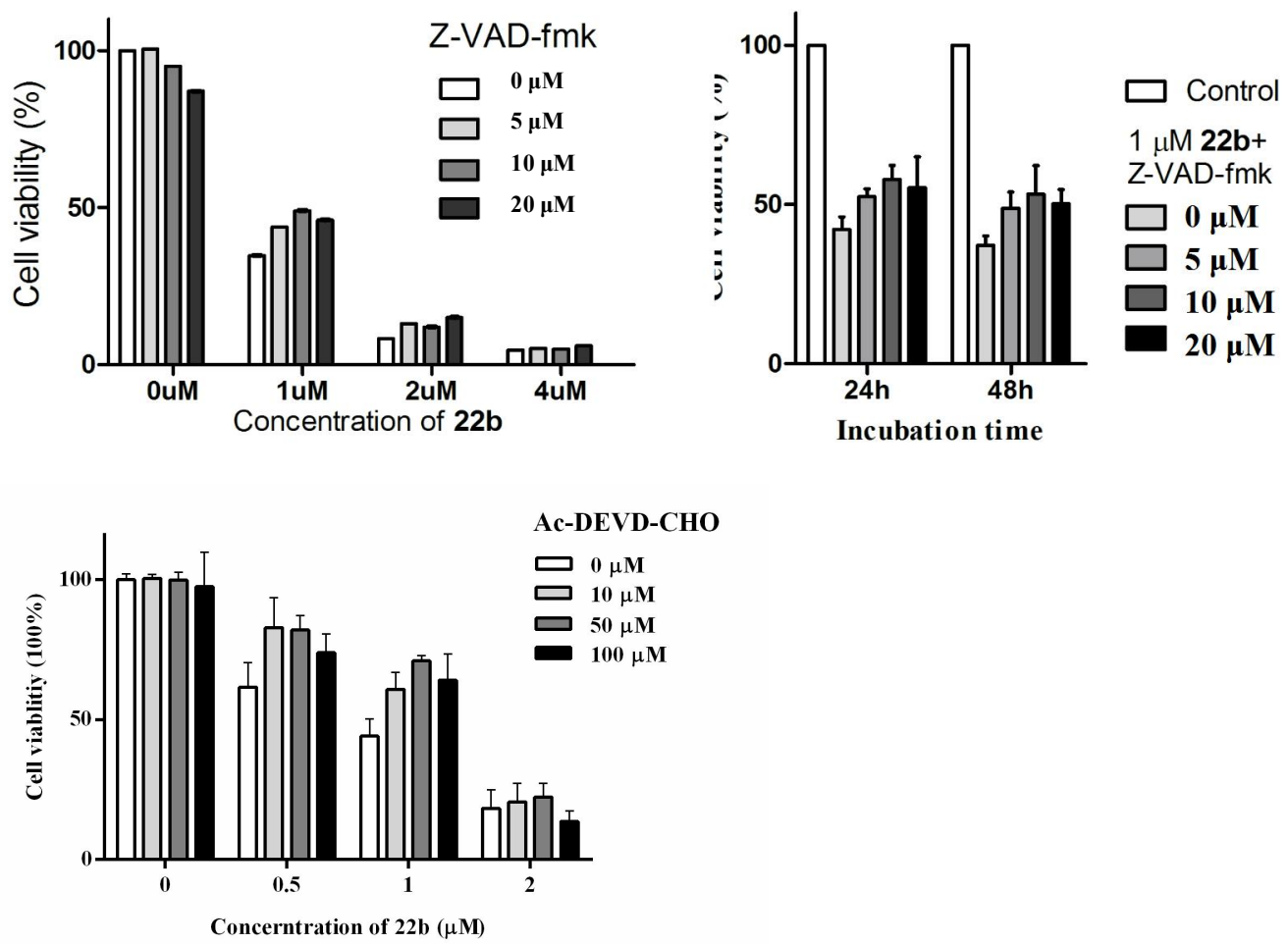

Figure S12. Optimization of the Z-VAD-fmk and Ac-DEVD-CHO assays. PC3 cells were pretreated with different concentration of Z-VAd-fmk or Ac-DEVD-CHO, and then explosed to 22b for corresponding times. Cell viability was monitored by MTT assays.

\section{Westernblot analysis of decrease of IkB-a and p-P65 reponse to CHX and ActD.}

Cells was treated with $10 \mu \mathrm{M} \mathrm{CHX}$ or $5 \mu \mathrm{M}$ ActD, and then westernblot analysis of IkB-a and its substrate p-P65 was performed. The significantly decrease of IkB-a ind p-P65 were obtained.

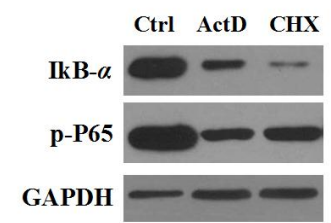

Figure S13. Westernblot analysis of IkB-a and p-P65 reponse to CHX and ActD. pretreated with $10 \mu \mathrm{M}$ ActD, or $5 \mu \mathrm{M}$ CHX, then subjected to 22b for $24 \mathrm{~h}$. MTT assays Westernblot analysis of IkB-a and p-P65 was shown above. 


\section{CI values calculated by CompuSyn software}

Table S6. Effects and CI values treated with combination of $\mathrm{Mg} 132$ and 22b. ${ }^{\text {a }}$

\begin{tabular}{|c|c|c|c|}
\hline Dose $\operatorname{Mg} 132^{\mathrm{b}}(\mu \mathrm{M})$ & Dose $22 \mathbf{b}(\mu \mathrm{M})$ & Effect ${ }^{c}$ & $\mathrm{CI}^{\mathrm{d}}$ \\
\hline 一 & 0.3125 & 0.2290 & - \\
\hline - & 0.625 & 0.4425 & - \\
\hline - & 1.25 & 0.5433 & - \\
\hline 一 & 2.5 & 0.5817 & - \\
\hline 1.25 & - & 0.0549 & - \\
\hline 2.5 & - & 0.0567 & - \\
\hline 5 & - & 0.1220 & - \\
\hline 10 & - & 0.2318 & - \\
\hline 1.25 & 0.3125 & 0.3137 & 0.7285 \\
\hline 2.5 & 0.3125 & 0.4161 & 0.20394 \\
\hline 5 & 0.3125 & 0.5432 & 0.16376 \\
\hline 10 & 0.3125 & 0.5650 & 2.19769 \\
\hline 1.25 & 0.625 & 0.4425 & 1.11447 \\
\hline 2.5 & 0.625 & 0.5063 & 0.58936 \\
\hline 5 & 0.625 & 0.5585 & 0.34974 \\
\hline 10 & 0.625 & 0.5852 & 0.26666 \\
\hline 1.25 & 1.25 & 0.4586 & 1.87721 \\
\hline 2.5 & 1.25 & 0.5561 & 0.7166 \\
\hline 5 & 1.25 & 0.5847 & 0.53605 \\
\hline 10 & 1.25 & 0.5908 & 0.50352 \\
\hline 1.25 & 2.5 & 0.7953 & 0.08592 \\
\hline 2.5 & 2.5 & 0.8474 & $\mathbf{0 . 0 3 5 3 5}$ \\
\hline 5 & 2.5 & 0.8852 & 0.01563 \\
\hline 10 & 2.5 & 0.8768 & 0.01907 \\
\hline
\end{tabular}

${ }^{\text {aPC } 3}$ cells treated diiferent concentration of $\mathrm{Mg} 132$ and $\mathbf{2 2 b}$. ${ }^{\mathrm{b}} \mathrm{Mg} 132$, a proteasome inihibitor, $\mathrm{IC}_{50}=17.14 \pm 0.30 \mu \mathrm{M}$. ${ }^{\mathbf{c}} \mathrm{Cell}$ viability was determined by MTTassay. ${ }^{\mathrm{d}} \mathrm{CI}$ values calculated by CompuSyn software. 
Table S7. Effects and CI values treated with combination of Bortezomib and $\mathbf{2 2 b}$. $^{\text {a }}$

\begin{tabular}{|c|c|c|c|}
\hline Dose Bortezomib $^{\mathrm{b}}(\mu \mathrm{M})$ & Dose 22b $(\mu \mathrm{M})$ & Effect $^{\mathrm{c}}$ & $\mathrm{CI}^{\mathrm{d}}$ \\
\hline- & 0.3125 & 0.3172 & - \\
\hline - & 0.625 & 0.4480 & - \\
\hline - & 1.25 & 0.5658 & - \\
\hline - & 2.5 & 0.7043 & - \\
\hline 0.0625 & - & 0.5168 & - \\
\hline 0.125 & - & 0.5333 & - \\
\hline 0.25 & - & 0.5412 & - \\
\hline 0.5 & - & 0.5677 & - \\
\hline 0.0625 & 0.3125 & 0.6220 & 0.2203 \\
\hline 0.125 & 0.3125 & 0.6380 & 0.1967 \\
\hline 0.25 & 0.3125 & 0.6597 & 0.1749 \\
\hline 0.5 & 0.3125 & 0.6741 & 0.1309 \\
\hline 0.0625 & 0.625 & 0.7488 & 0.1792 \\
\hline 0.125 & 0.625 & 0.7315 & 0.2065 \\
\hline 0.25 & 0.625 & 0.7371 & 0.1928 \\
\hline 0.5 & 0.625 & 0.7455 & 0.1797 \\
\hline 0.0625 & 1.25 & 0.9394 & 0.0385 \\
\hline 0.125 & 1.25 & 0.9355 & 0.0385 \\
\hline 0.25 & 1.25 & 0.9414 & 0.0385 \\
\hline 0.5 & 1.25 & 0.9354 & 0.0385 \\
\hline 0.0625 & 2.5 & 0.9501 & 0.0593 \\
\hline 0.125 & 2.5 & 0.9529 & 0.0593 \\
\hline 0.25 & 2.5 & 0.9519 & 0.0593 \\
\hline 0.5 & 2.5 & 0.9523 & 0.0593 \\
\hline
\end{tabular}

${ }^{a} \mathrm{PC} 3$ cells treated diiferent concentration of Bortezomib and 22b. ${ }^{b}$ Bortezomib, a proteasome inihibitor, inhibition for PC3 cells was approx. $50 \%$ from $0.0625 \mu \mathrm{M}$ to $0.5 \mu \mathrm{M}$. ${ }^{\mathrm{c} C e l l}$ viability was determined by MTTassay. ${ }^{\mathrm{d}} \mathrm{CI}$ values calculated by CompuSyn software.

\section{Weight of individual excised tumor.}

Table S8. Weight of individual excised tumor.

\begin{tabular}{ccc}
\hline Placebo (g) & 22b (g) & Docetaxol (g) \\
\hline 0.89 & 0.56 & 0.08 \\
0.83 & 0.53 & 0.06 \\
0.65 & 0.52 & 0.05 \\
0.58 & 0.39 & 0.02 \\
0.57 & 0.09 & \\
\hline
\end{tabular}


8. Figures of HR-ESI-MS, IR, ${ }^{1} \mathrm{H},{ }^{13} \mathrm{C}$ and $2 \mathrm{D}$ NMR spectra for derivatives.

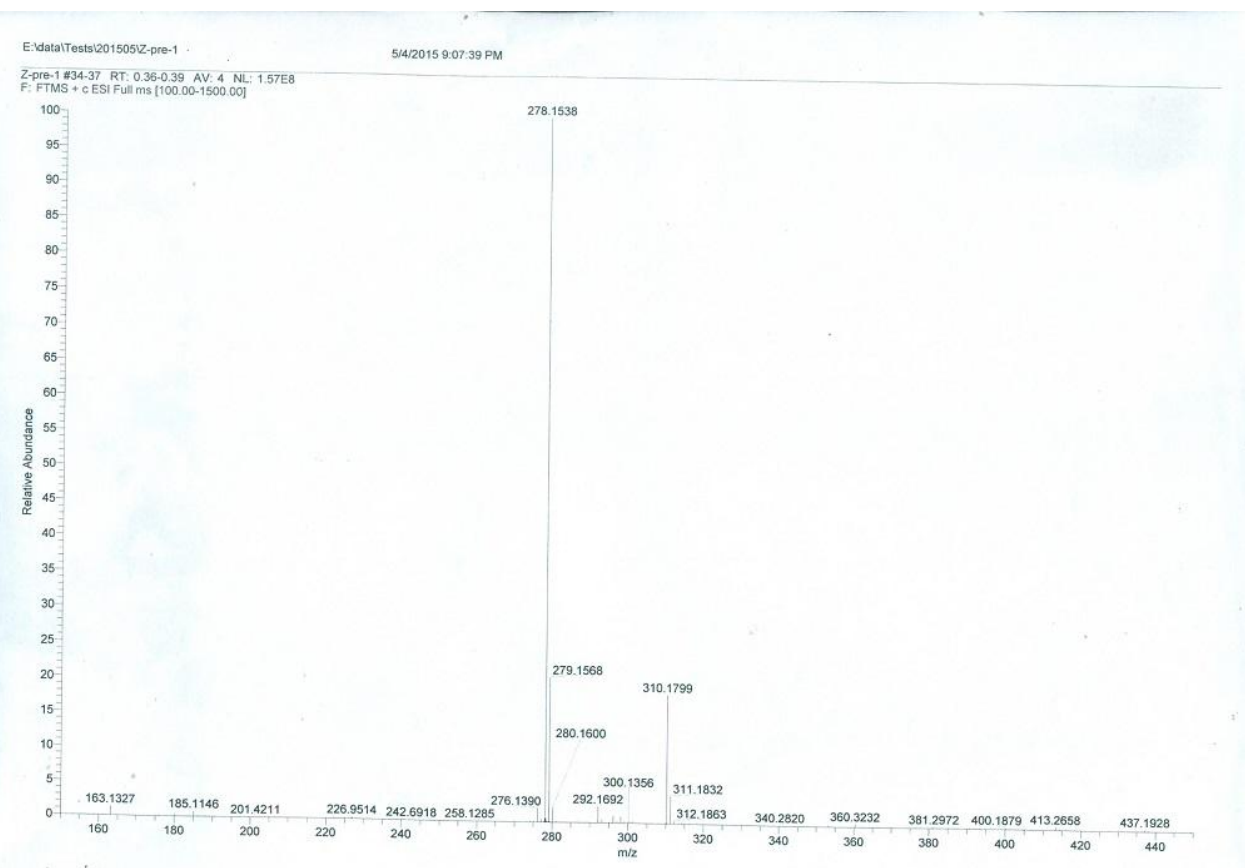

Figure S14. HRESIMS spectrum of SD1.
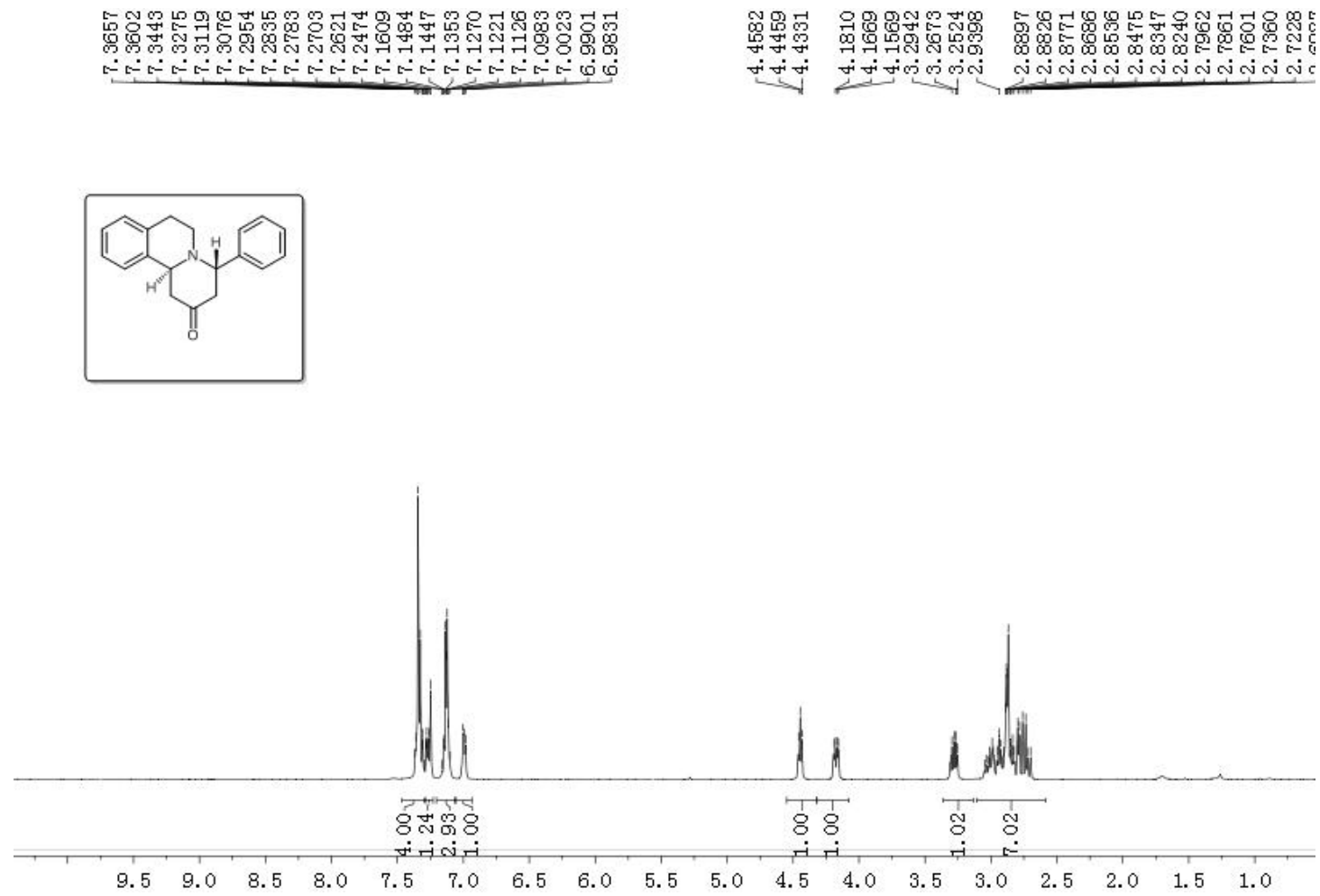

Figure S15. ${ }^{1} \mathrm{H}$ NMR spectrum of SD1. 

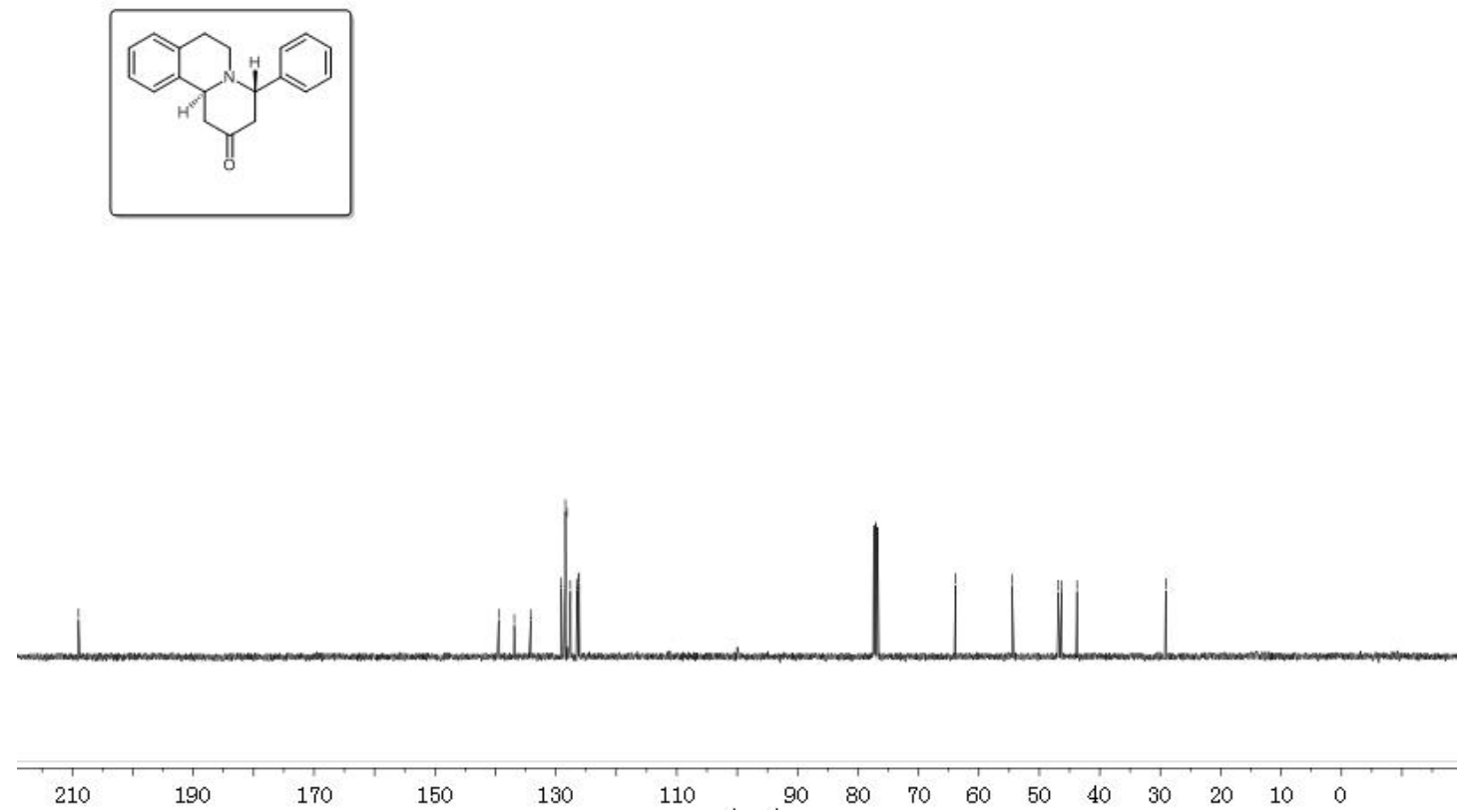

Figure S16. ${ }^{13} \mathrm{C}$ NMR spectrum of SD1.

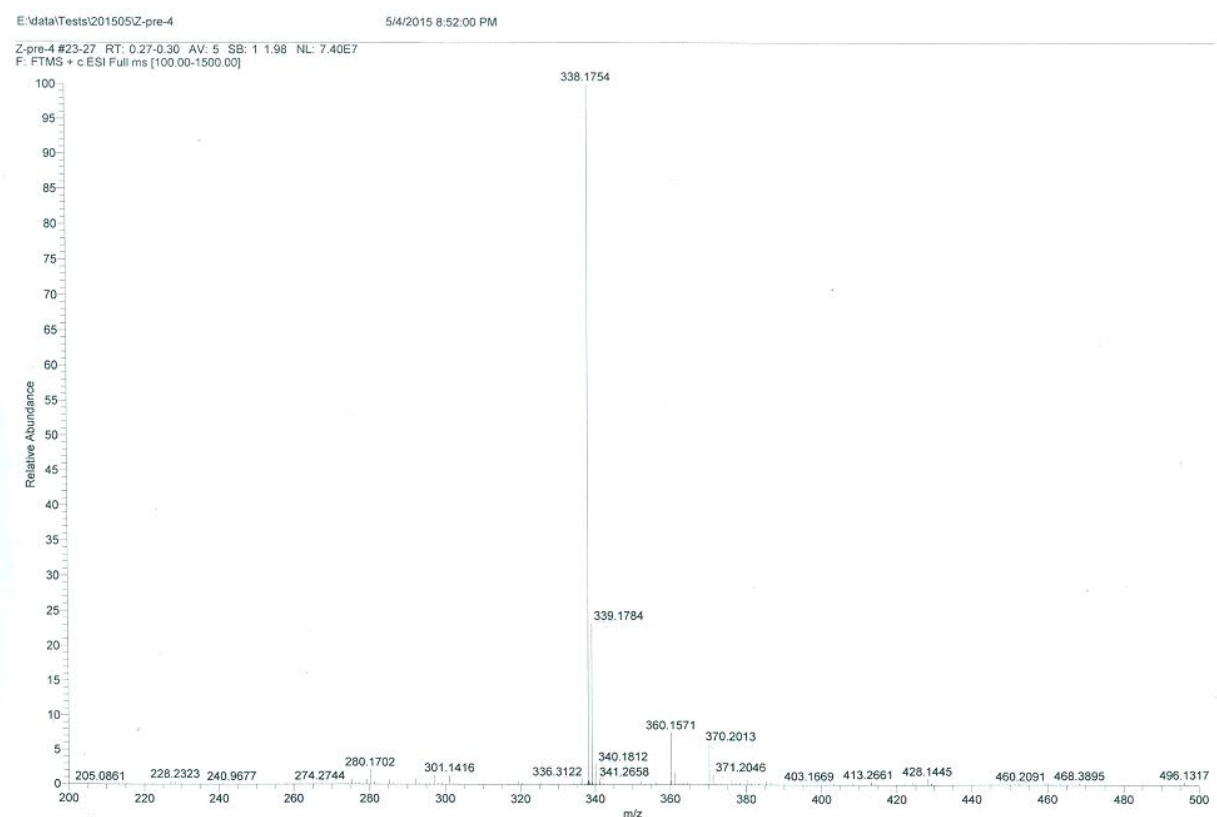

Figure S17. HRESIMS spectrum of SD2. 

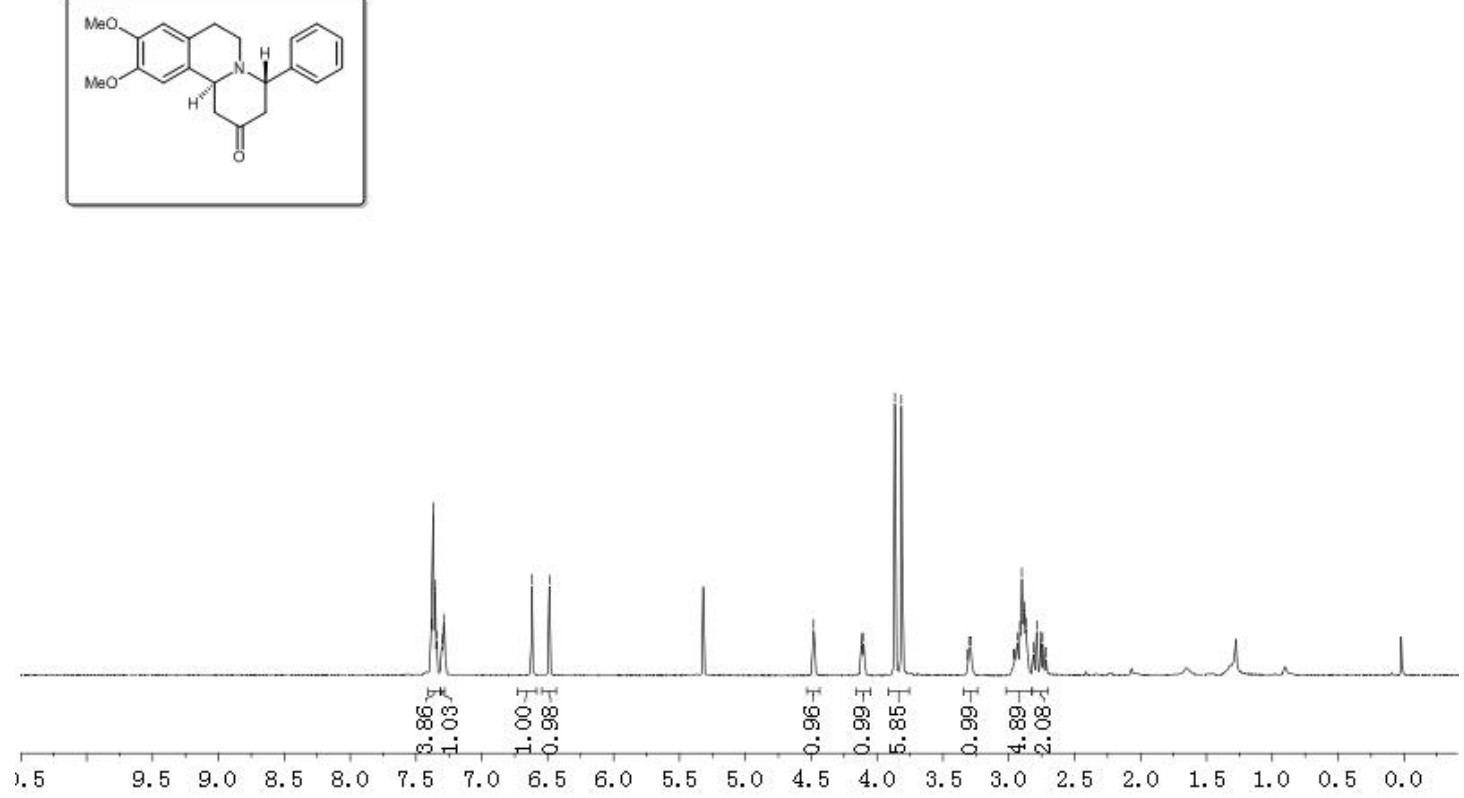

Figure S18. ${ }^{1} \mathrm{H}$ NMR spectrum of SD2.

จุ.
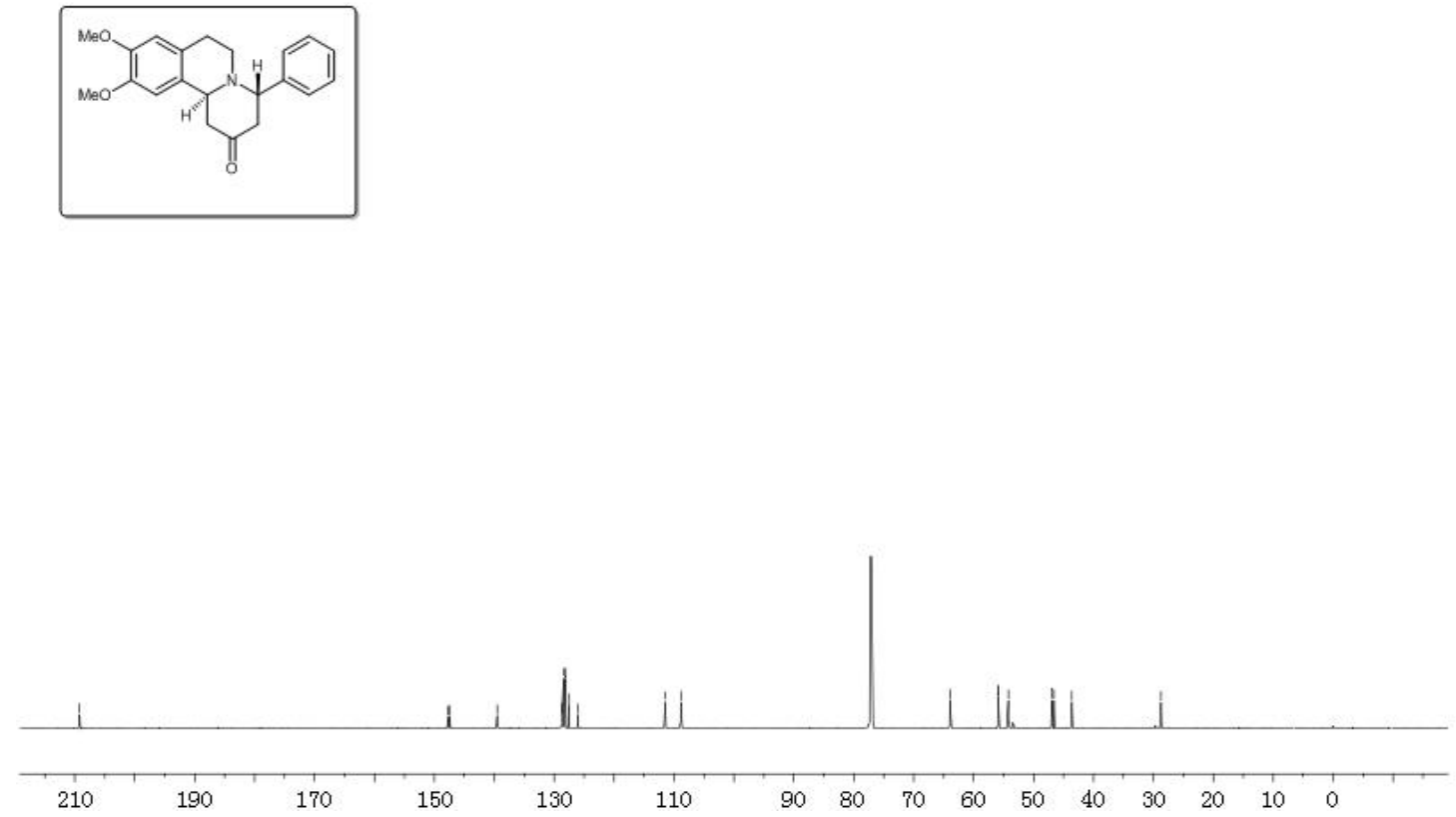

Figure S19. ${ }^{13} \mathrm{C}$ NMR spectrum of SD2. 


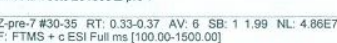

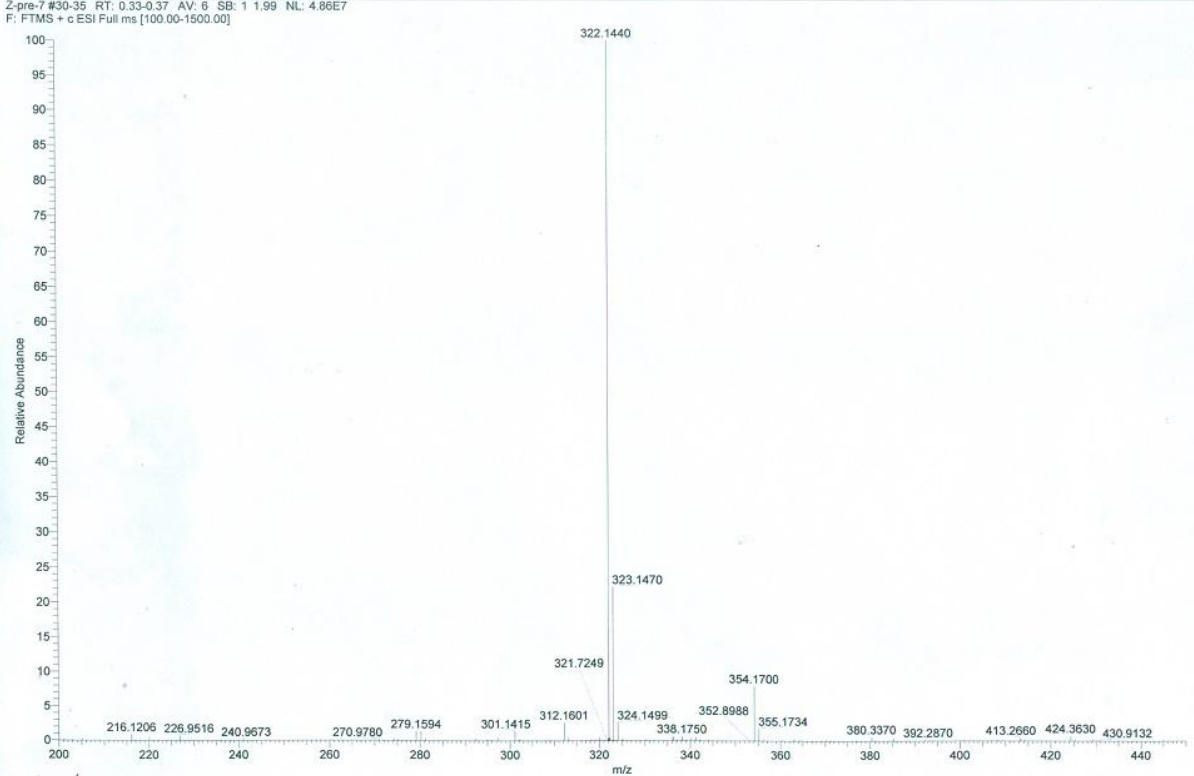

Figure S20. HRESIMS spectrum of SD3.

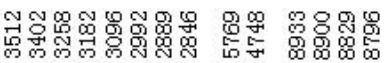

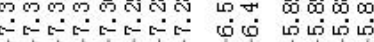

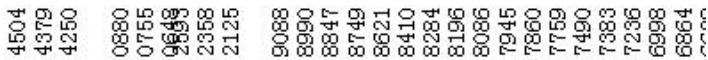

पंu
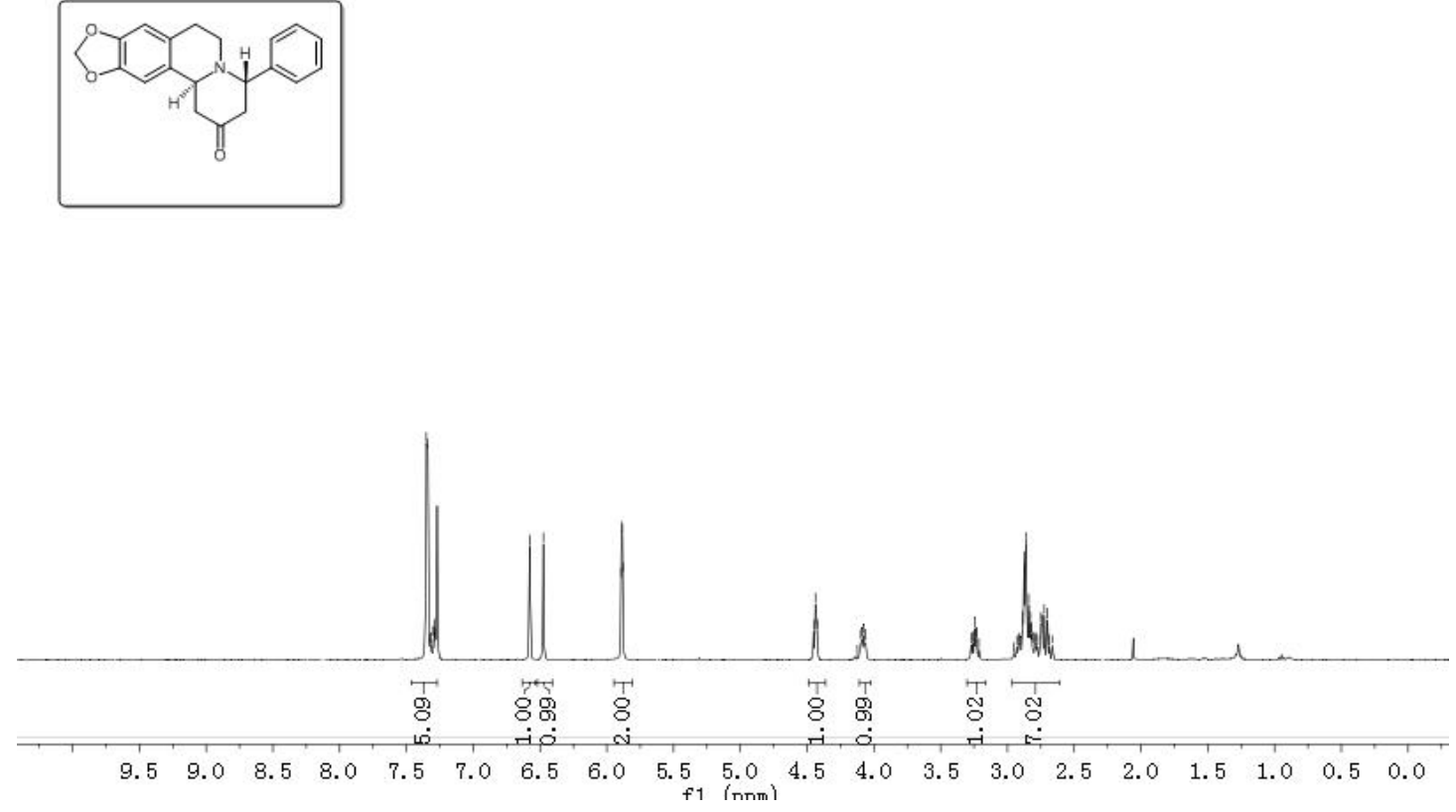

Figure S21. ${ }^{1}$ H NMR spectrum of SD3. 


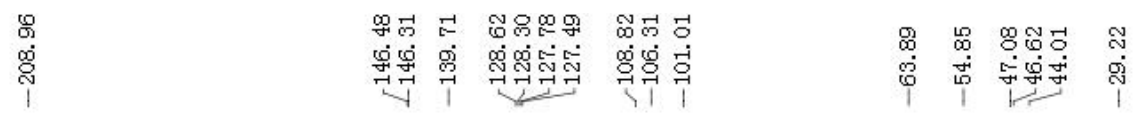
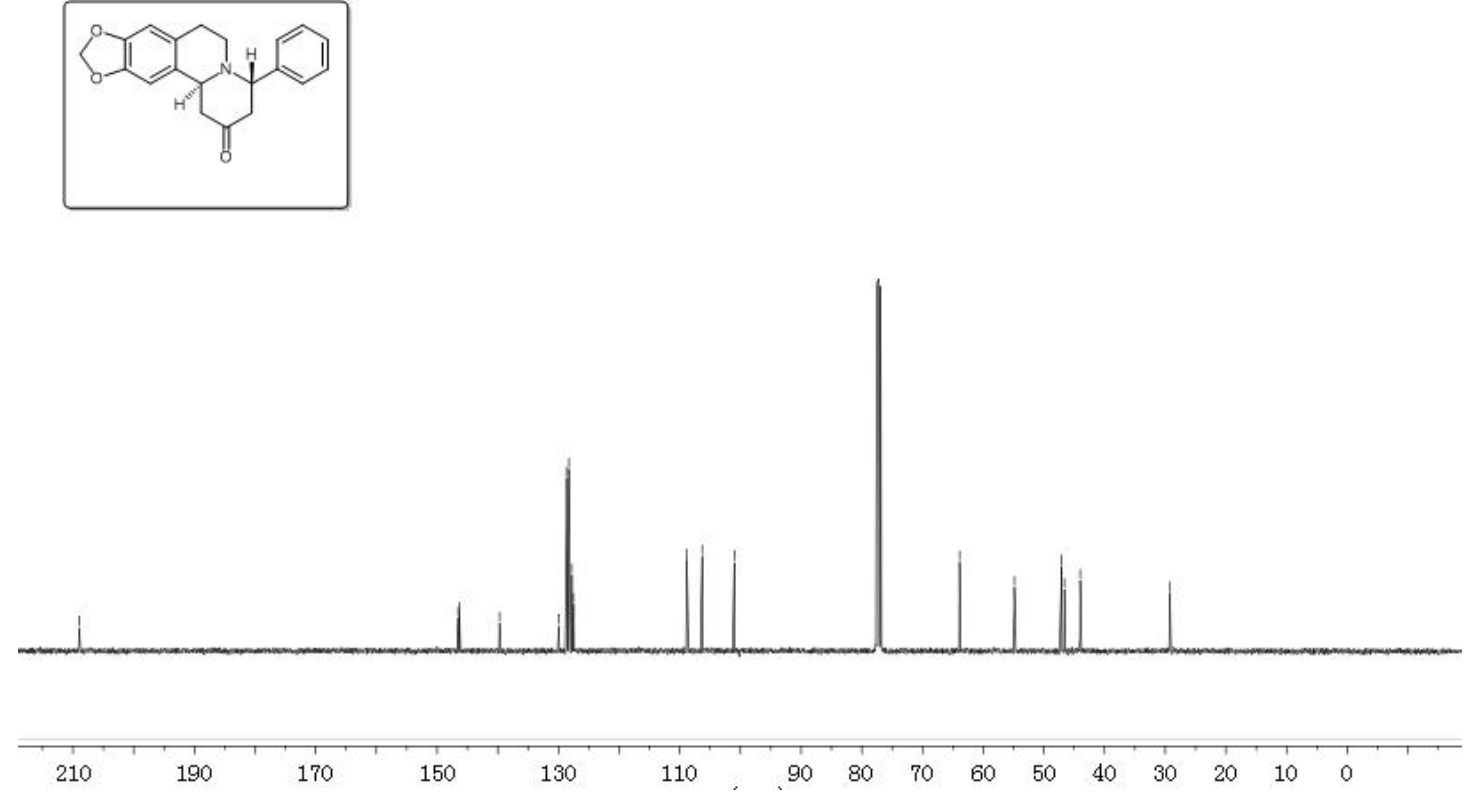

Figure S22. ${ }^{13} \mathrm{C}$ NMR spectrum of SD3.

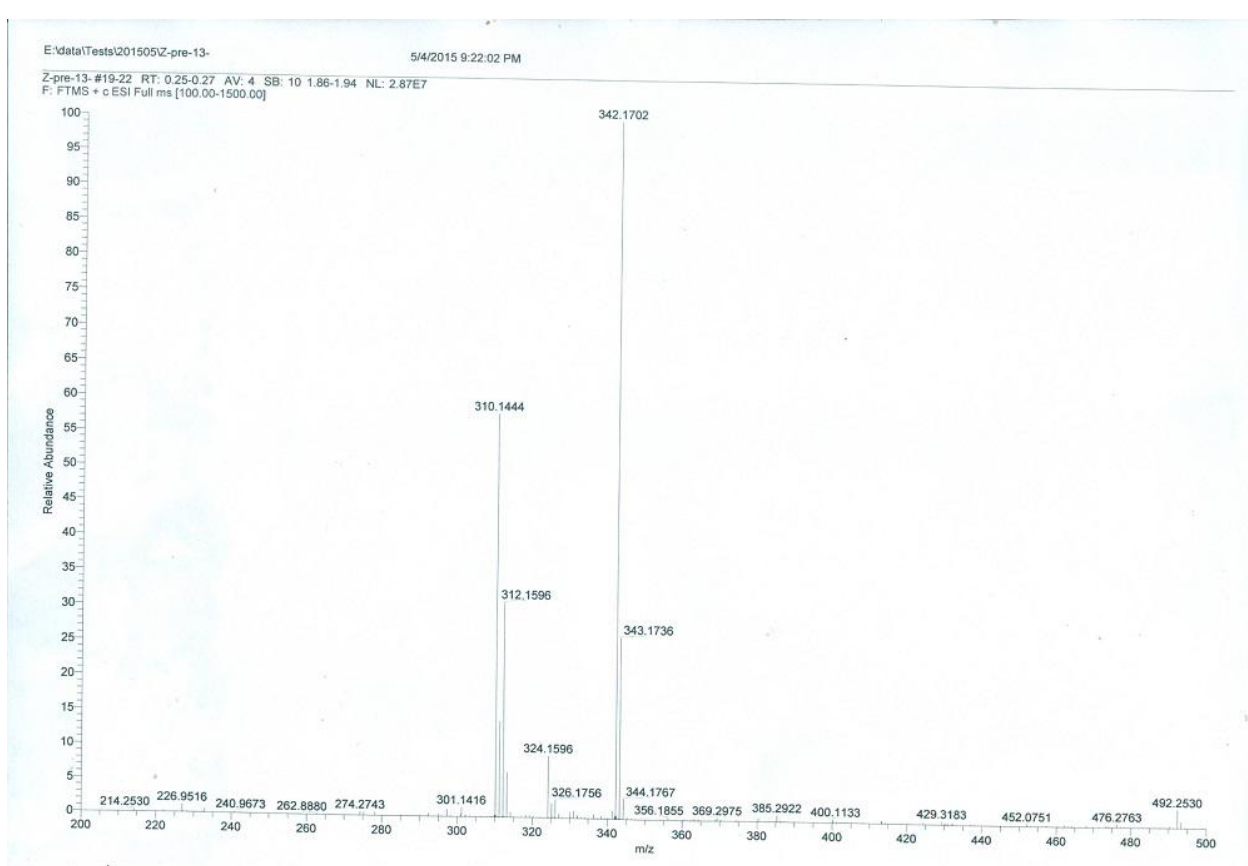

Figure S23. HRESIMS spectrum of SD4. 

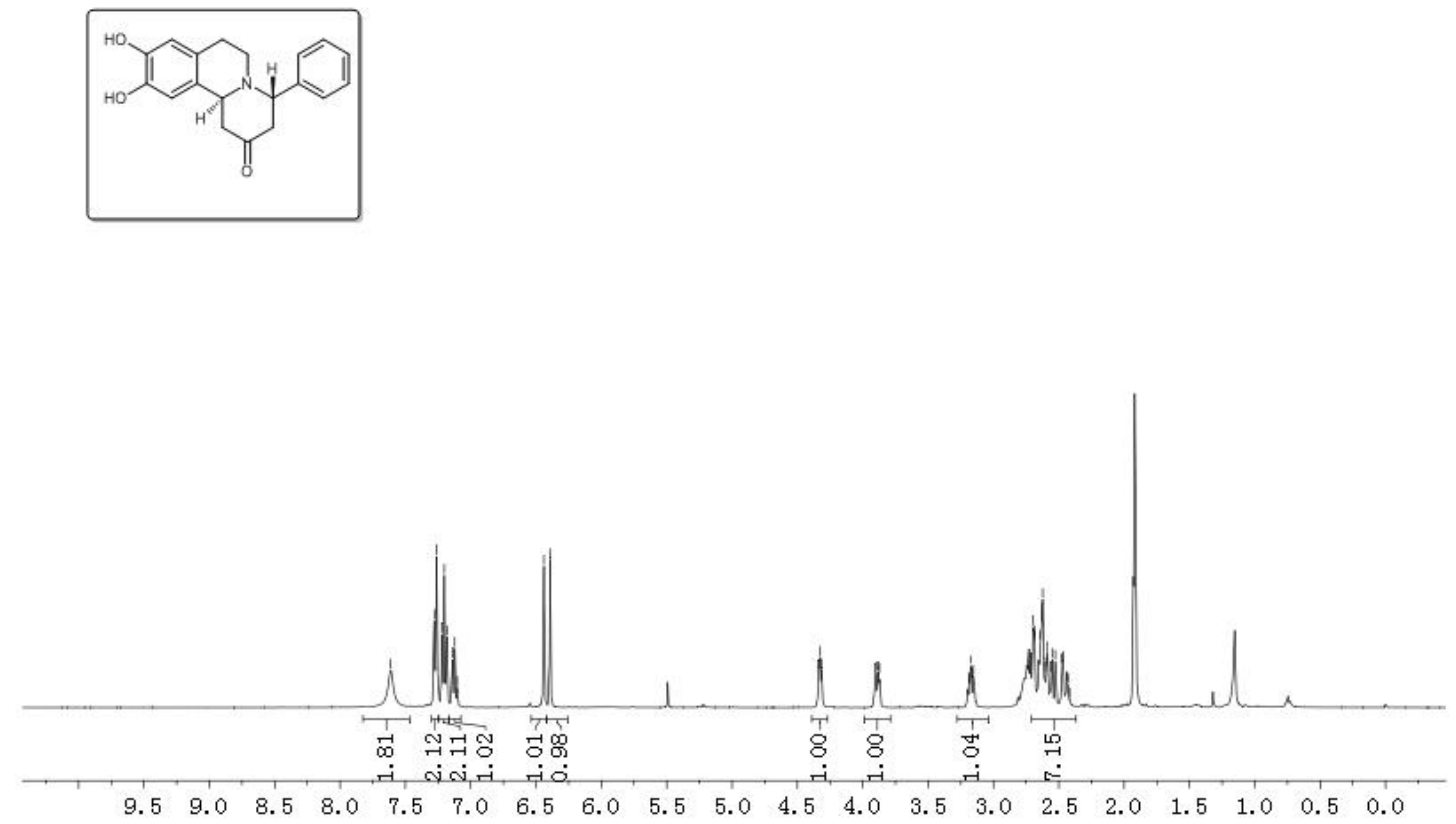

Figure S24. ${ }^{1} \mathrm{H}$ NMR spectrum of SD4.

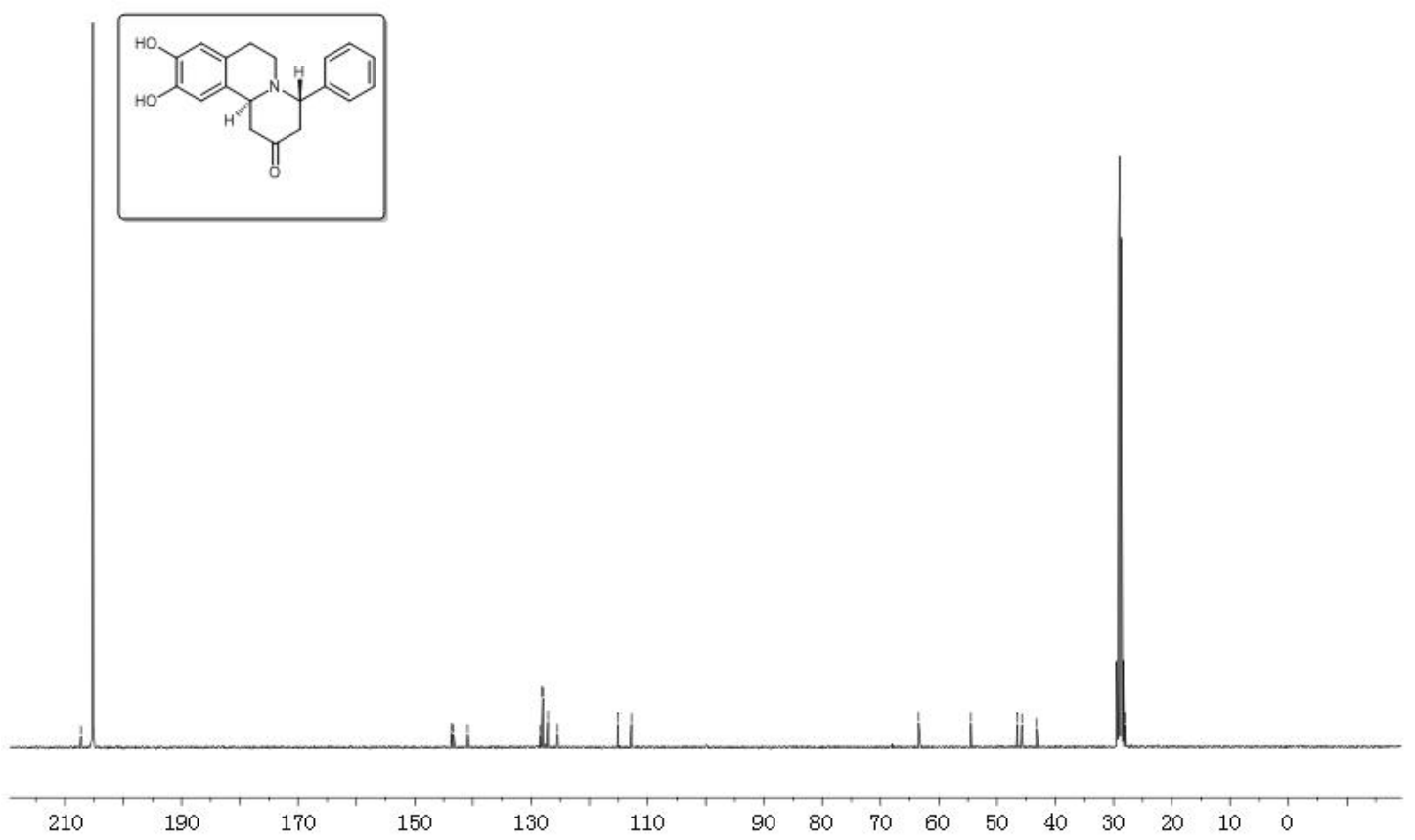

Figure S25. ${ }^{13} \mathrm{C}$ NMR spectrum of SD4. 


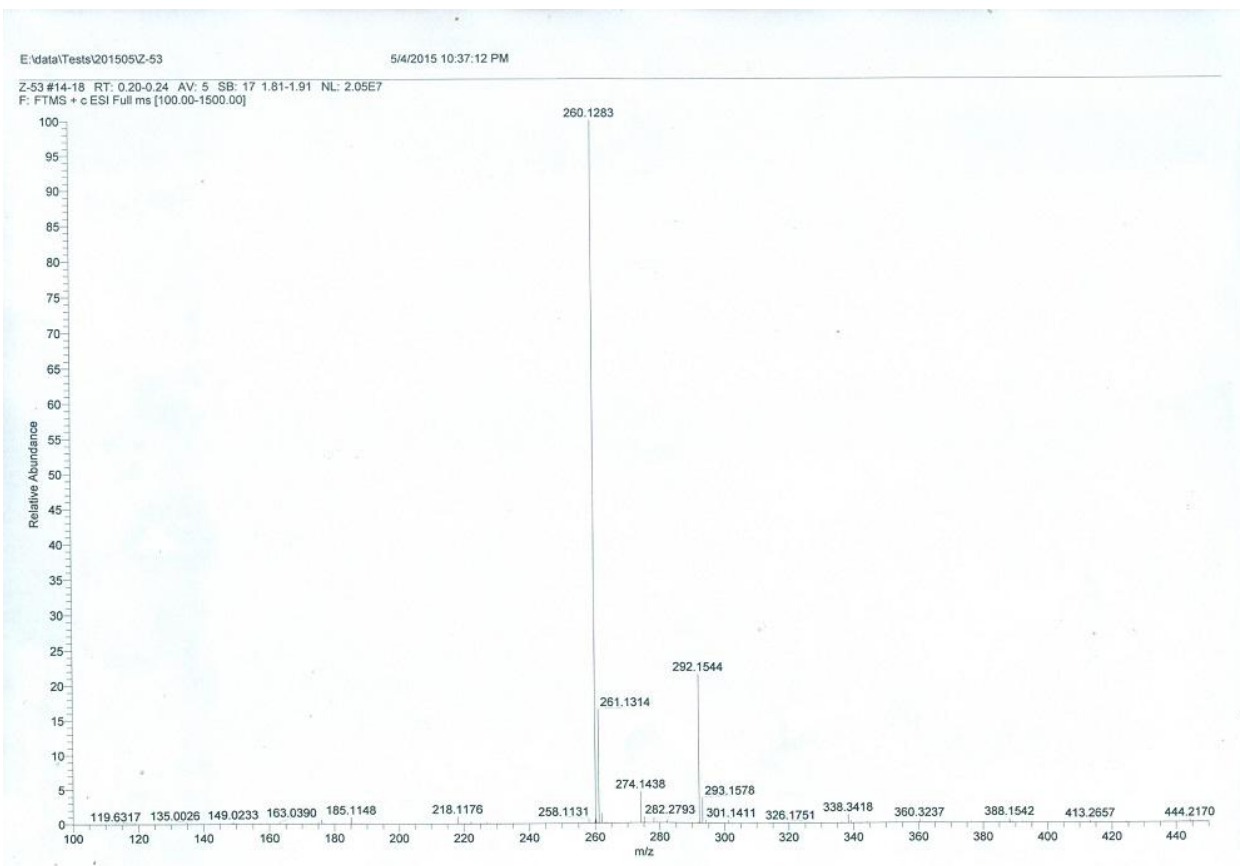

Figure S26. HRESIMS spectrum of SD5.
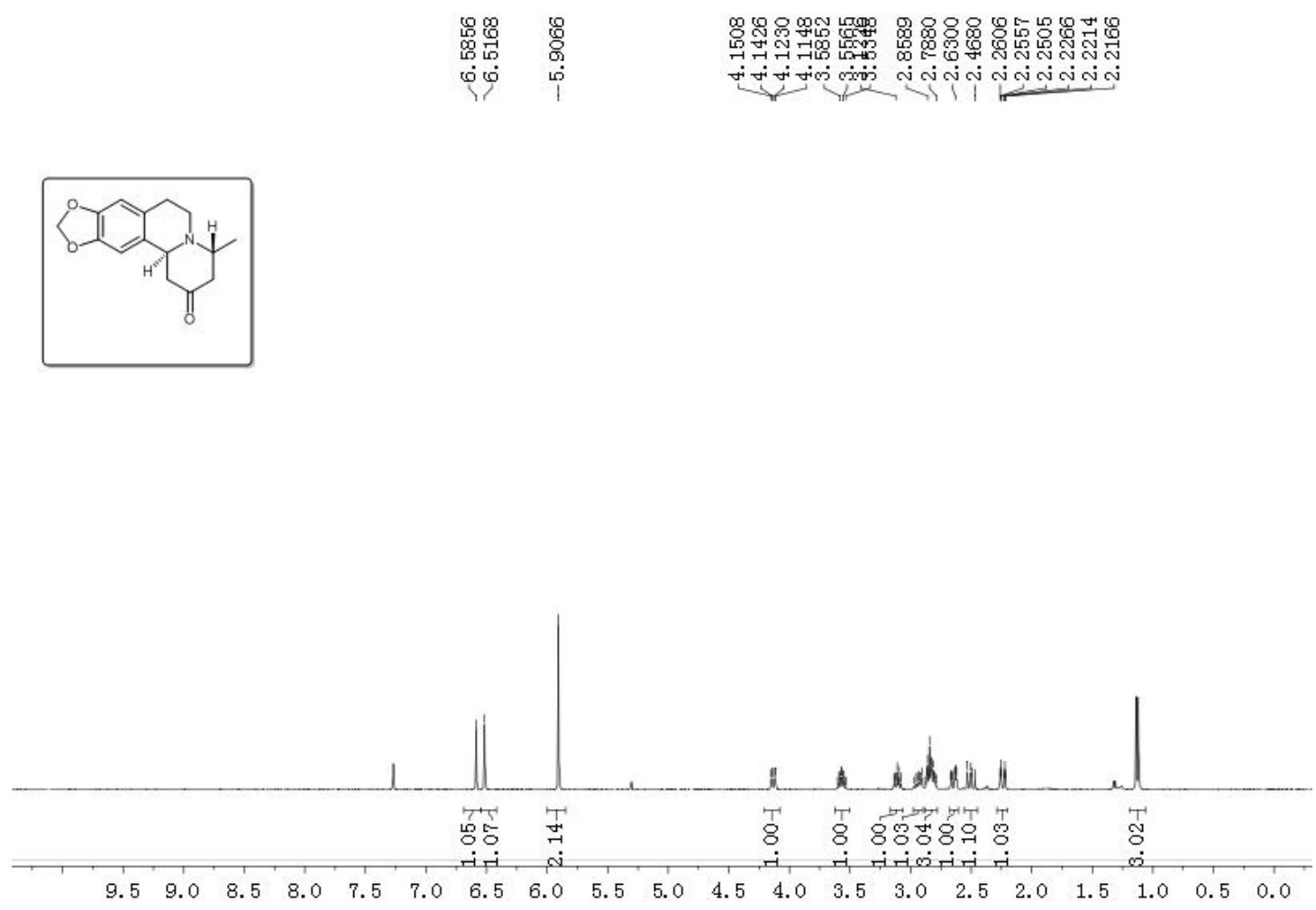

Figure S27. ${ }^{1} \mathrm{H}$ NMR spectrum of SD5. 


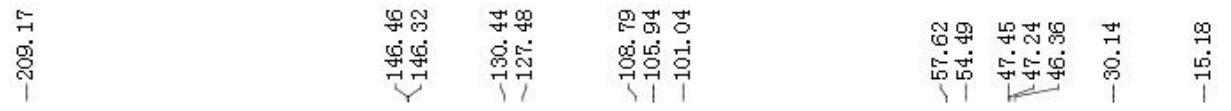

$$
\text { 11 }
$$
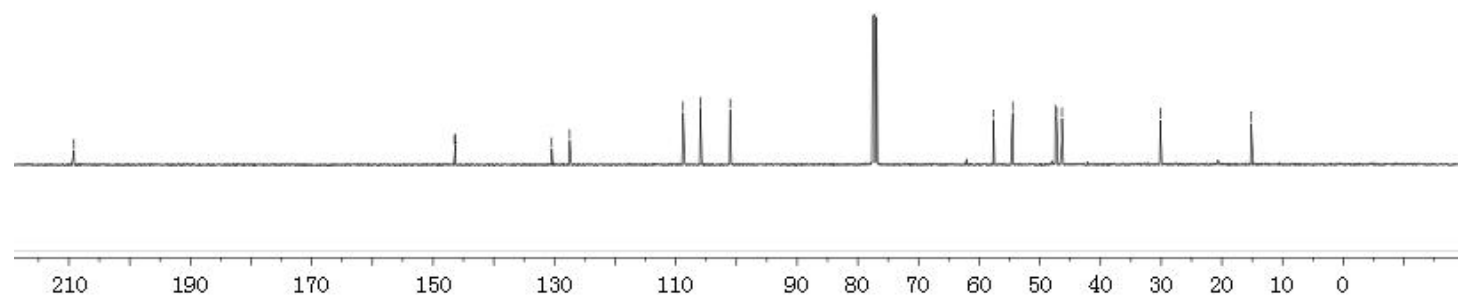

Figure S28. ${ }^{13} \mathrm{C}$ NMR spectrum of SD5.

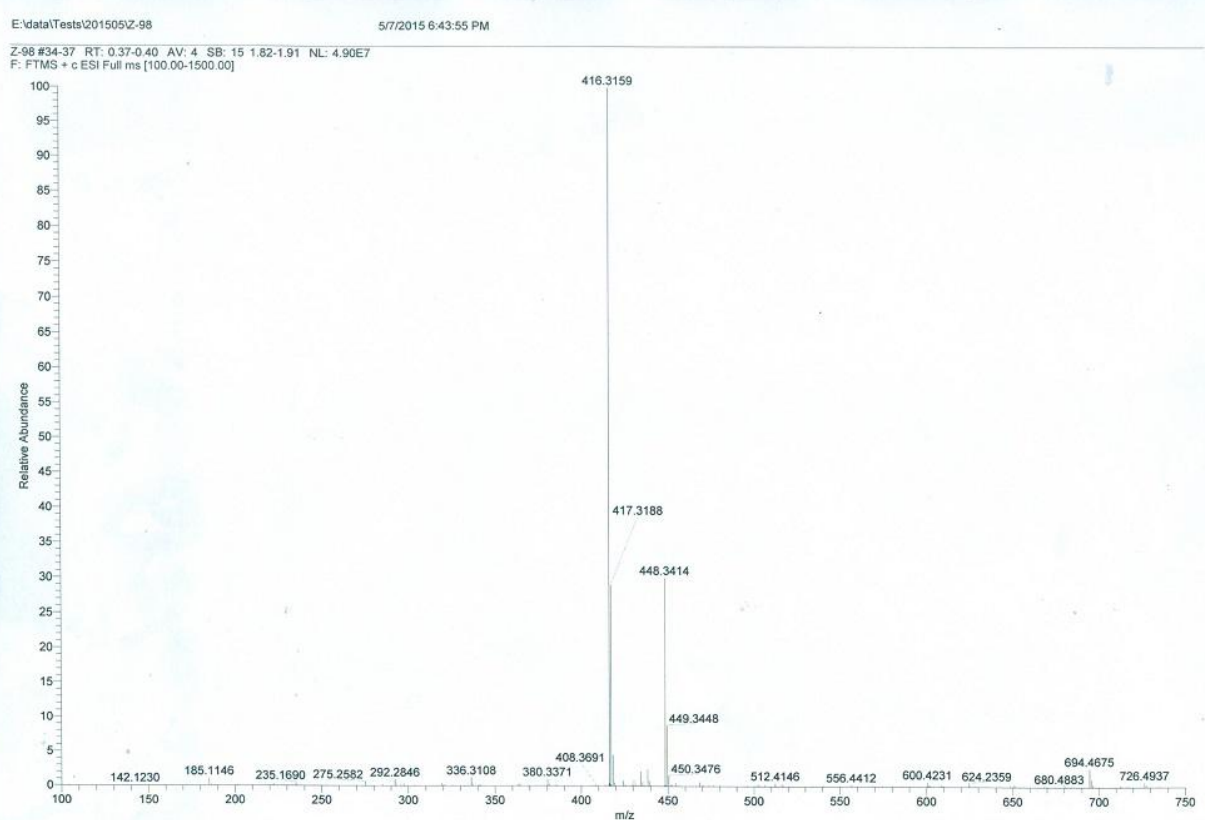

Figure S29. HRESIMS spectrum of SD6. 

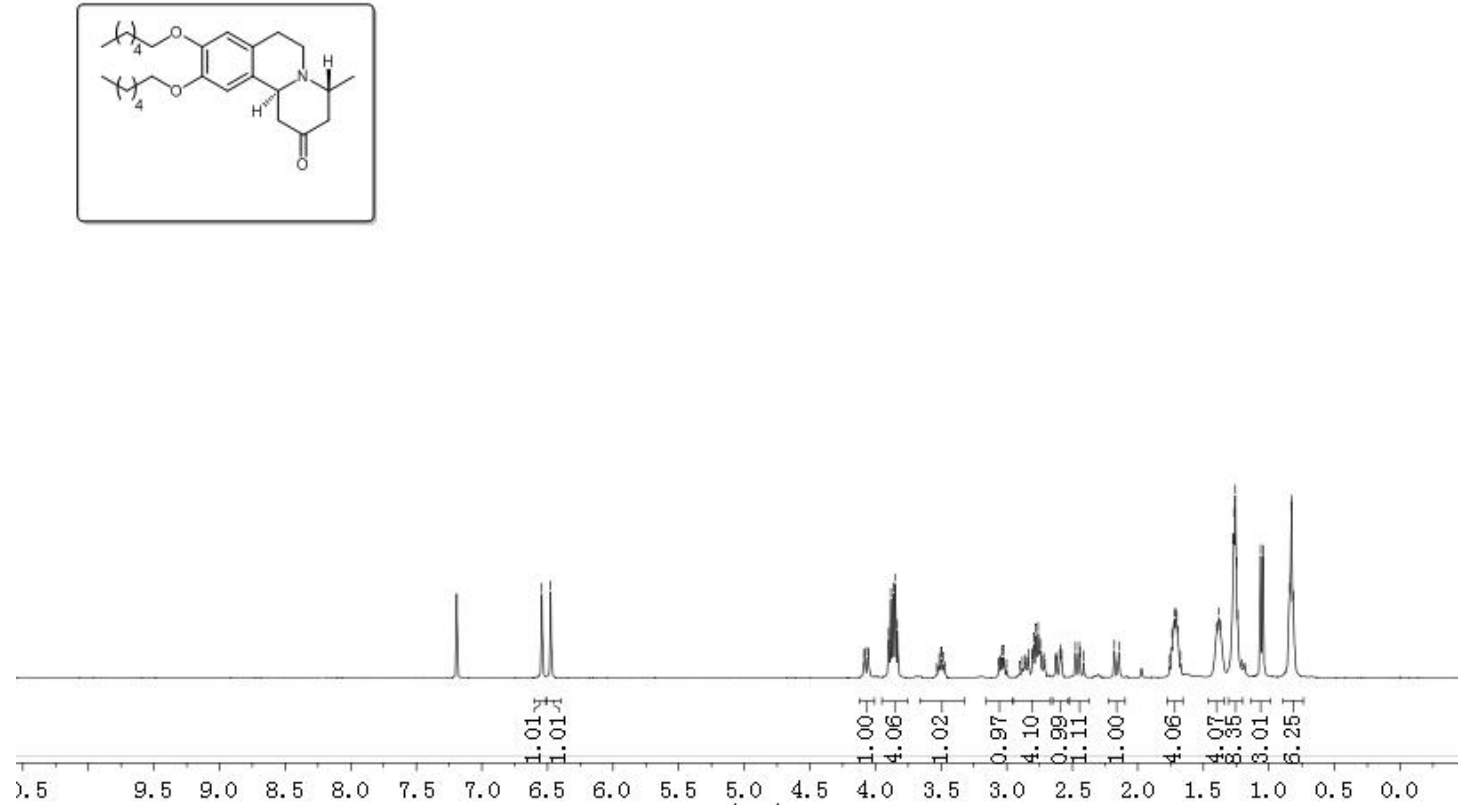

Figure S30.. ${ }^{1} \mathrm{H}$ NMR spectrum of SD6.

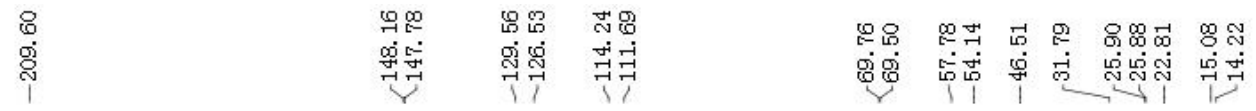
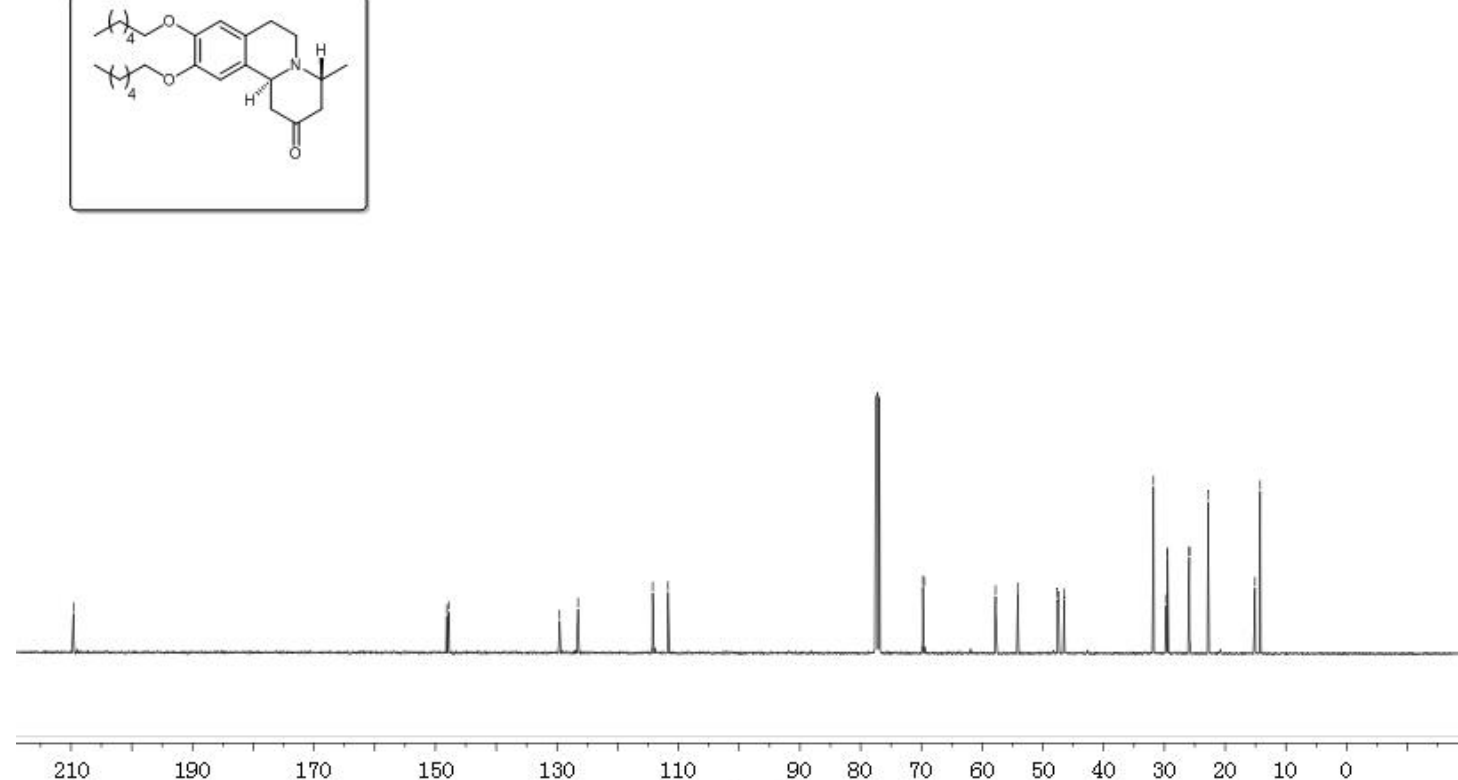

150

Figure S31. ${ }^{13} \mathrm{C}$ NMR spectrum of SD6. 


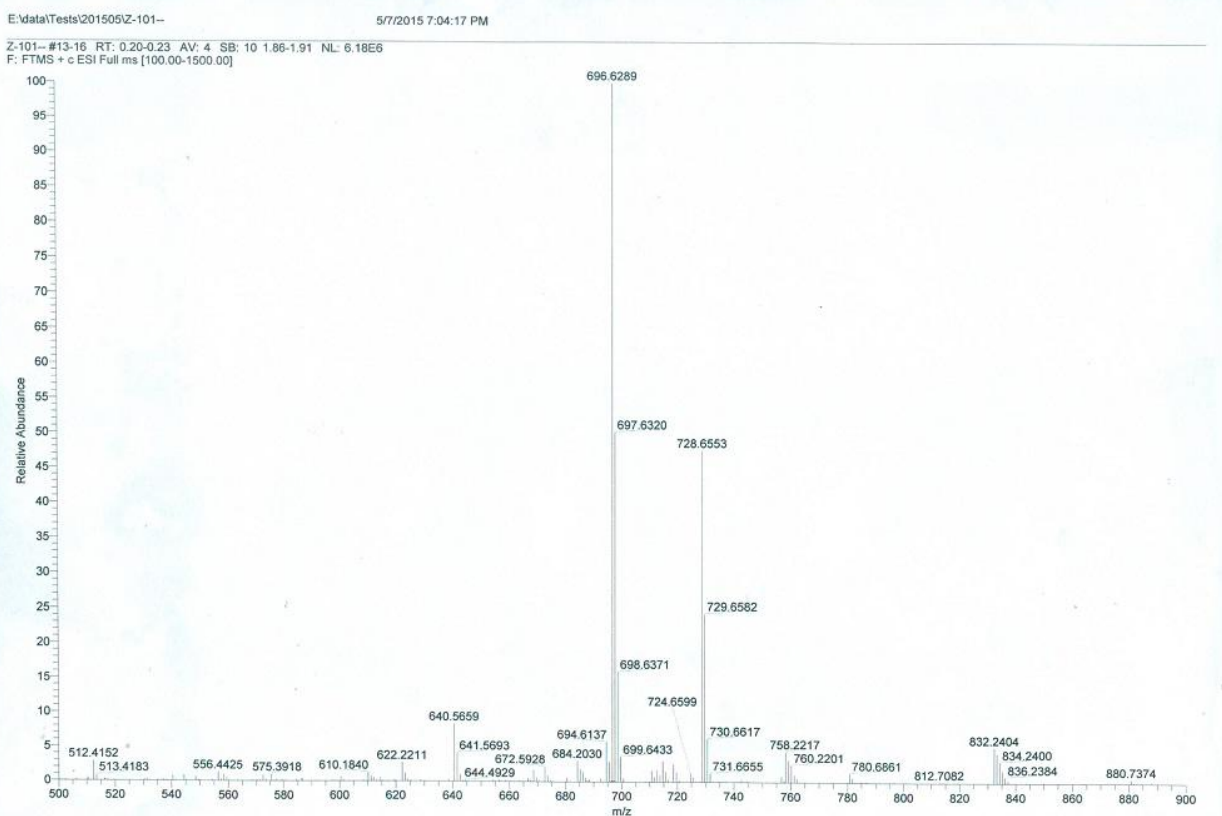

Figure S32. HRESIMS spectrum of SD7.
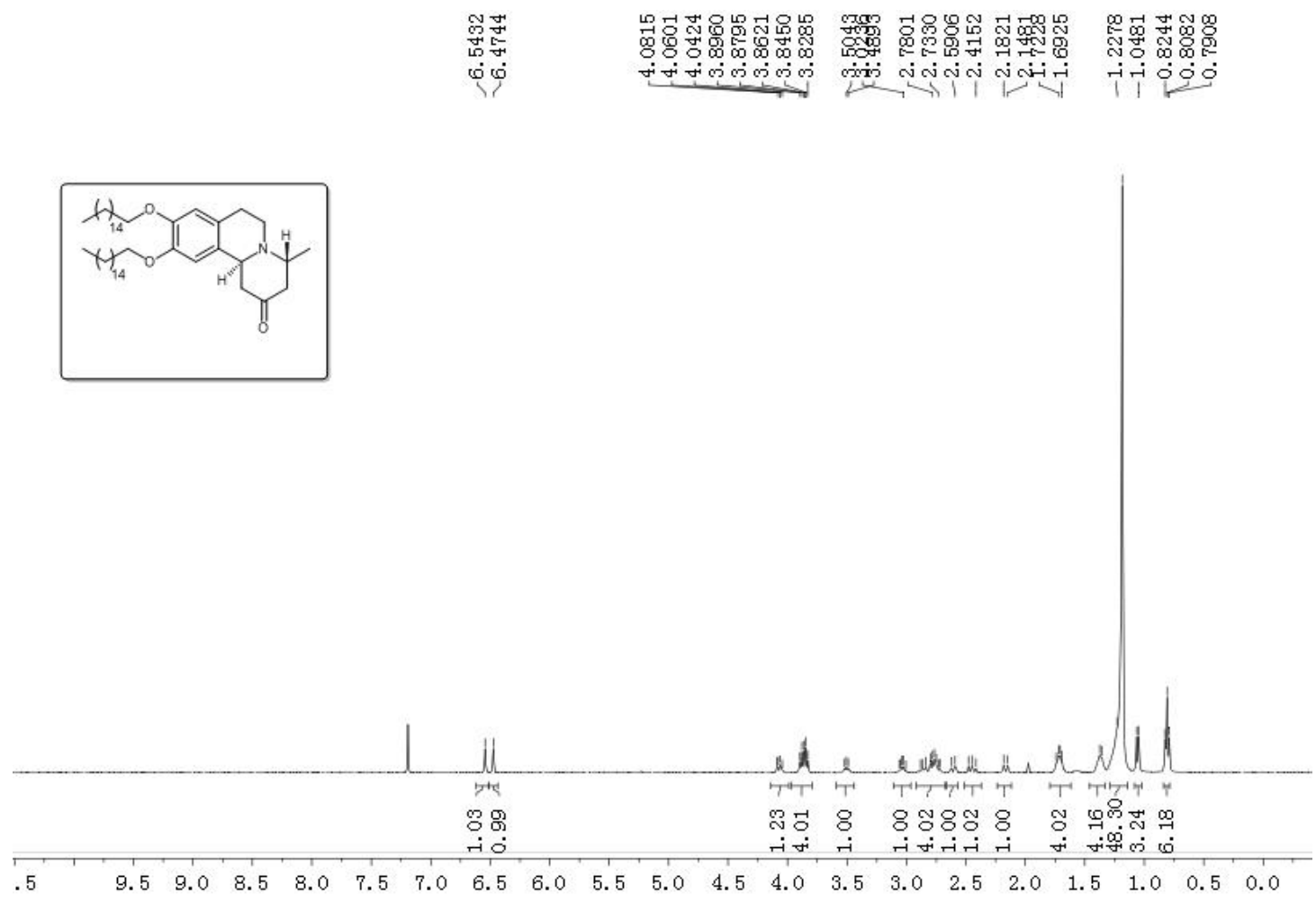

Figure S33. ${ }^{1} \mathrm{H}$ NMR spectrum of SD7. 


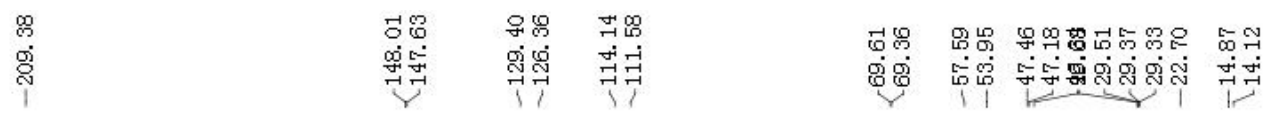
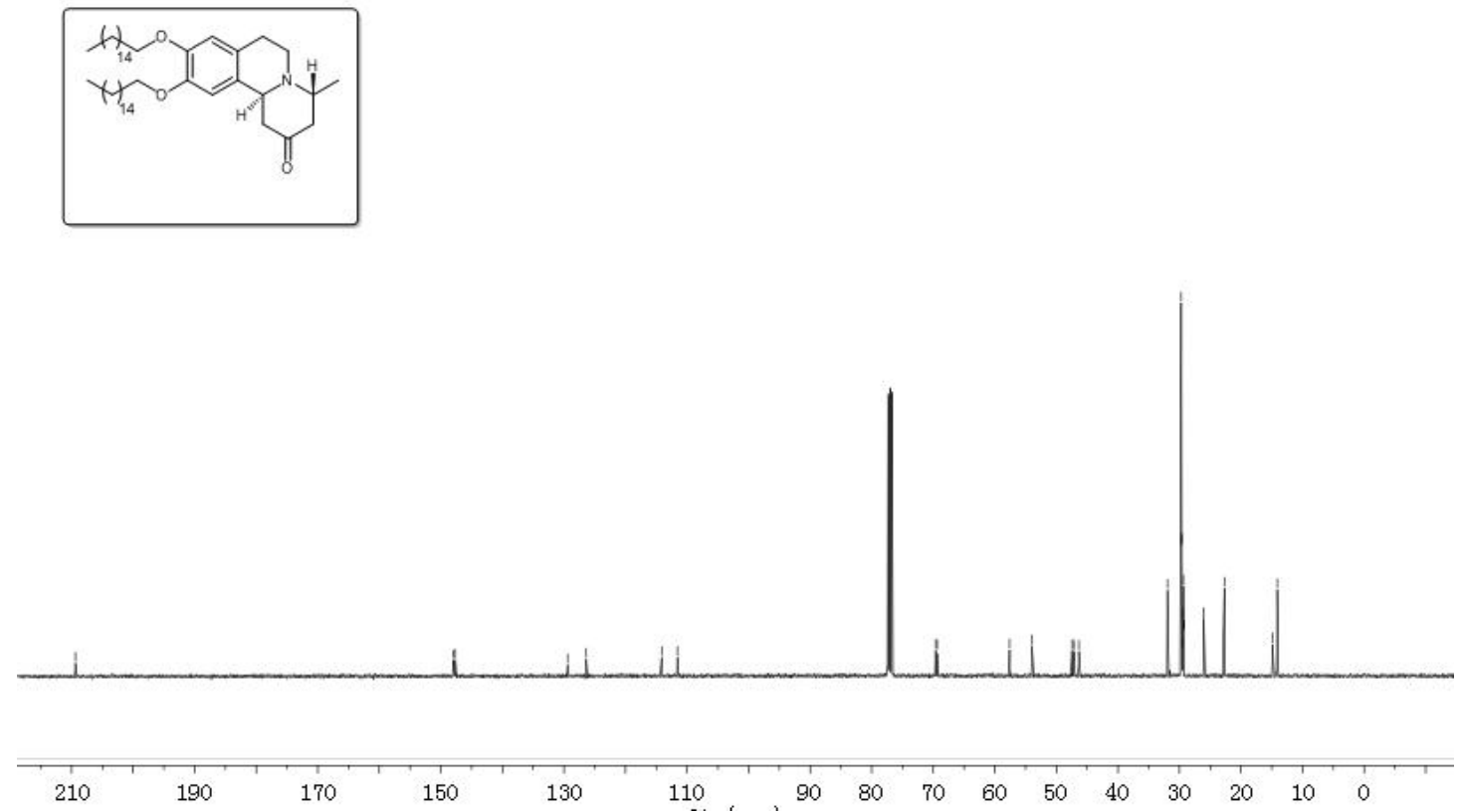

Figure S34. ${ }^{13} \mathrm{C}$ NMR spectrum of SD7.

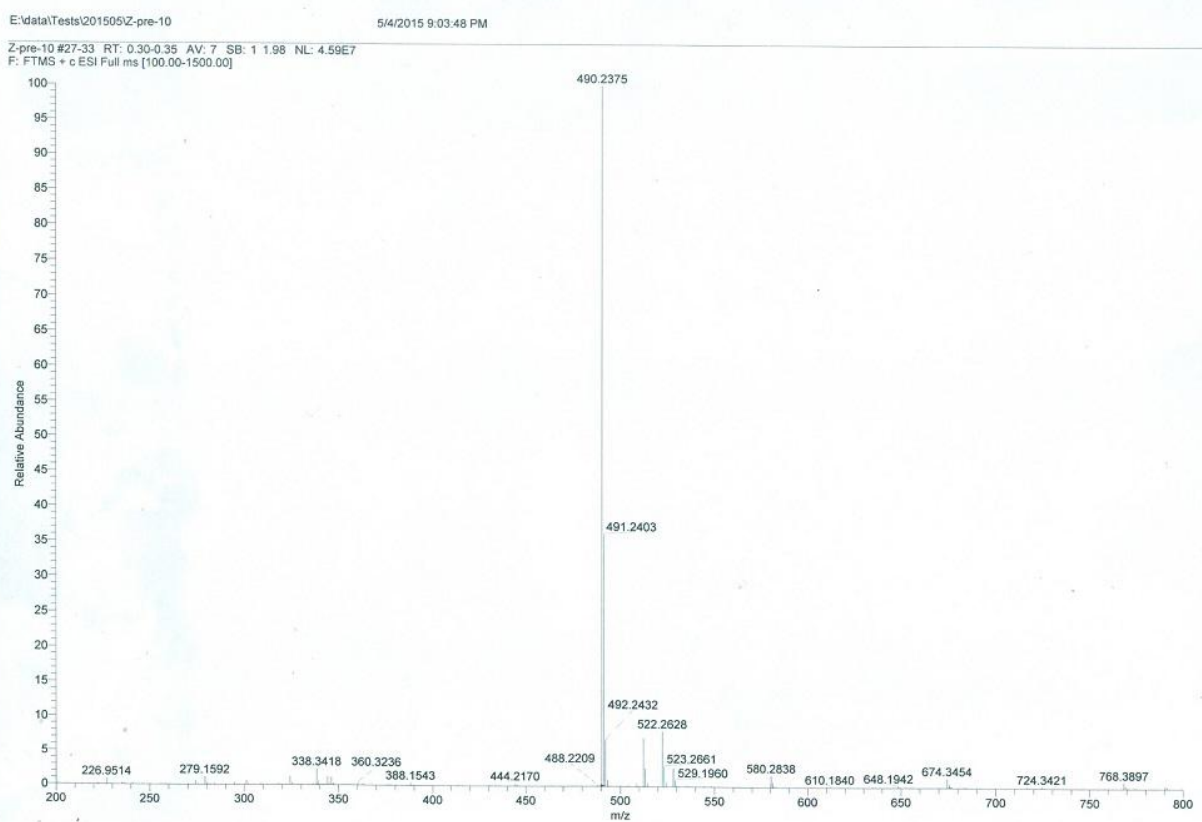

Figure S35. HRESIMS spectrum of SD8. 

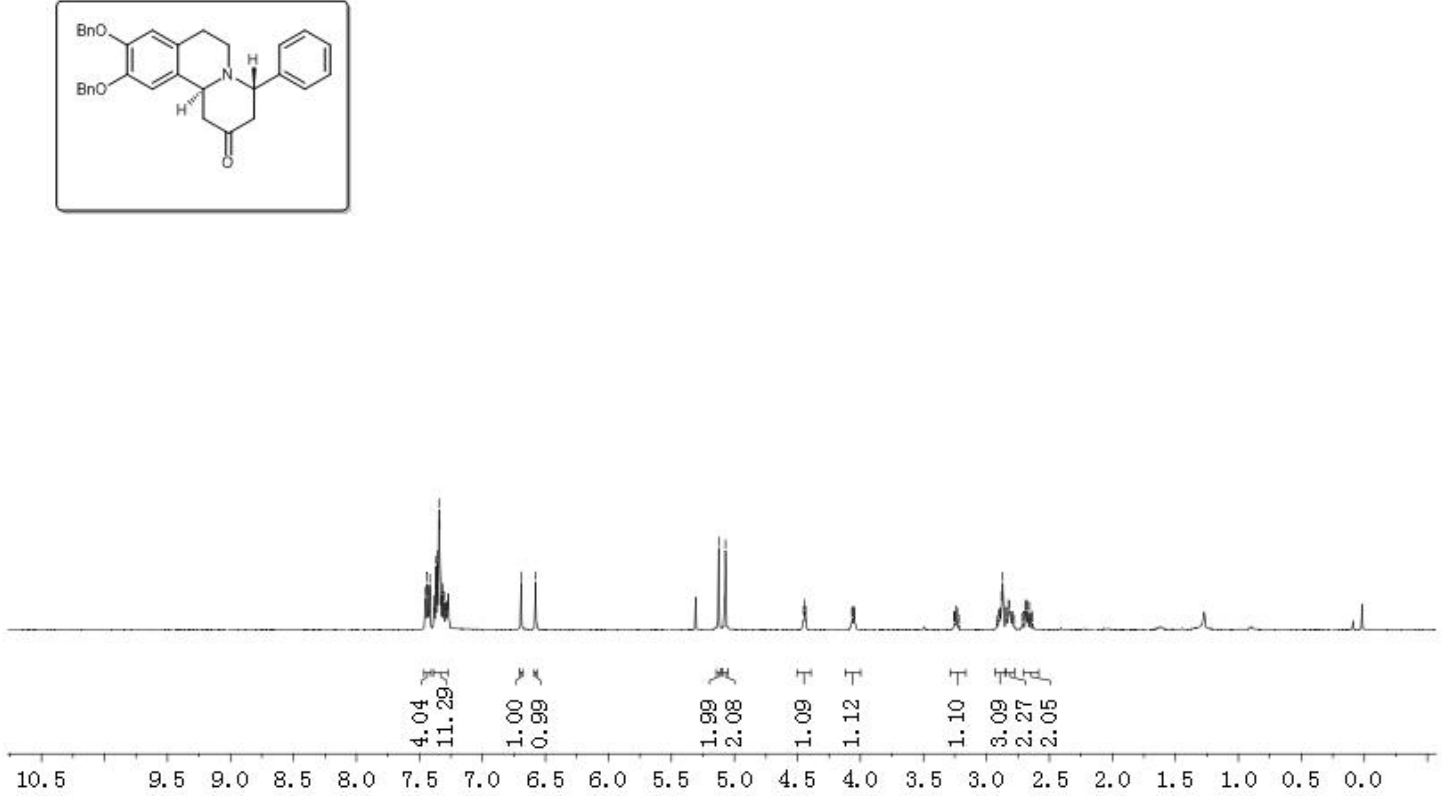

Figure S36. ${ }^{1} \mathrm{H}$ NMR spectrum of SD8

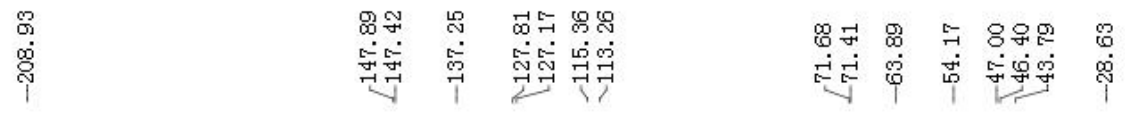
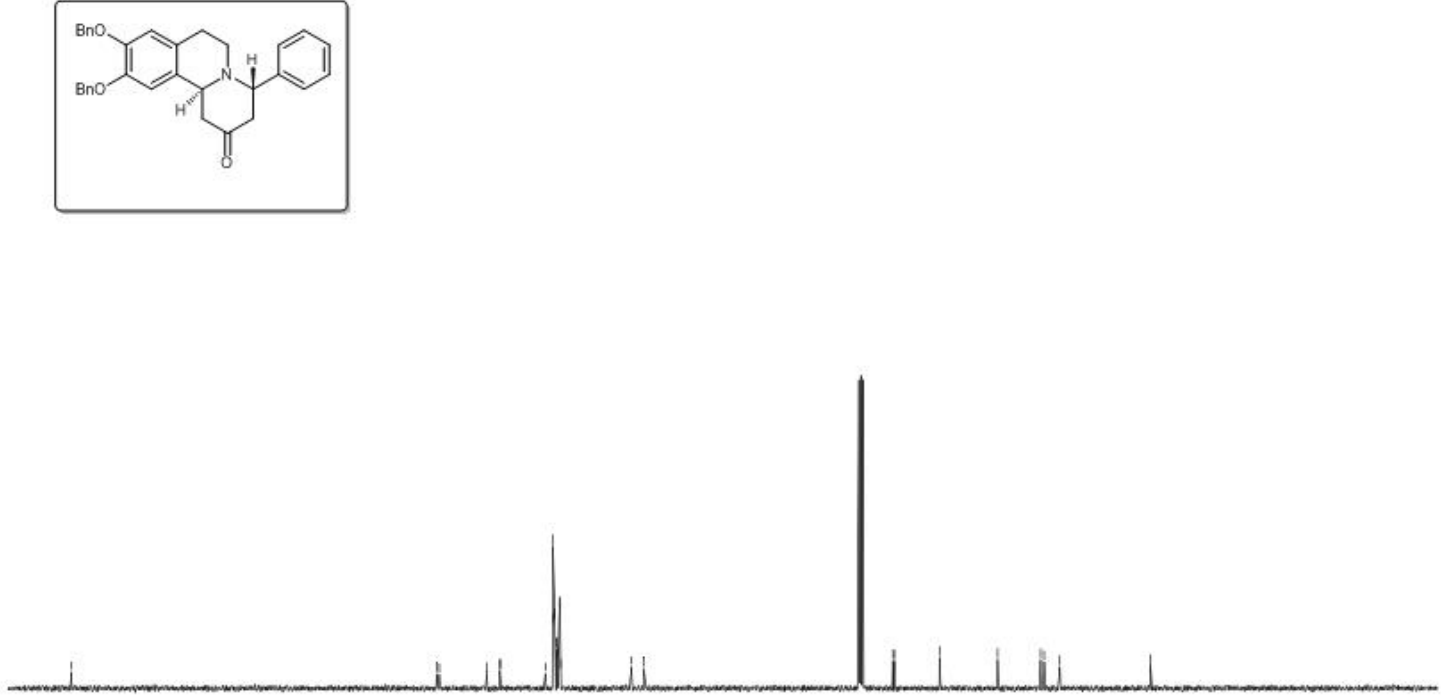

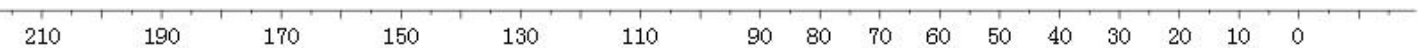

Figure S37. ${ }^{13} \mathrm{C}$ NMR spectrum of SD8. 


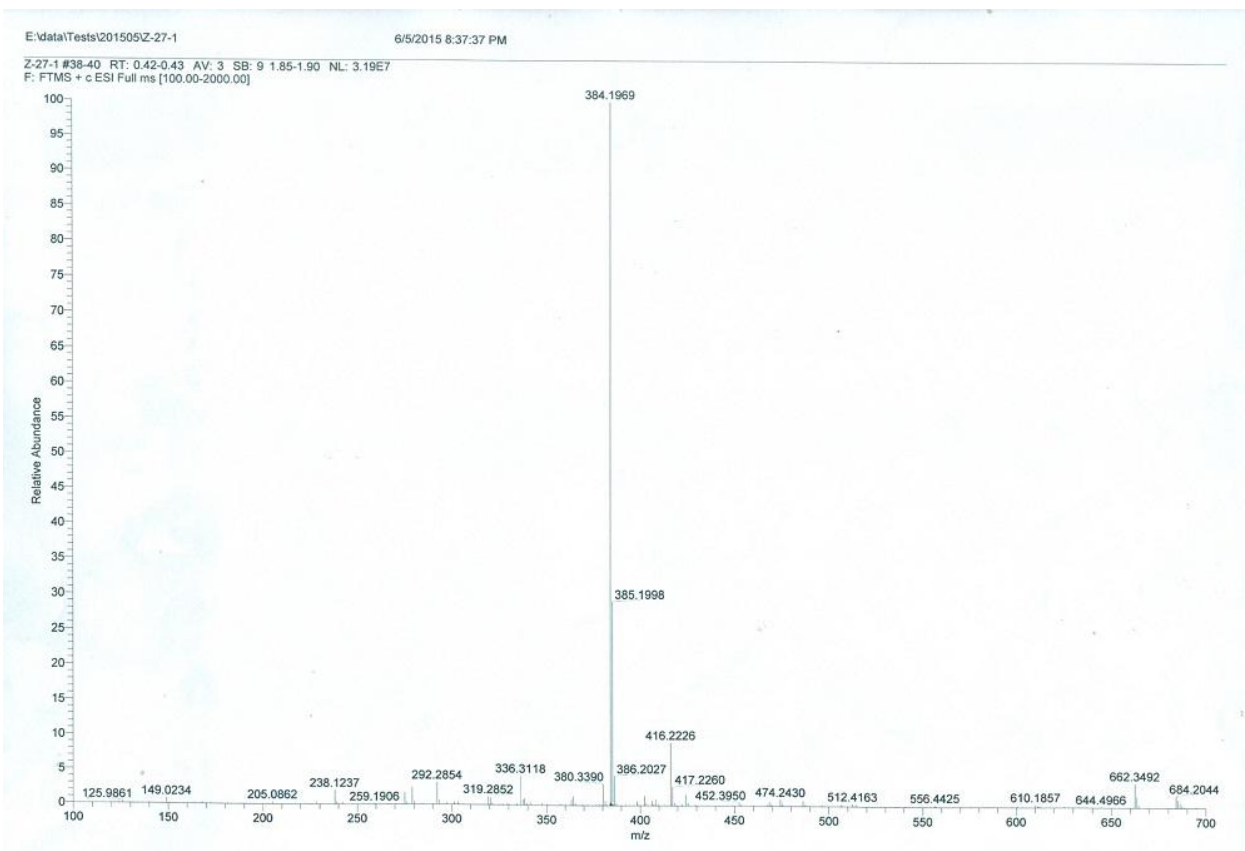

Figure S38. HRESIMS spectrum of SD9.
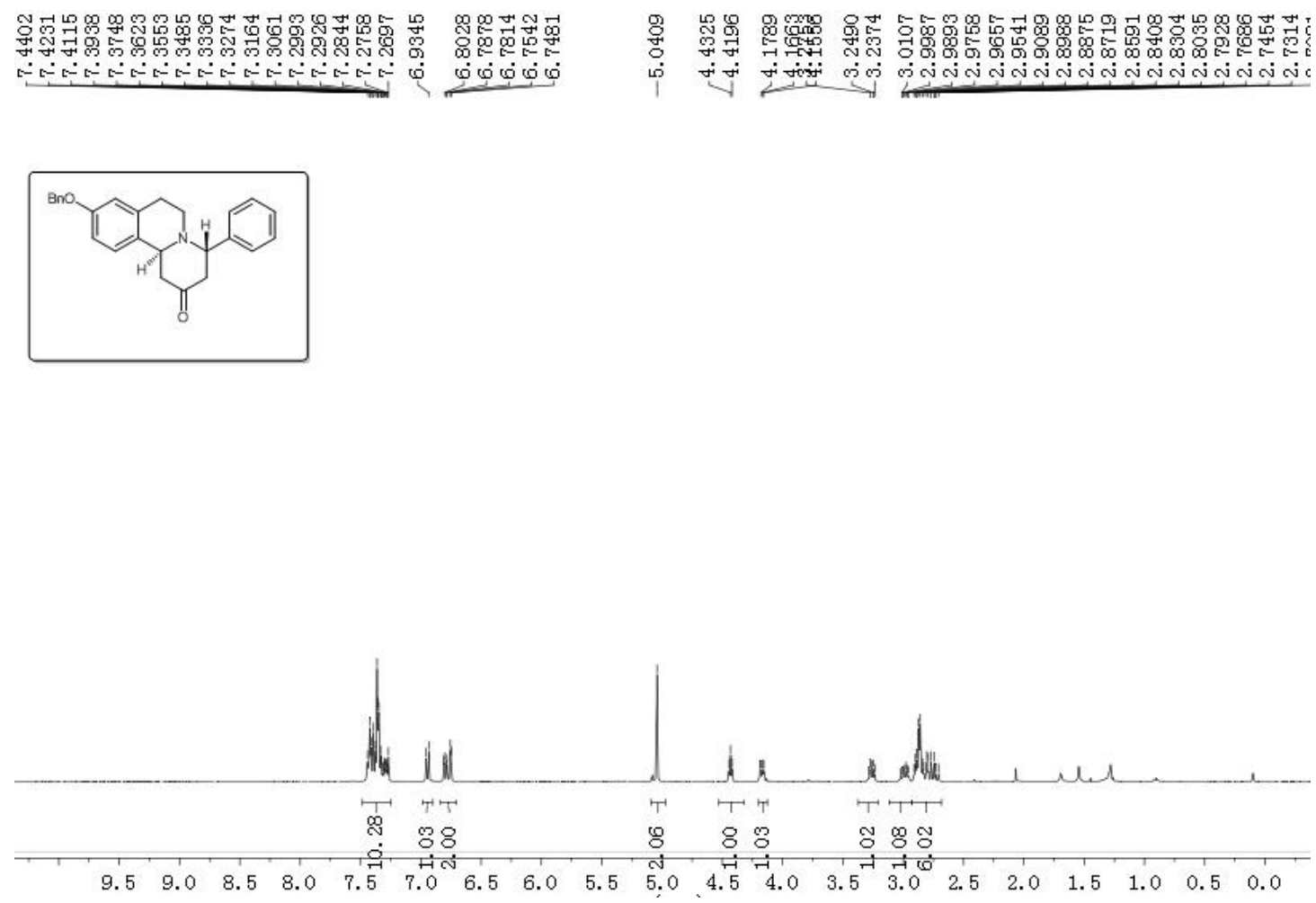

Figure S39. ${ }^{1} \mathrm{H}$ NMR spectrum of SD9. 


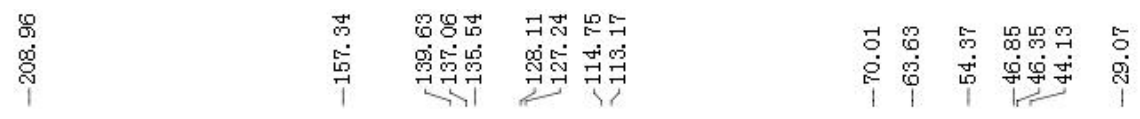
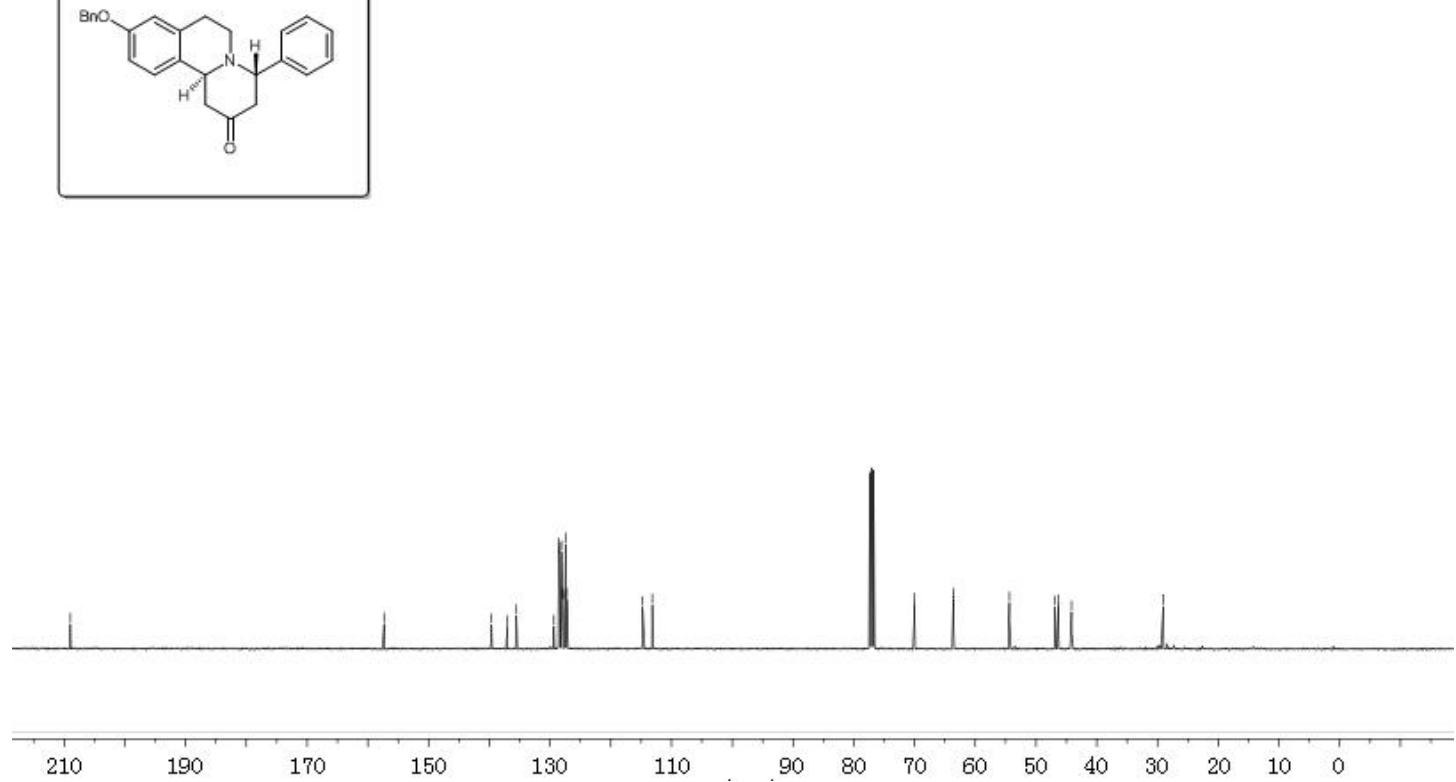

Figure S40. ${ }^{13} \mathrm{C}$ NMR spectrum of SD9.

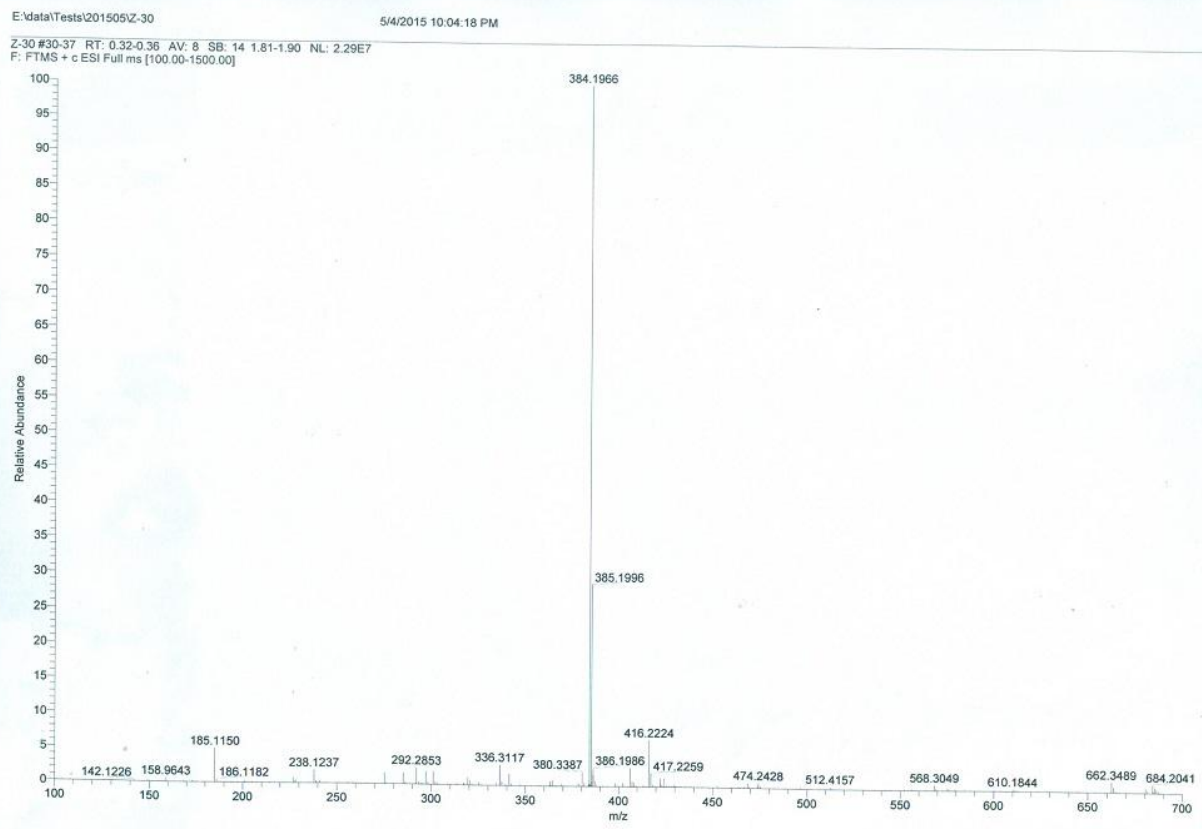

Figure S41. HRESIMS spectrum of SD10. 

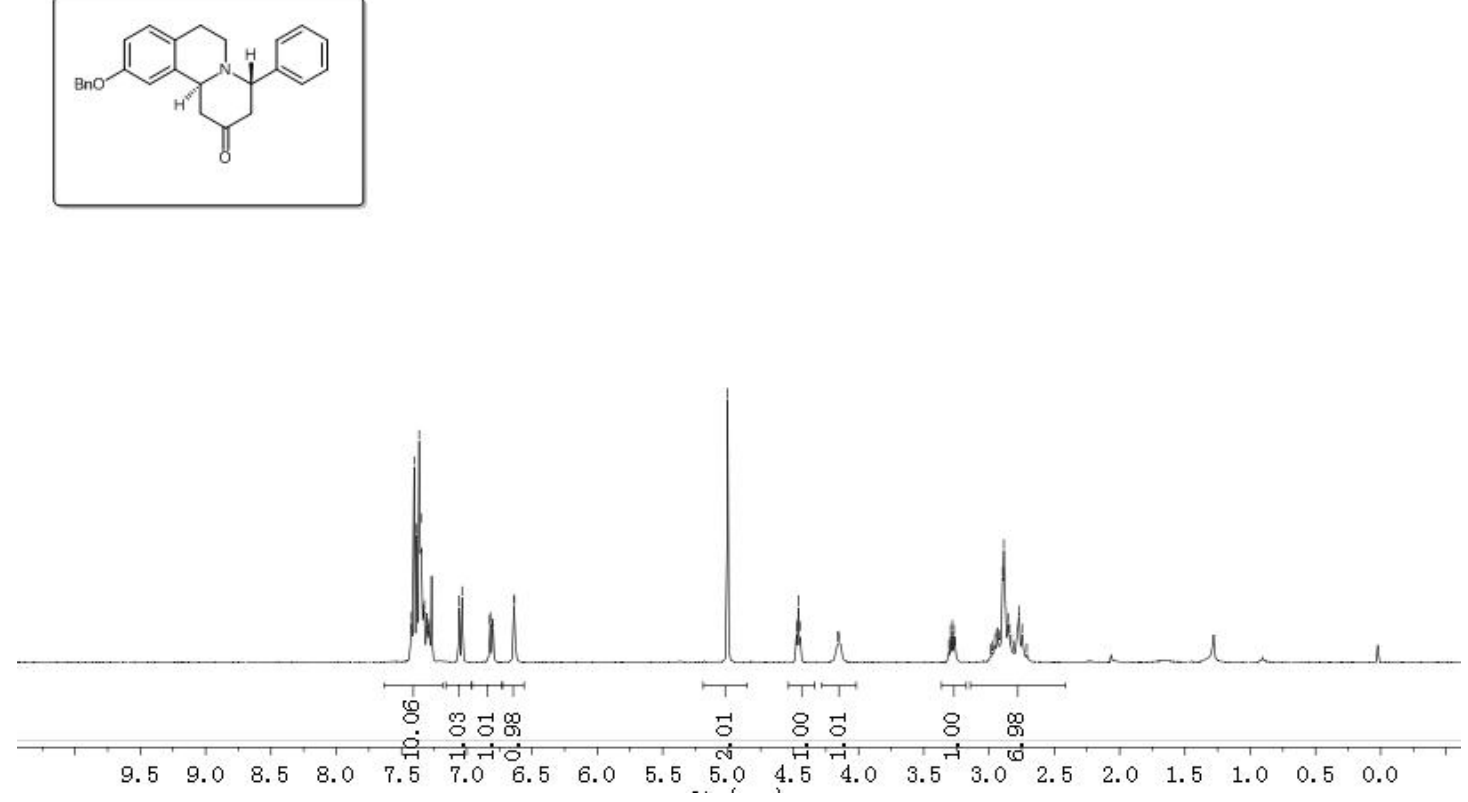

Figure S42. ${ }^{1} \mathrm{H}$ NMR spectrum of SD10.

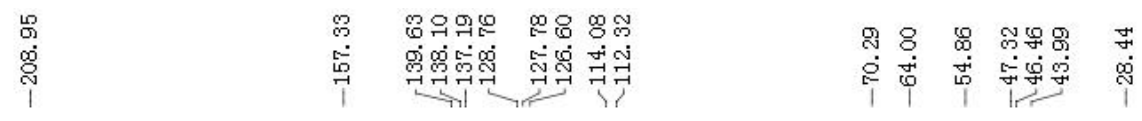
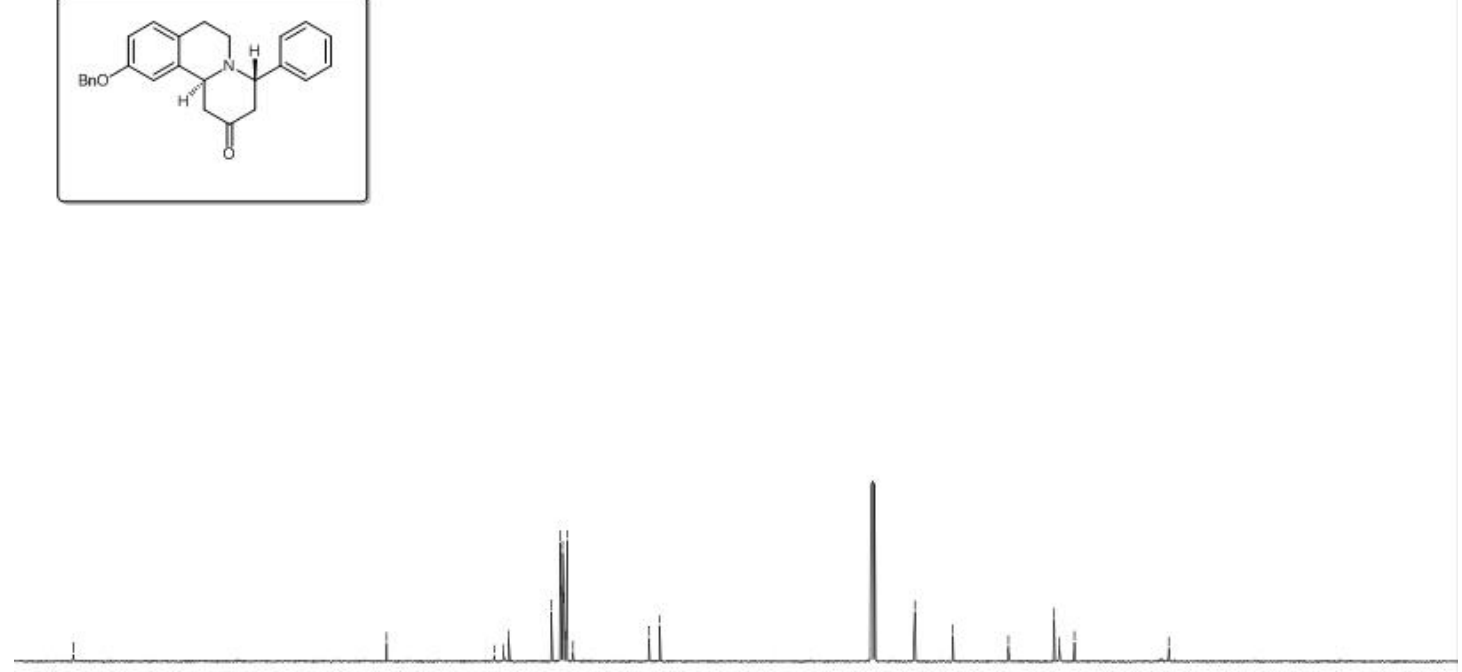

Figure S43. ${ }^{13} \mathrm{C}$ NMR spectrum of SD10. 

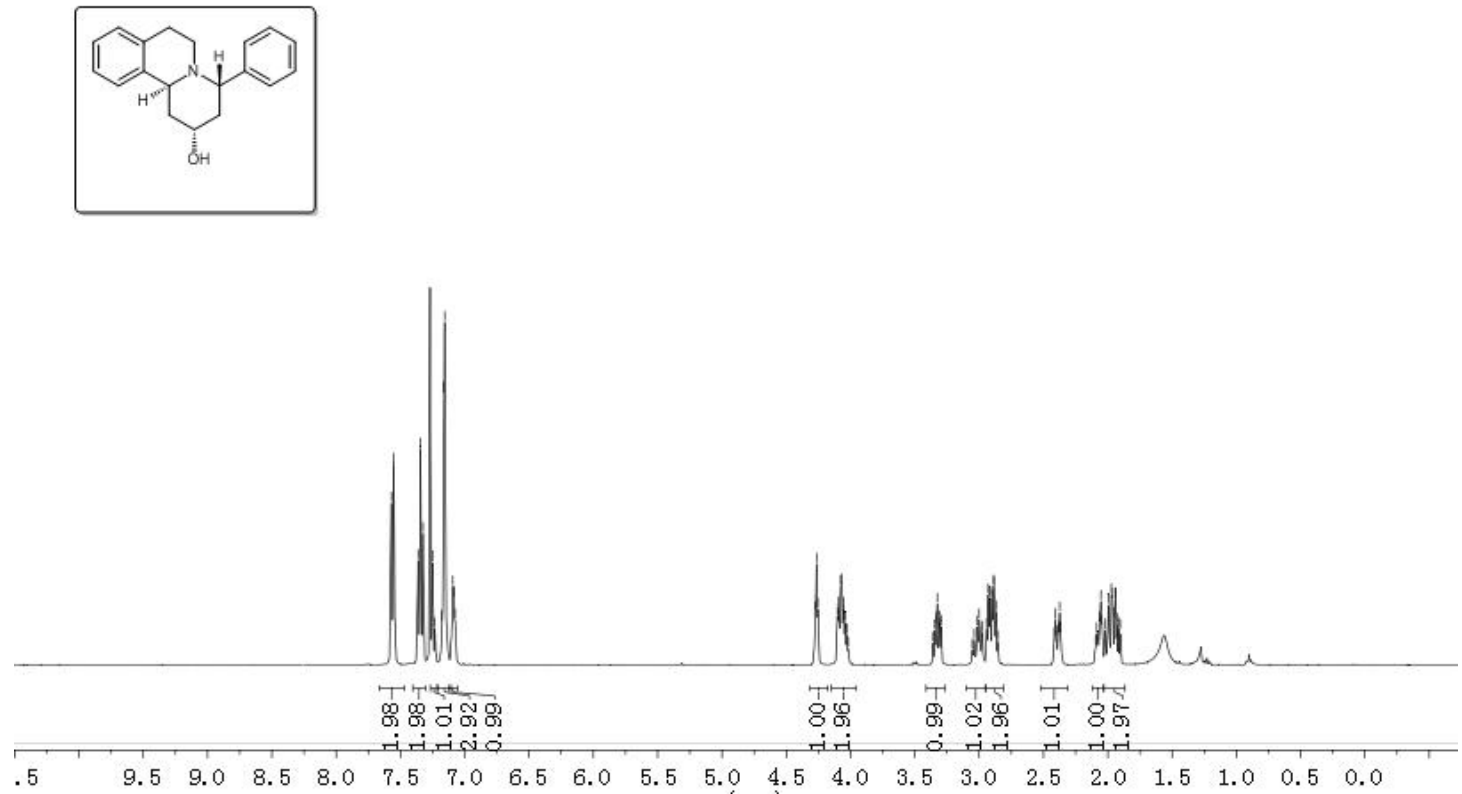

Figure S44. ${ }^{1} \mathrm{H}$ NMR spectrum of SE1a

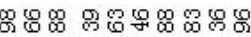

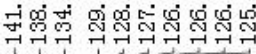

당 매

घं
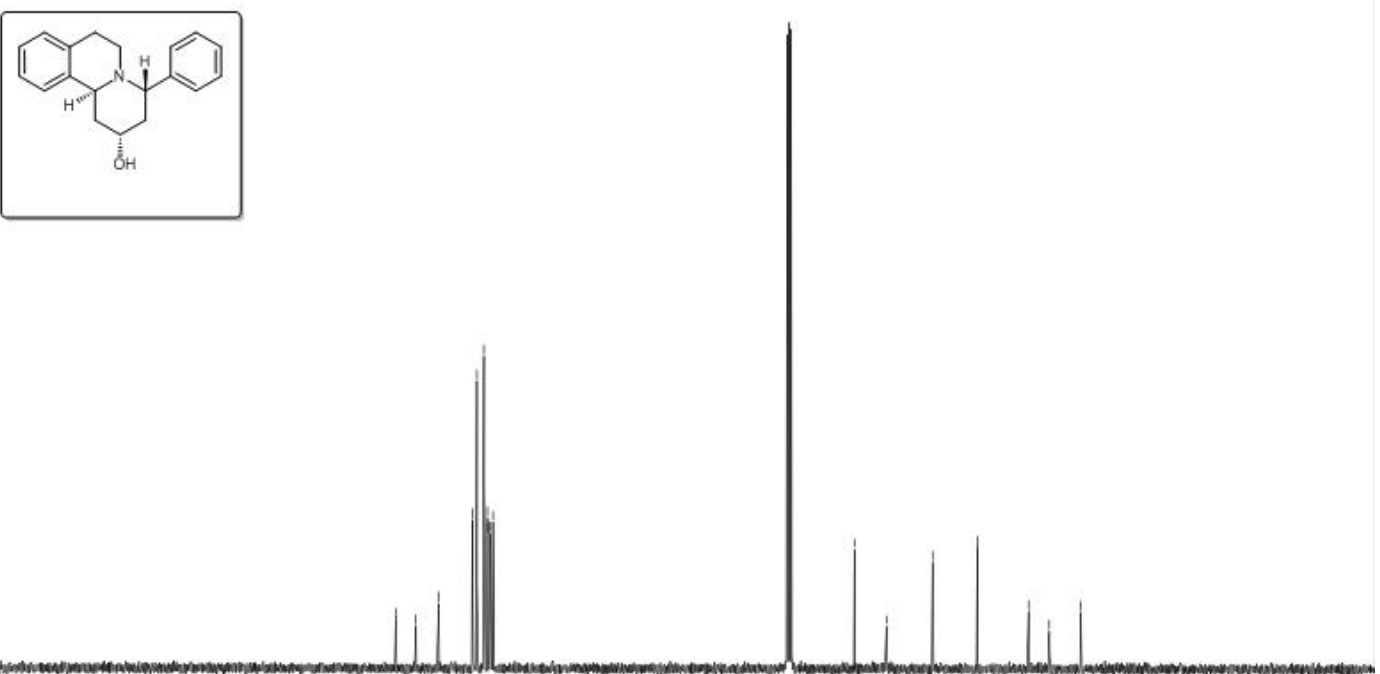

Figure S45. ${ }^{13} \mathrm{C}$ NMR spectrum of SE1a. 


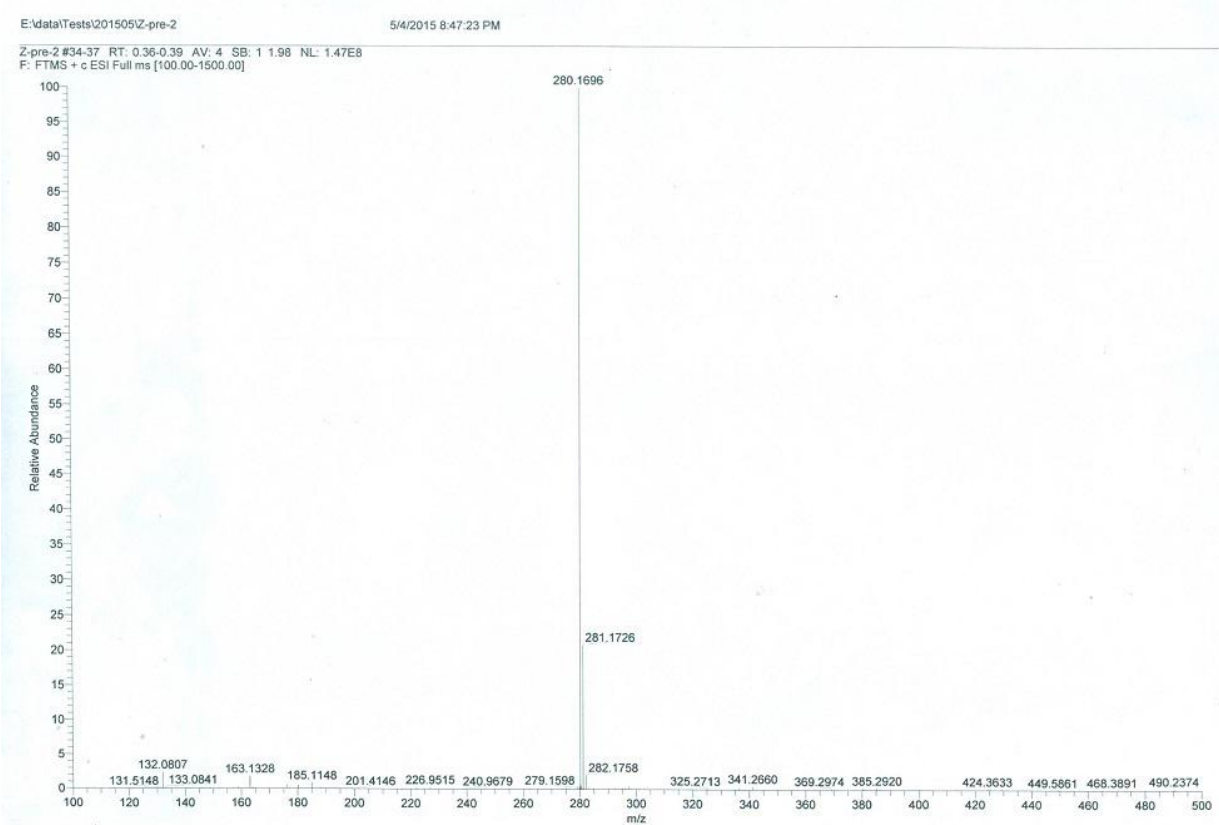

Figure S46. HRESIMS spectrum of SE1b.
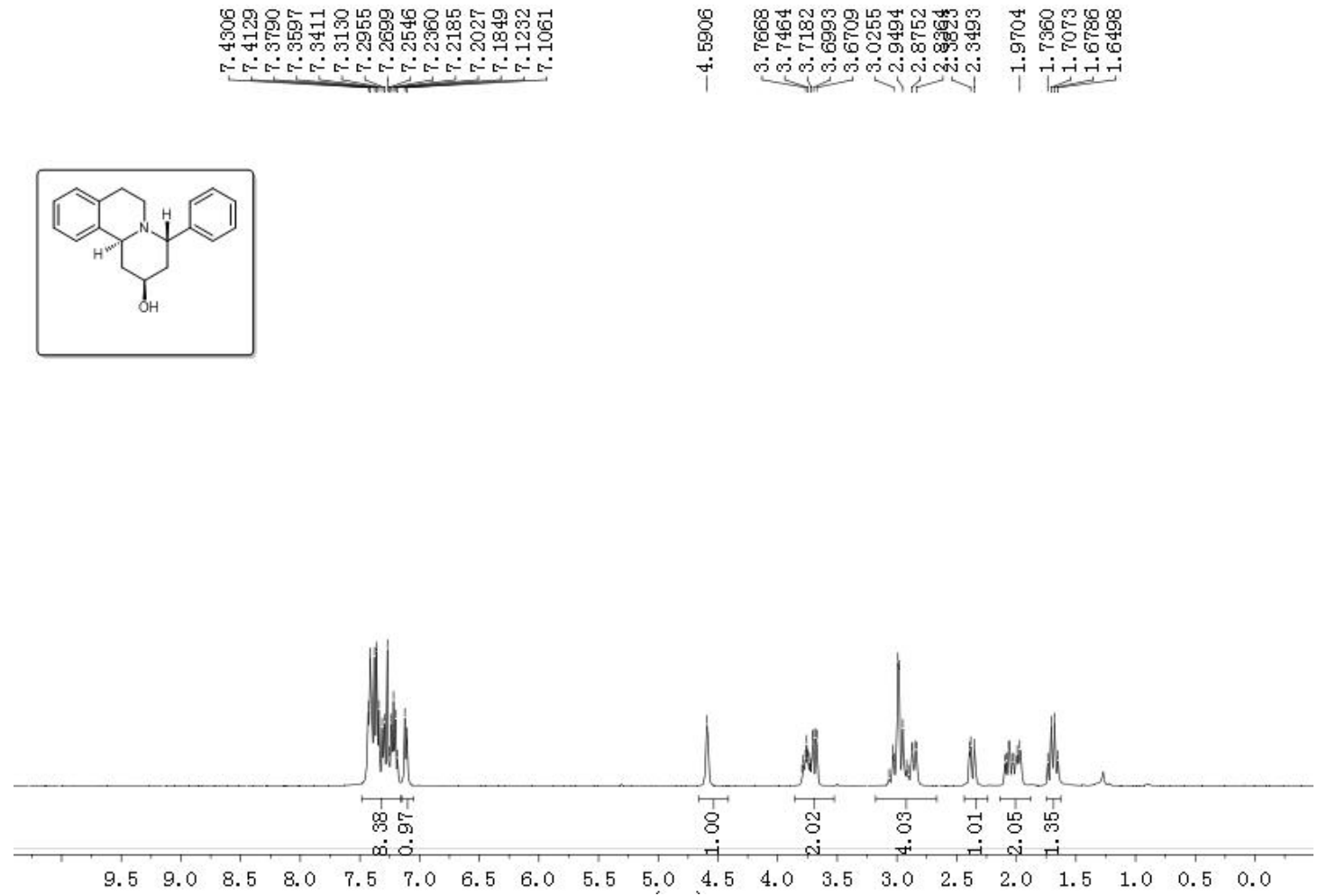

Figure S47. ${ }^{1} \mathrm{H}$ NMR spectrum of SE1b. 

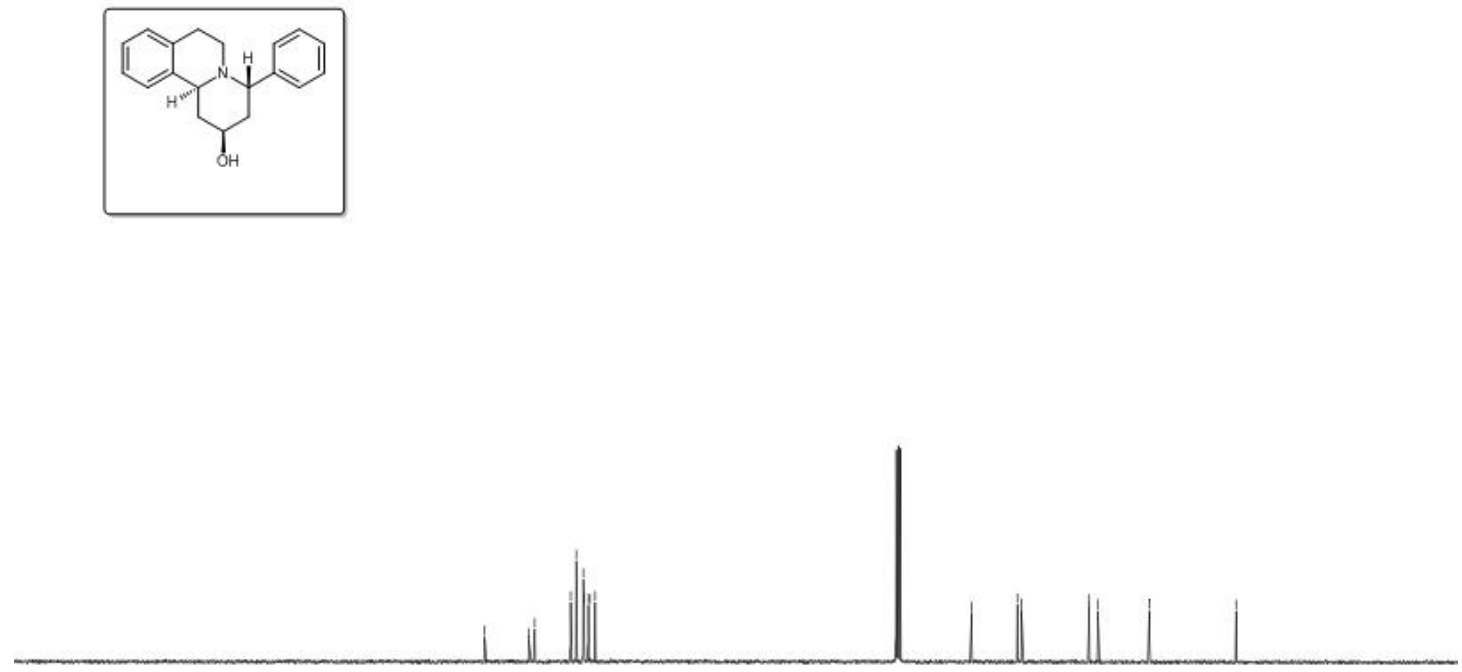

$\begin{array}{lllllllllllllllllllllllllllllll}210 & 200 & 190 & 180 & 170 & 160 & 150 & 140 & 130 & 120 & 110 & 100 & 90 & 80 & 70 & 60 & 50 & 40 & 30 & 20 & 10 & 0 & -10\end{array}$

Figure S48. ${ }^{13} \mathrm{C}$ NMR spectrum of SE1b.

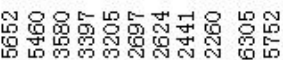

$\therefore$

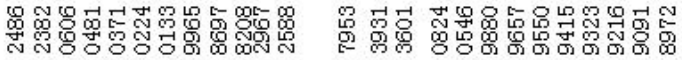

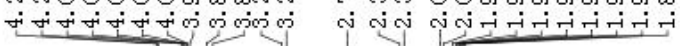
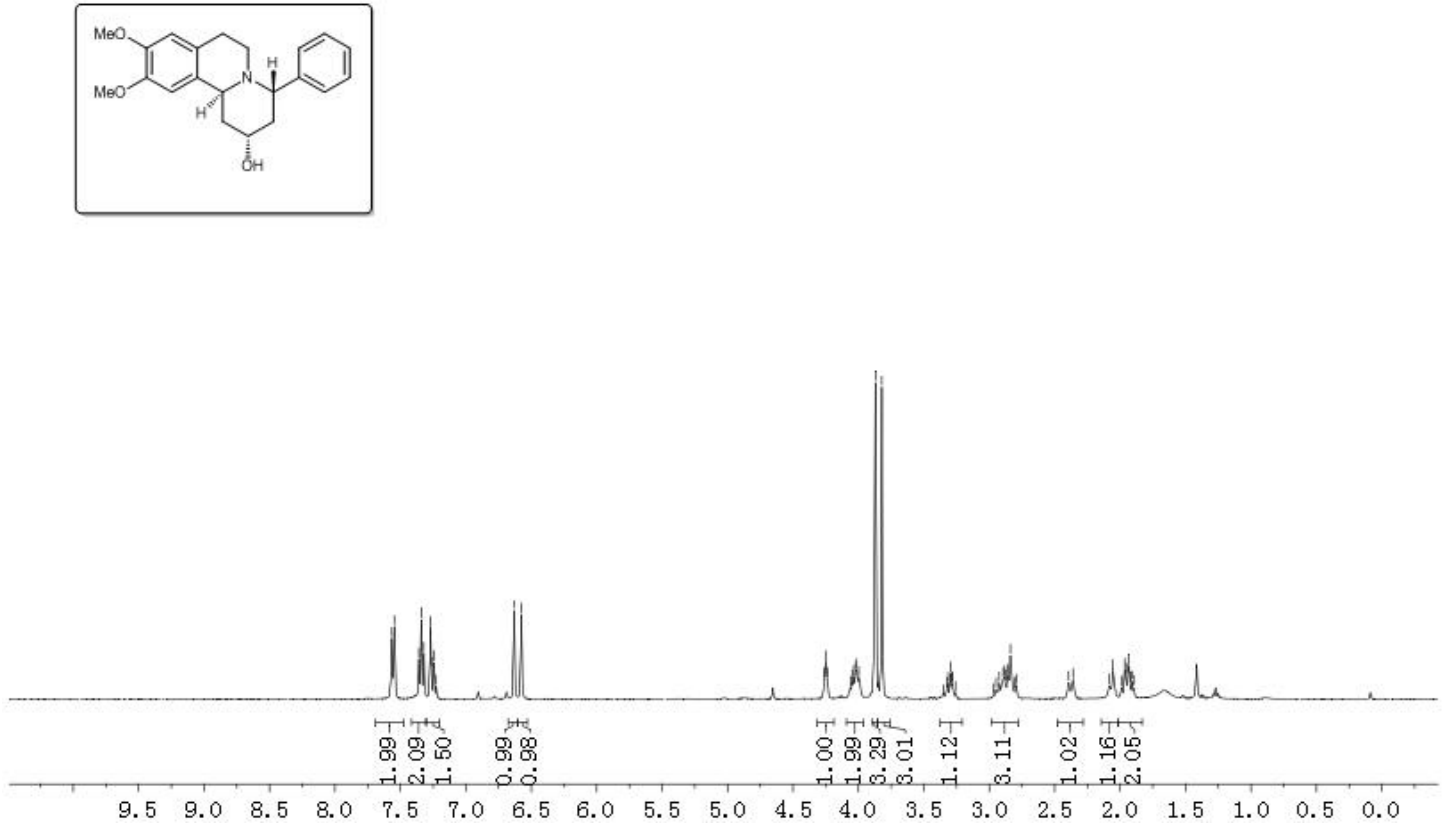

Figure S49. ${ }^{1} \mathrm{H}$ NMR spectrum of SE2a. 


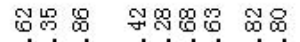

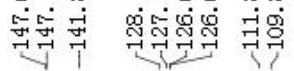

织

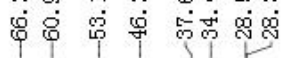
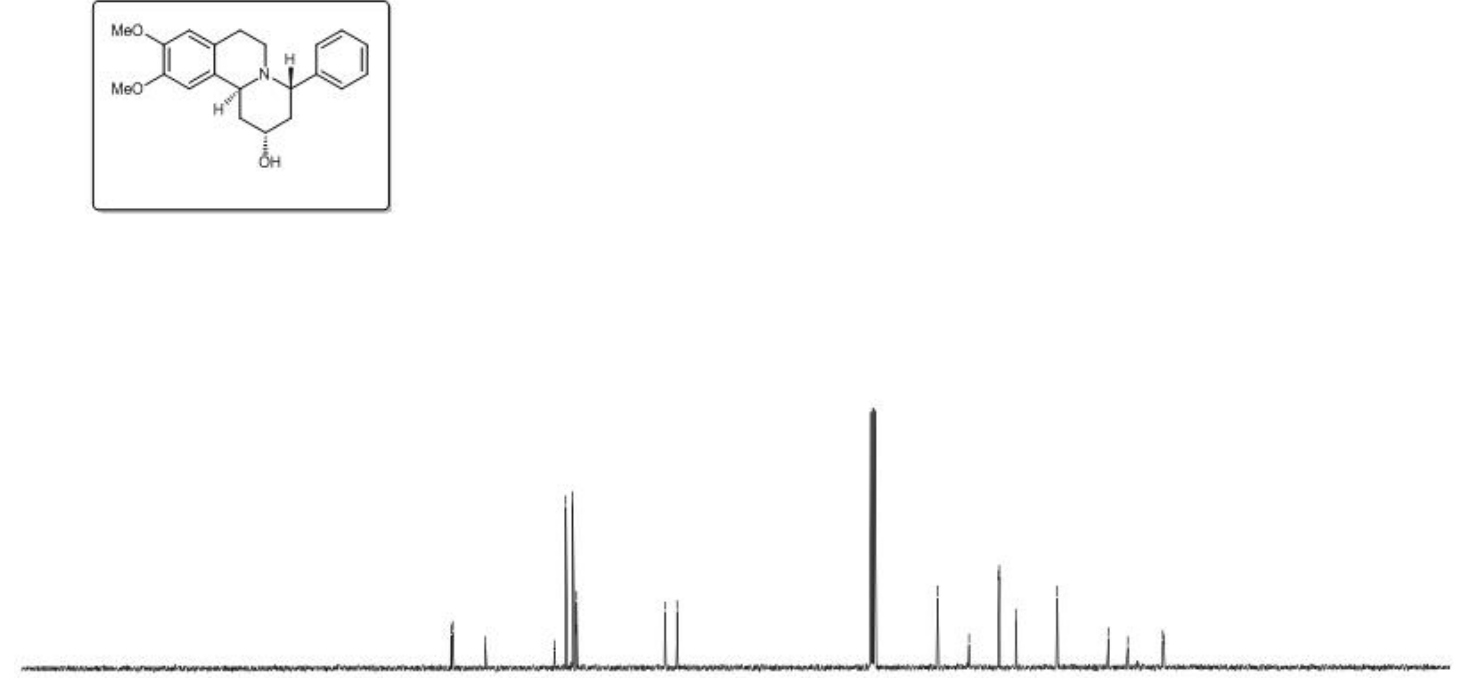

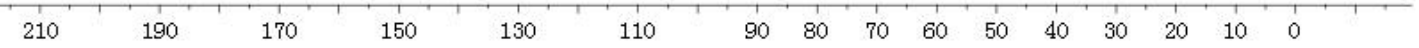

Figure S50. ${ }^{13} \mathrm{C}$ NMR spectrum of SE2a.

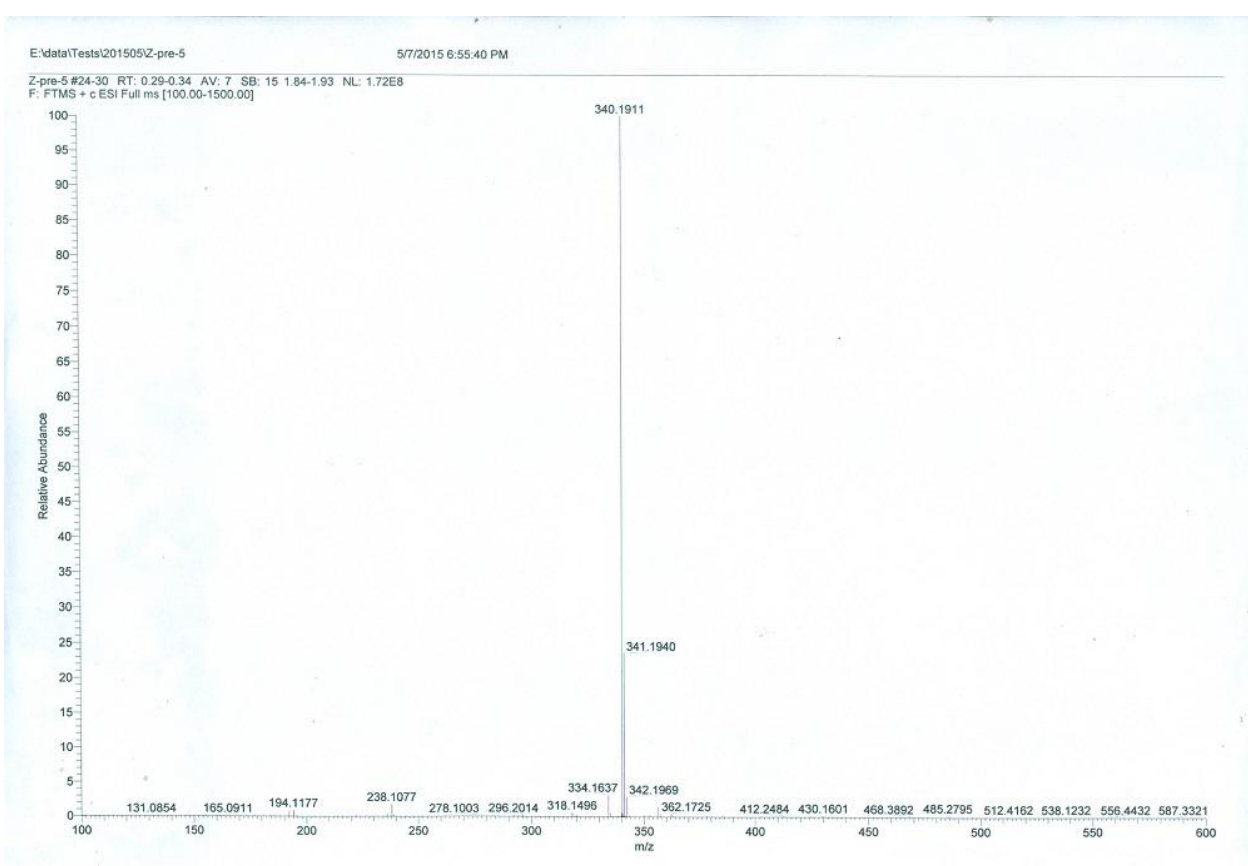

Figure S51. HRESIMS spectrum of SE2b. 


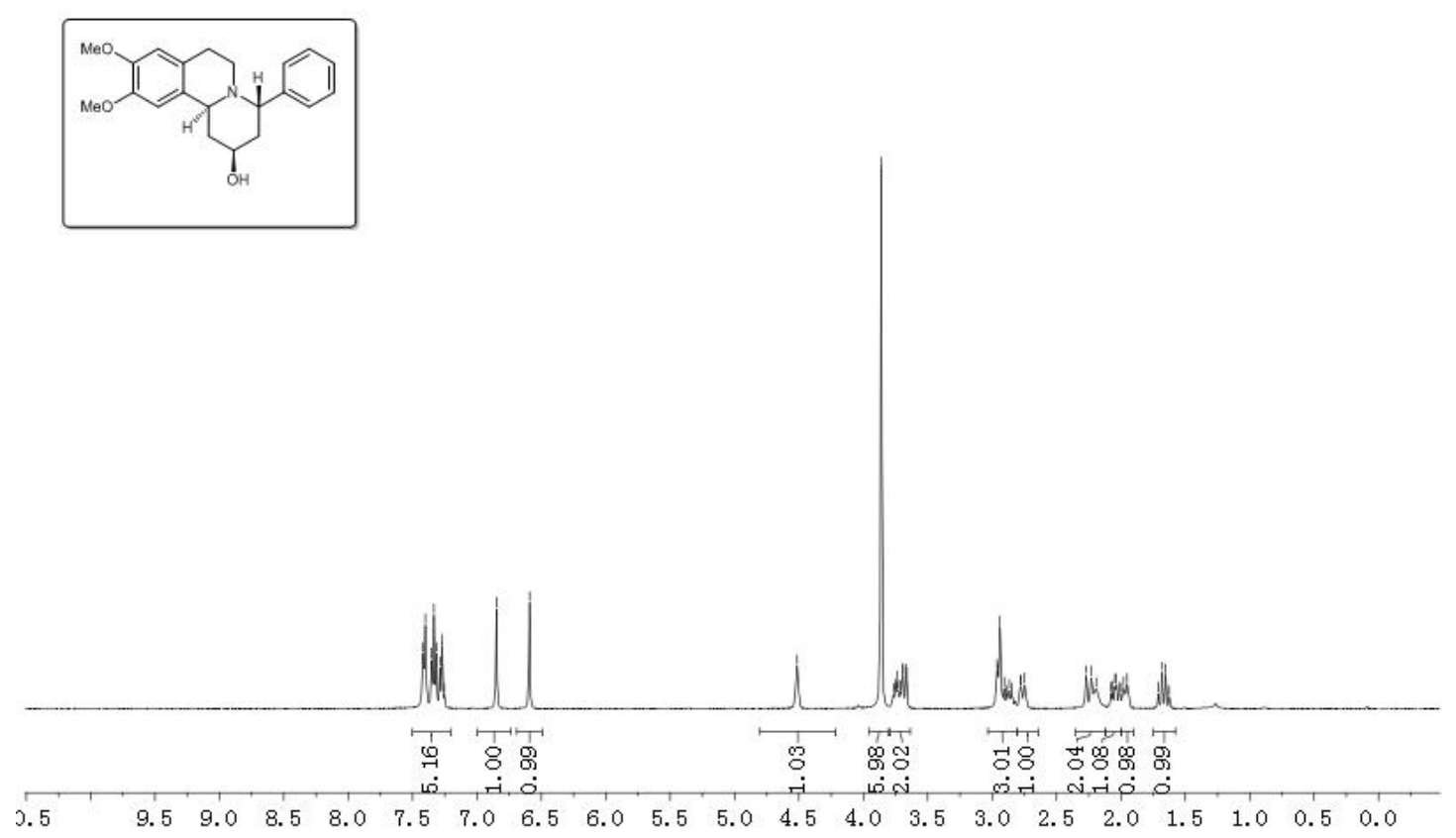

Figure S52. ${ }^{1} \mathrm{H}$ NMR spectrum of SE2b.

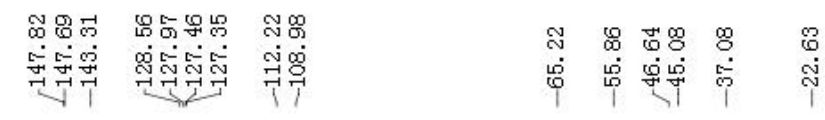
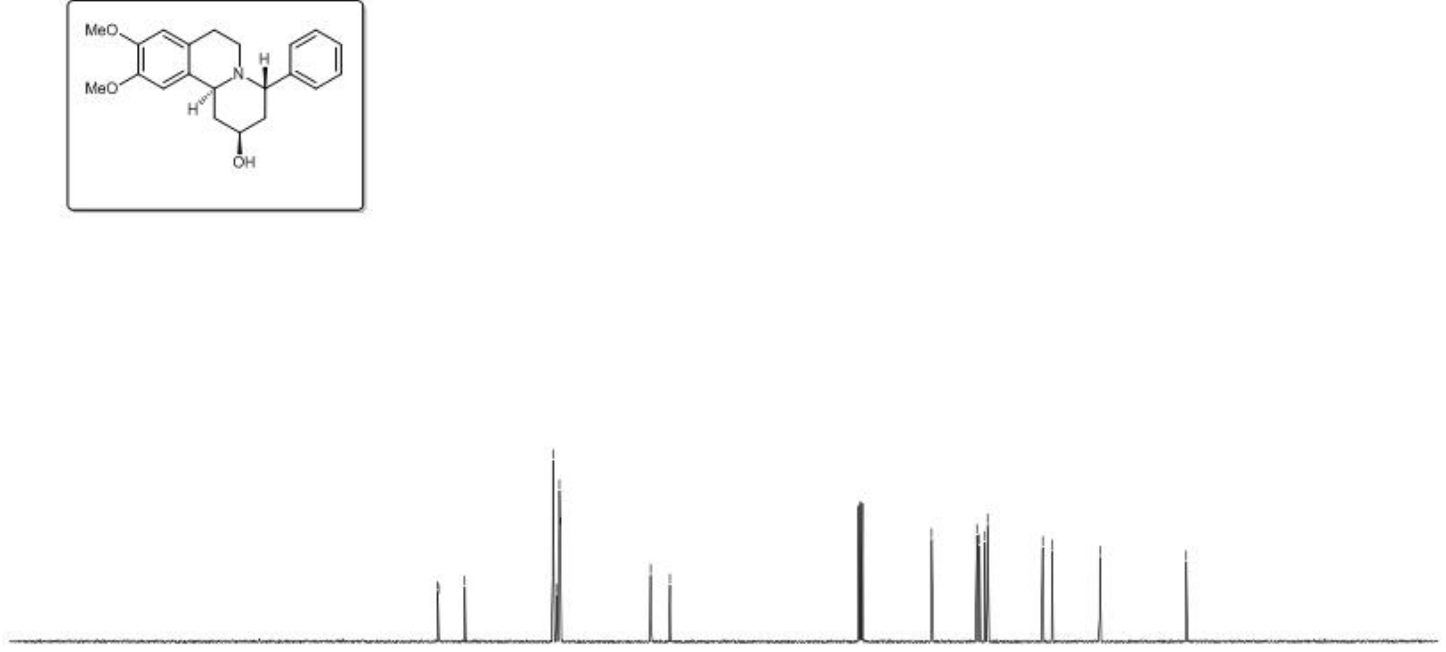

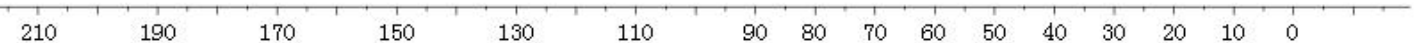

Figure S53. ${ }^{13} \mathrm{C}$ NMR spectrum of SE2b. 

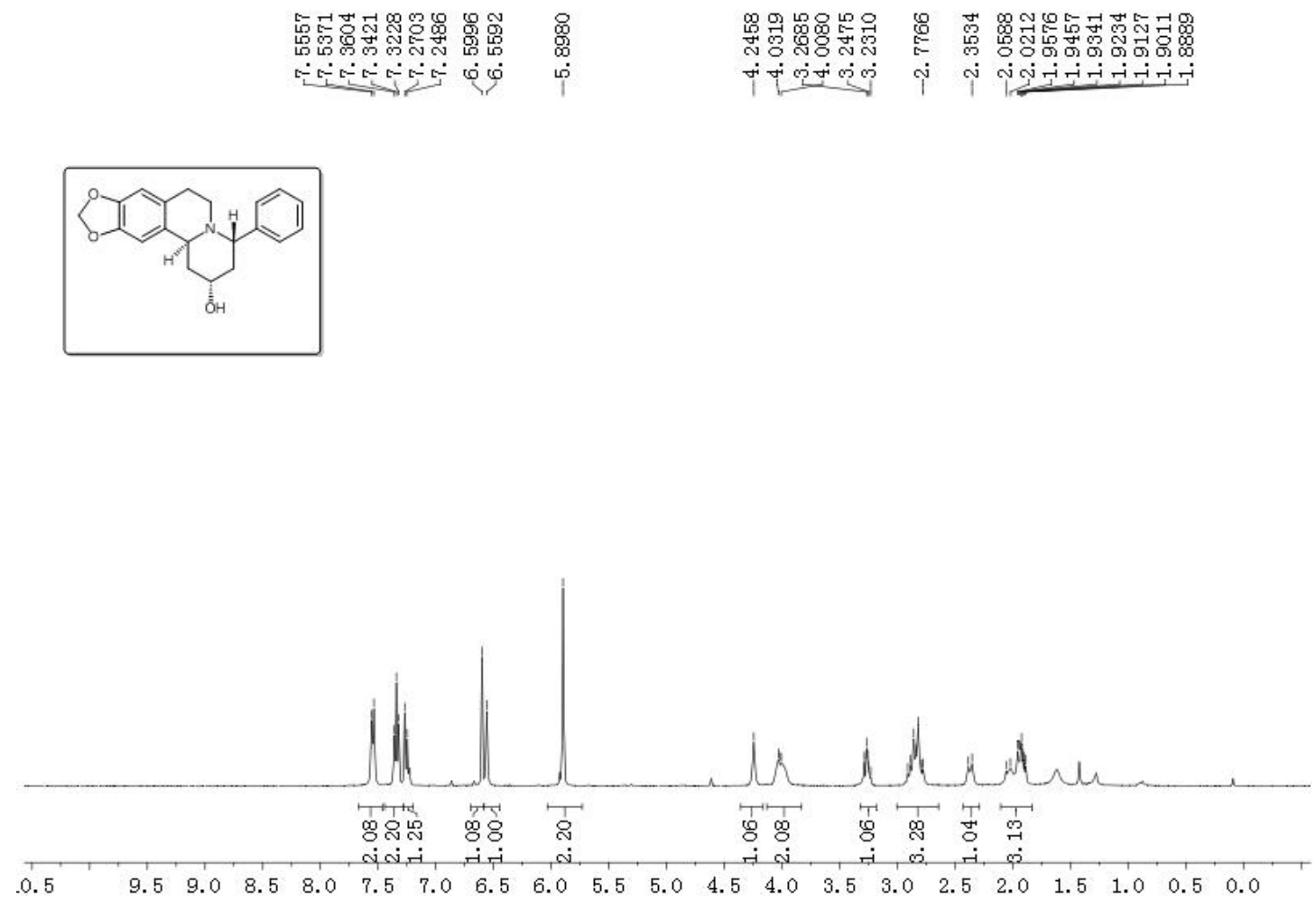

Figure S54. ${ }^{1} \mathrm{H}$ NMR spectrum of SE3a.

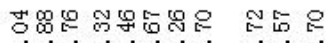

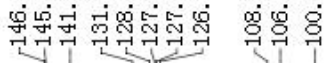

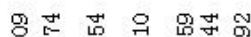

ச்
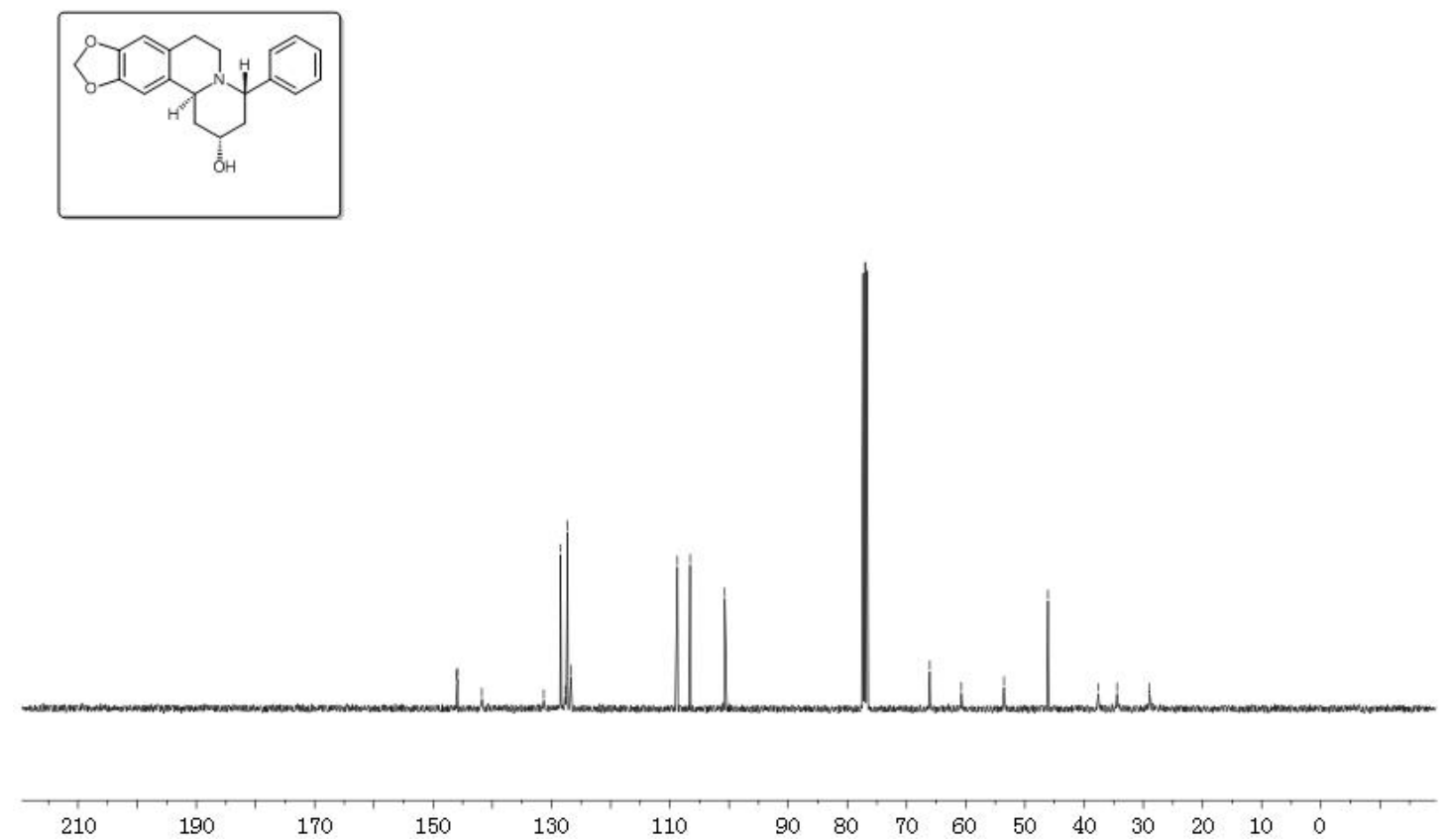

Figure S55. ${ }^{13} \mathrm{C}$ NMR spectrum of SE3a. 


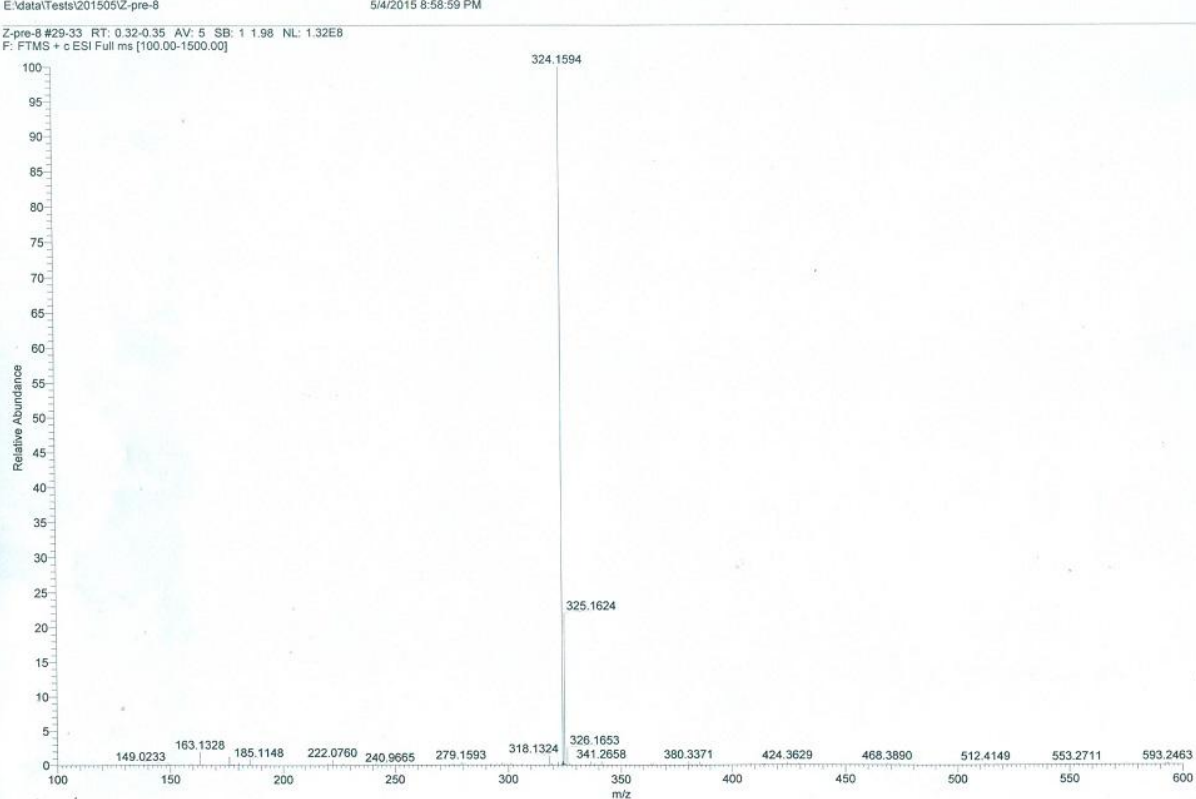

Figure S56. HRESIMS spectrum of SE3b.

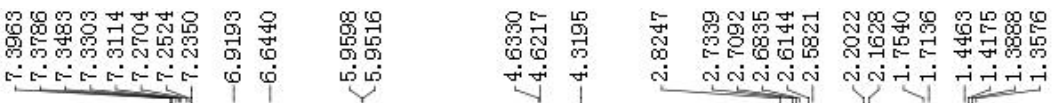

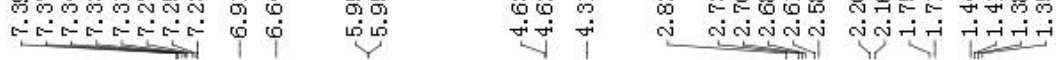
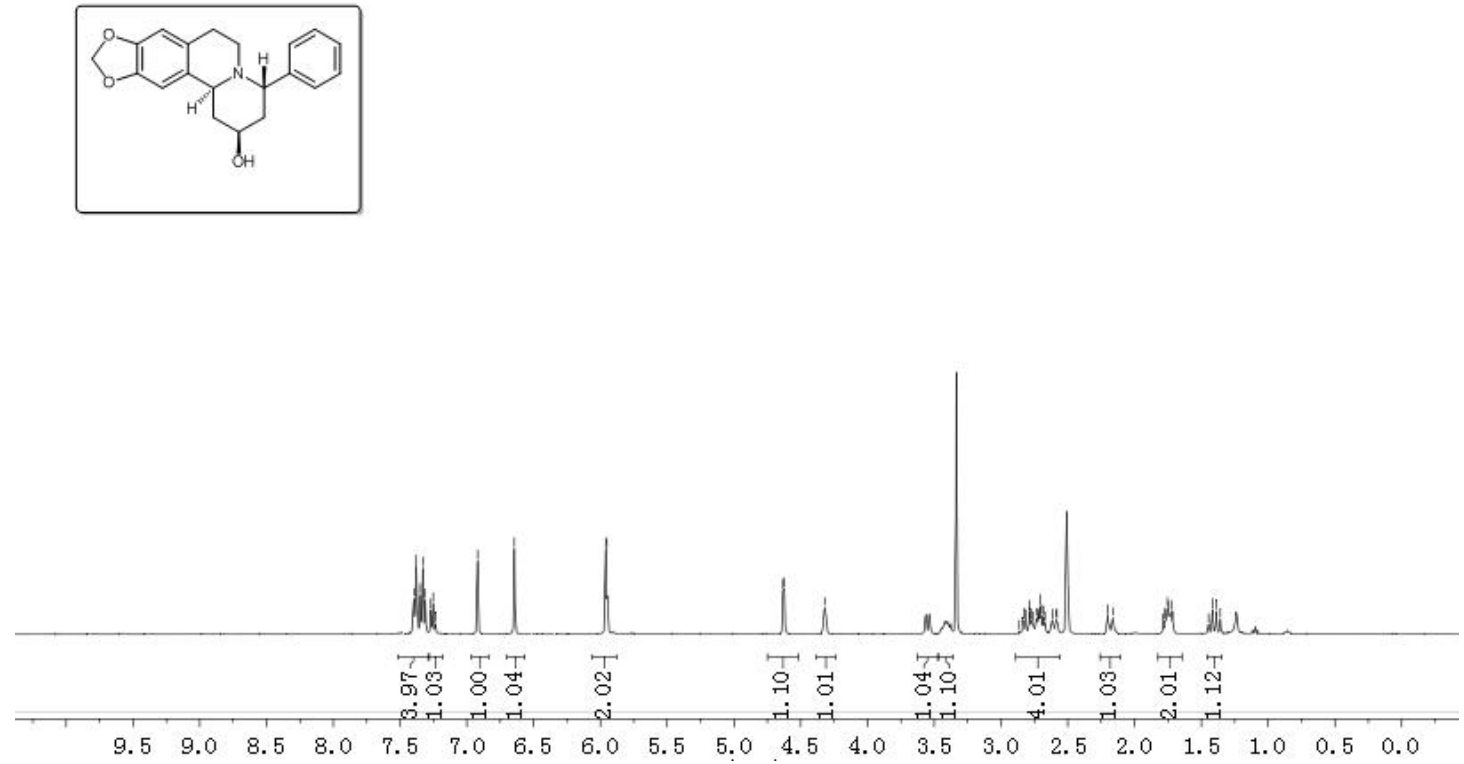

Figure S57. ${ }^{1} \mathrm{H}$ NMR spectrum of SE3b. 


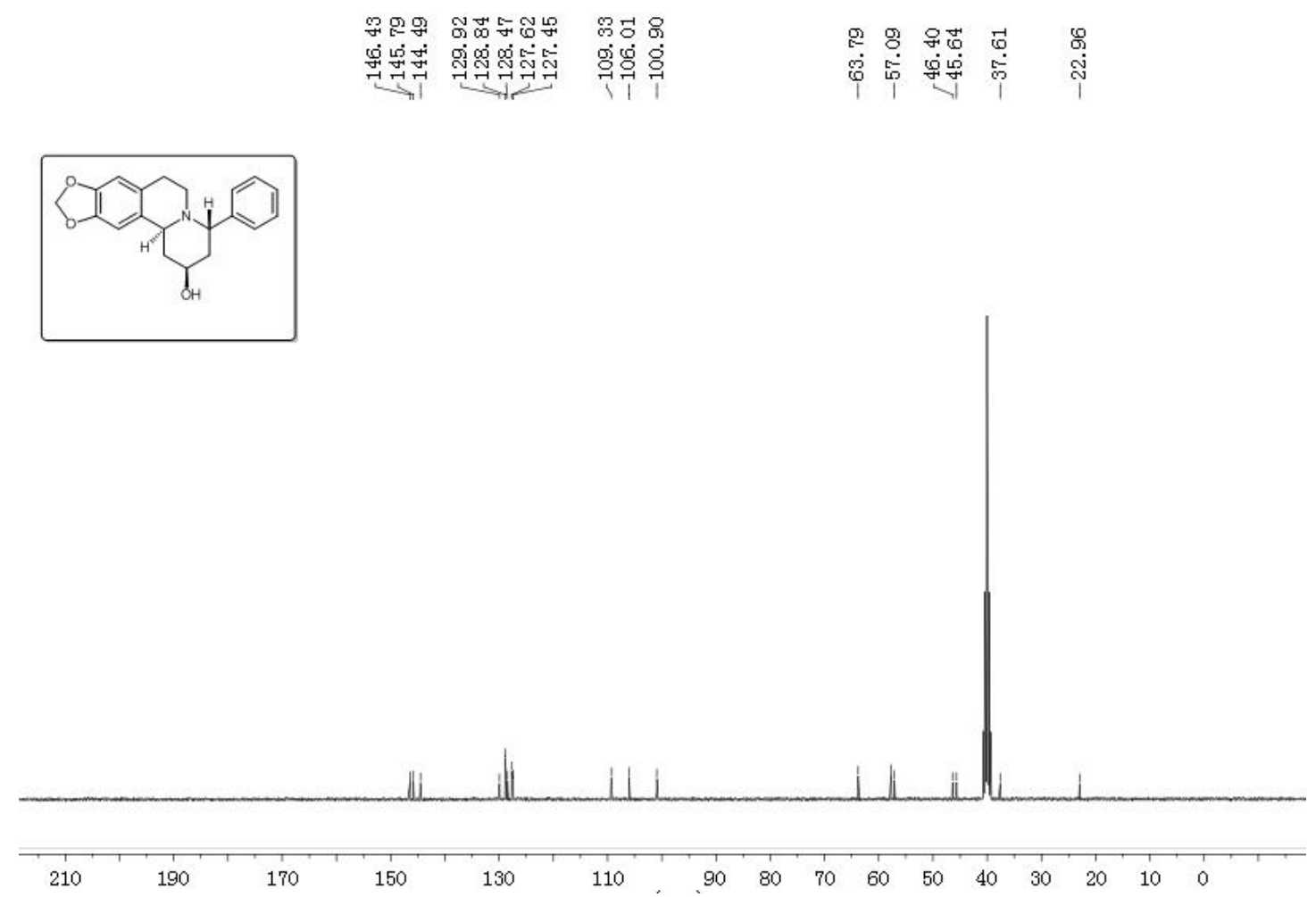

Figure S58. ${ }^{13} \mathrm{C}$ NMR spectrum of SE3b.

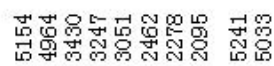

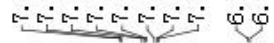

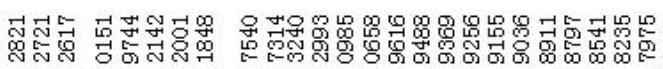

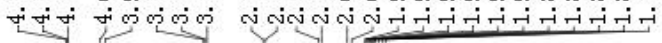
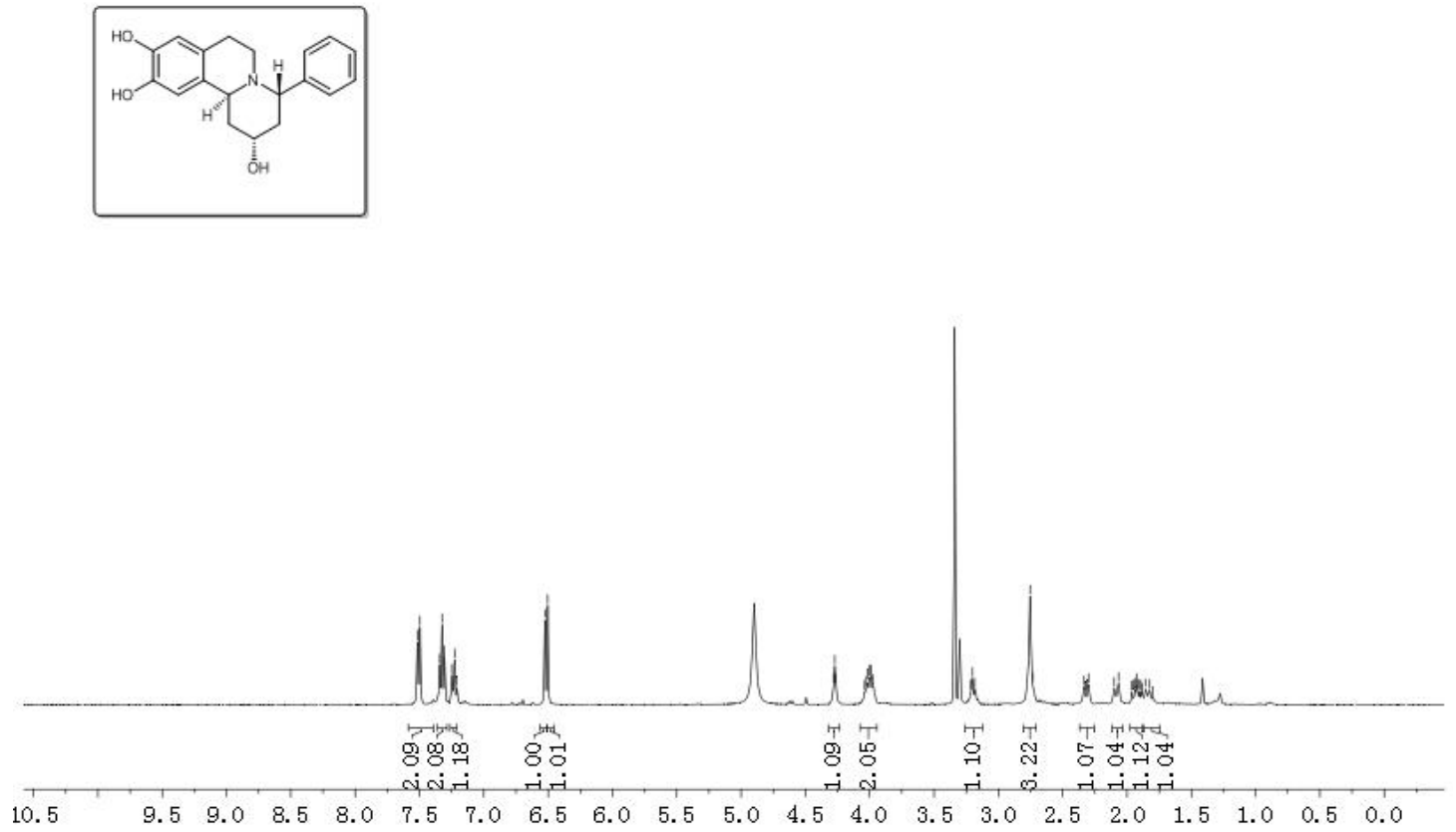

Figure S69. ${ }^{1} \mathrm{H}$ NMR spectrum of SE4a 

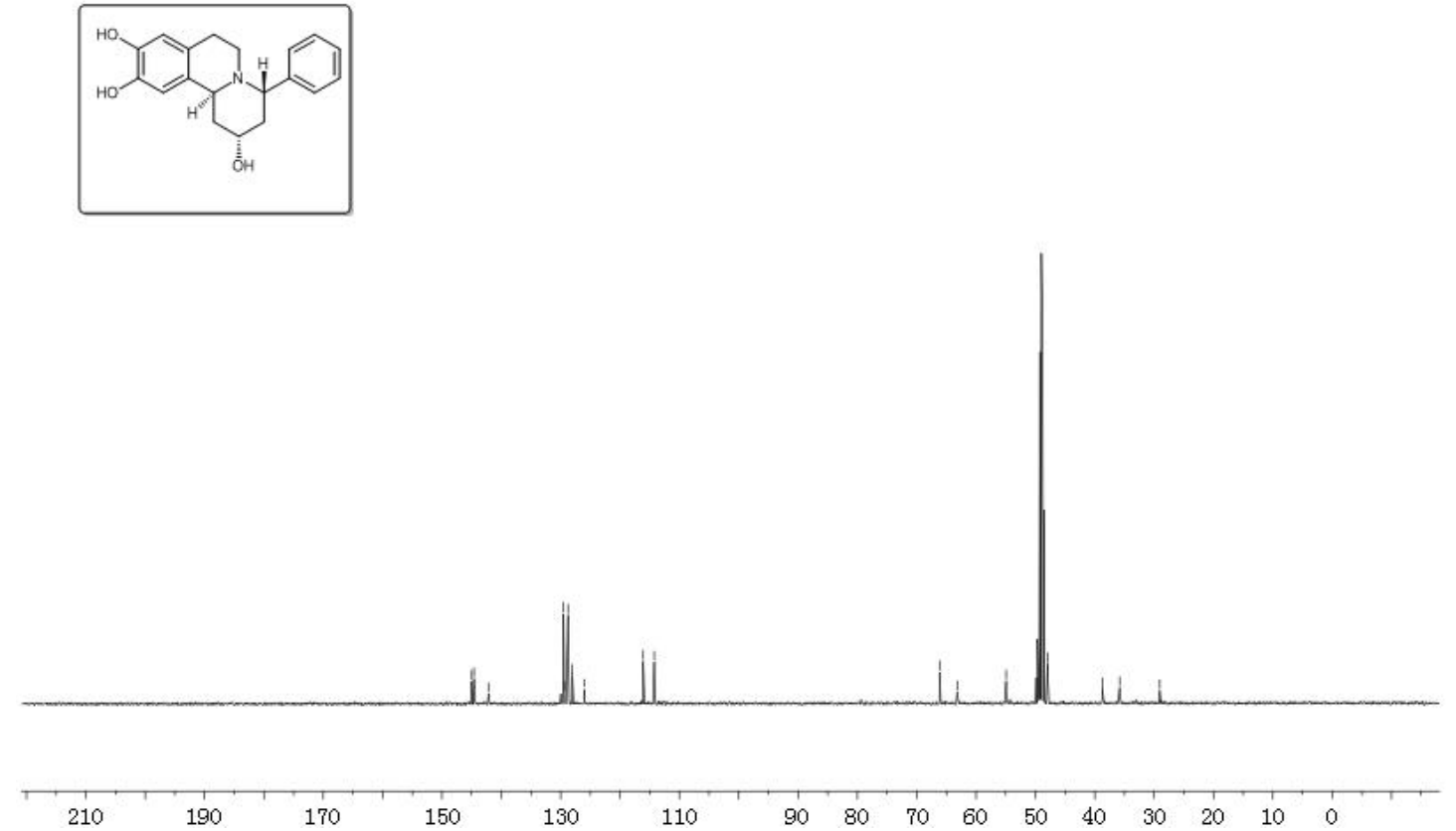

Figure S60. ${ }^{13} \mathrm{C}$ NMR spectrum of SE4a.

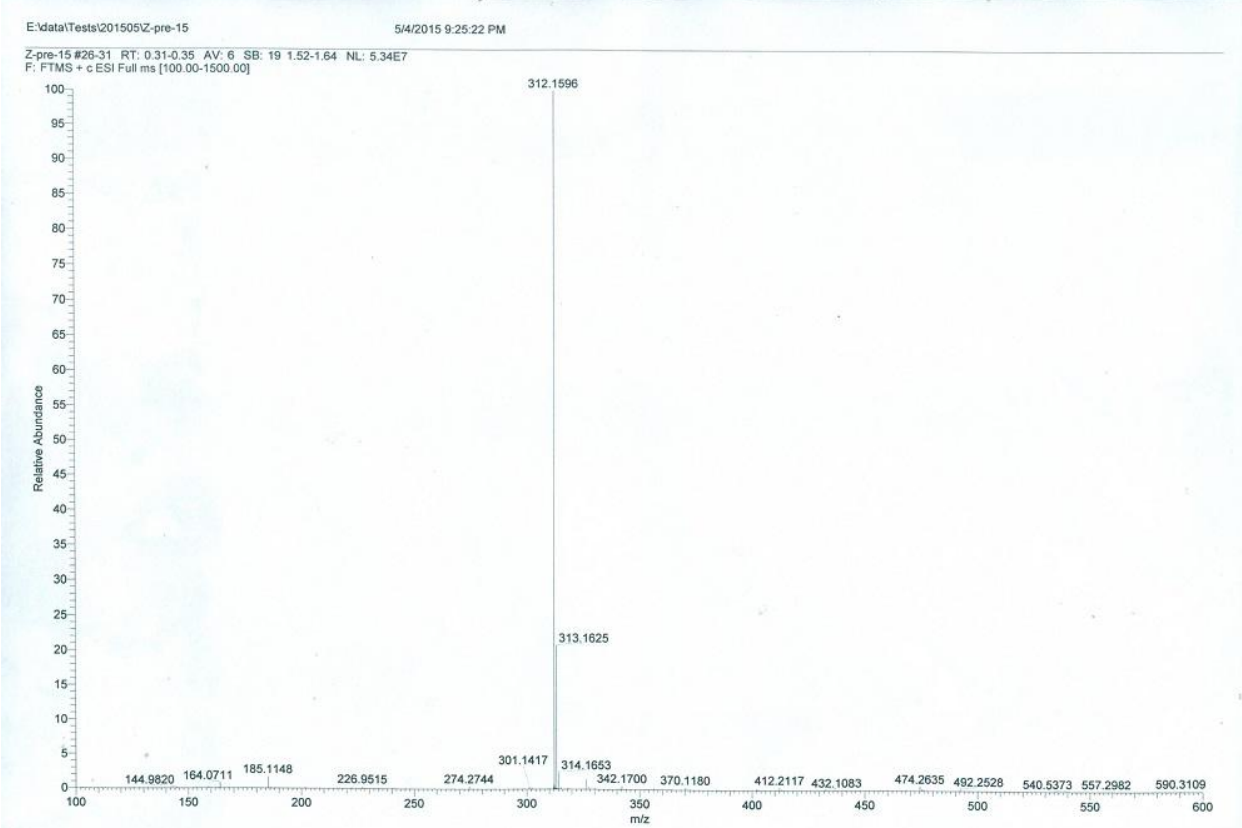

Figure S61. HRESIMS spectrum of SE4b. 


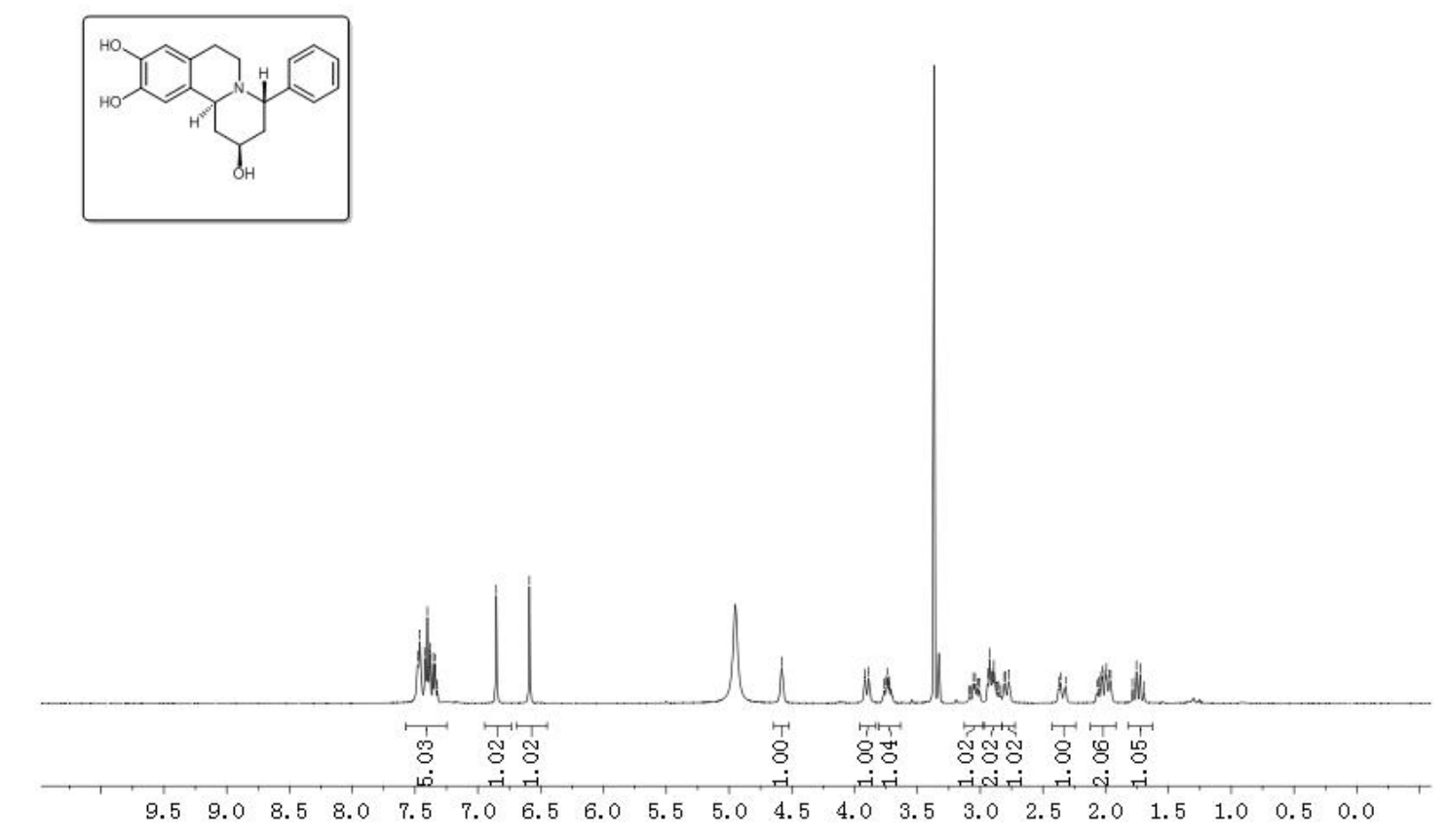

Figure S62. ${ }^{1} \mathrm{H}$ NMR spectrum of SE4b.

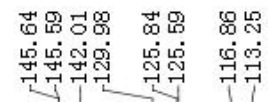

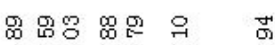

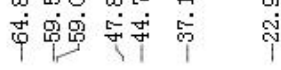
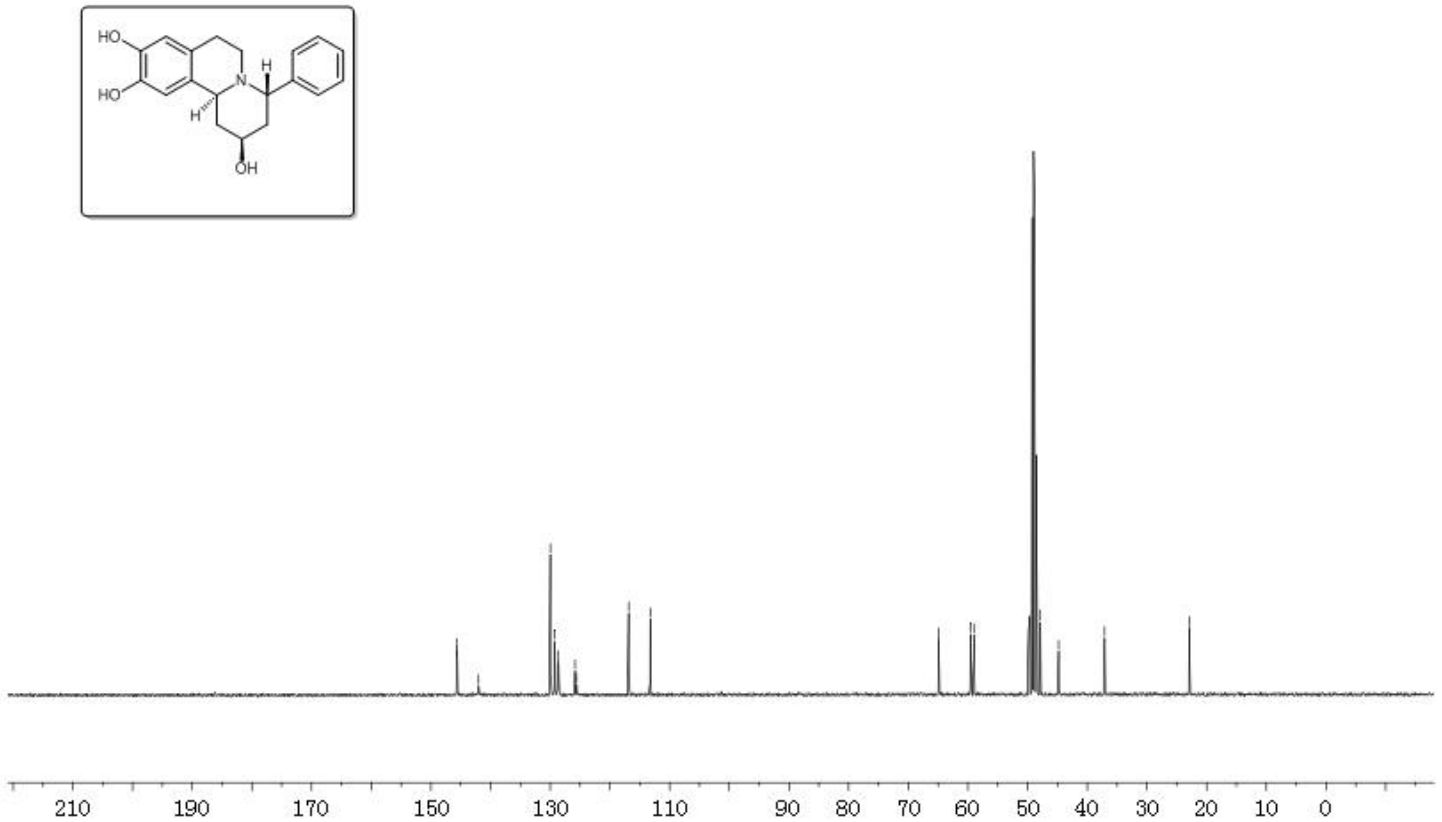

Figure S63. ${ }^{13} \mathrm{C}$ NMR spectrum of SE4b. 

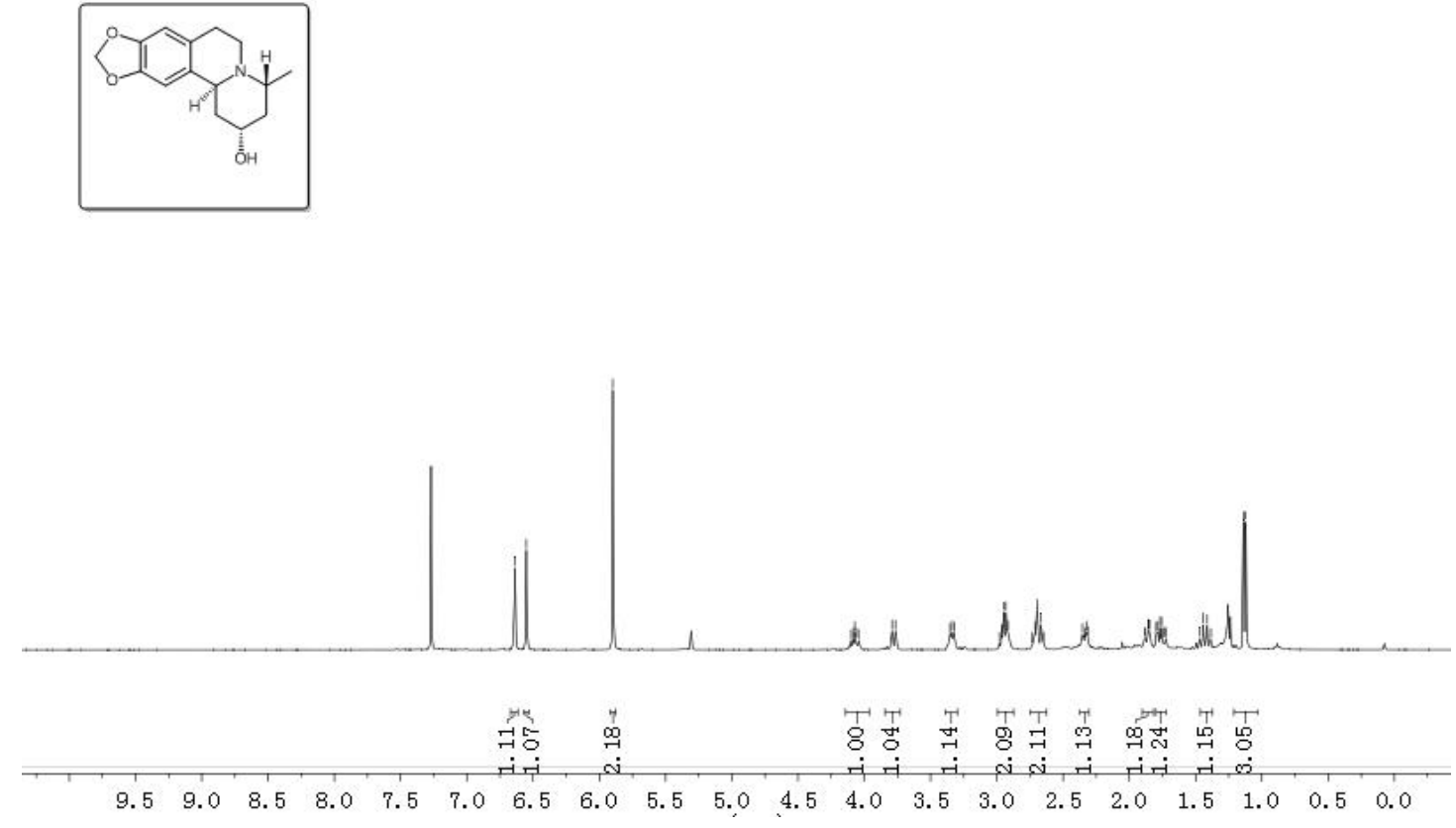

Figure S64. ${ }^{1} \mathrm{H}$ NMR spectrum of SE5a.

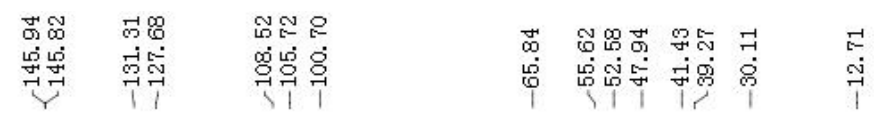

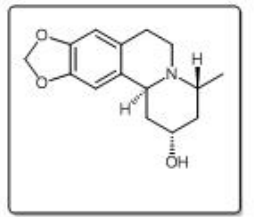

가

곤

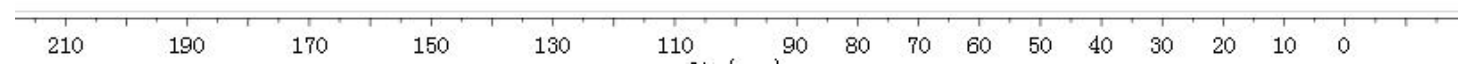

Figure S65. ${ }^{13} \mathrm{C}$ NMR spectrum of SE5a. 


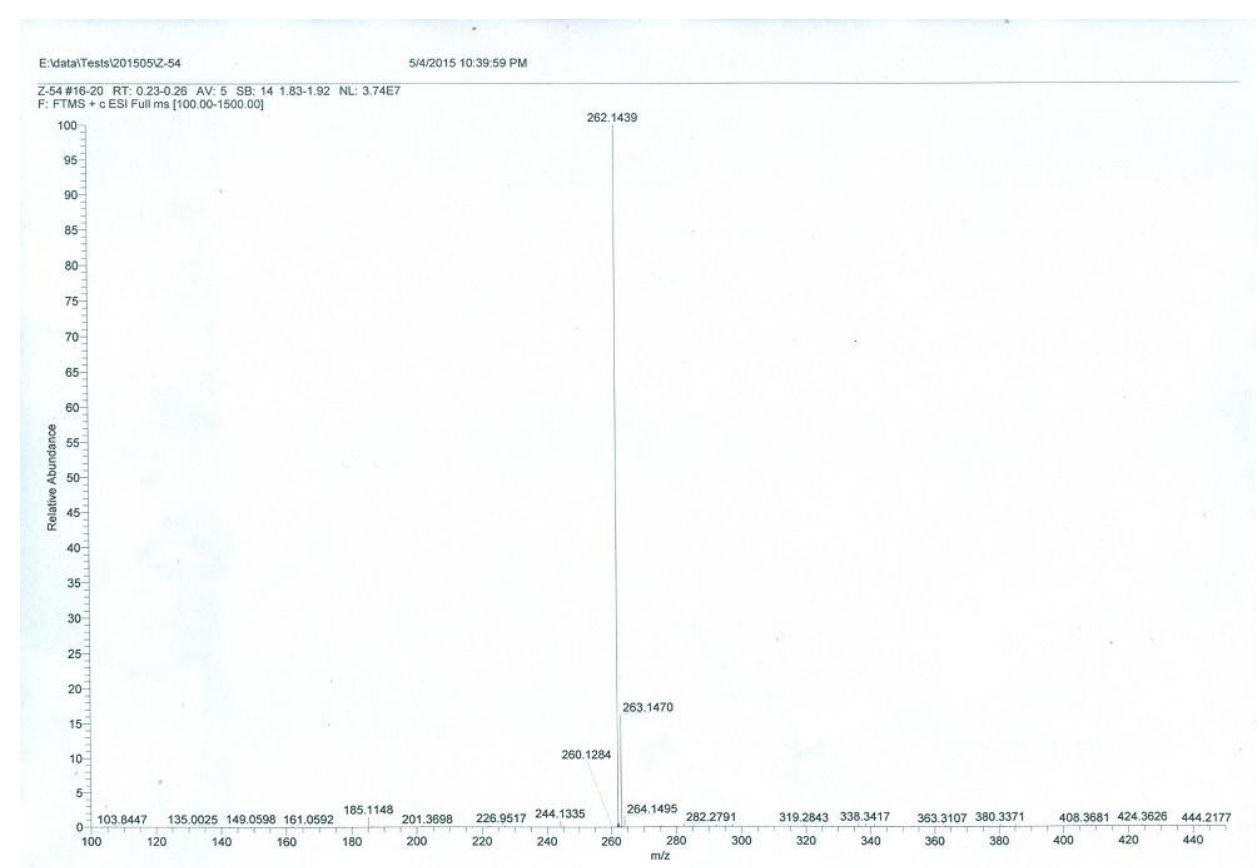

Figure S66. HRESIMS spectrum of SE5b.
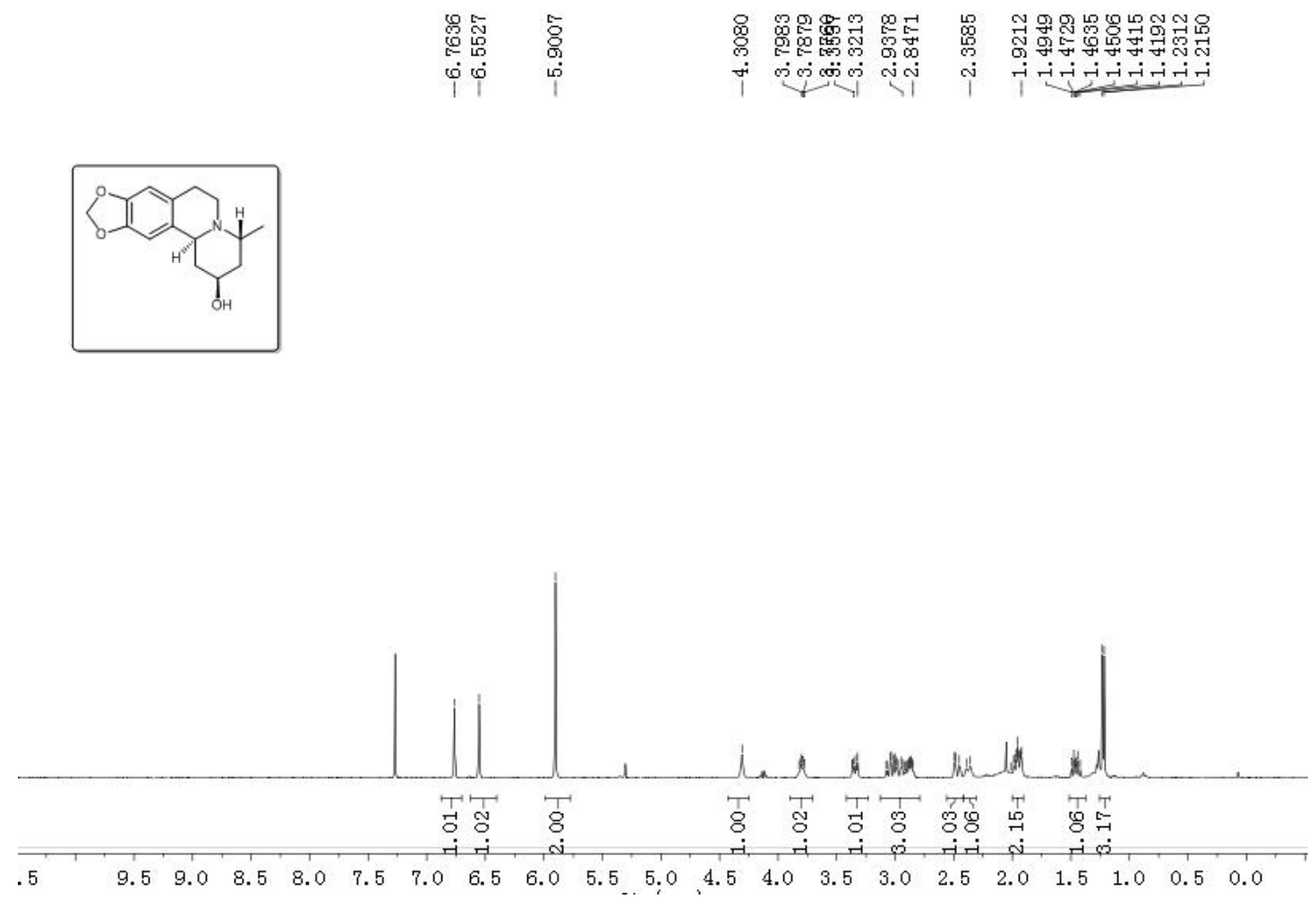

Figure S67. ${ }^{1} \mathrm{H}$ NMR spectrum of SE5b. 


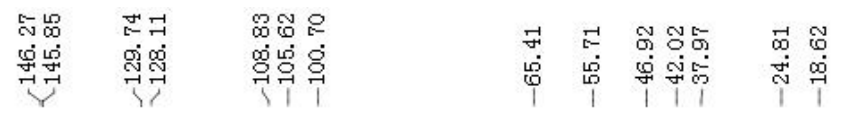
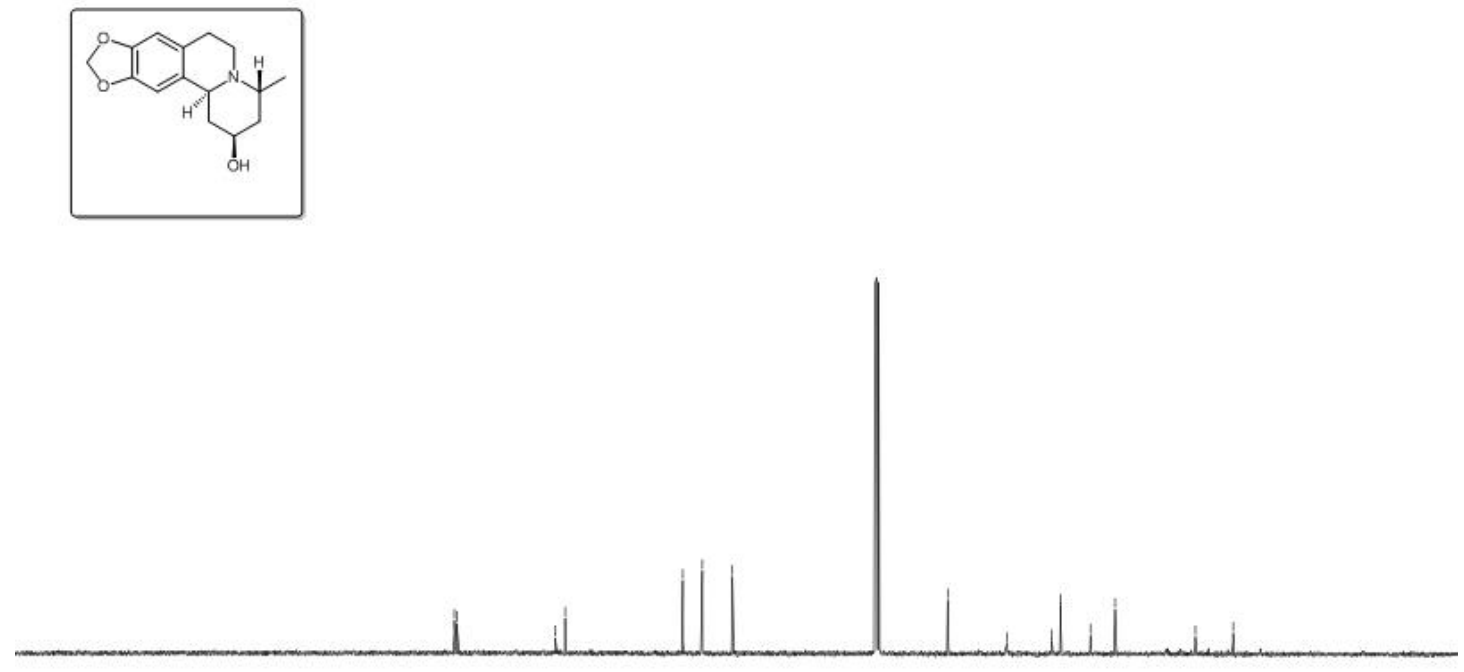

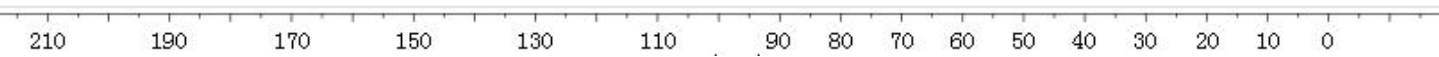

Figure S68. ${ }^{13} \mathrm{C}$ NMR spectrum of SE5b.
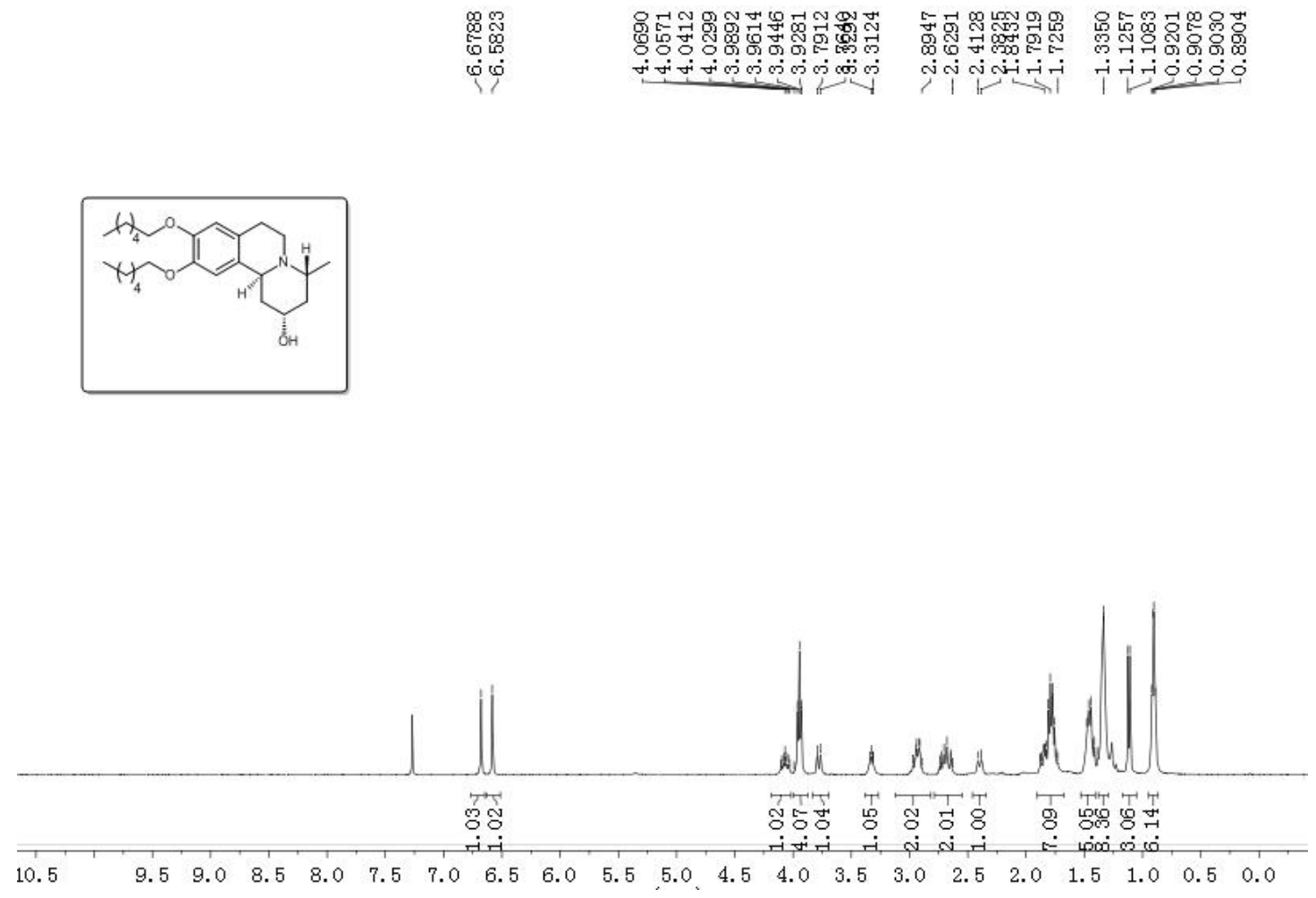

Figure S69.. ${ }^{1} \mathrm{H}$ NMR spectrum of SE6a. 

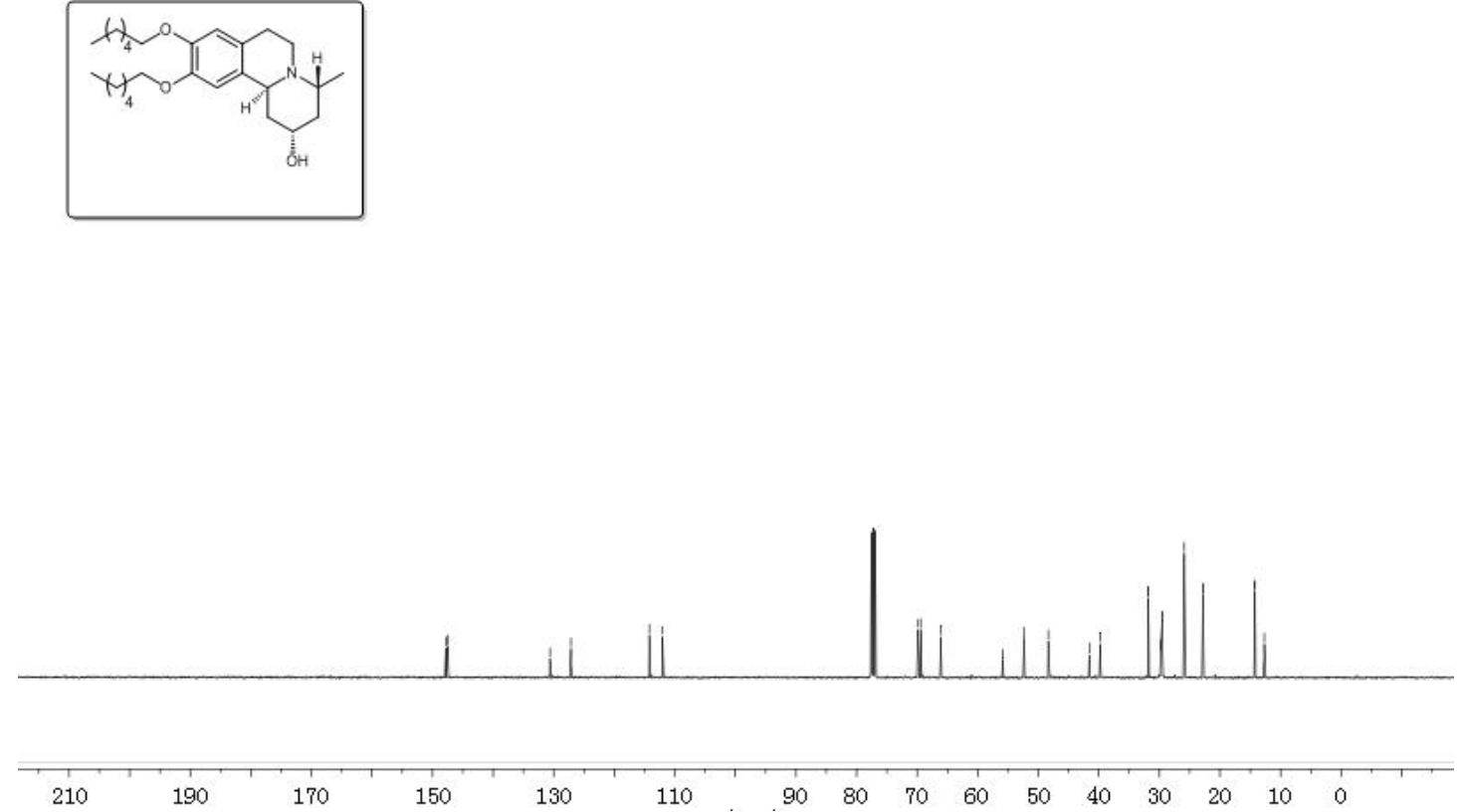

Figure S70. ${ }^{13} \mathrm{C}$ NMR spectrum of SE6a.

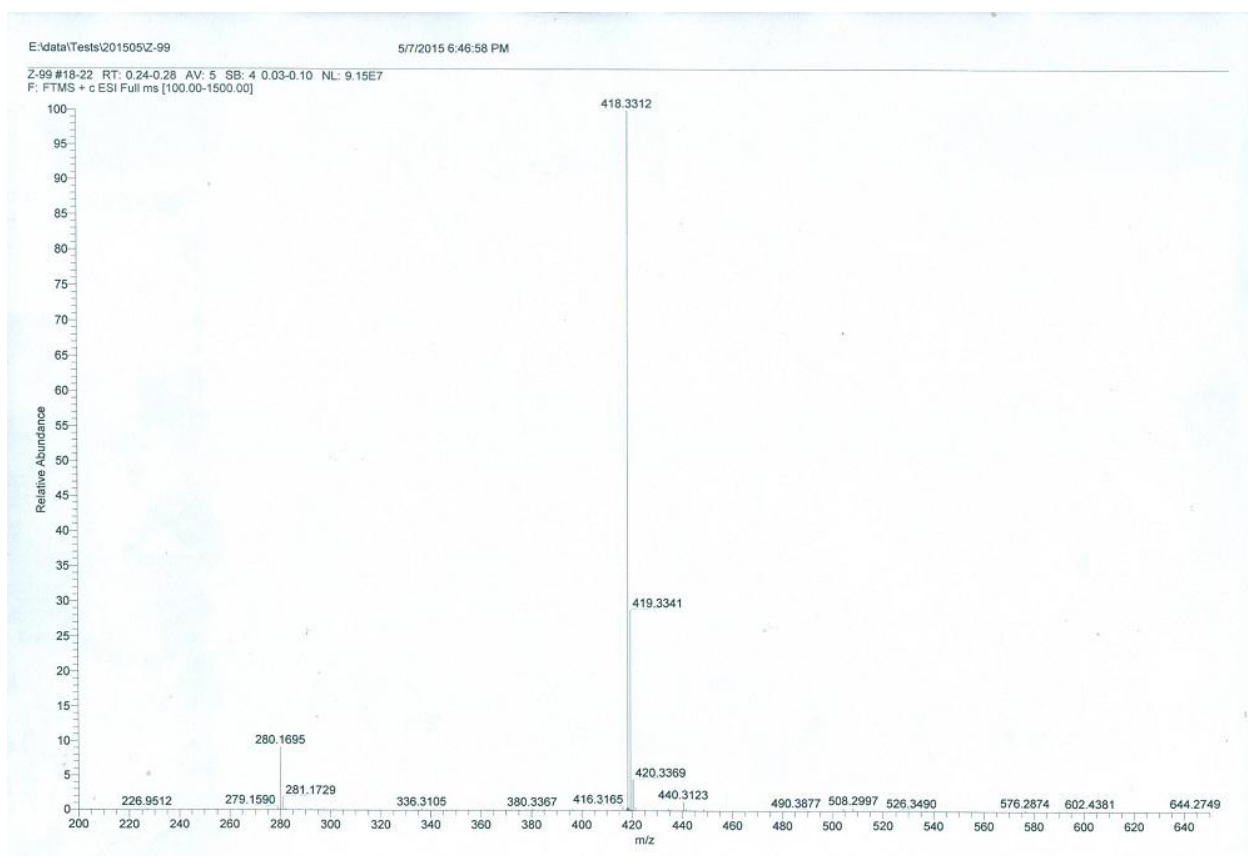

Figure S71. HRESIMS spectrum of SE6b. 


\section{型}

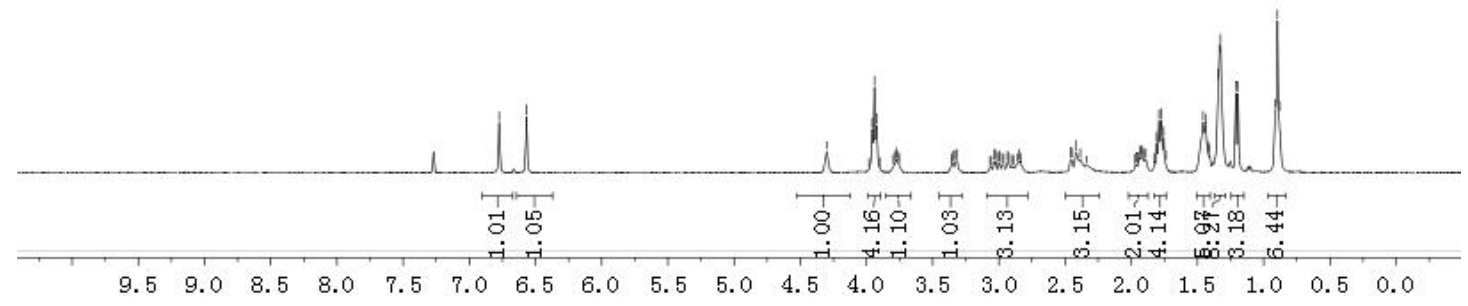

Figure S72. ${ }^{1} \mathrm{H}$ NMR spectrum of SE6b.
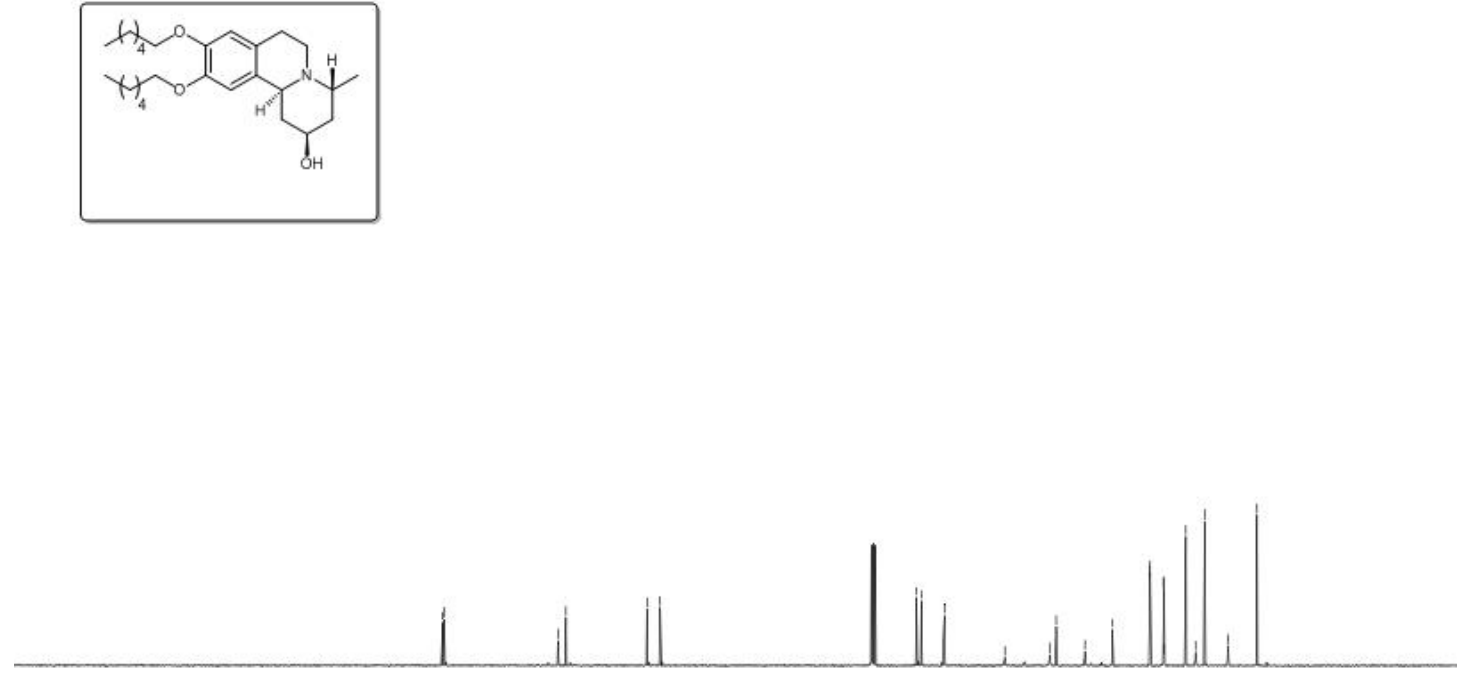

Figure S73. ${ }^{13} \mathrm{C}$ NMR spectrum of SE6b. 


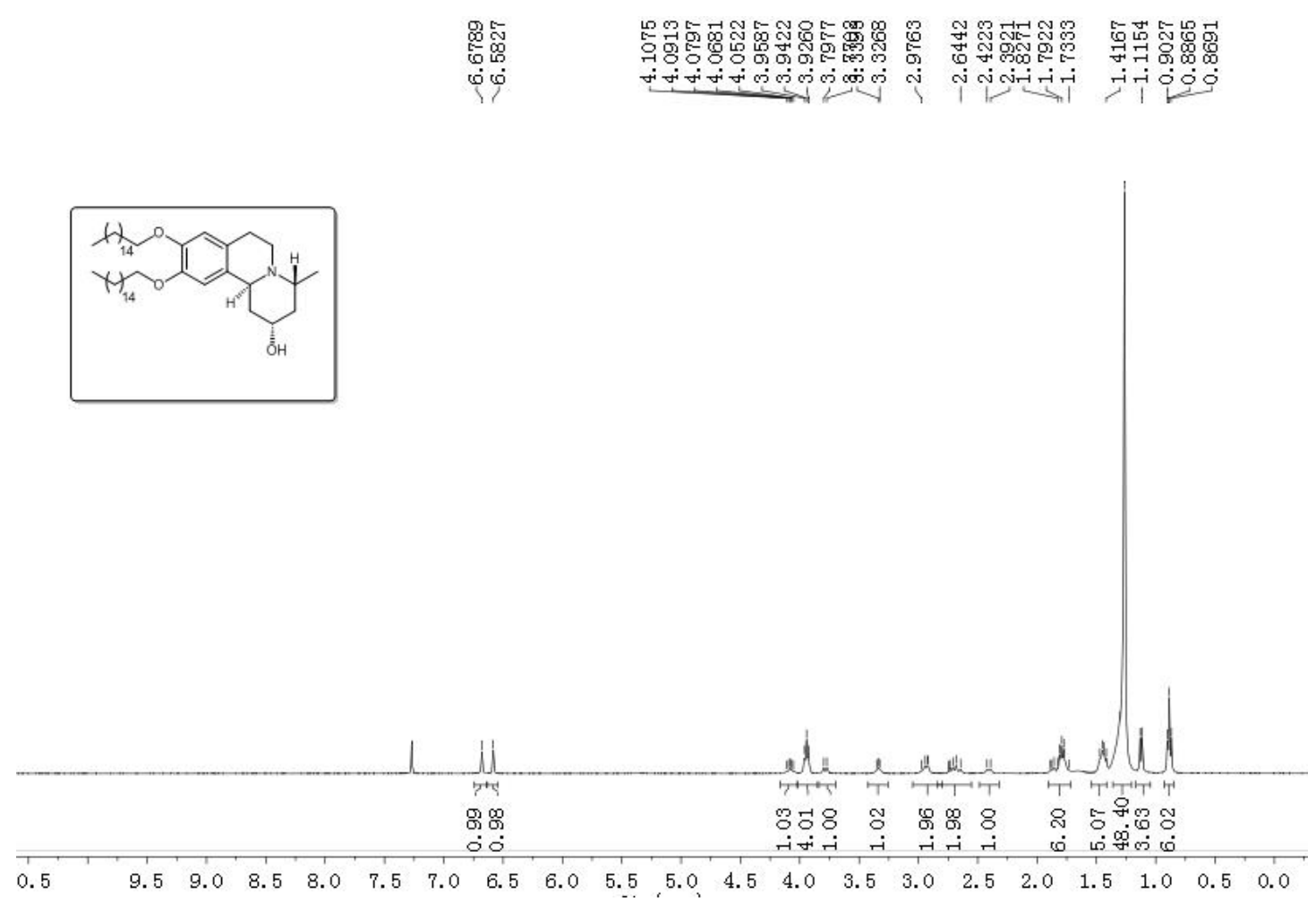

Figure S74. ${ }^{1} \mathrm{H}$ NMR spectrum of SE7a.

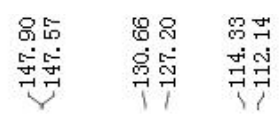

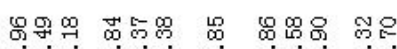

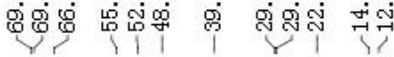
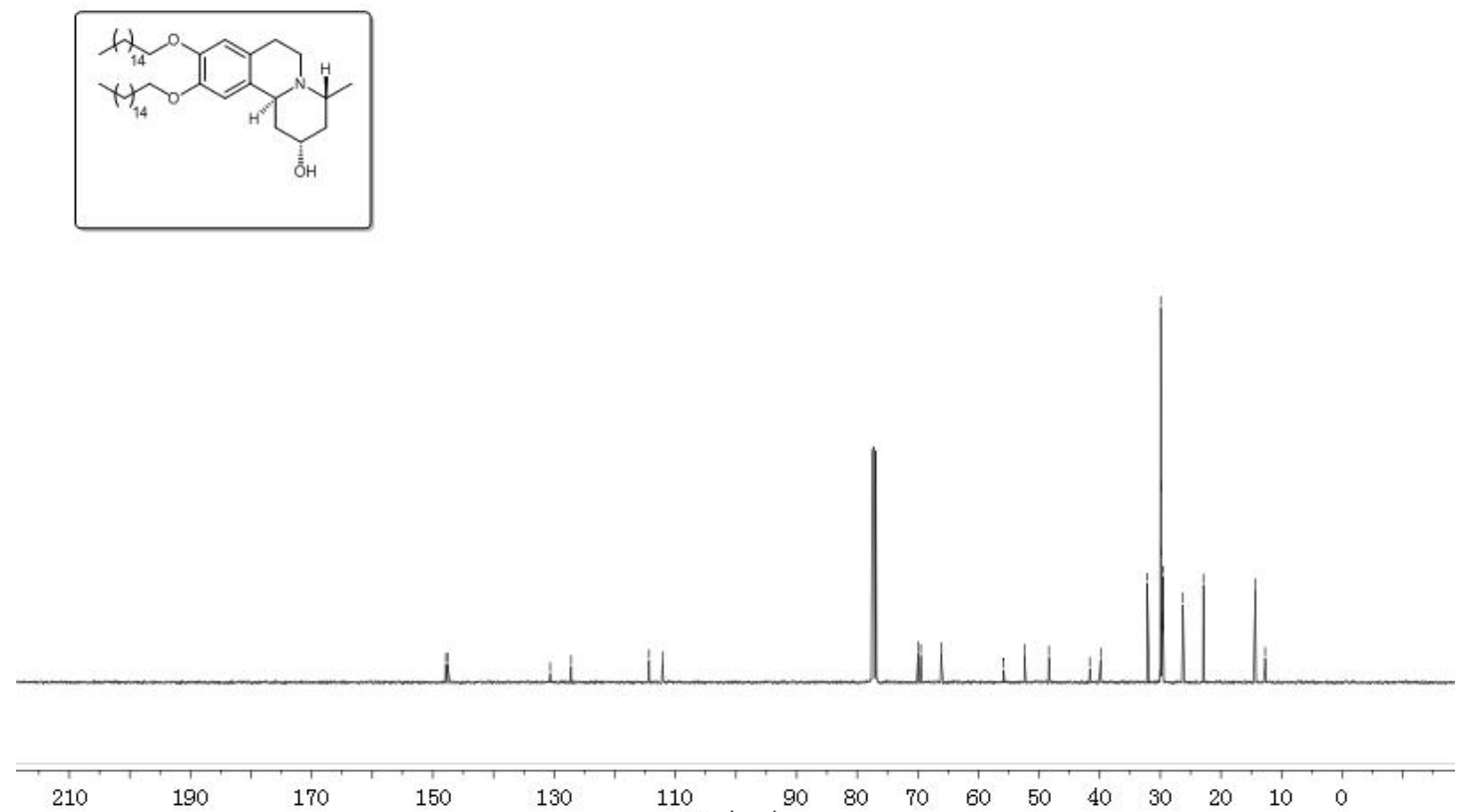

Figure S75. ${ }^{13} \mathrm{C}$ NMR spectrum of SE7a. 


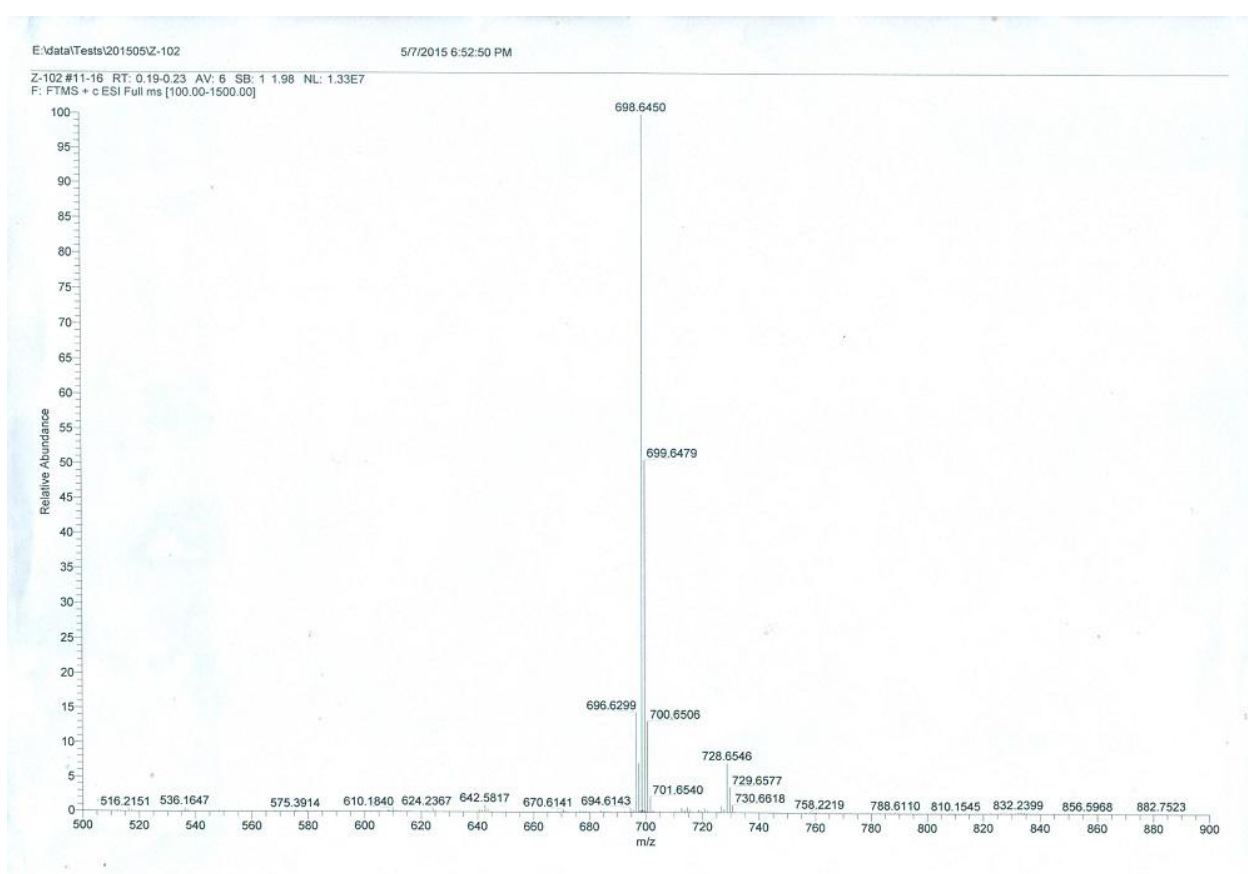

Figure S76. HRESIMS spectrum of SE7b.

58
98
0
0
1
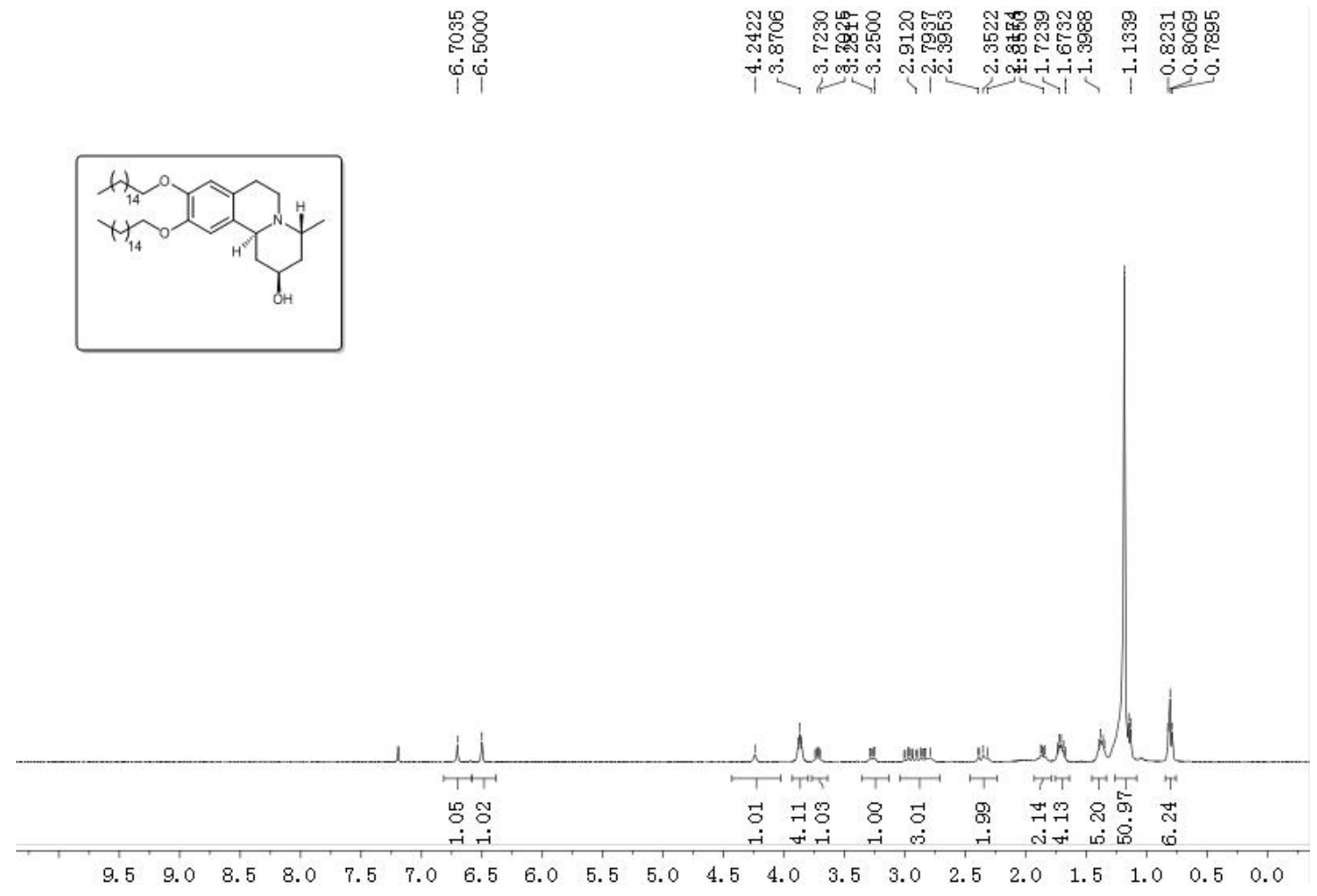

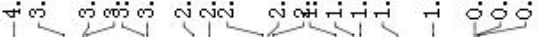

Figure S77. ${ }^{1} \mathrm{H}$ NMR spectrum of SE7b. 


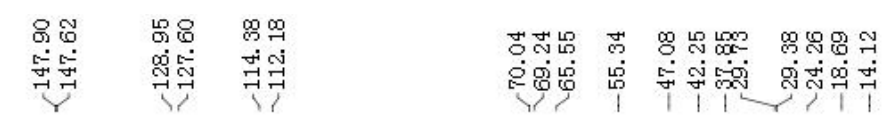
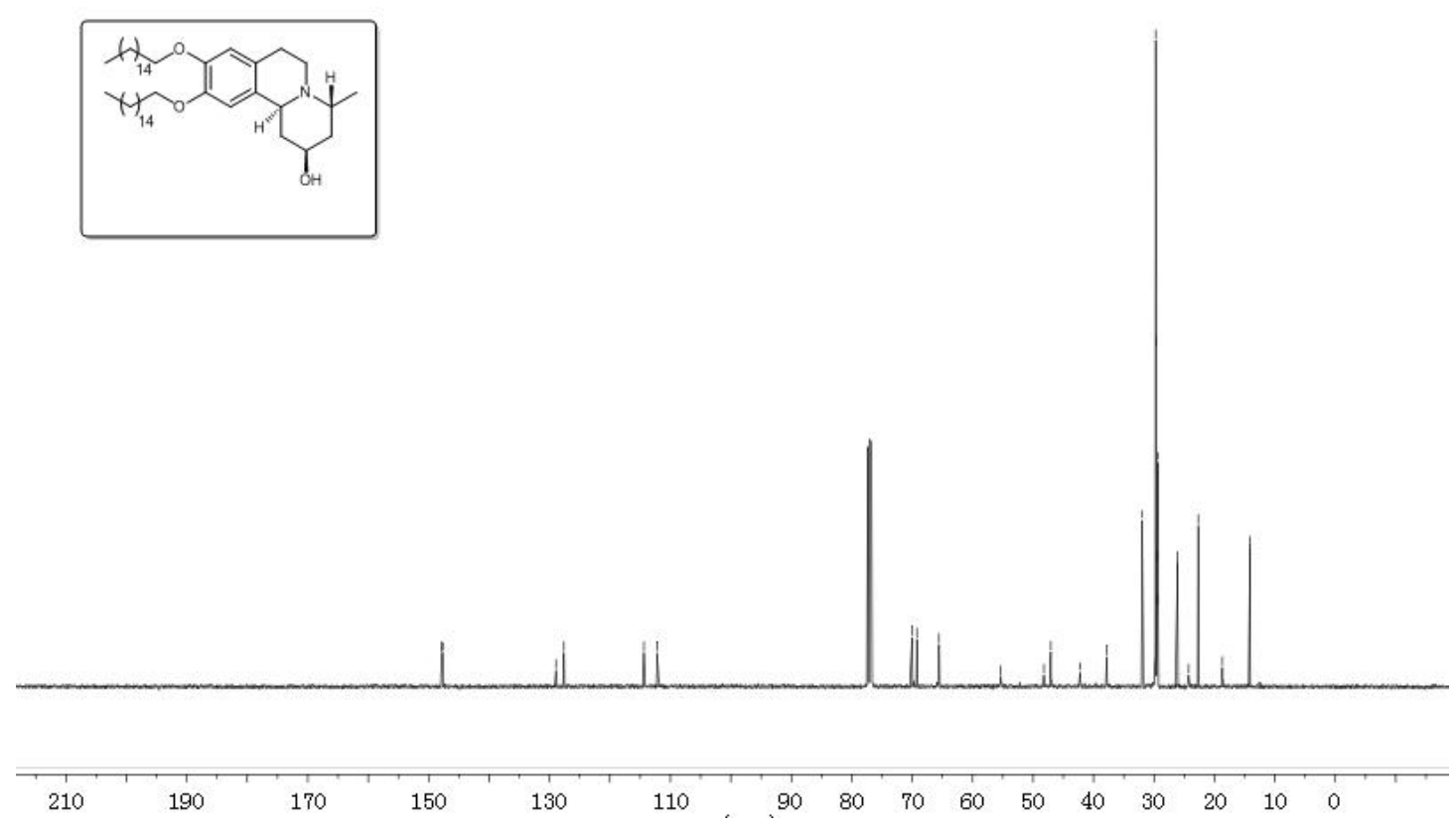

Figure S78. ${ }^{13} \mathrm{C}$ NMR spectrum of SE7b.
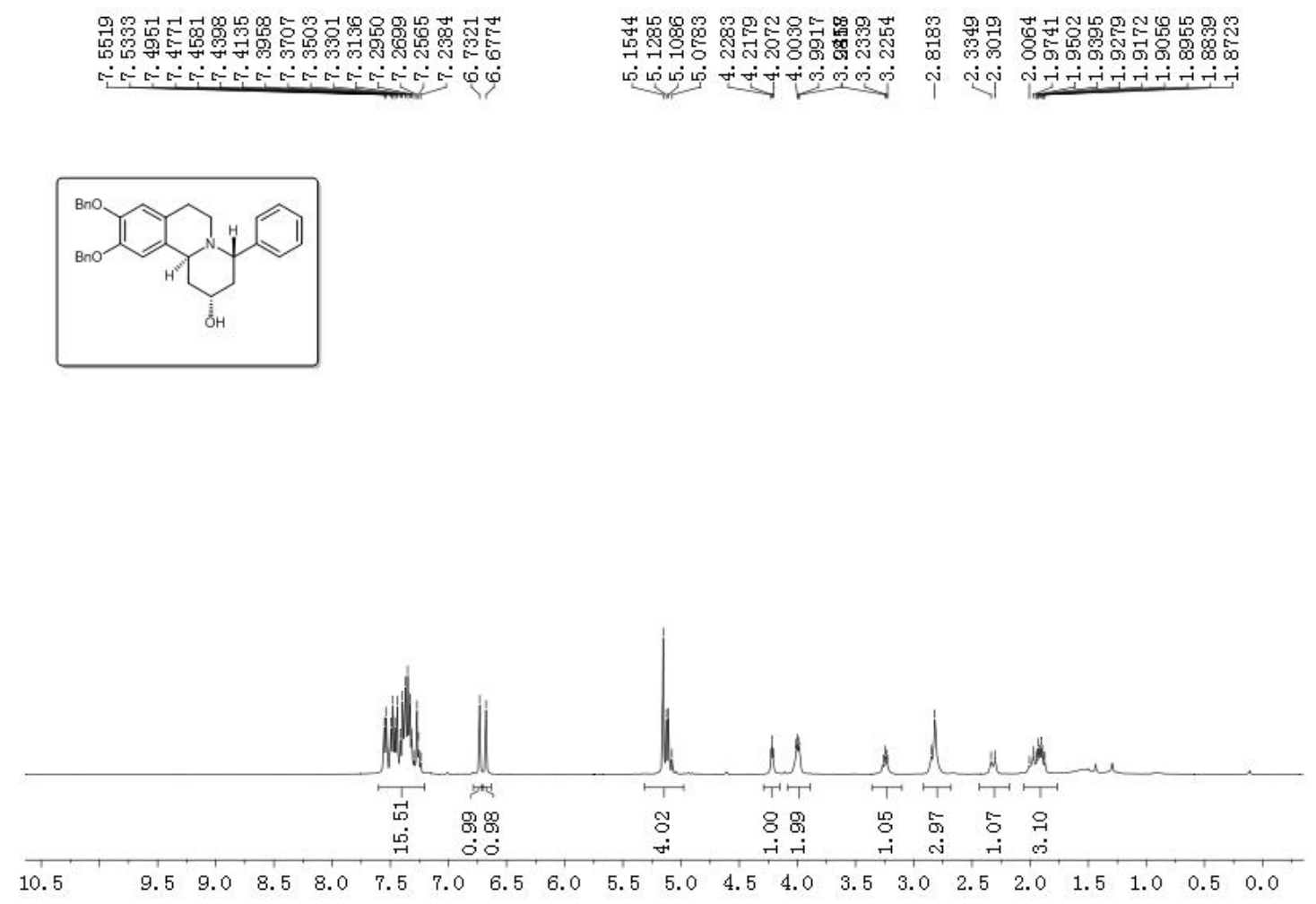

Figure S79. ${ }^{1} \mathrm{H}$ NMR spectrum of SE8a. 


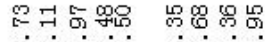

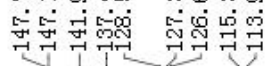

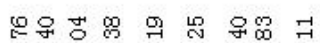

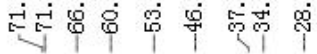<smiles>CC1Cc2cc(O)c(O)cc2CN(c2ccccc2)C1</smiles>

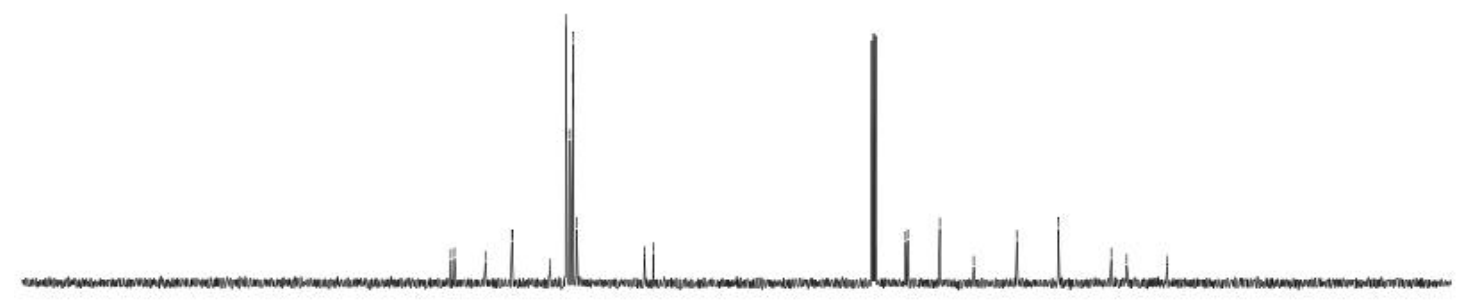

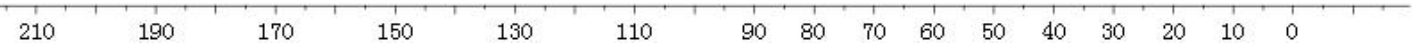

Figure S80. ${ }^{13} \mathrm{C}$ NMR spectrum of SE8a.

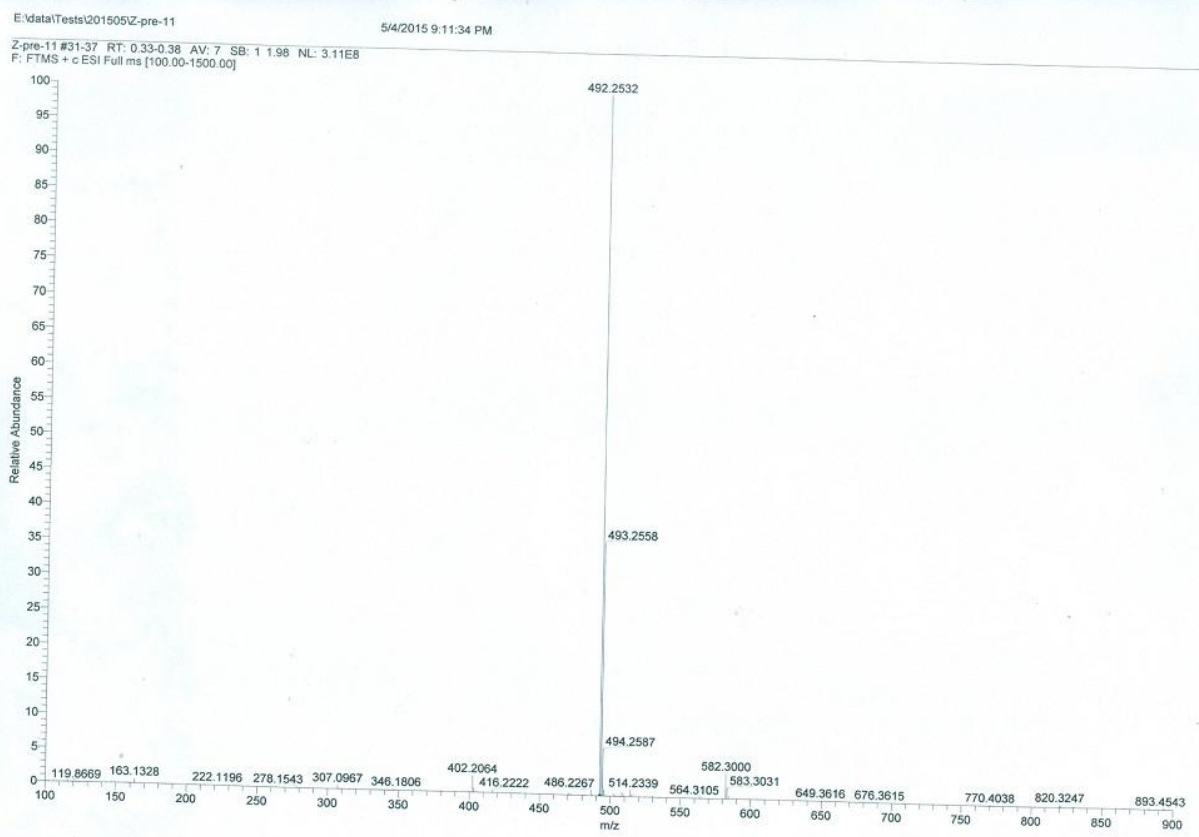

Figure S81. HRESIMS spectrum of $\mathbf{1}$. 

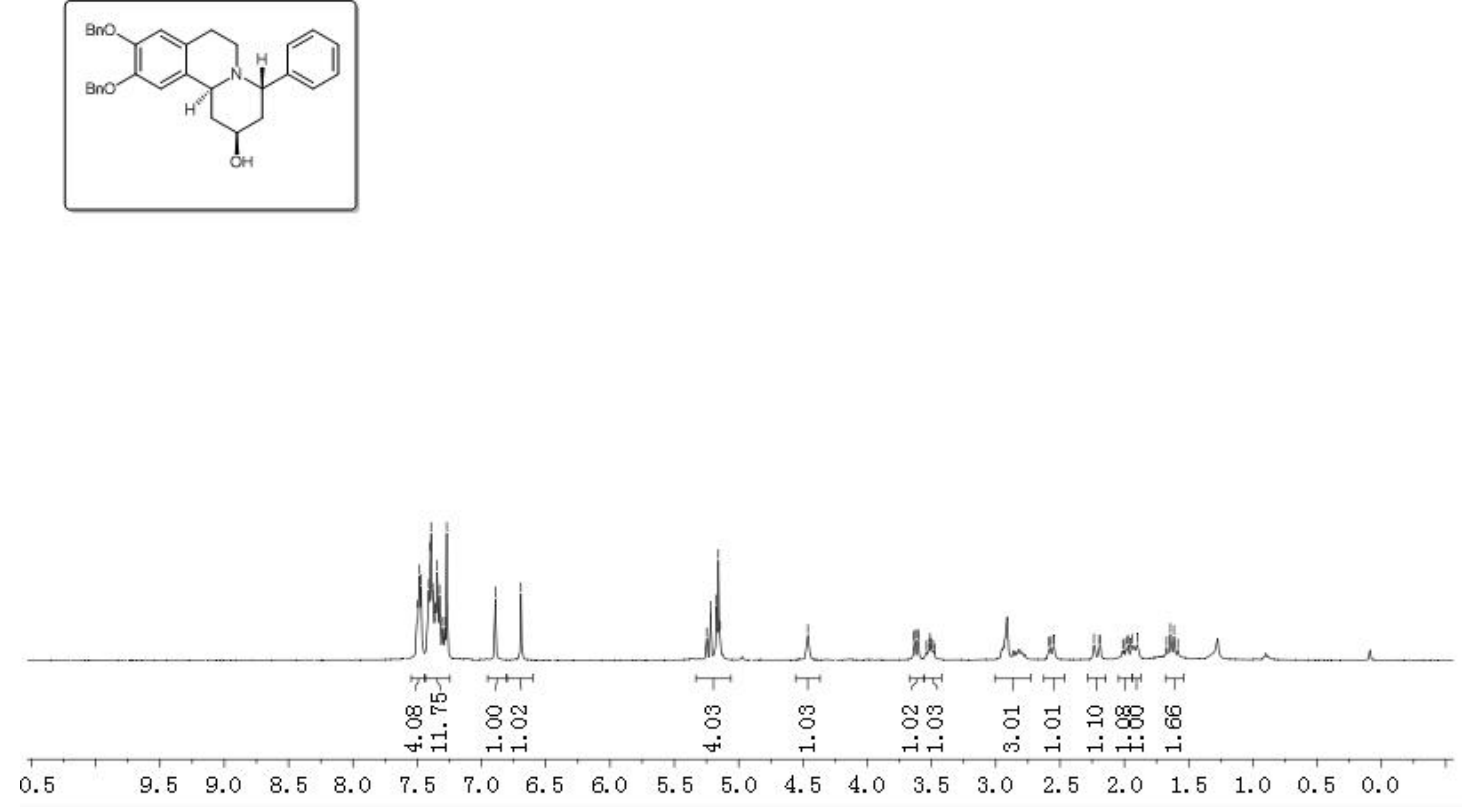

Figure S82. ${ }^{1} \mathrm{H}$ NMR spectrum of $\mathbf{1}$.

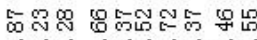

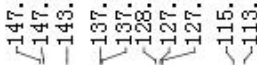

สำ ㄱำ

संत्र
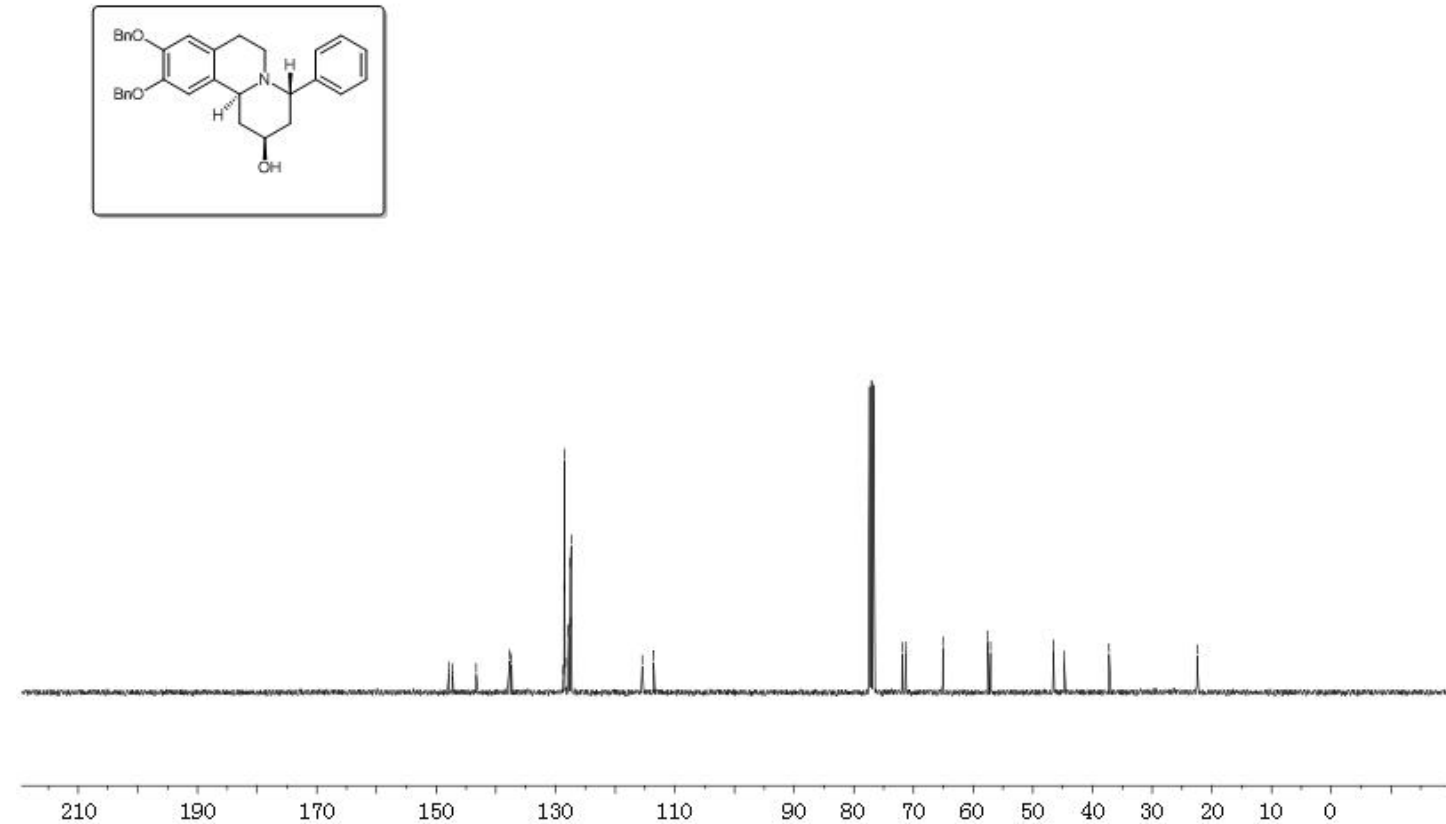

150

130

$90 \quad 80$

Figure S83. ${ }^{13} \mathrm{C}$ NMR spectrum of $\mathbf{1}$. 


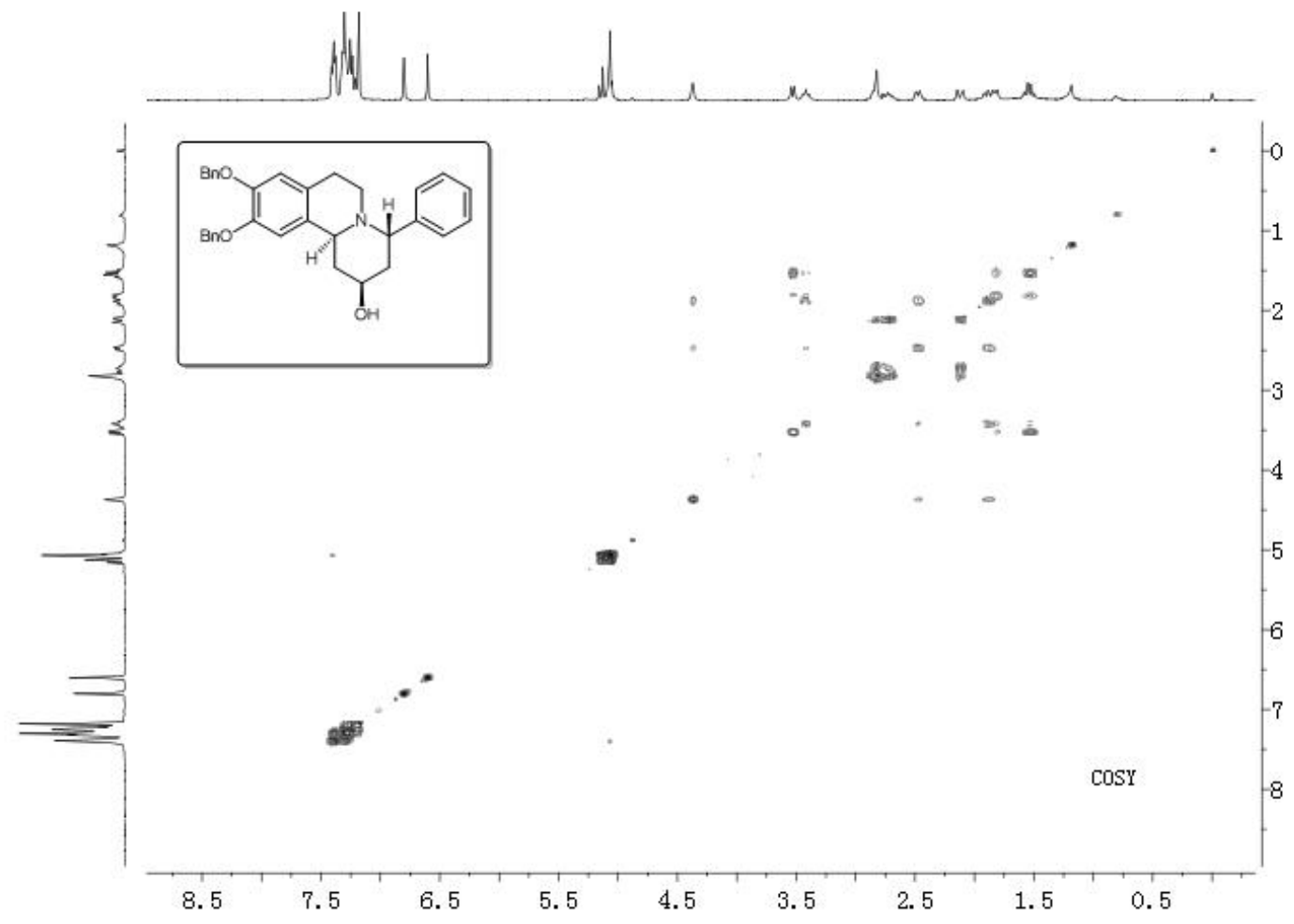

Figure S84. COSY spectrum of $\mathbf{1}$.

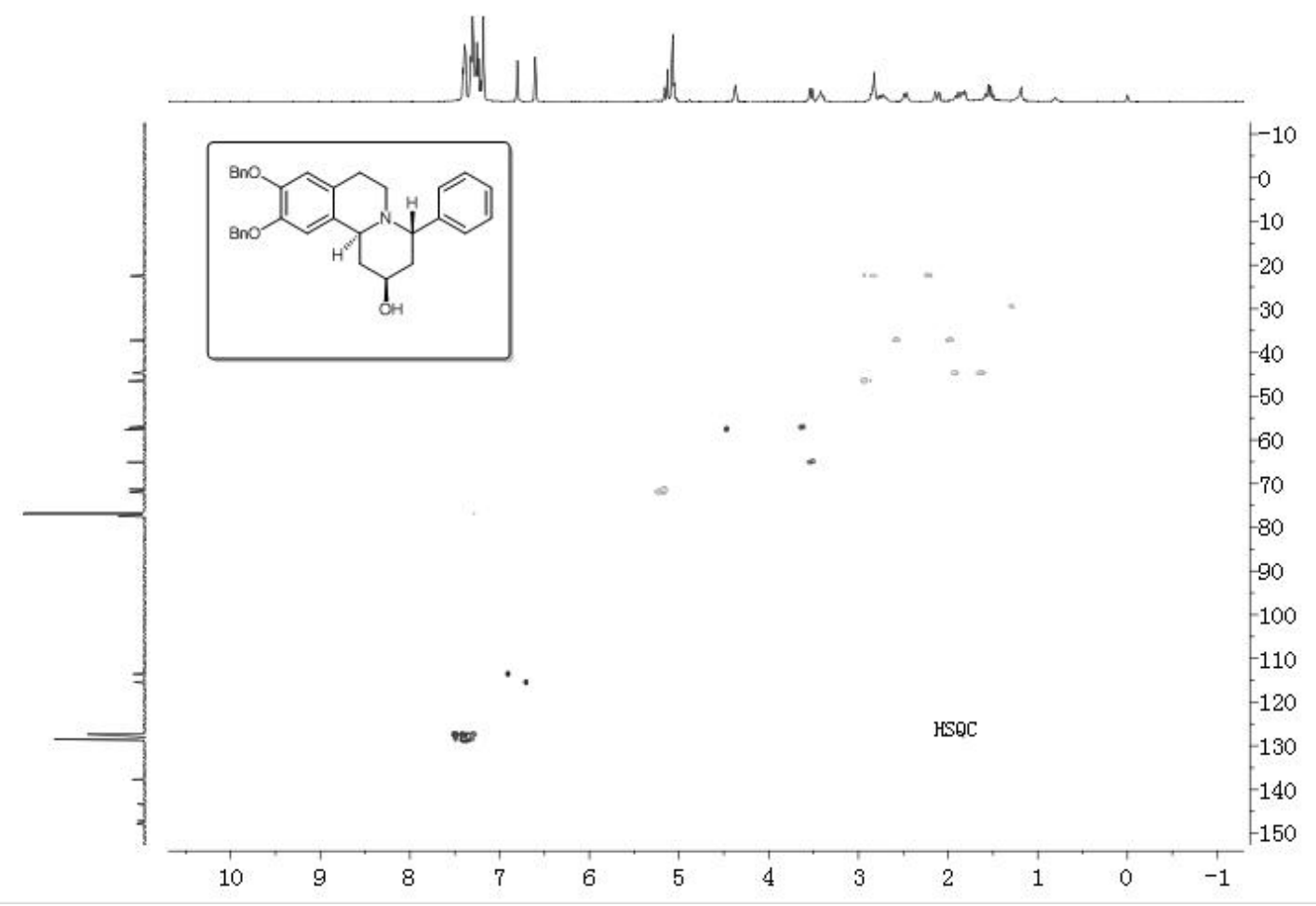

Figure S85. HSQC spectrum of $\mathbf{1}$ 


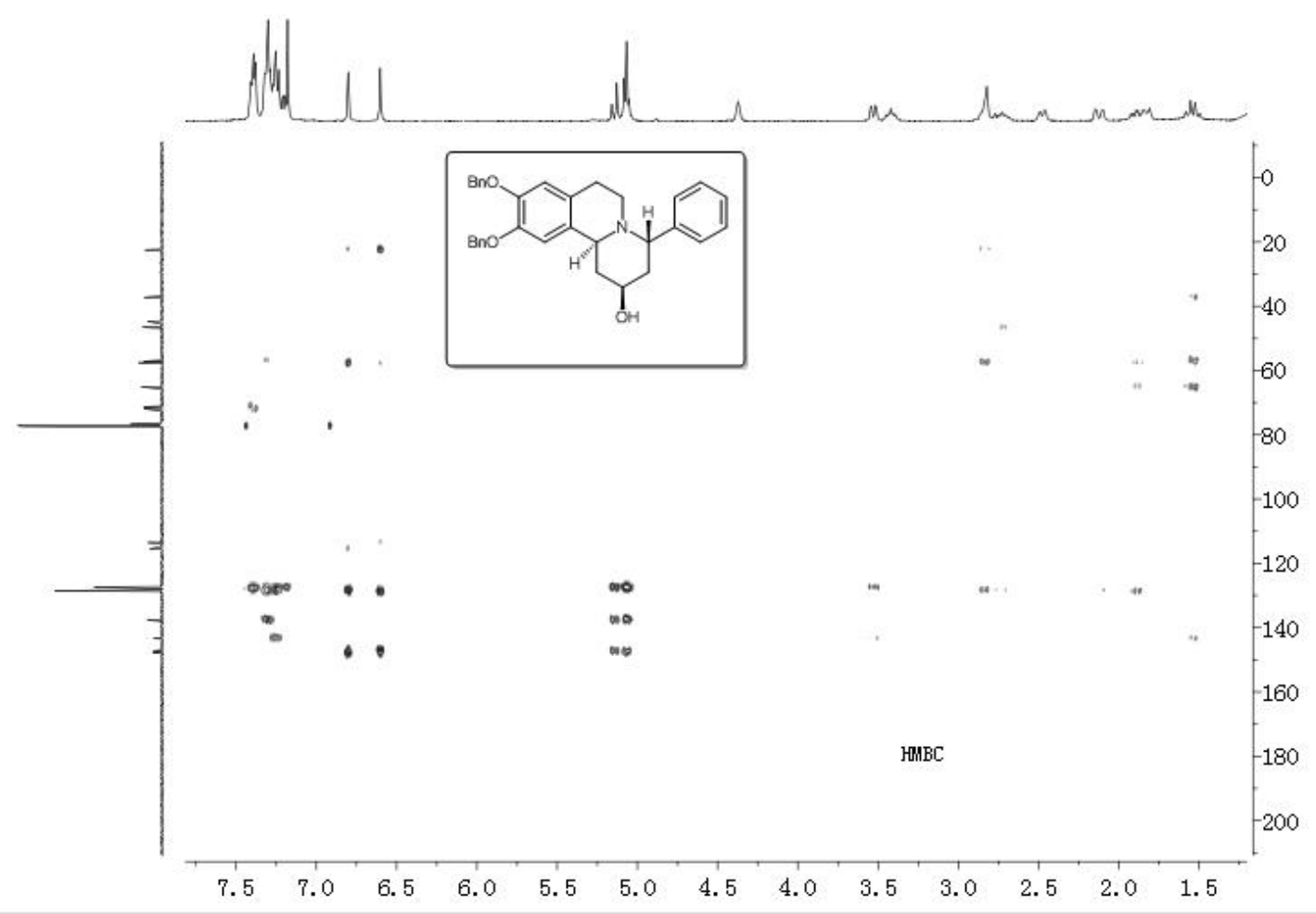

Figure S86. HMBC spectrum of $\mathbf{1}$.

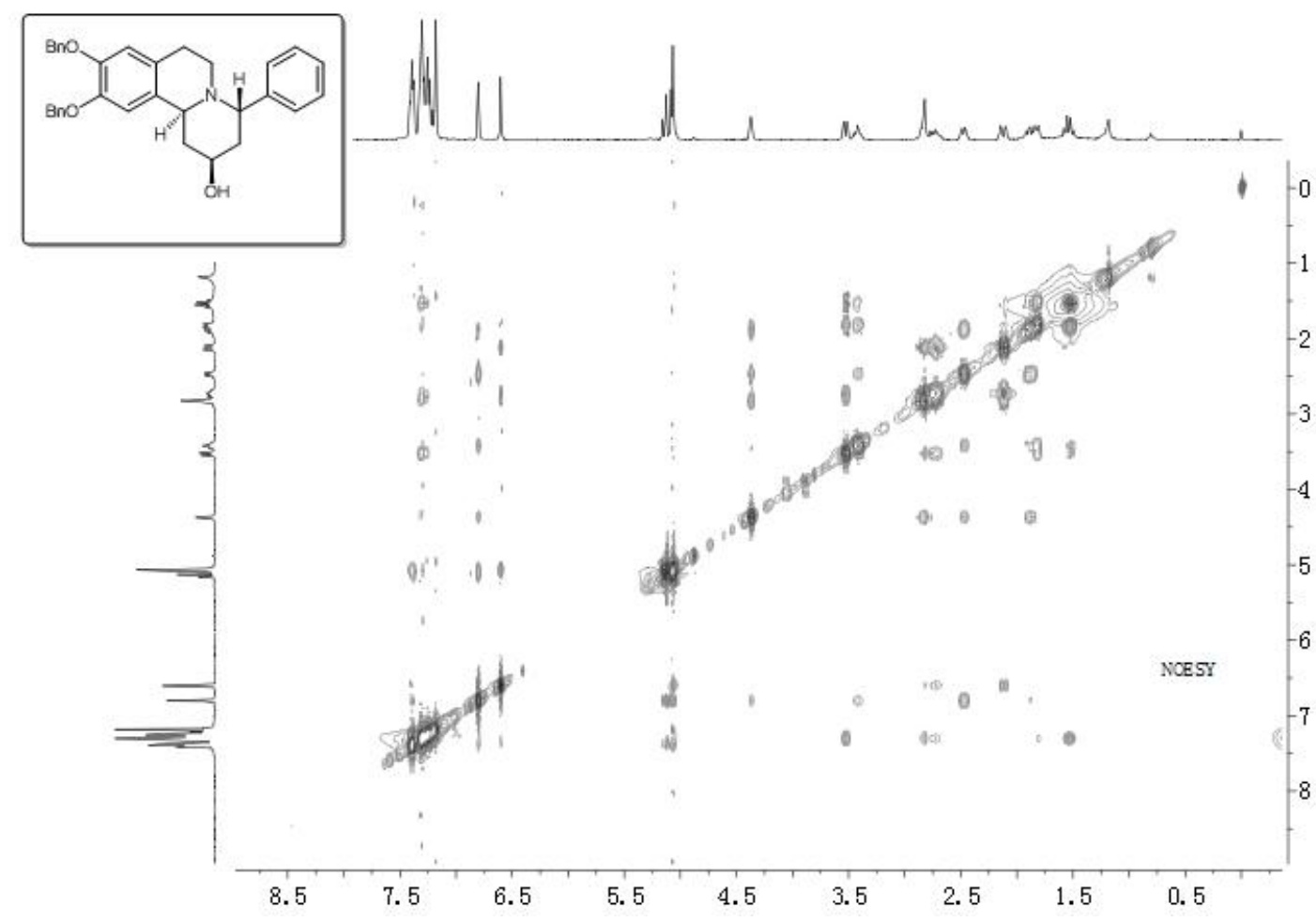

Figure S87. NOESY spectrum of 1. 

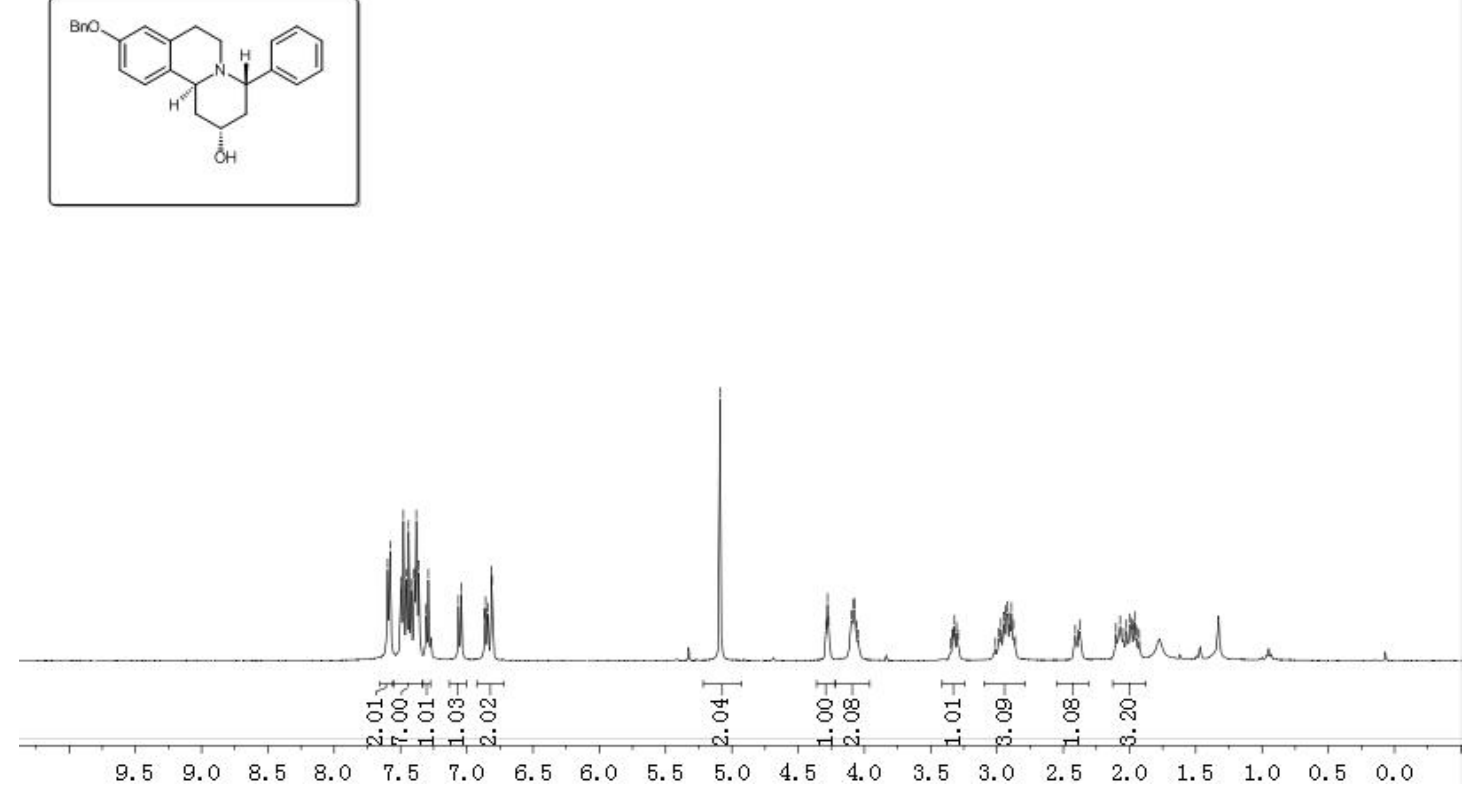

Figure S88. ${ }^{1} \mathrm{H}$ NMR spectrum of SE9a
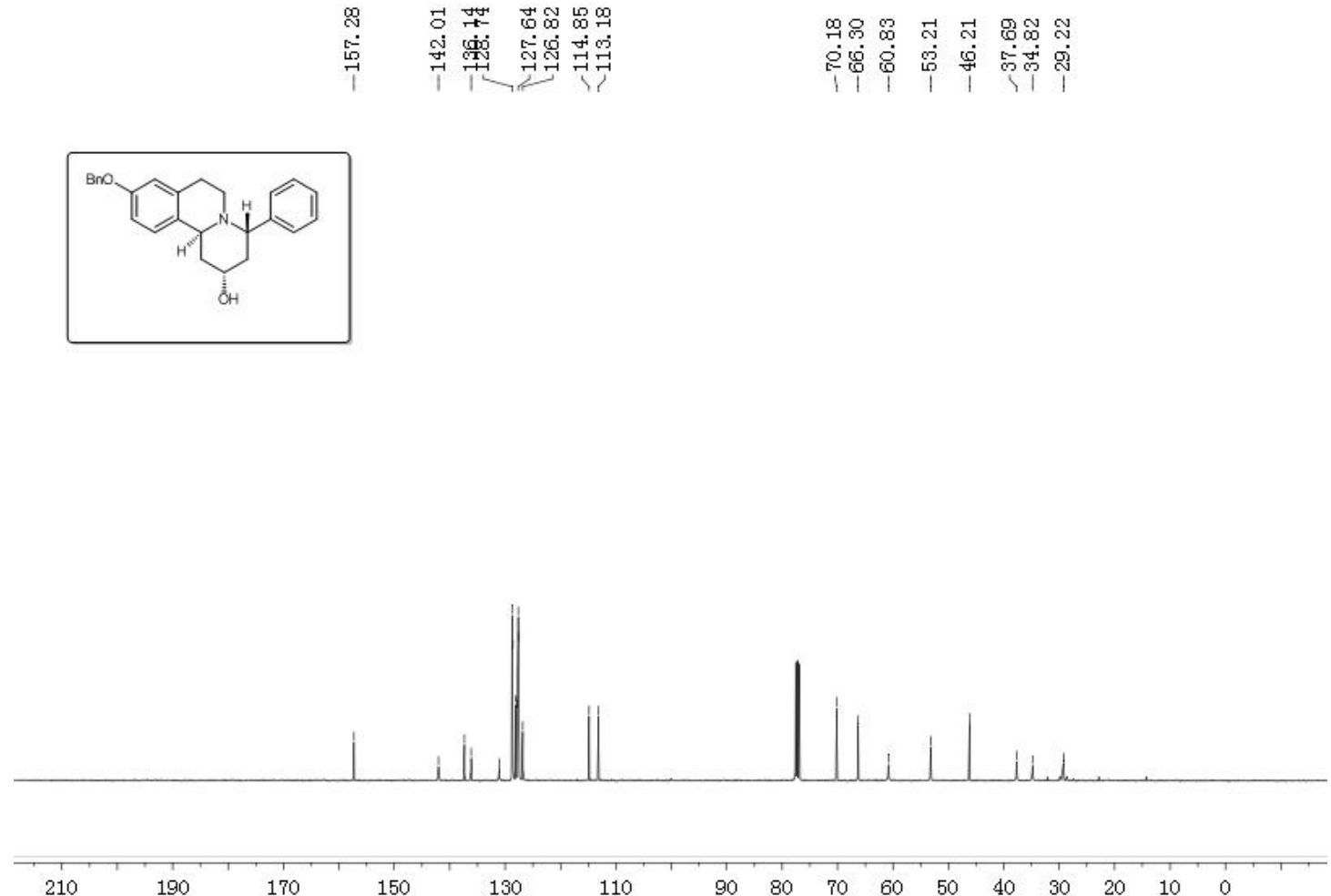

$150 \quad 130$

Figure S89. ${ }^{13} \mathrm{C}$ NMR spectrum of SE9a. 


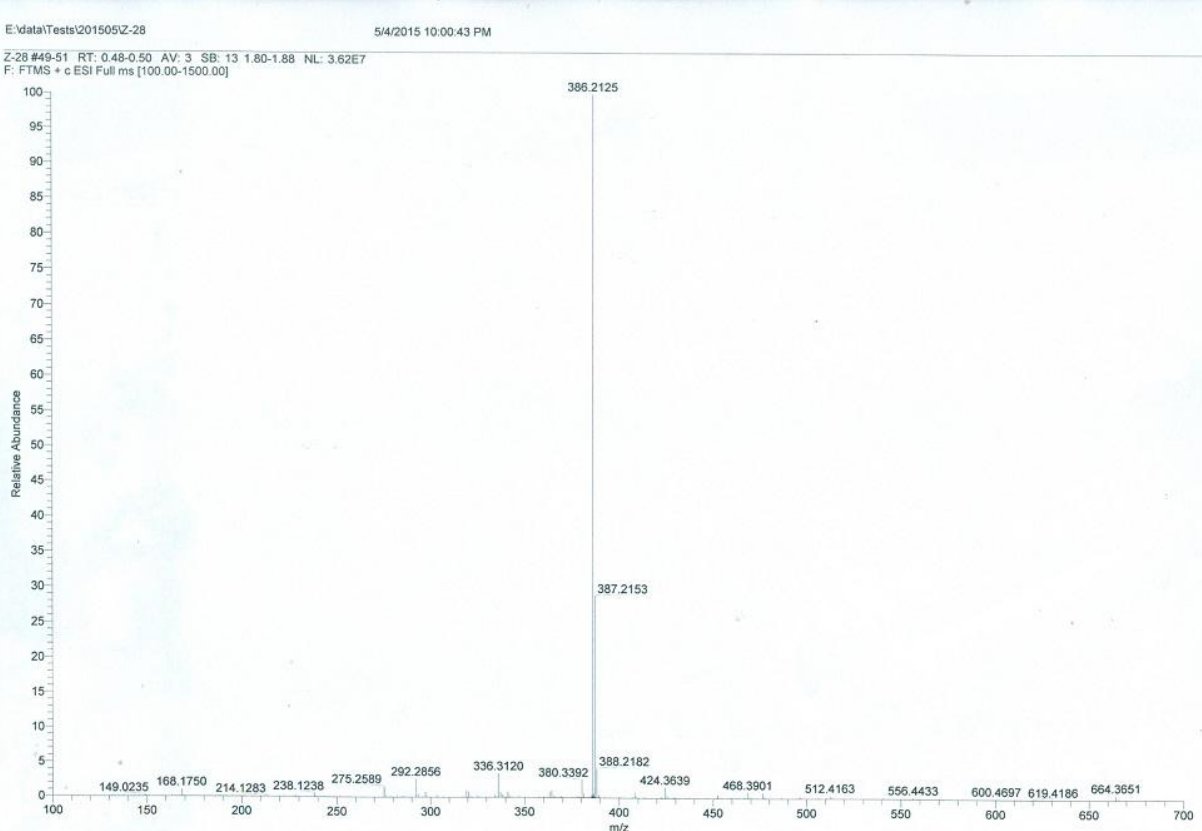

Figure S90. HRESIMS spectrum of 2.
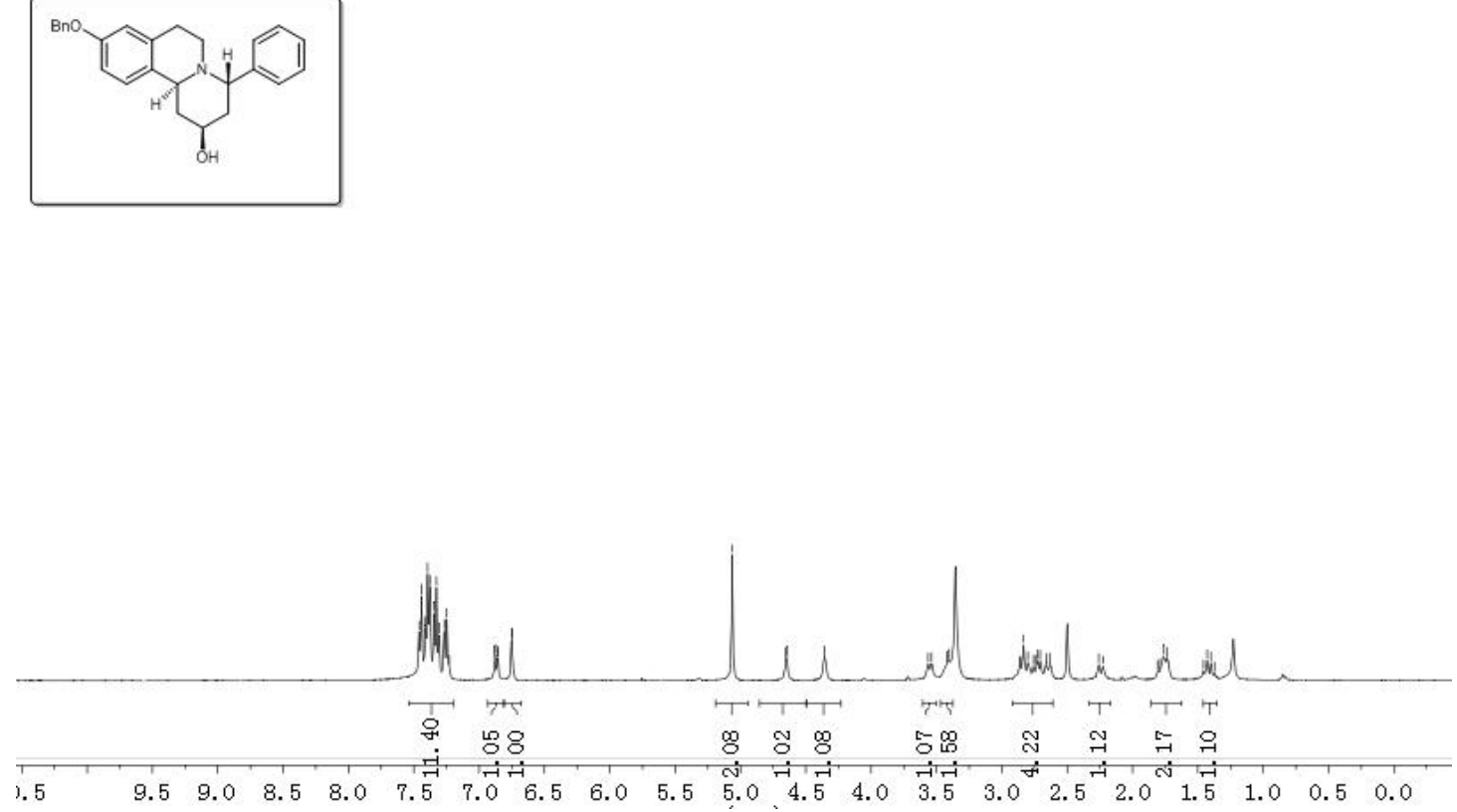

Figure S91. ${ }^{1} \mathrm{H}$ NMR spectrum of 2. 

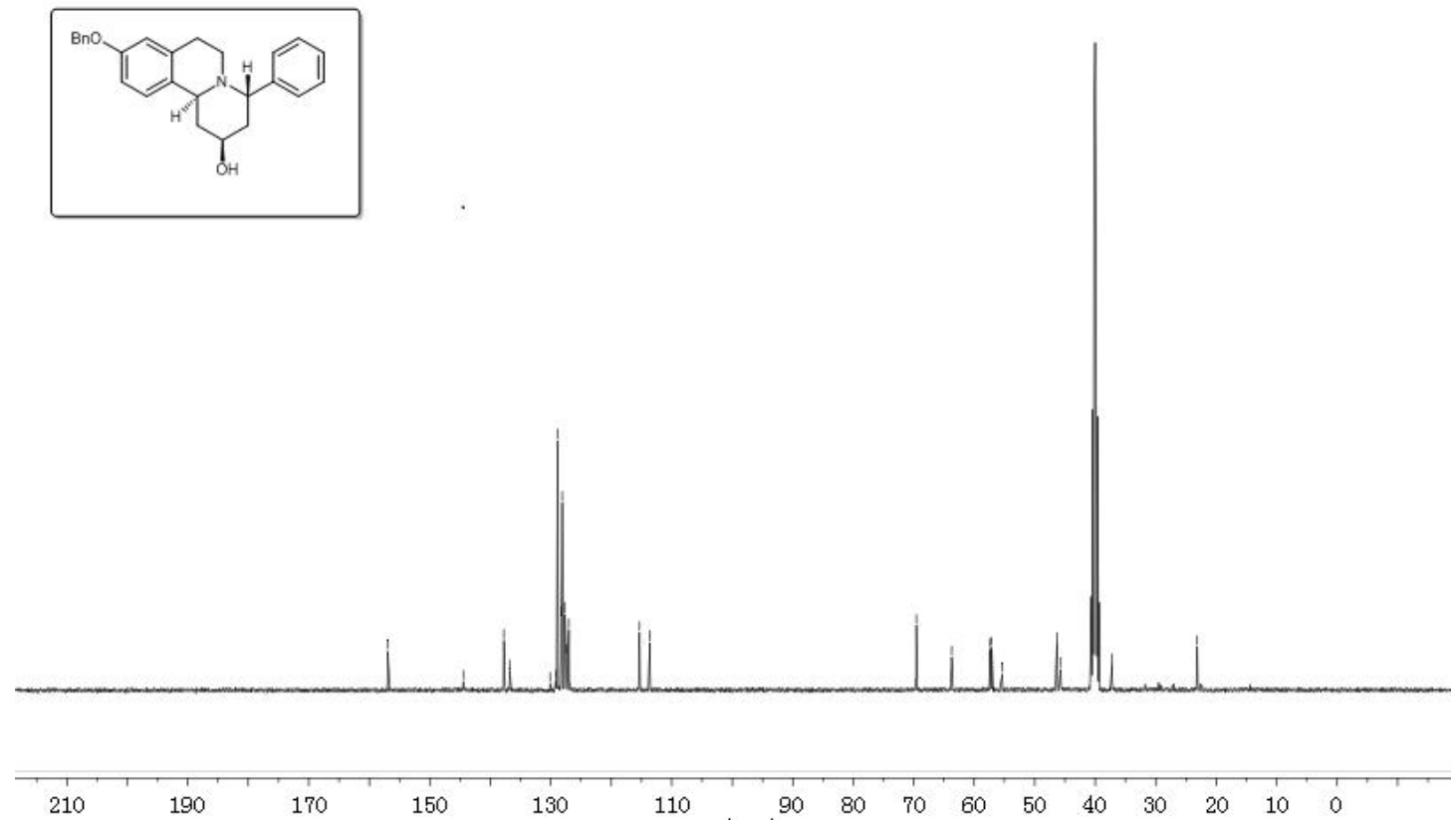

Figure S92. ${ }^{13} \mathrm{C}$ NMR spectrum of 2 .

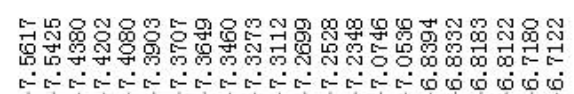

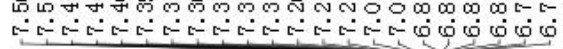
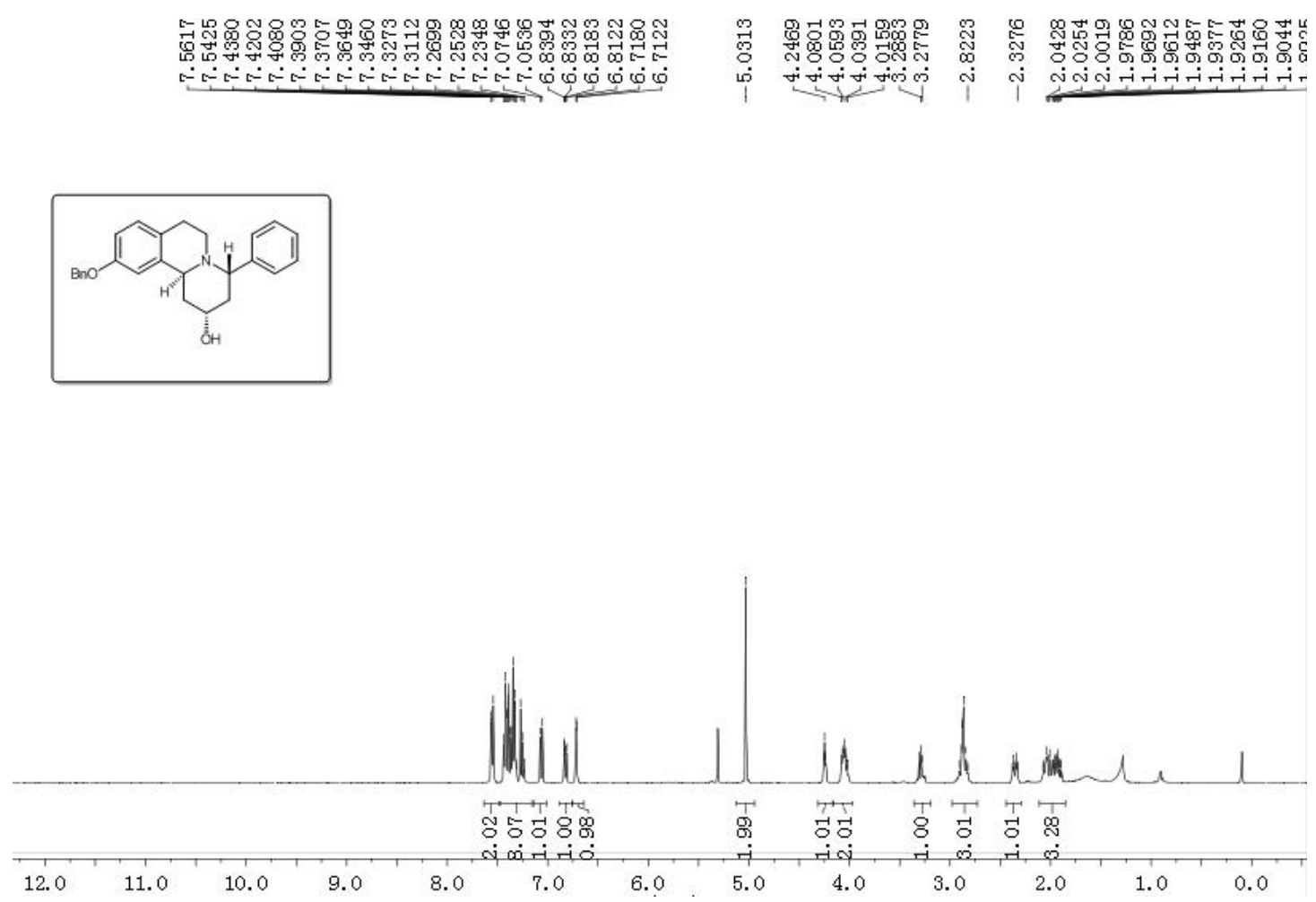

Figure S93. ${ }^{1} \mathrm{H}$ NMR spectrum of SE10a. 

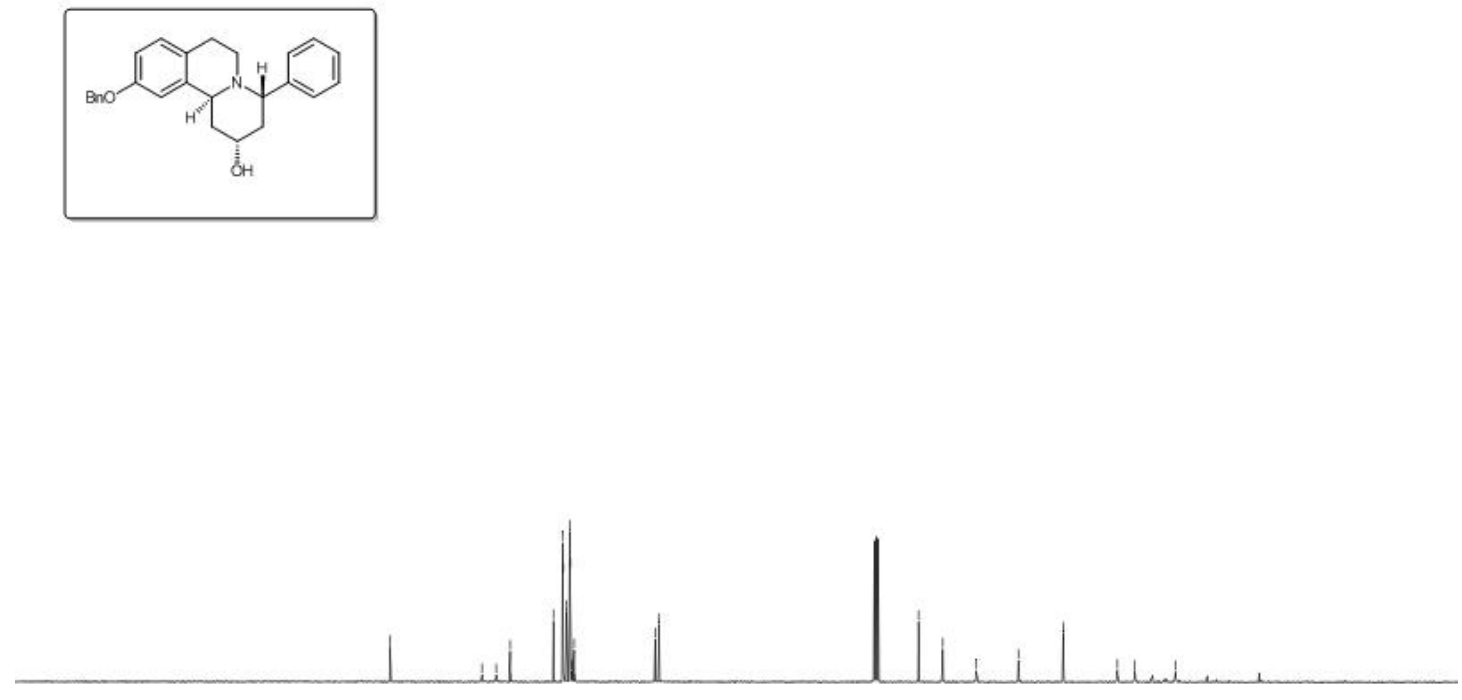

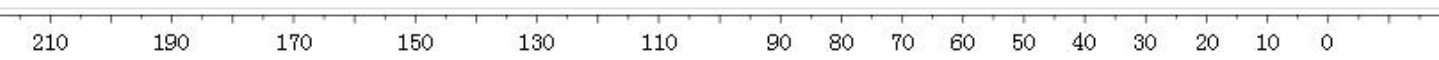

Figure S94. ${ }^{13} \mathrm{C}$ NMR spectrum of SE10a.

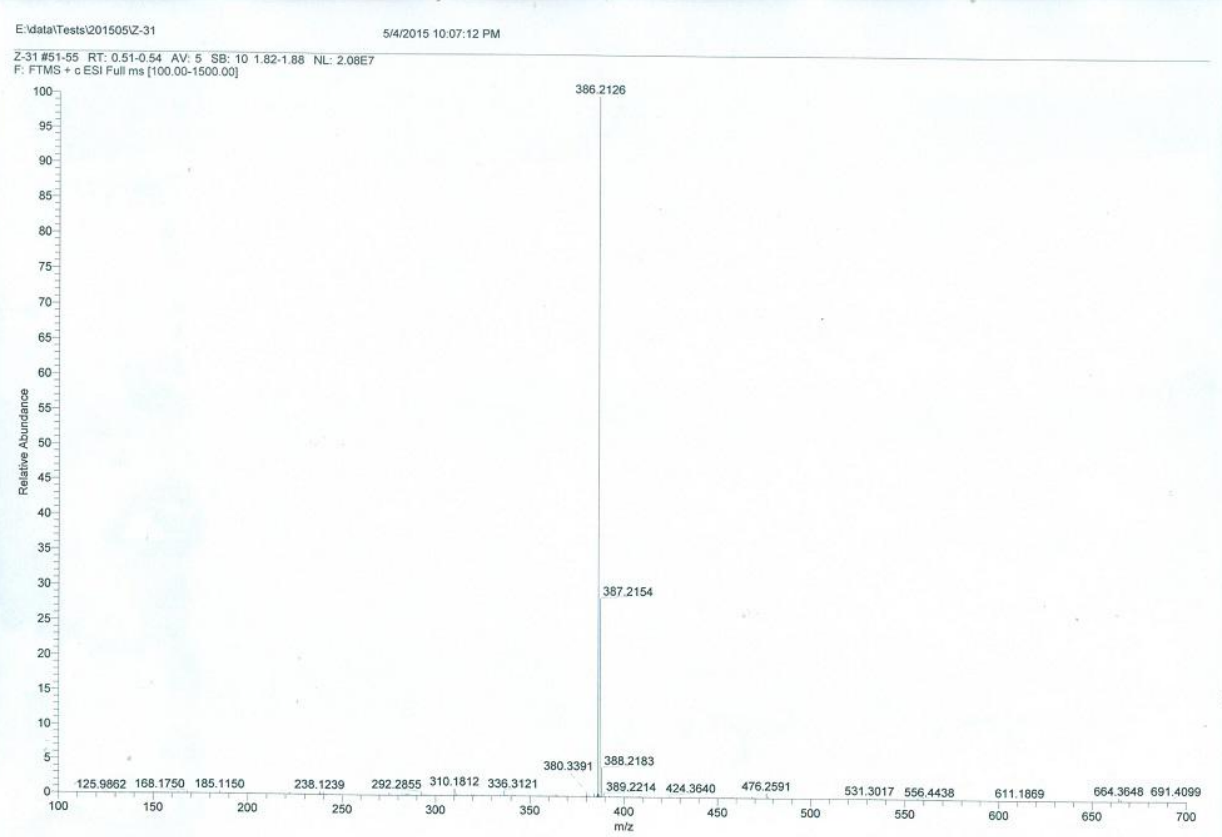

Figure S95. HRESIMS spectrum of 3. 

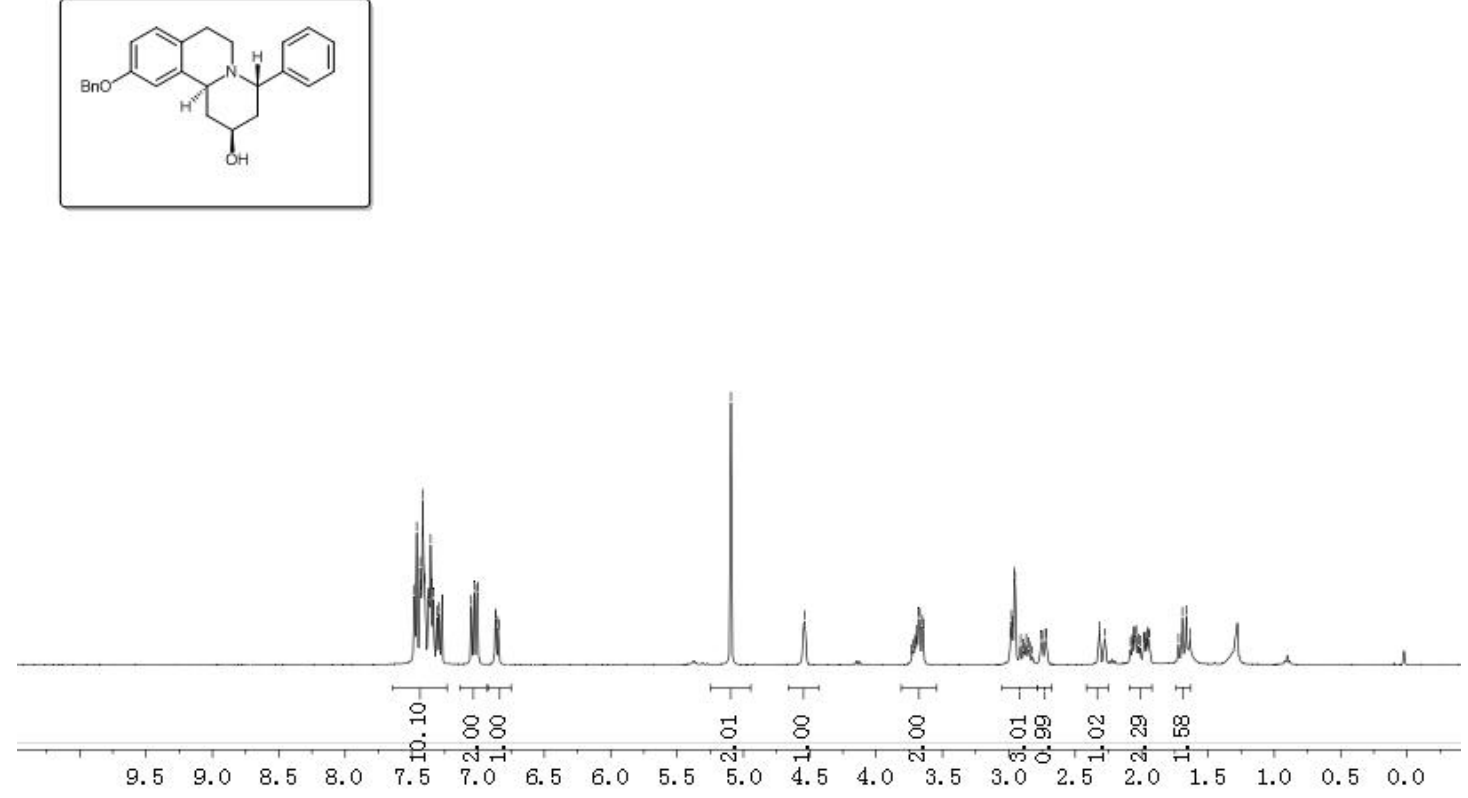

Figure S96. ${ }^{1} \mathrm{H}$ NMR spectrum of 3 .

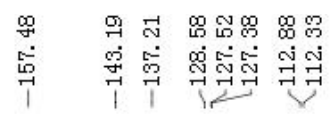

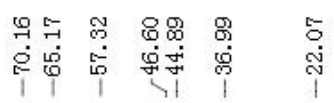
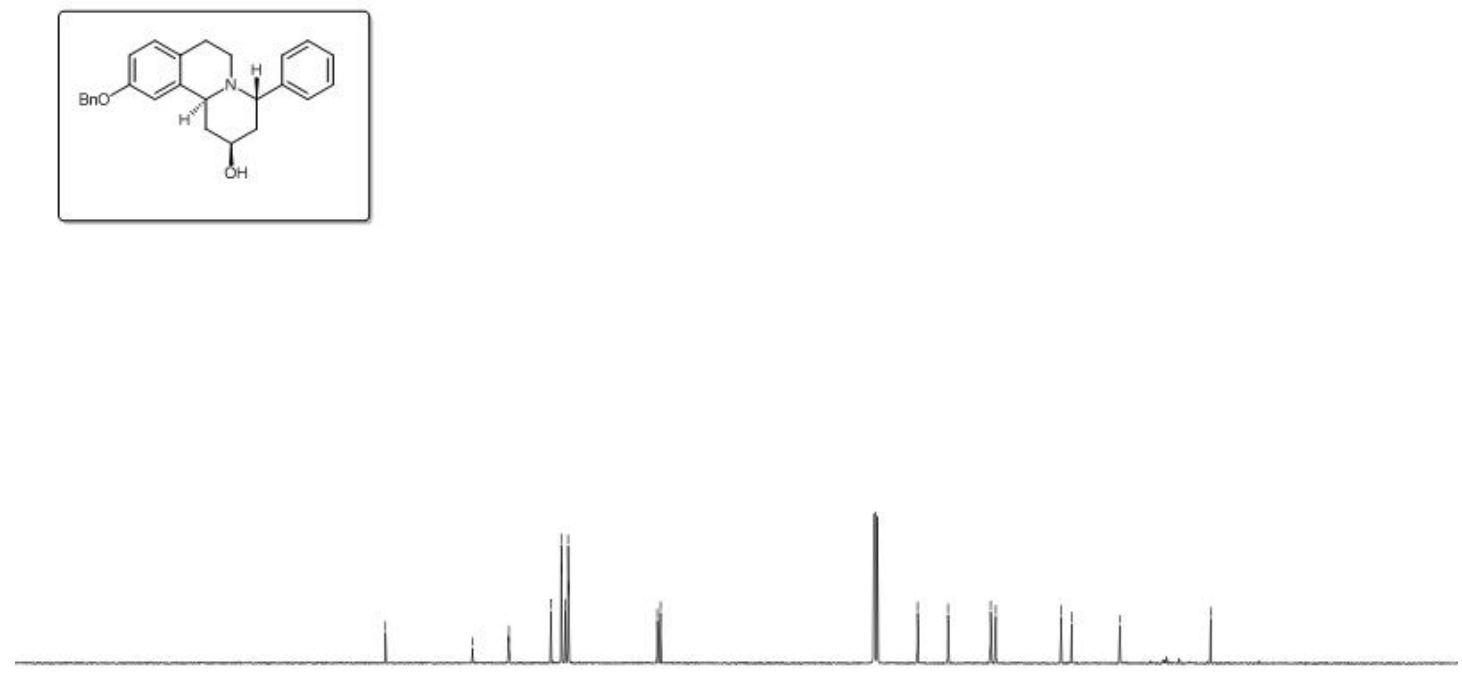

Figure S97. ${ }^{13} \mathrm{C}$ NMR spectrum of 3 . 


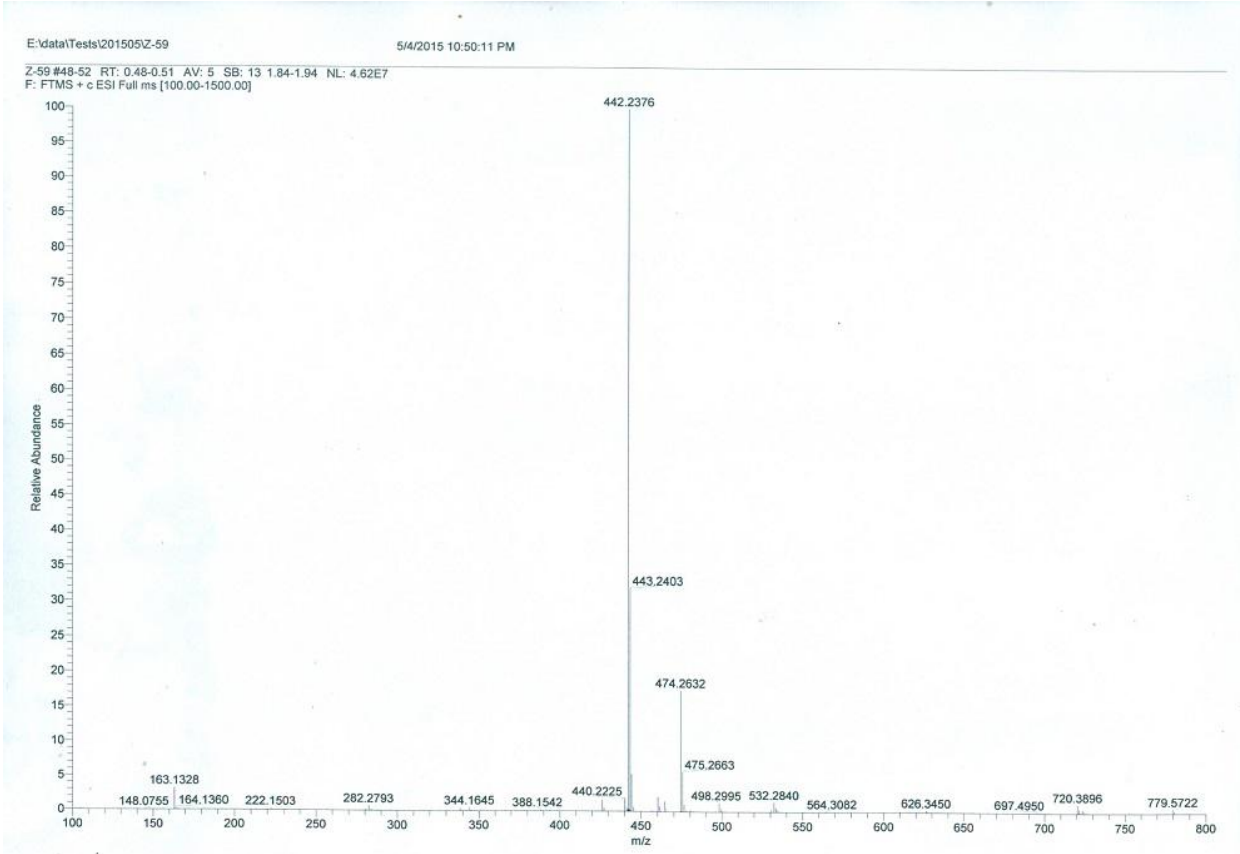

Figure S98. HRESIMS spectrum of SF1.
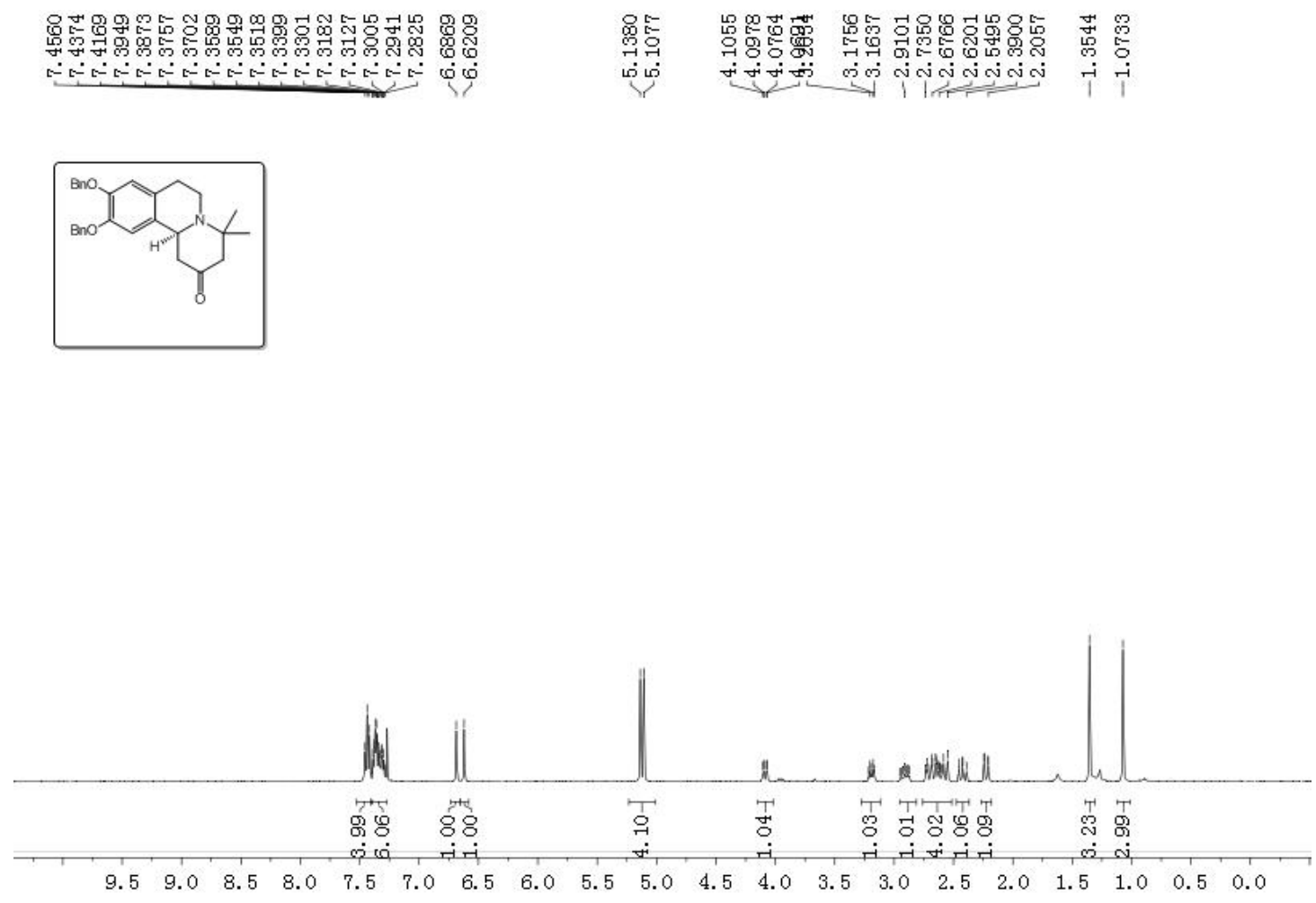

Figure S99. ${ }^{1} \mathrm{H}$ NMR spectrum of SF1. 


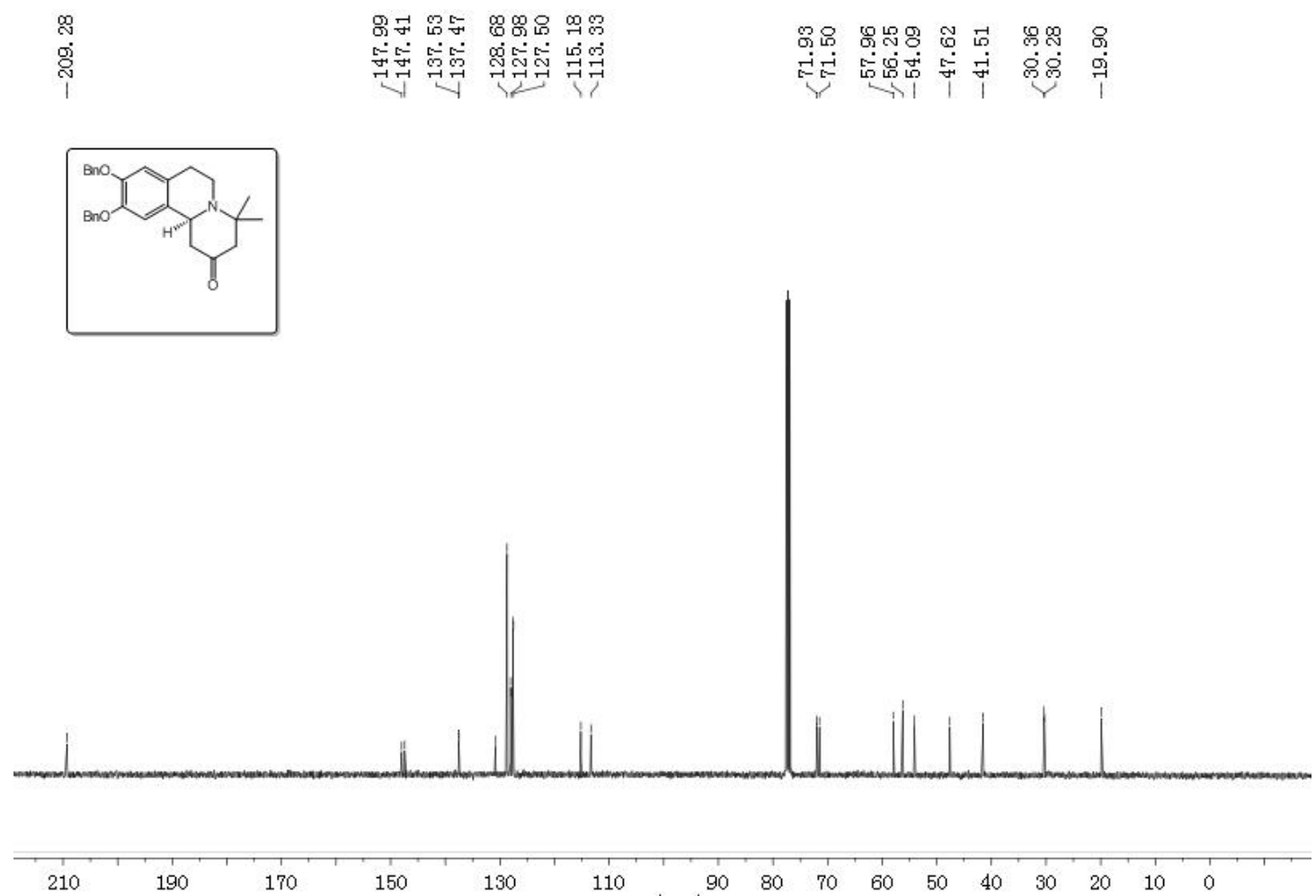

Figure S100. ${ }^{13} \mathrm{C}$ NMR spectrum of SF1.

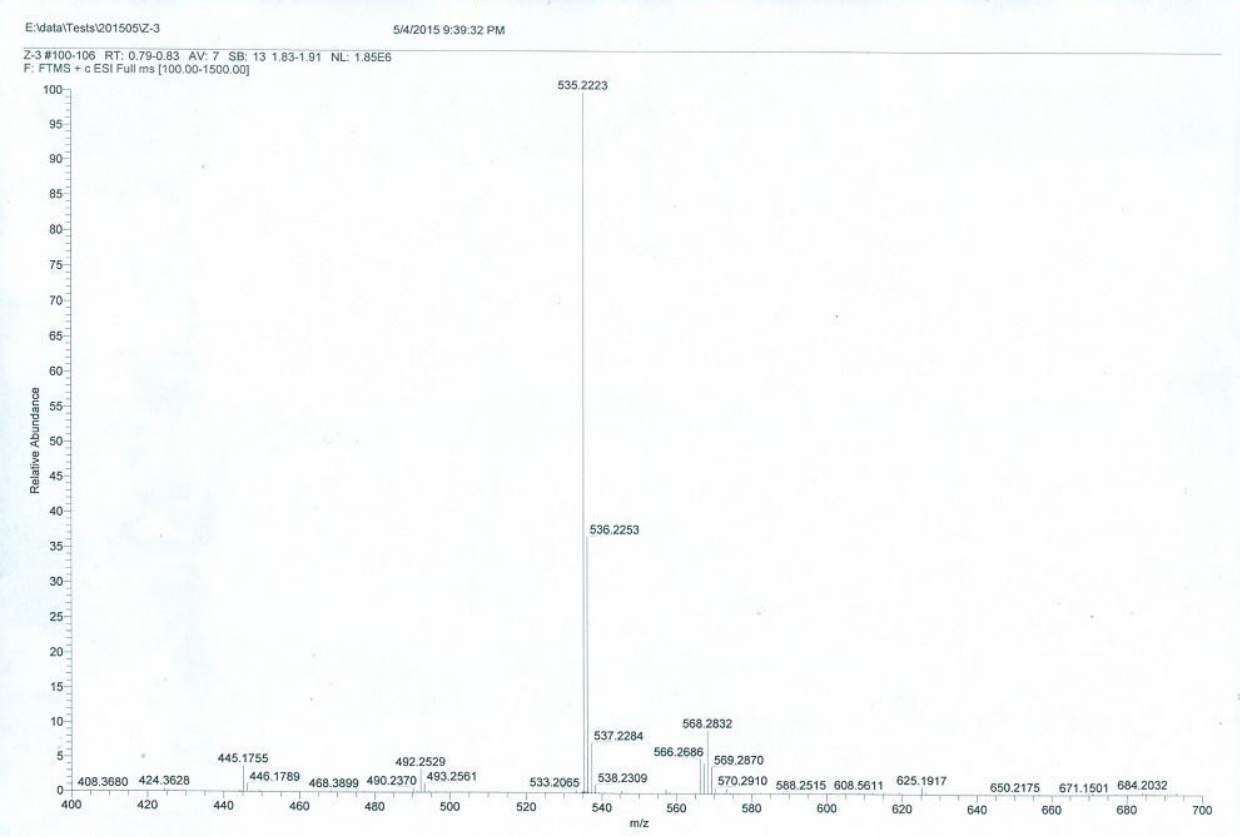

Figure S101. HRESIMS spectrum of SF2. 

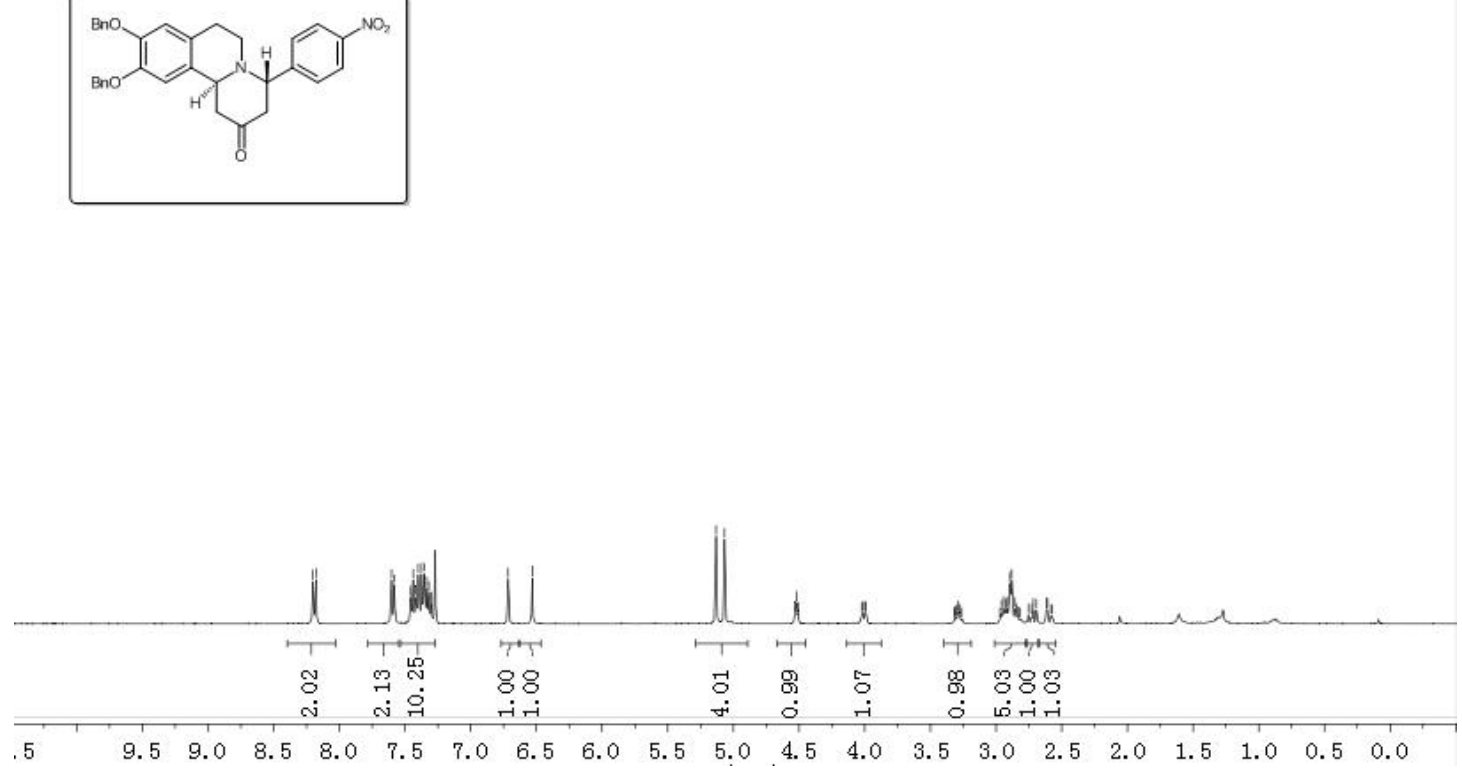

Figure S102. ${ }^{1} \mathrm{H}$ NMR spectrum of SF2

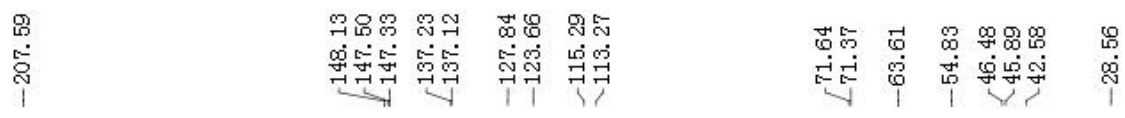
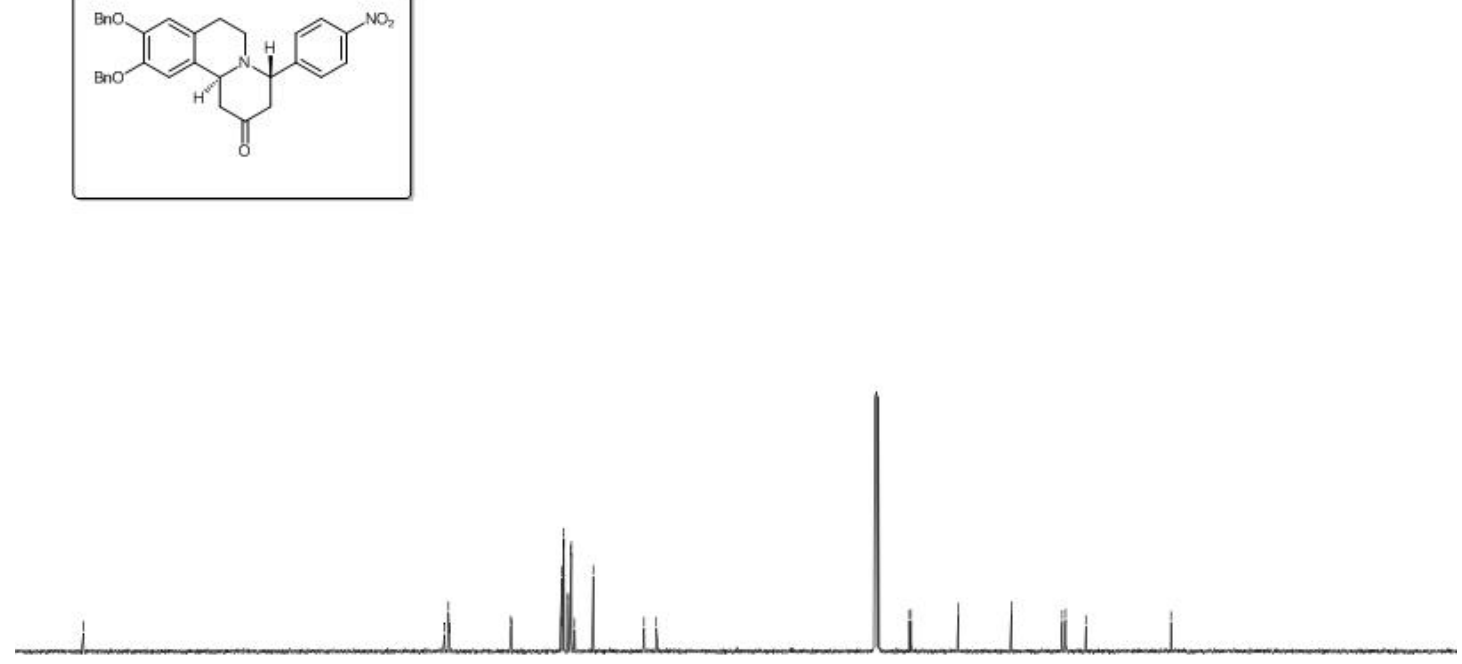

$210 \quad 190 \quad 170$

Figure S103. ${ }^{13} \mathrm{C}$ NMR spectrum of SF2. 


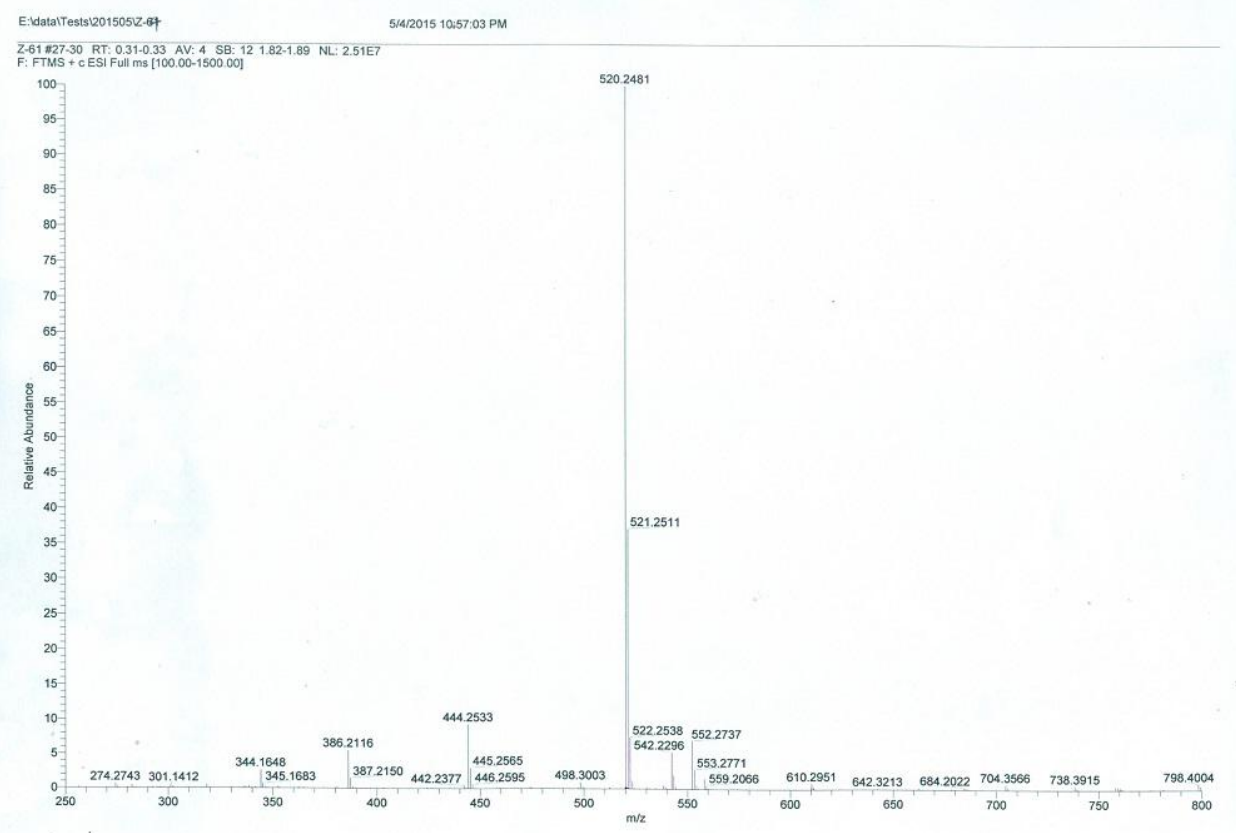

Figure S104. HRESIMS spectrum of SF3.
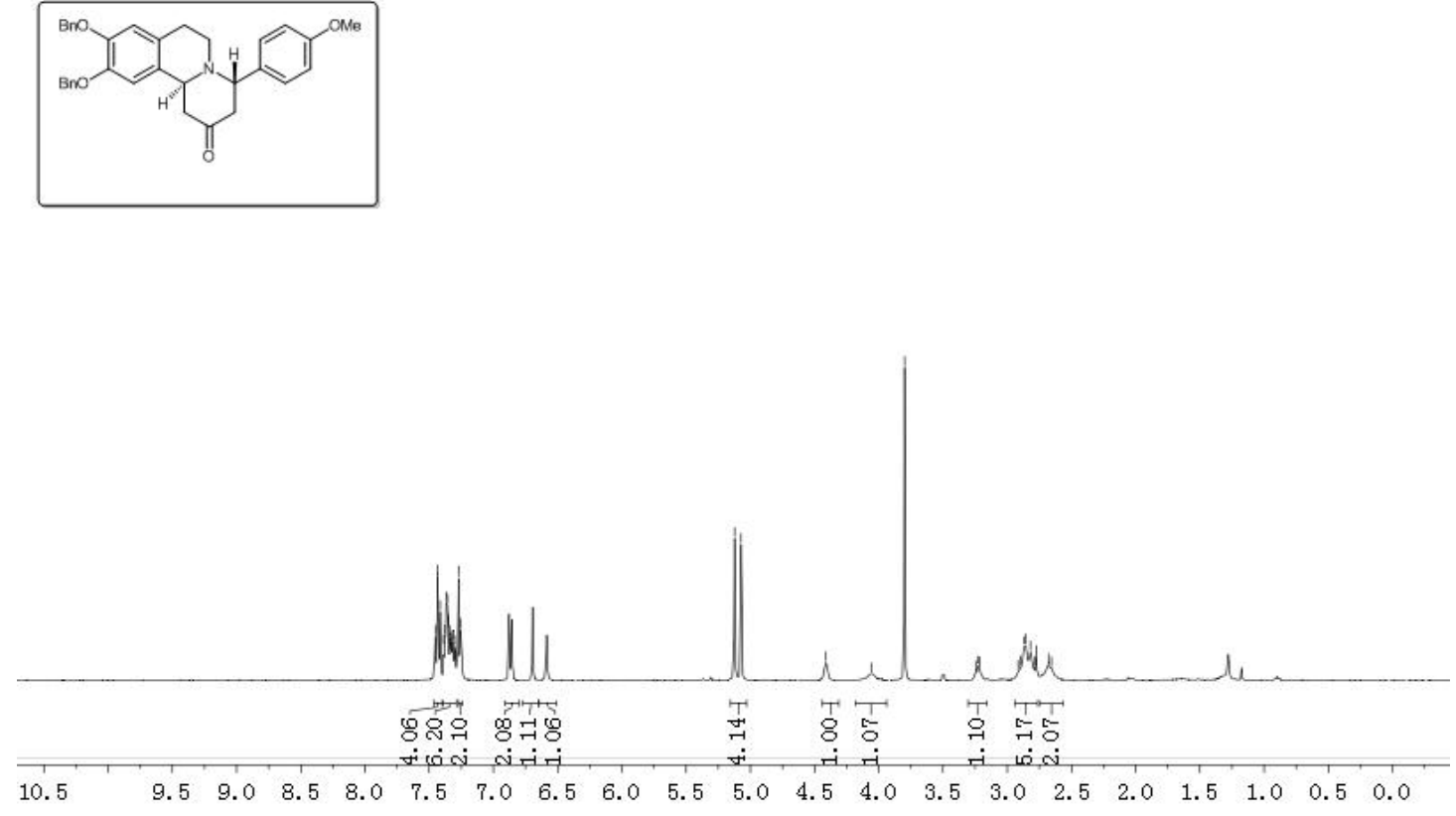

Figure S105. ${ }^{1} \mathrm{H}$ NMR spectrum of SF3. 

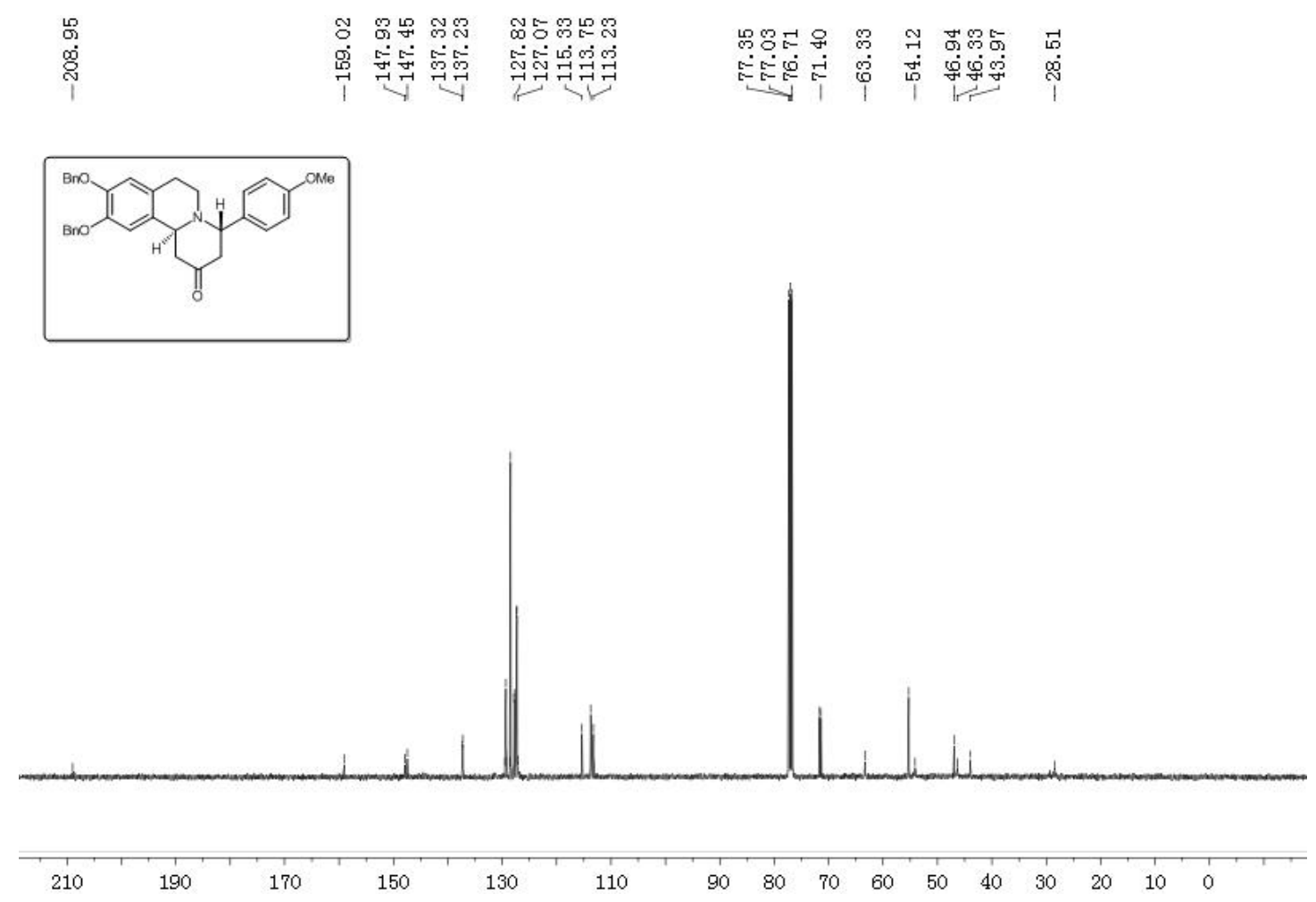

Figure S106. ${ }^{13} \mathrm{C}$ NMR spectrum of SF3.

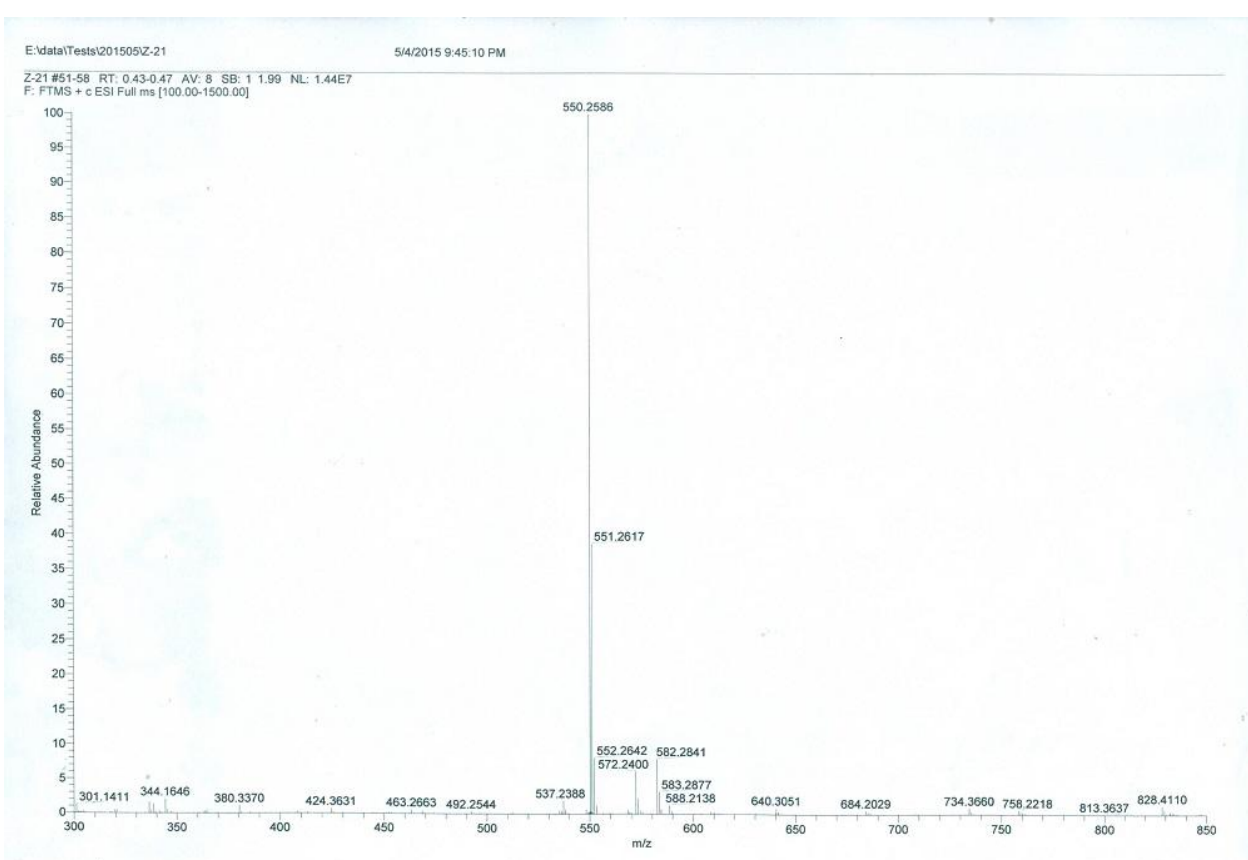

Figure S107. HRESIMS spectrum of SF4. 

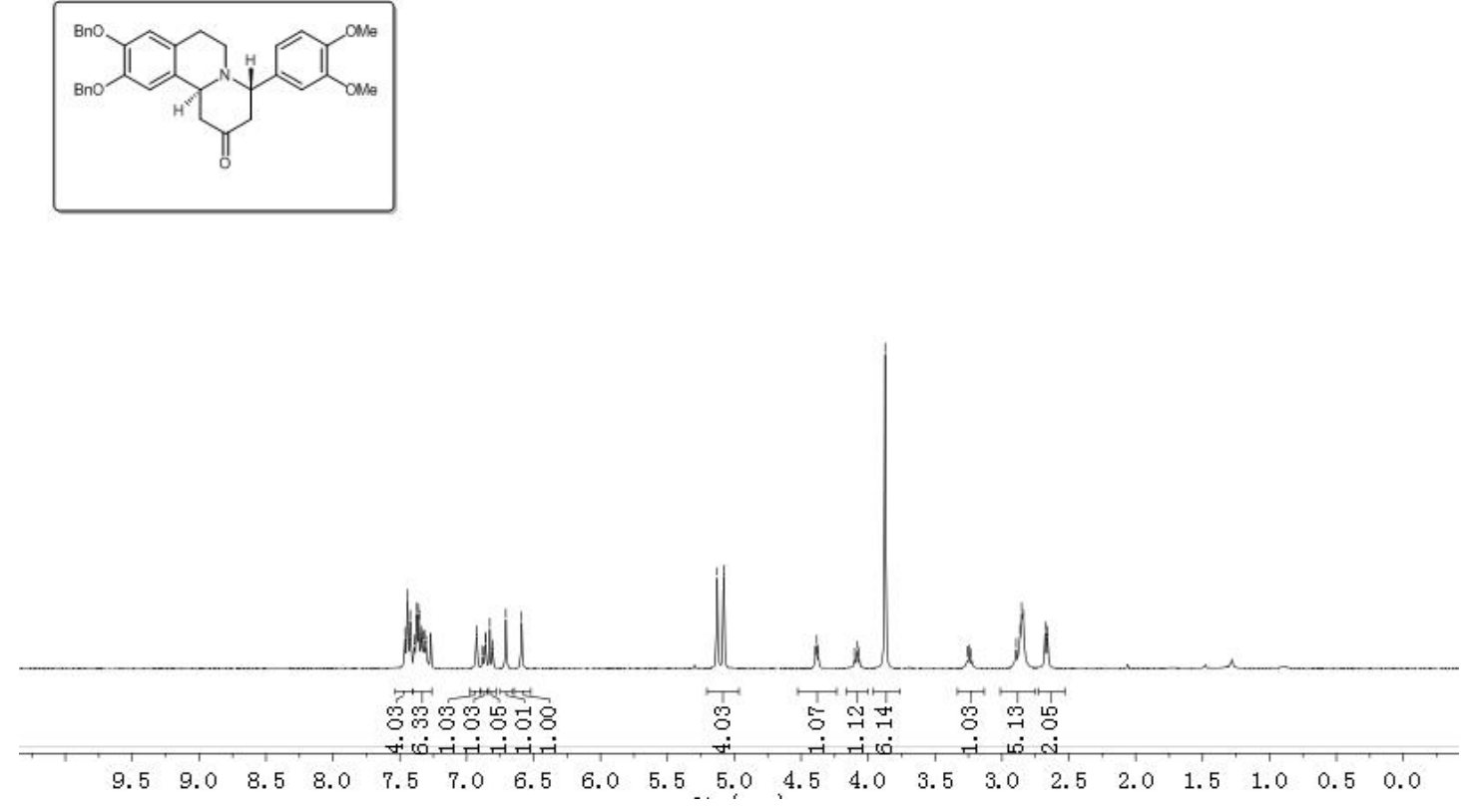

Figure S108. ${ }^{1} \mathrm{H}$ NMR spectrum of SF4.

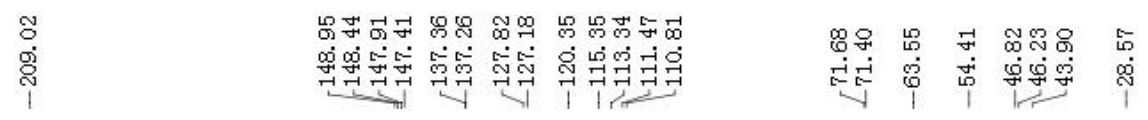
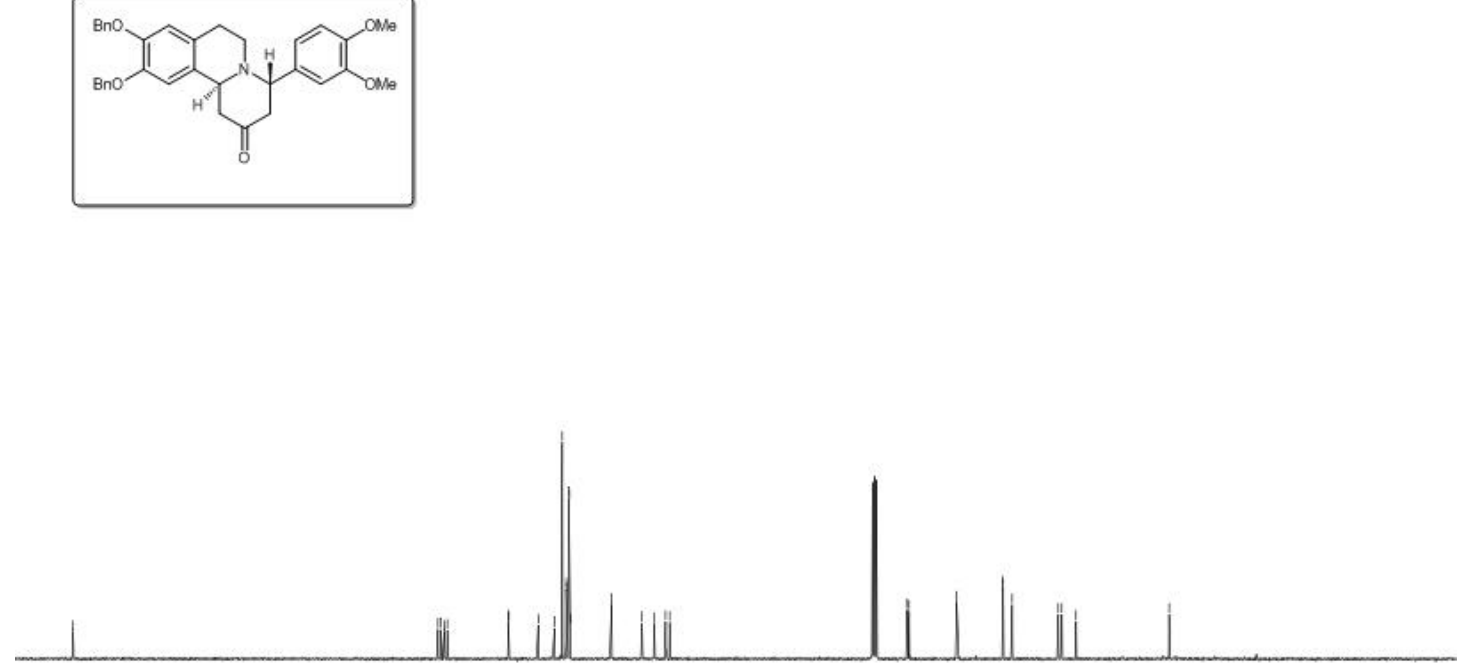

Figure S109. ${ }^{13} \mathrm{C}$ NMR spectrum of SF4. 


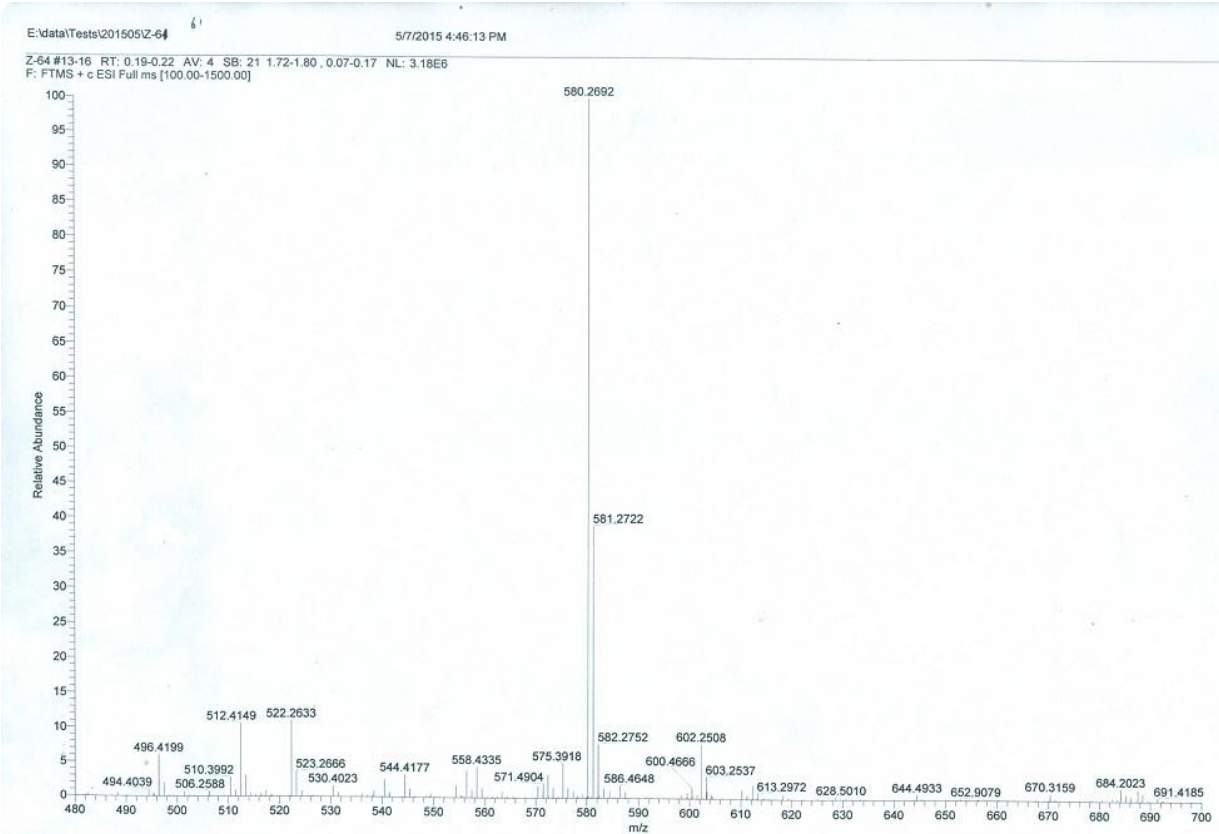

Figure S110. HRESIMS spectrum of SF5.

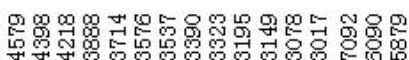

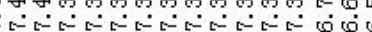

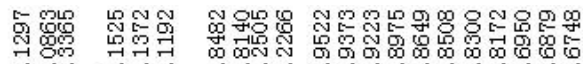

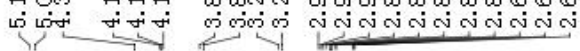
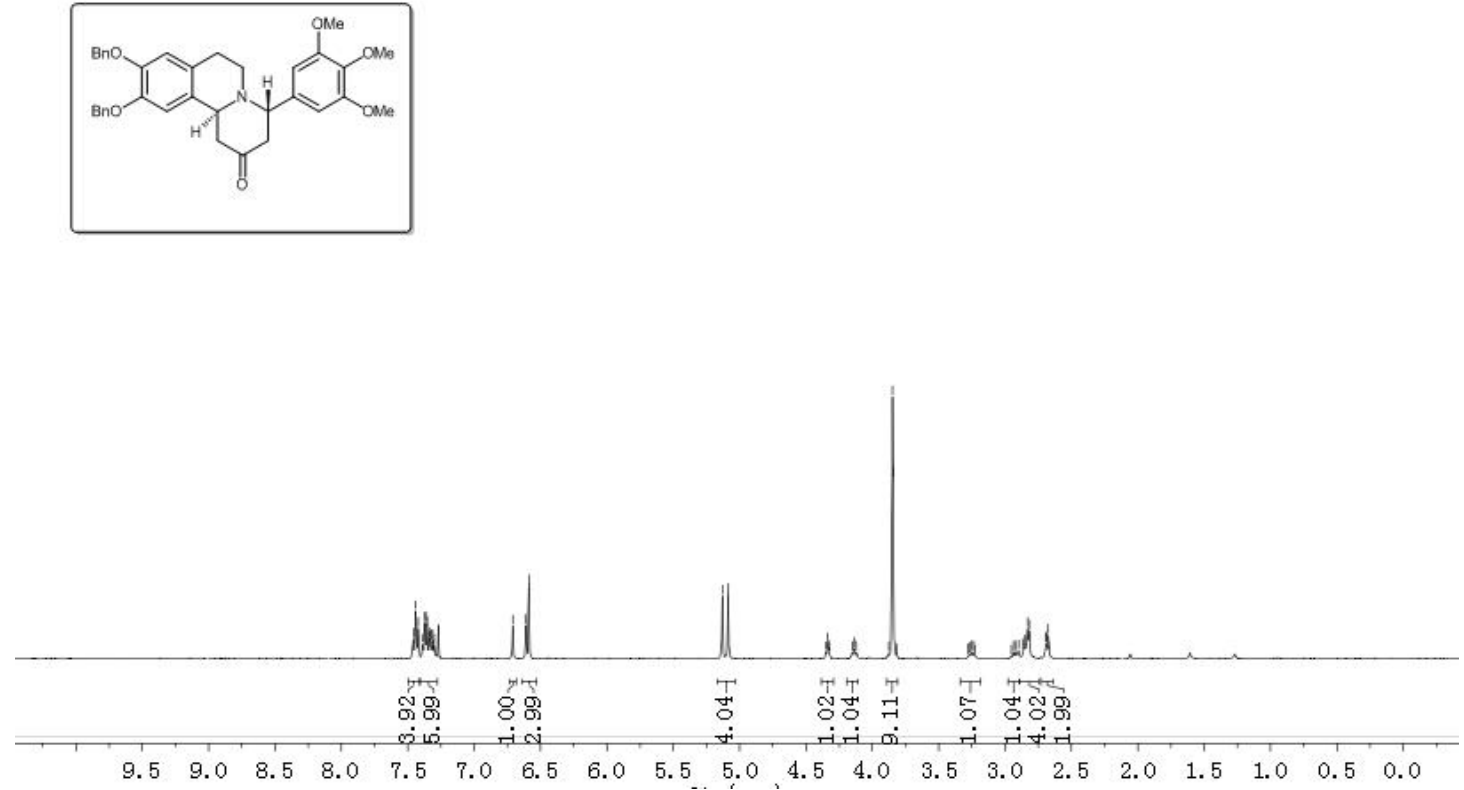

Figure S111. ${ }^{1} \mathrm{H}$ NMR spectrum of SF5. 


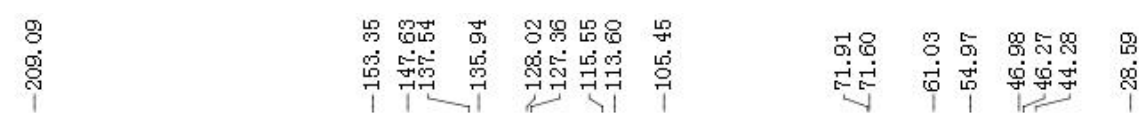
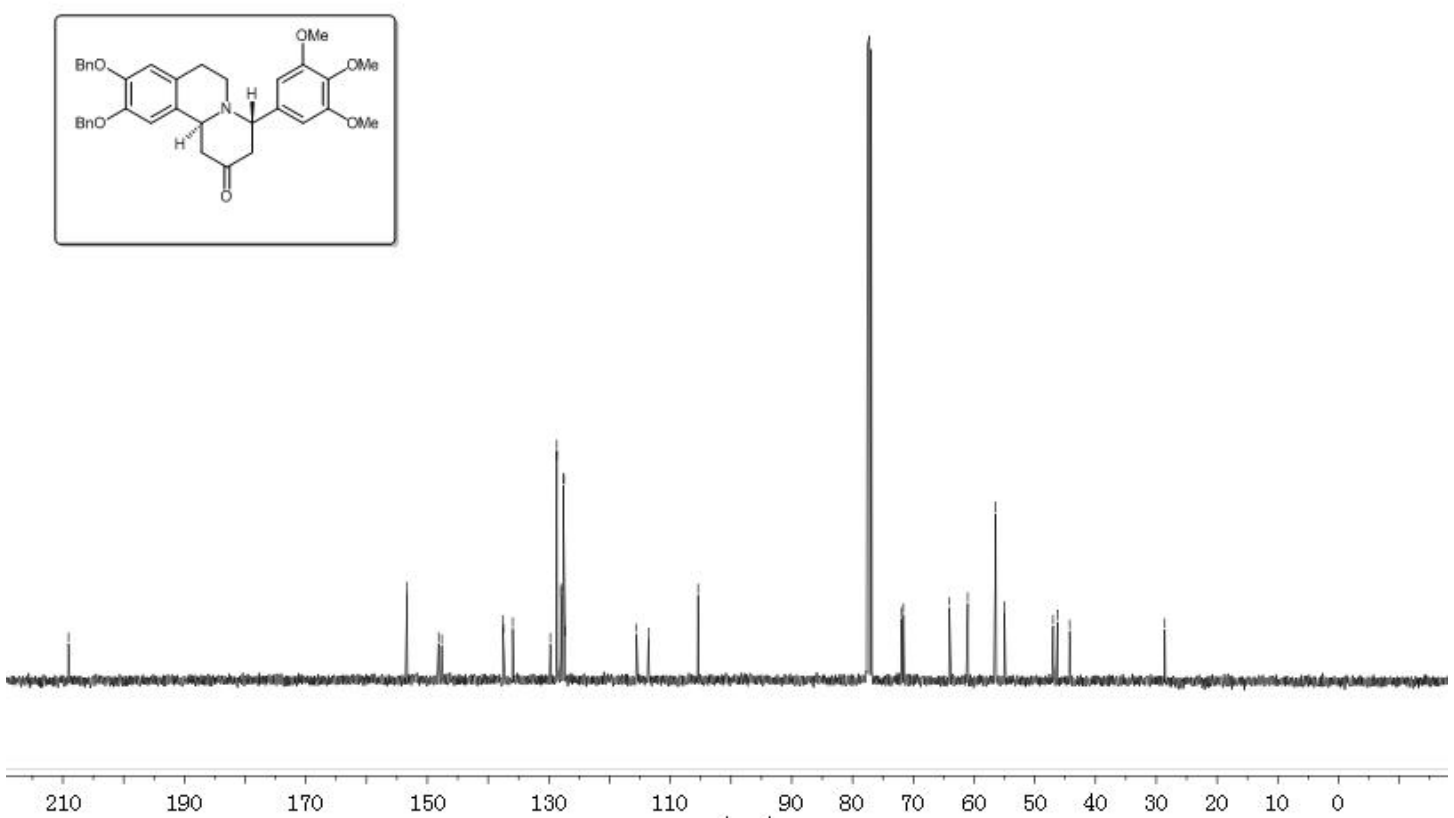

Figure S112. ${ }^{13} \mathrm{C}$ NMR spectrum of SF5.

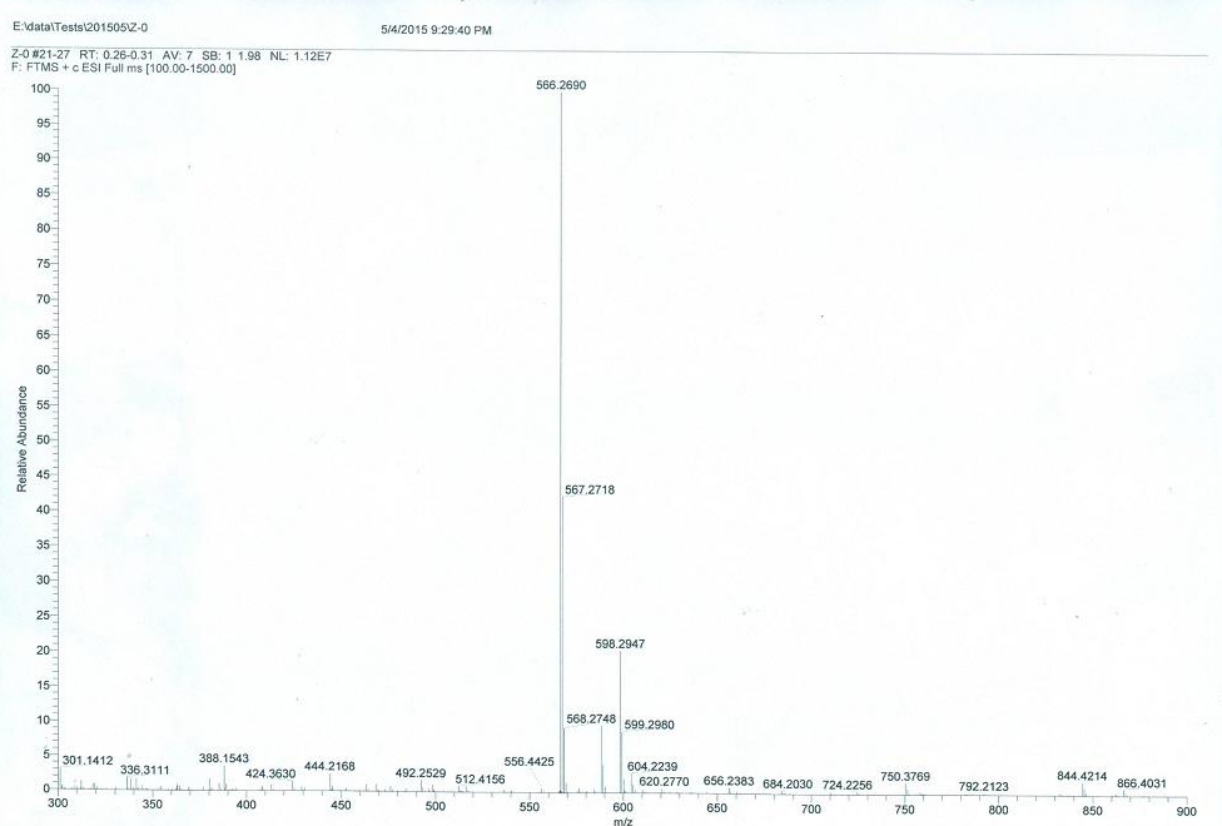

Figure S113. HRESIMS spectrum of SF6. 

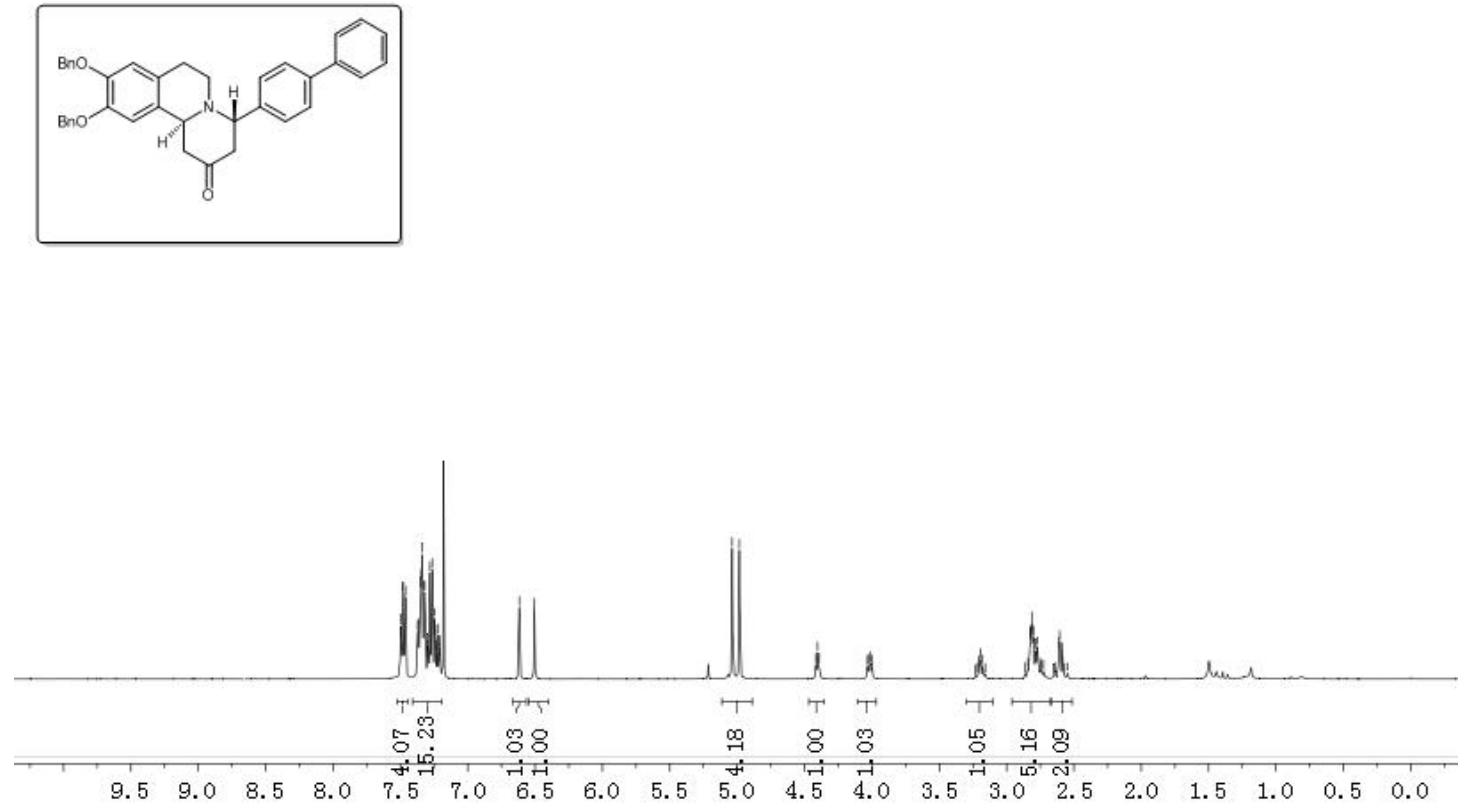

Figure S114. ${ }^{1} \mathrm{H}$ NMR spectrum of SF6.

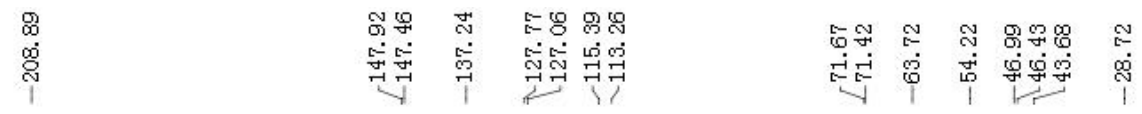
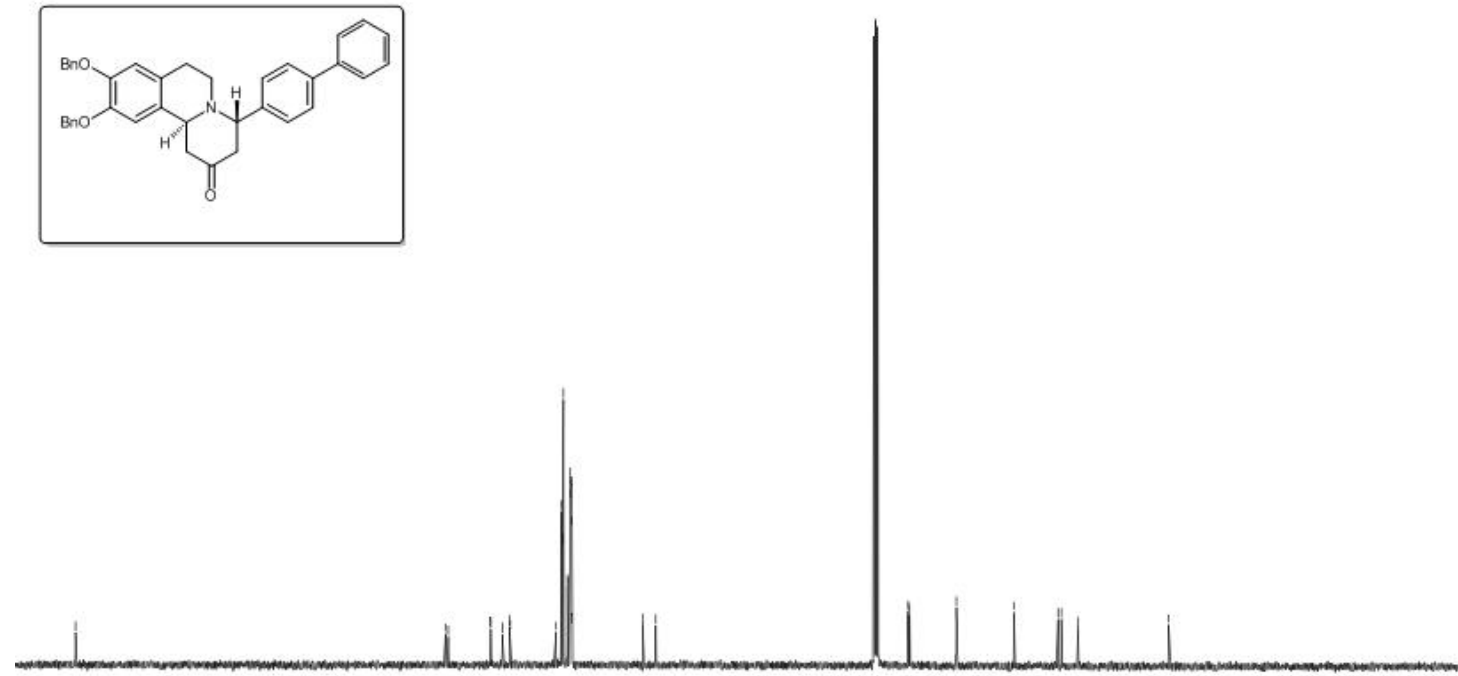

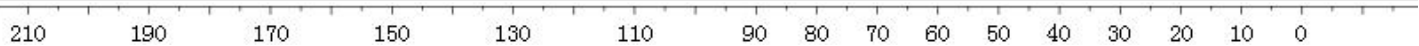

Figure S115. ${ }^{13} \mathrm{C}$ NMR spectrum of SF6. 


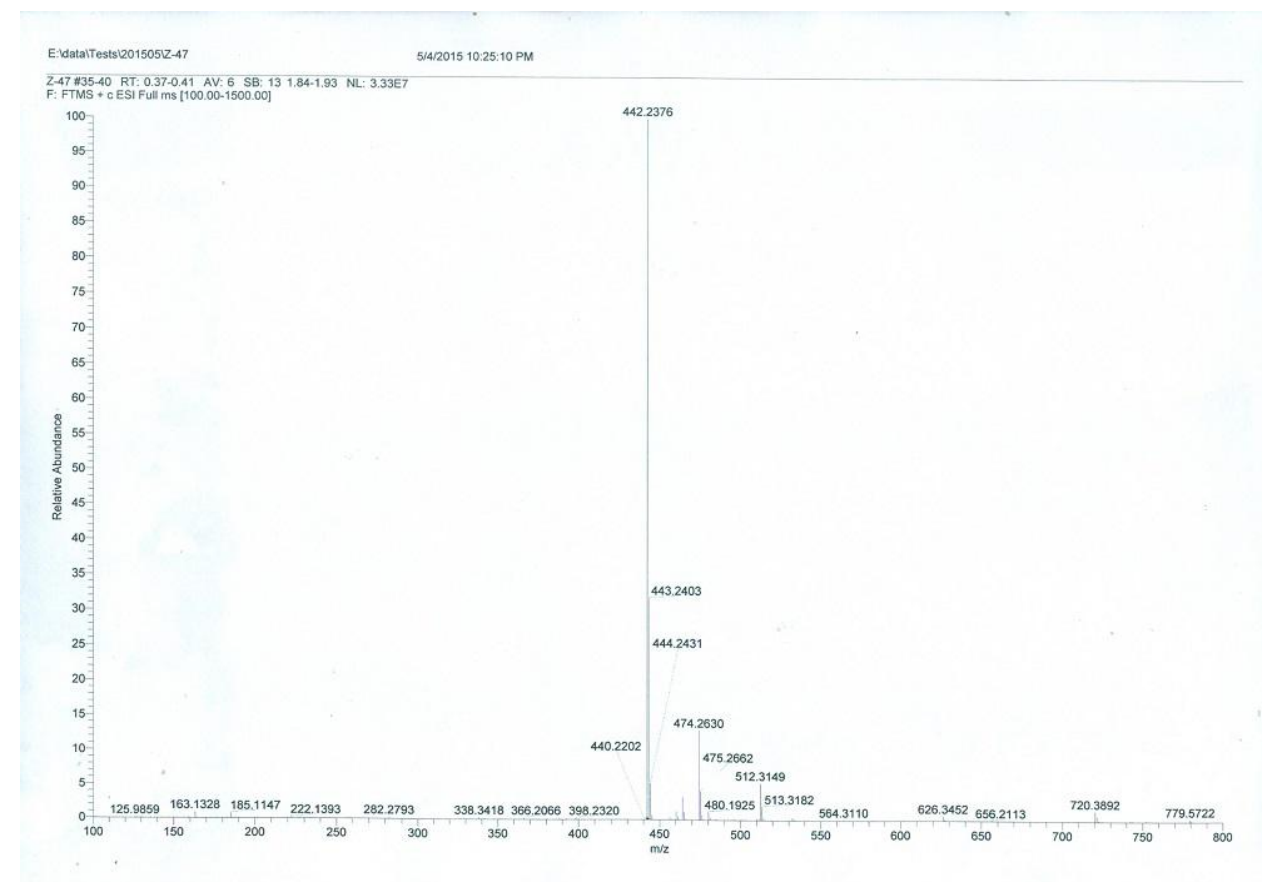

Figure S116. HRESIMS spectrum of SF7.

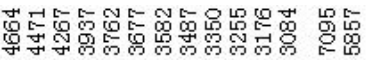

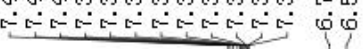

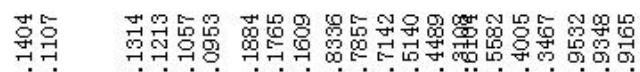

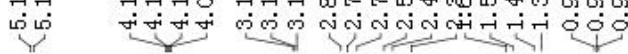
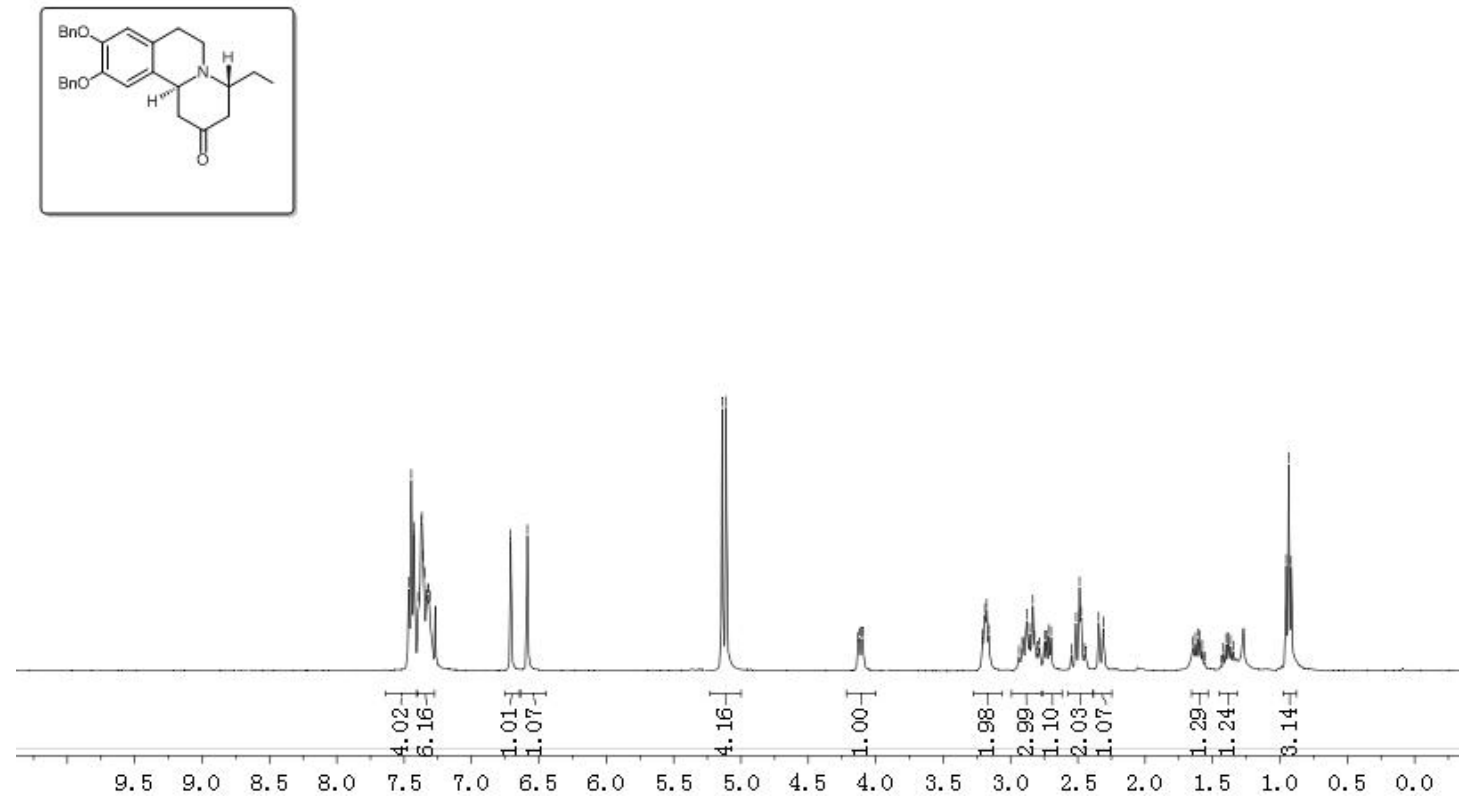

Figure S117. ${ }^{1} \mathrm{H}$ NMR spectrum of SF7. 


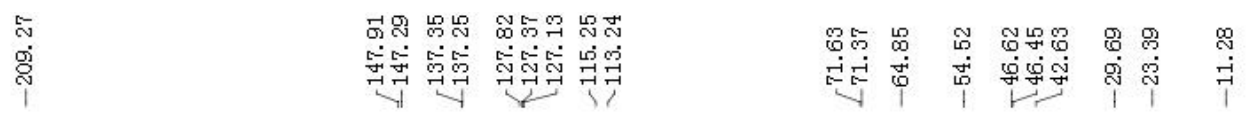
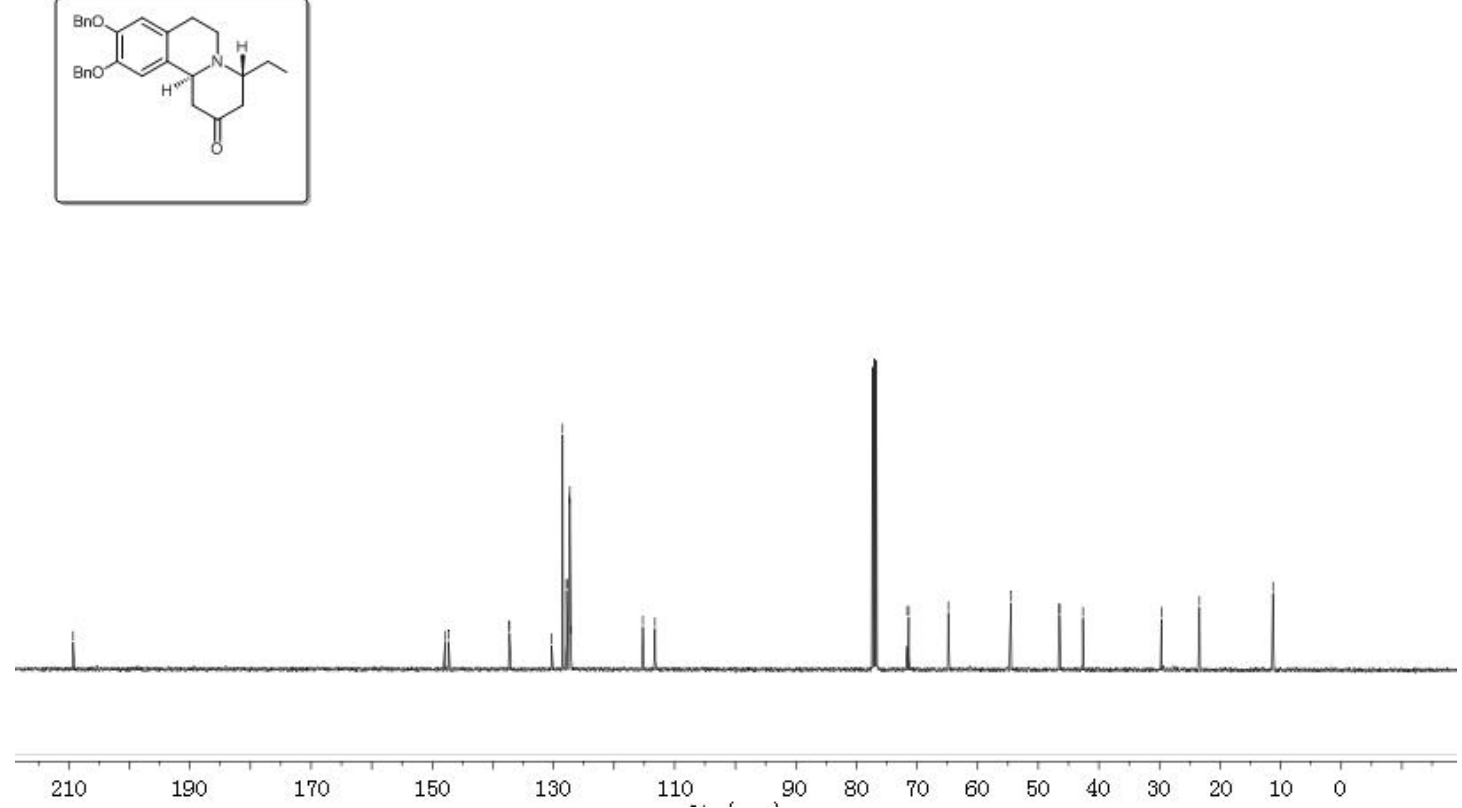

Figure S118. ${ }^{13} \mathrm{C}$ NMR spectrum of SF7.

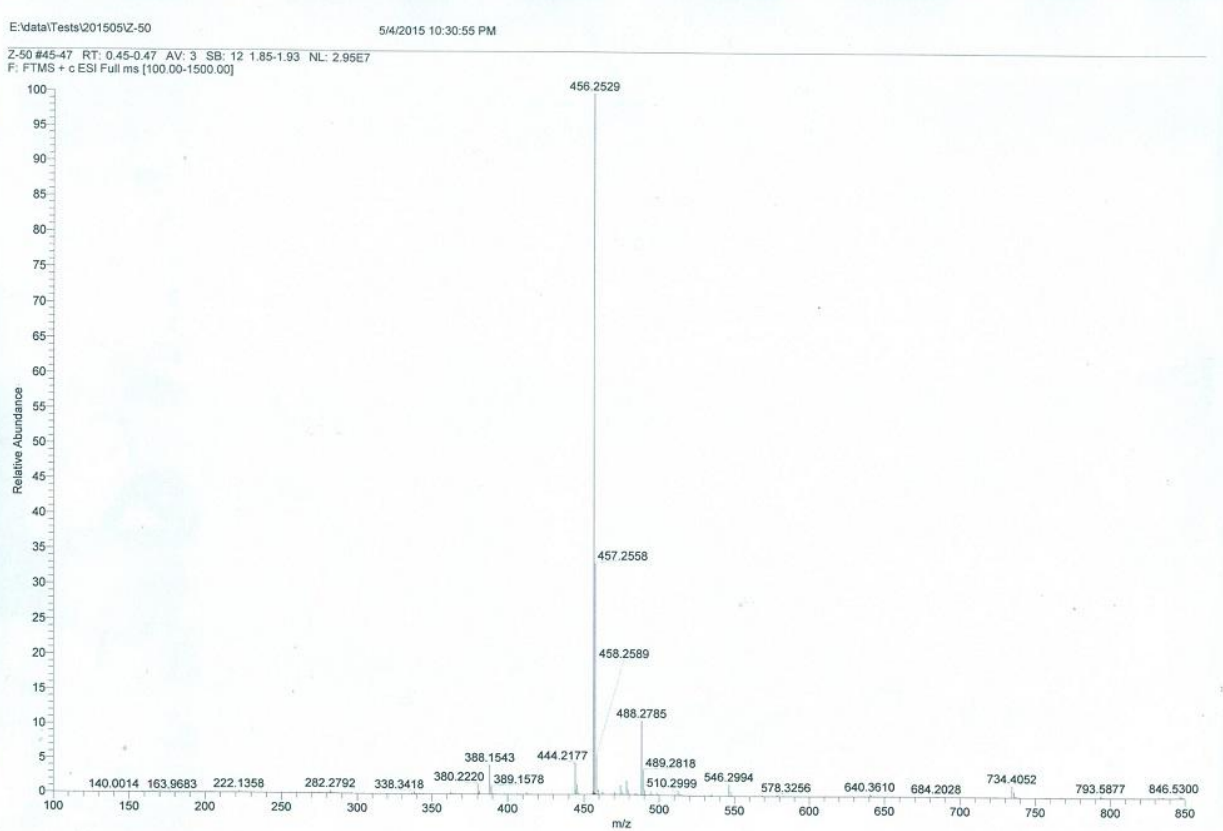

Figure S119. HRESIMS spectrum of SF8. 

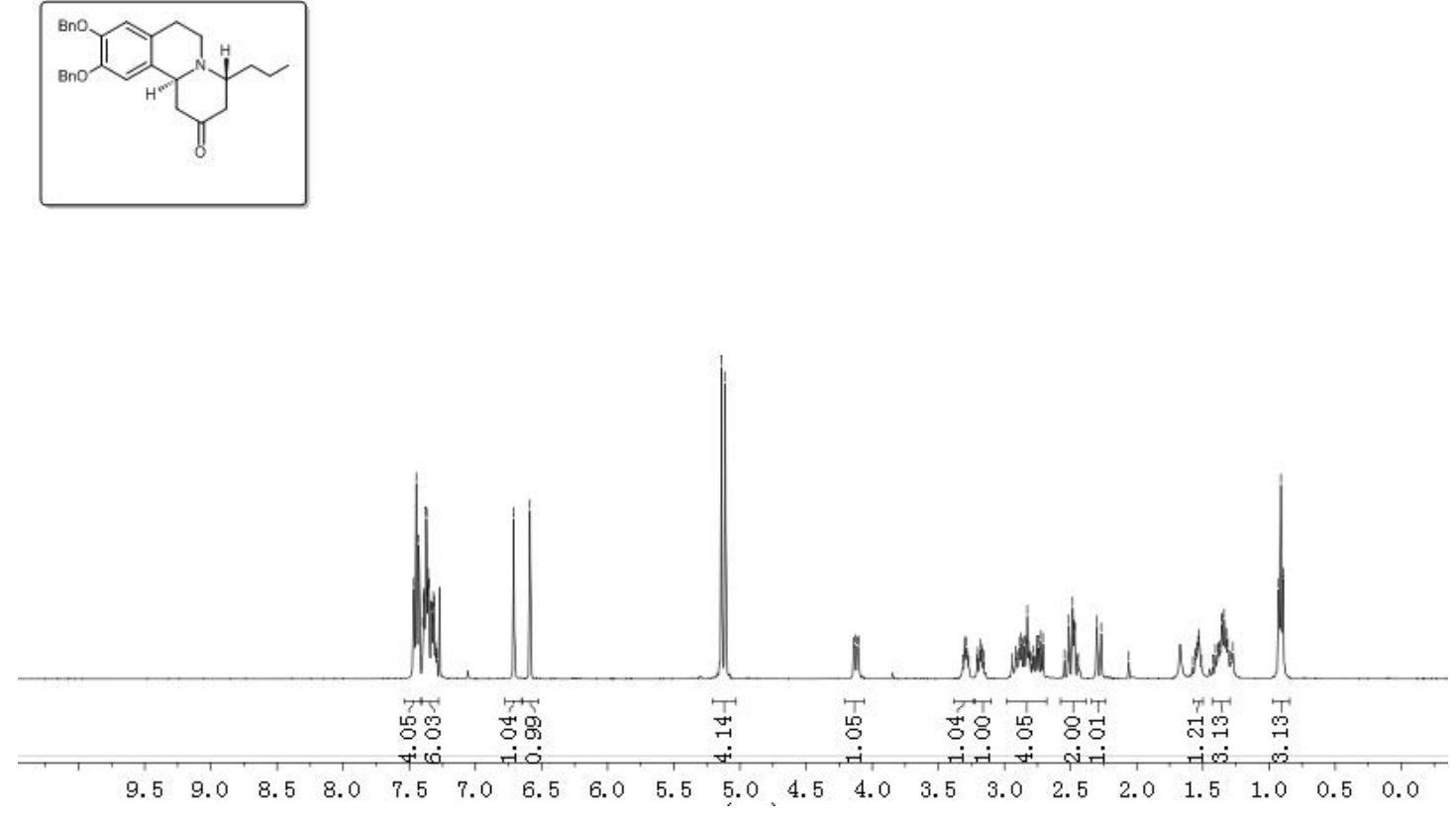

Figure S120. ${ }^{1} \mathrm{H}$ NMR spectrum of SF8
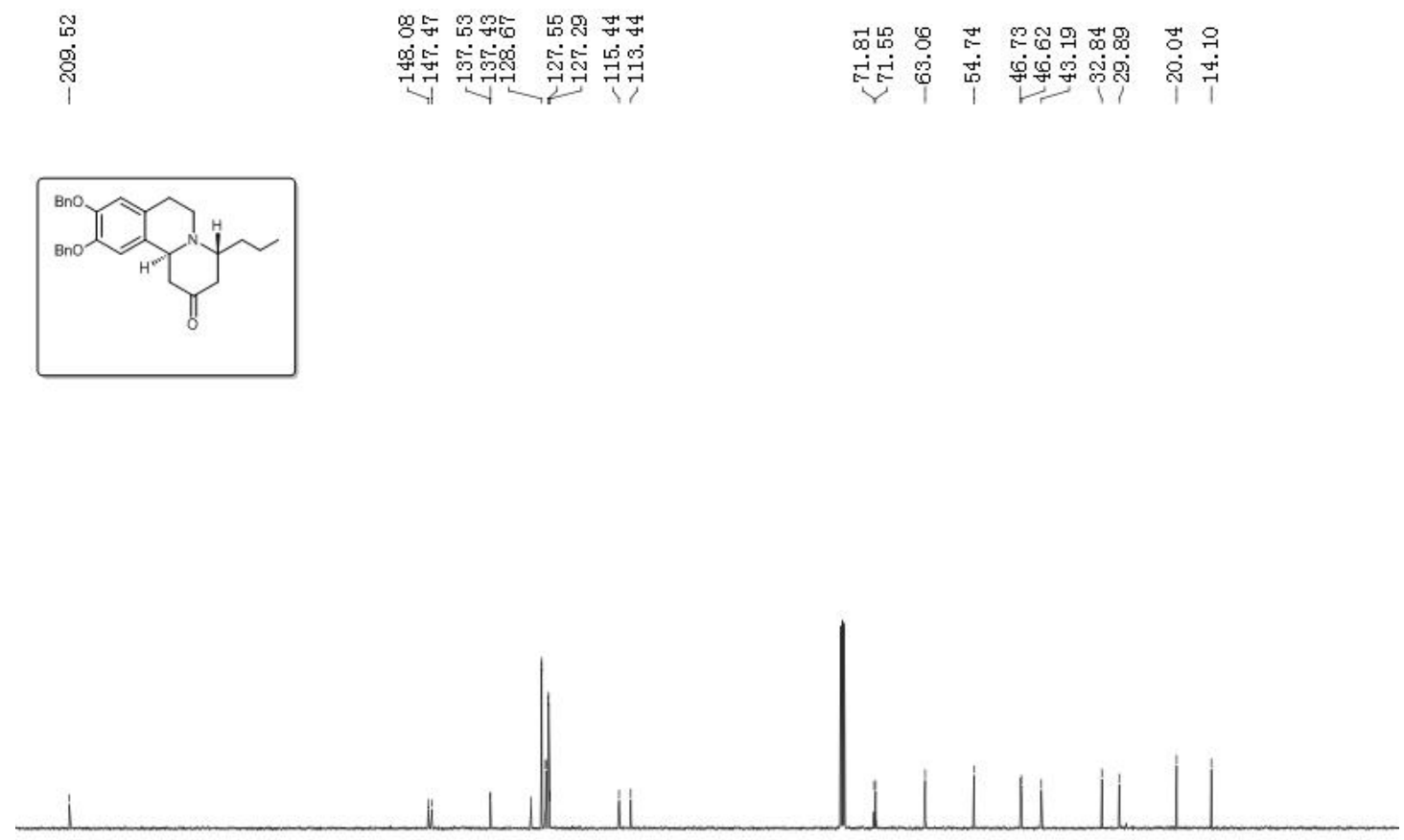

$210 \quad 190 \quad 170 \quad 15$

Figure S121. ${ }^{13} \mathrm{C}$ NMR spectrum of SF8. 


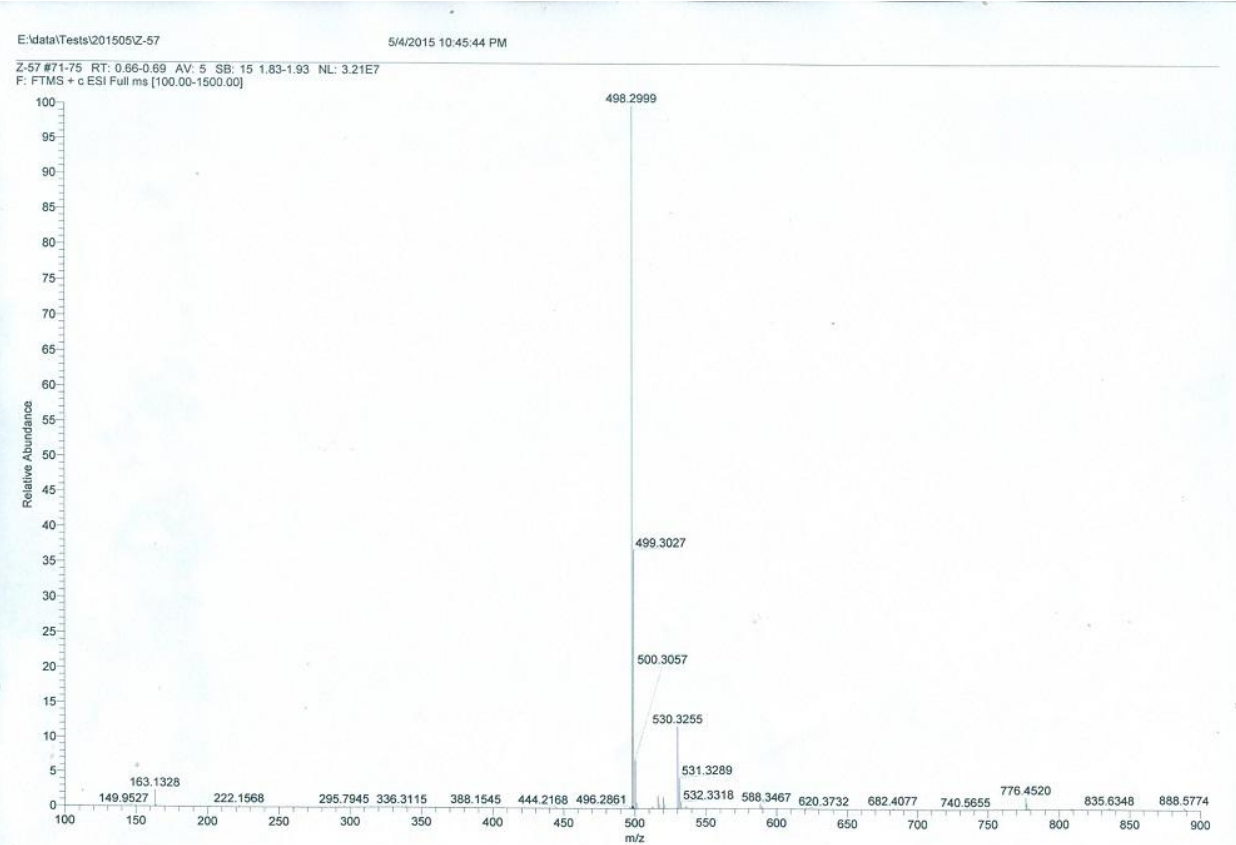

Figure S122. HRESIMS spectrum of SF9.
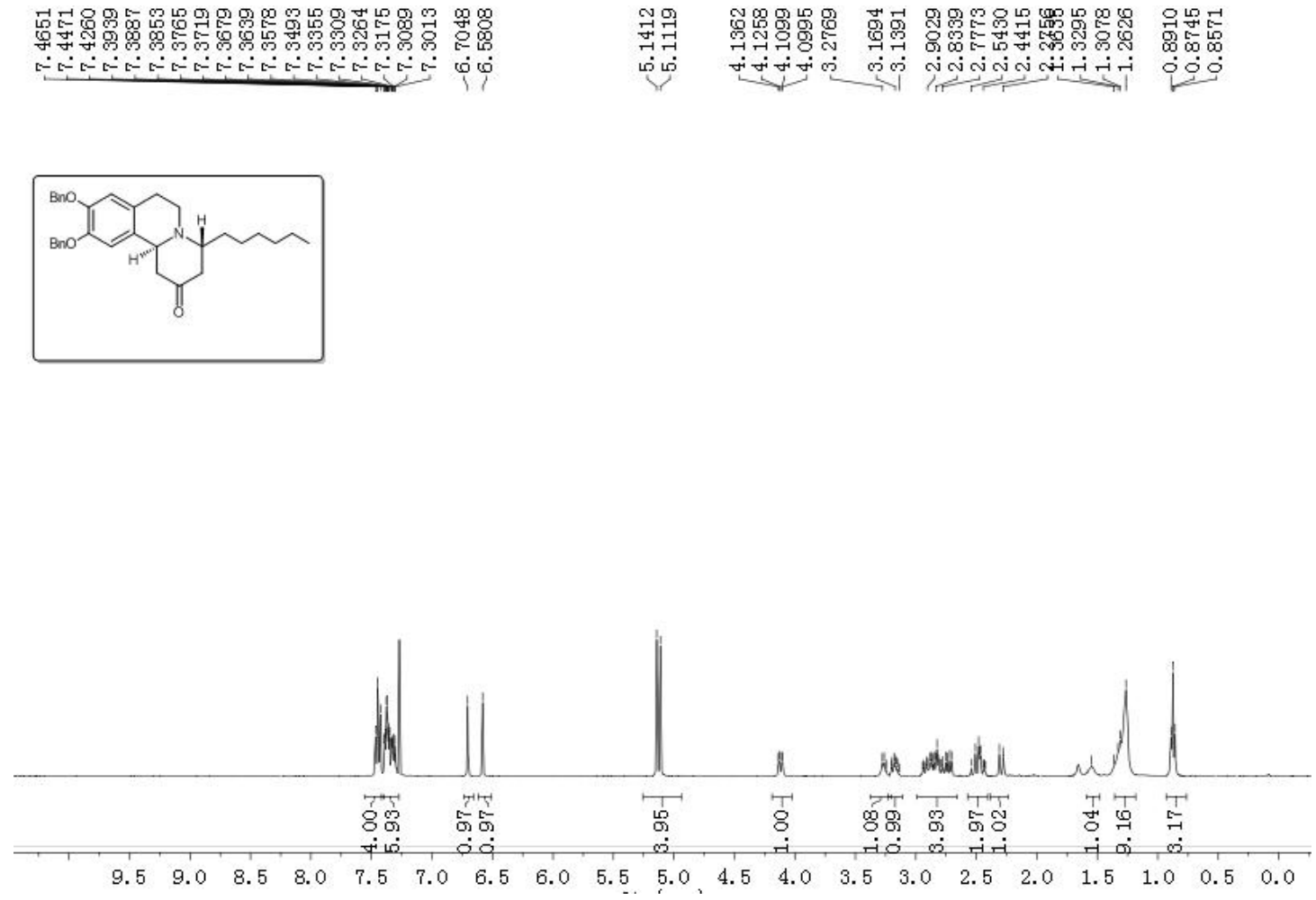

Figure S123. ${ }^{1} \mathrm{H}$ NMR spectrum of SF9. 


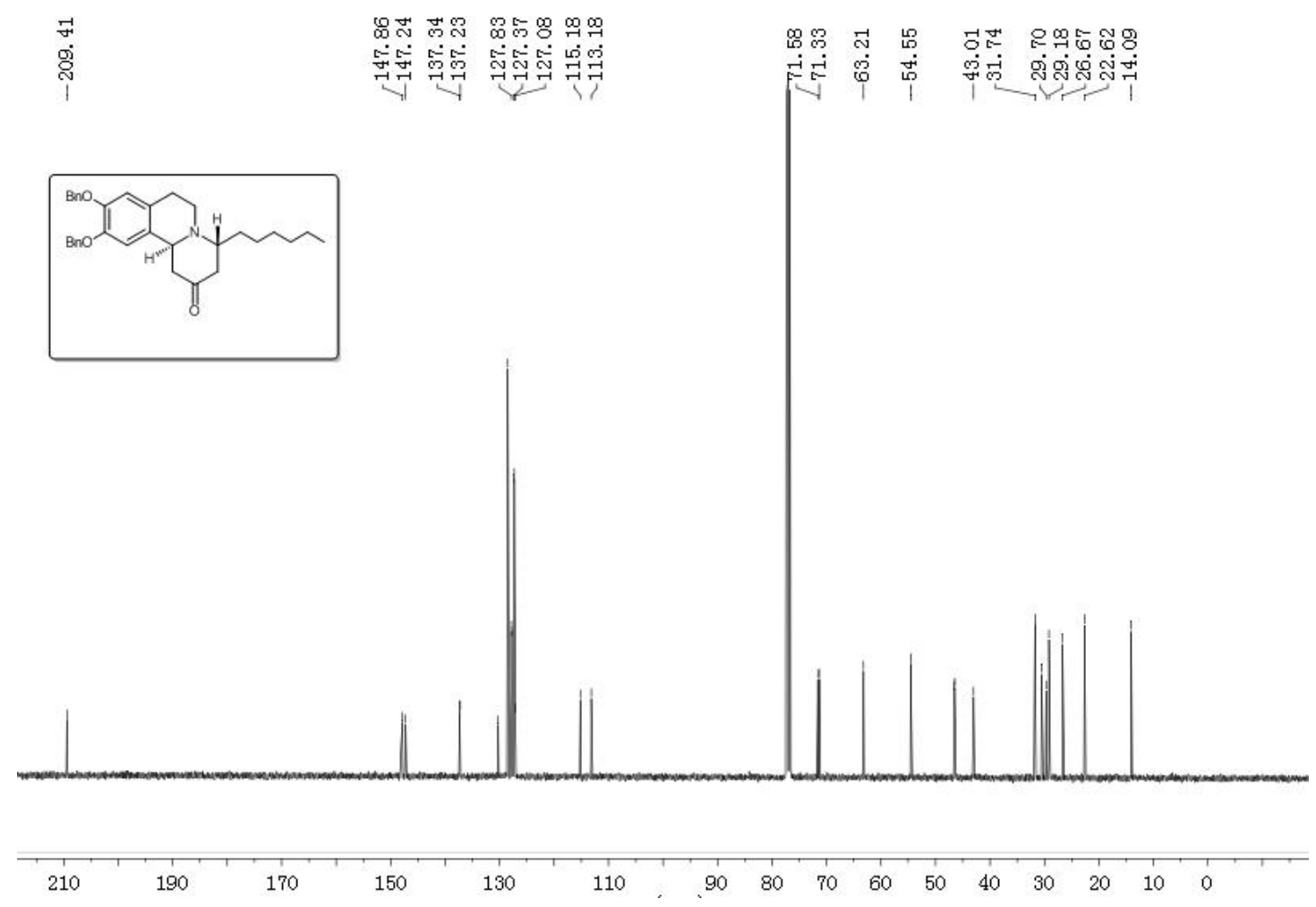

Figure S124. ${ }^{13} \mathrm{C}$ NMR spectrum of SF9.

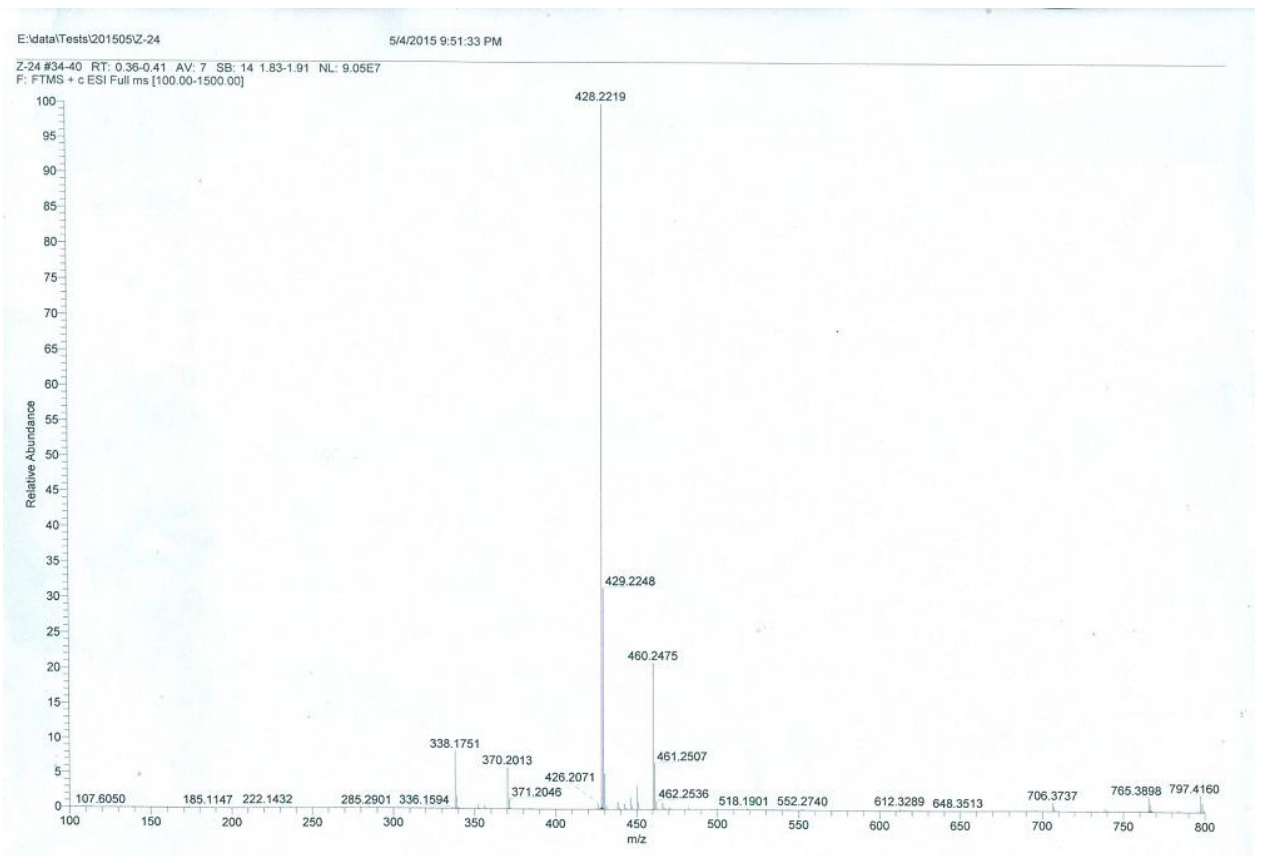

Figure S125. HRESIMS spectrum of $\mathbf{3 4}$. 

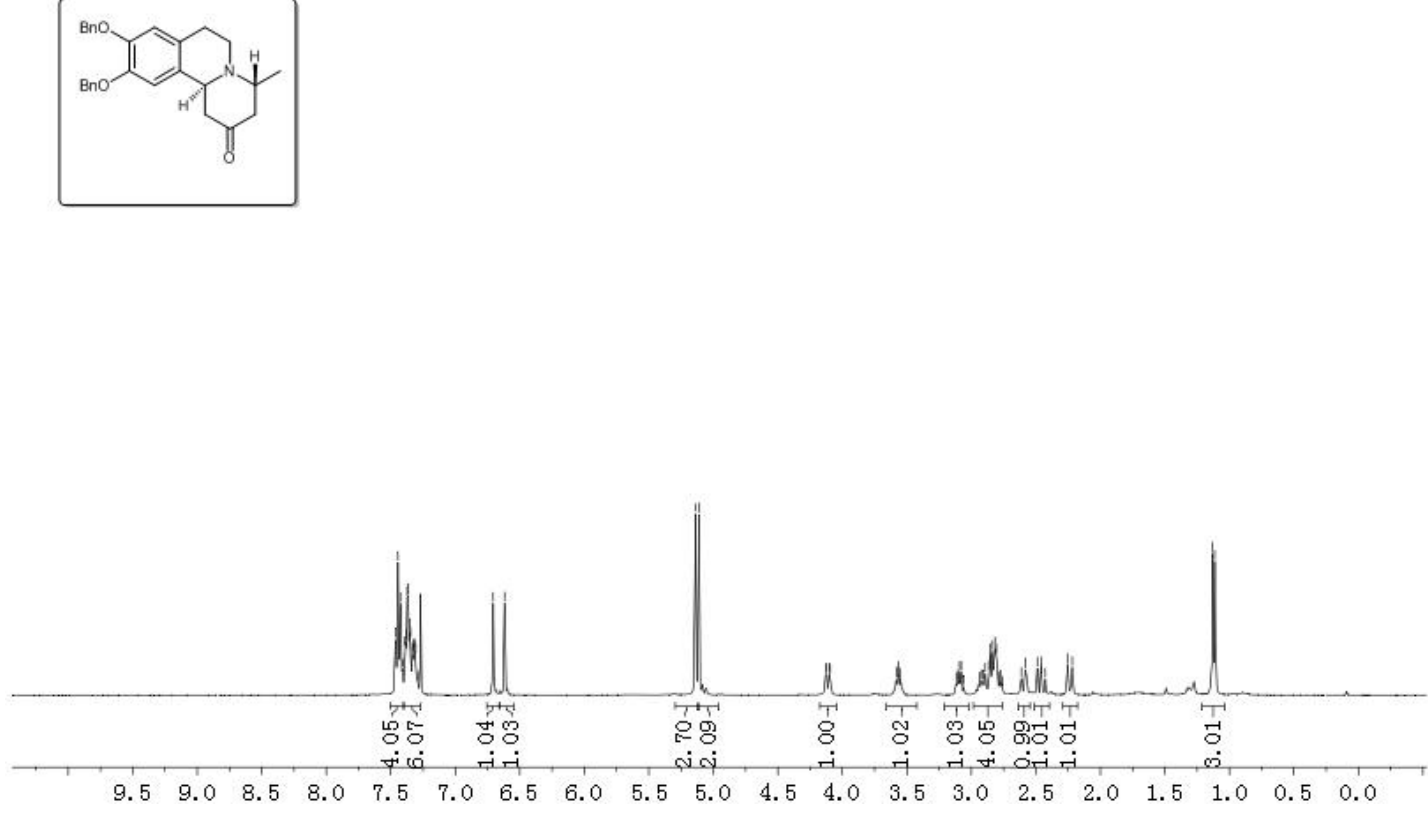

Figure S126. ${ }^{1} \mathrm{H}$ NMR spectrum of 34 .

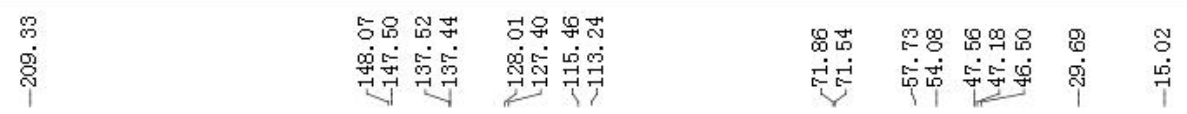<smiles>CC1(C)CC(=O)N2CCc3cc(O)c(O)cc3[C@H]2C1</smiles>
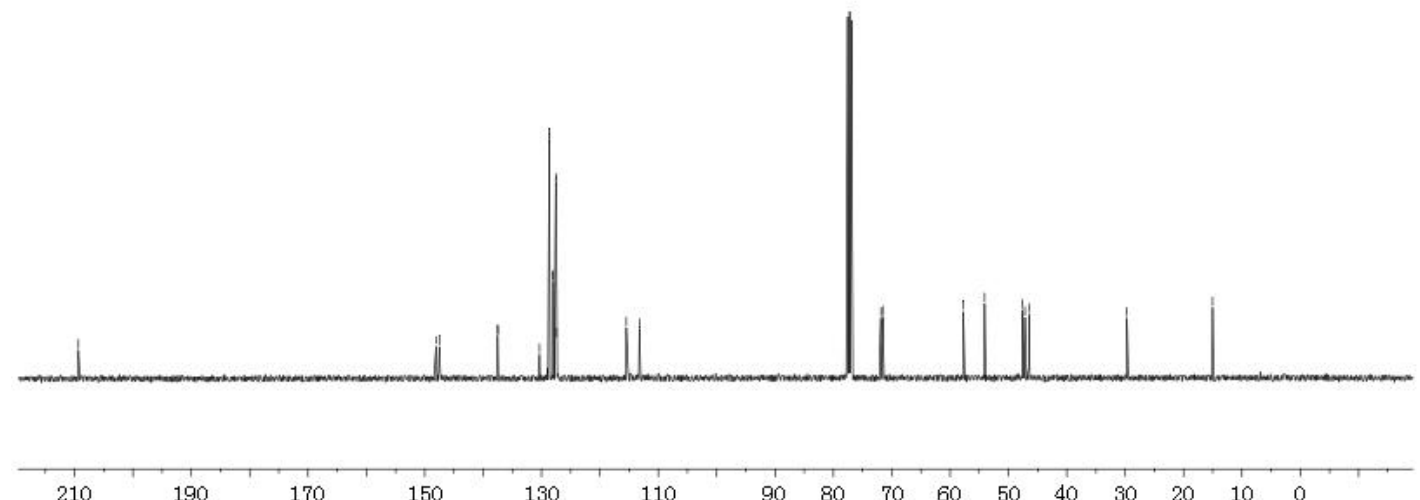

190

Figure S127. ${ }^{13} \mathrm{C}$ NMR spectrum of 34 . 


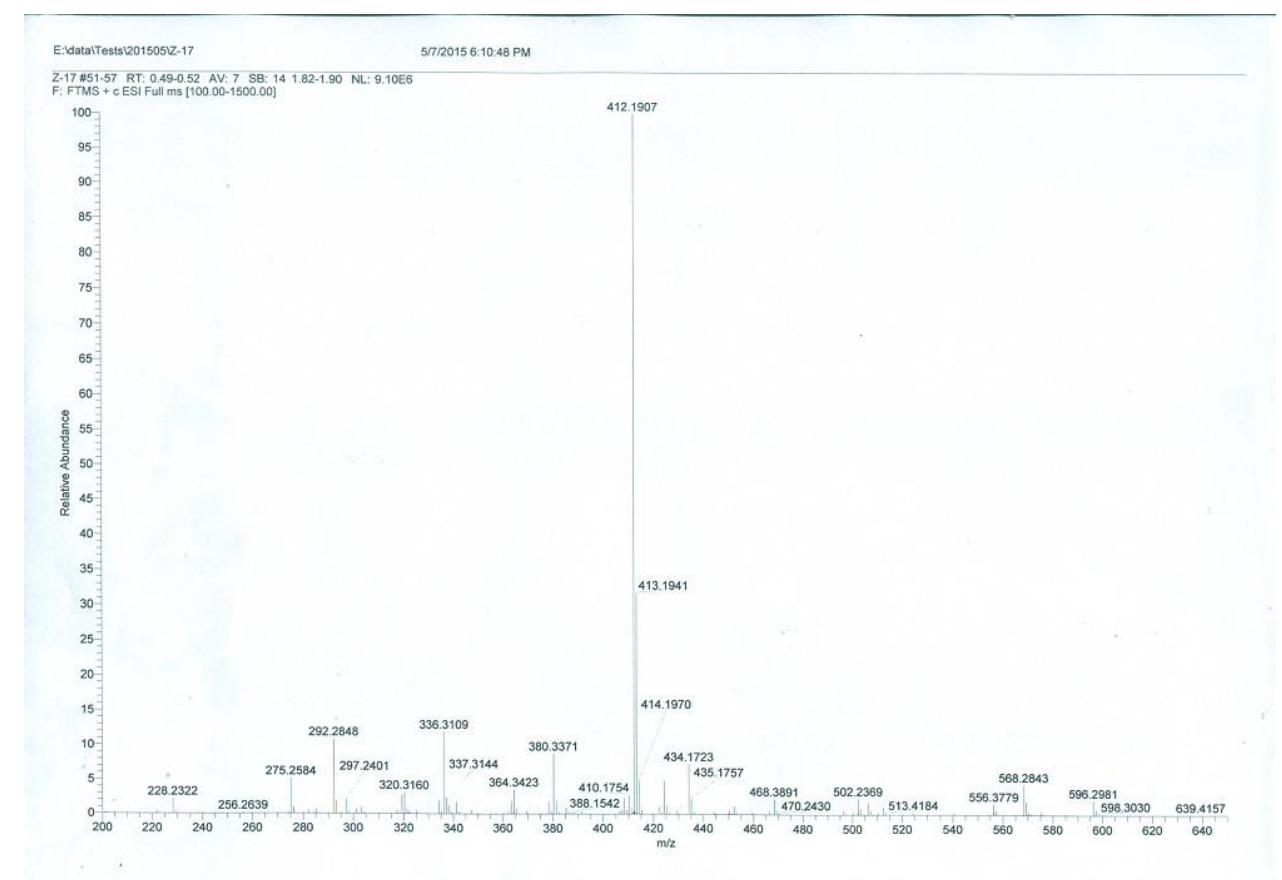

Figure S128. HRESIMS spectrum of $\mathbf{3 6}$.
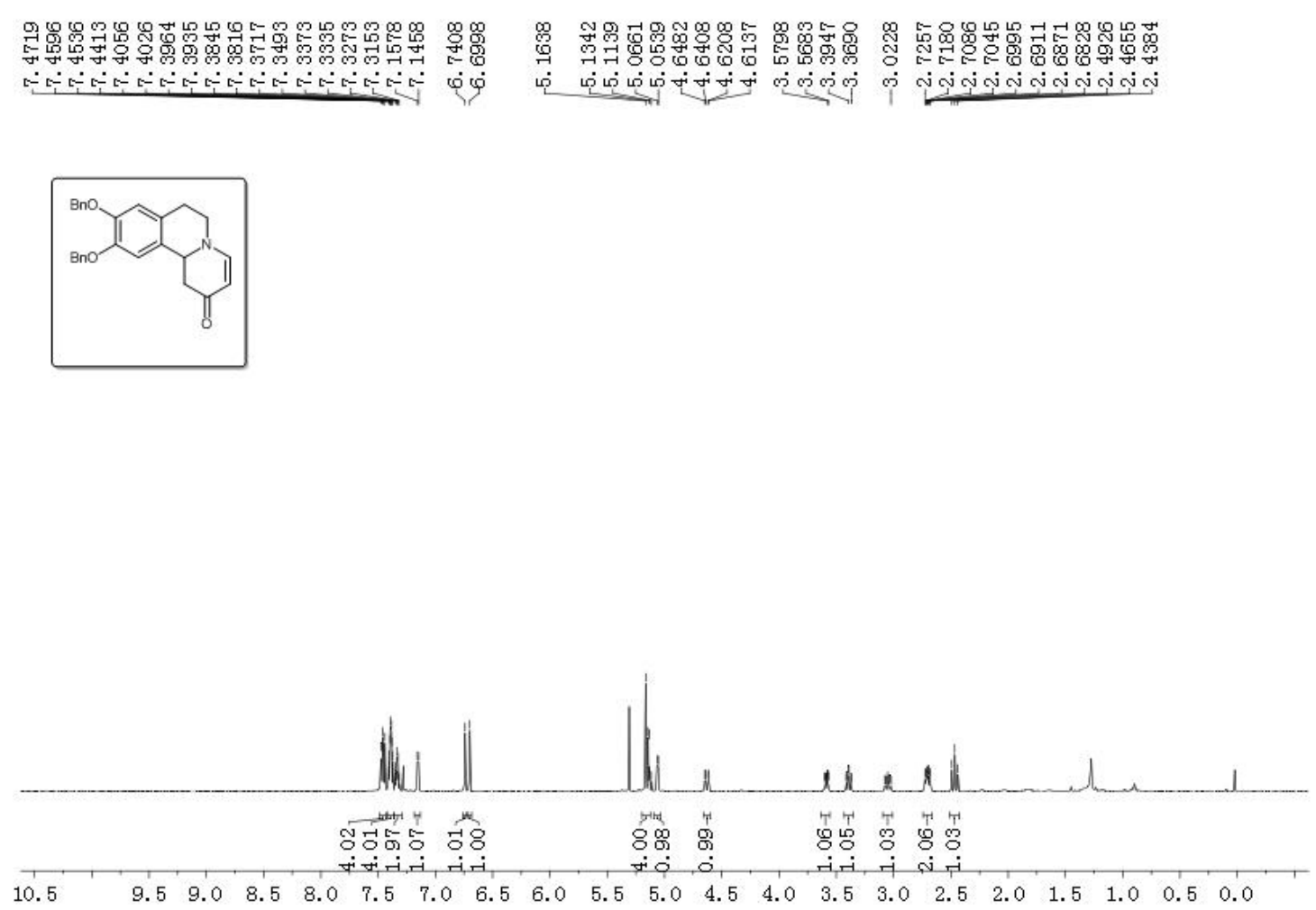

Figure S129. ${ }^{1} \mathrm{H}$ NMR spectrum of $\mathbf{3 6}$. 

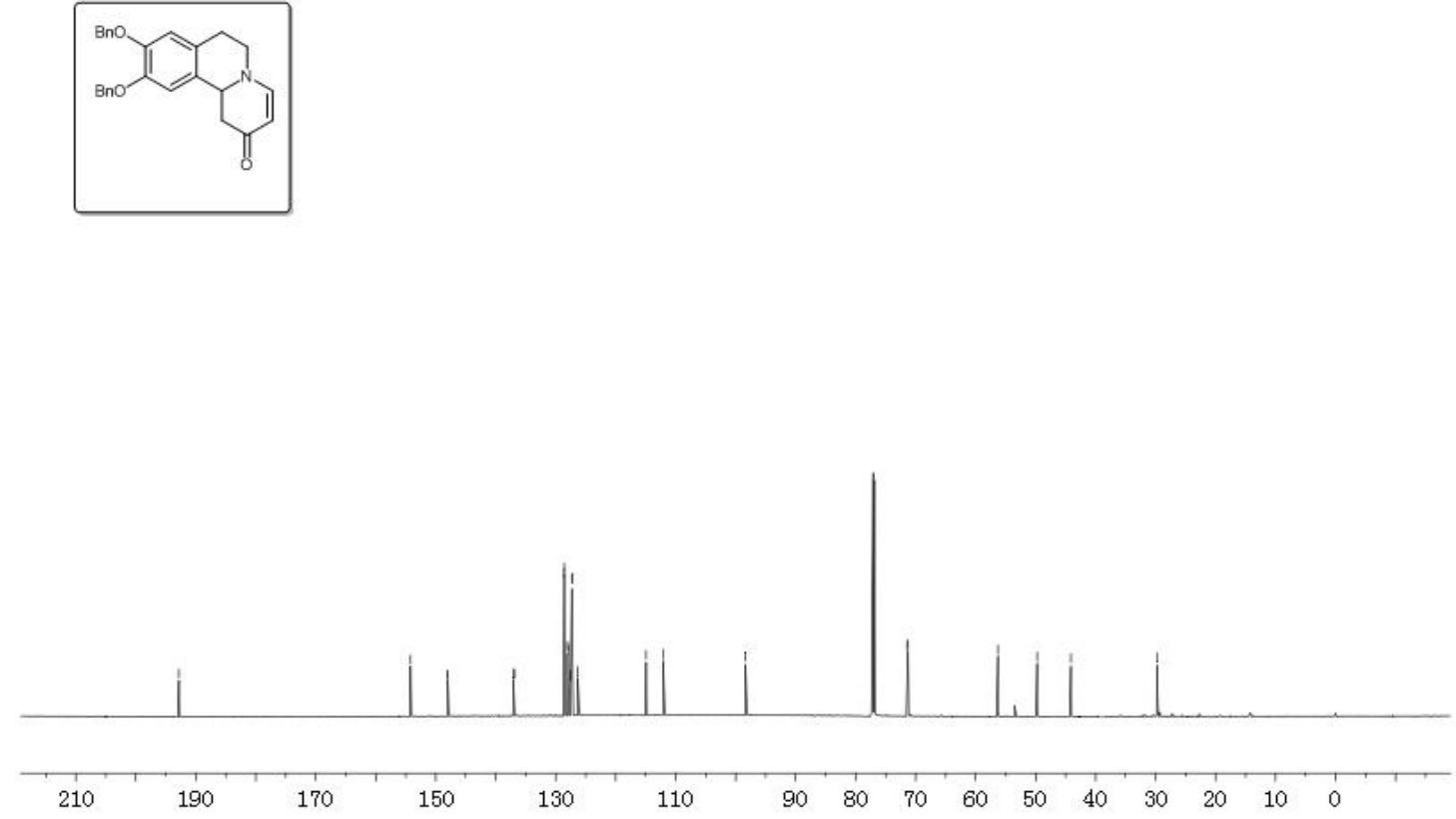

Figure S130. ${ }^{13} \mathrm{C}$ NMR spectrum of $\mathbf{3 6}$.

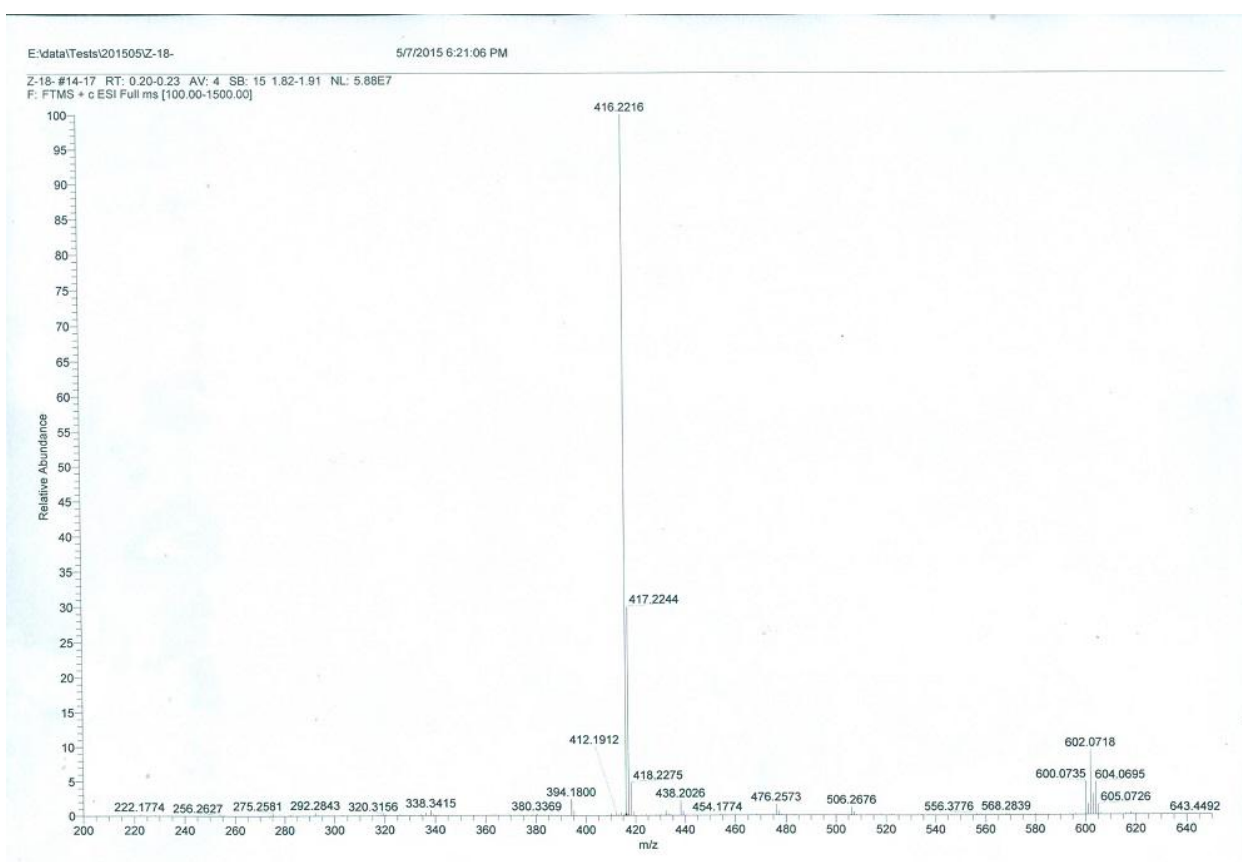

Figure S131. HRESIMS spectrum of 4. 


\section{(non}

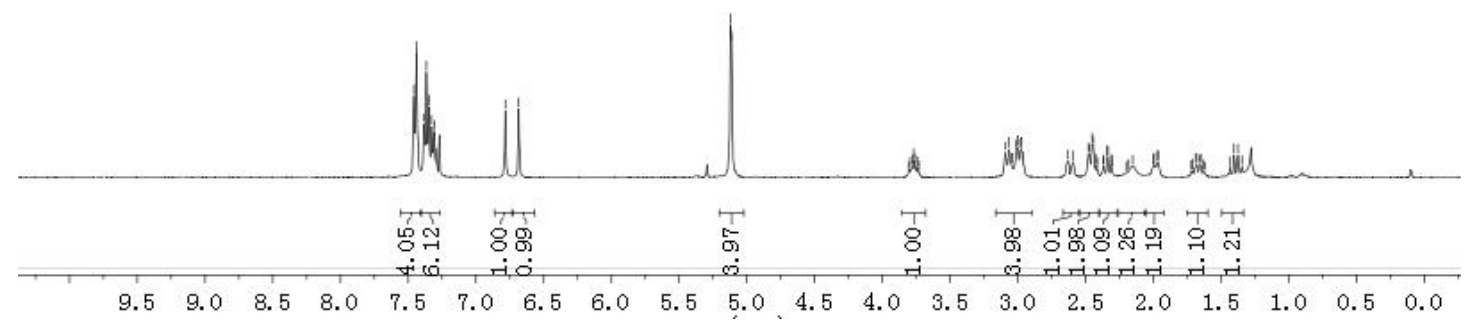

Figure S132. ${ }^{1} \mathrm{H}$ NMR spectrum of 4 .

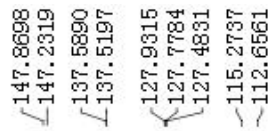

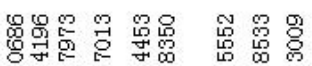

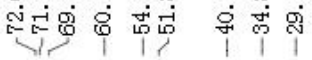
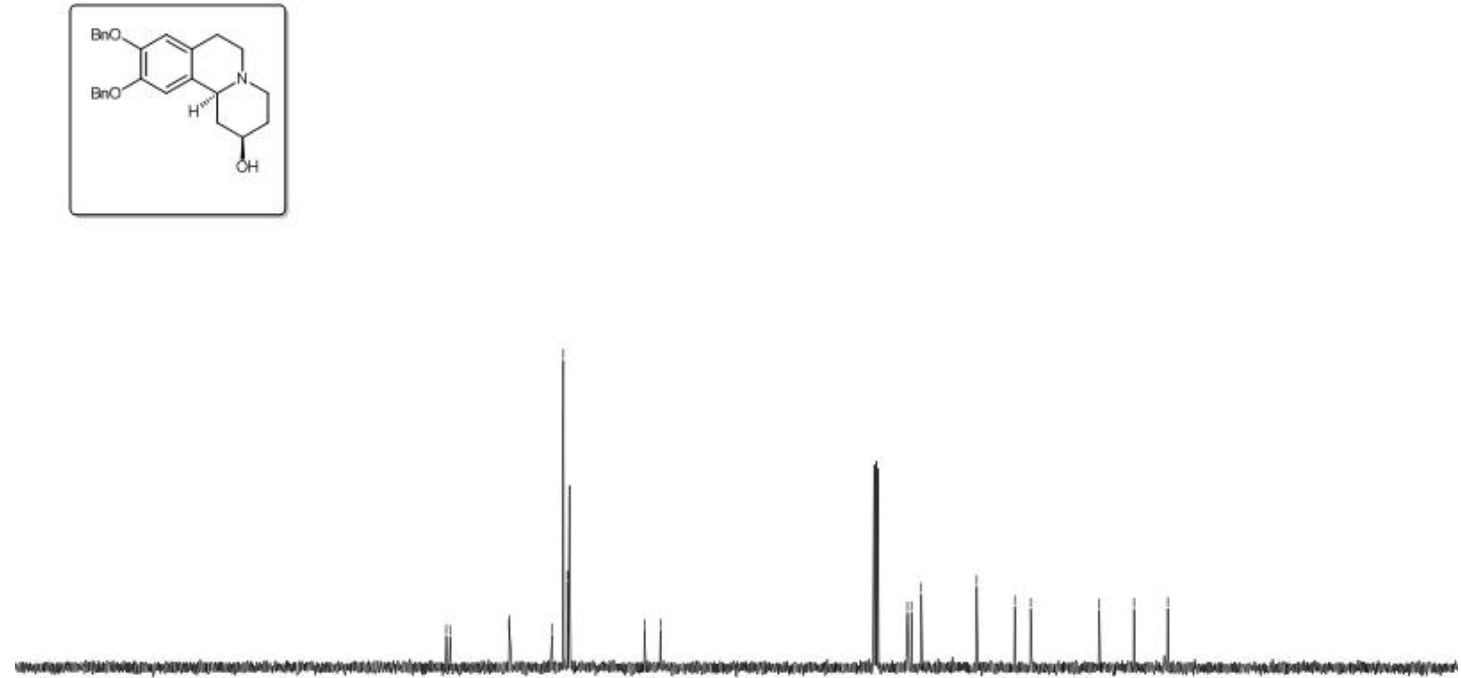

Figure S133. ${ }^{13} \mathrm{C}$ NMR spectrum of 4 . 


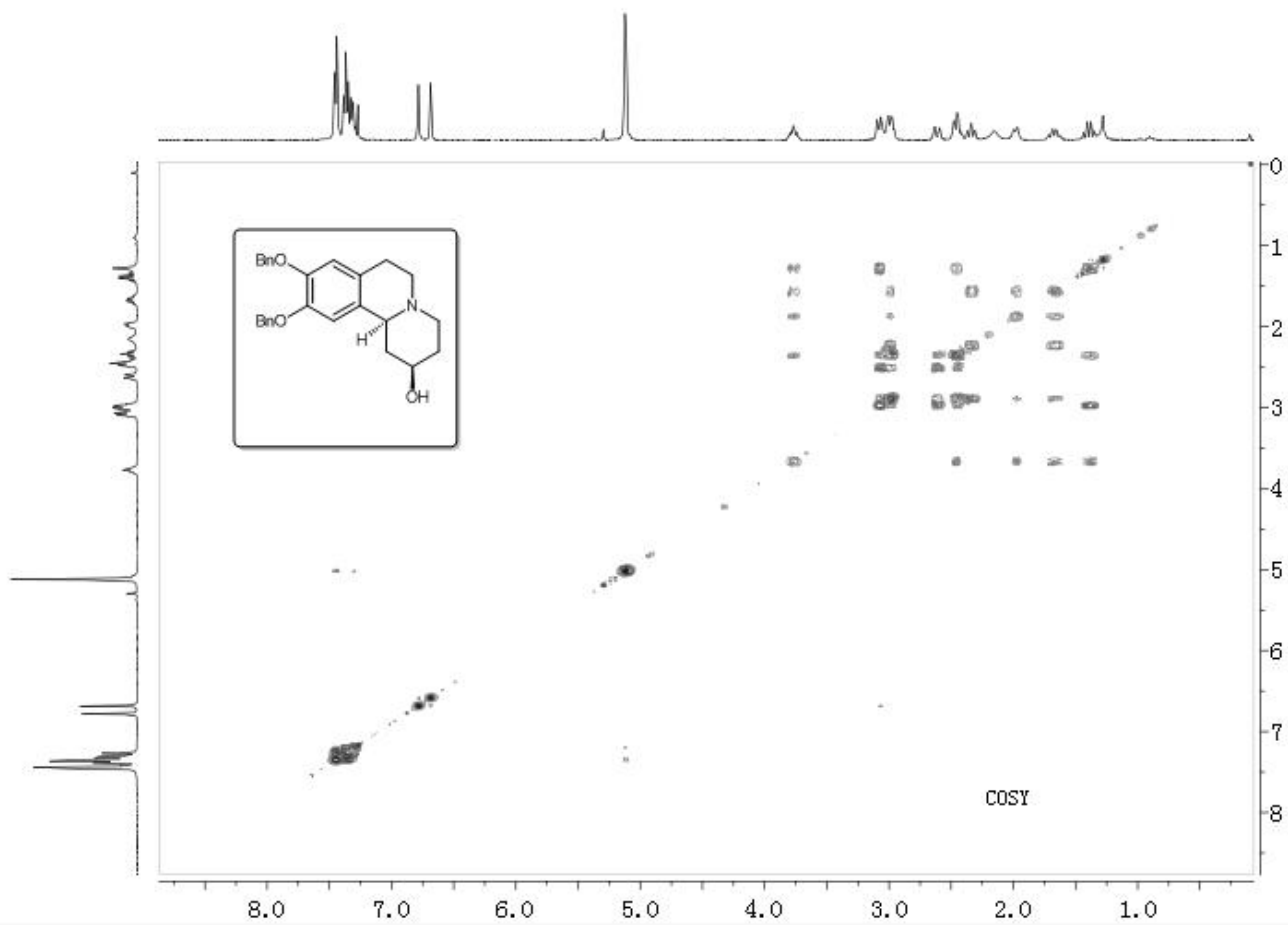

Figure S134. COSY spectrum of 4.

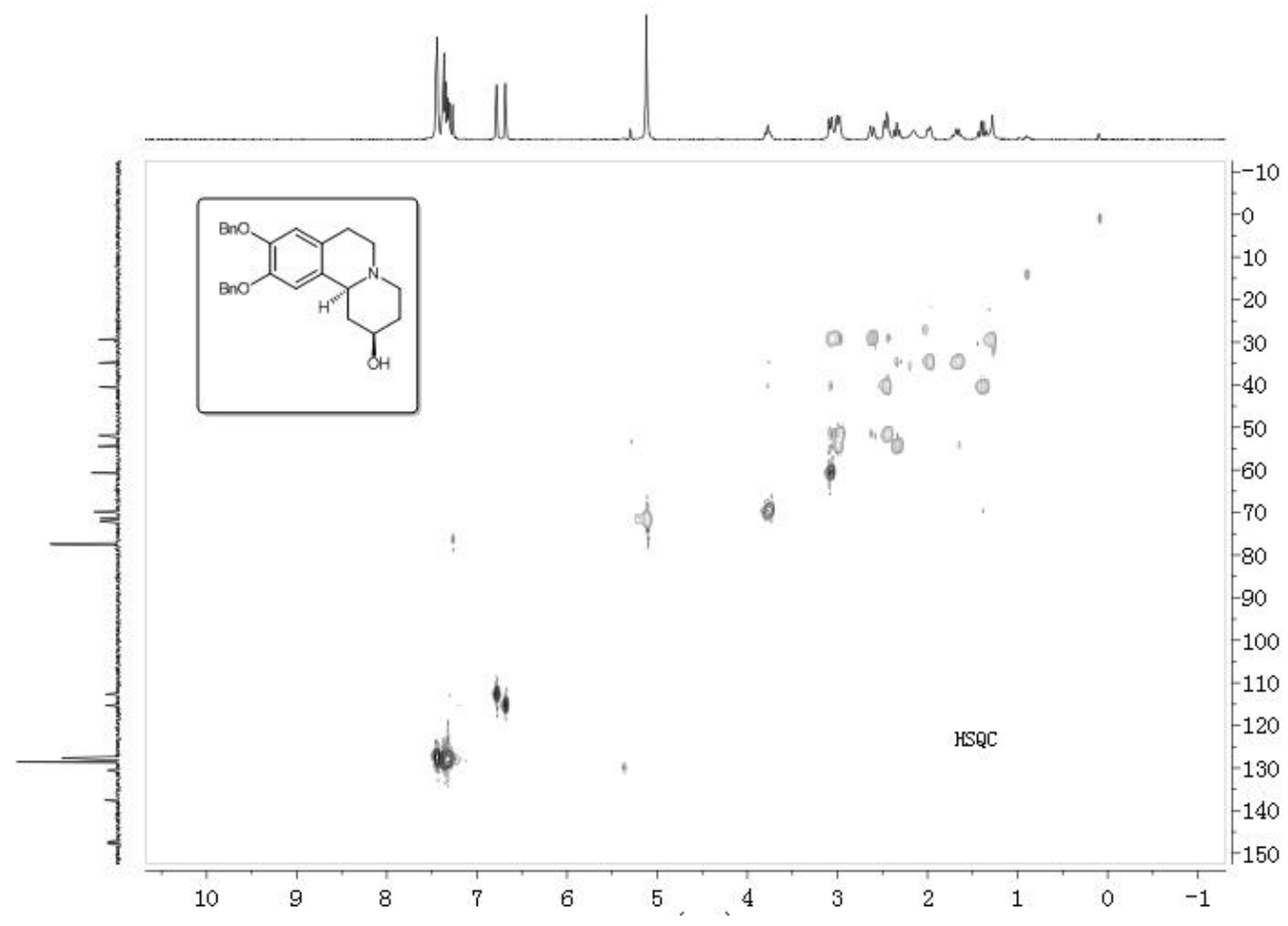

Figure S135. HSQC spectrum of 4. 


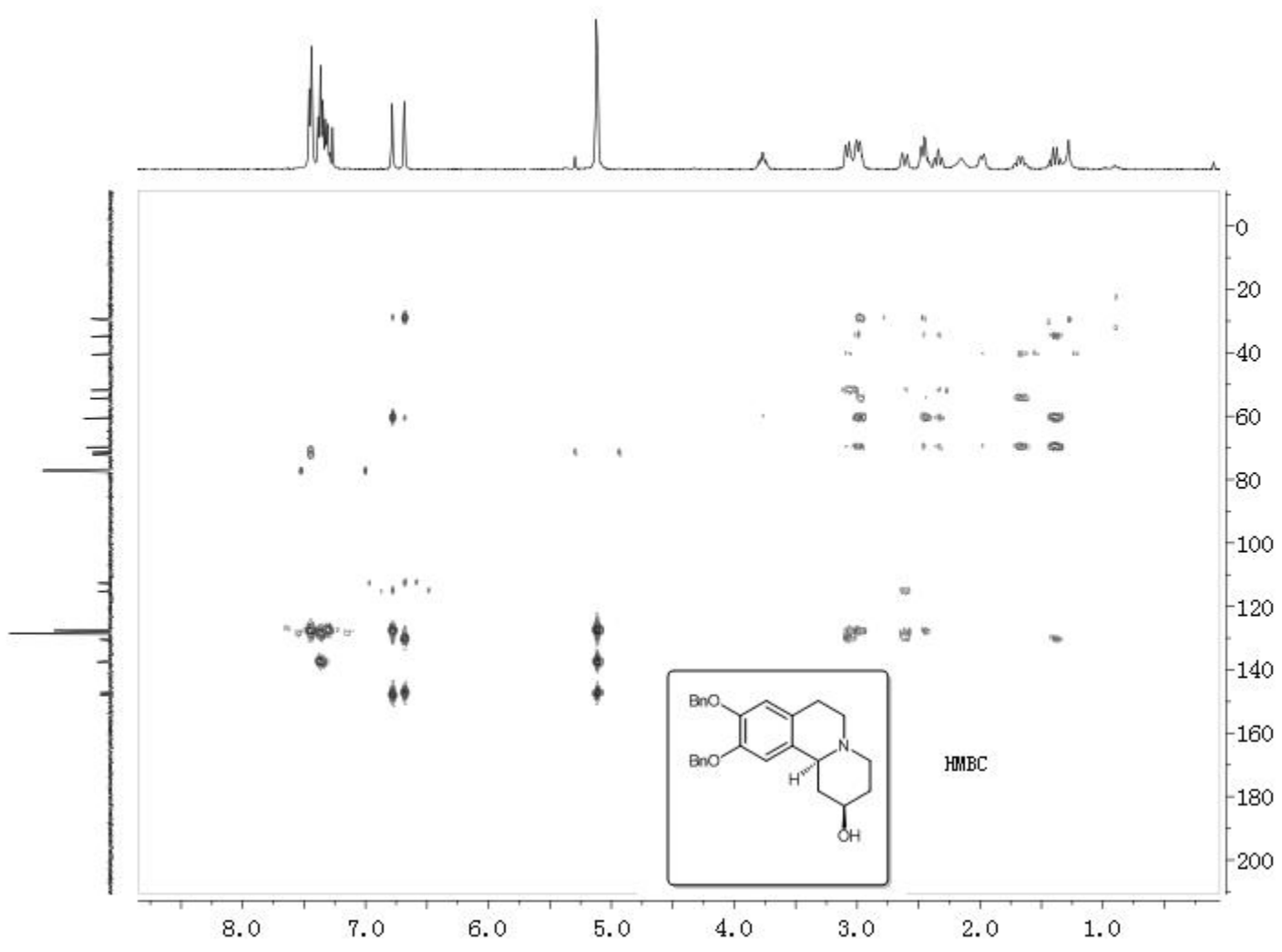

Figure S136. HMBC spectrum of 4.

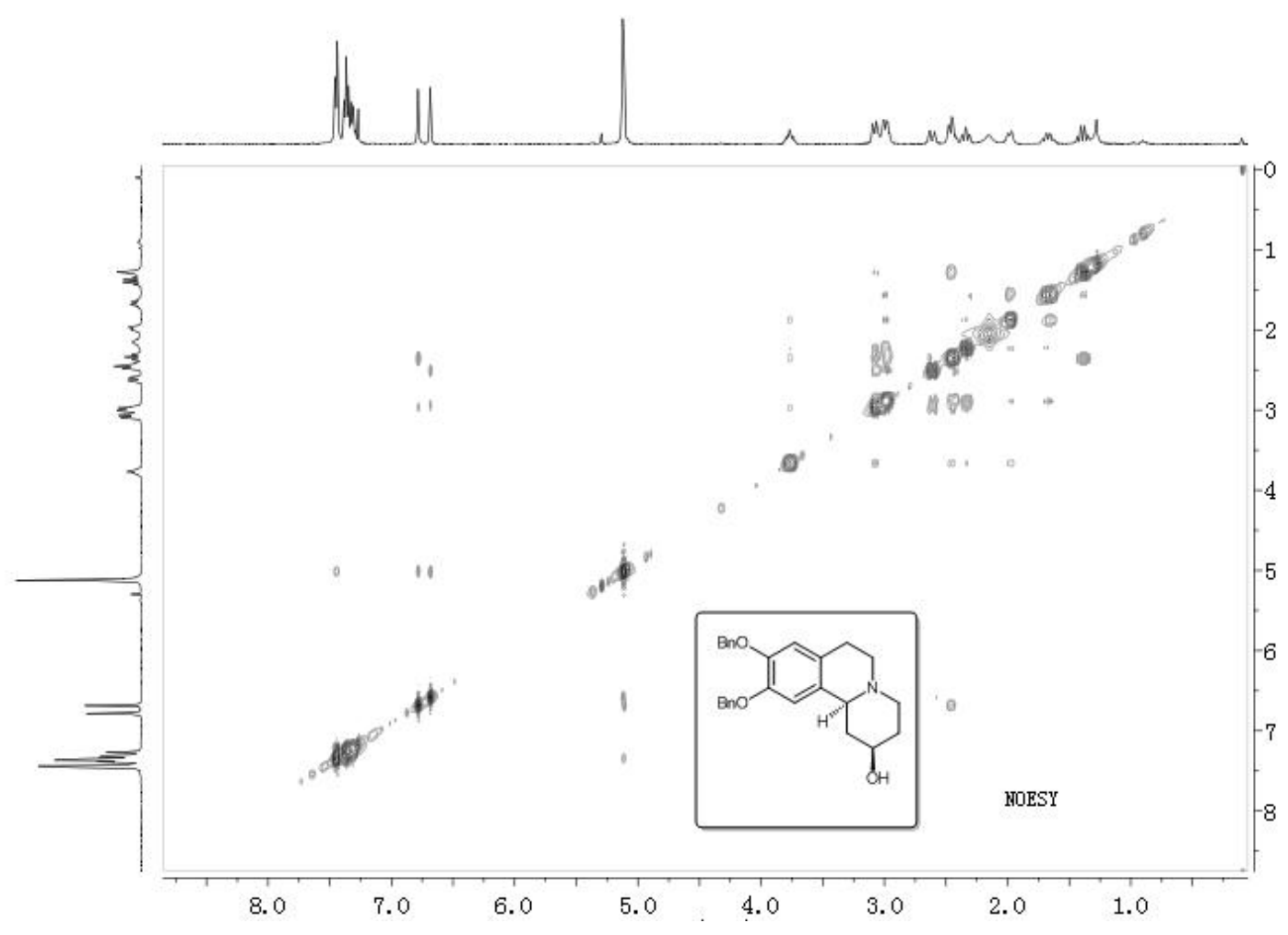

Figure S137. NOESY spectrum of 4. 


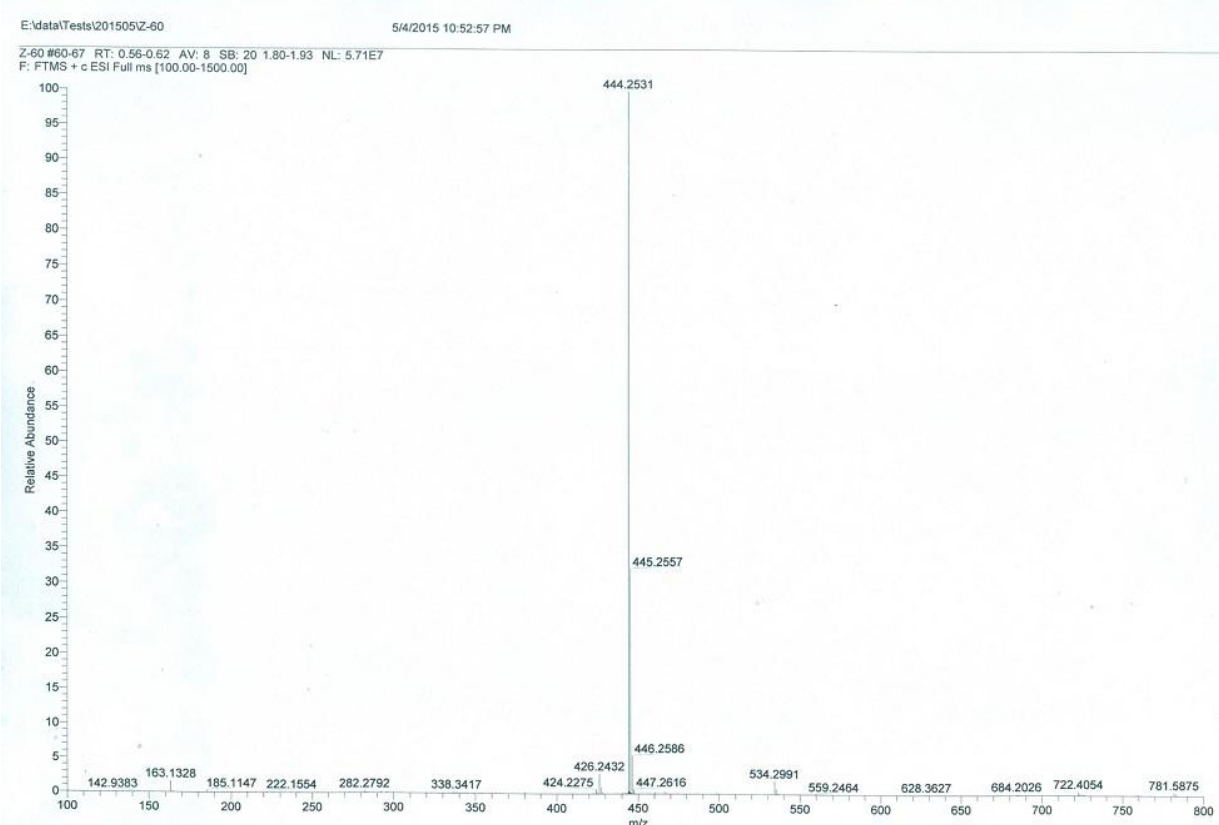

Figure S138. HRESIMS spectrum of 5.
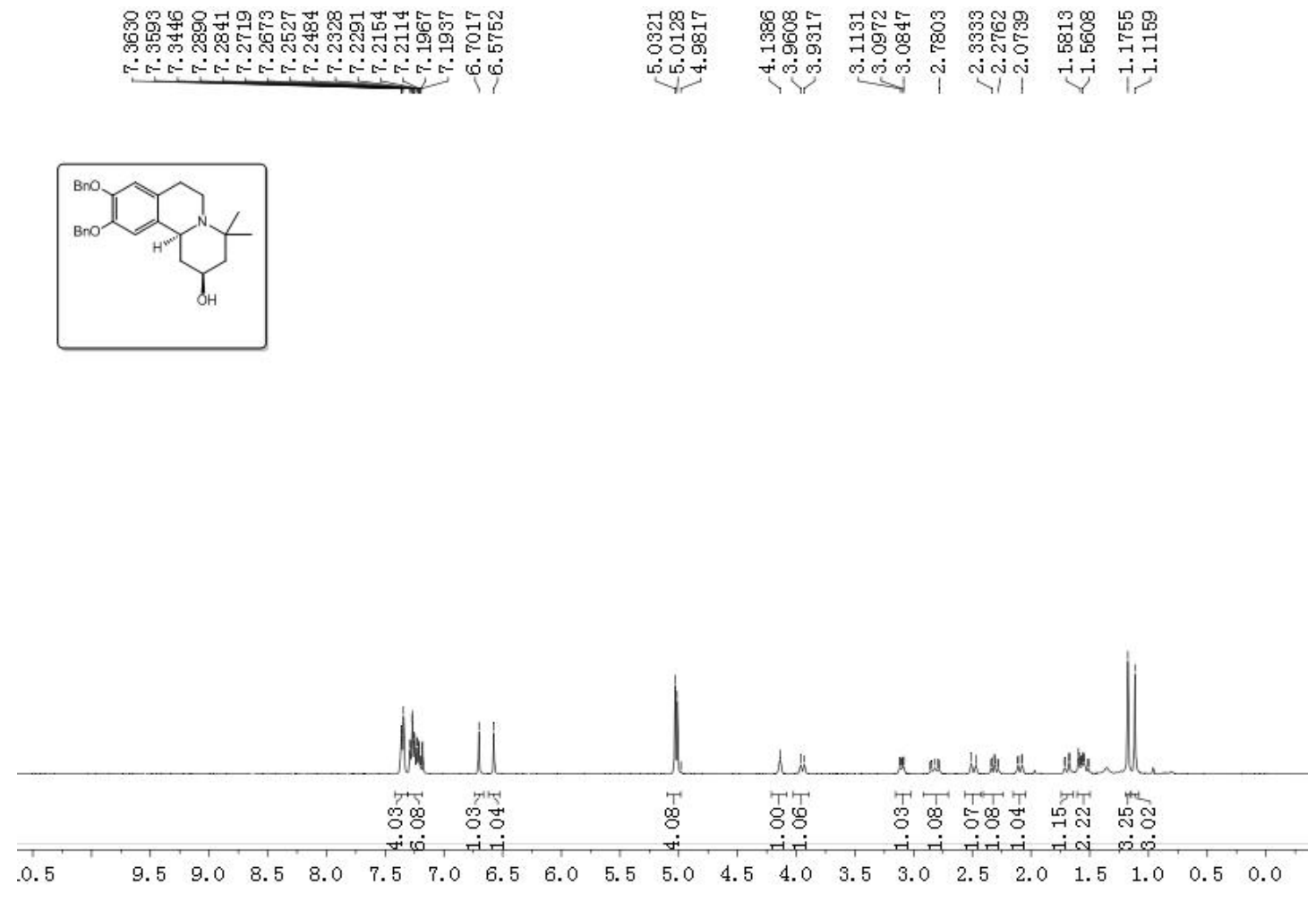

Figure S139. ${ }^{1} \mathrm{H}$ NMR spectrum of 5 . 

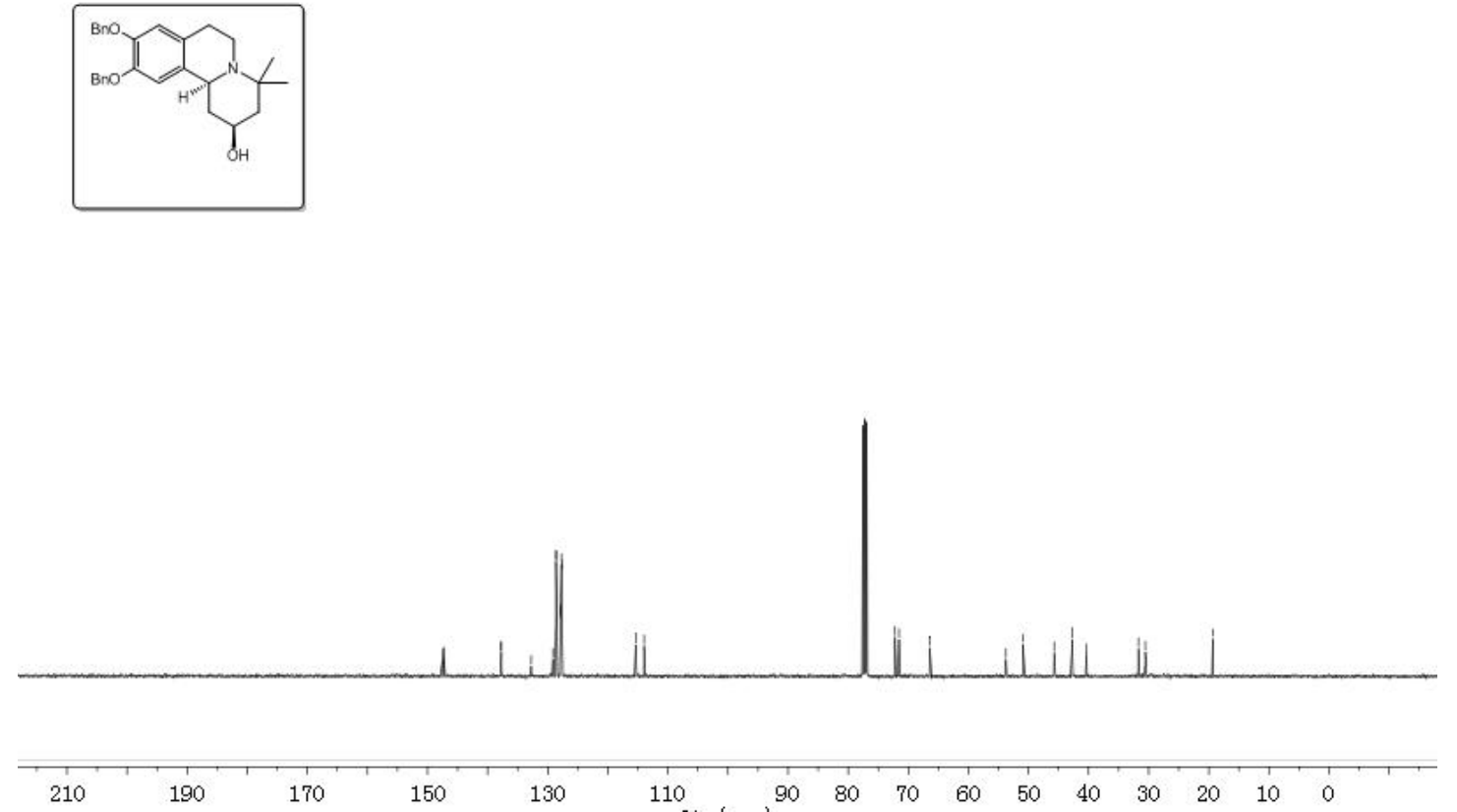

Figure S140. ${ }^{13} \mathrm{C}$ NMR spectrum of 5 .

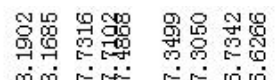

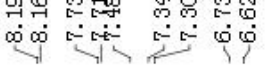

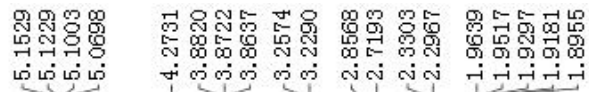

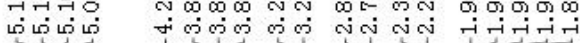
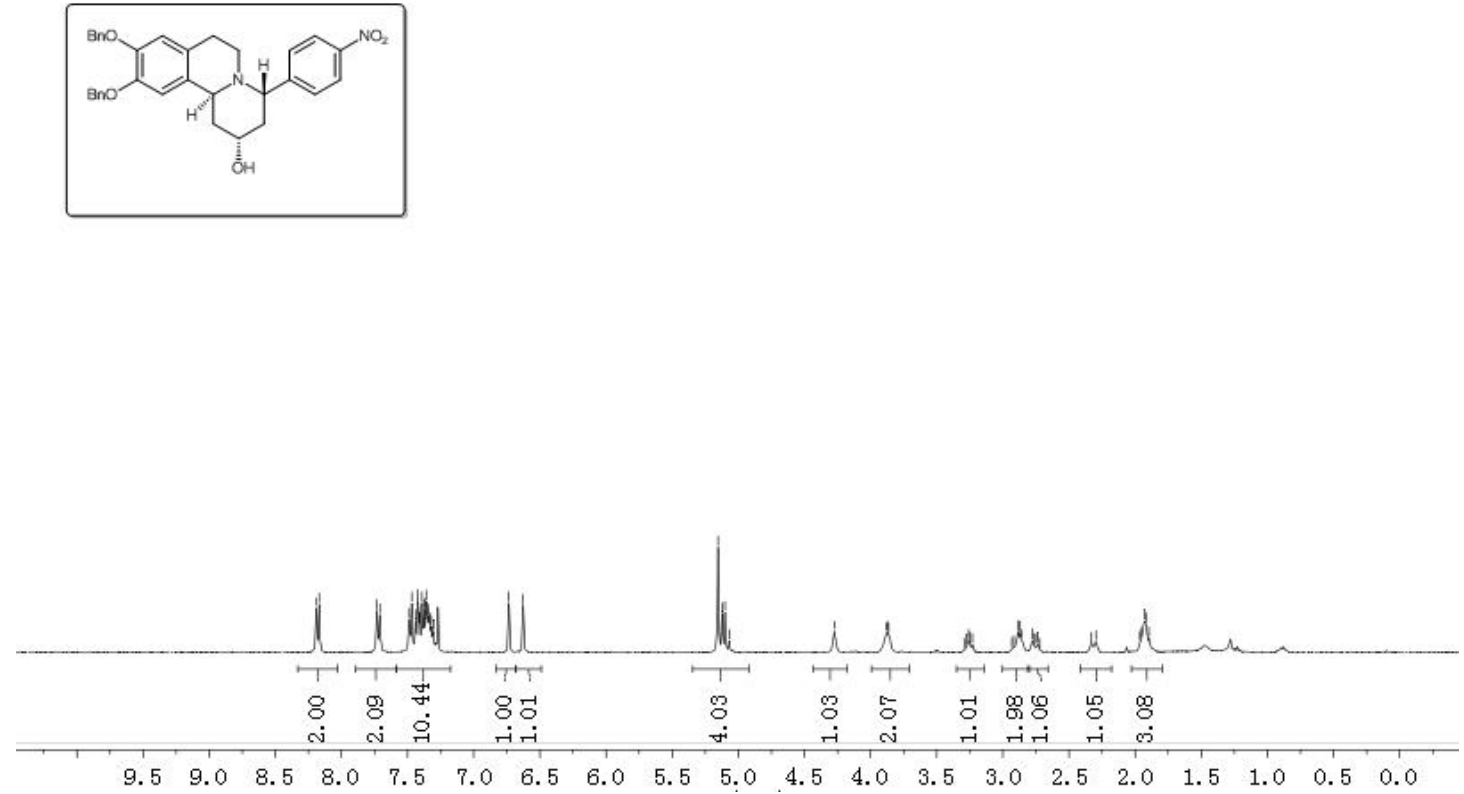

Figure S141. ${ }^{1} \mathrm{H}$ NMR spectrum of $\mathbf{6 a}$. 

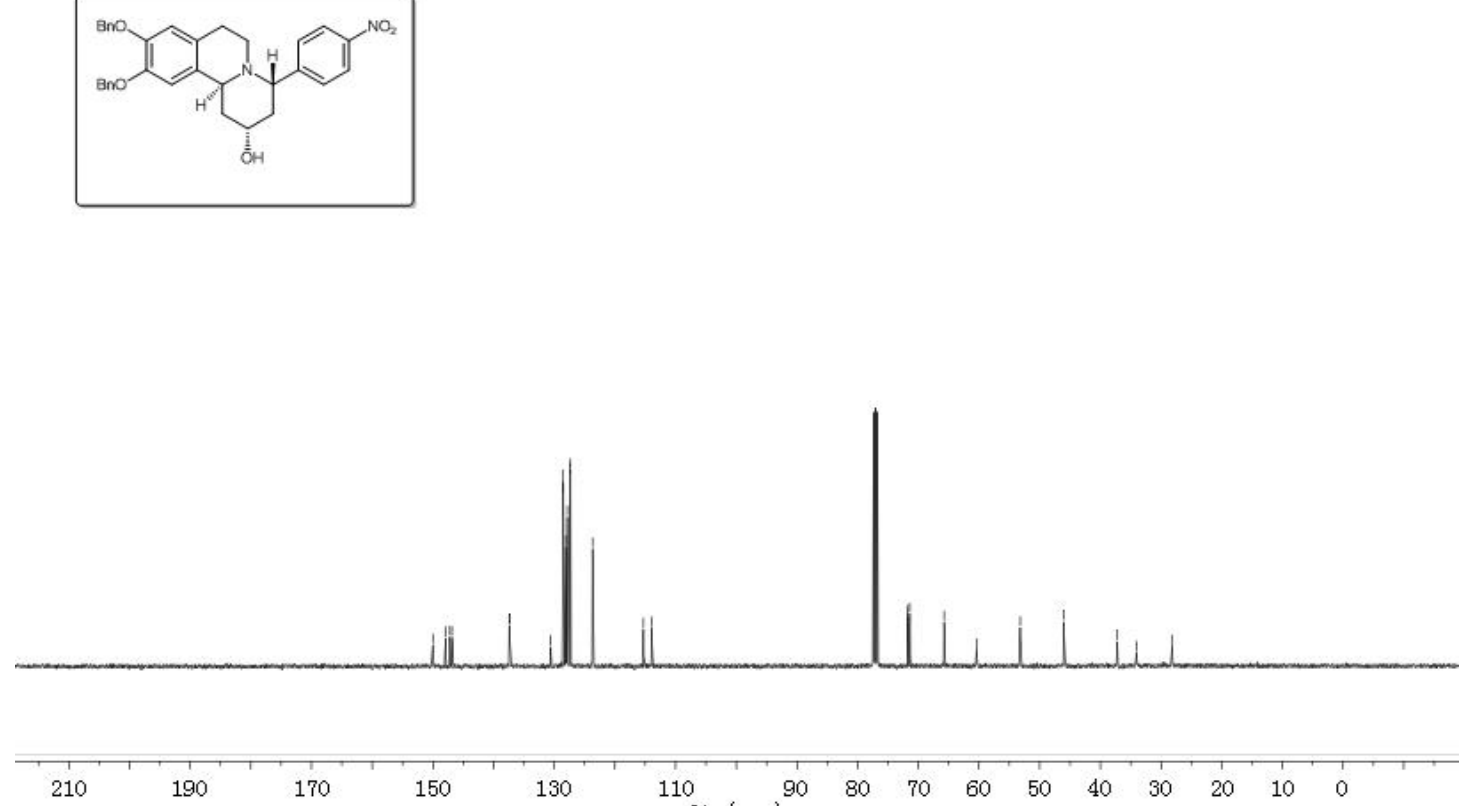

Figure $\mathbf{S 1 4 2 .}{ }^{13} \mathrm{C}$ NMR spectrum of $\mathbf{6 a}$.

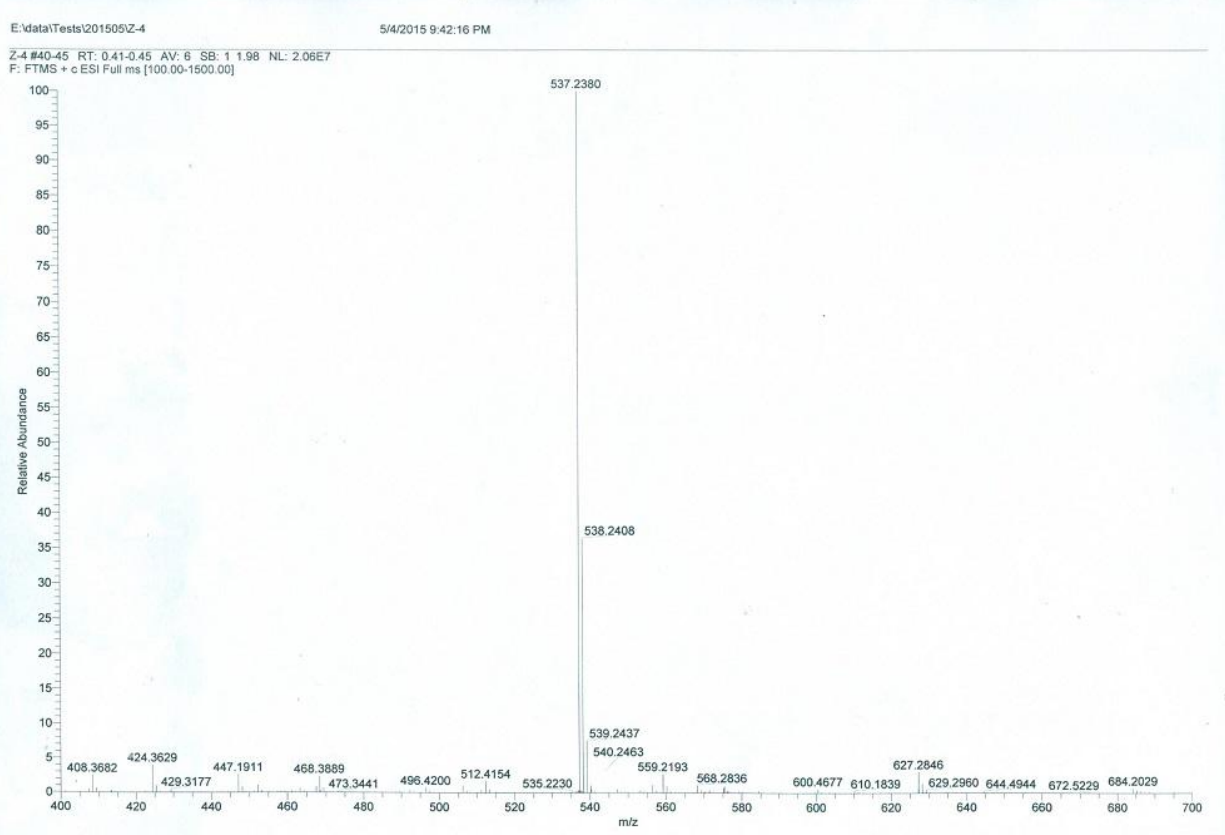

Figure S143. HRESIMS spectrum of $\mathbf{6 b}$. 
胑产 का को $=0$

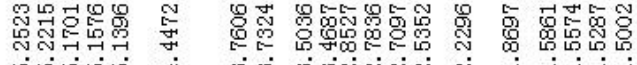

में
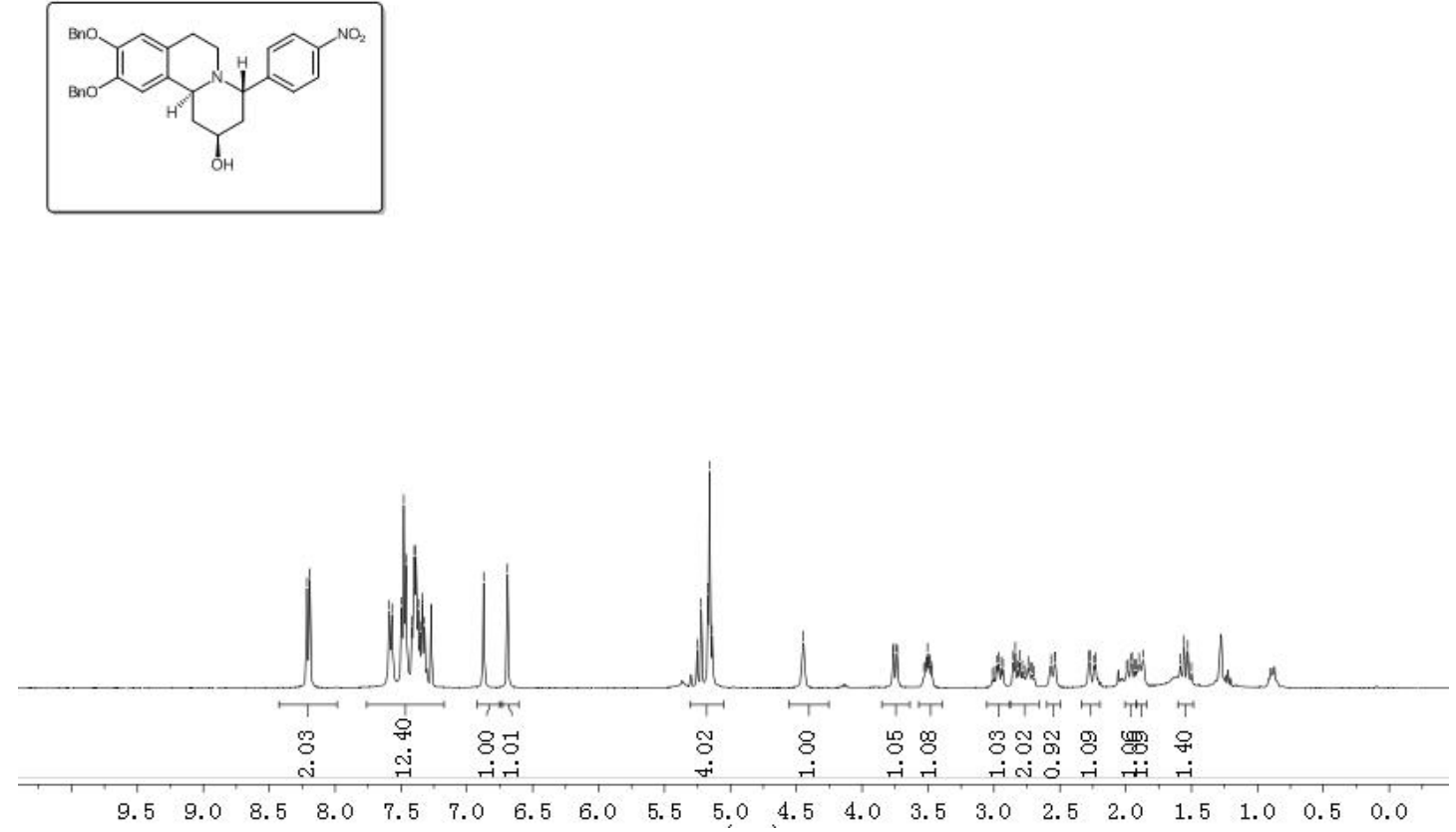

Figure S144. ${ }^{1} \mathrm{H}$ NMR spectrum of $\mathbf{6 b}$.

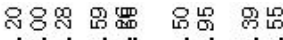

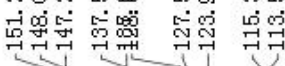

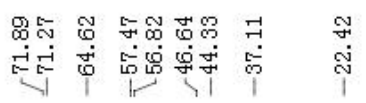
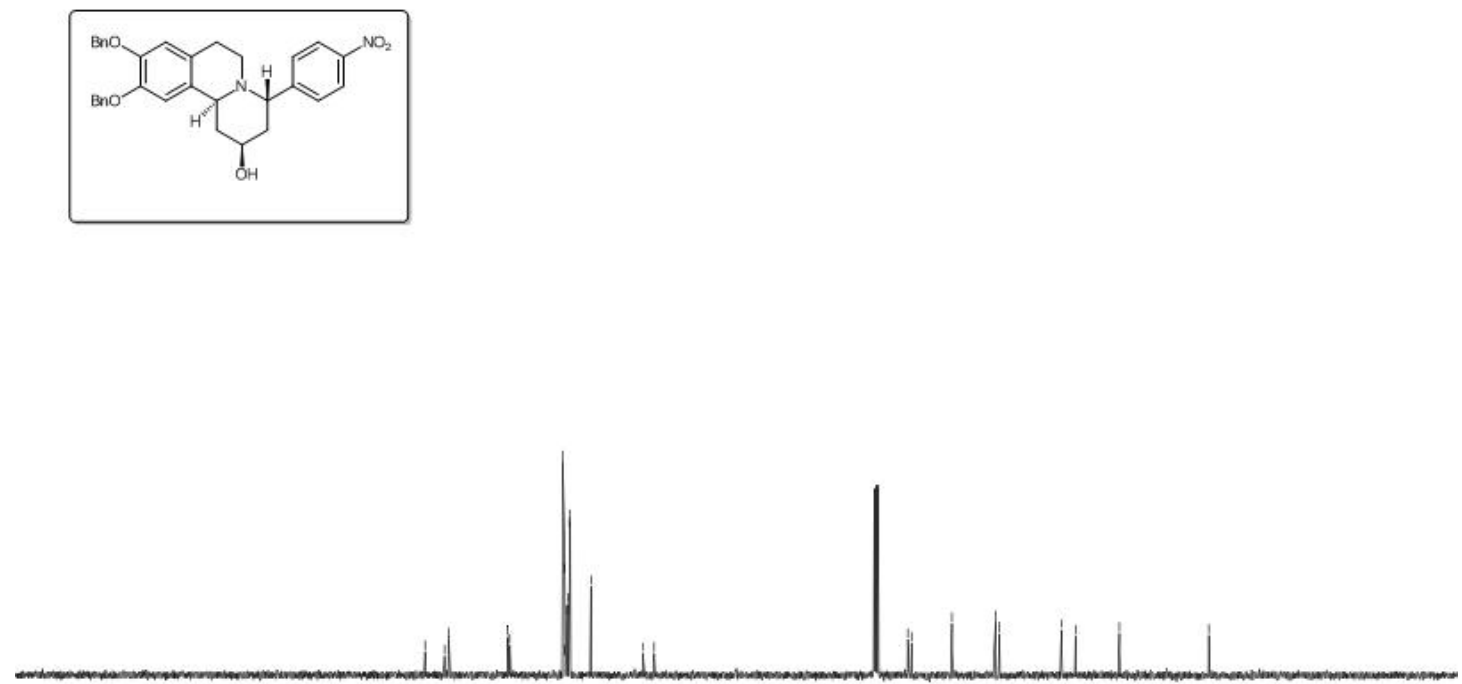

Figure S145. ${ }^{13} \mathrm{C}$ NMR spectrum of $6 \mathbf{b}$. 

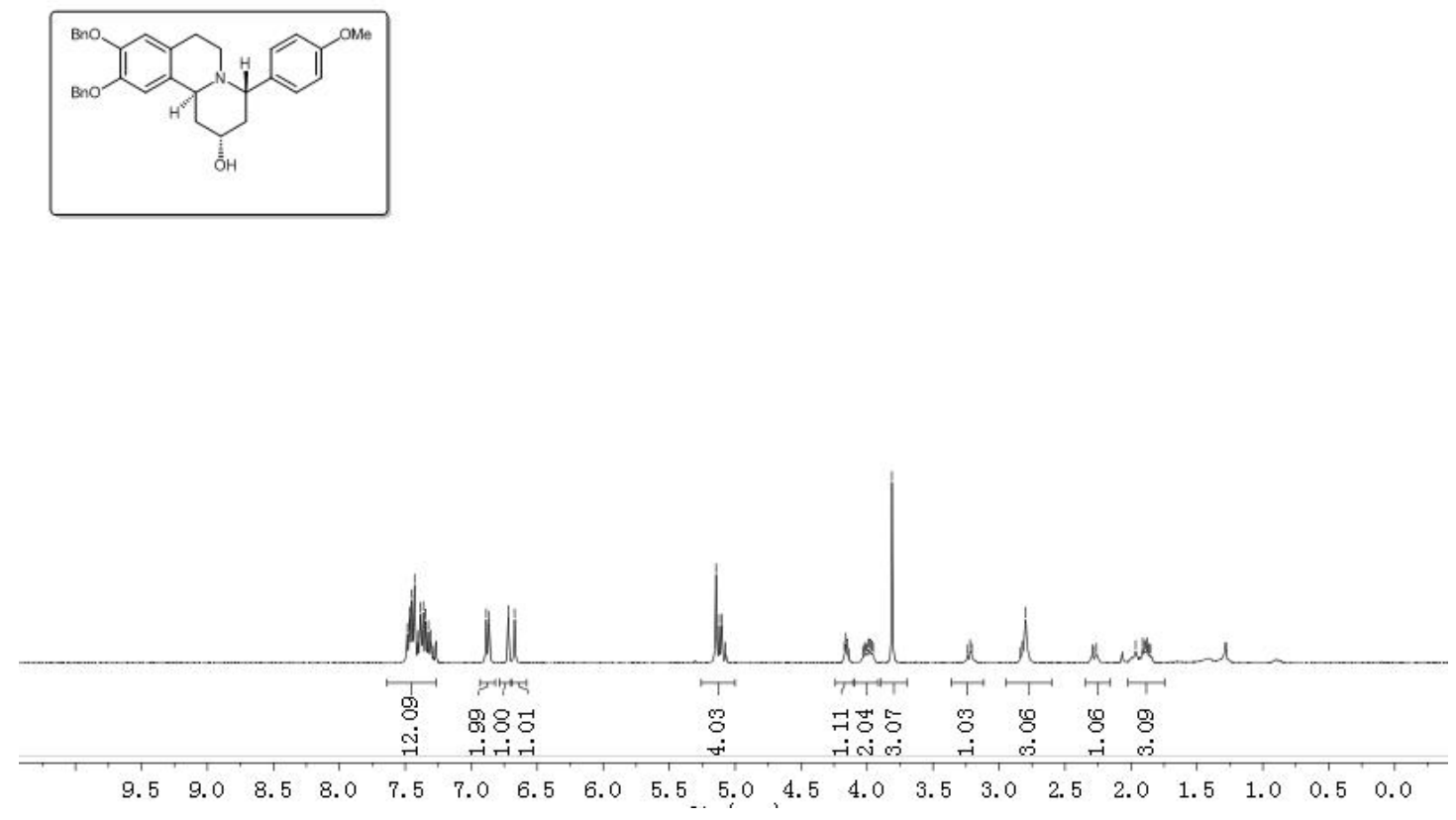

Figure S146. ${ }^{1} \mathrm{H}$ NMR spectrum of $7 \mathbf{a}$.
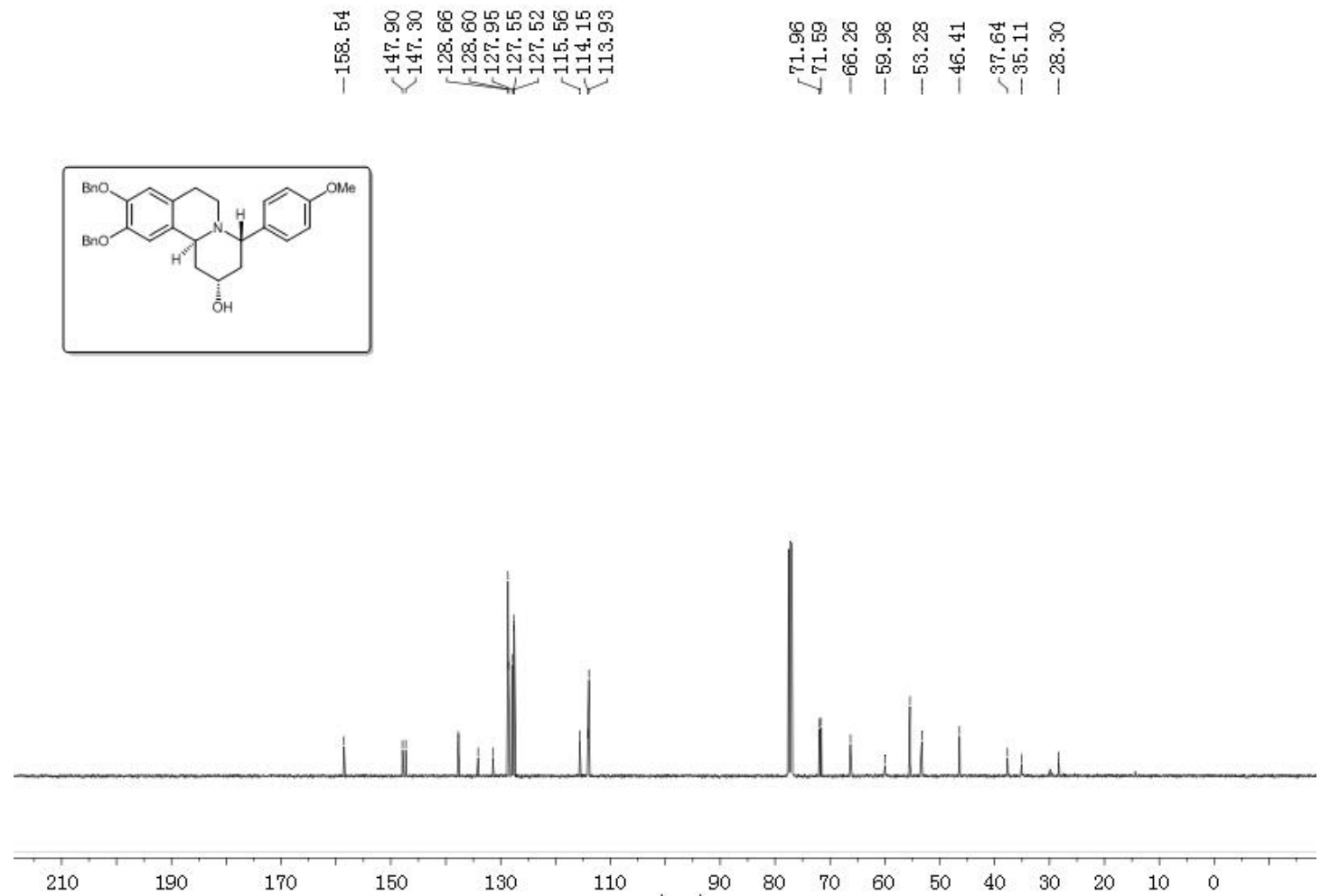

Figure S147. ${ }^{13} \mathrm{C}$ NMR spectrum of $7 a$. 


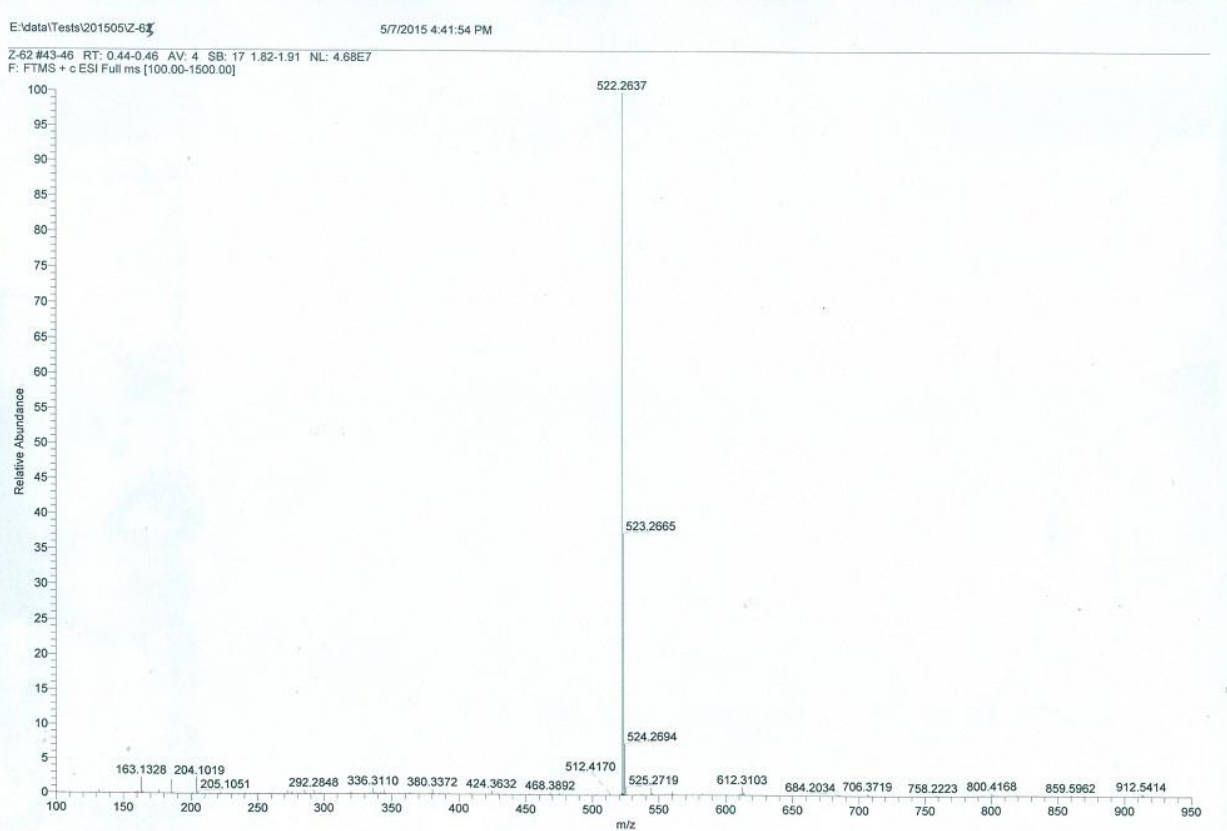

Figure S148. HRESIMS spectrum of $\mathbf{7 b}$.
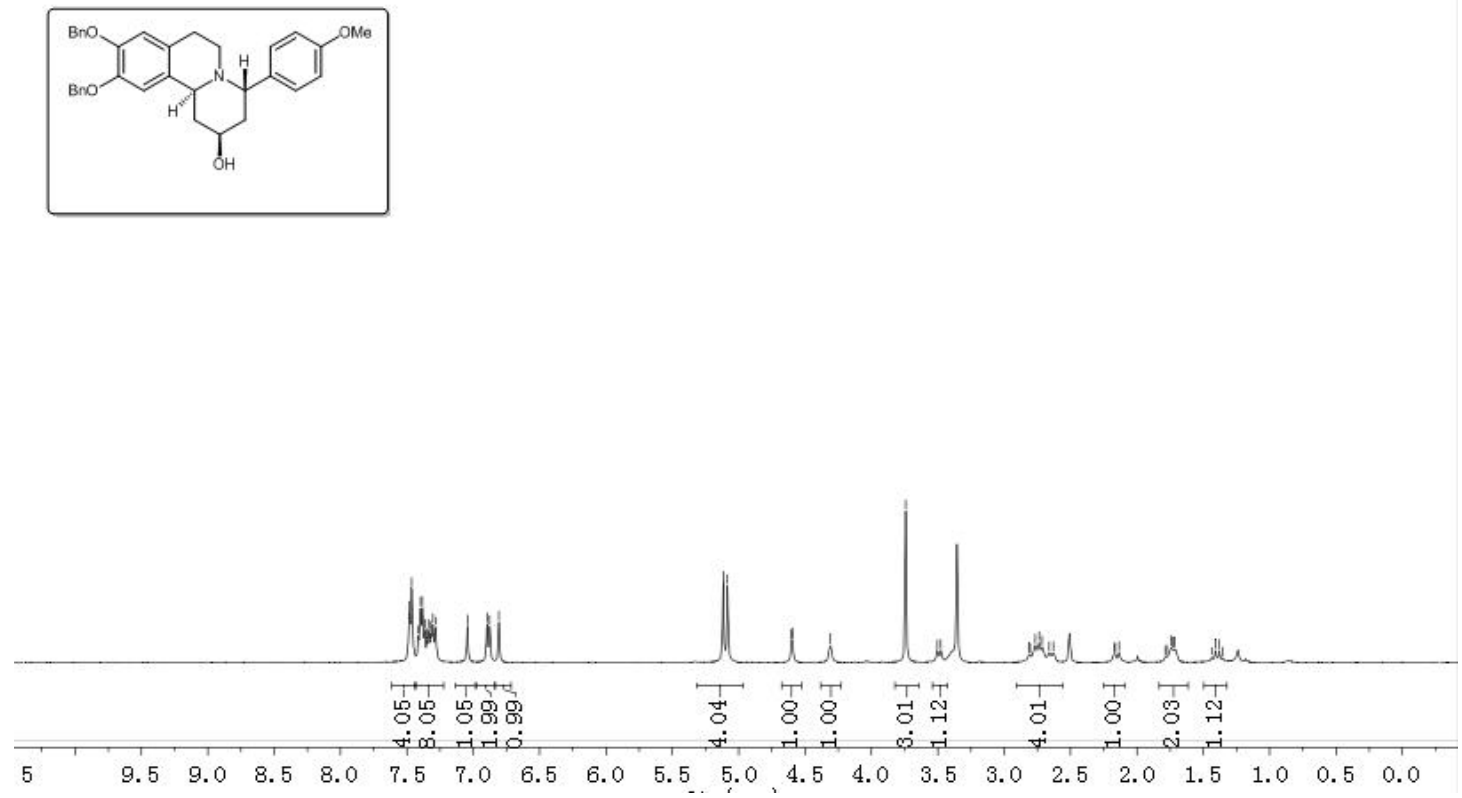

Figure S149. ${ }^{1} \mathrm{H}$ NMR spectrum of $\mathbf{7 b}$. 

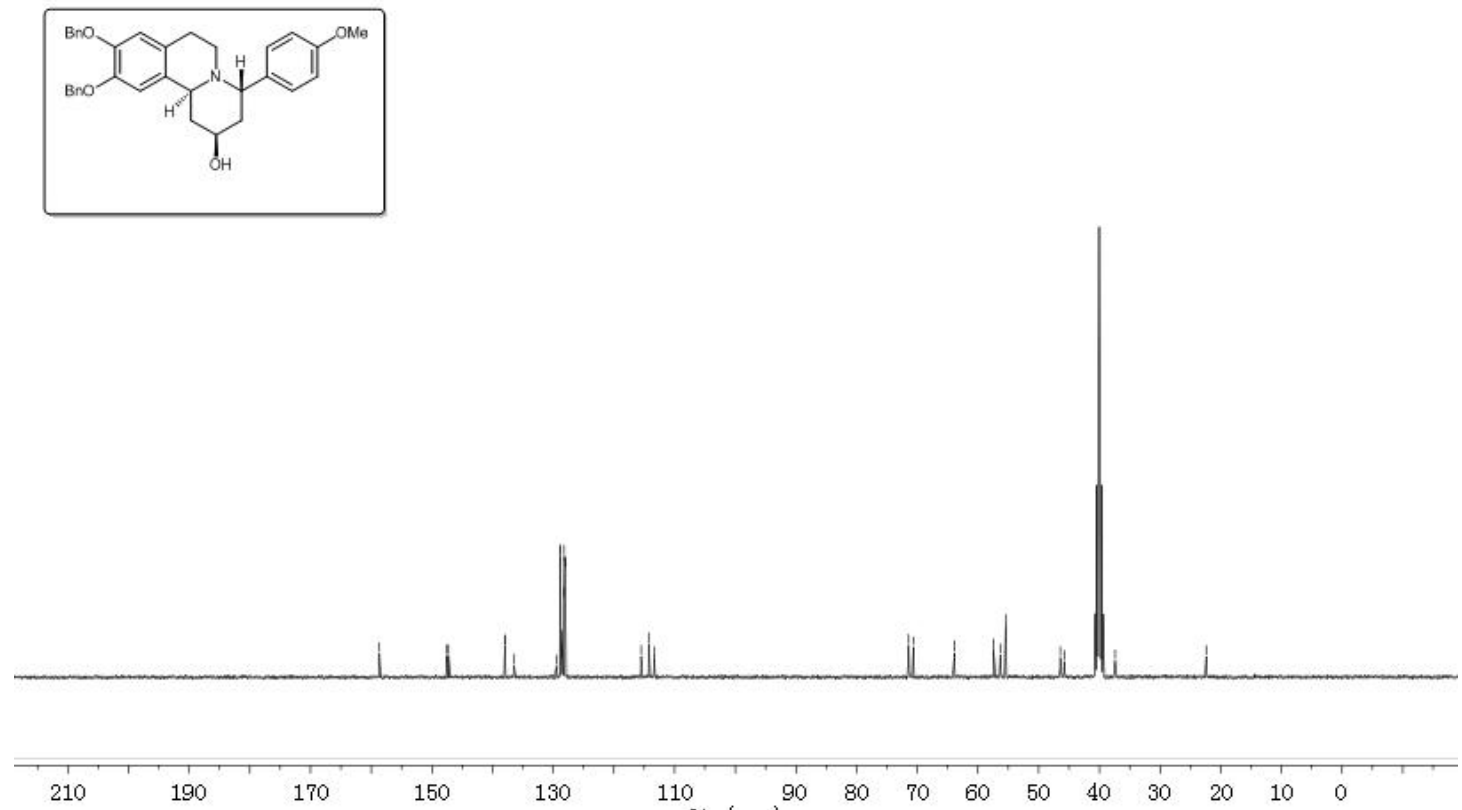

Figure S150. ${ }^{13} \mathrm{C}$ NMR spectrum of $7 \mathbf{b}$.

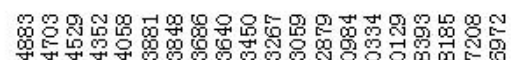

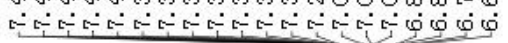

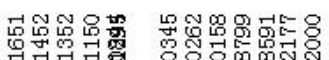

政

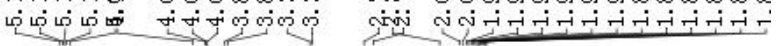
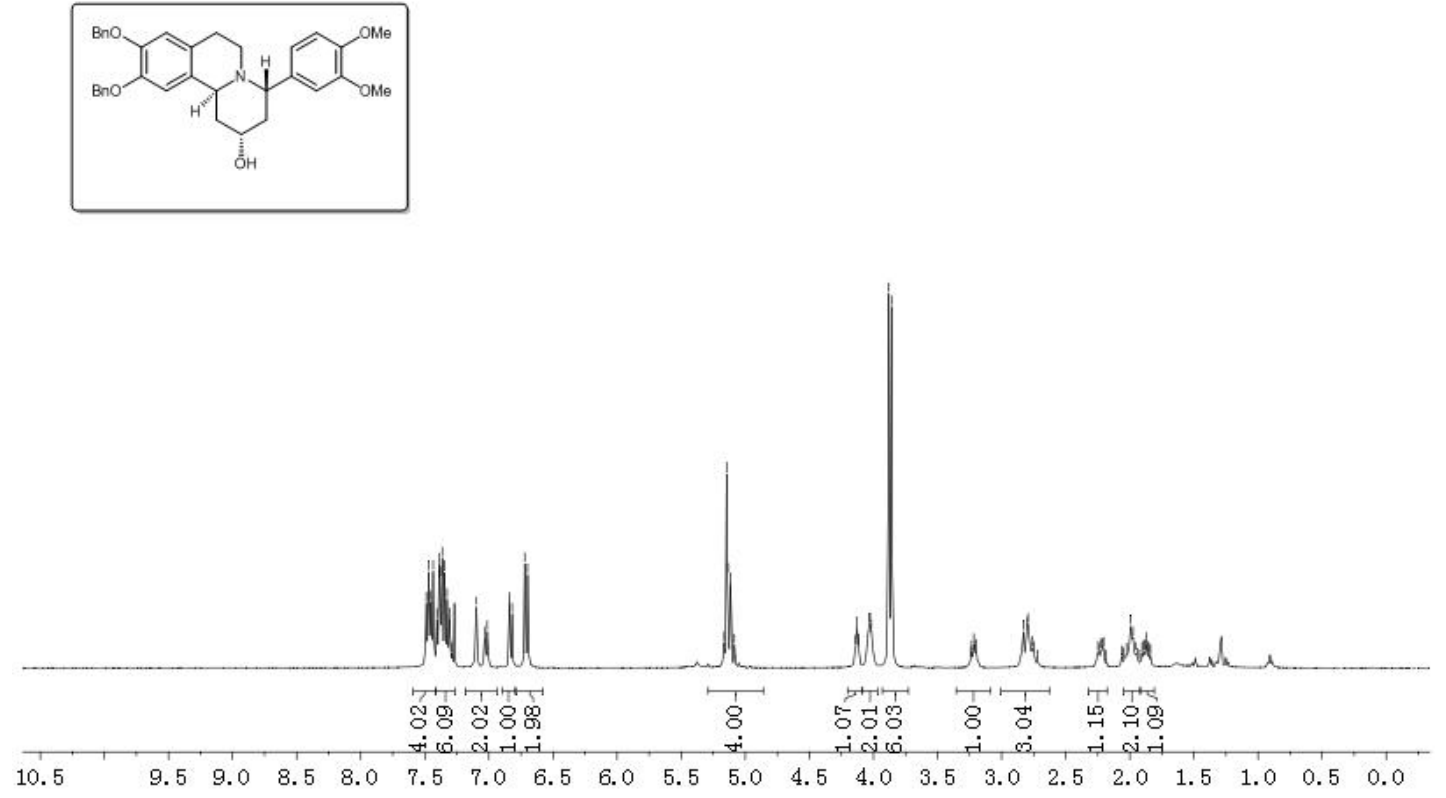

Figure S151. ${ }^{1} \mathrm{H}$ NMR spectrum of $\mathbf{8 a}$. 


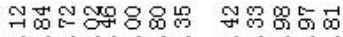

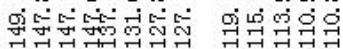

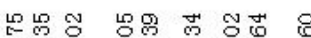

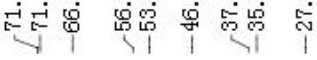
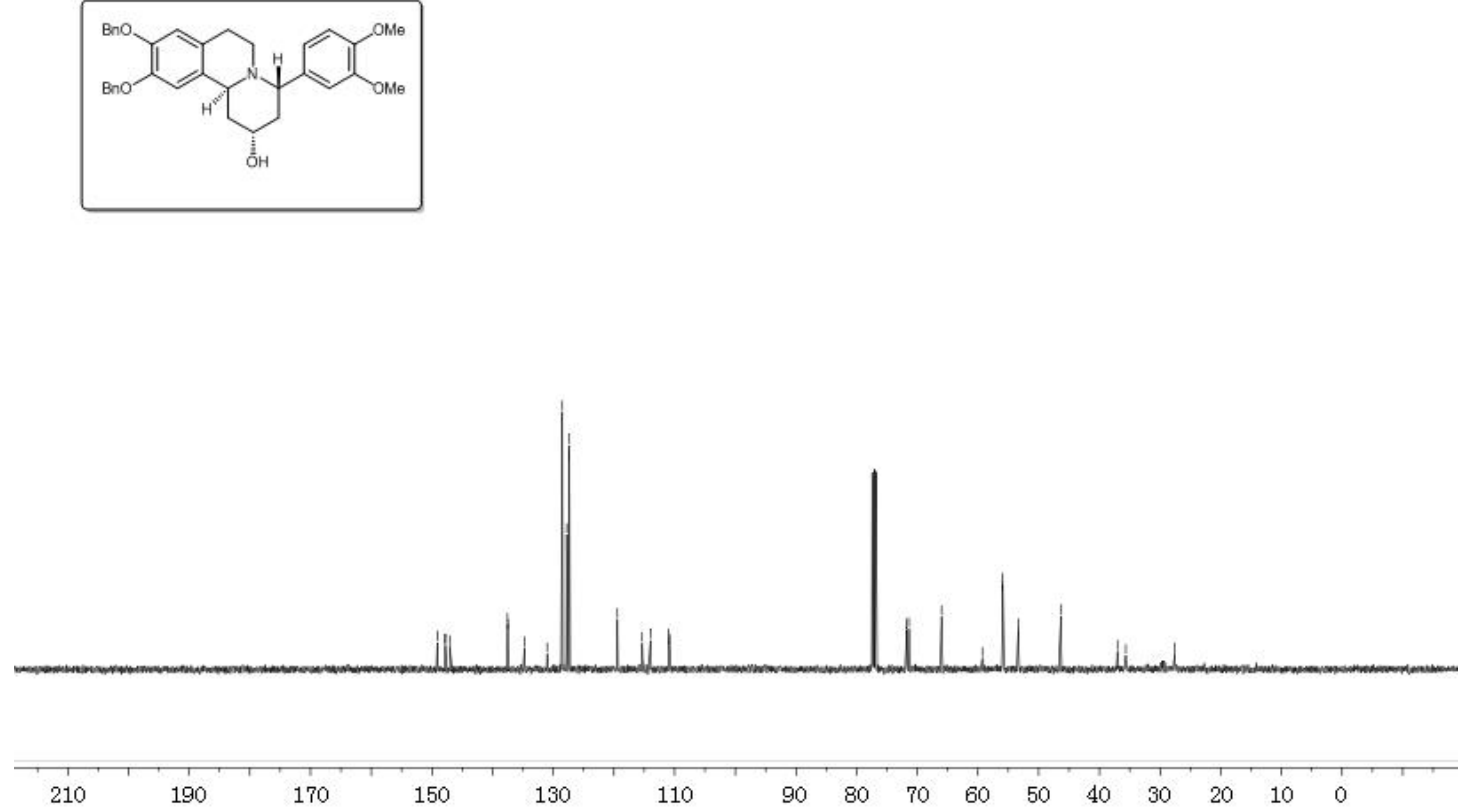

Figure S152. ${ }^{13} \mathrm{C}$ NMR spectrum of $\mathbf{8 a}$.

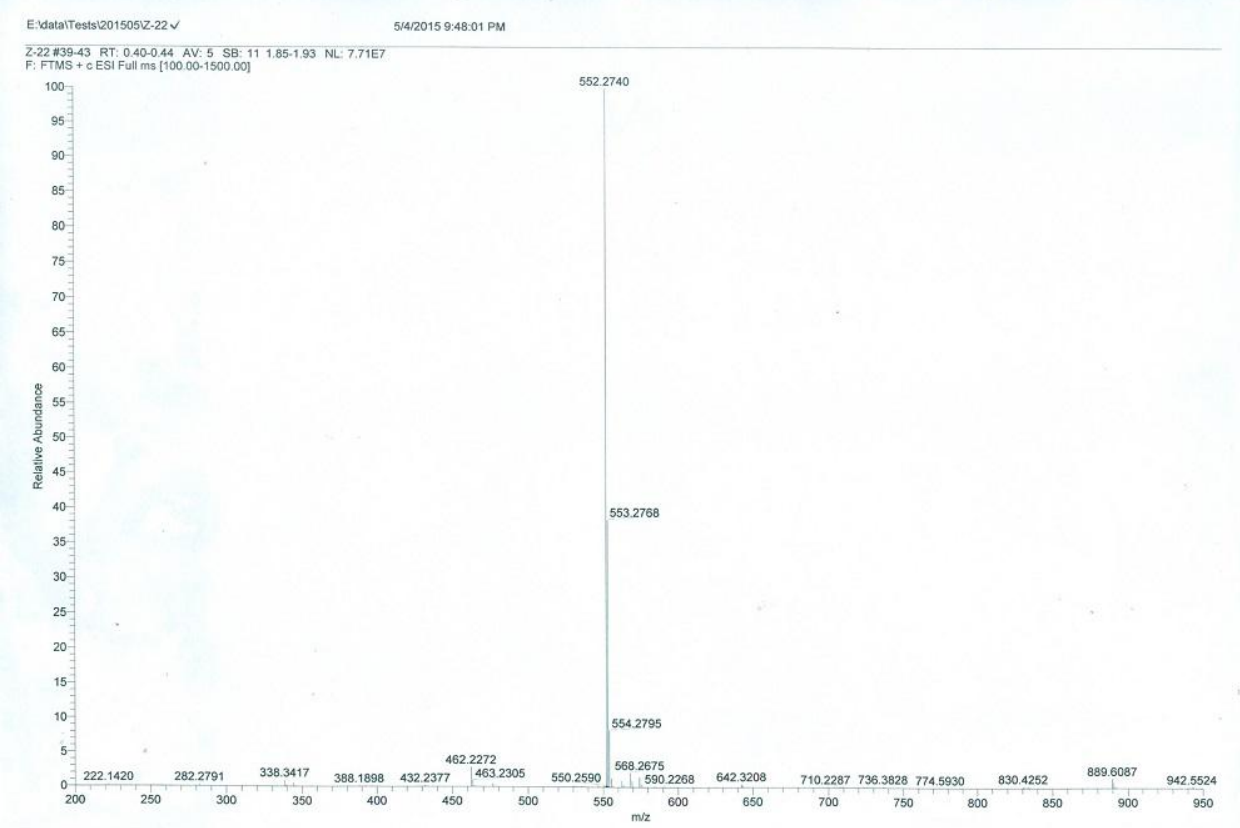

Figure S153. HRESIMS spectrum of $\mathbf{8 b}$. 

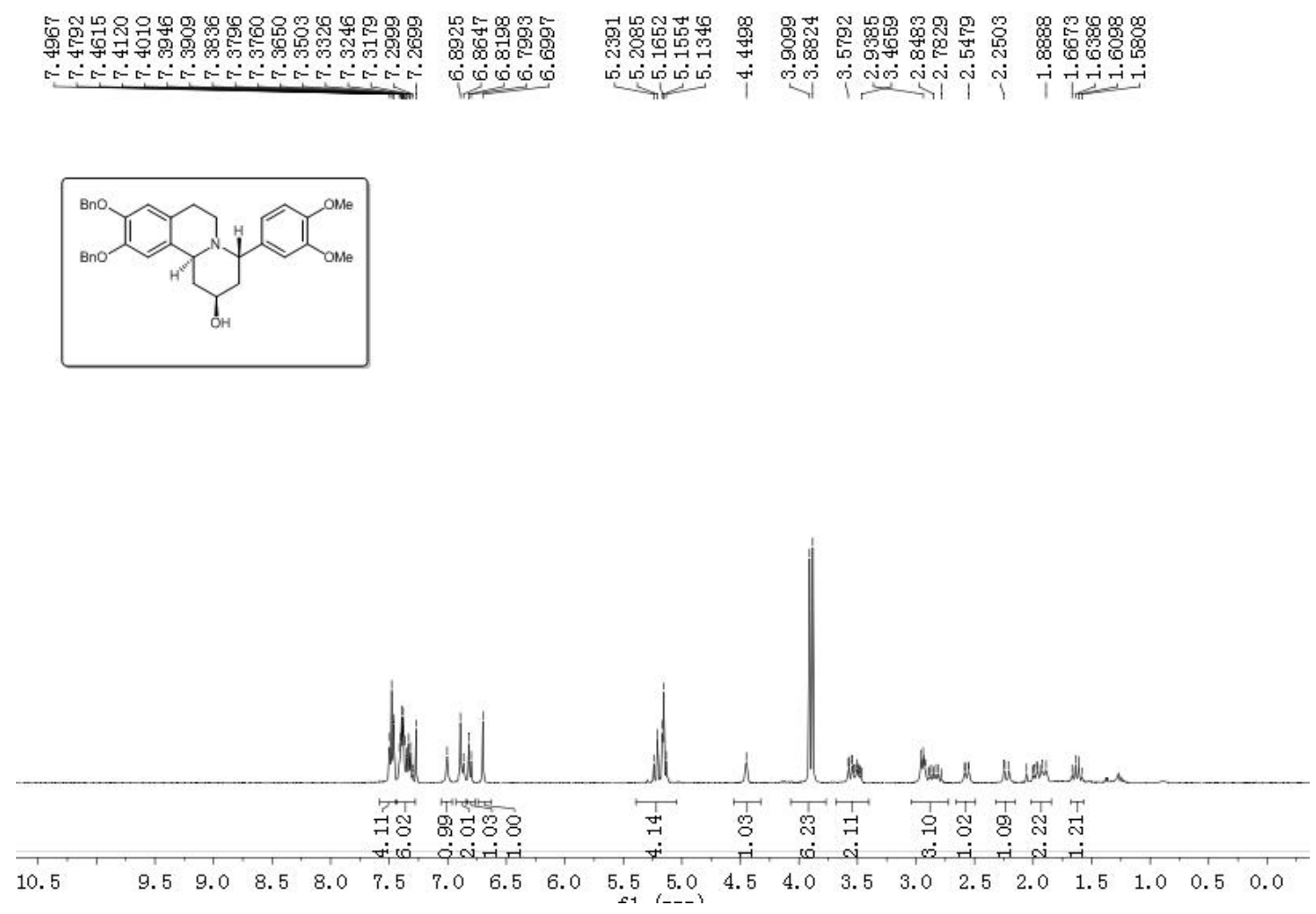

Figure S154. ${ }^{1} \mathrm{H}$ NMR spectrum of $\mathbf{8 b}$.

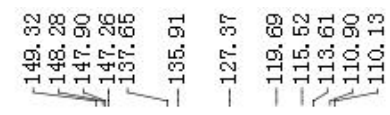

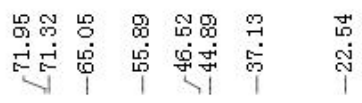
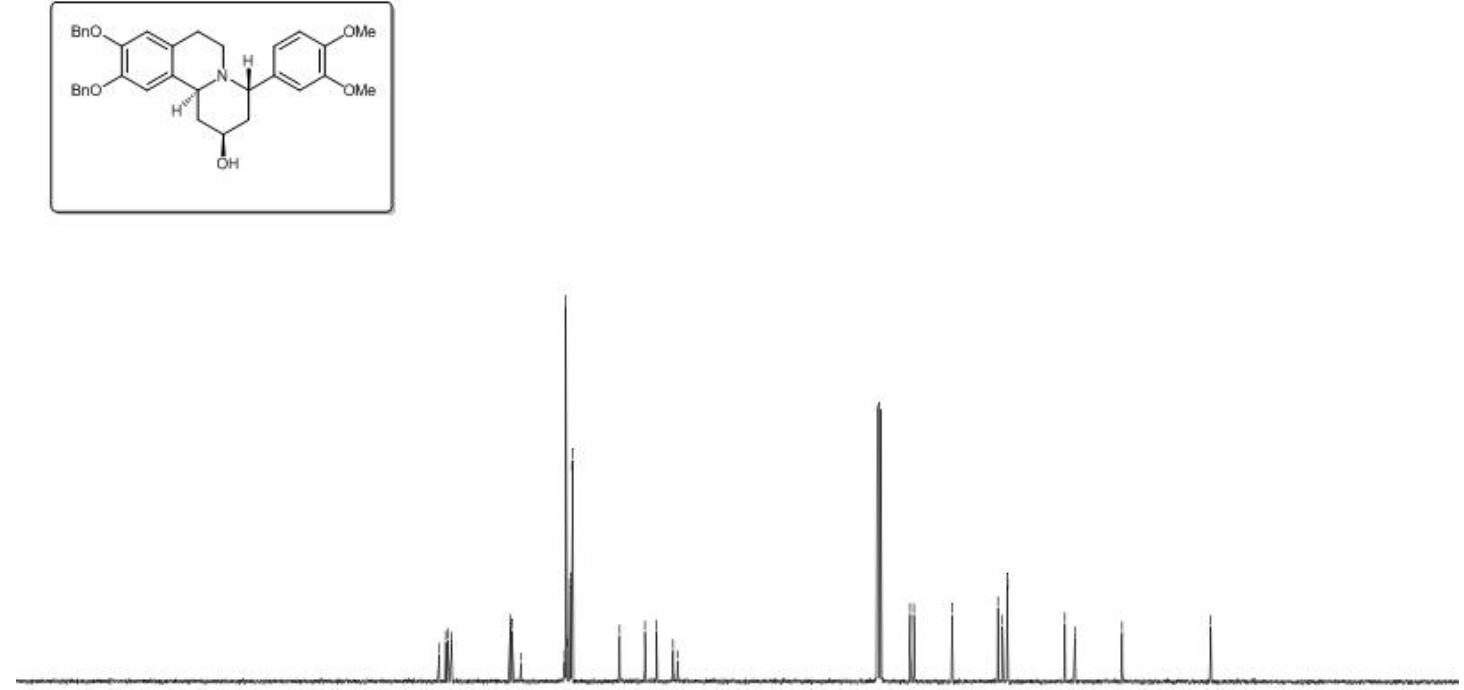

$\begin{array}{llllll}210 & 190 & 170 & 150 & 130 & 110\end{array}$

Figure $\mathbf{S 1 5 5} .{ }^{13} \mathrm{C}$ NMR spectrum of $\mathbf{8 b}$. 

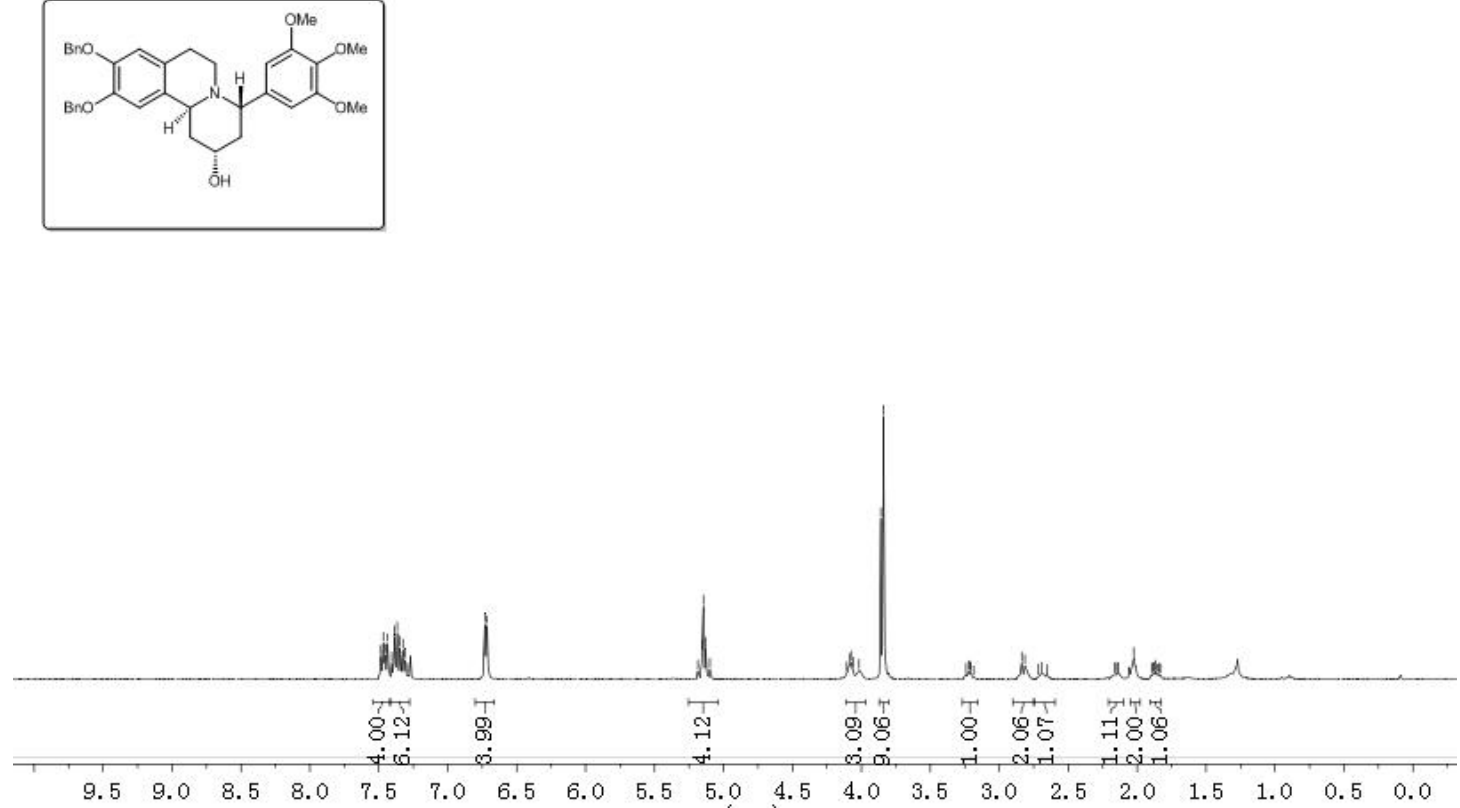

Figure S156. ${ }^{1} \mathrm{H}$ NMR spectrum of 9 a.

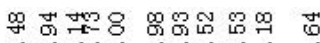

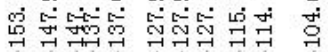
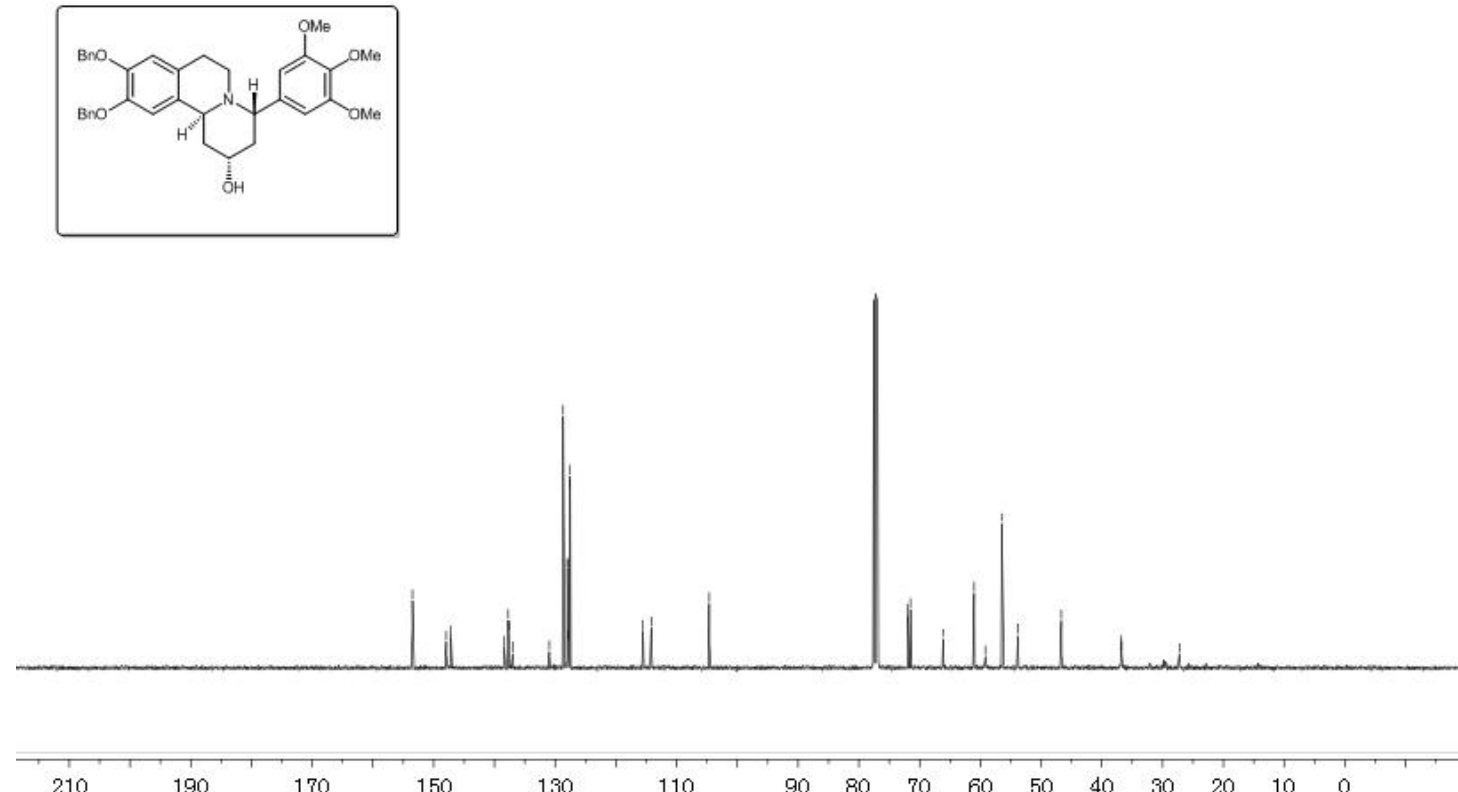

Figure $\mathbf{S 1 5 7} .{ }^{13} \mathrm{C}$ NMR spectrum of $9 \mathbf{a}$. 


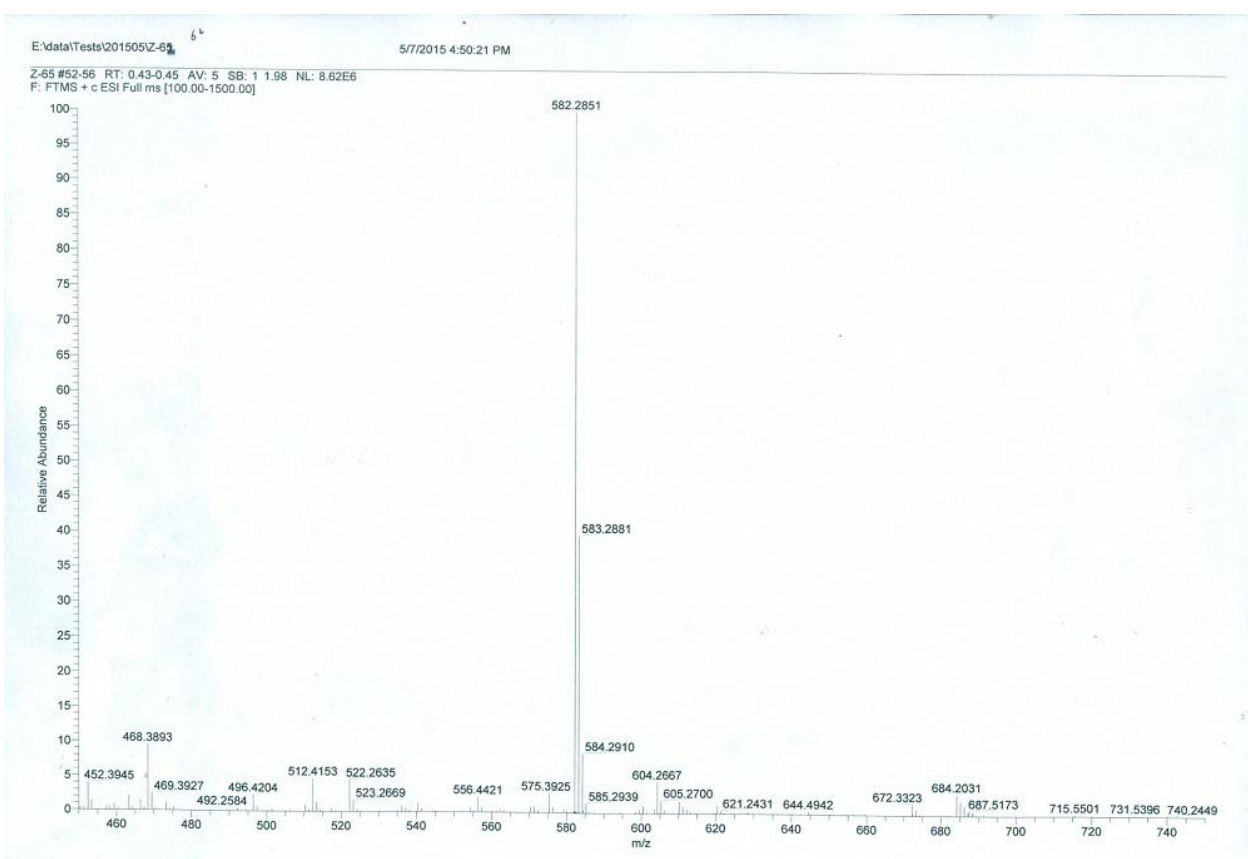

Figure S158. HRESIMS spectrum of $\mathbf{9 b}$.
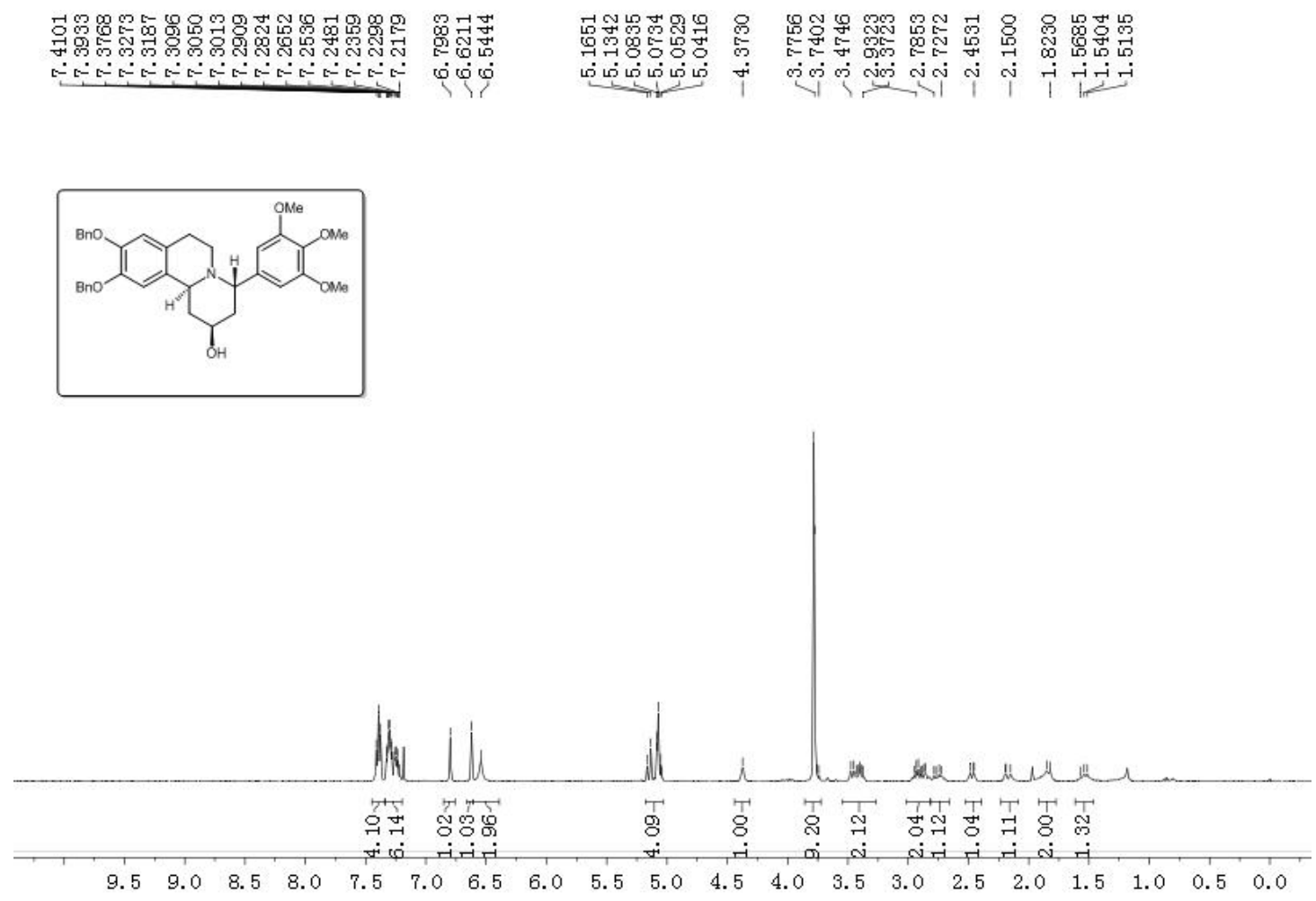

Figure S159. ${ }^{1} \mathrm{H}$ NMR spectrum of $\mathbf{9 b}$. 

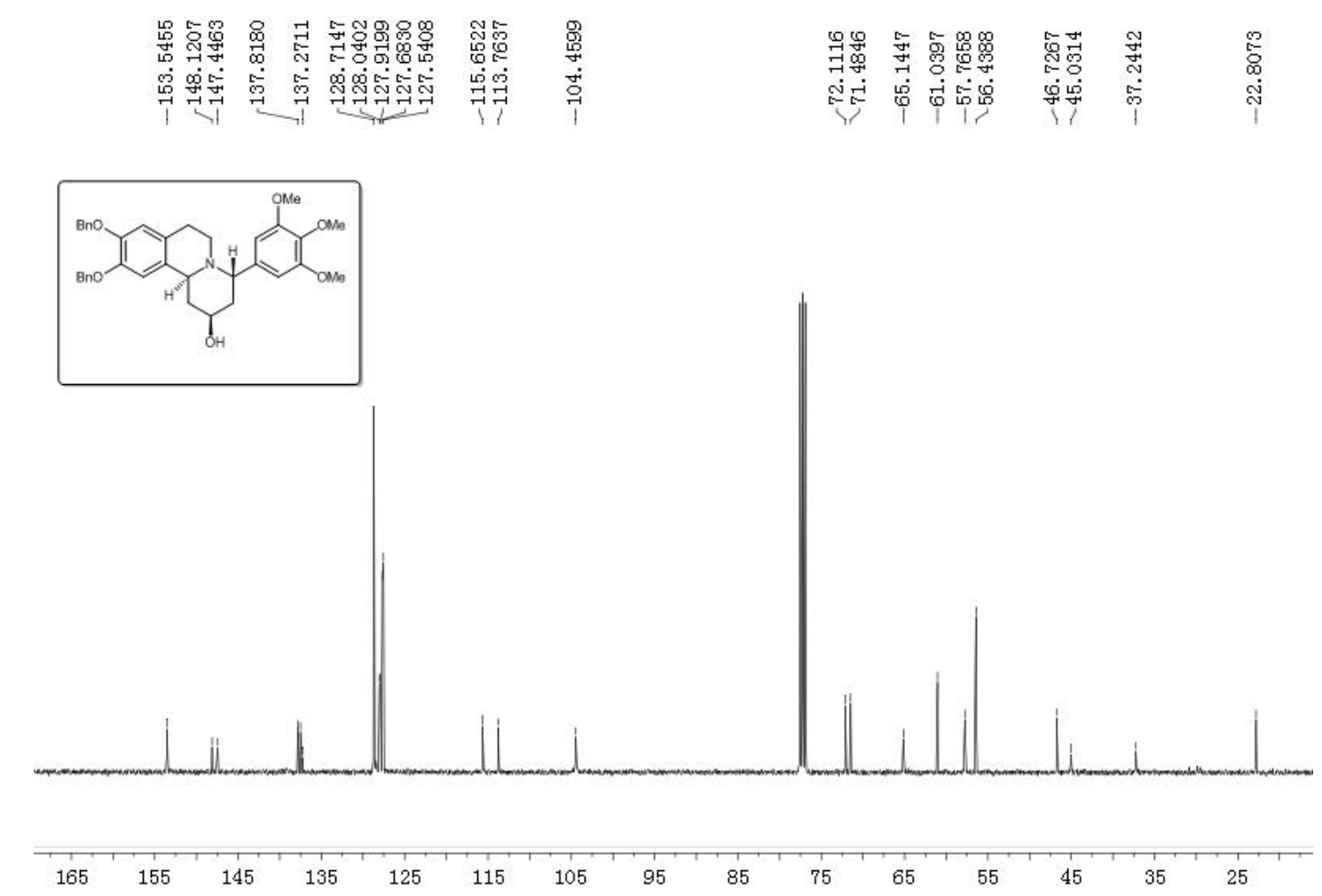

Figure S160. ${ }^{13} \mathrm{C}$ NMR spectrum of $9 \mathbf{b}$.
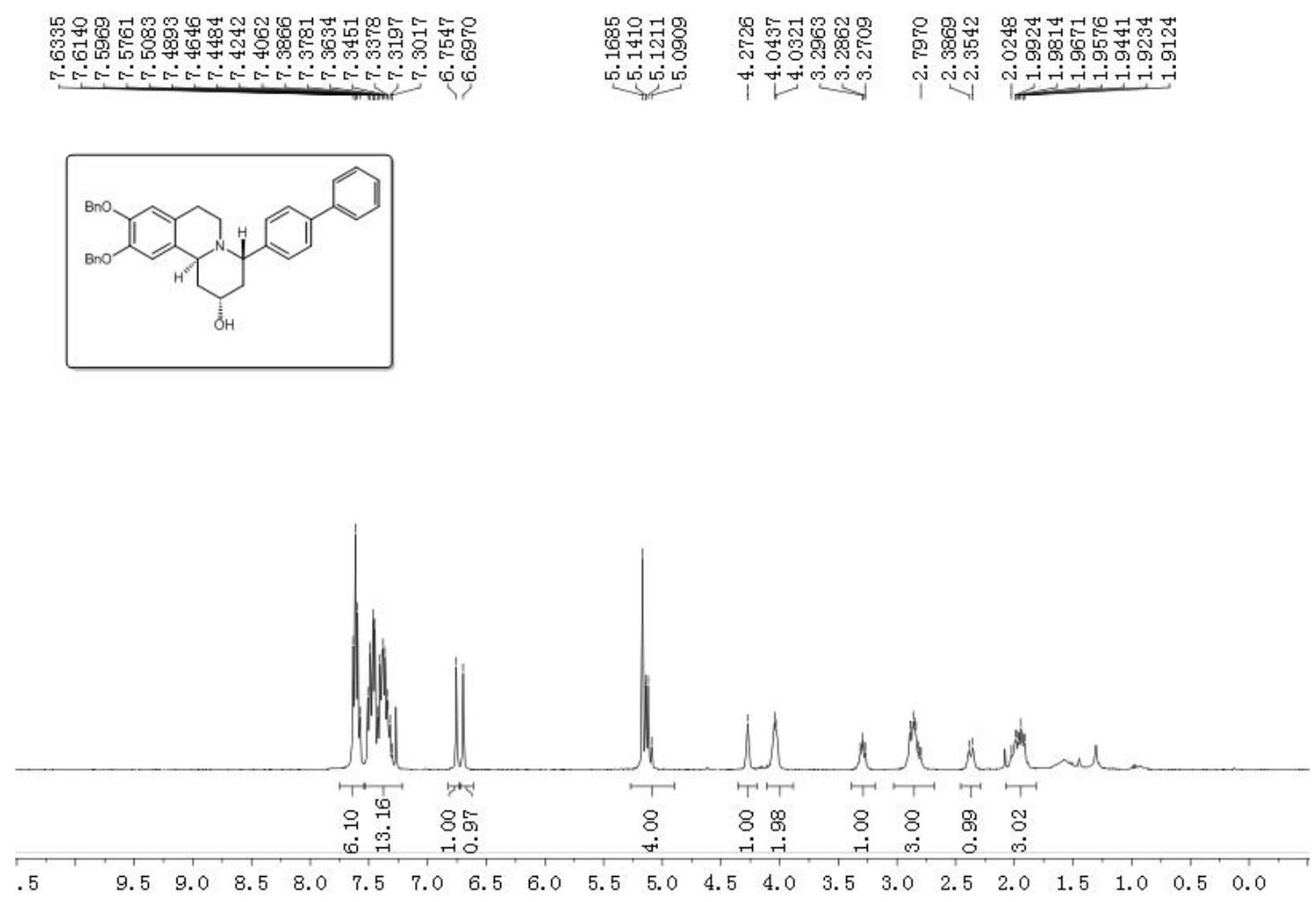

Figure S161. ${ }^{1} \mathrm{H}$ NMR spectrum of 10a. 

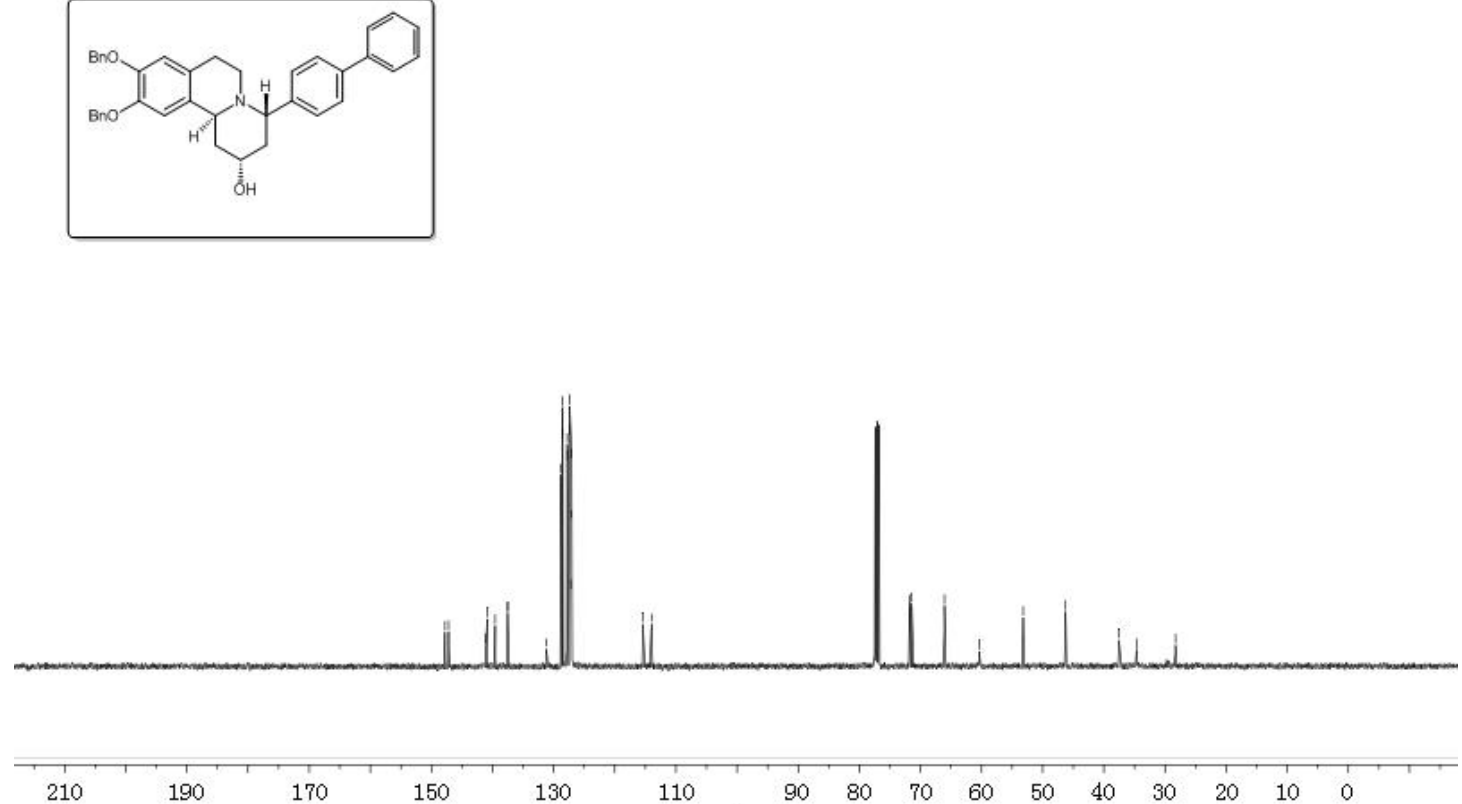

Figure S162. ${ }^{13} \mathrm{C}$ NMR spectrum of 10a.

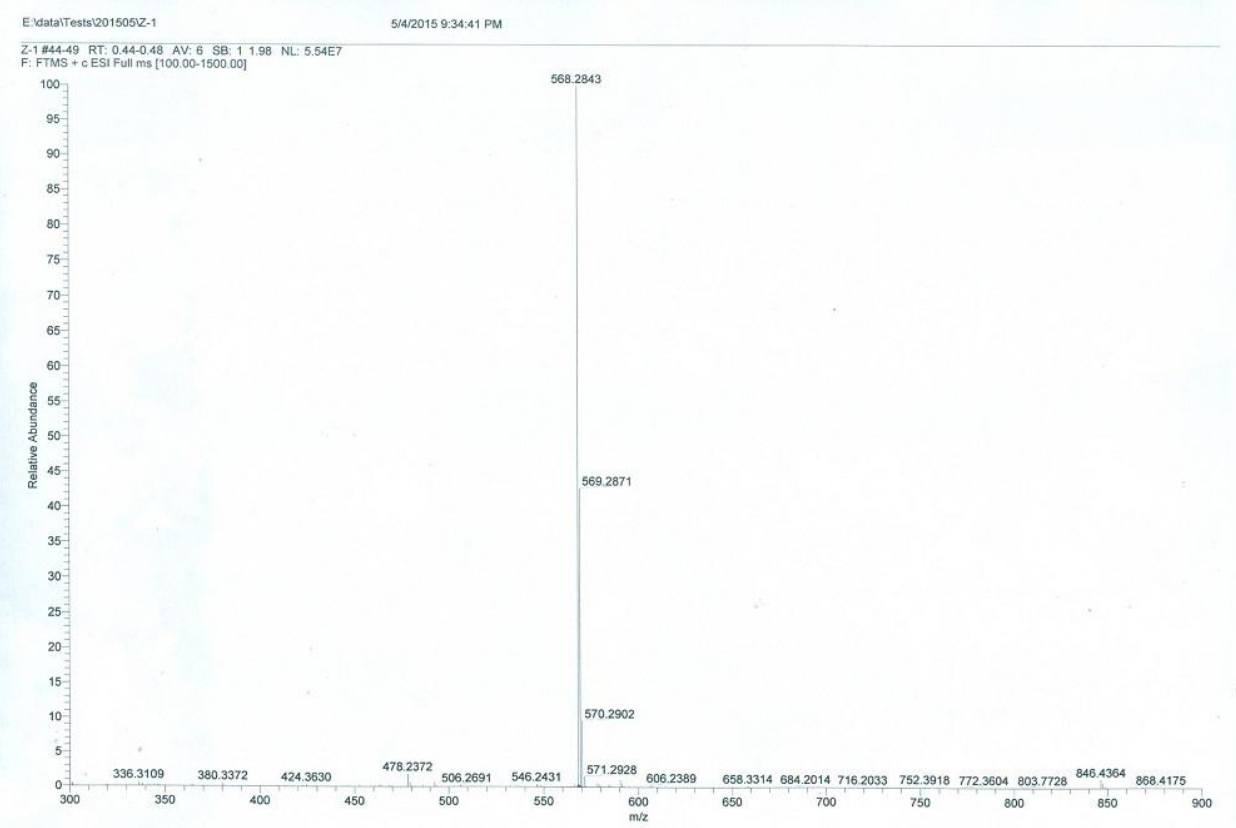

Figure S163. HRESIMS spectrum of $\mathbf{1 0 b}$. 

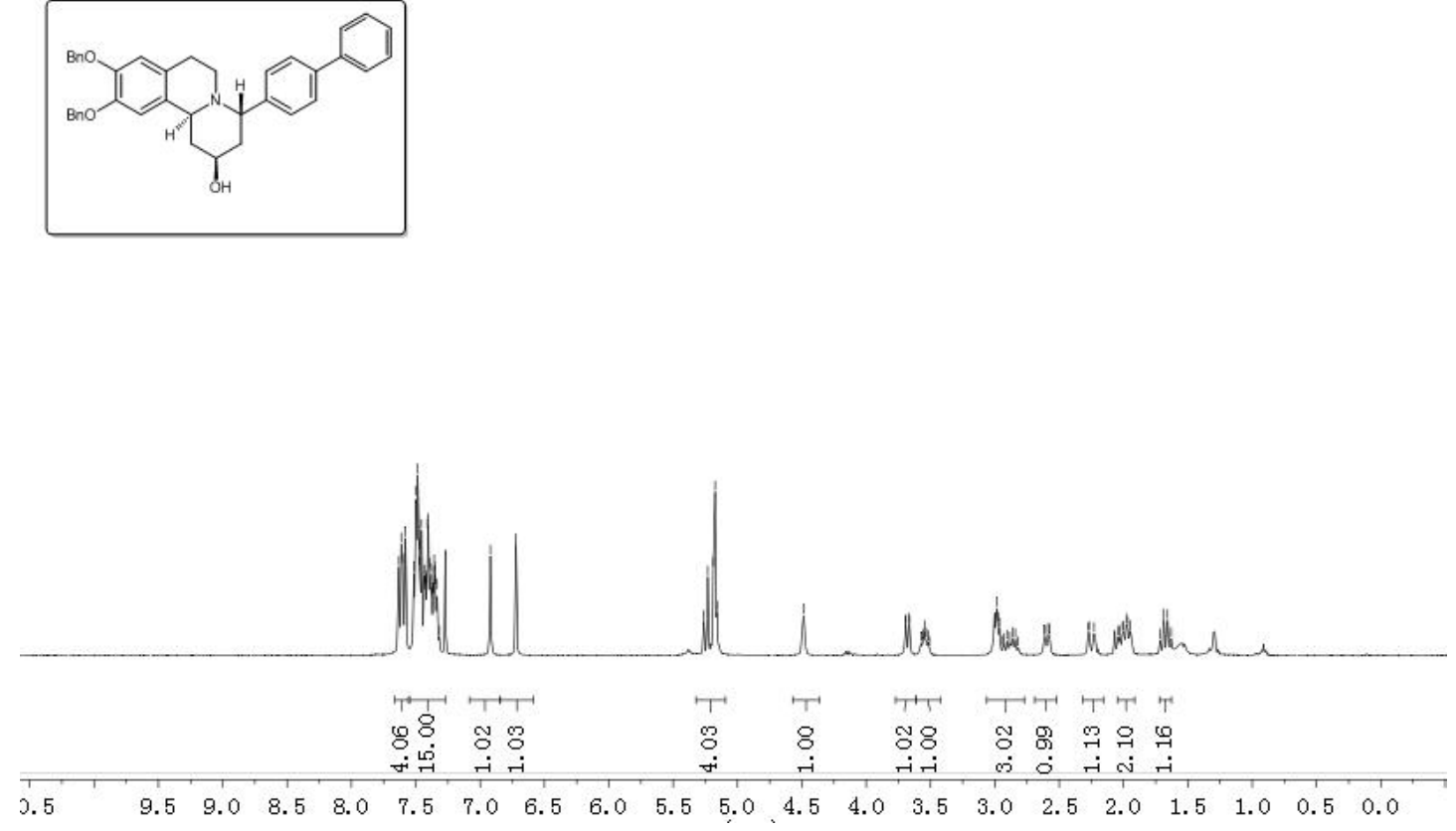

Figure S164. ${ }^{1} \mathrm{H}$ NMR spectrum of $\mathbf{1 0 b}$

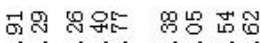

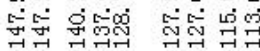

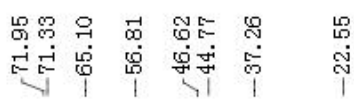
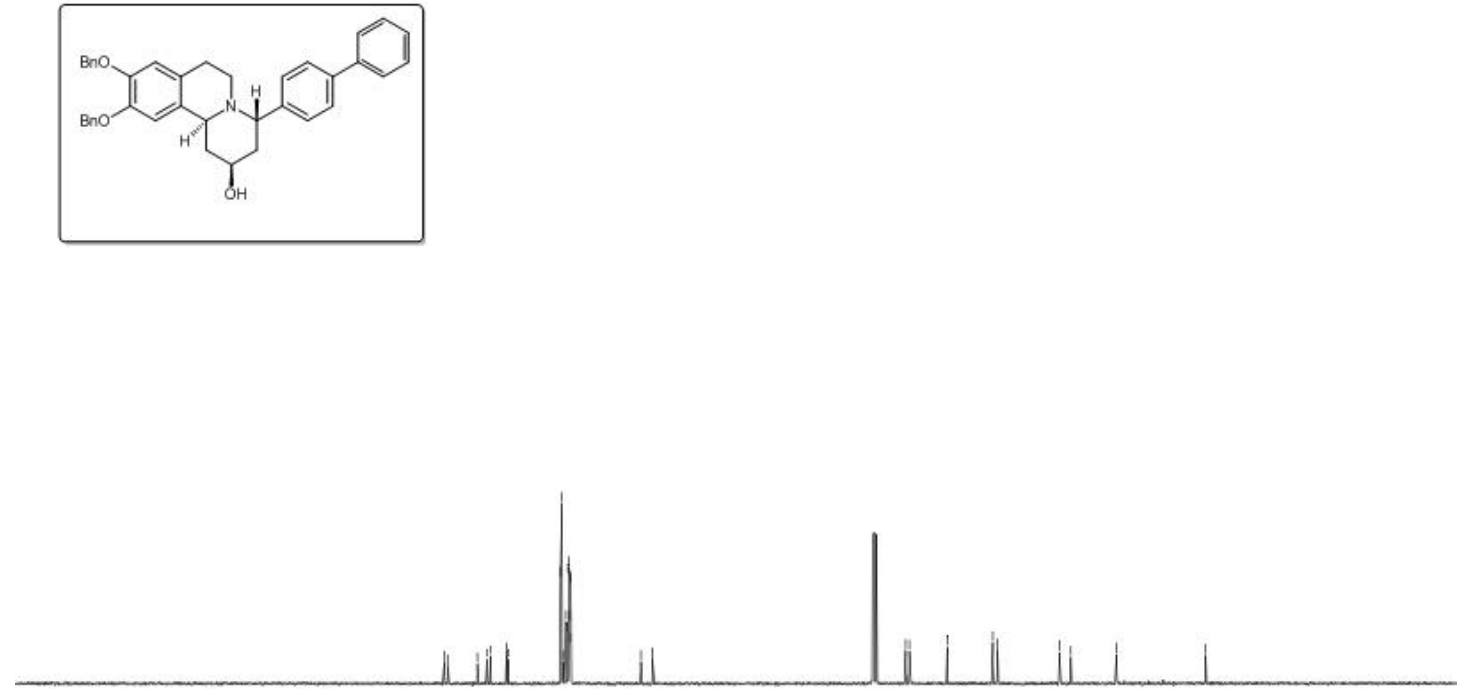

Figure S165. ${ }^{13} \mathrm{C}$ NMR spectrum of $\mathbf{1 0 b}$. 


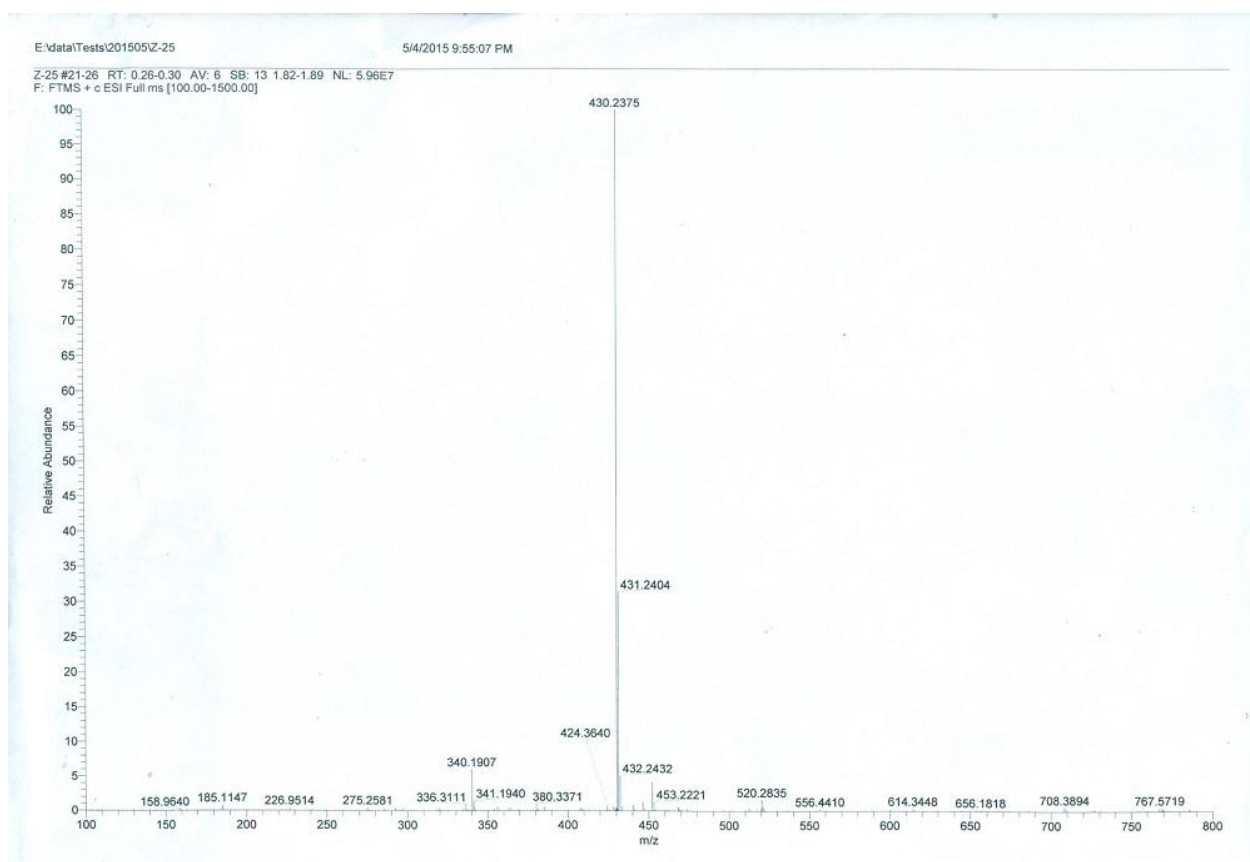

Figure S166. HRESIMS spectrum of 11a.

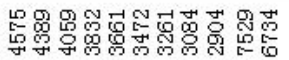

$\therefore<-60$
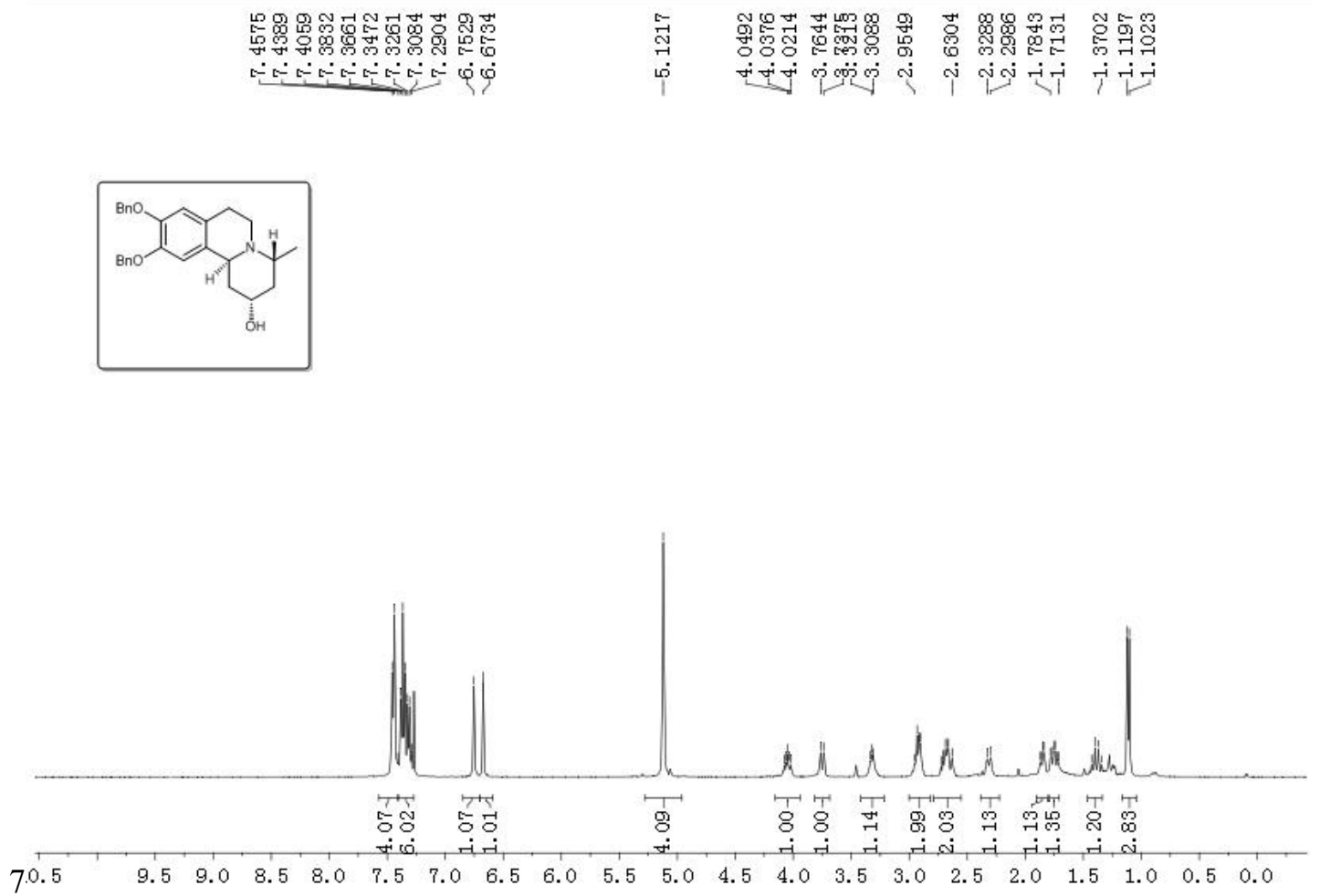

Figure S167. ${ }^{1} \mathrm{H}$ NMR spectrum of $\mathbf{1 1 a}$. 

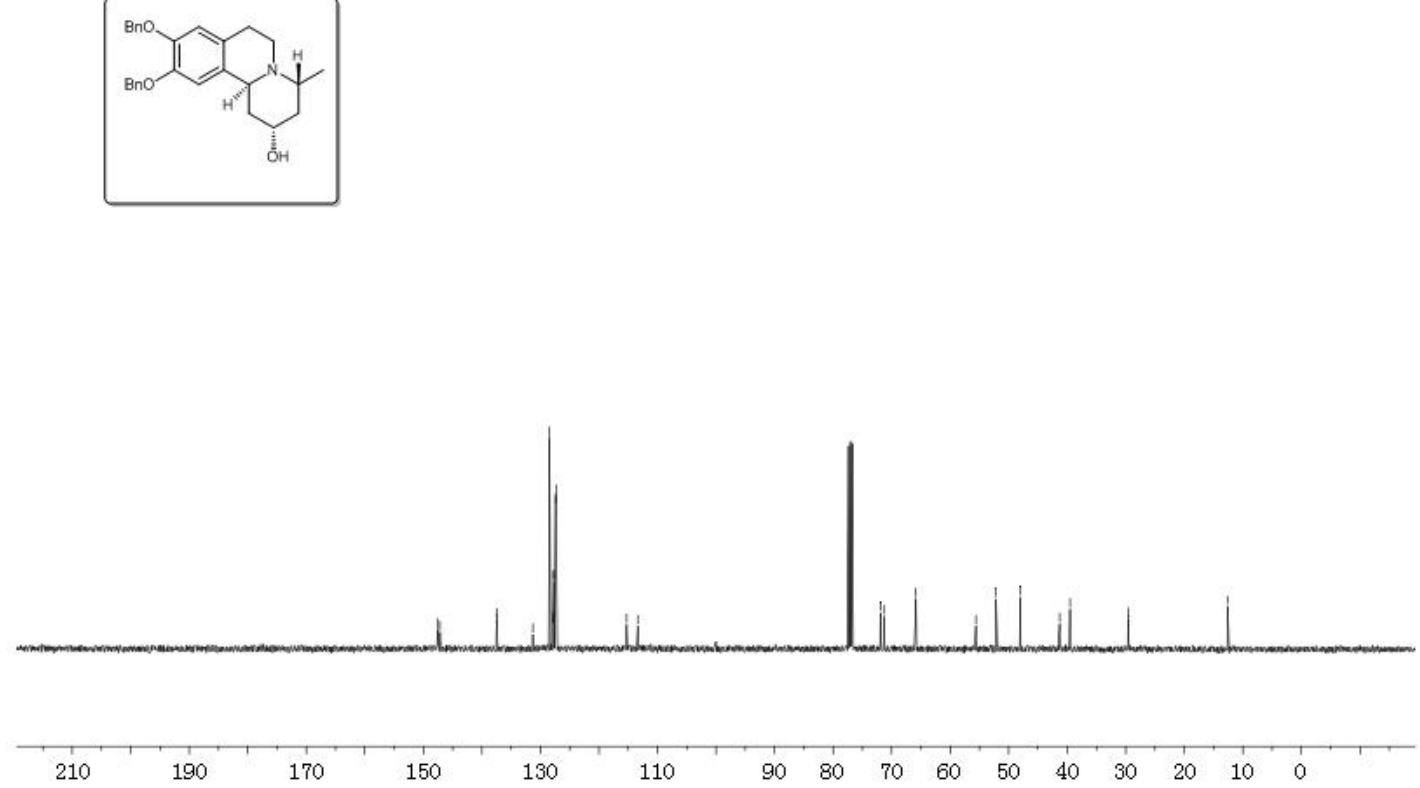

Figure S168. ${ }^{13} \mathrm{C}$ NMR spectrum of $\mathbf{1 1 a}$.

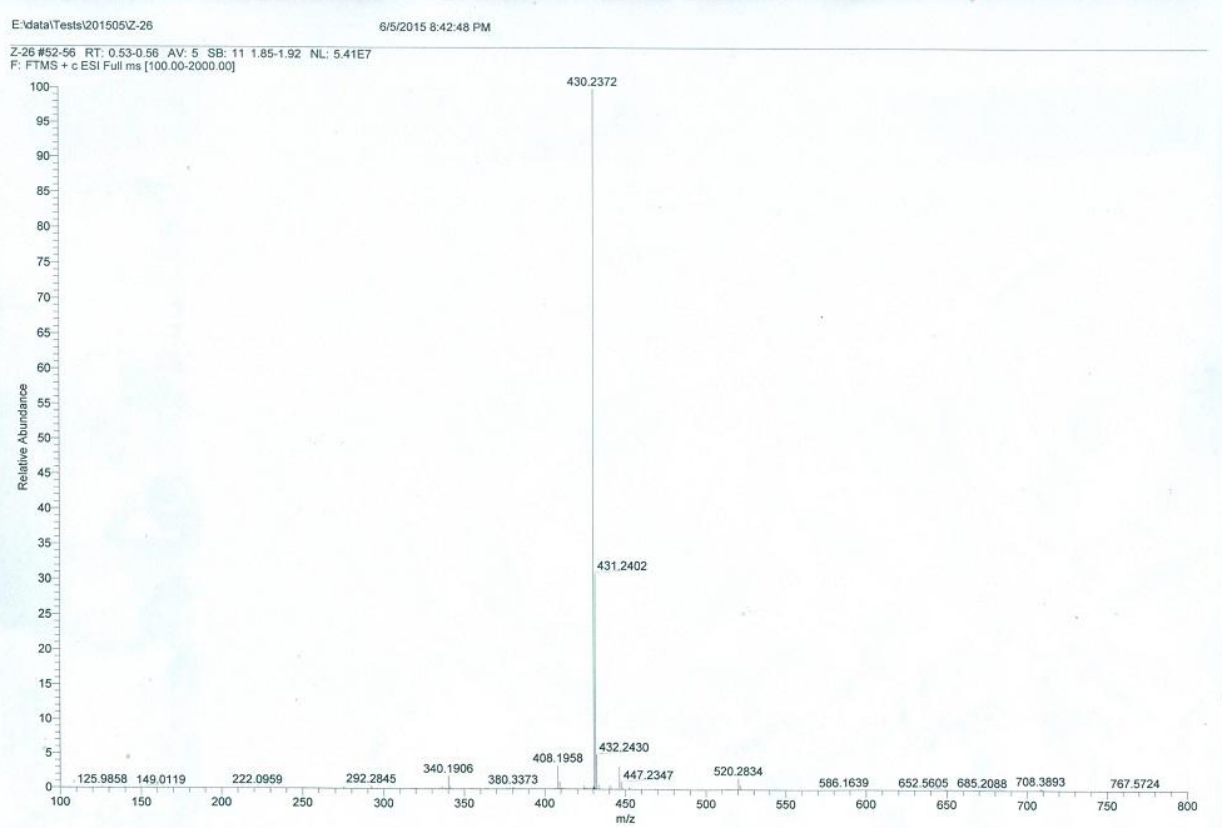

Figure S169. HRESIMS spectrum of $\mathbf{1 1 b}$. 

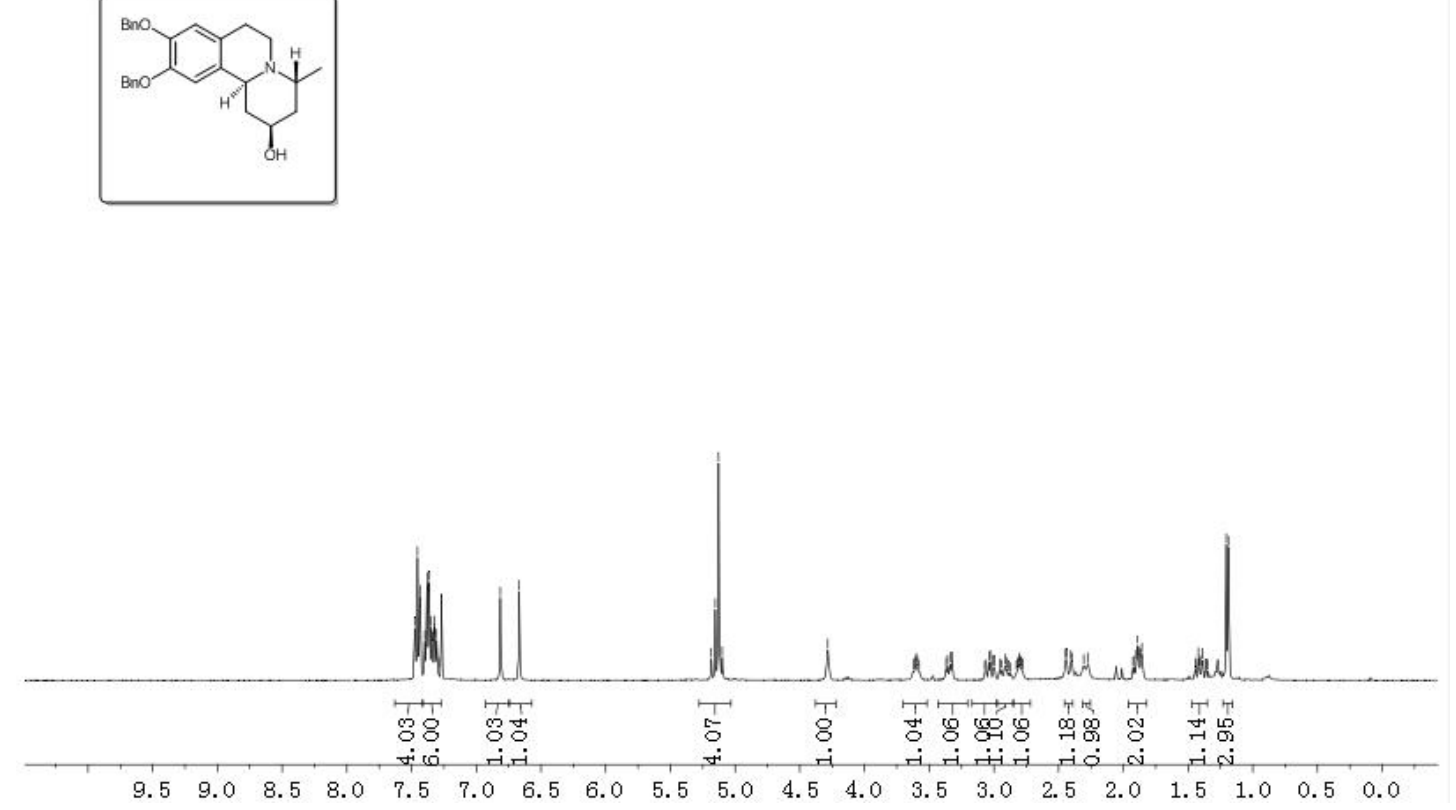

Figure S170. ${ }^{1} \mathrm{H}$ NMR spectrum of $\mathbf{1 1 b}$.
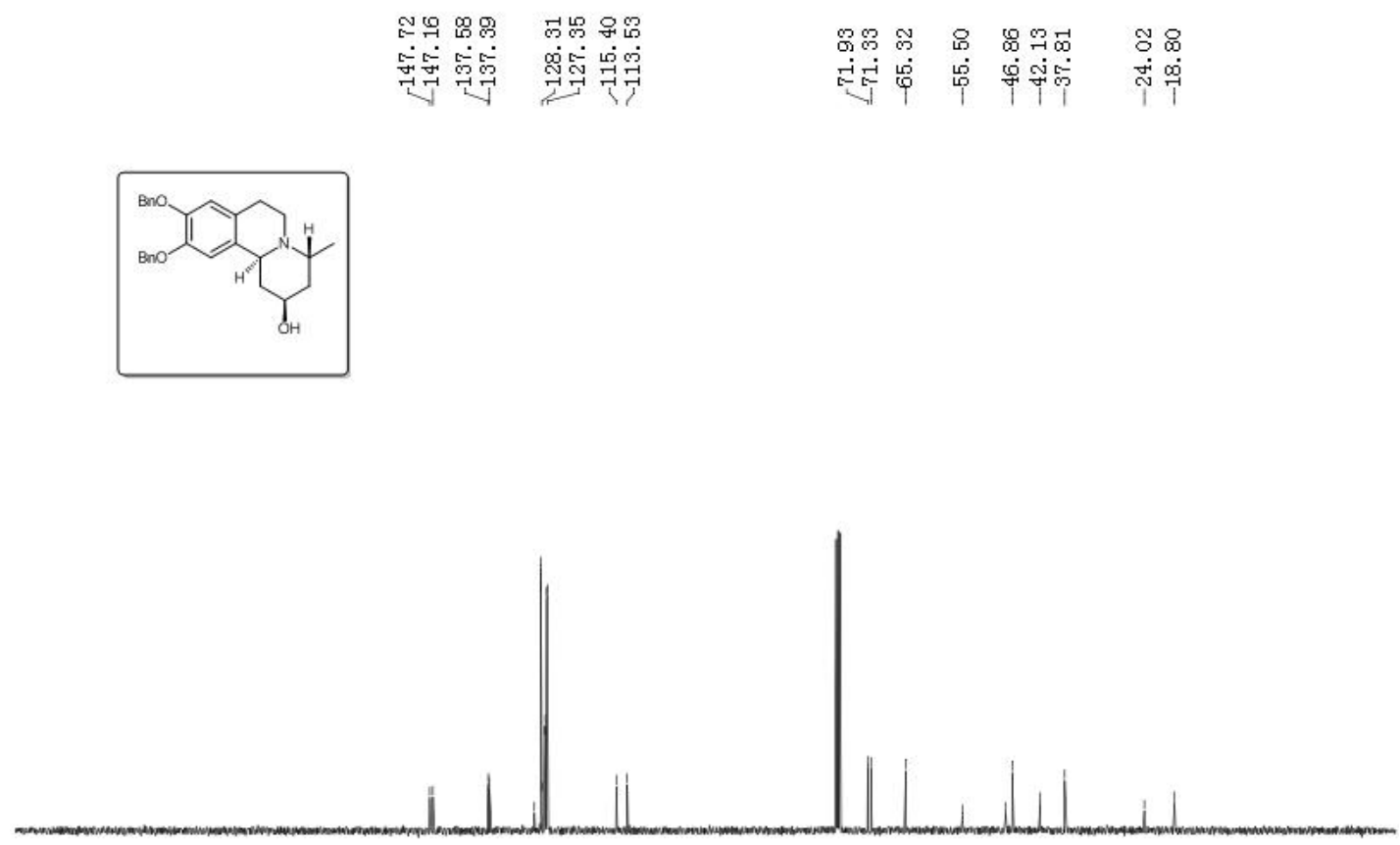

$210 \quad 190 \quad 170 \quad 150$

Figure S171. ${ }^{13} \mathrm{C}$ NMR spectrum of $\mathbf{1 1 b}$. 


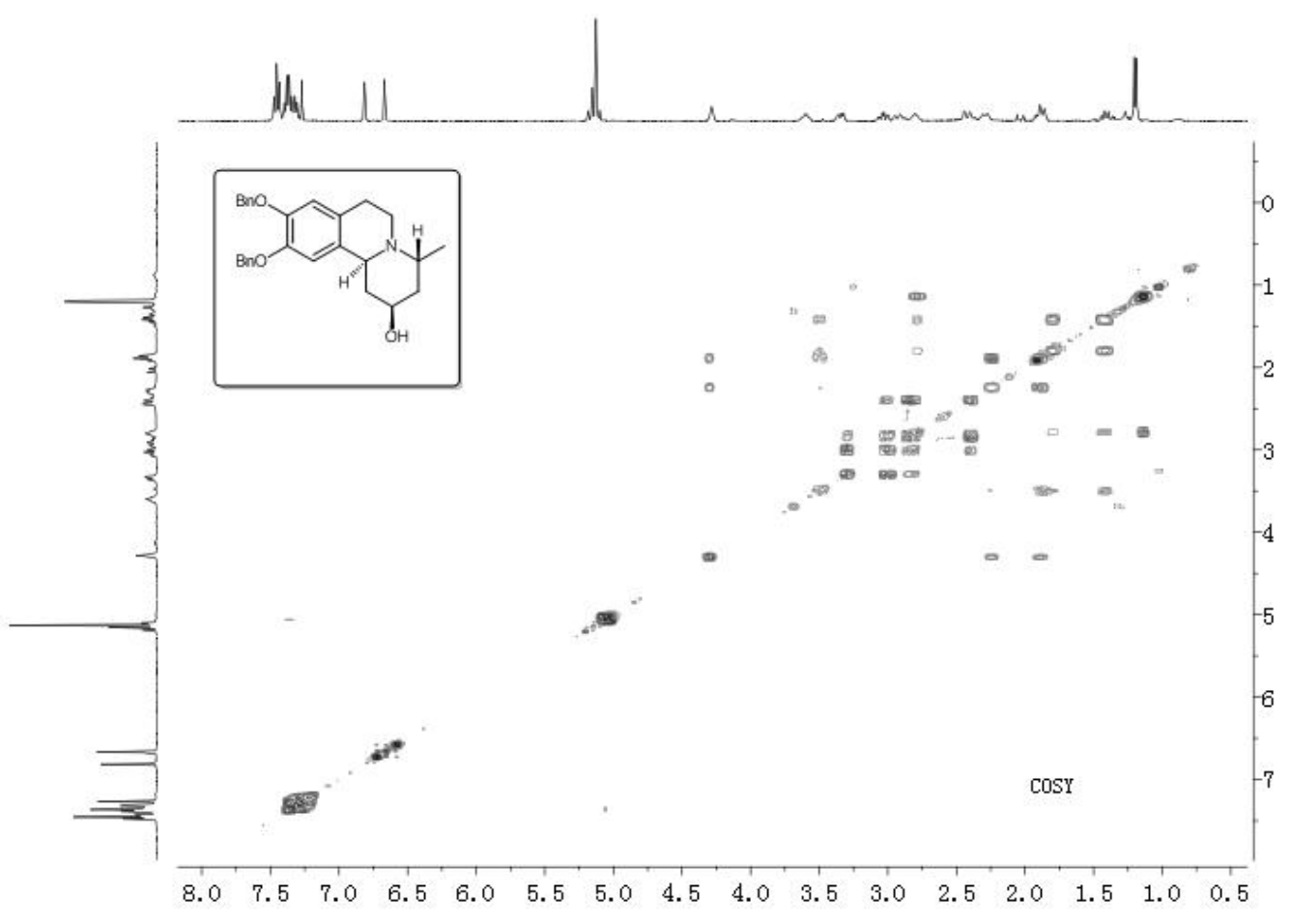

Figure S172. COSY spectrum of $\mathbf{1 1 b .}$

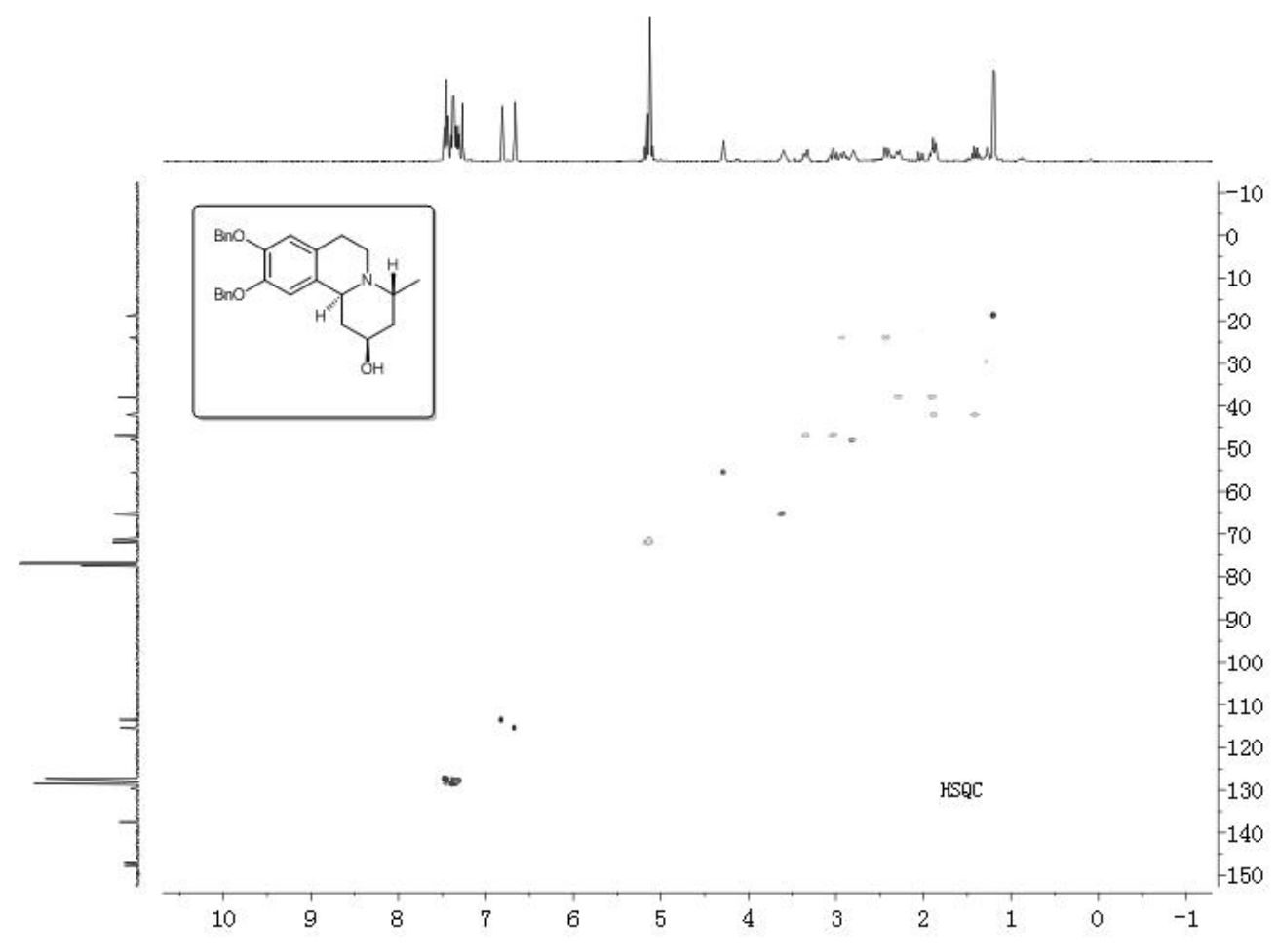

Figure S173. HSQC spectrum of $\mathbf{1 1 b}$. 


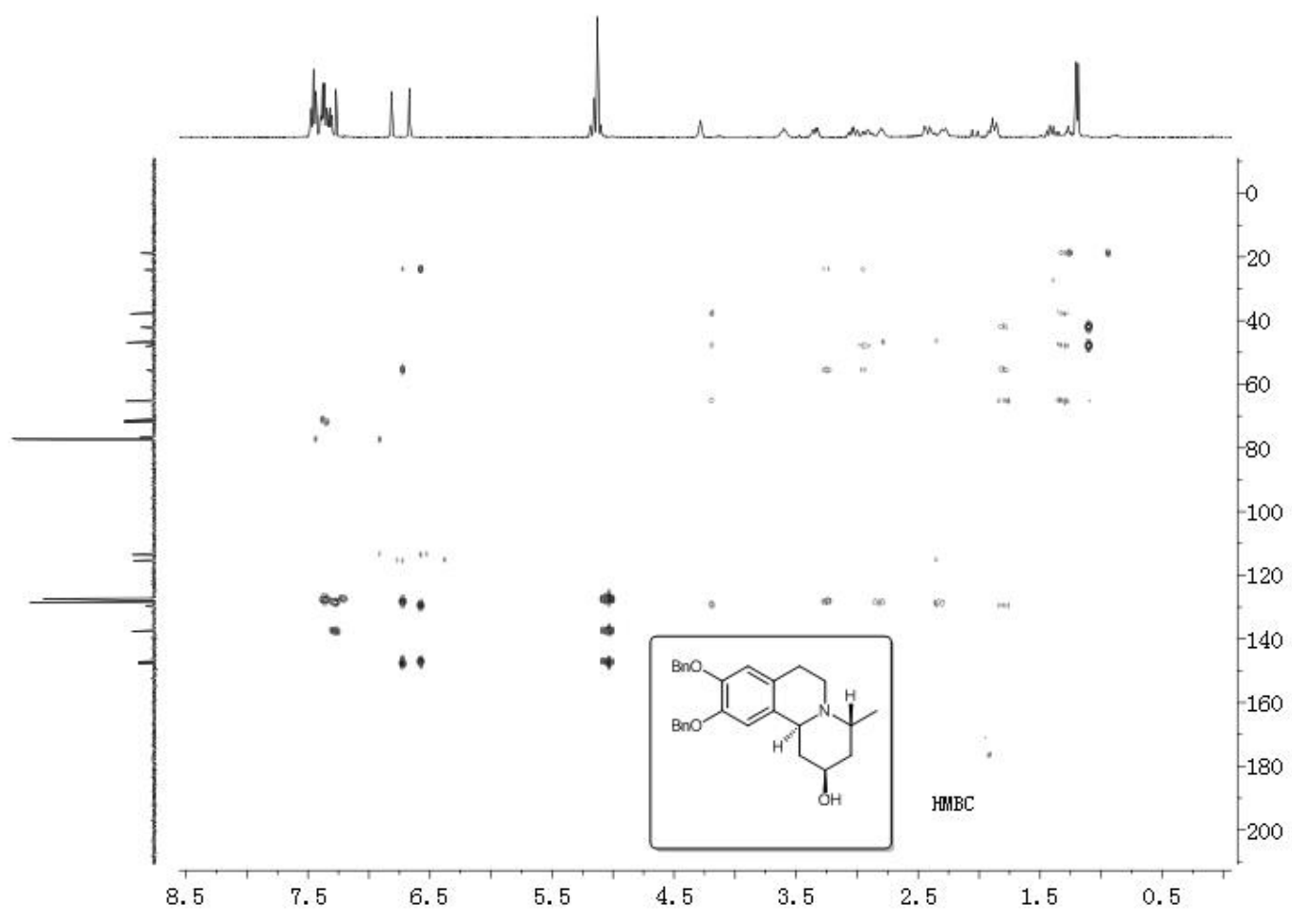

Figure S174. HMBC spectrum of 11b.

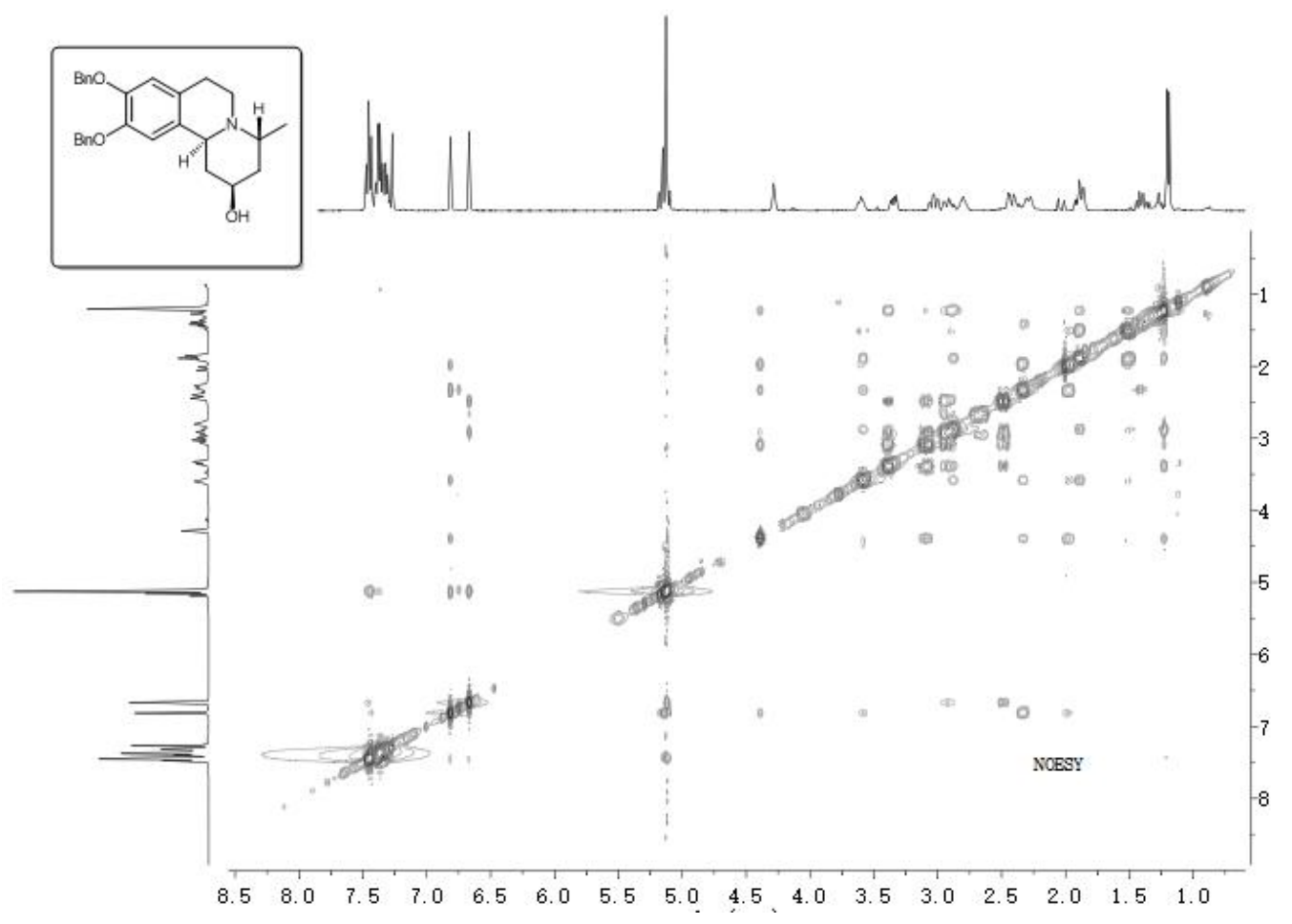

Figure S175. NOESY spectrum of $\mathbf{1 1 b}$. 

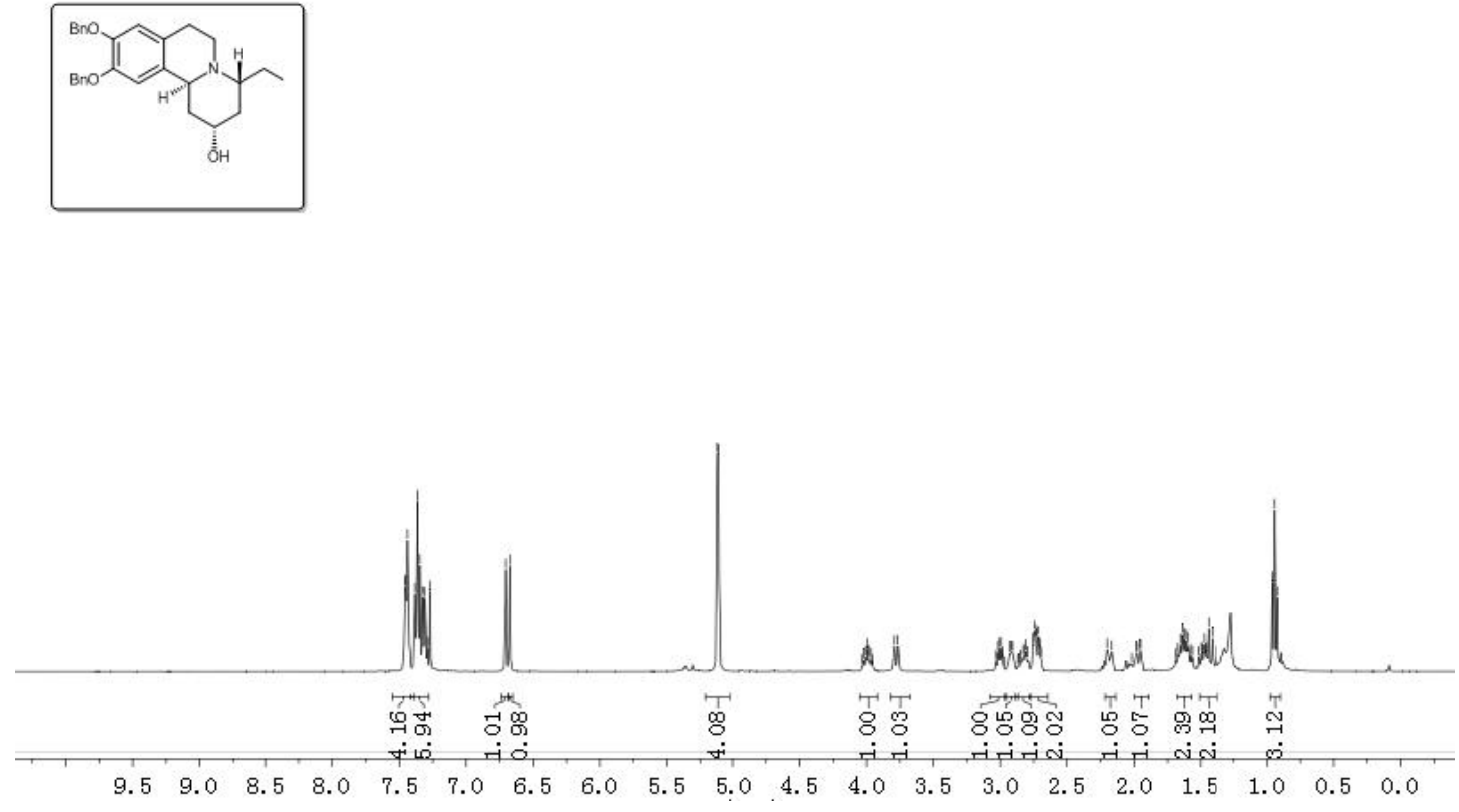

Figure S176. ${ }^{1} \mathrm{H}$ NMR spectrum of 12a.

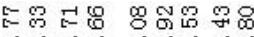

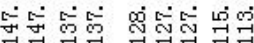

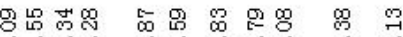

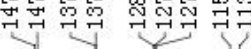

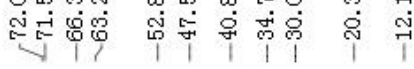
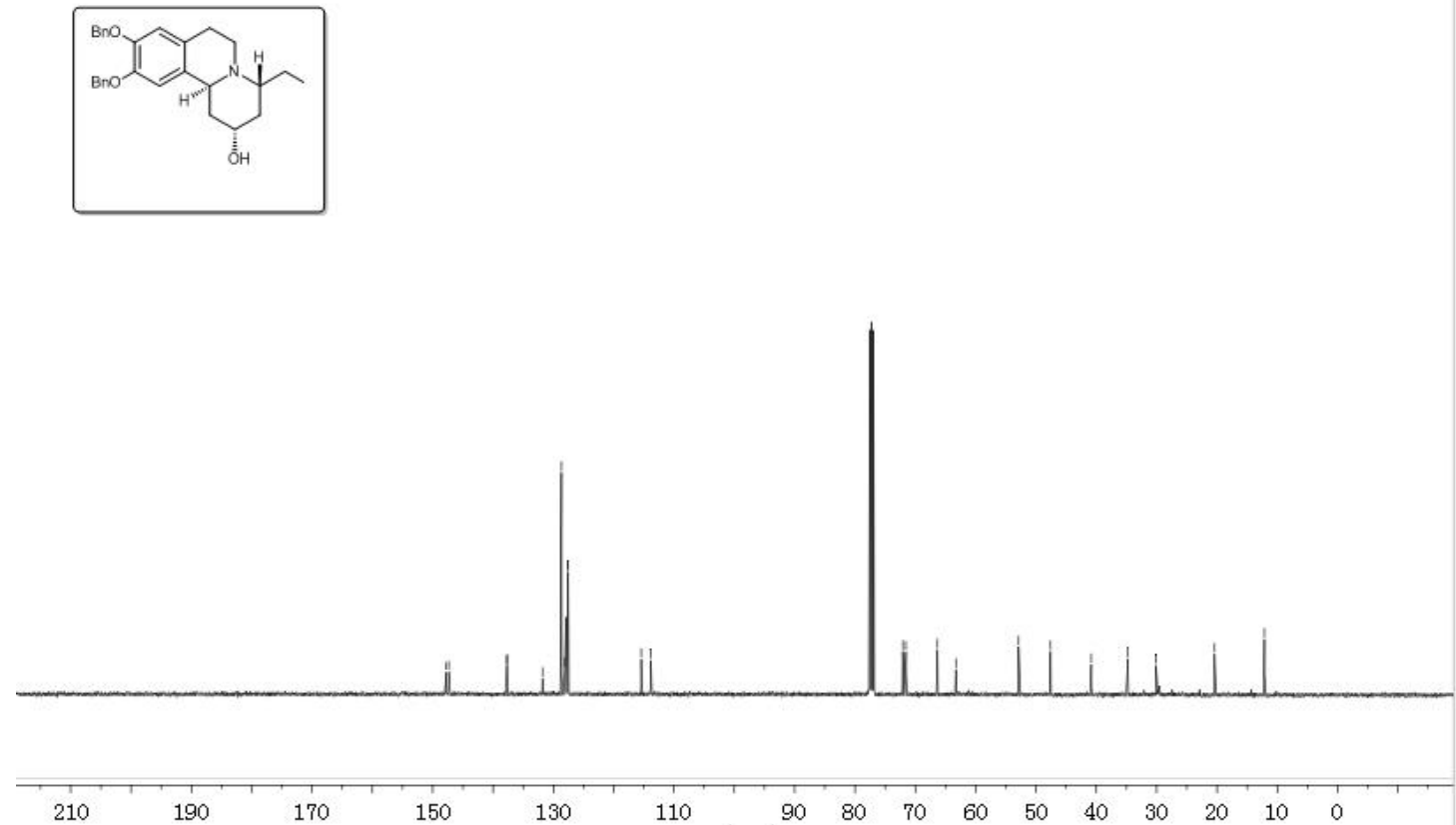

Figure S177. ${ }^{13} \mathrm{C}$ NMR spectrum of $\mathbf{1 2 a}$ 


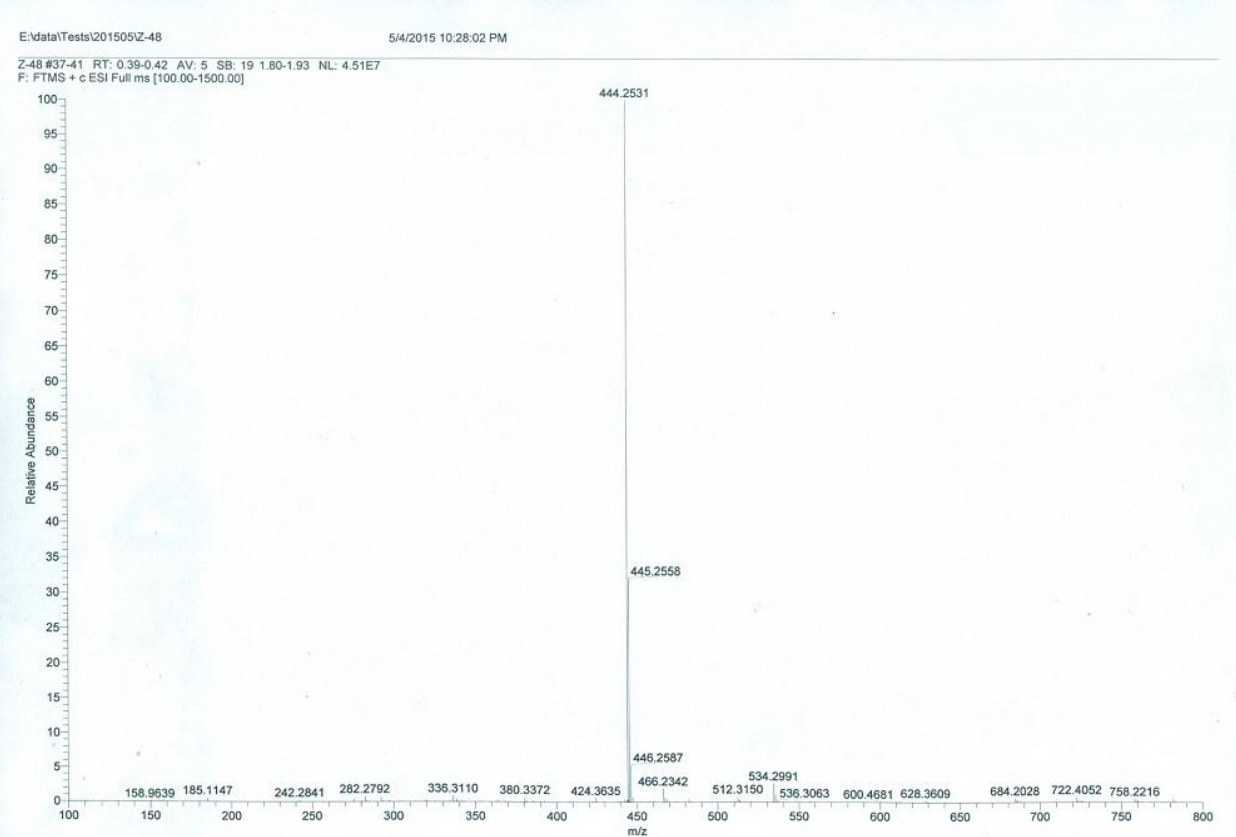

Figure S178. HRESIMS spectrum of $\mathbf{1 2 b}$.
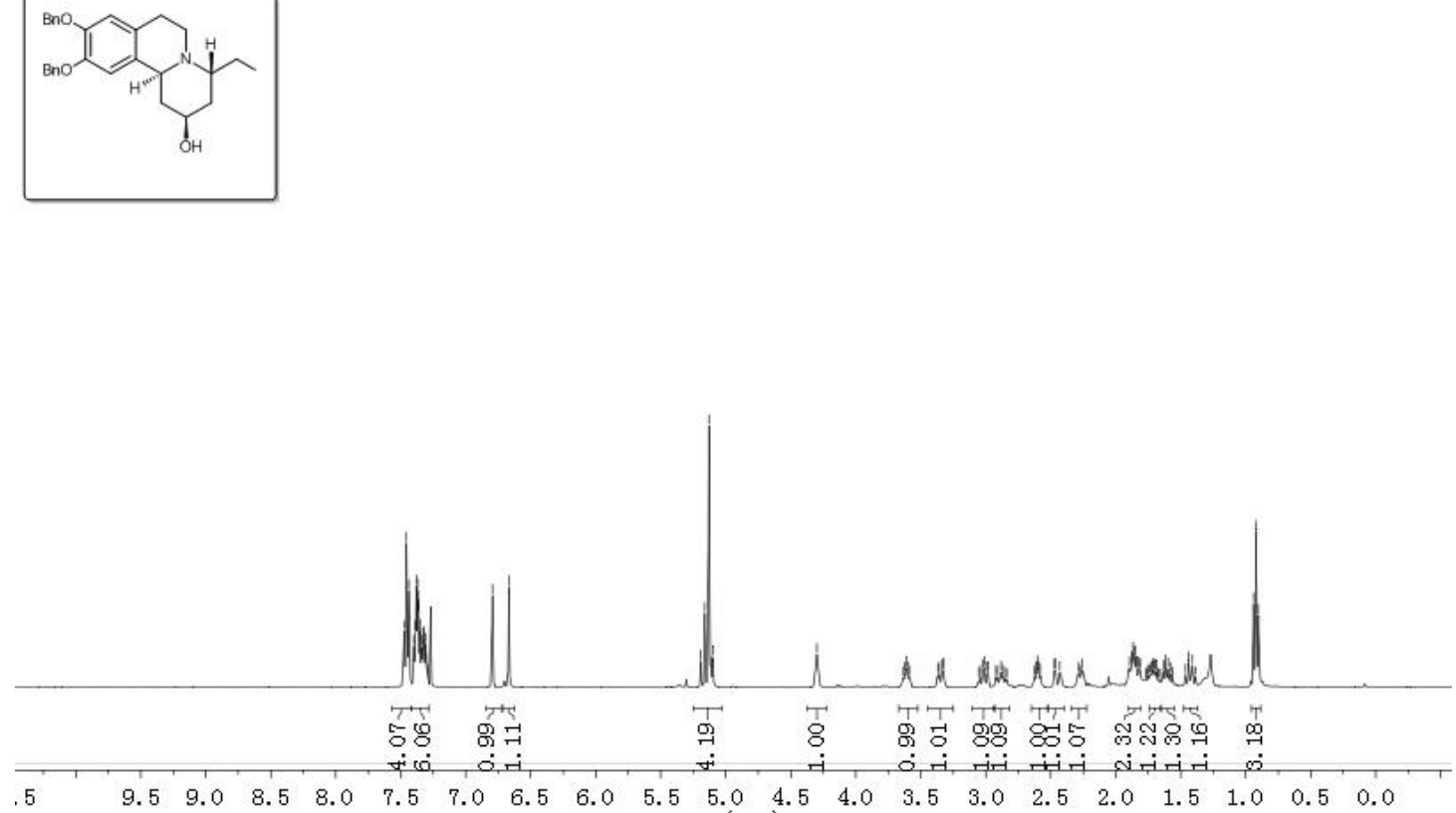

Figure S179. ${ }^{1} \mathrm{H}$ NMR spectrum of $\mathbf{1 2 b}$ 

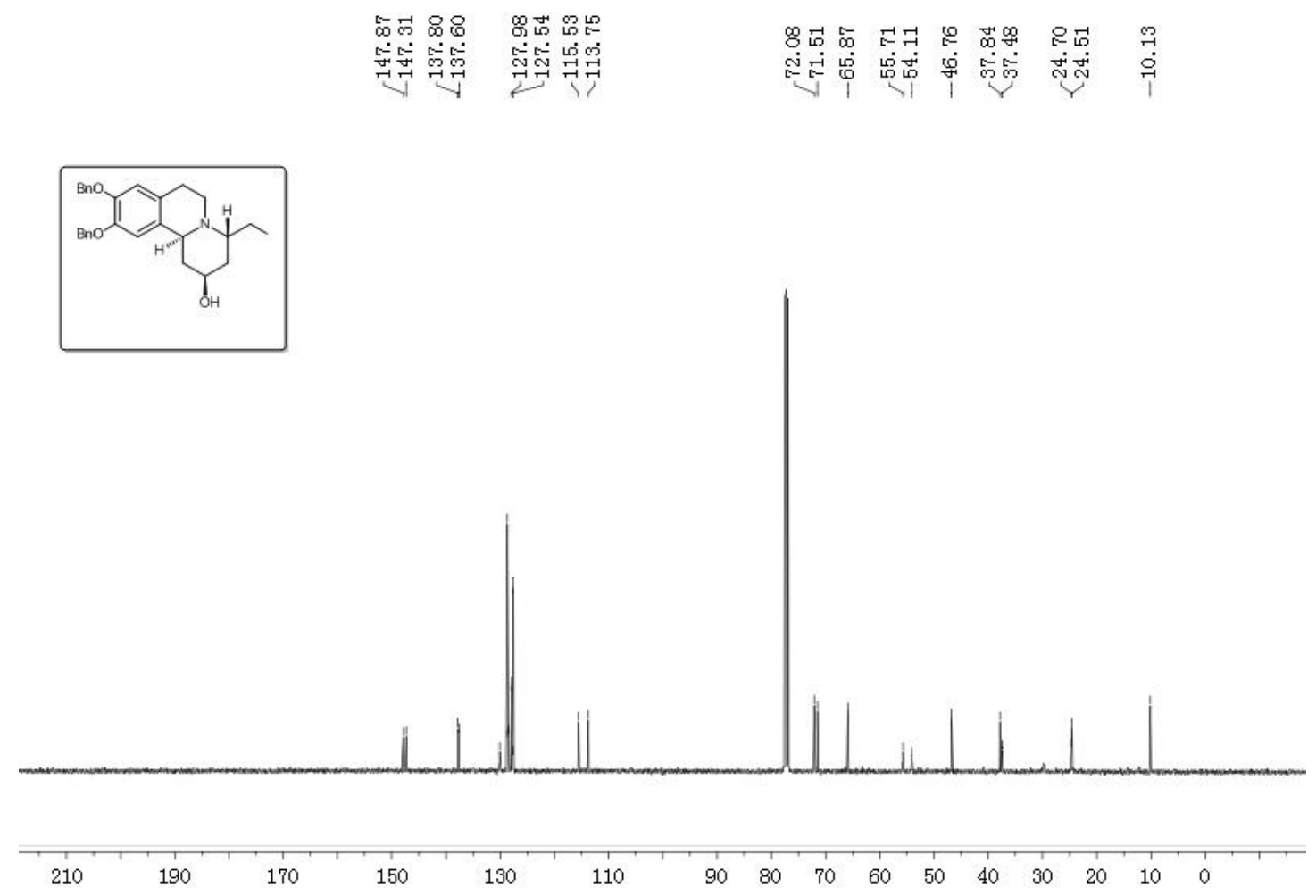

Figure S180. ${ }^{13} \mathrm{C}$ NMR spectrum of $\mathbf{1 2 b}$.
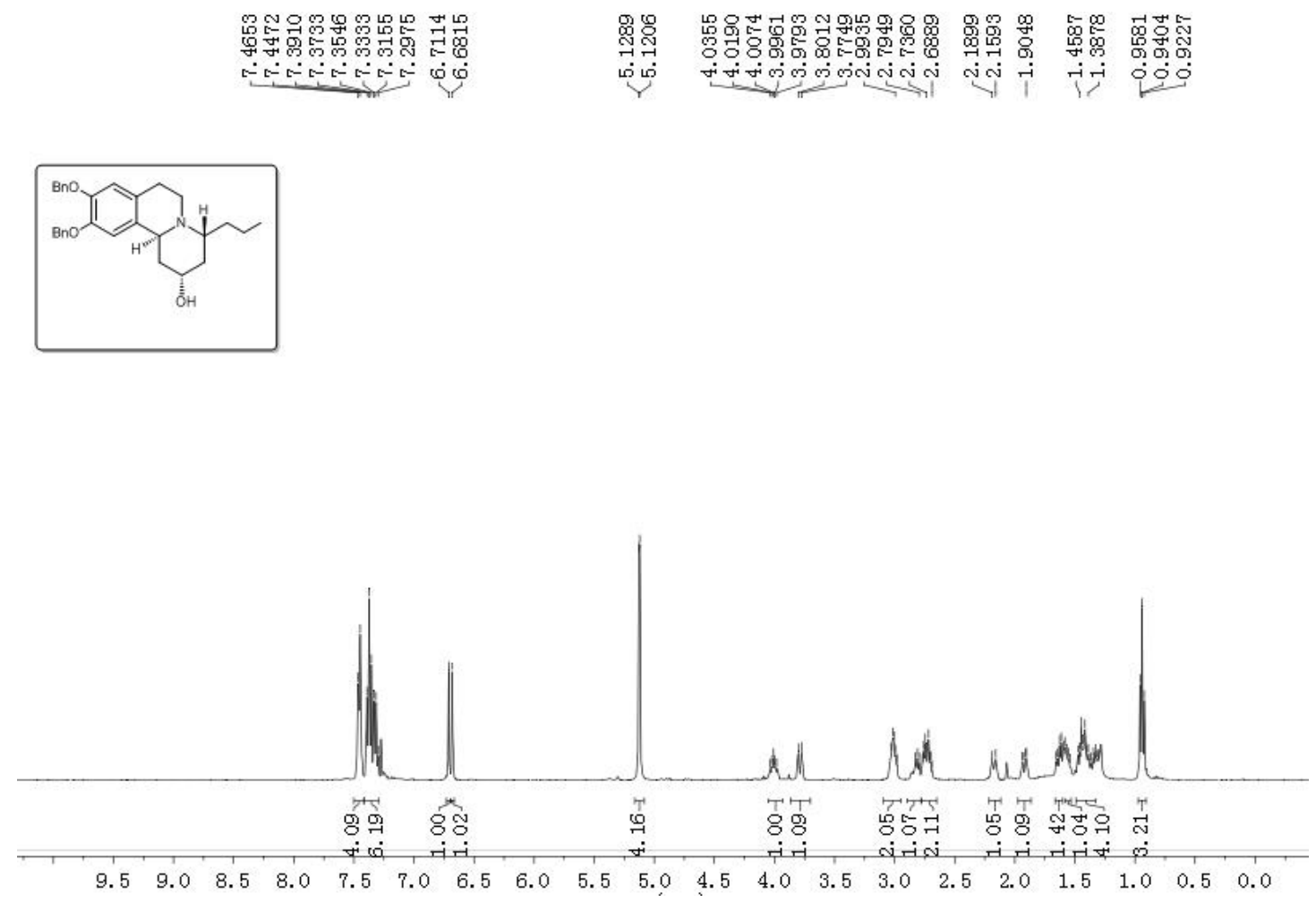

Figure S181. ${ }^{1} \mathrm{H}$ NMR spectrum of $\mathbf{1 3 a}$. 
모용

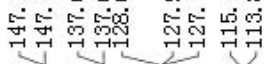

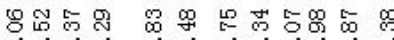

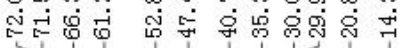
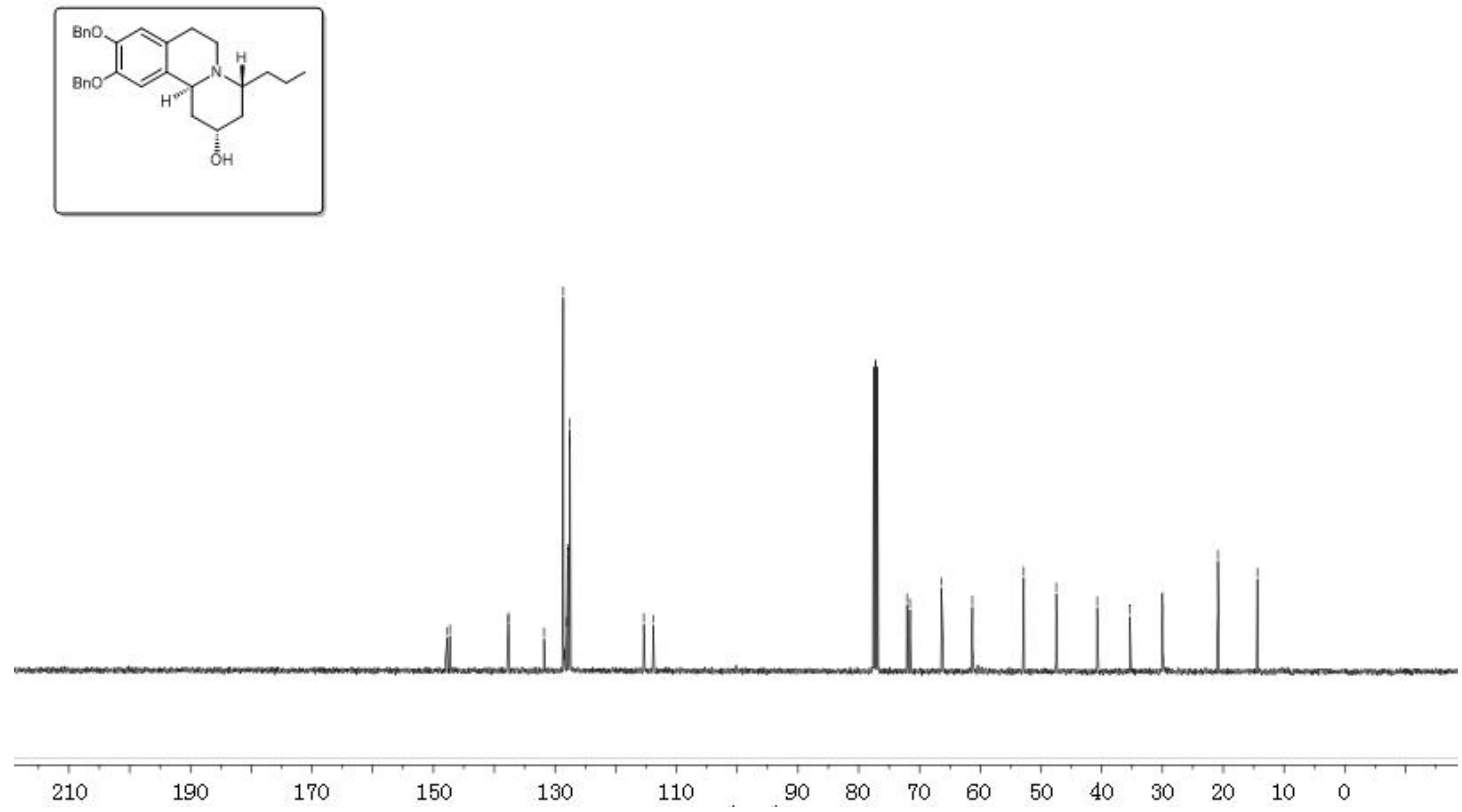

Figure S182. ${ }^{13} \mathrm{C}$ NMR spectrum of 13a.

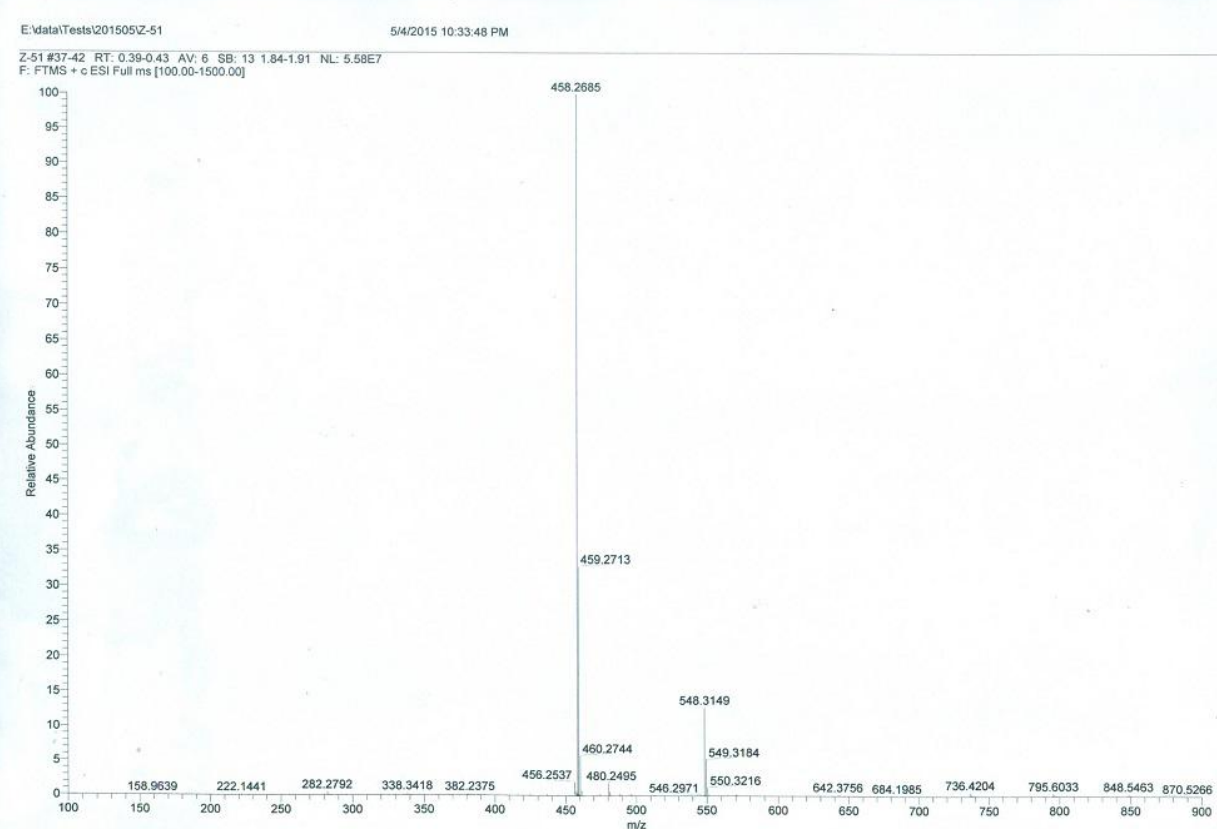

Figure S183. HRESIMS spectrum of $\mathbf{1 3 b}$. 

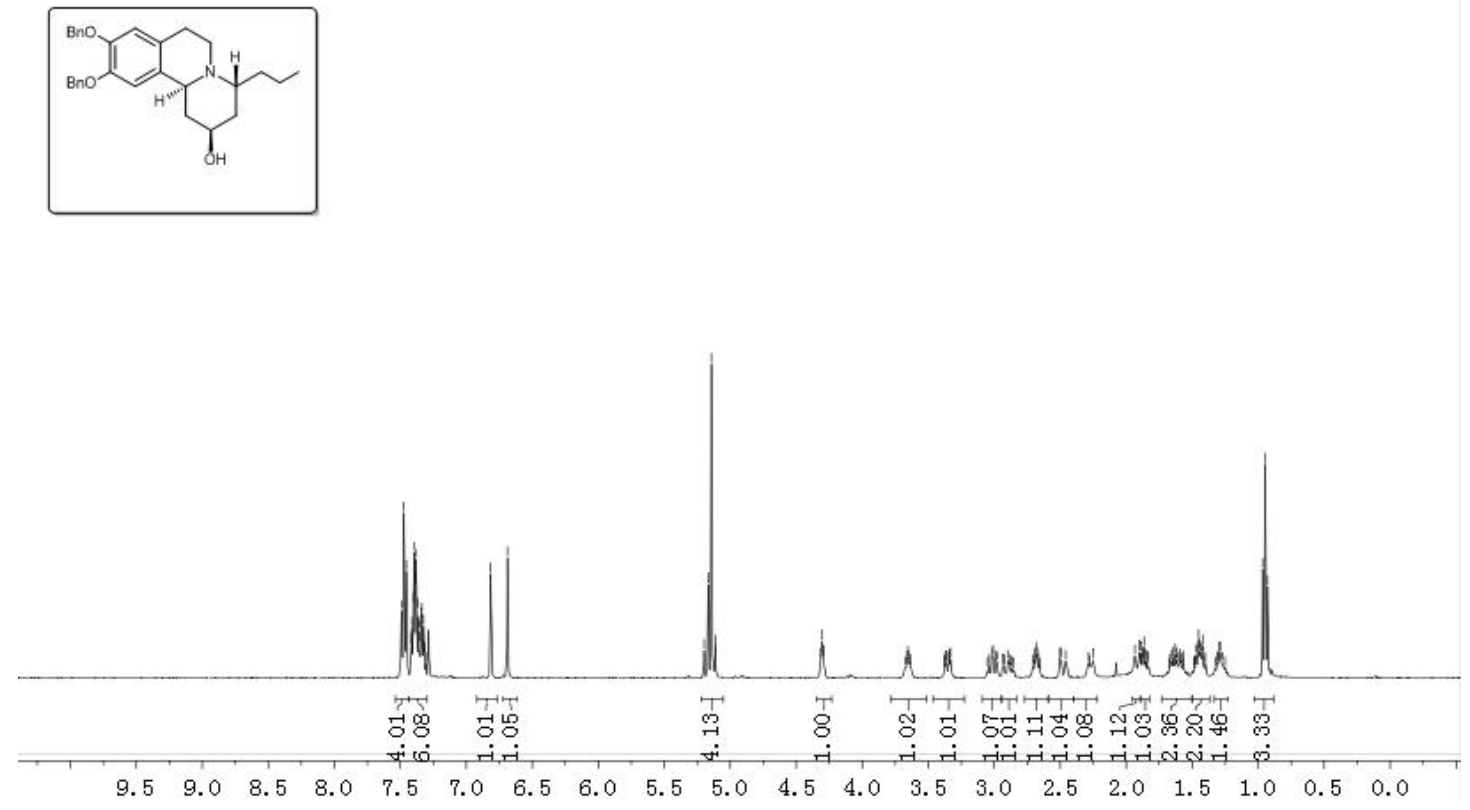

Figure S184. ${ }^{1} \mathrm{H}$ NMR spectrum of $\mathbf{1 3 b}$
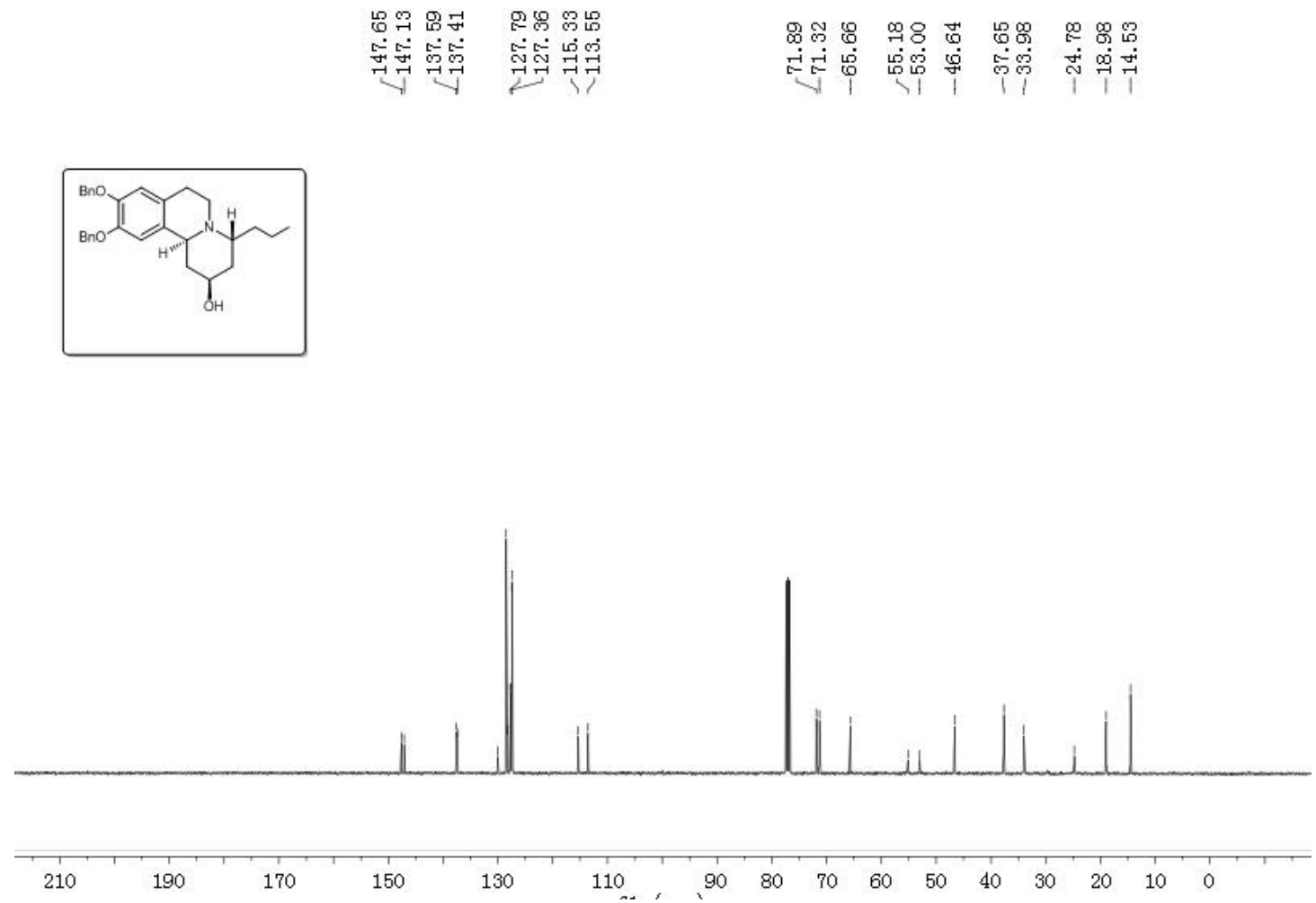

Figure S185. ${ }^{13} \mathrm{C}$ NMR spectrum of $\mathbf{1 3 b}$. 

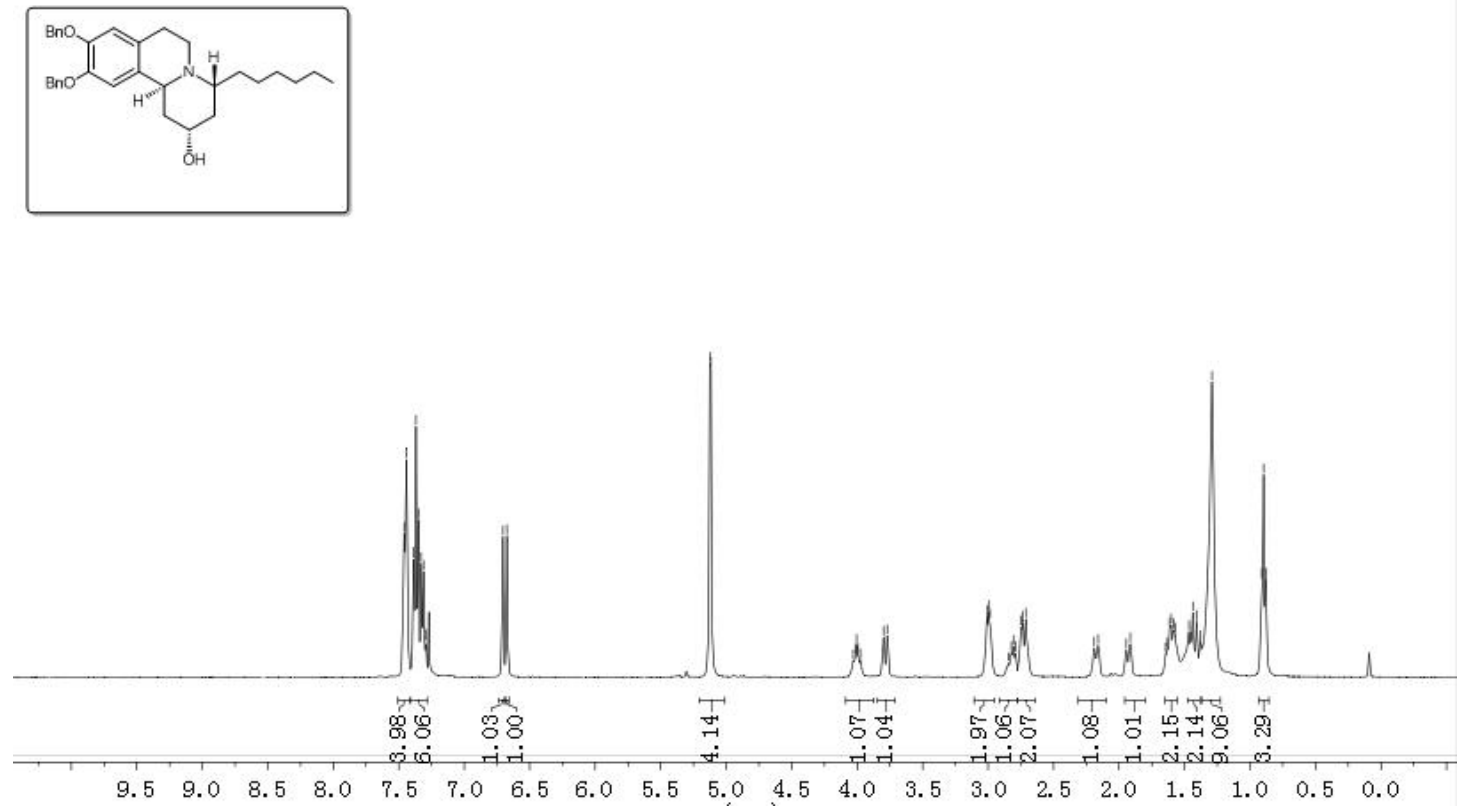

Figure S186. ${ }^{1} \mathrm{H}$ NMR spectrum of $\mathbf{1 4 a}$.

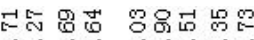

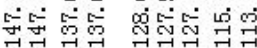

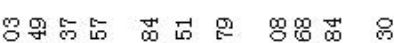

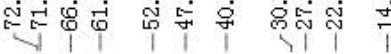
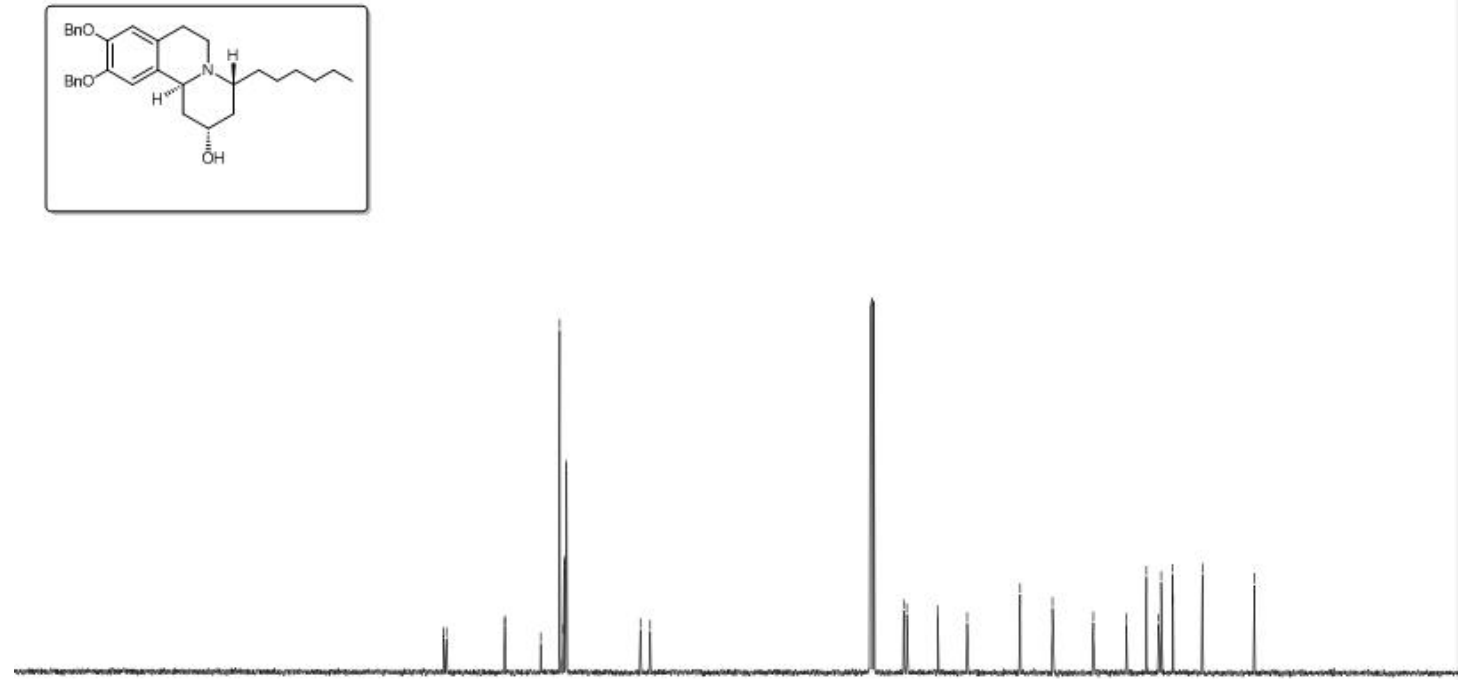

190

150

130

10

90

Figure S187. ${ }^{13} \mathrm{C}$ NMR spectrum of $14 a$. 


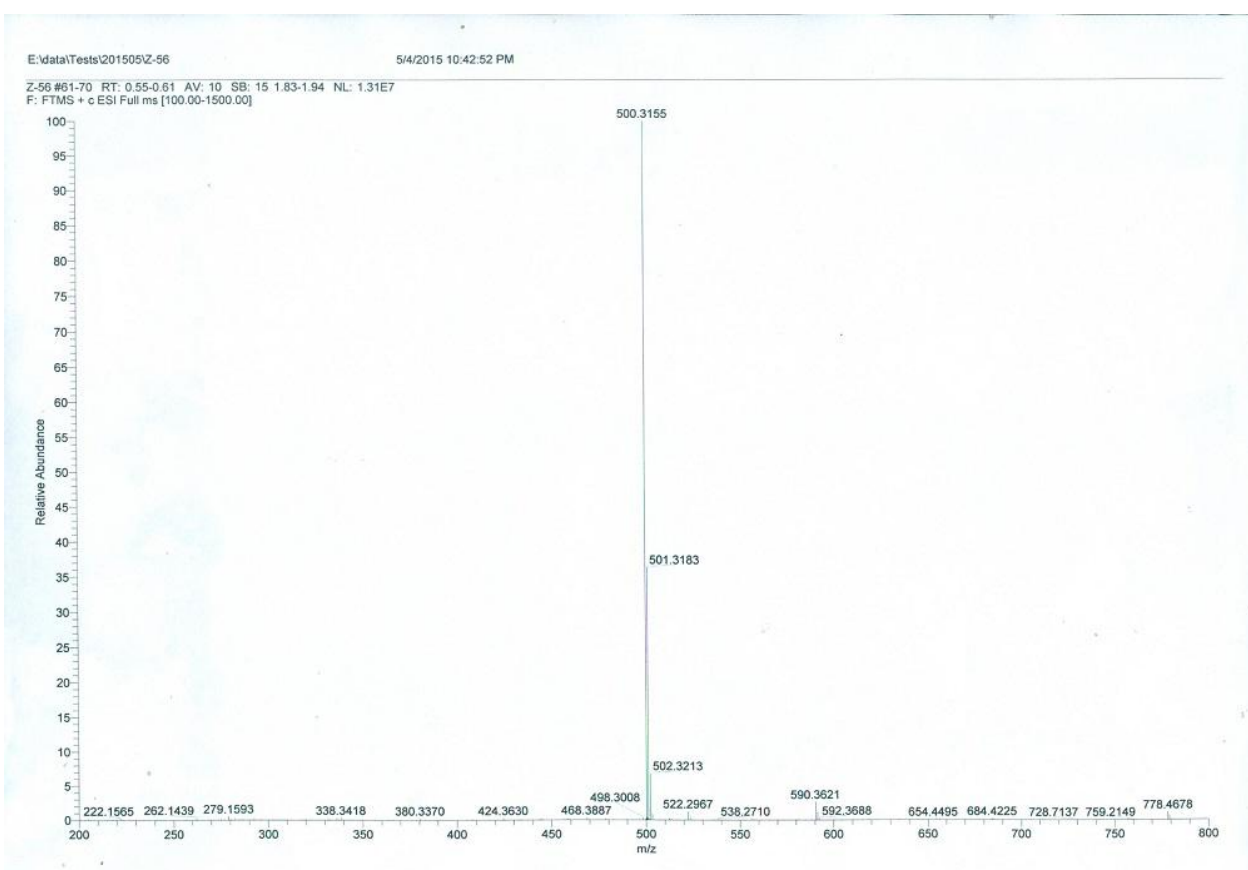

Figure S188. HRESIMS spectrum of $\mathbf{1 4 b}$.

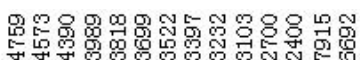

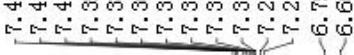

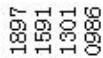

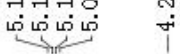

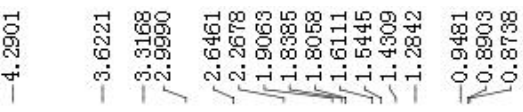<smiles>CCCCC1CC2CC(O)CC(CCCC)C2CN1</smiles>

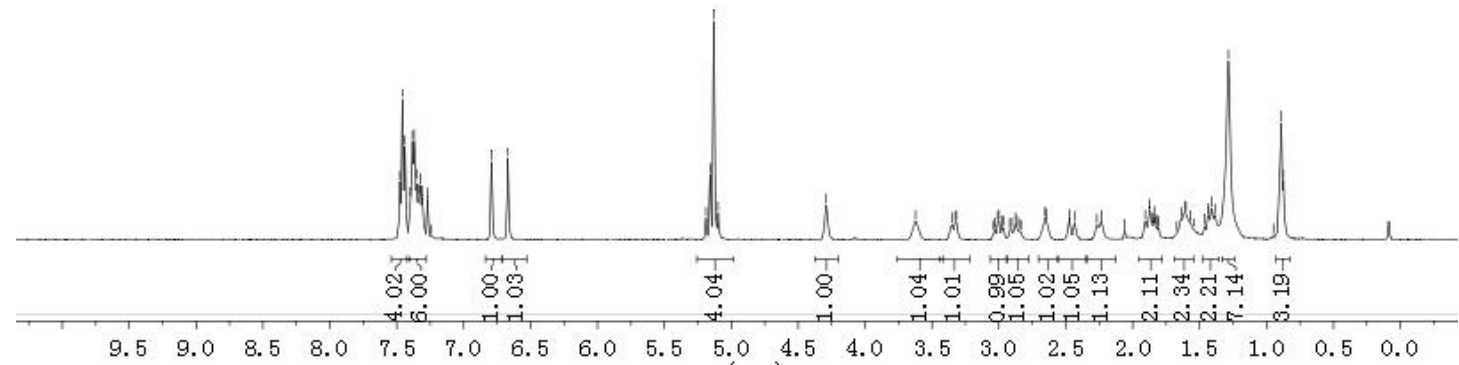

Figure S189. ${ }^{1} \mathrm{H}$ NMR spectrum of $\mathbf{1 4 b}$ 

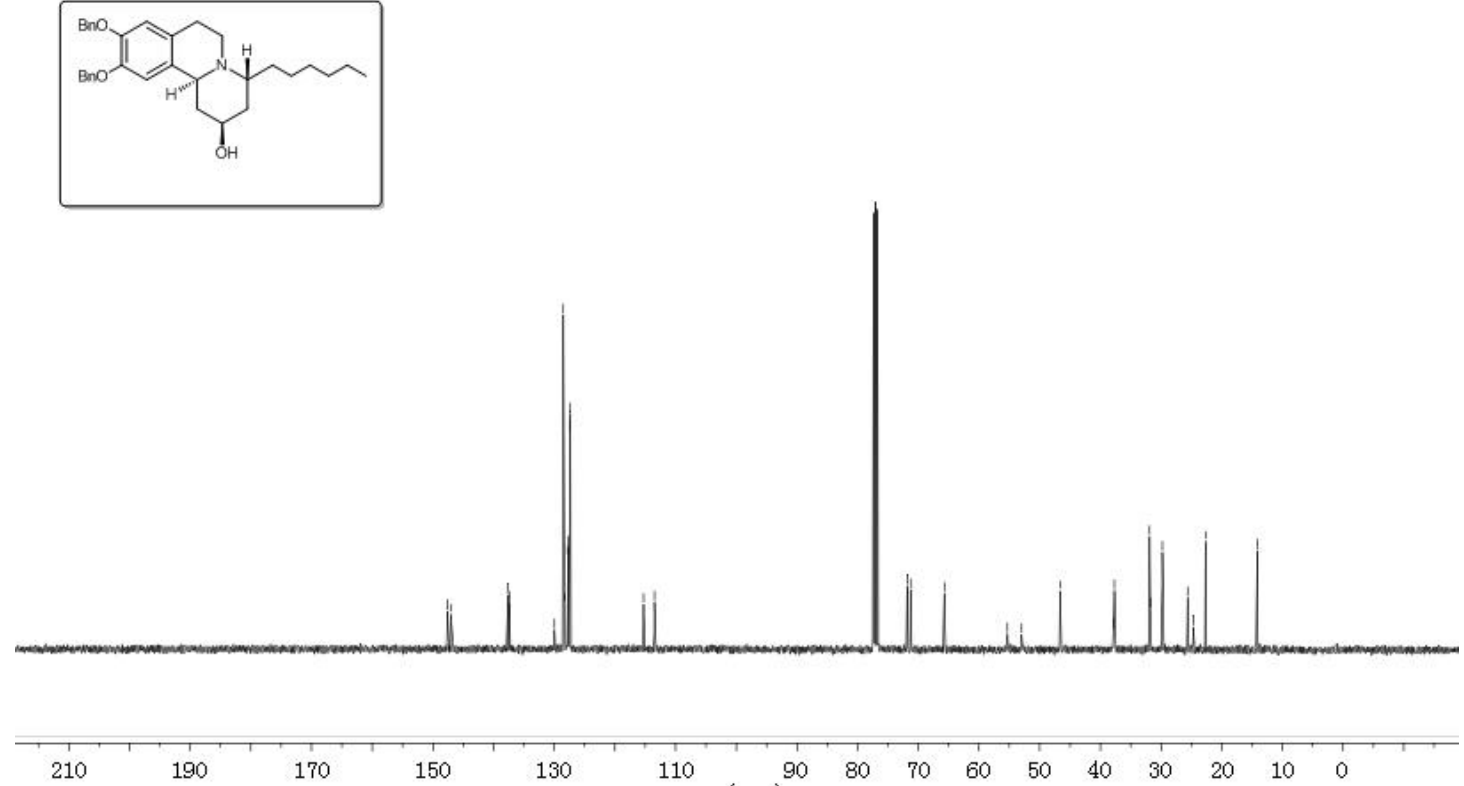

Figure S190. ${ }^{13} \mathrm{C}$ NMR spectrum of $\mathbf{1 4 b}$.

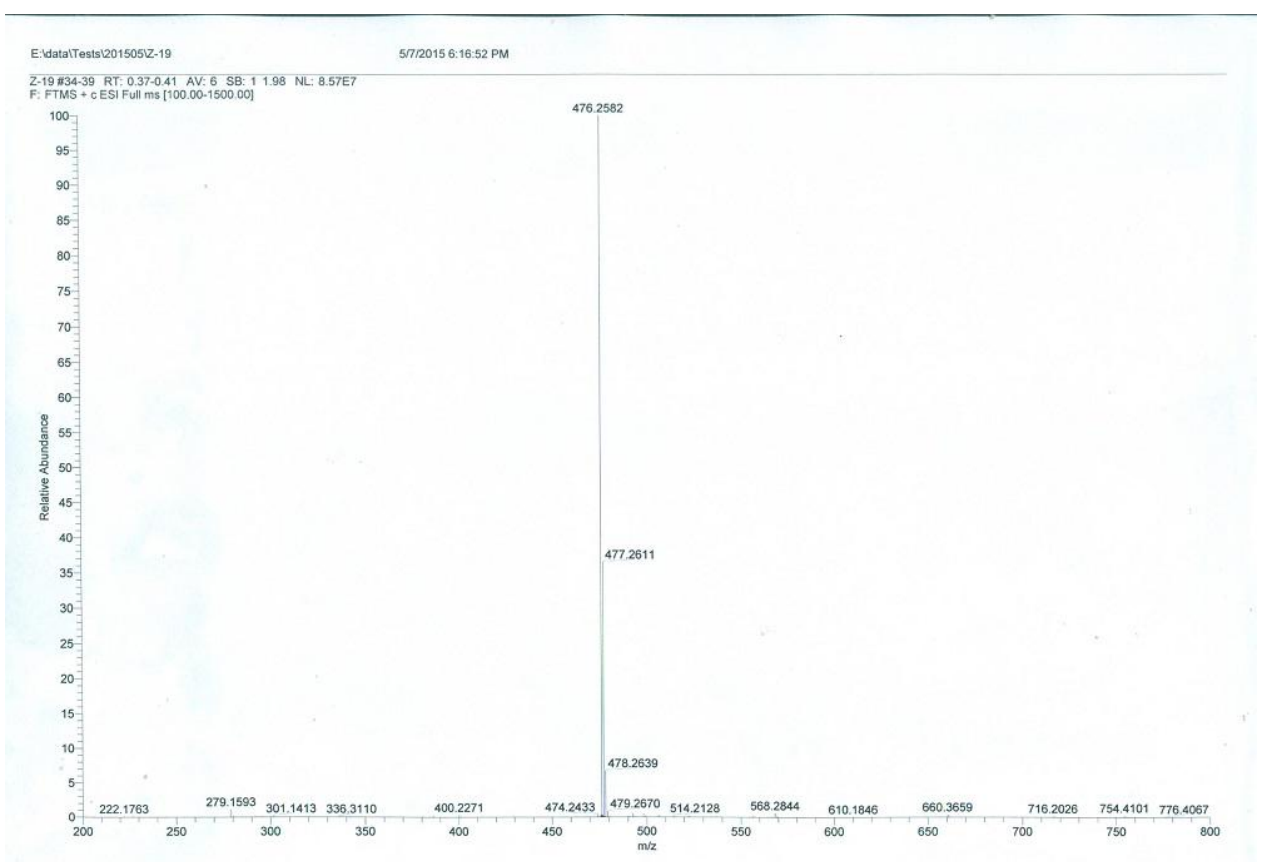

Figure S191. HRESIMS spectrum of $\mathbf{1 5 .}$ 
<smiles>Oc1cc2c(cc1O)[C@H]1CCCCN1[C@H](c1ccccc1)CC2</smiles>

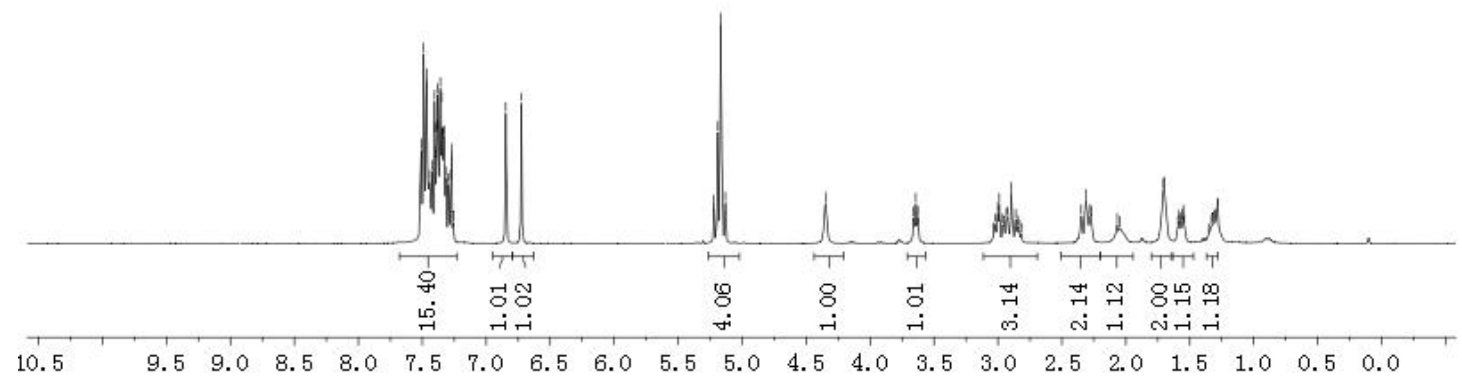

Figure S192. ${ }^{1} \mathrm{H}$ NMR spectrum of $\mathbf{1 5}$.
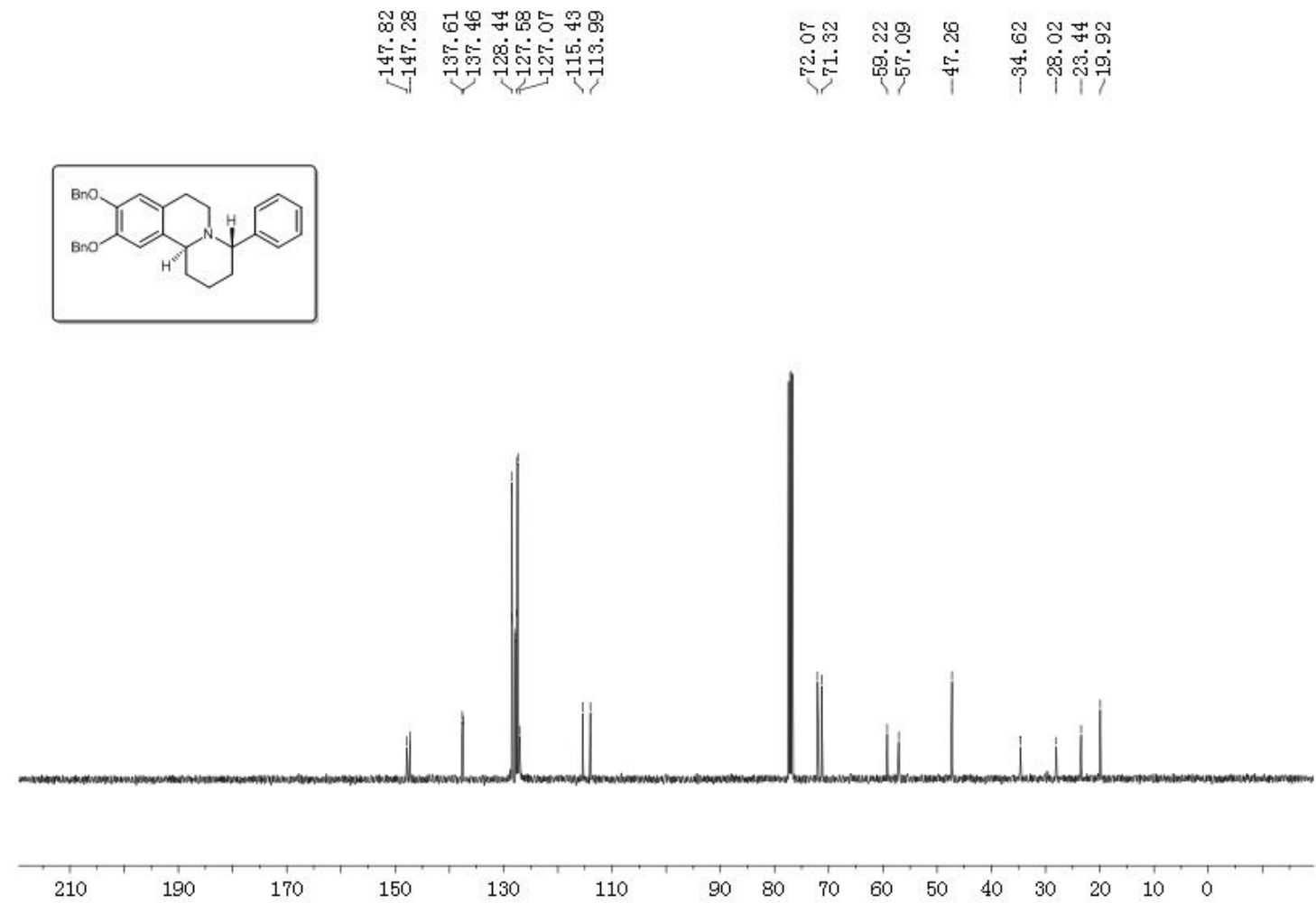

Figure S193. ${ }^{13} \mathrm{C}$ NMR spectrum of $\mathbf{1 5}$. 

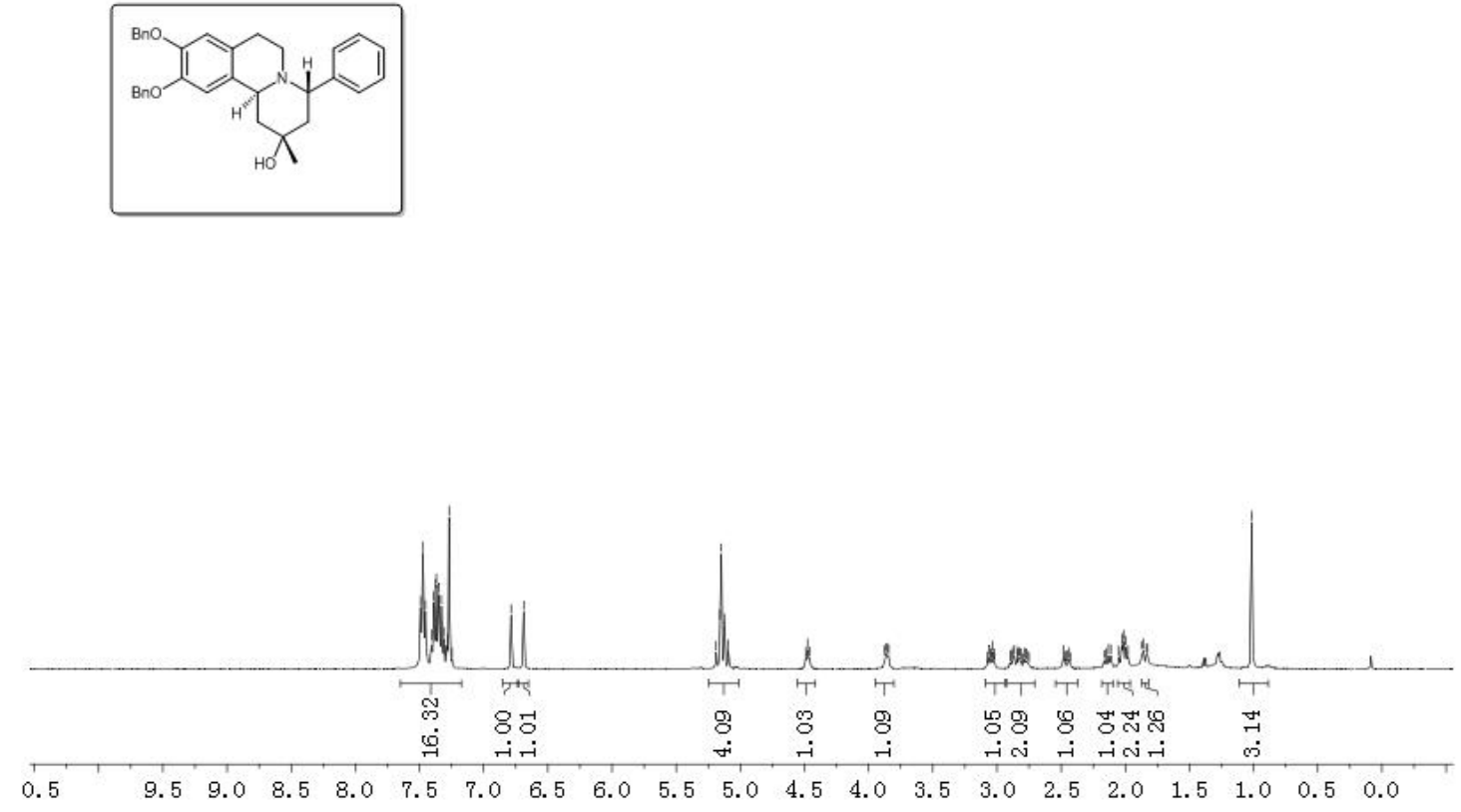

Figure S194. ${ }^{1} \mathrm{H}$ NMR spectrum of $\mathbf{1 6 a}$

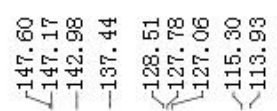

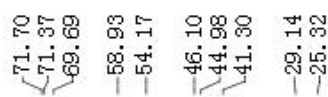
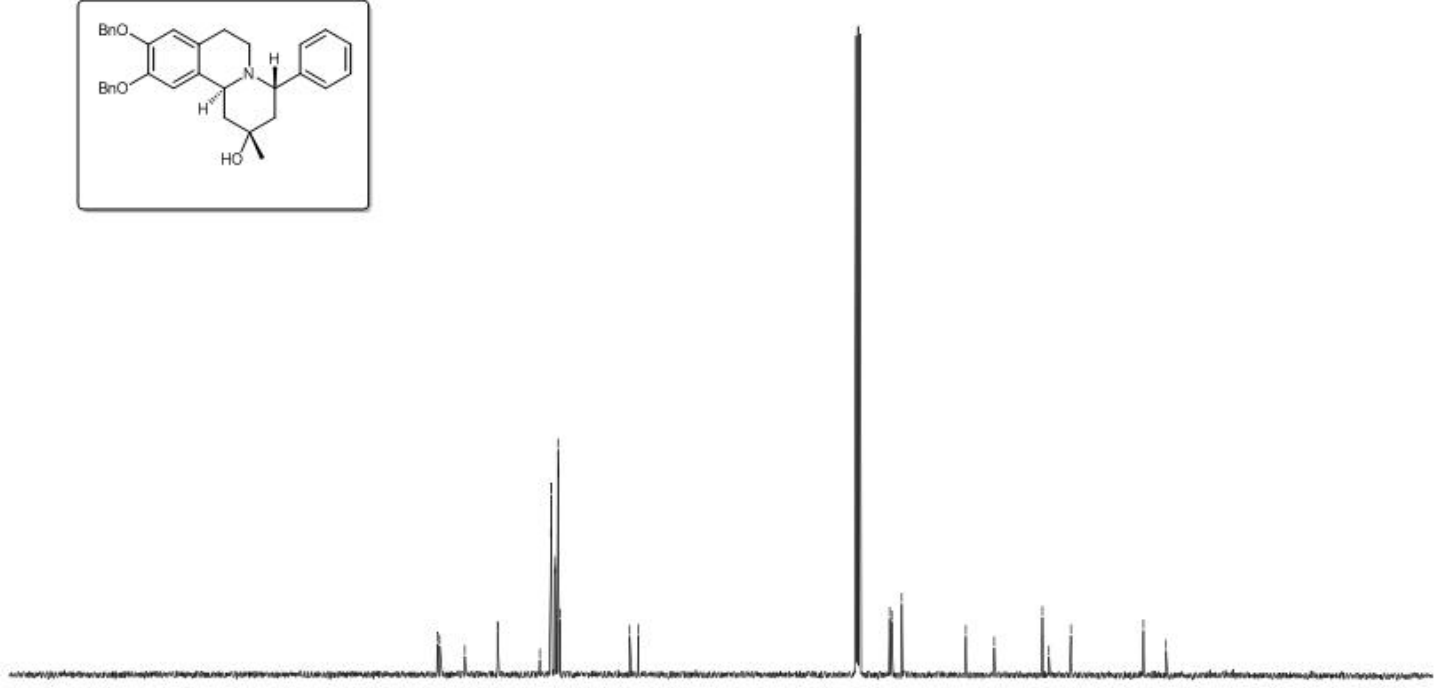

Figure S195. ${ }^{13} \mathrm{C}$ NMR spectrum of $\mathbf{1 6 a}$. 


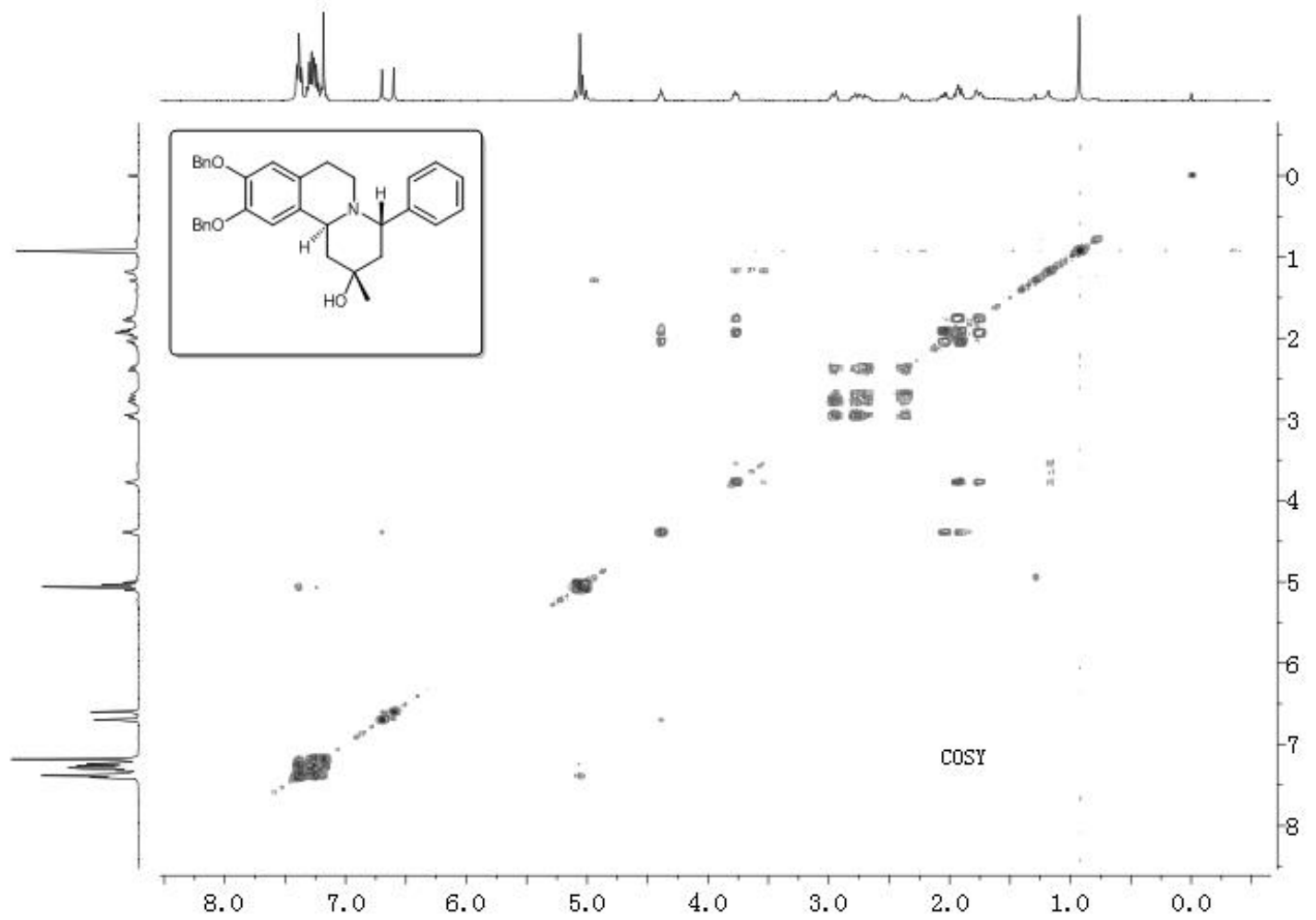

Figure S196. COSY spectrum of 16a.

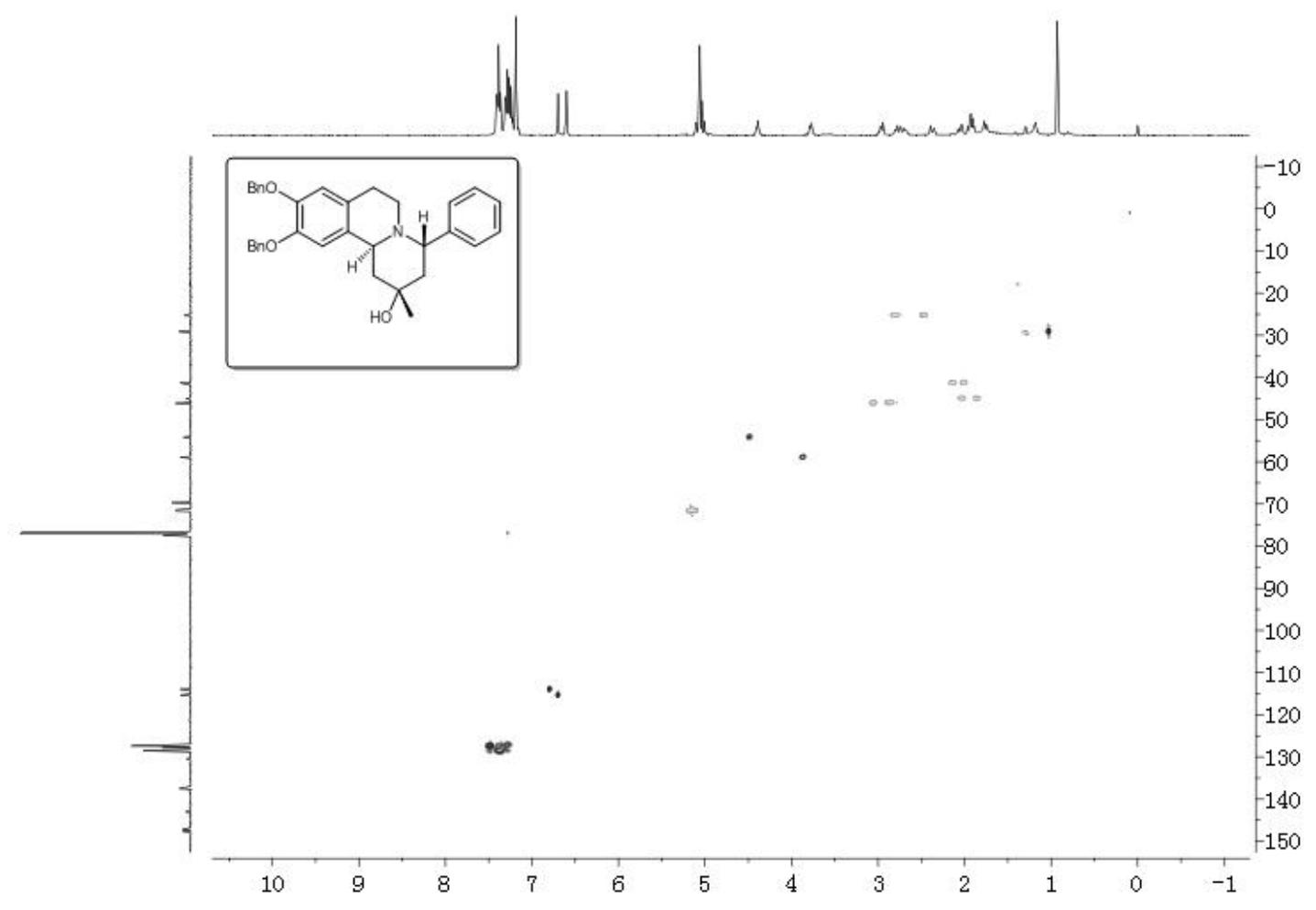

Figure S197. HSQC spectrum of 16a. 


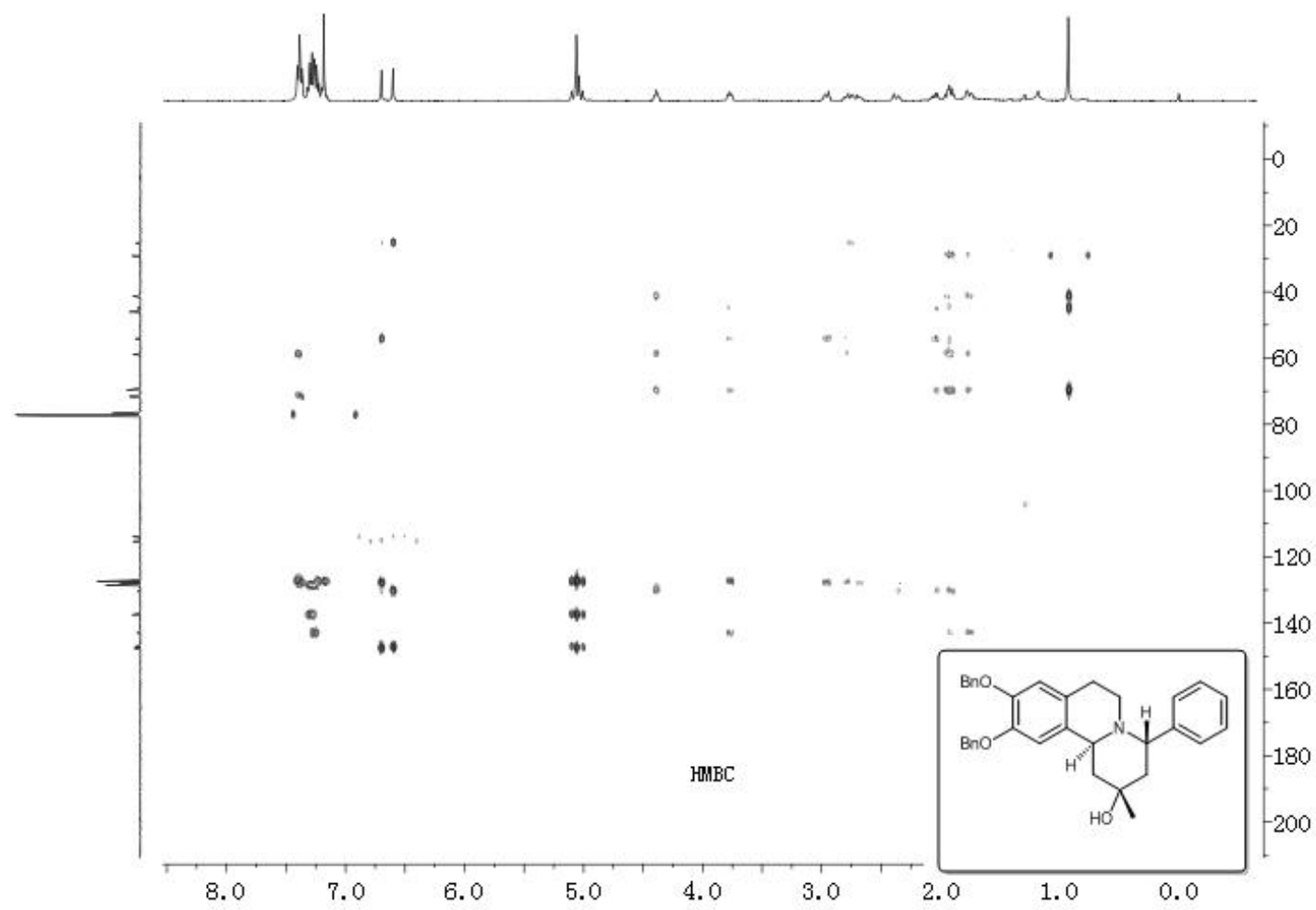

Figure S198. HMBC spectrum of 16a.

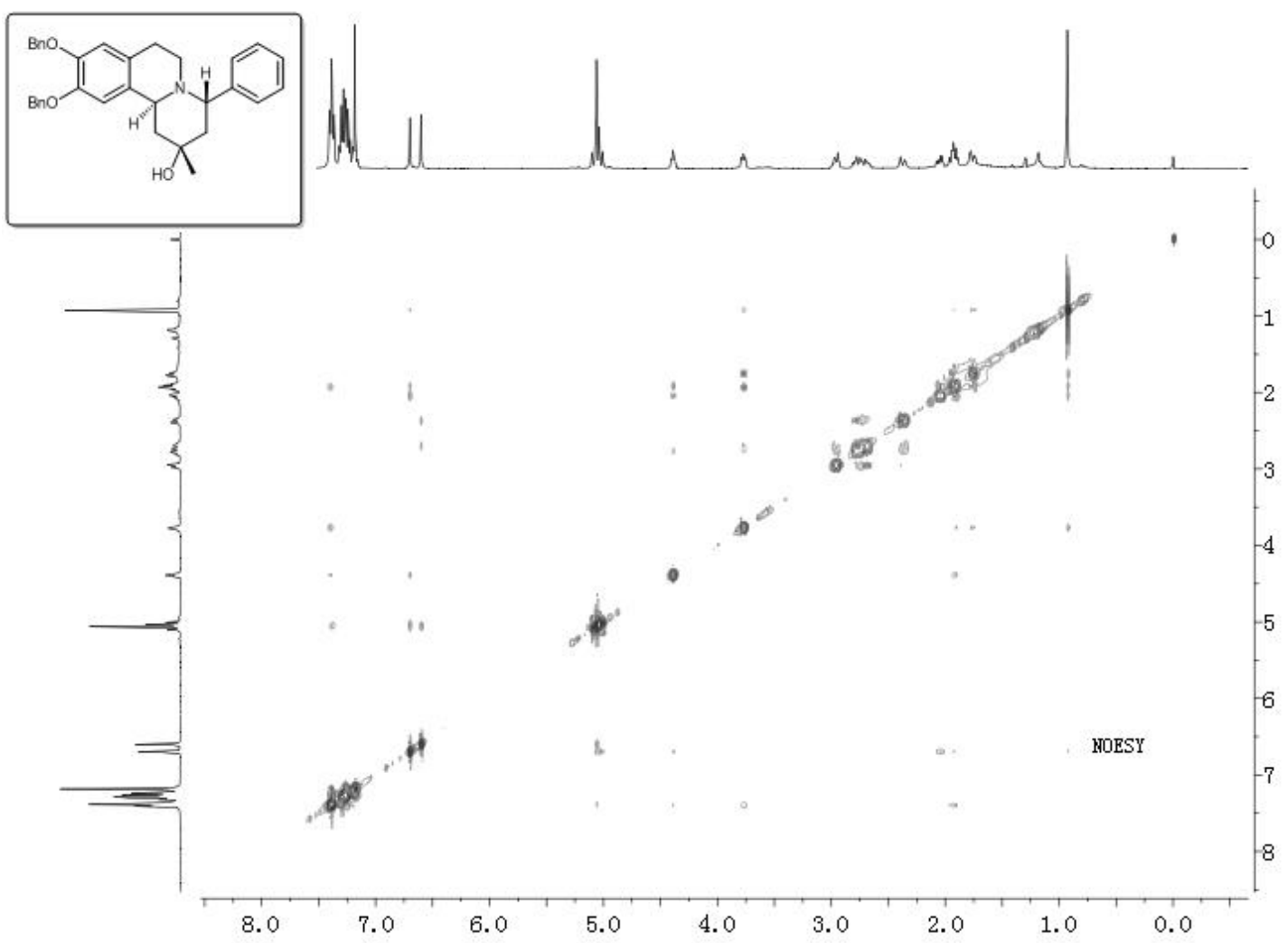

Figure S199. NOESY spectrum of $\mathbf{1 6 a}$. 


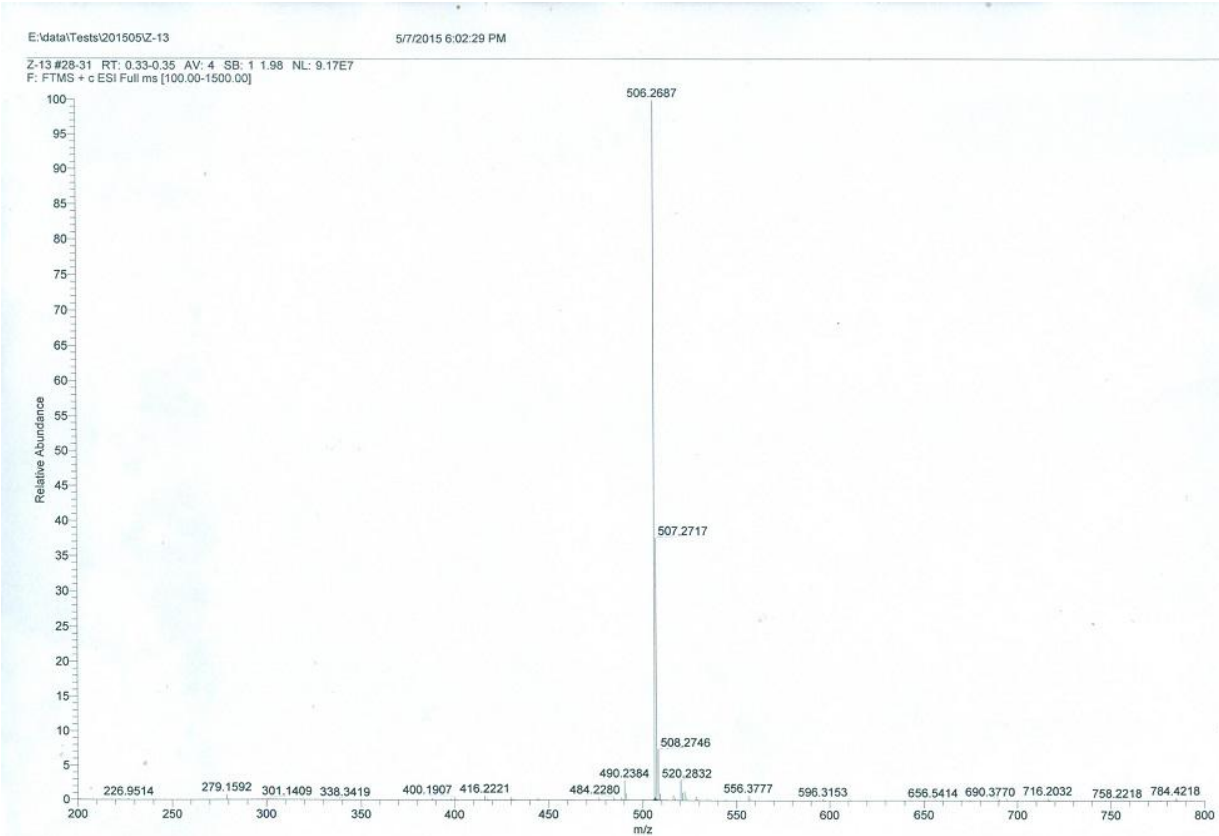

Figure S200. HRESIMS spectrum of $\mathbf{1 6 b}$.

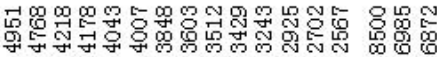

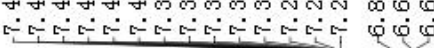

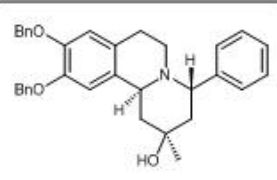

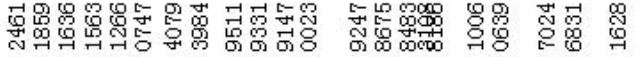

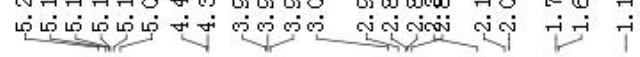

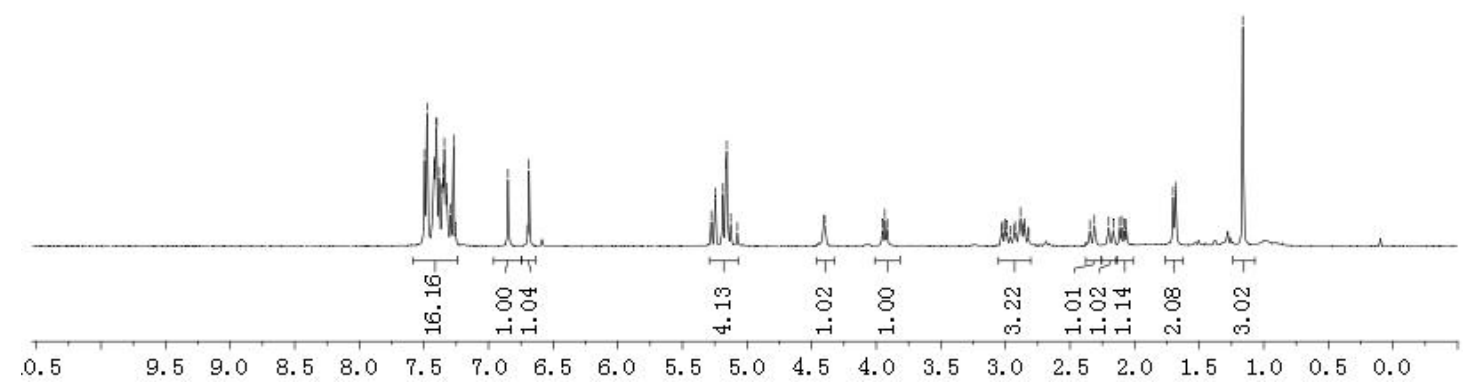

Figure S201. ${ }^{1} \mathrm{H}$ NMR spectrum of $\mathbf{1 6 b}$. 

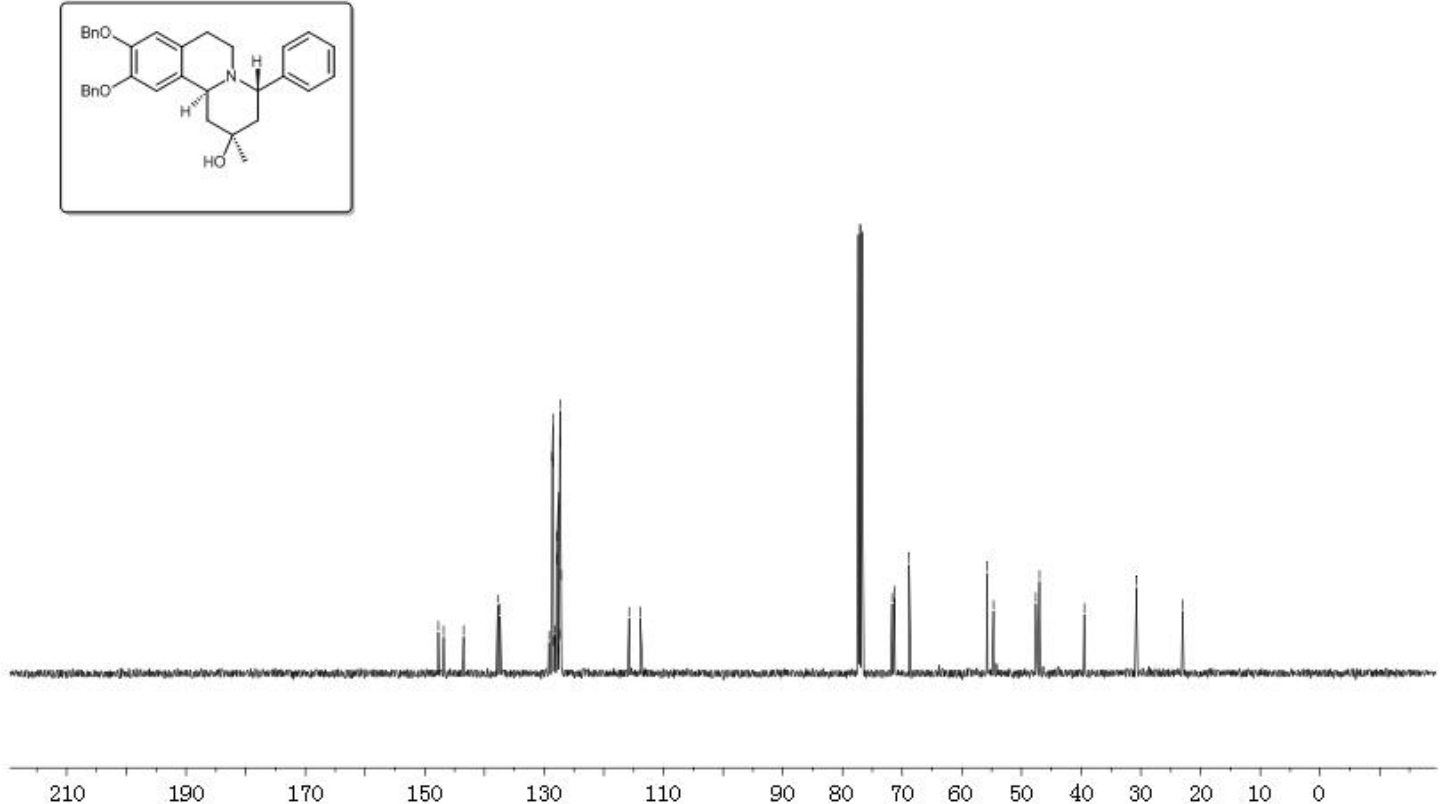

Figure S202. ${ }^{13} \mathrm{C}$ NMR spectrum of $\mathbf{1 6 b}$.
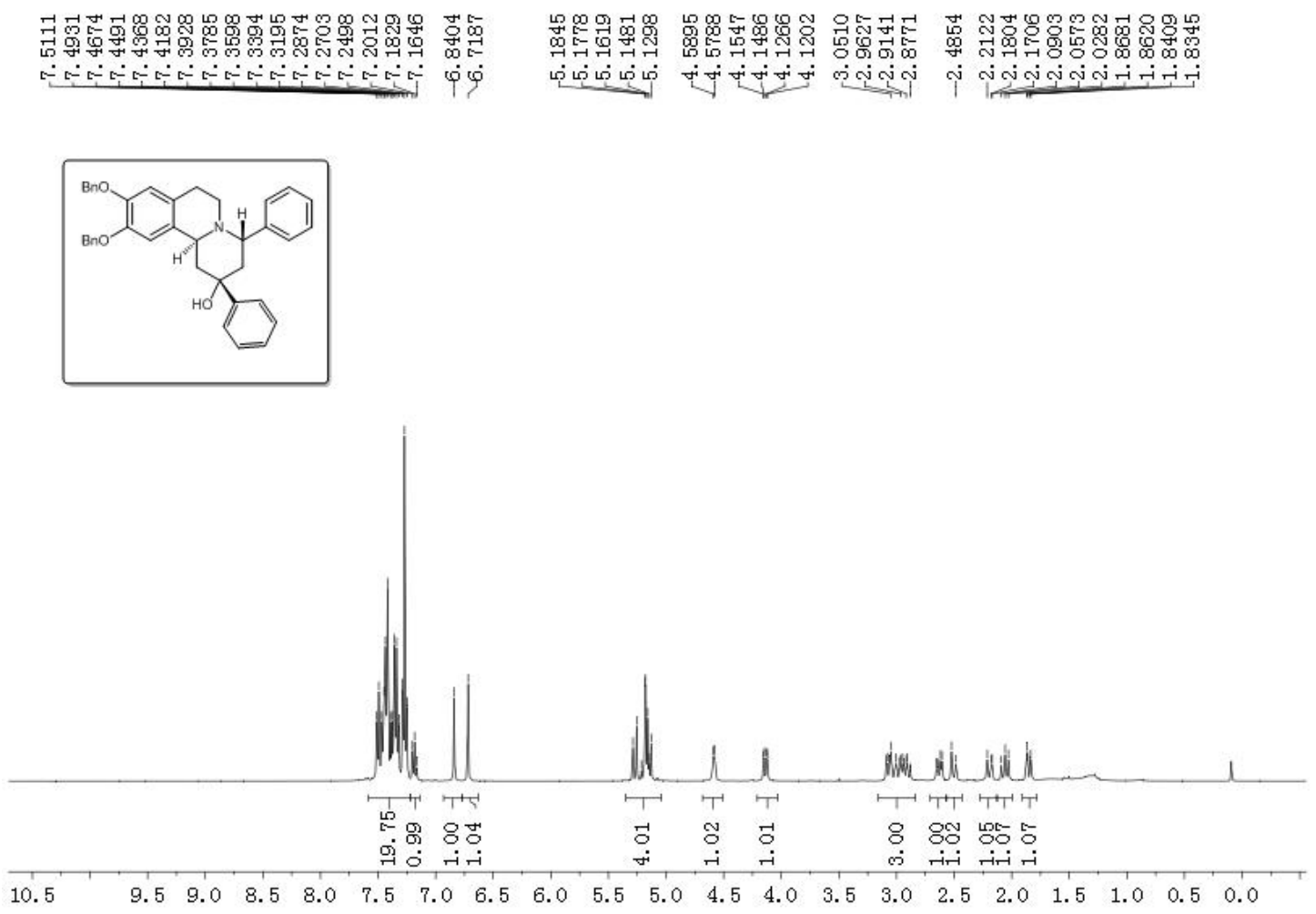

Figure S203. ${ }^{1} \mathrm{H}$ NMR spectrum of $\mathbf{1 7 a}$. 


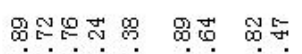

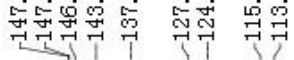

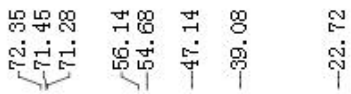
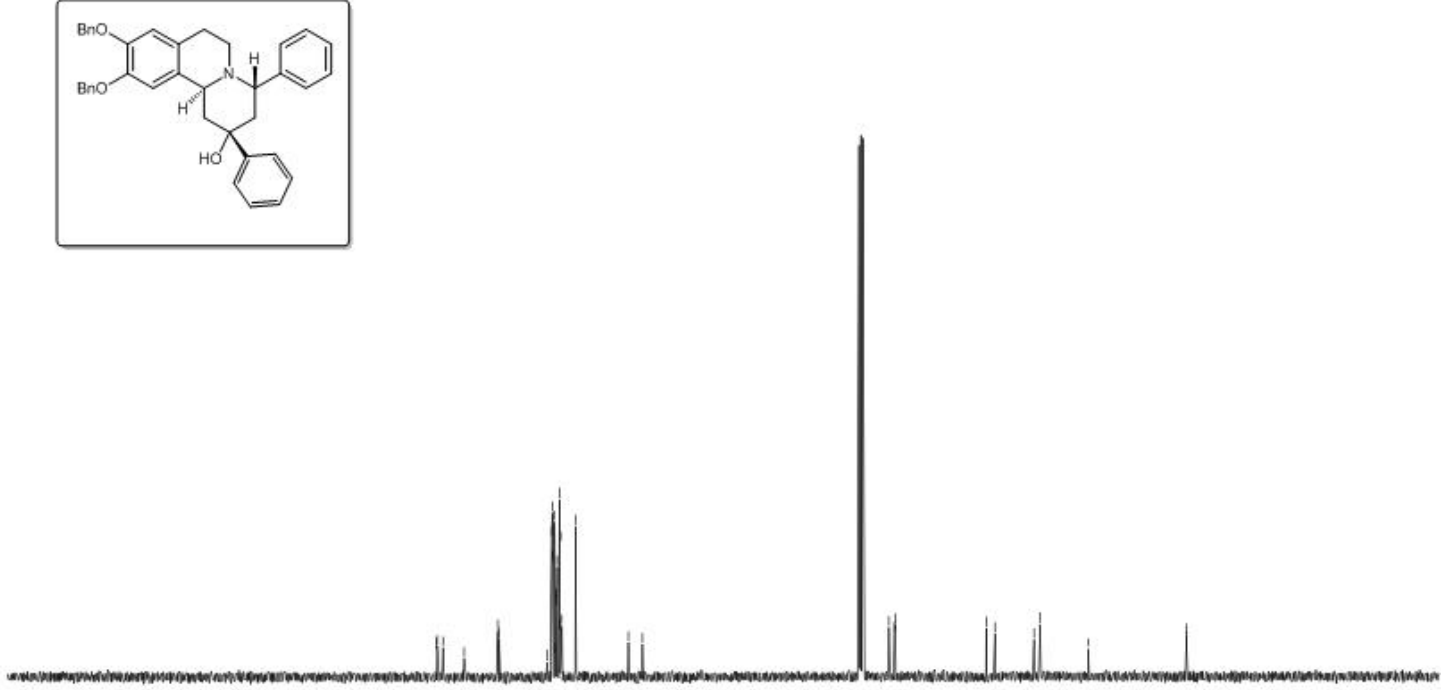

Figure S204. ${ }^{13} \mathrm{C}$ NMR spectrum of $17 \mathbf{a}$.

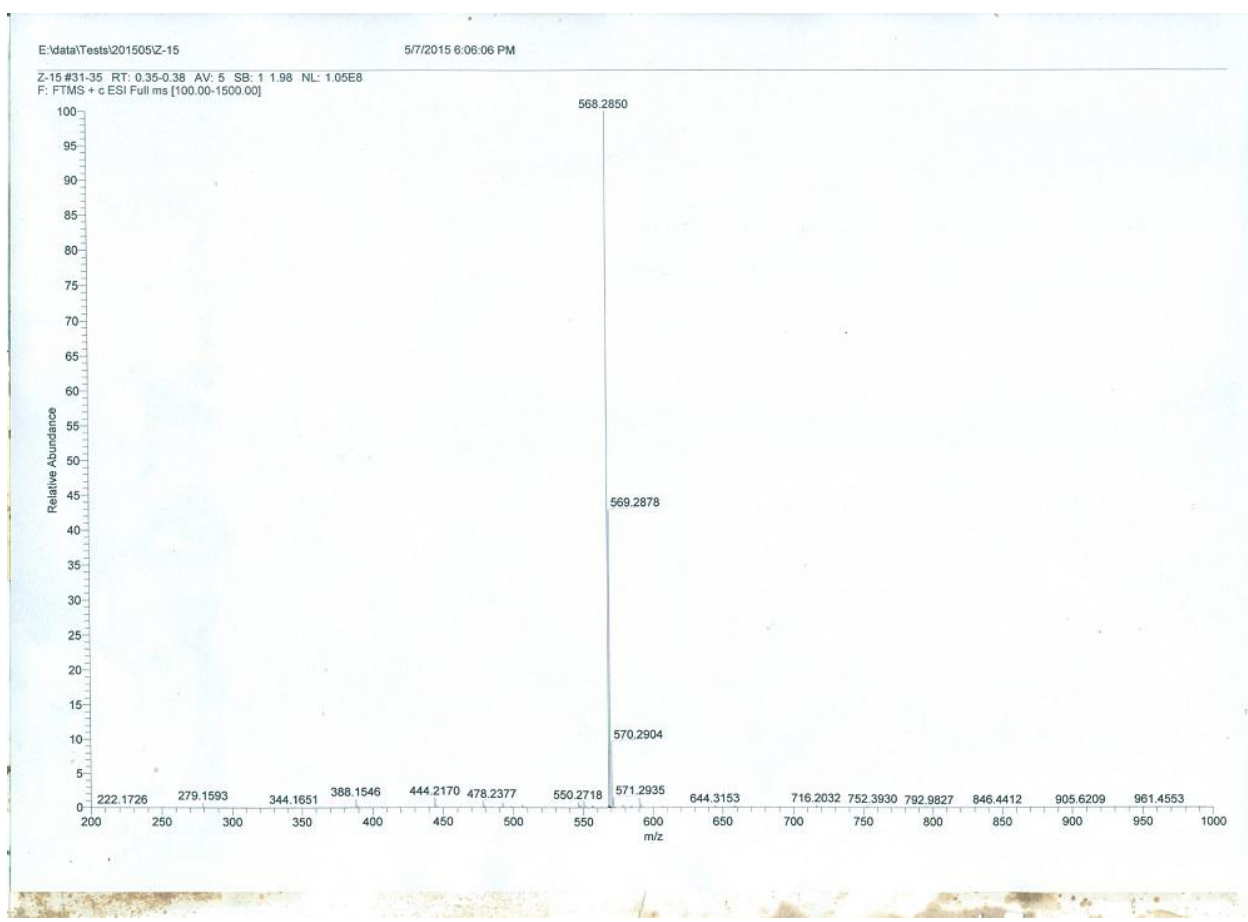

Figure S205. HRESIMS spectrum of $\mathbf{1 7 b}$. 

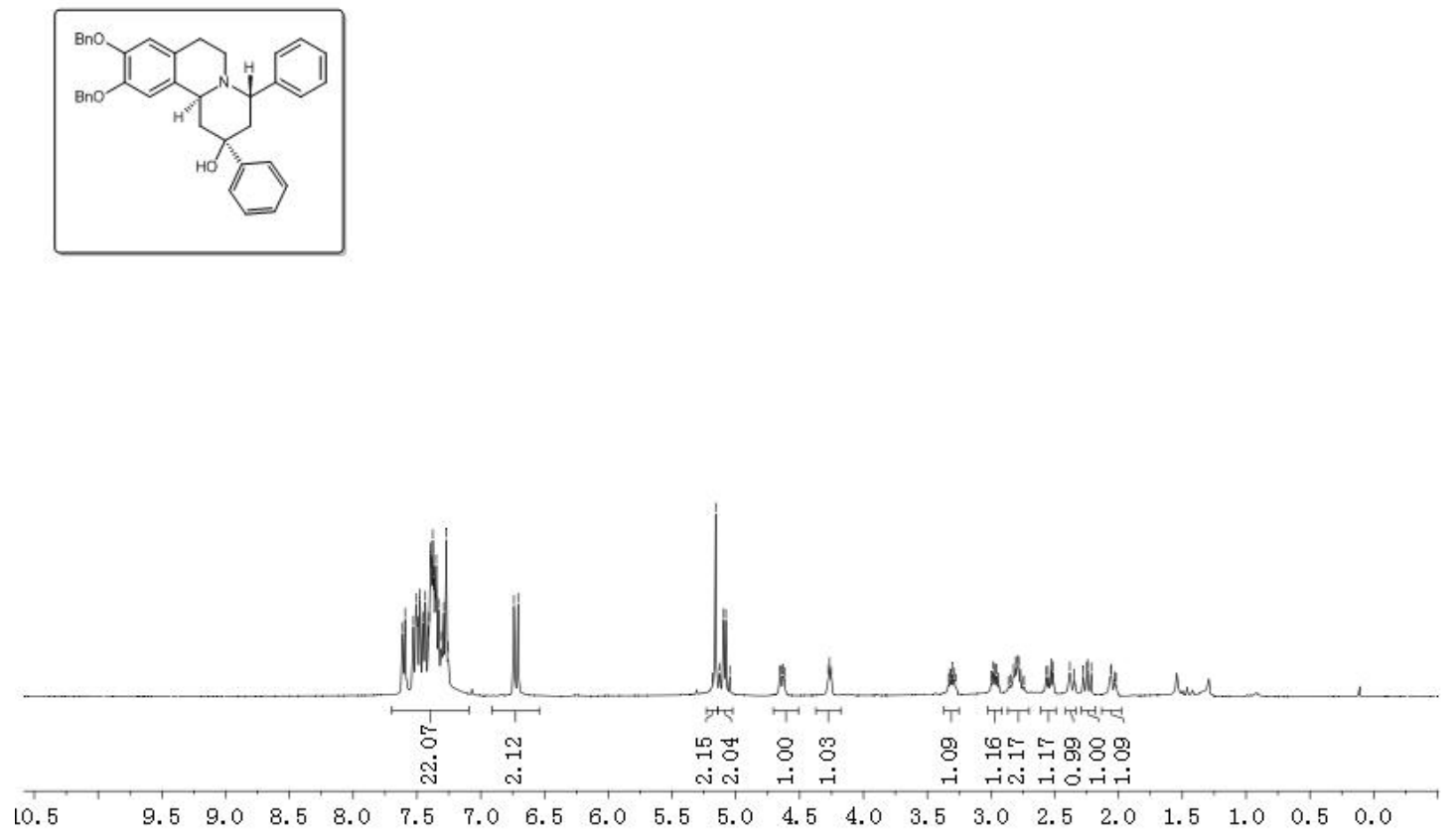

Figure S206. ${ }^{1} \mathrm{H}$ NMR spectrum of $\mathbf{1 7 b}$.
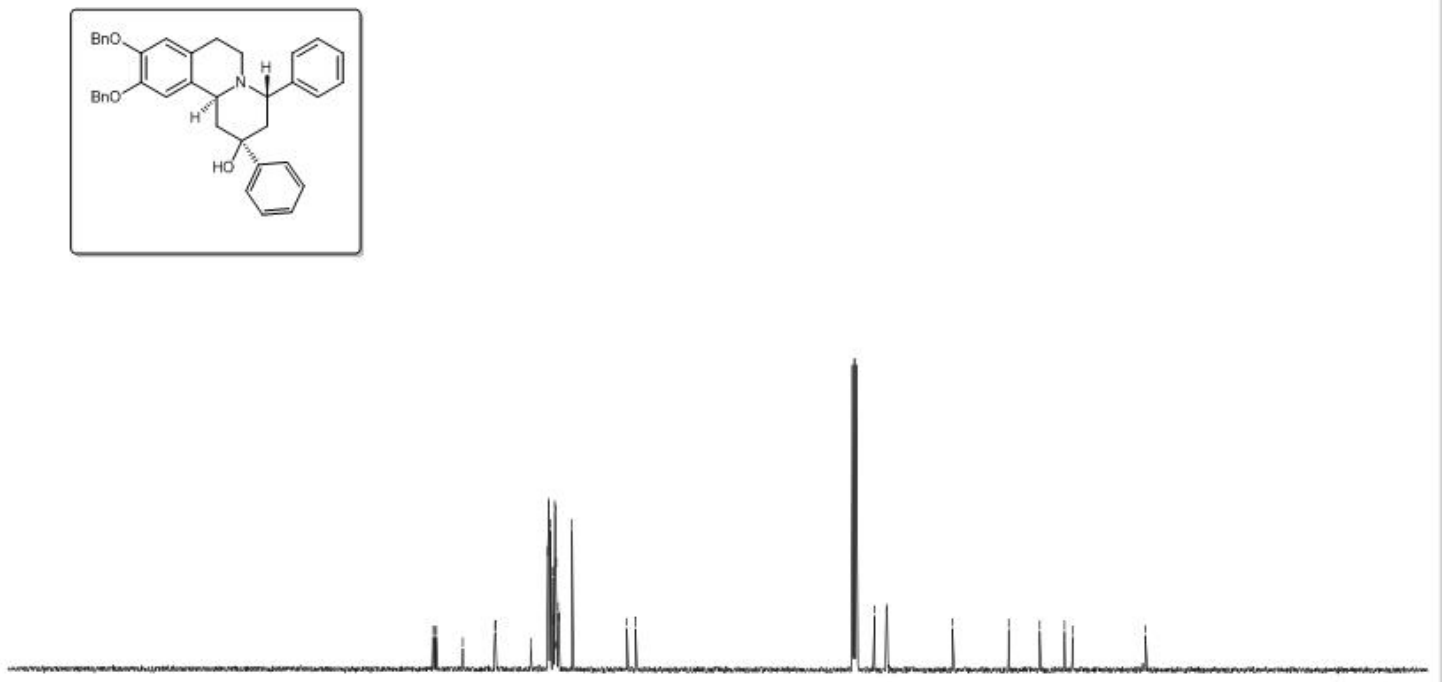

Figure S207. ${ }^{13} \mathrm{C}$ NMR spectrum of $\mathbf{1 7 b}$. 


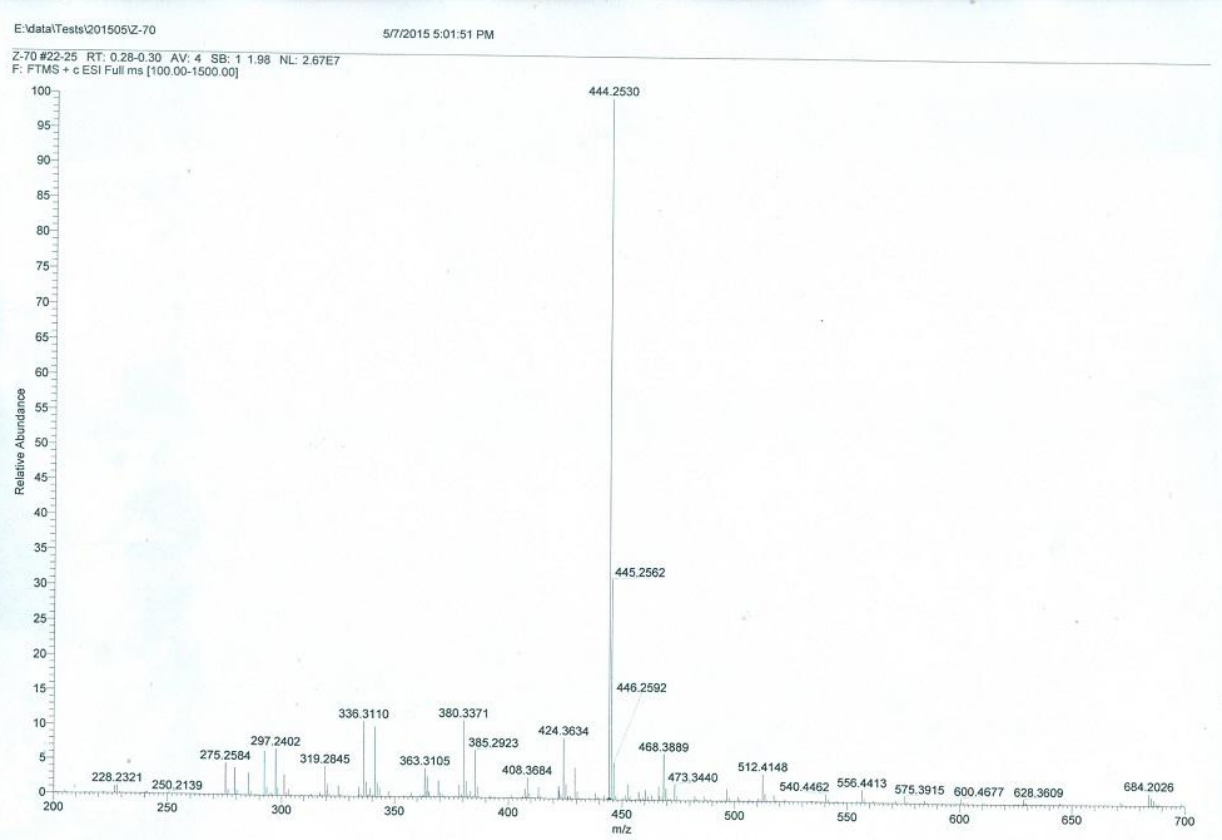

Figure S208. HRESIMS spectrum of $\mathbf{1 8 .}$

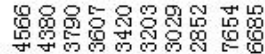

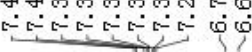
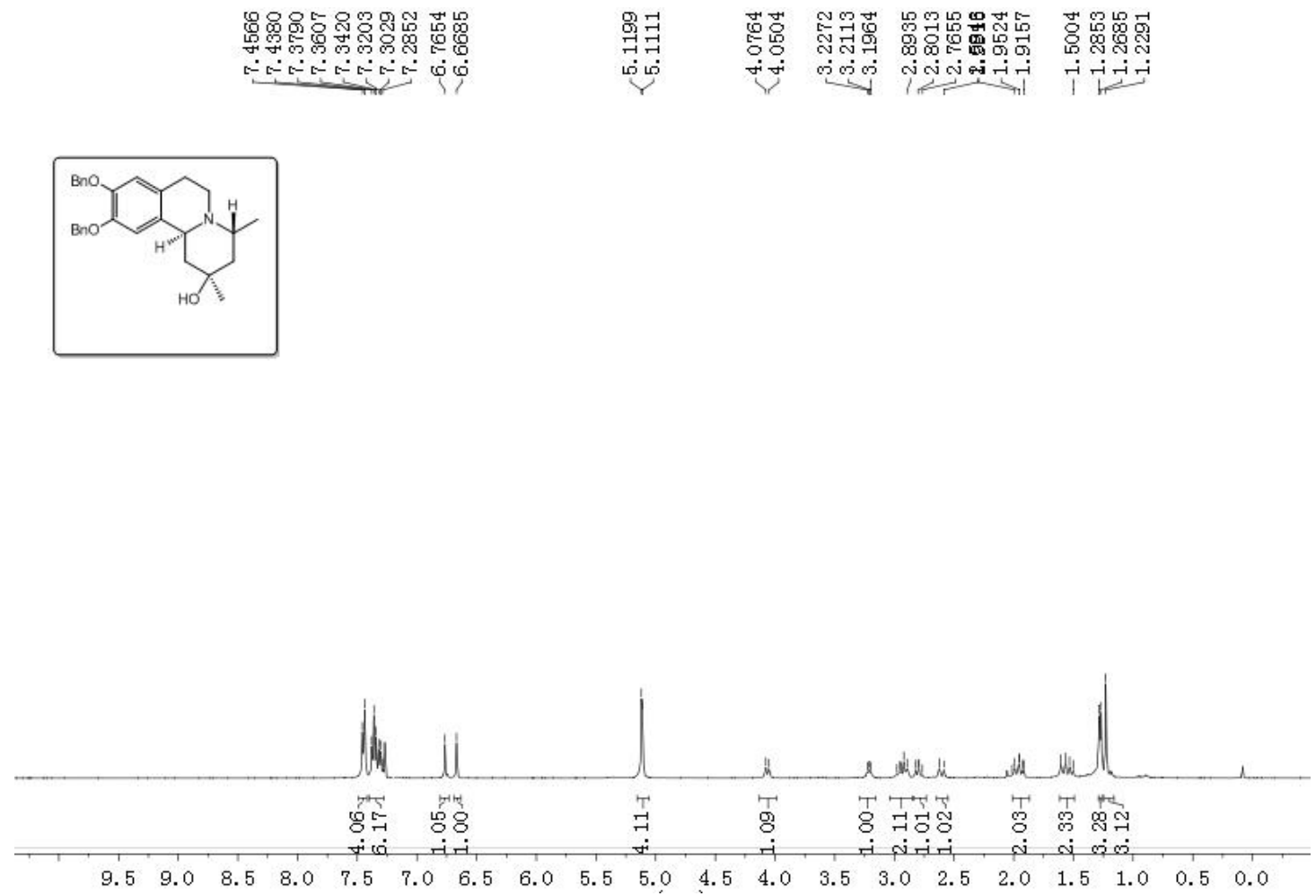

Figure S209. ${ }^{1} \mathrm{H}$ NMR spectrum of $\mathbf{1 8}$. 


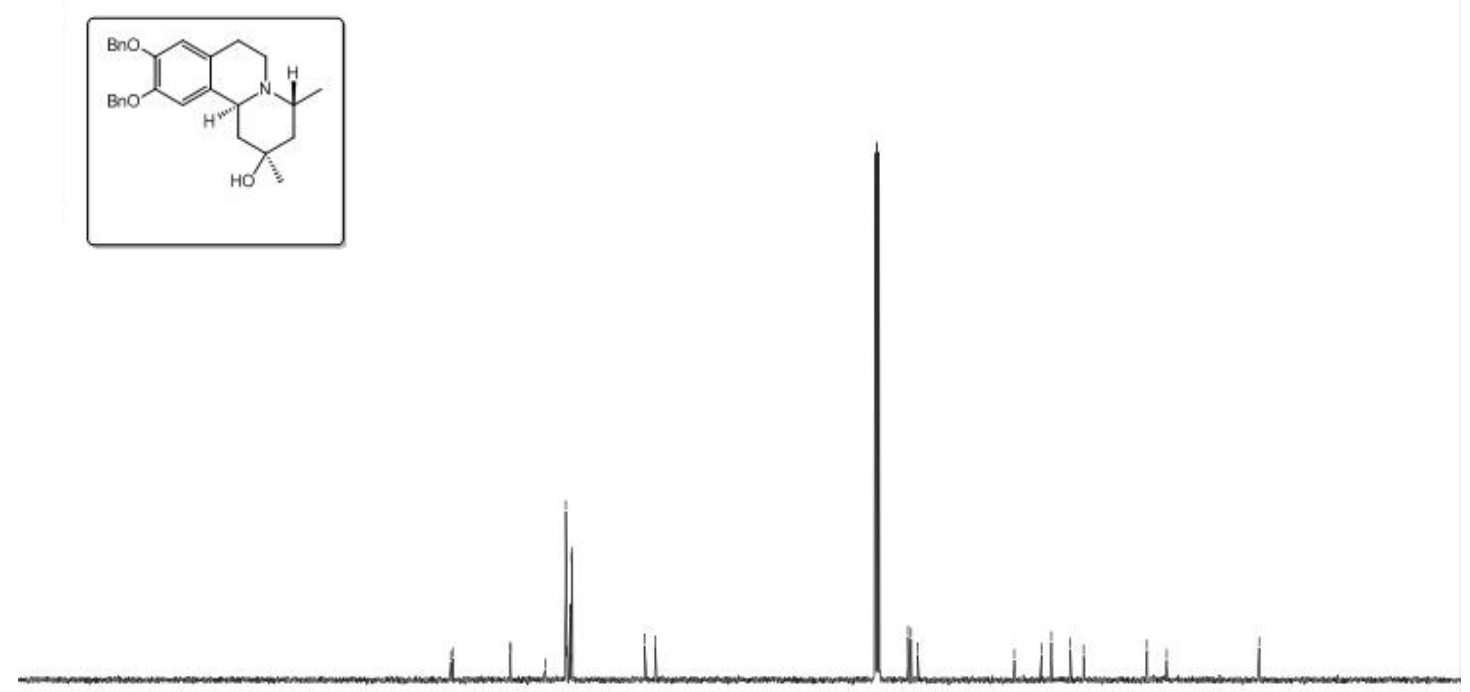

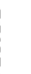

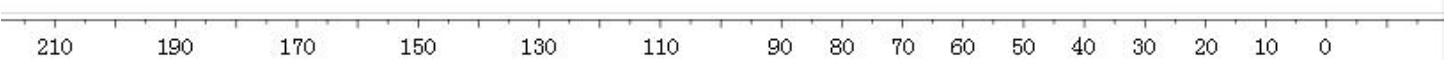

Figure S210. ${ }^{13} \mathrm{C}$ NMR spectrum of $\mathbf{1 8}$.

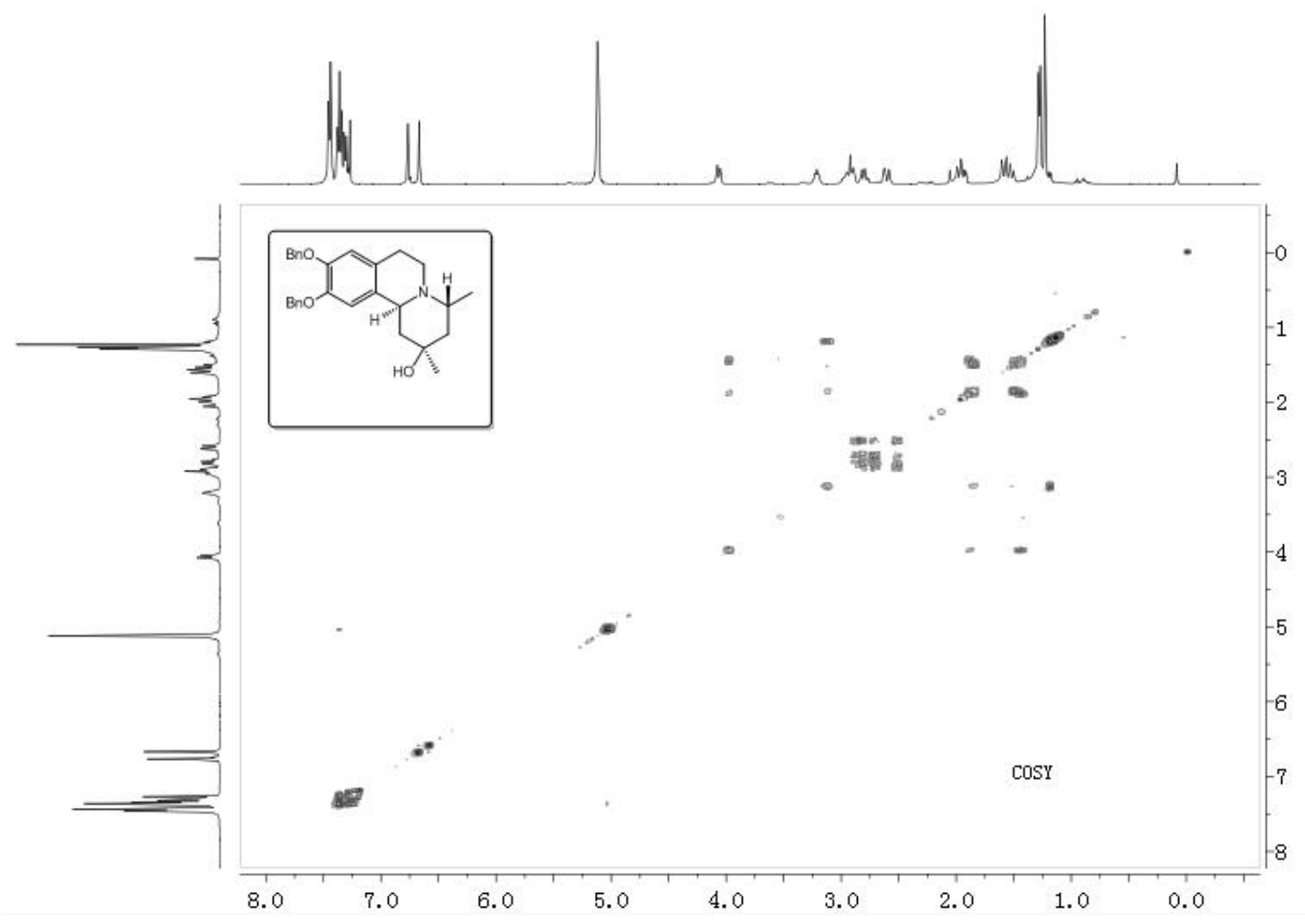

Figure S211. COSY spectrum of $\mathbf{1 8}$. 


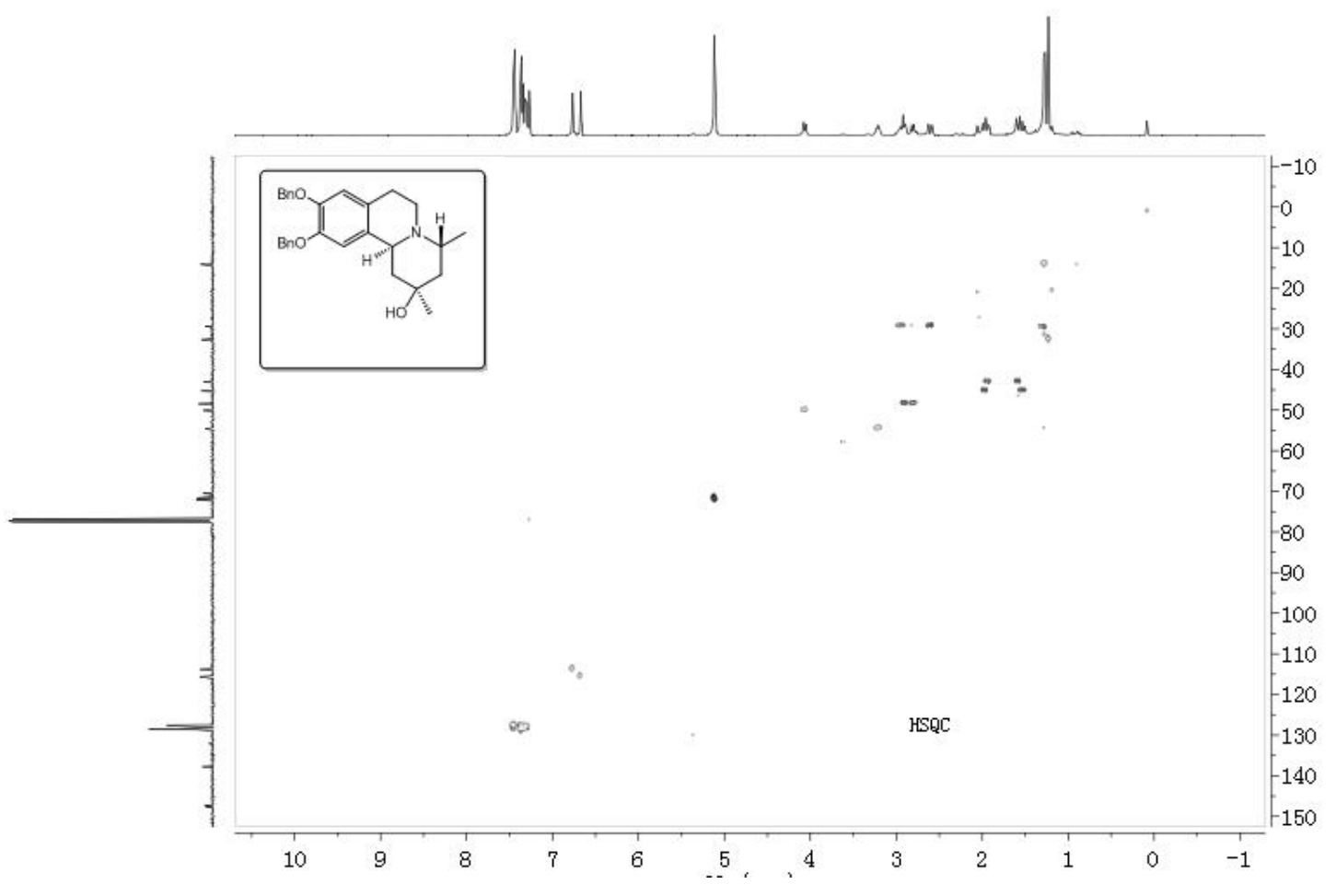

Figure S212. HSQC spectrum of $\mathbf{1 8 .}$

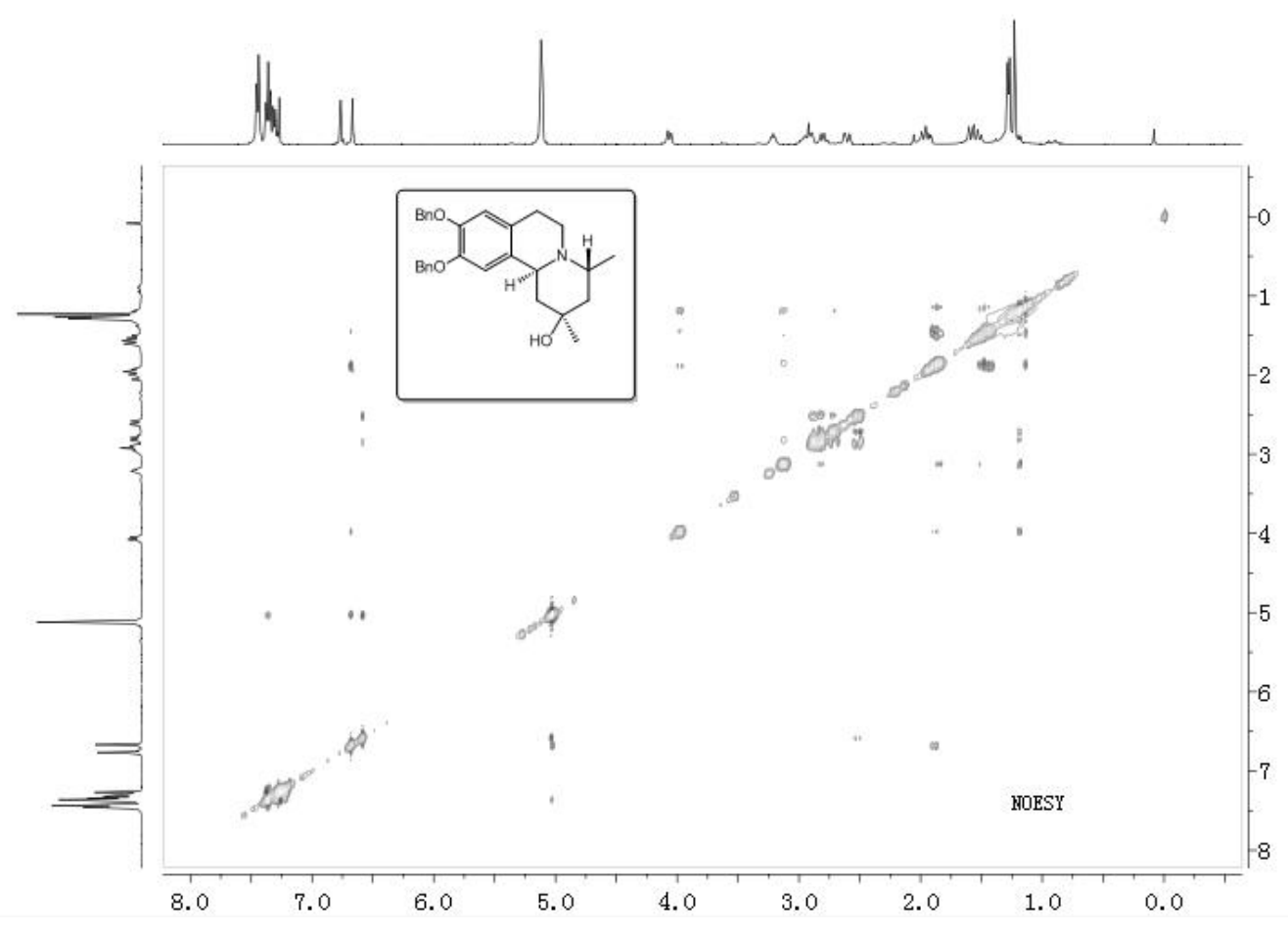

Figure S213. NOESY spectrum of 18. 


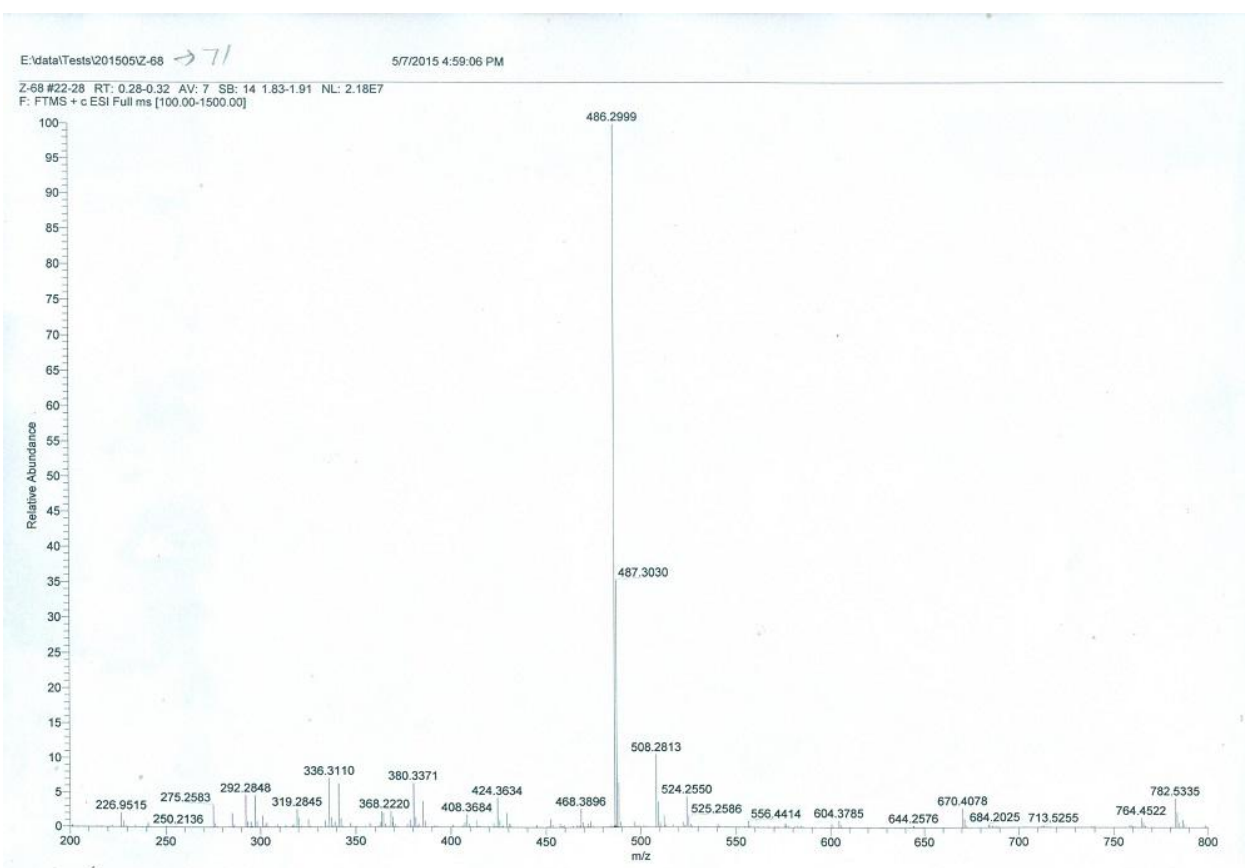

Figure S214. HRESIMS spectrum of 19.
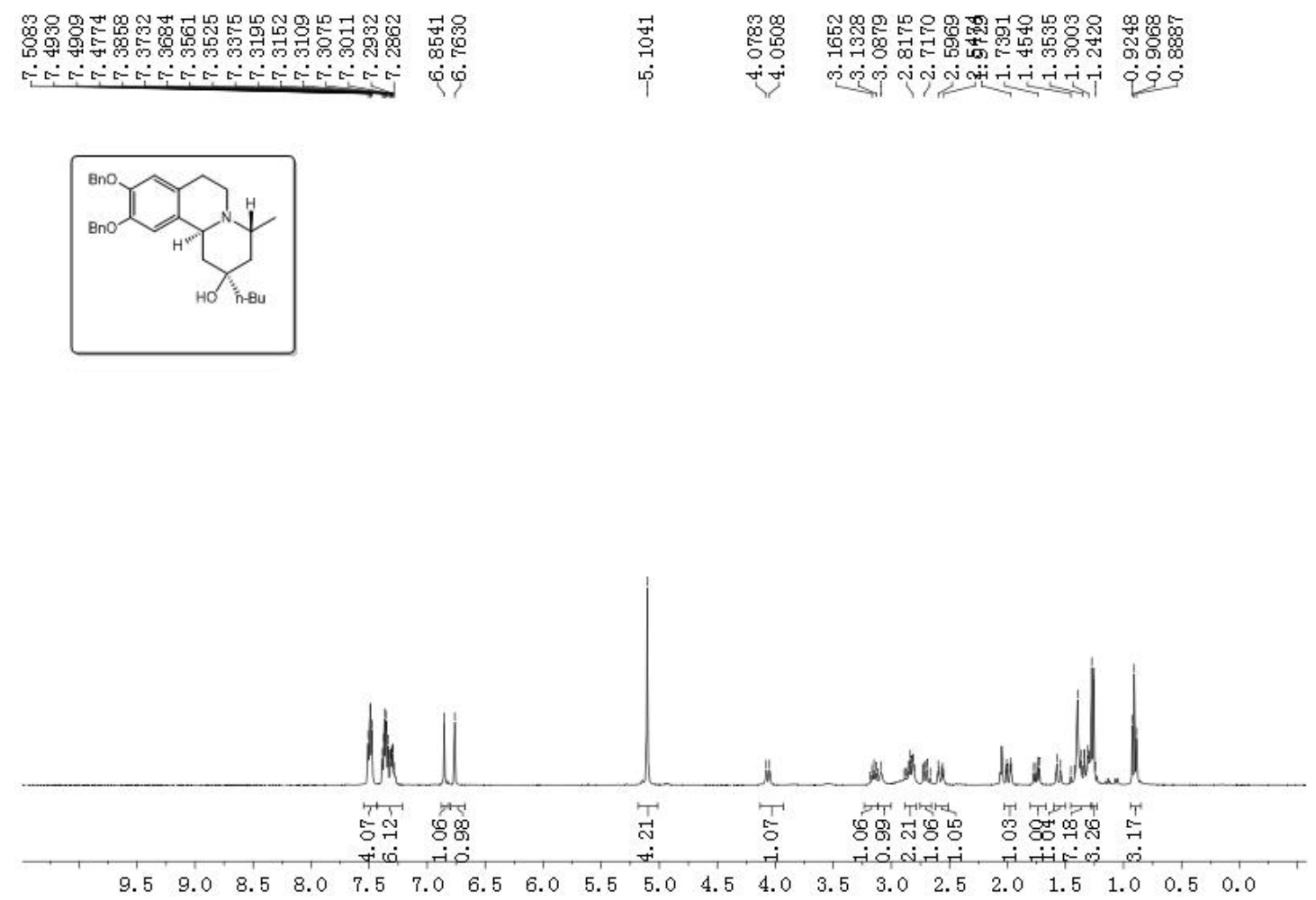

Figure S215. ${ }^{1} \mathrm{H}$ NMR spectrum of 19. 


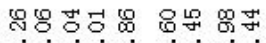

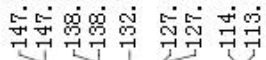

ㄱำ

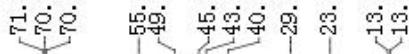
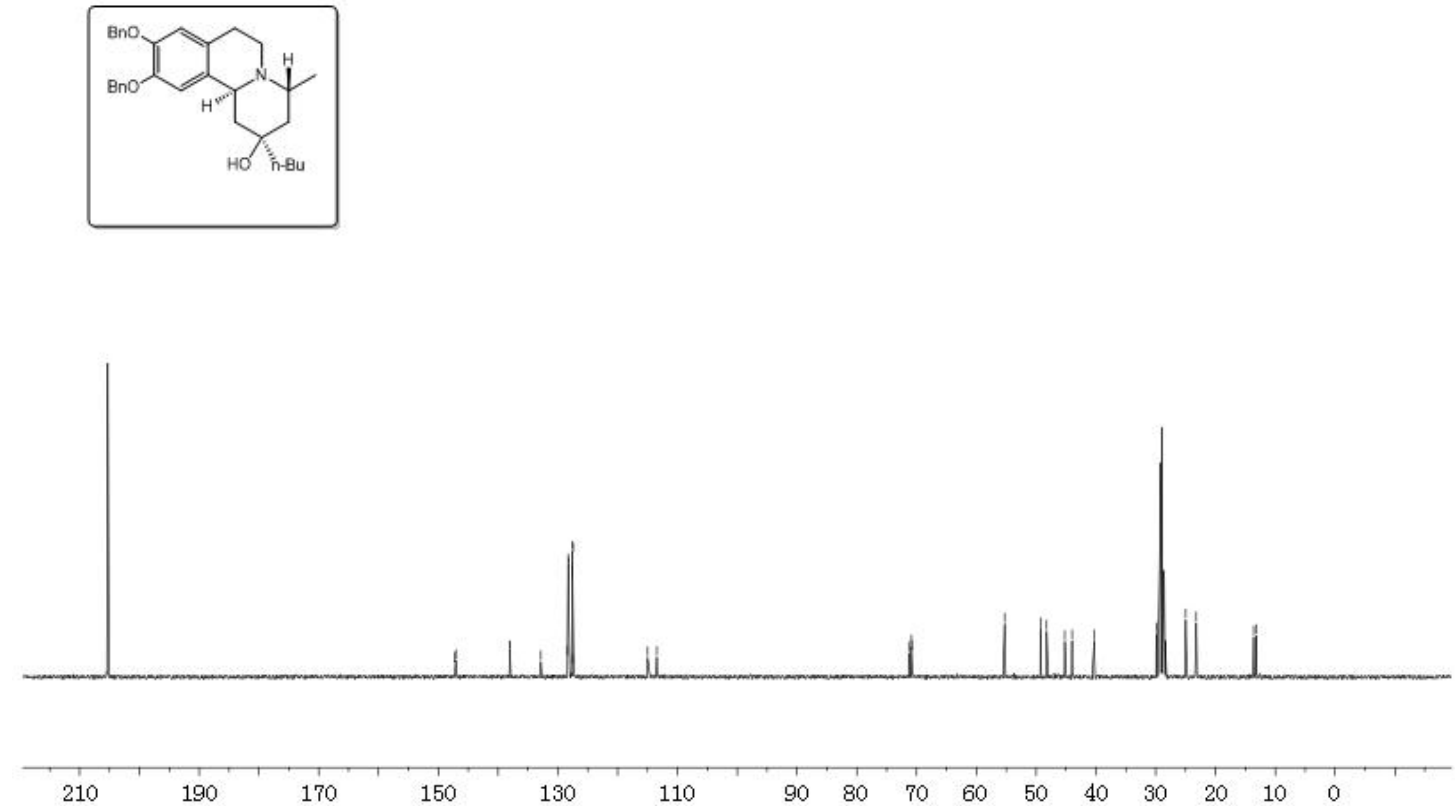

Figure S216. ${ }^{13} \mathrm{C}$ NMR spectrum of 19.

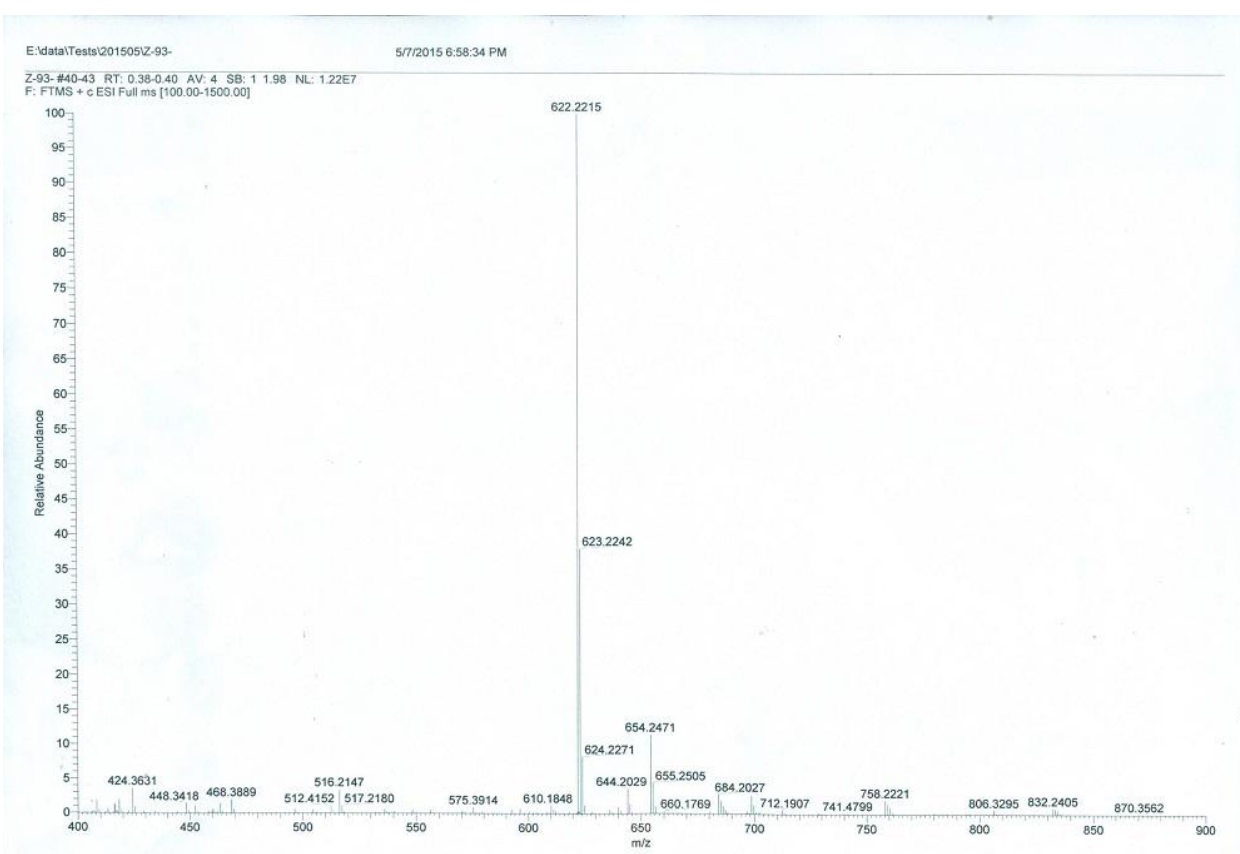

Figure S217. HRESIMS spectrum of SG1. 

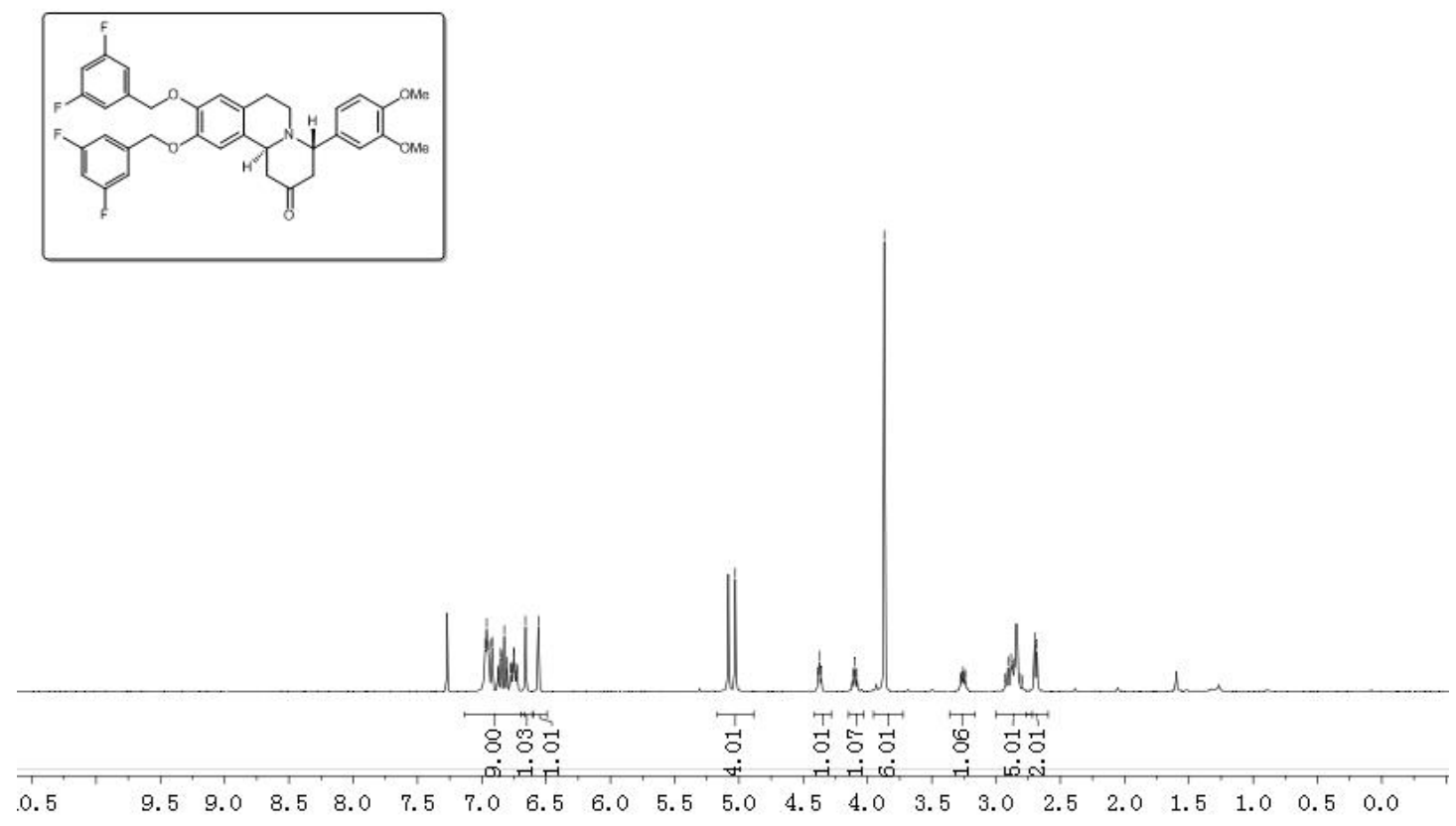

Figure S218. ${ }^{1} \mathrm{H}$ NMR spectrum of SG1.
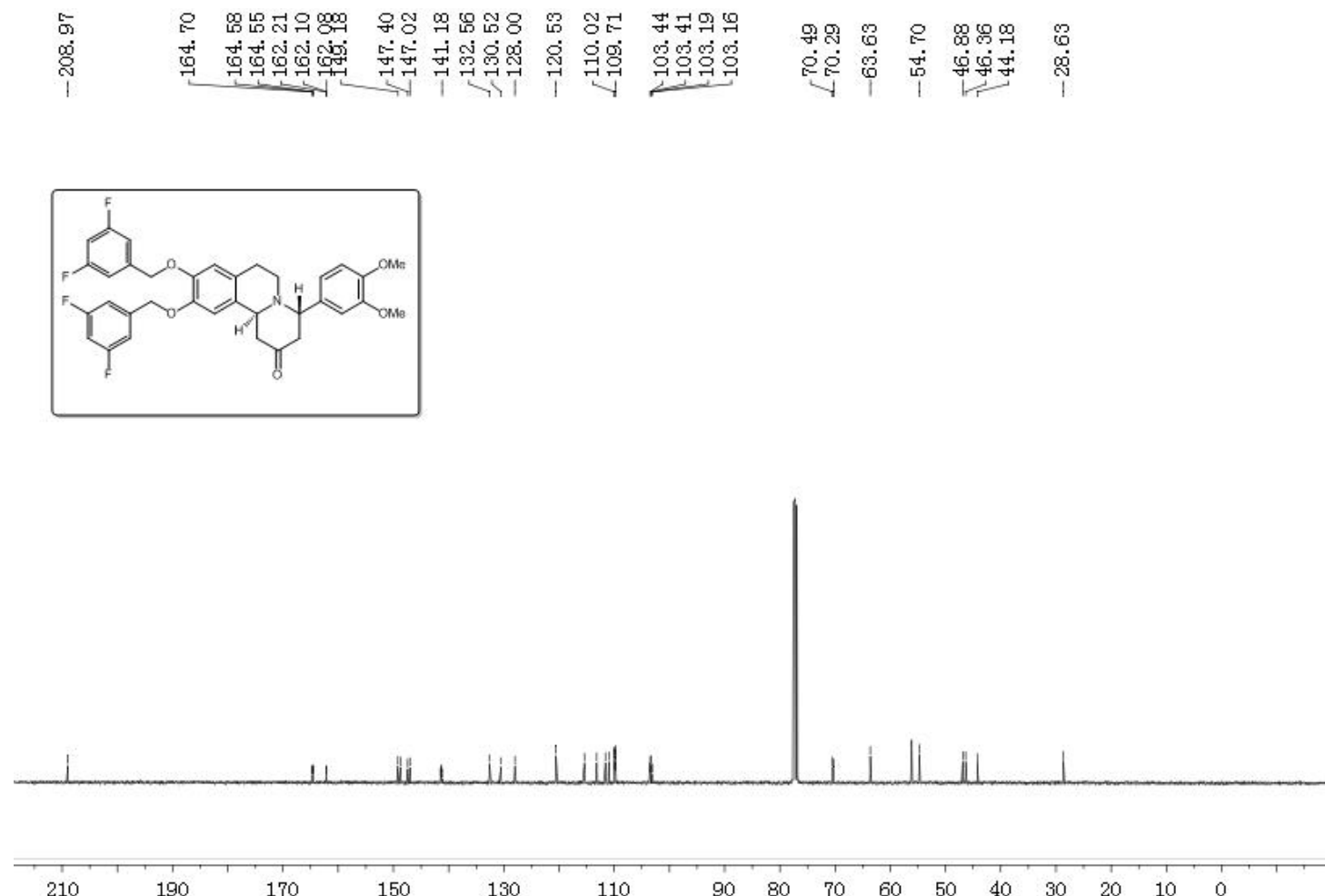

110

$\begin{array}{llll}90 & 80 \quad 70 \quad 60\end{array}$

$\begin{array}{lllll}50 & 40 & 30 & 20 & 10\end{array}$

Figure S219. ${ }^{13} \mathrm{C}$ NMR spectrum of SG1. 


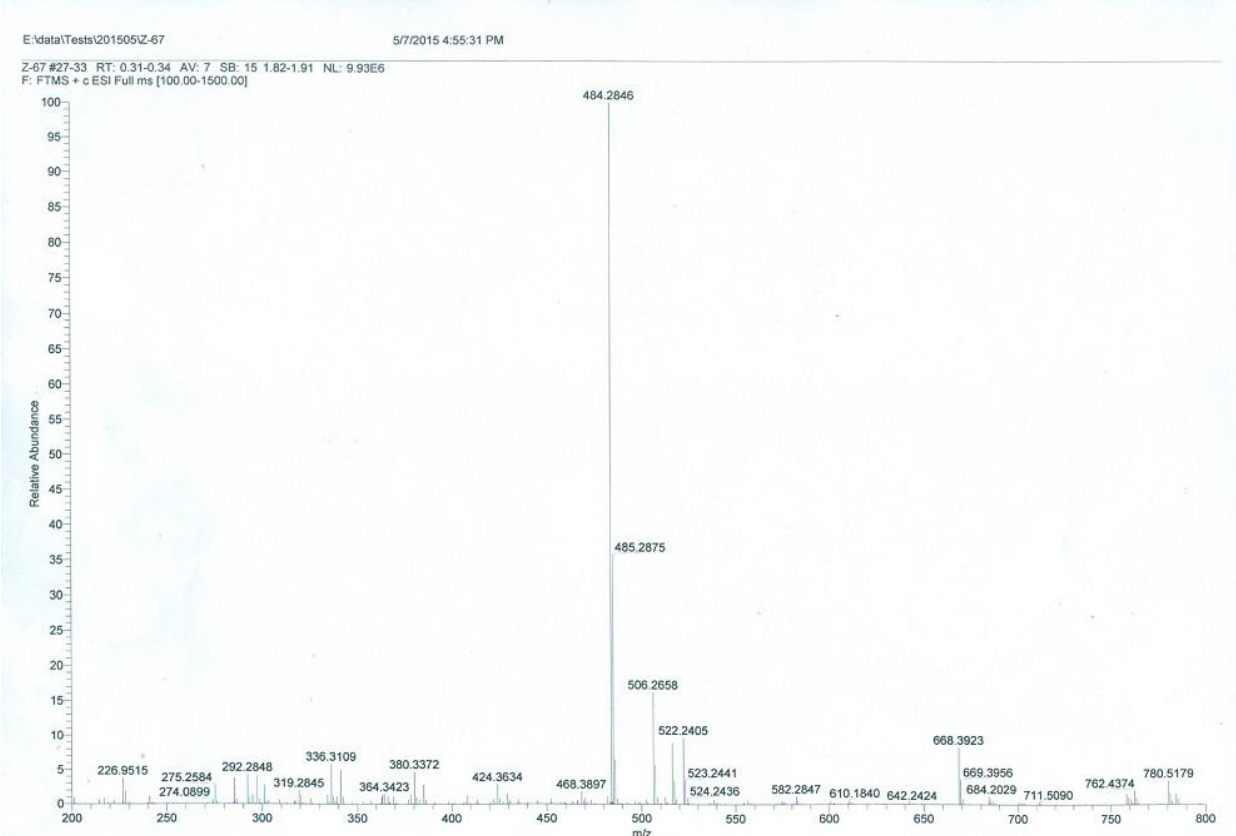

Figure S220. HRESIMS spectrum of SG2.
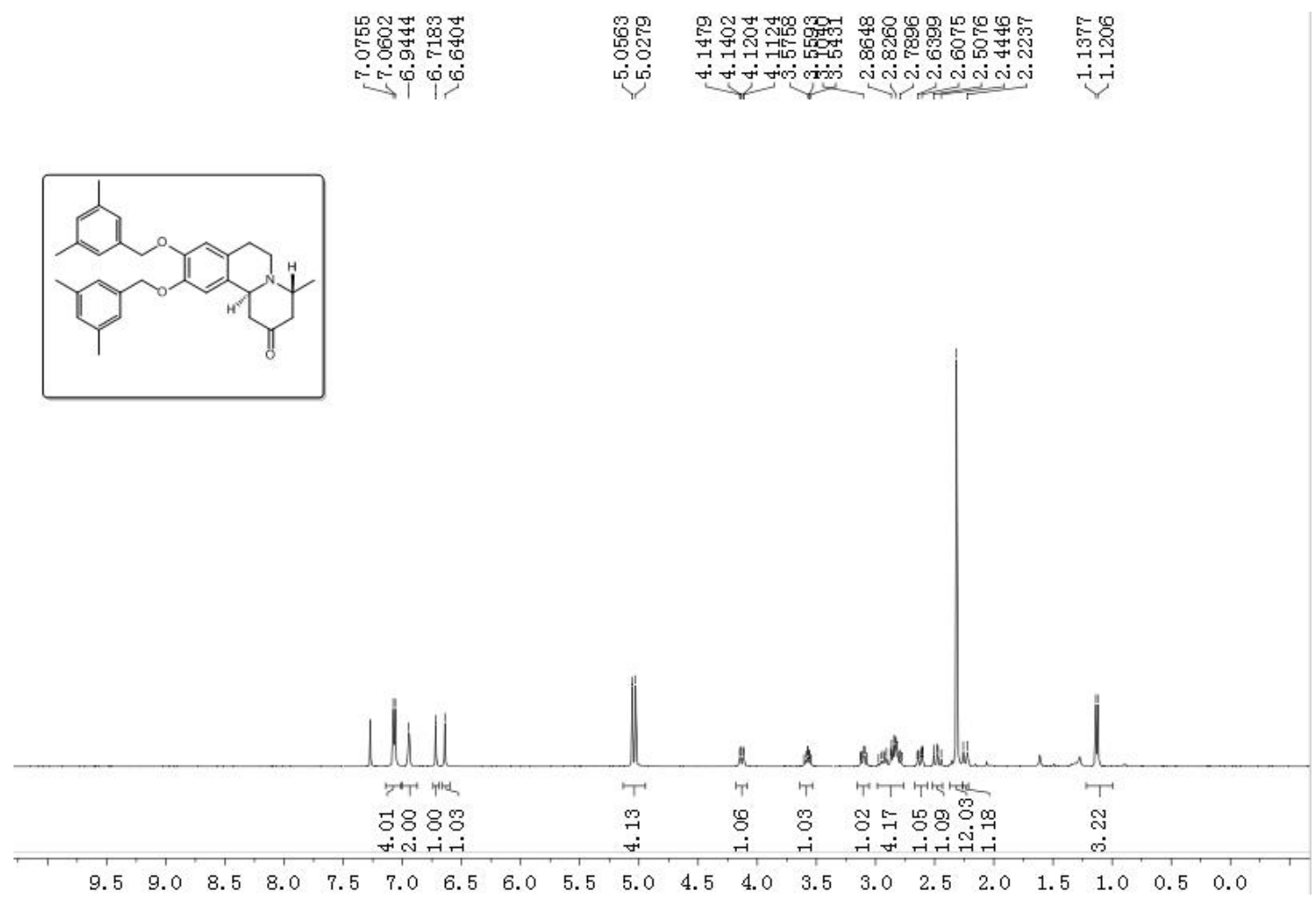

Figure S221. ${ }^{1} \mathrm{H}$ NMR spectrum of SG2. 


$$
\text { 감 }
$$
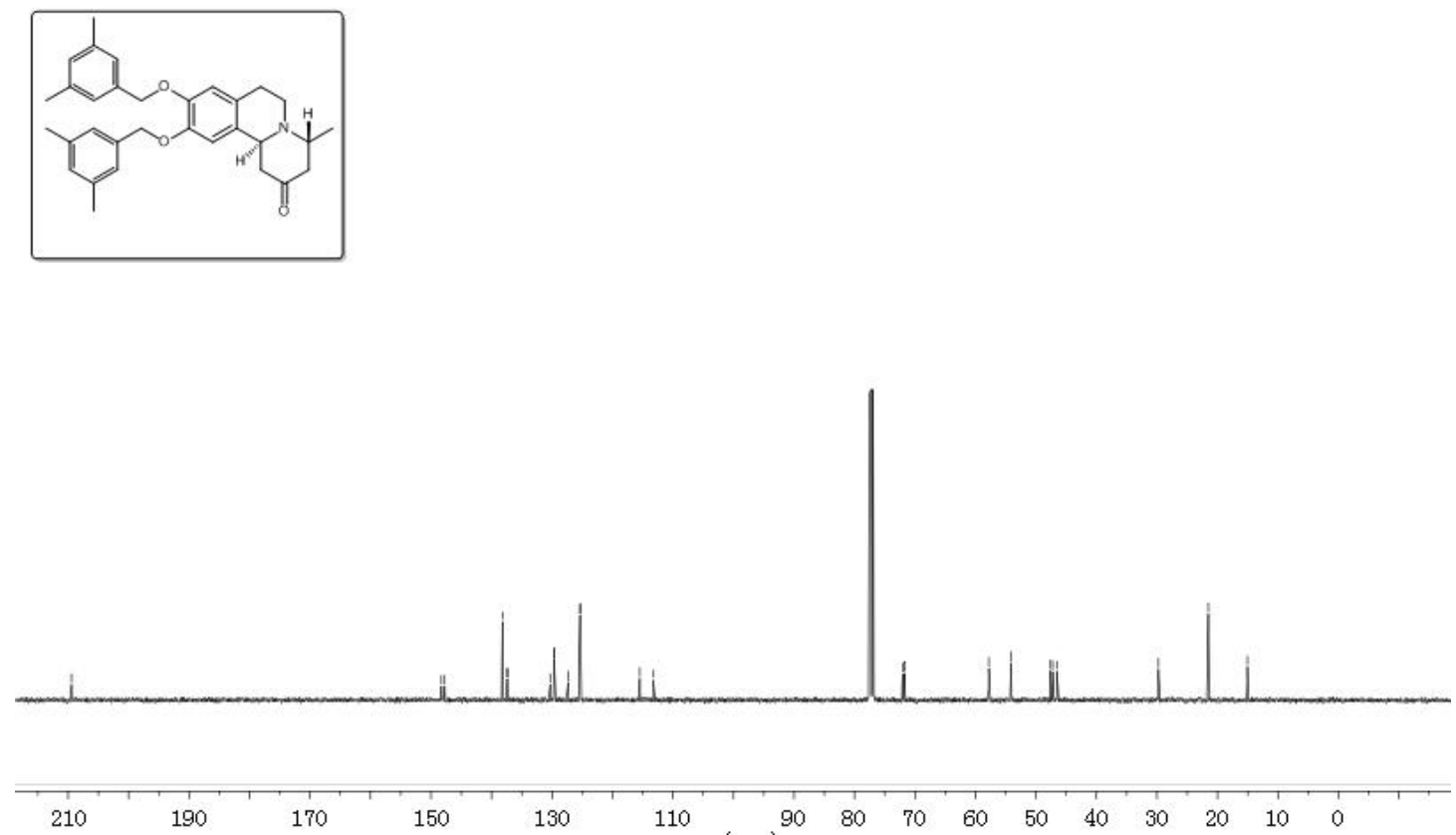

Figure S222. ${ }^{13} \mathrm{C}$ NMR spectrum of SG2.

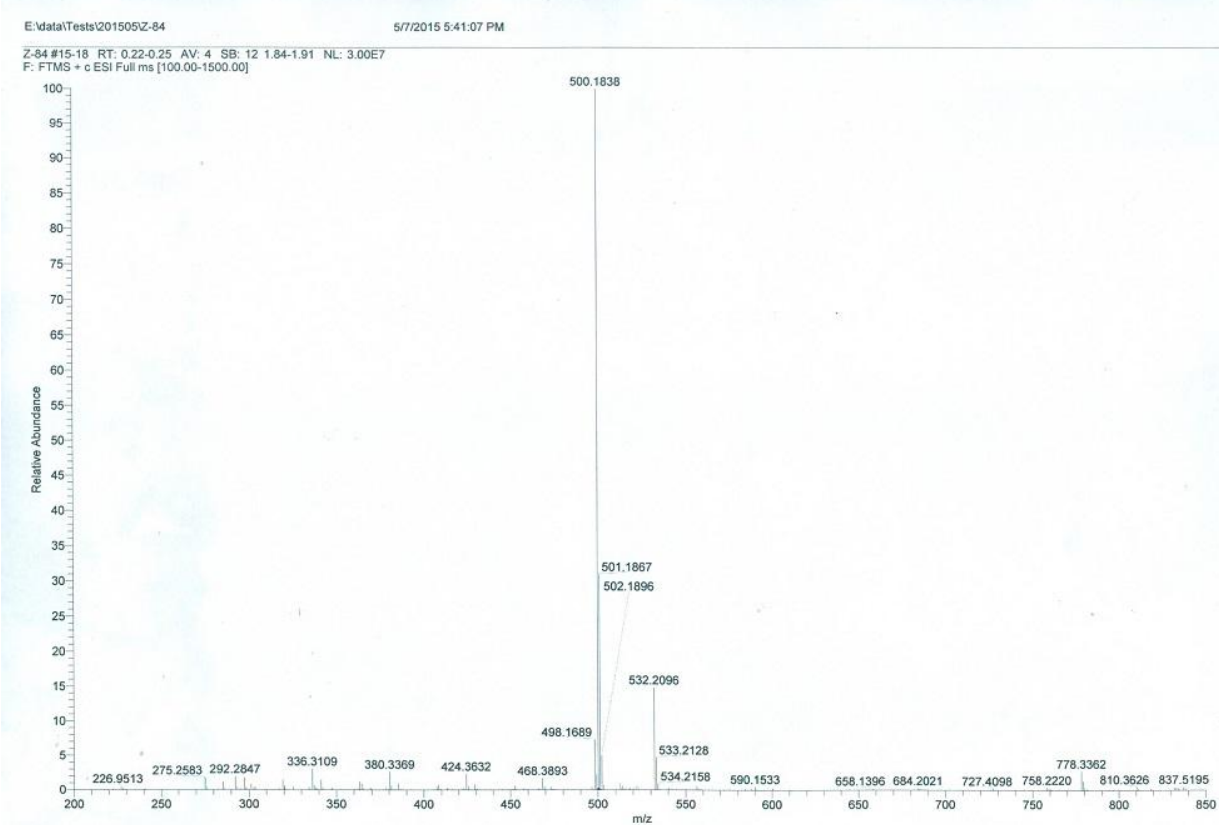

Figure S223. HRESIMS spectrum of SG3. 

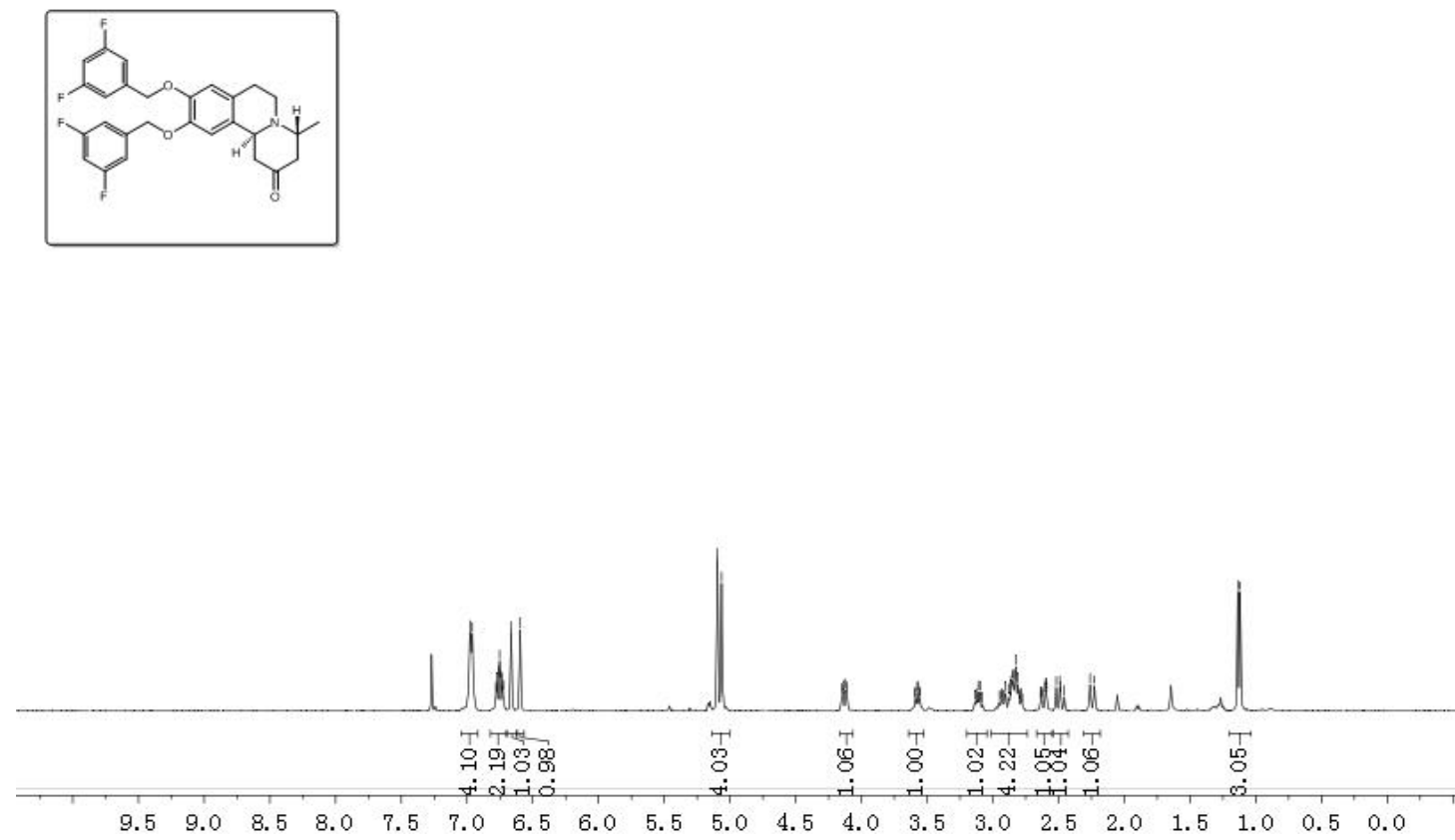

Figure S224. ${ }^{1} \mathrm{H}$ NMR spectrum of SG3.

던ㄷㄴ옥

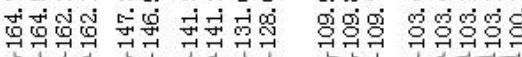
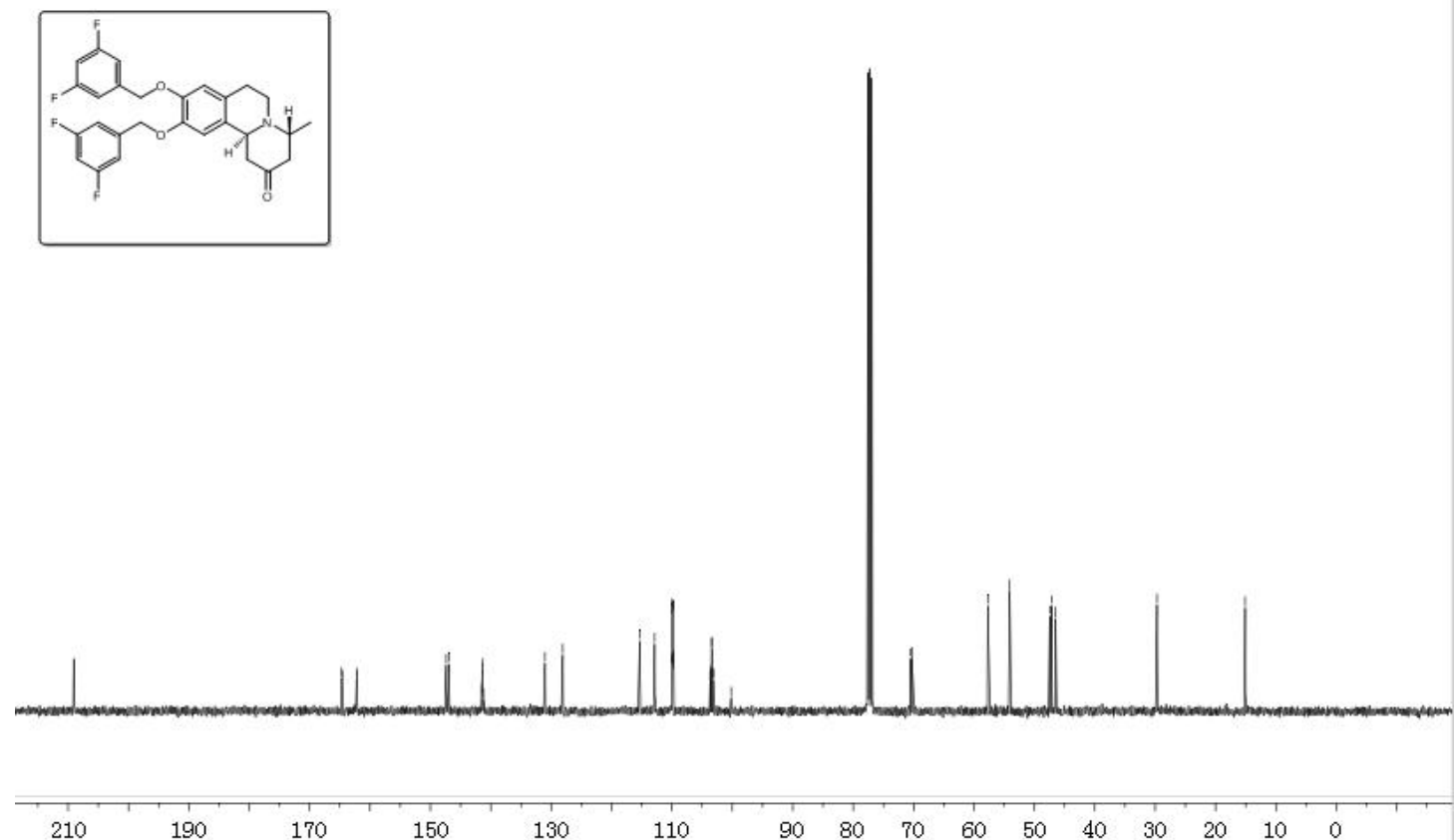

Figure S225. ${ }^{13} \mathrm{C}$ NMR spectrum of SG3. 


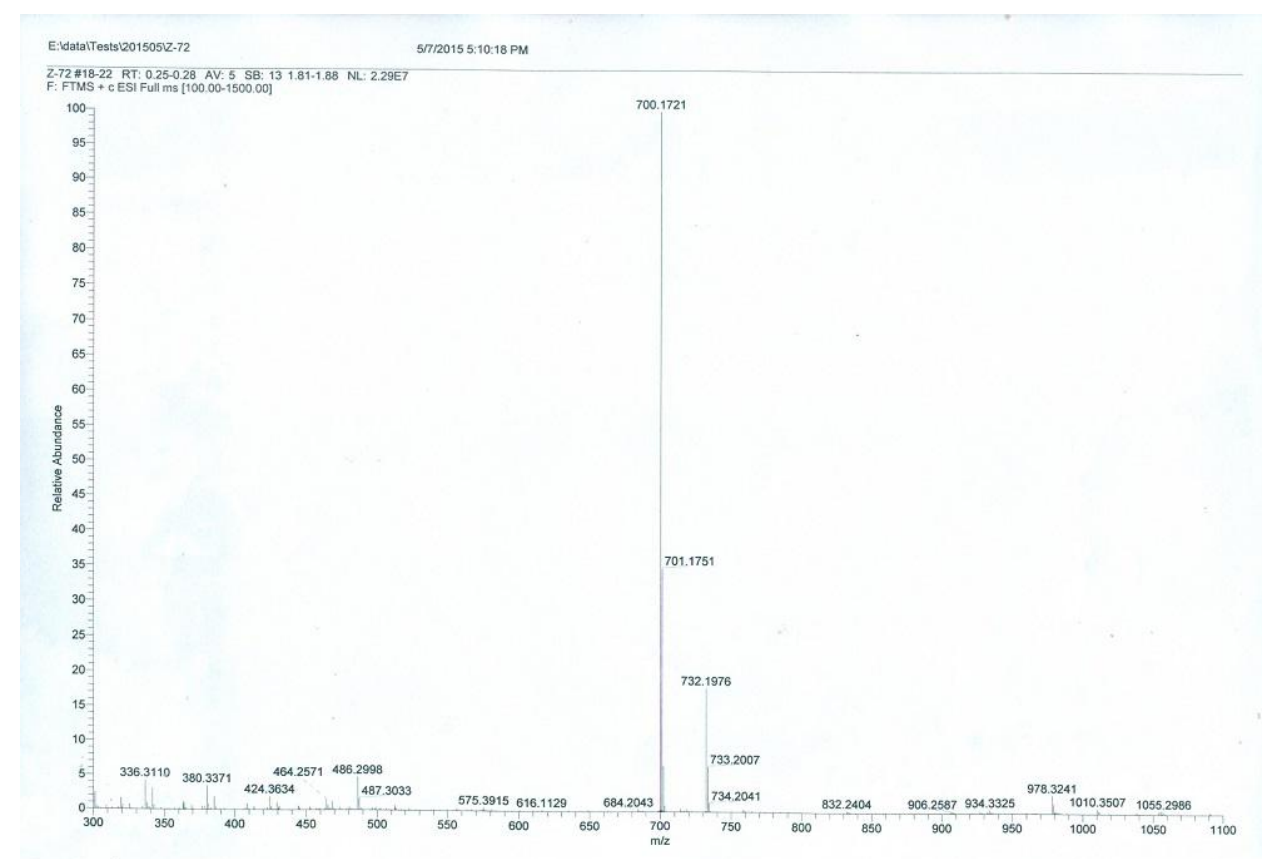

Figure S226. HRESIMS spectrum of SG4.
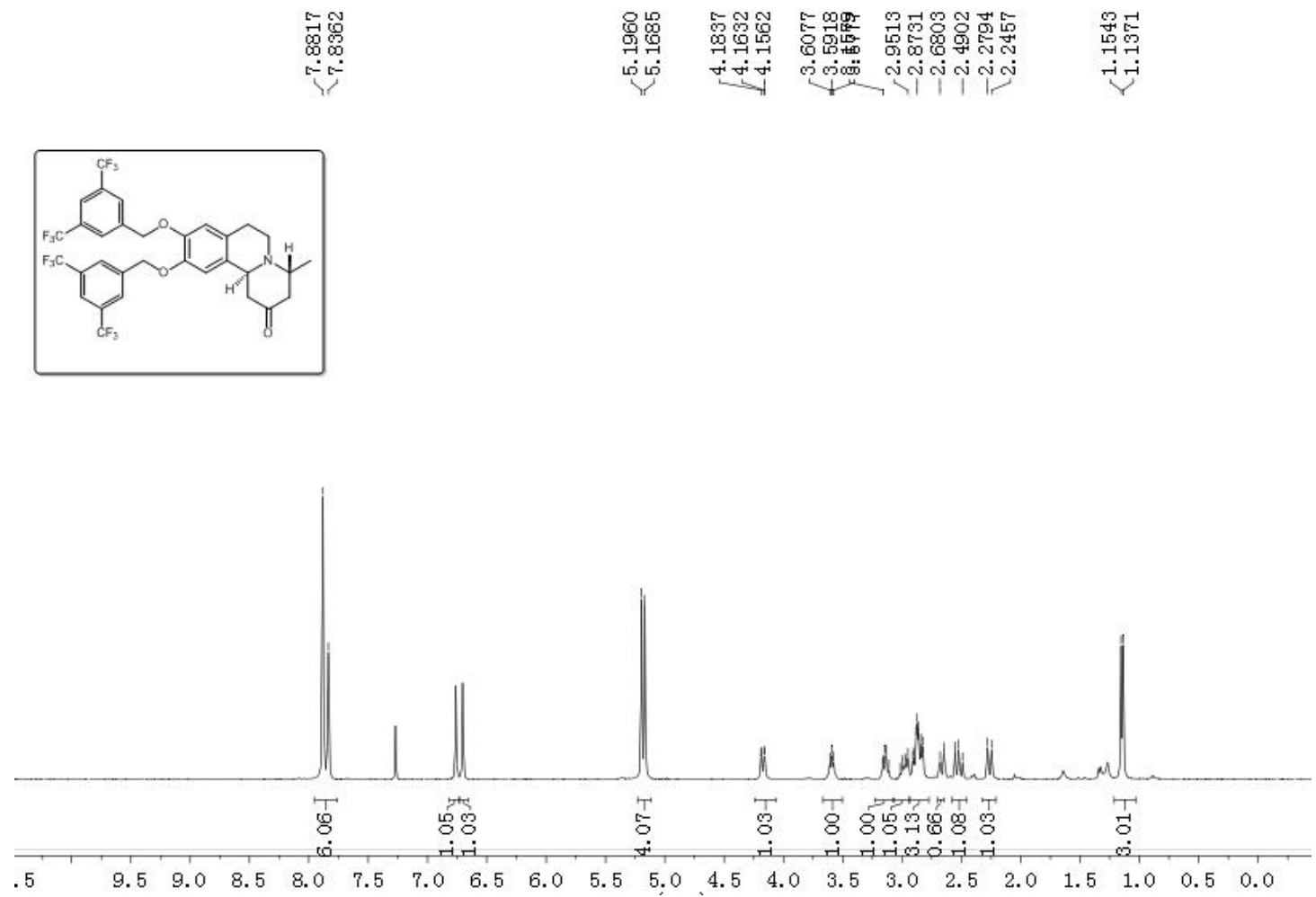

Figure S227. ${ }^{1}$ H NMR spectrum of SG4. 

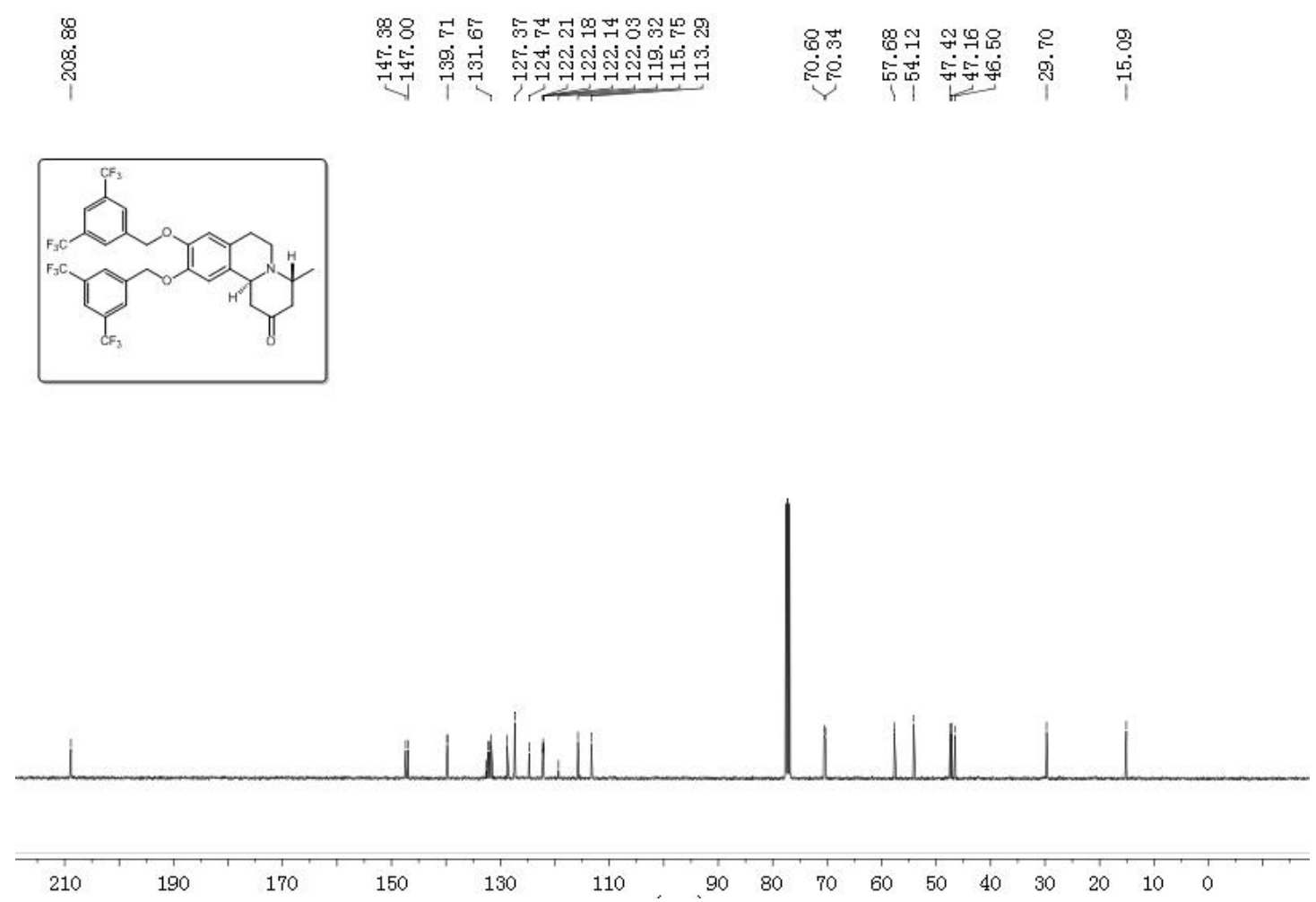

Figure S228. ${ }^{13} \mathrm{C}$ NMR spectrum of SG4.

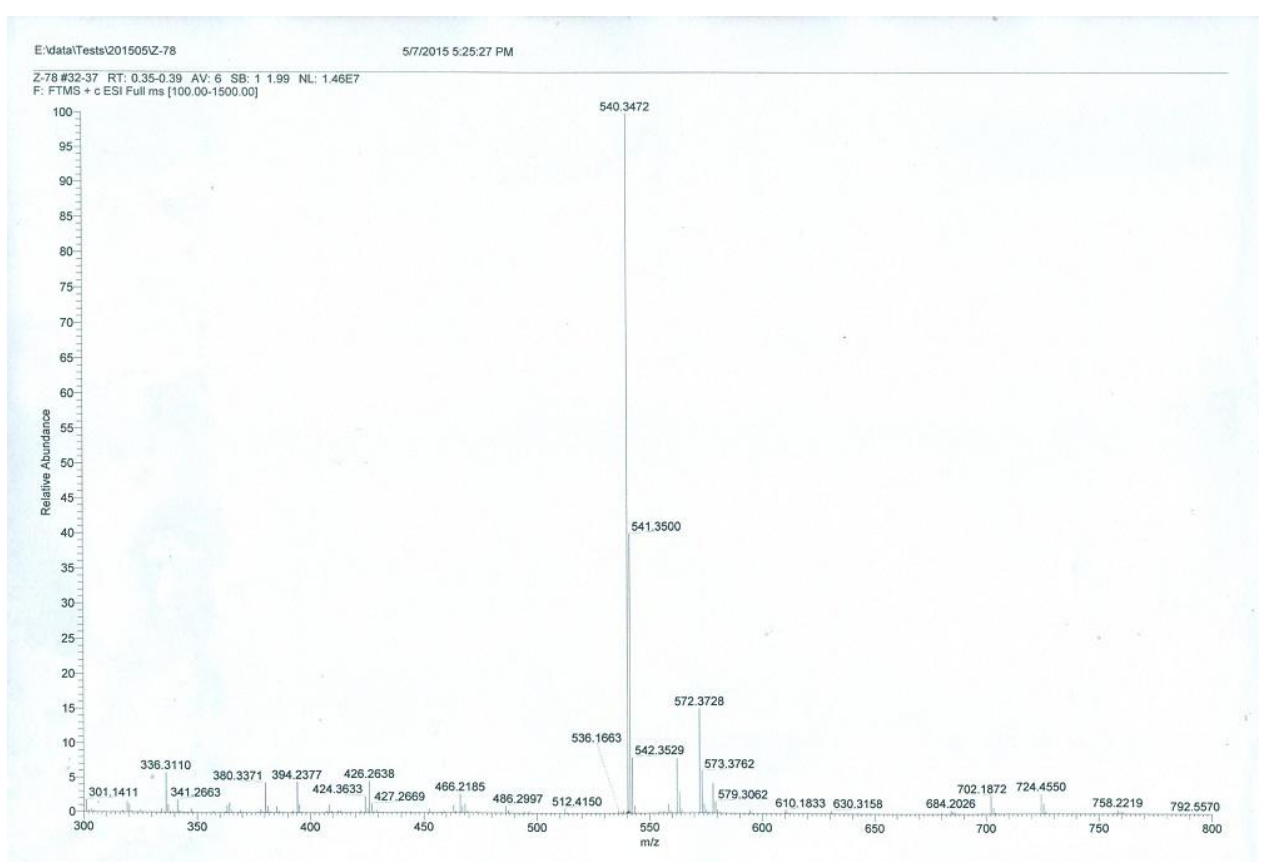

Figure S229. HRESIMS spectrum of SG5. 

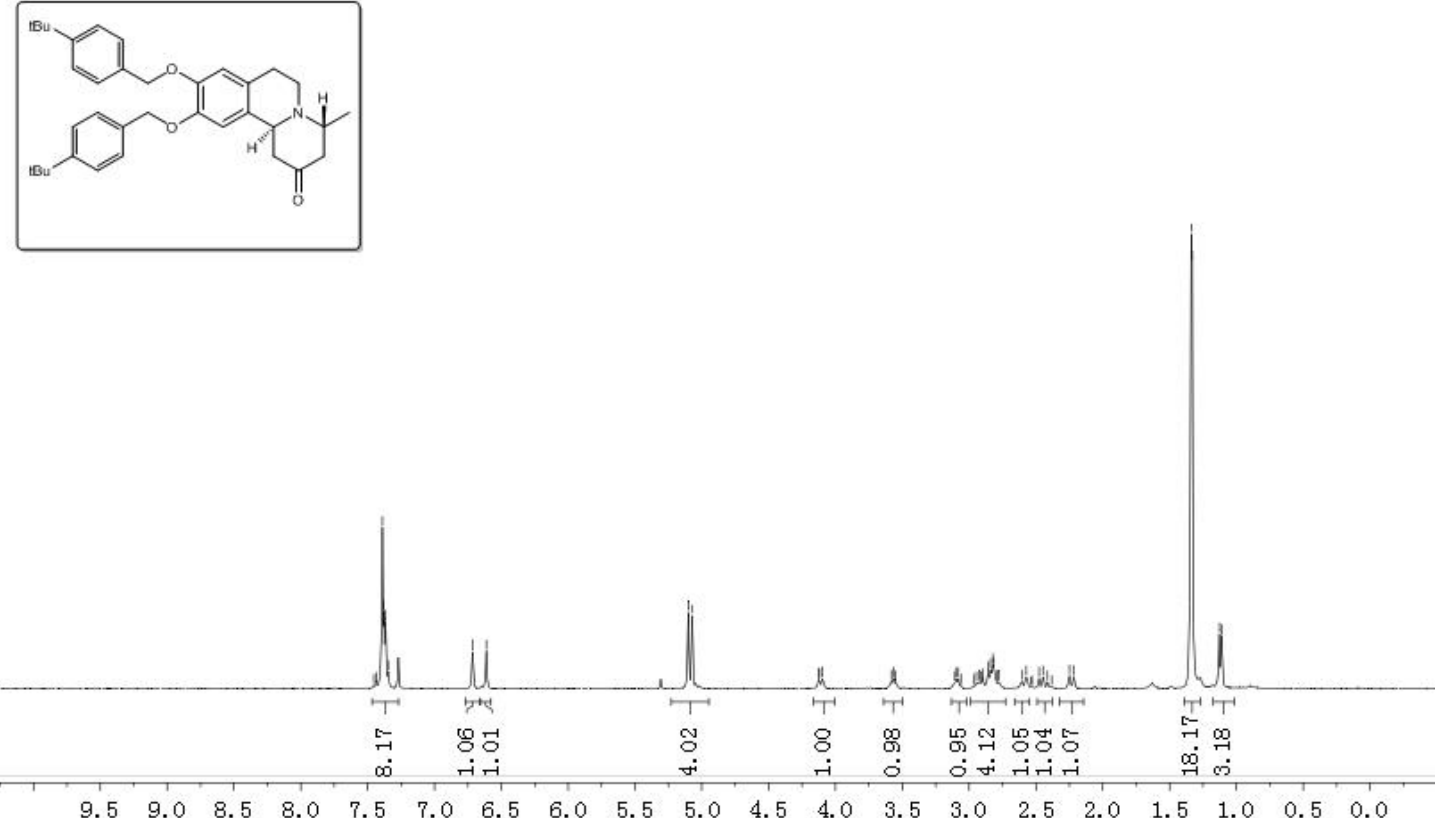

Figure S230. ${ }^{1} \mathrm{H}$ NMR spectrum of SG5.

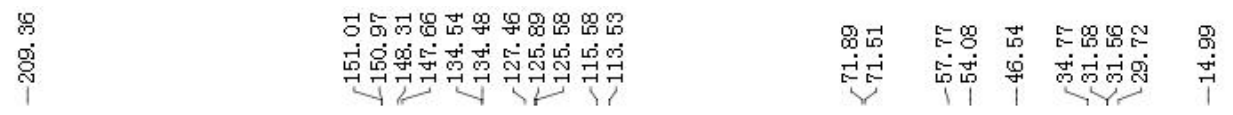
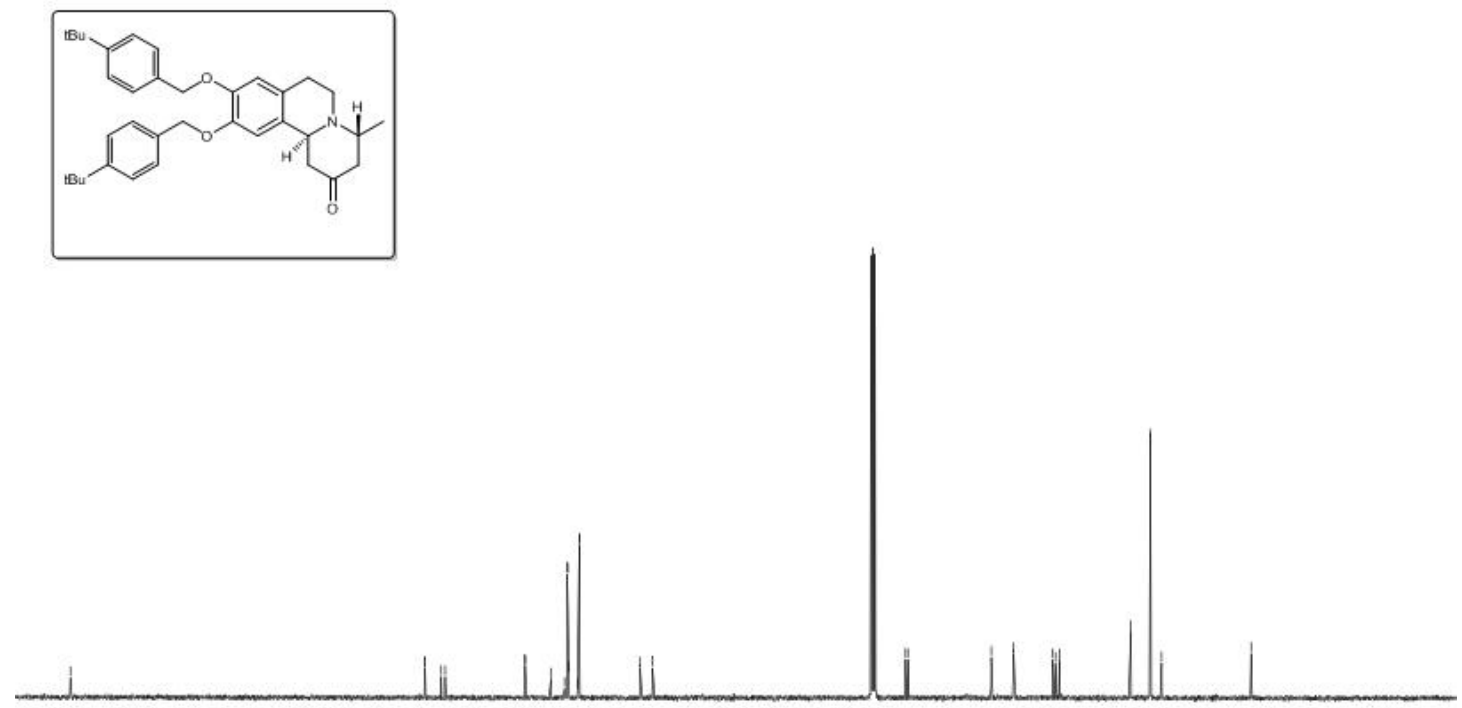

Figure S231. ${ }^{13} \mathrm{C}$ NMR spectrum of SG5. 


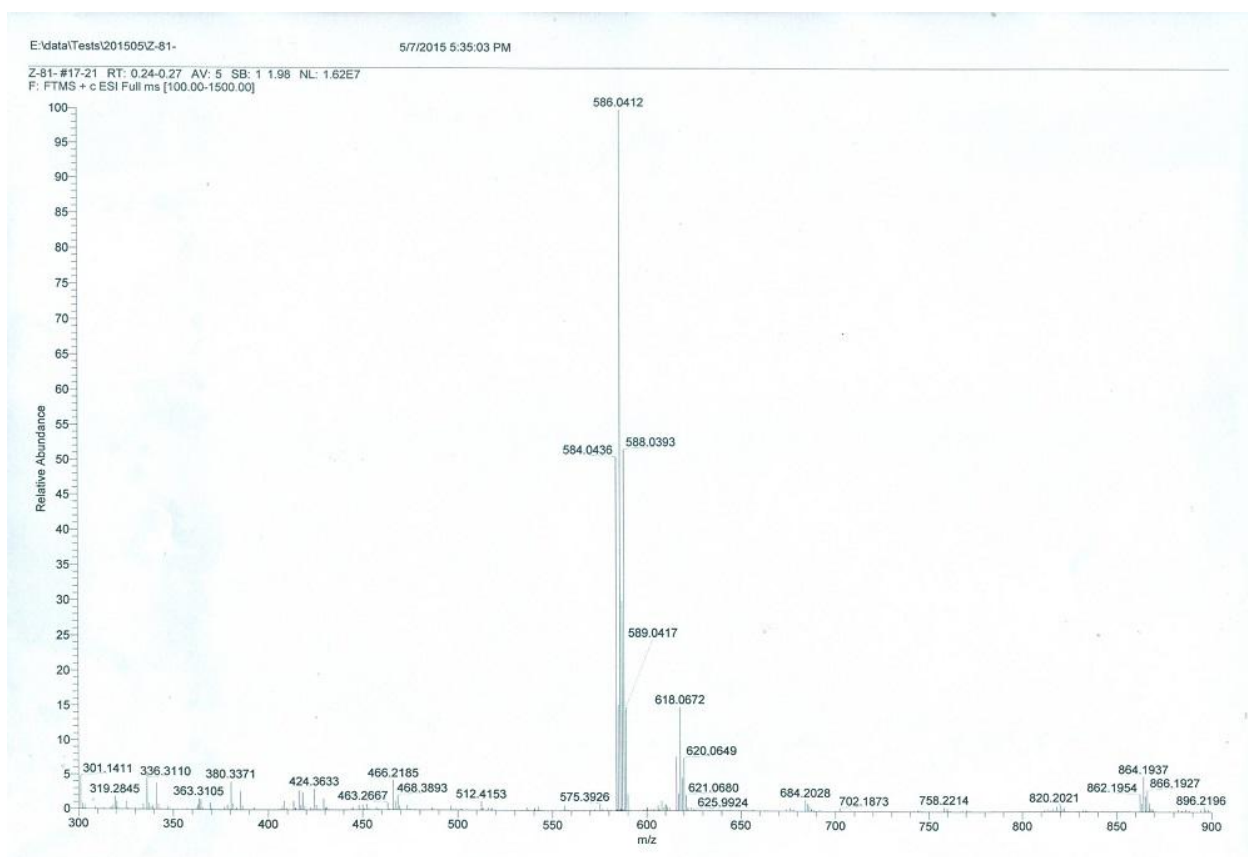

Figure S232. HRESIMS spectrum of SG6.
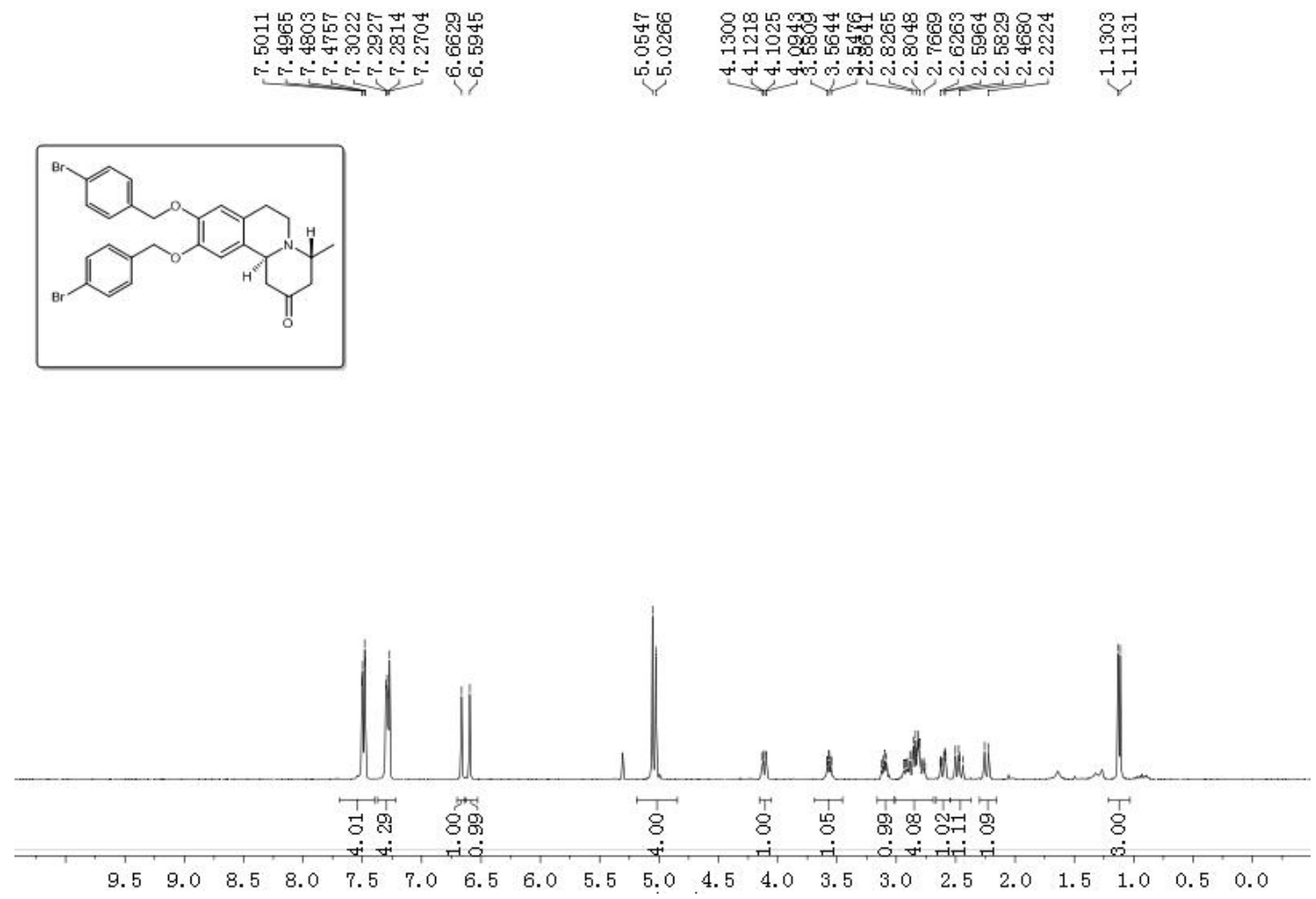

Figure S233. ${ }^{1} \mathrm{H}$ NMR spectrum of SG6 

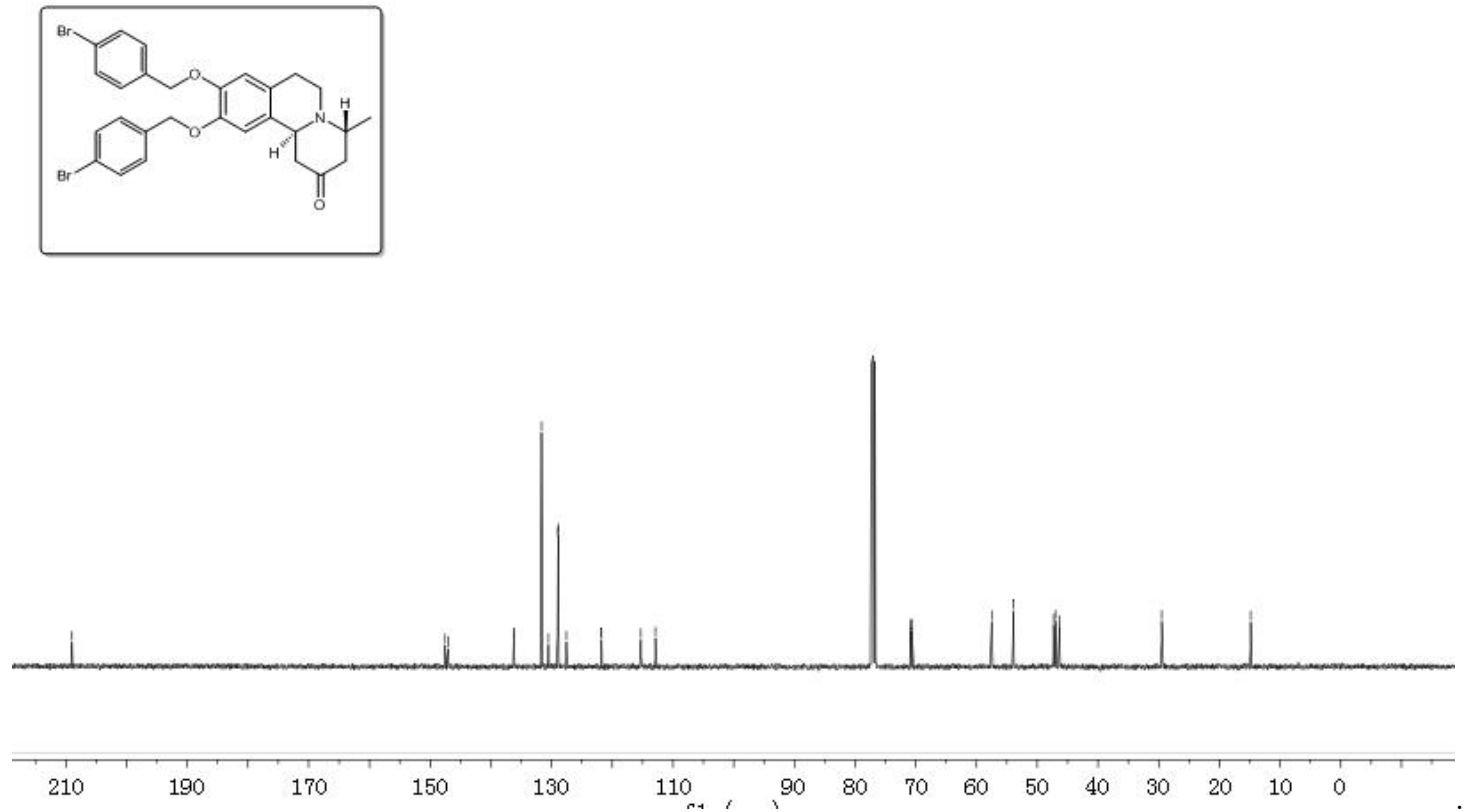

Figure S234. ${ }^{13} \mathrm{C}$ NMR spectrum of SG6

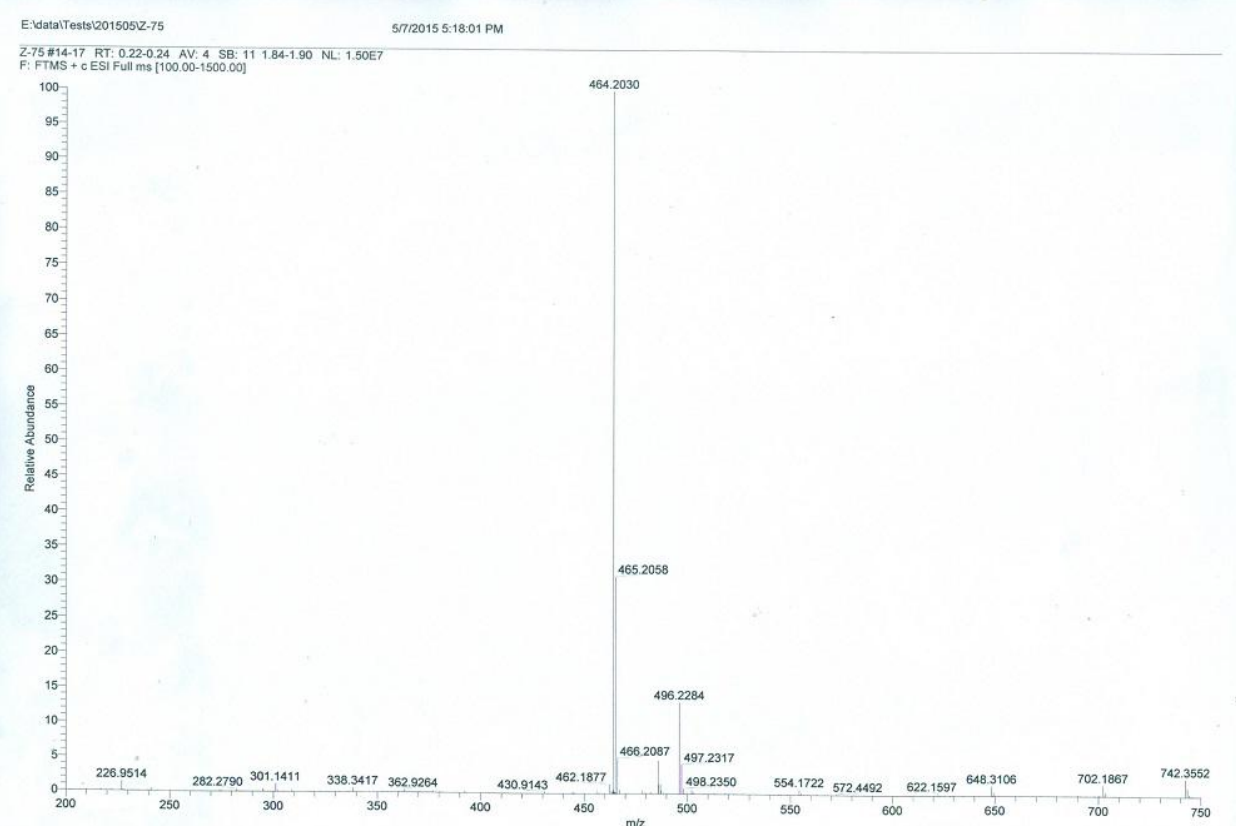

Figure S235. HRESIMS spectrum of 35 . 

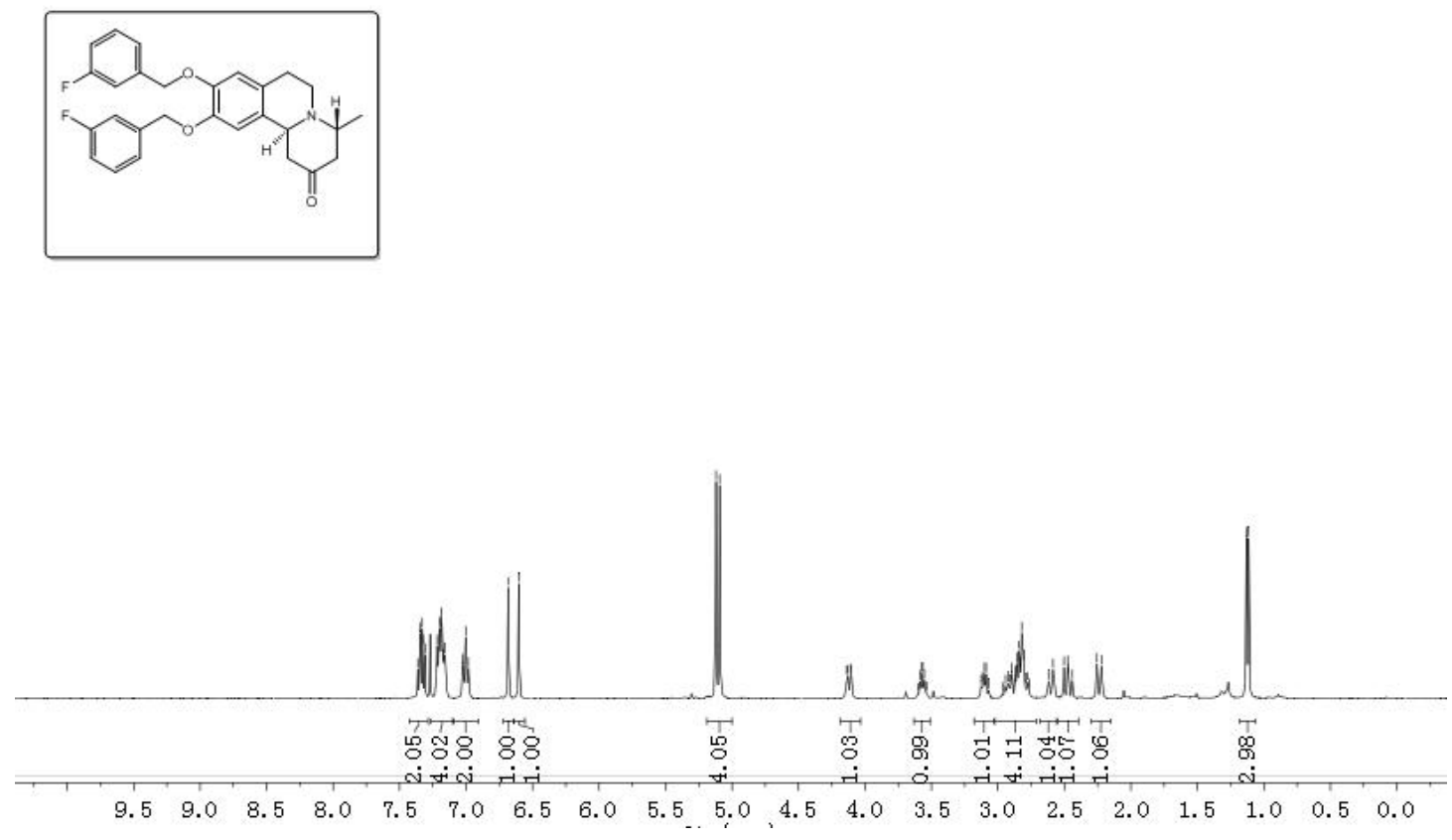

Figure S236. ${ }^{1} \mathrm{H}$ NMR spectrum of 35 .
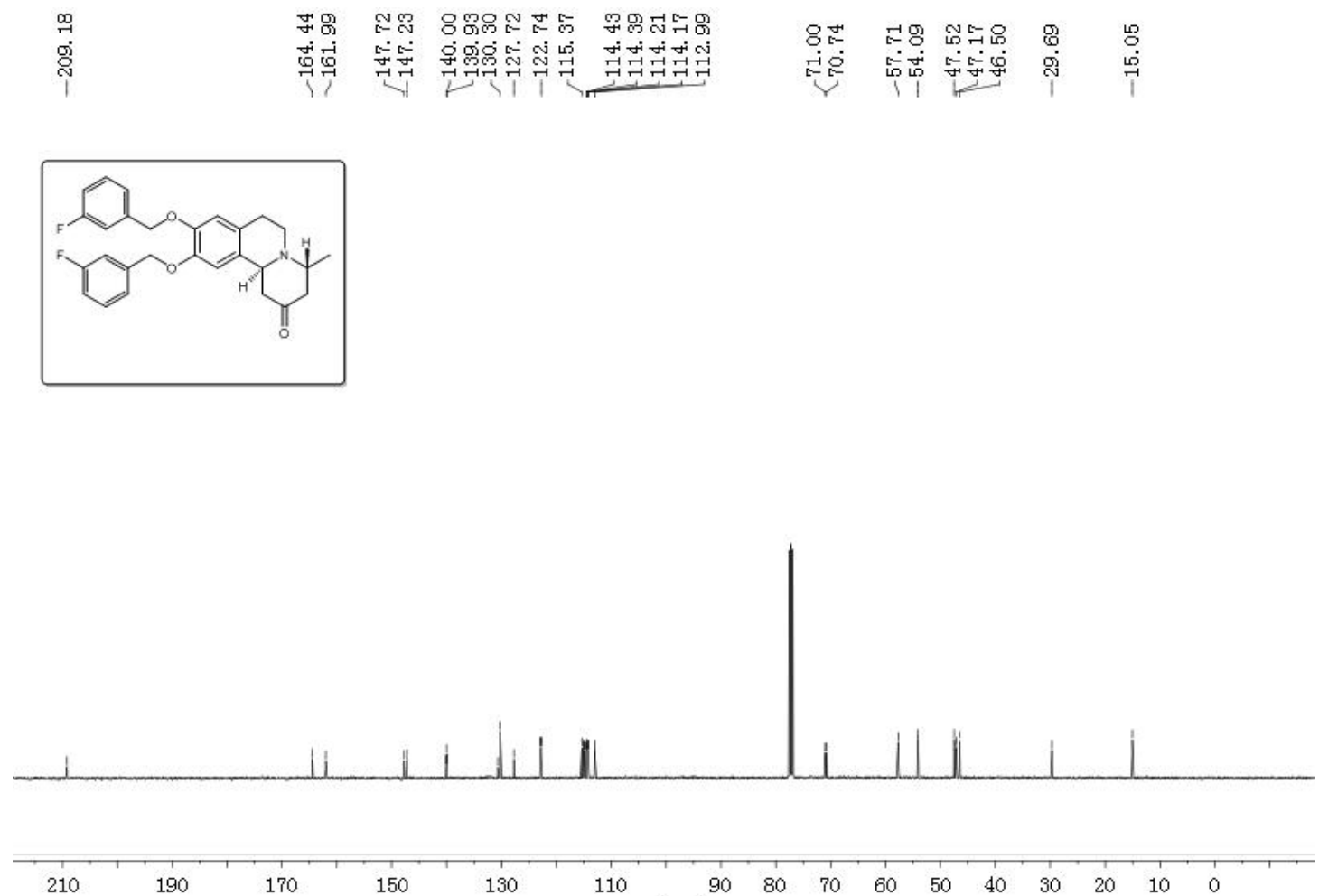

Figure S237. ${ }^{13} \mathrm{C}$ NMR spectrum of 35 . 

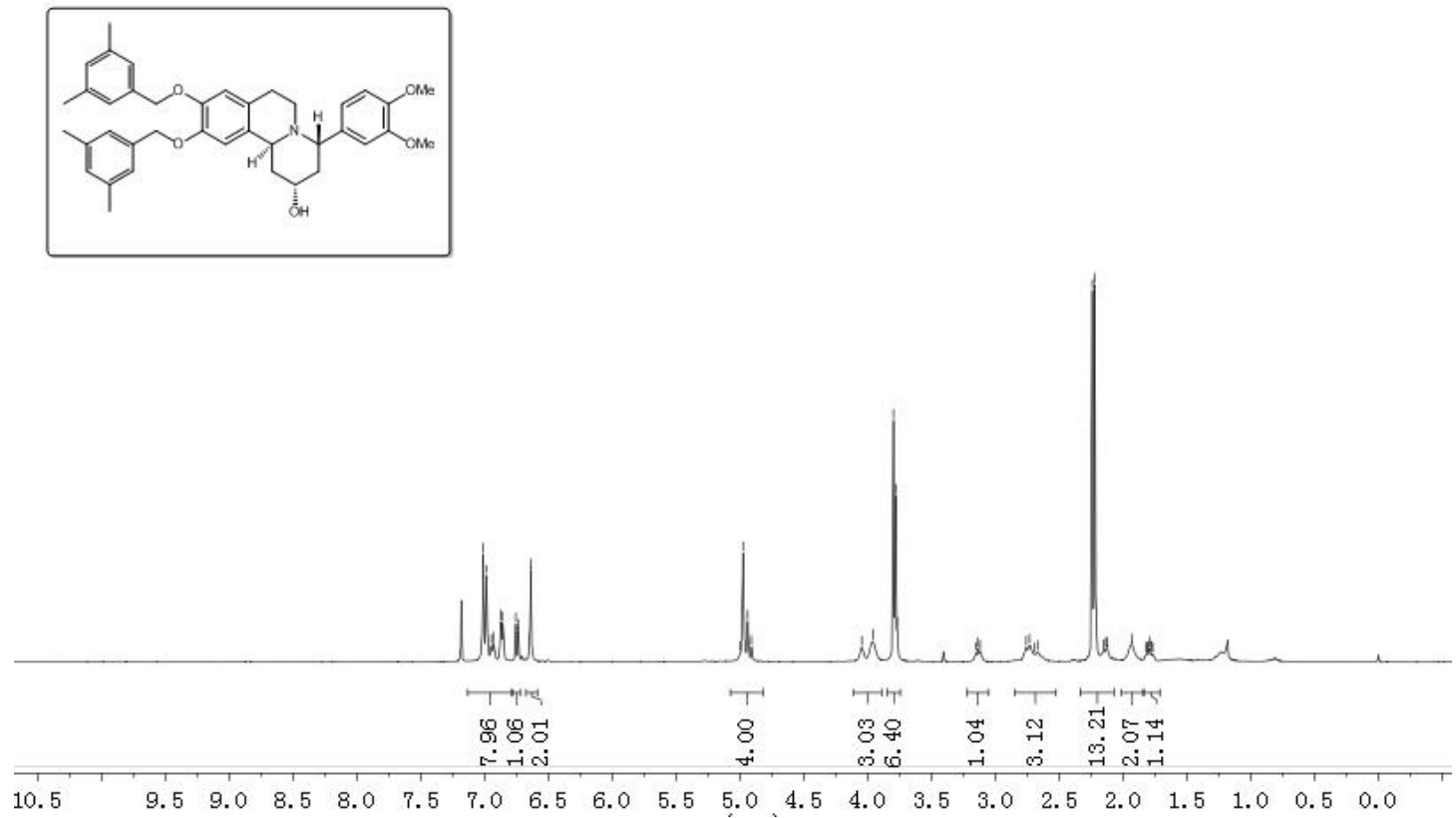

Figure S238. ${ }^{1} \mathrm{H}$ NMR spectrum of 20a.

ल죽워

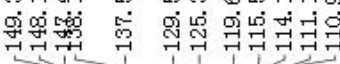

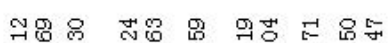

선려
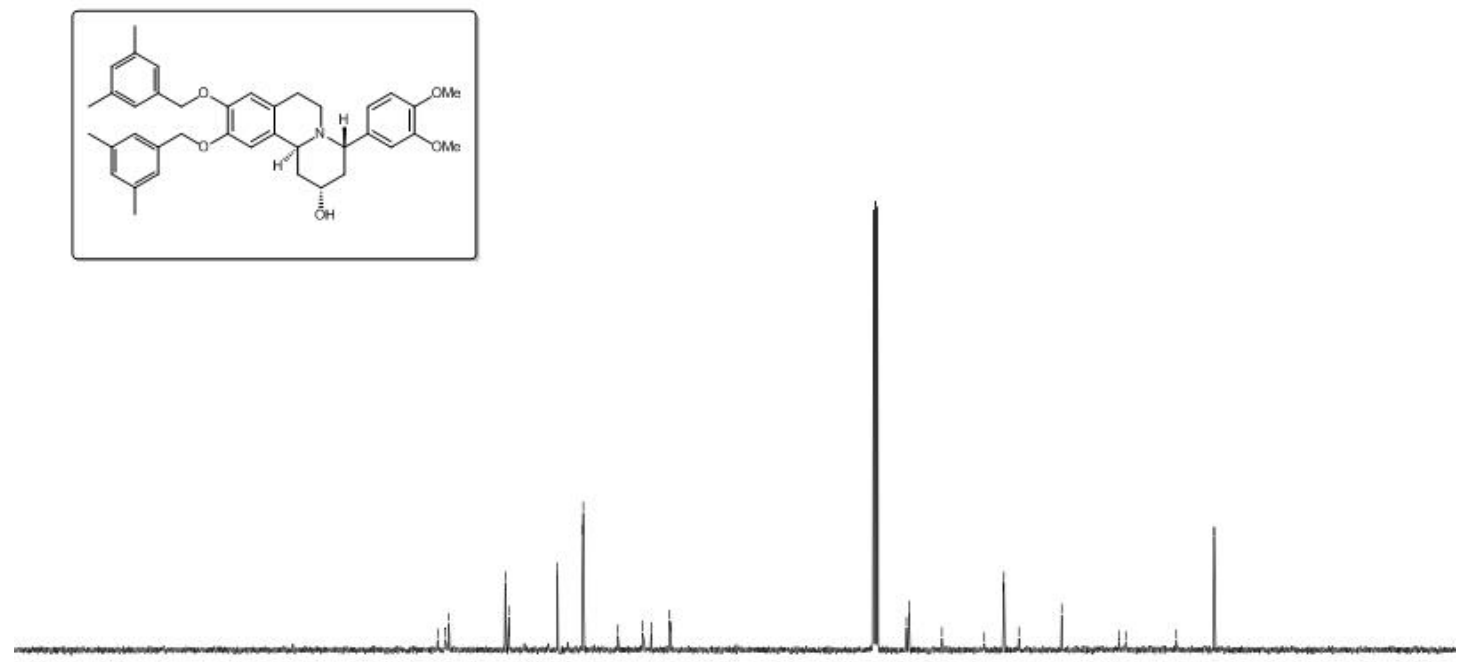

210

190

150

130

110

80

50

Figure S239. ${ }^{13} \mathrm{C}$ NMR spectrum of $\mathbf{2 0 a}$. 


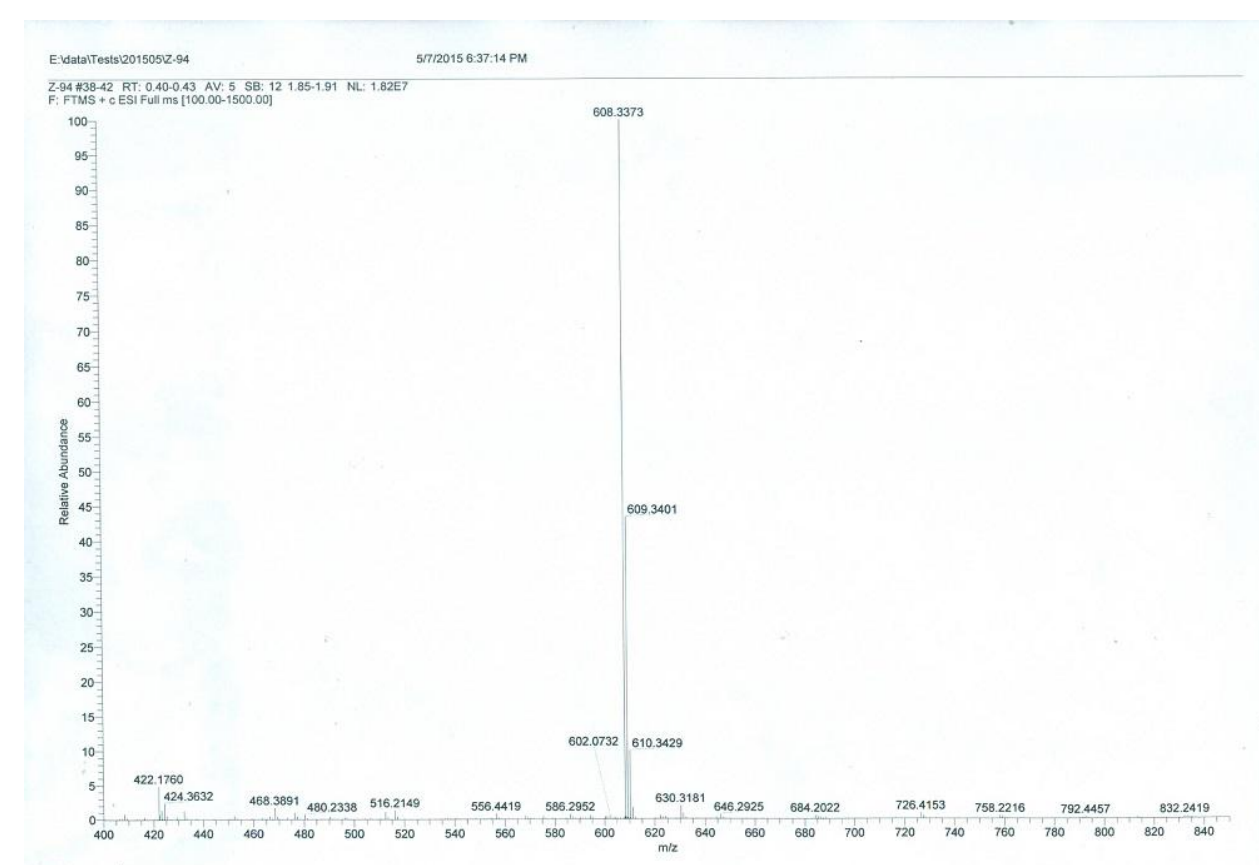

Figure S240. HRESIMS spectrum of $\mathbf{2 0 b}$.

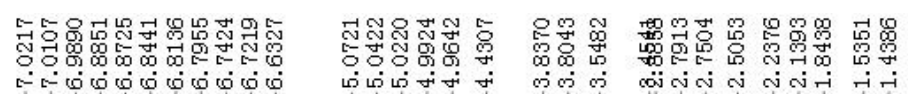

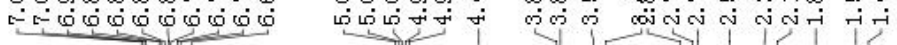

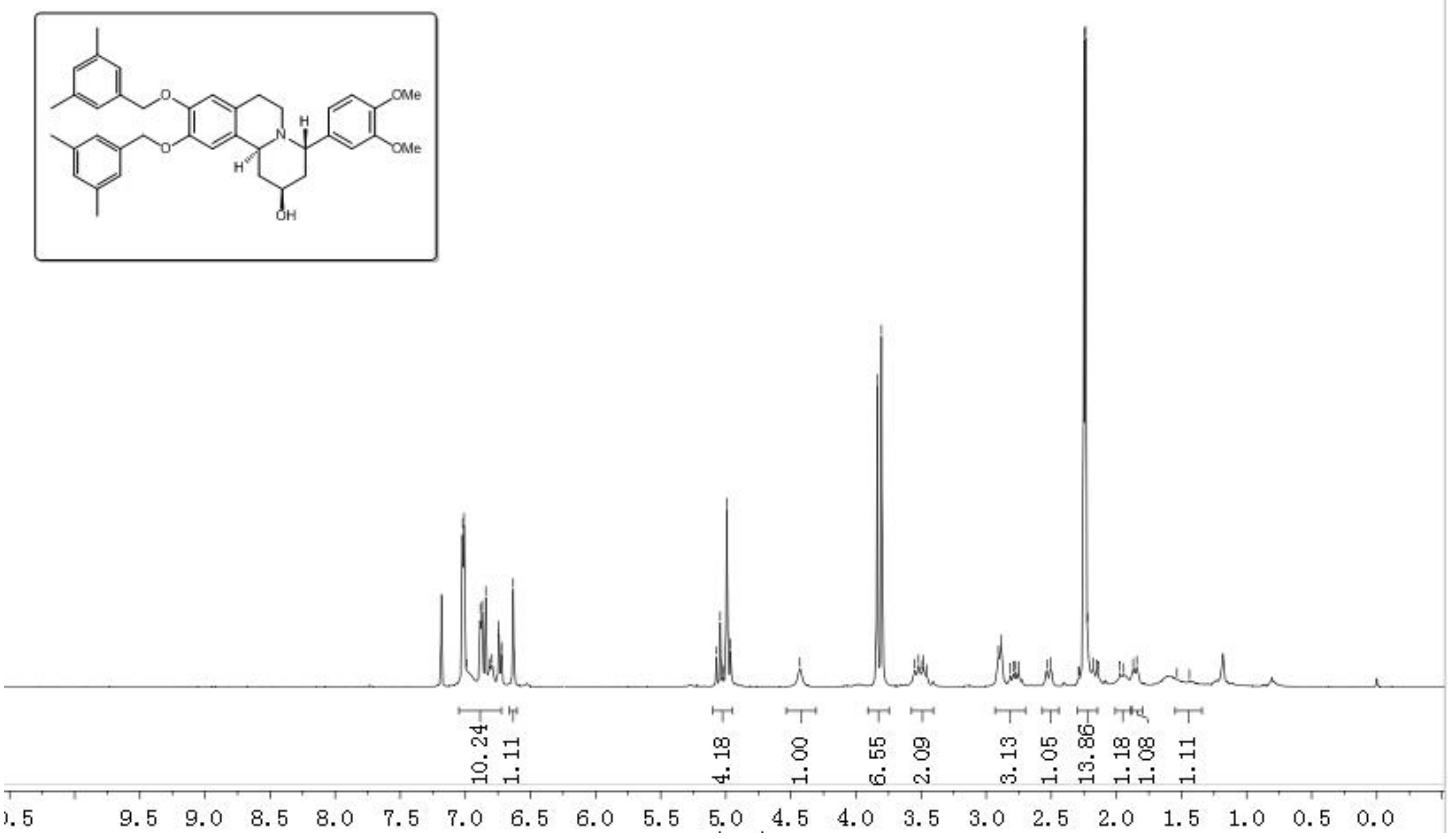

Figure S241. ${ }^{1} \mathrm{H}$ NMR spectrum of 20b. 

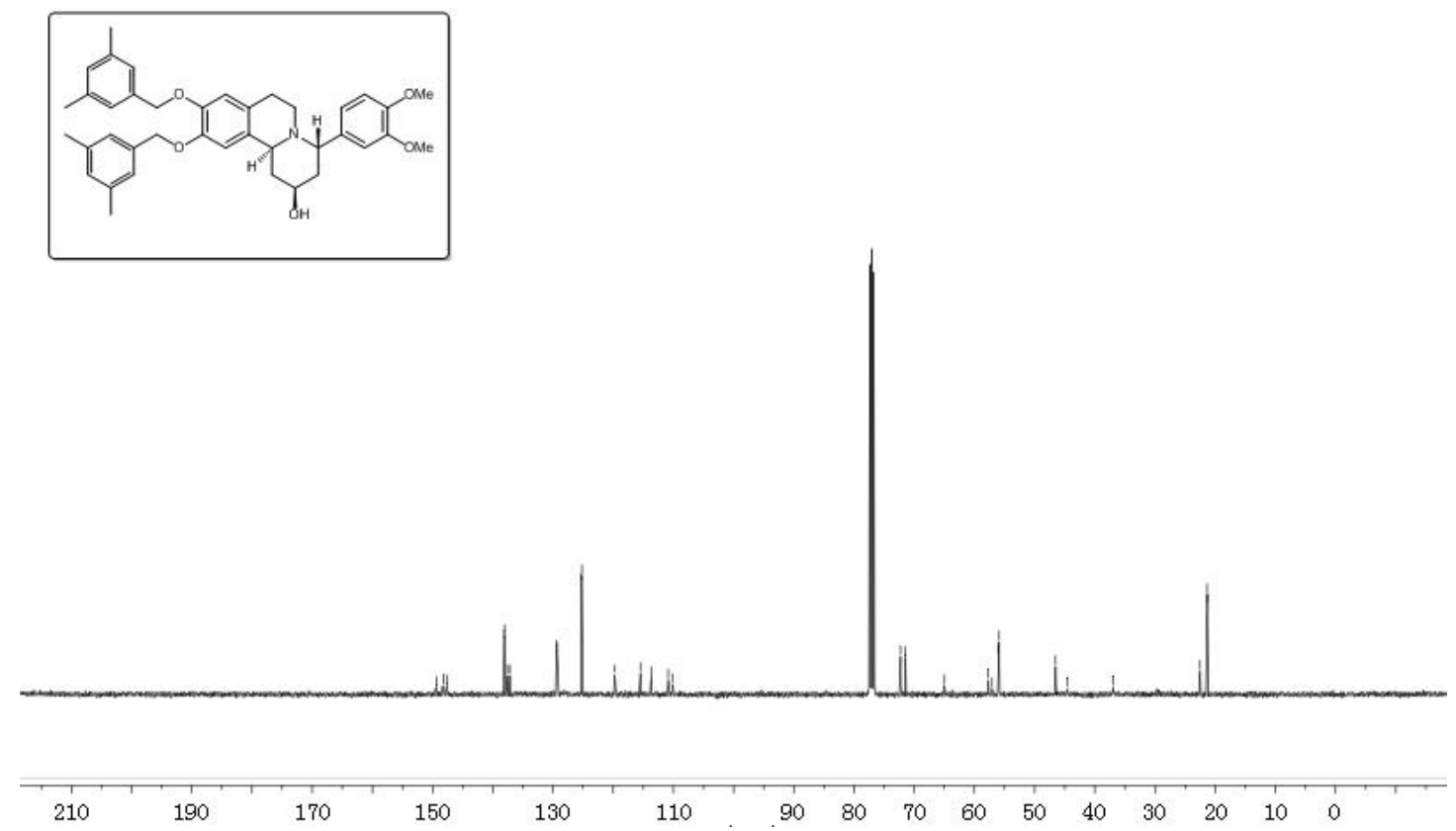

Figure S242. ${ }^{13} \mathrm{C}$ NMR spectrum of $\mathbf{2 0 b}$.

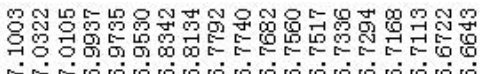
$\therefore$ ए
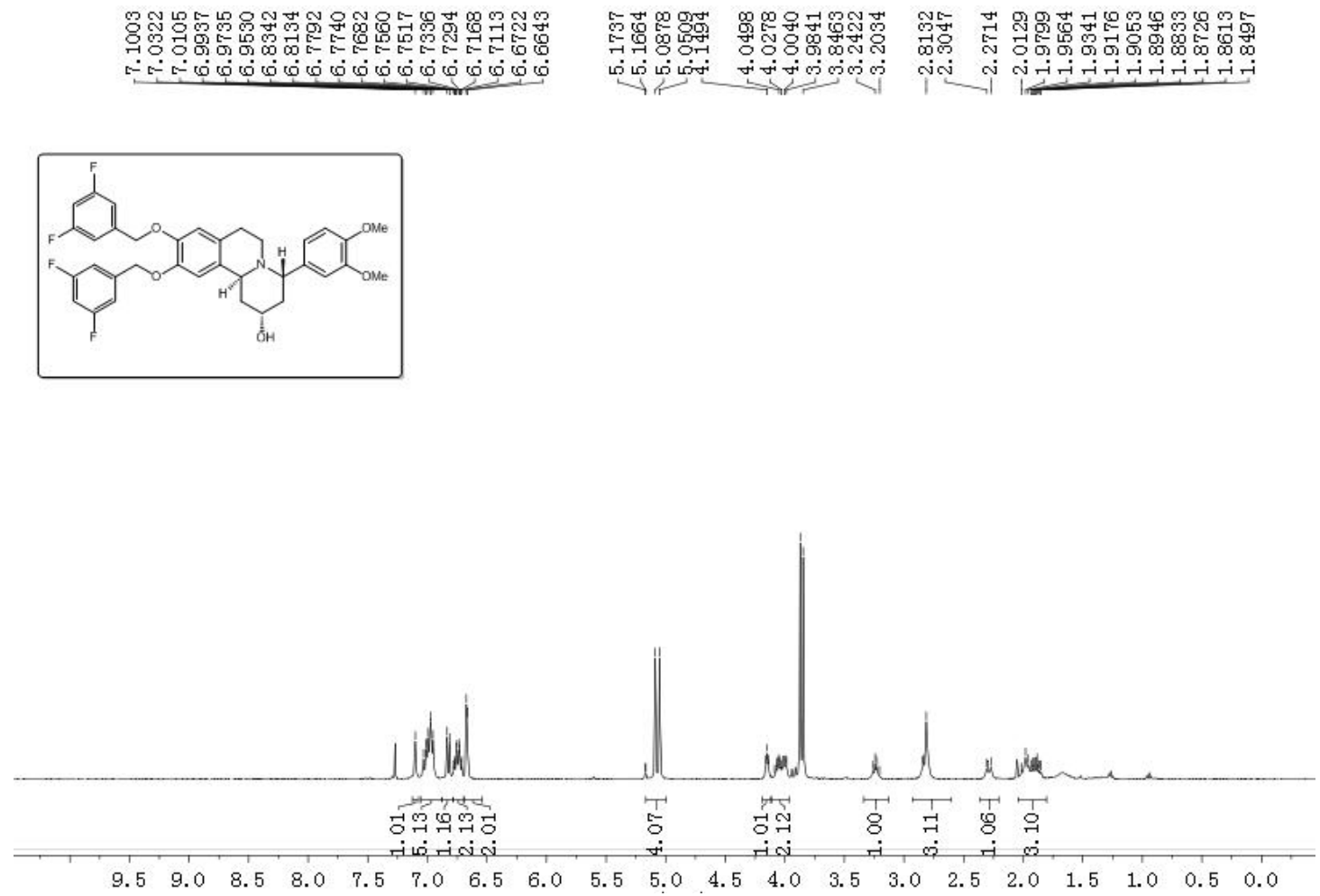

Figure S243. ${ }^{1} \mathrm{H}$ NMR spectrum of 21a. 

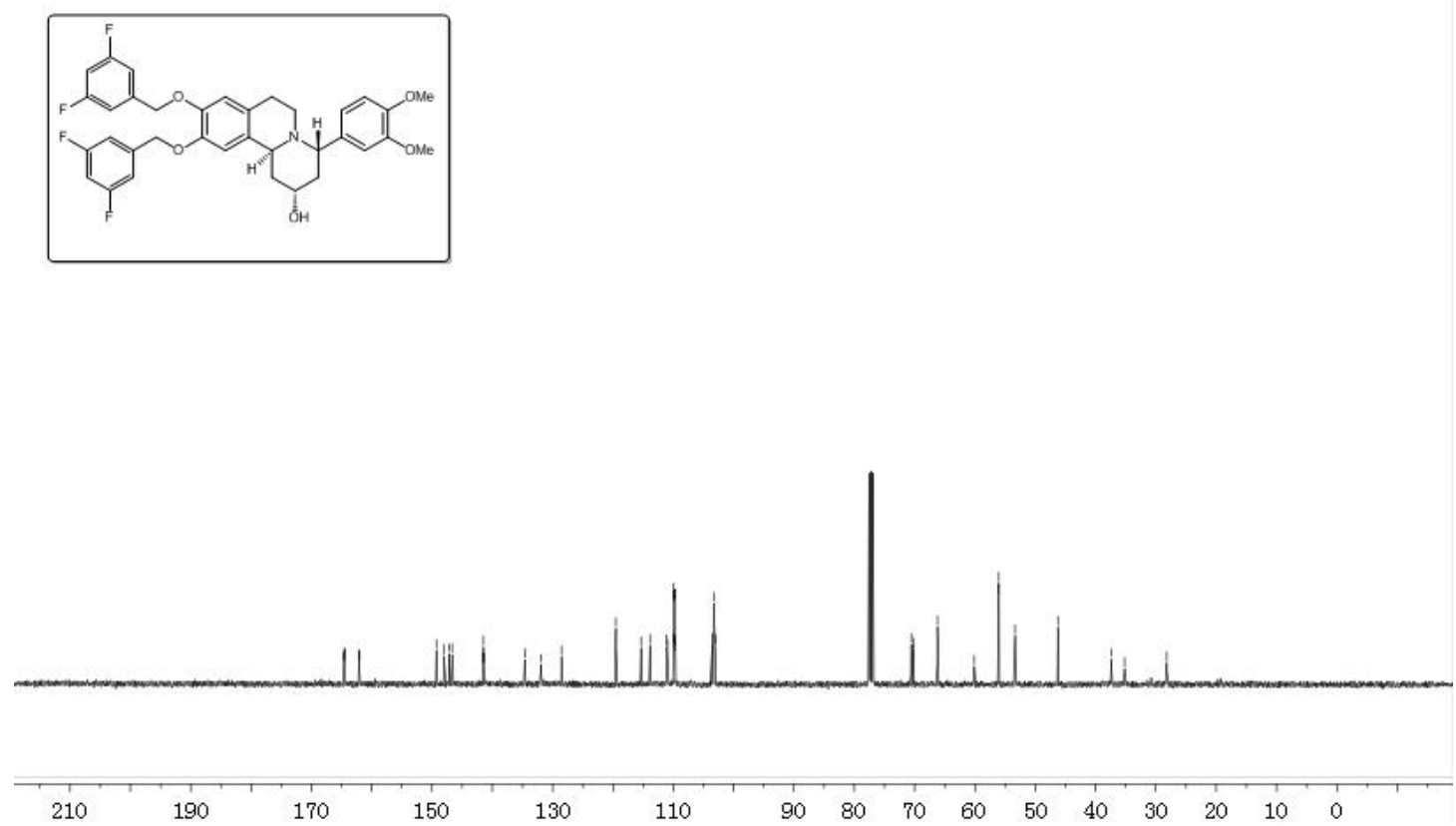

Figure S244. ${ }^{13} \mathrm{C}$ NMR spectrum of 21a.

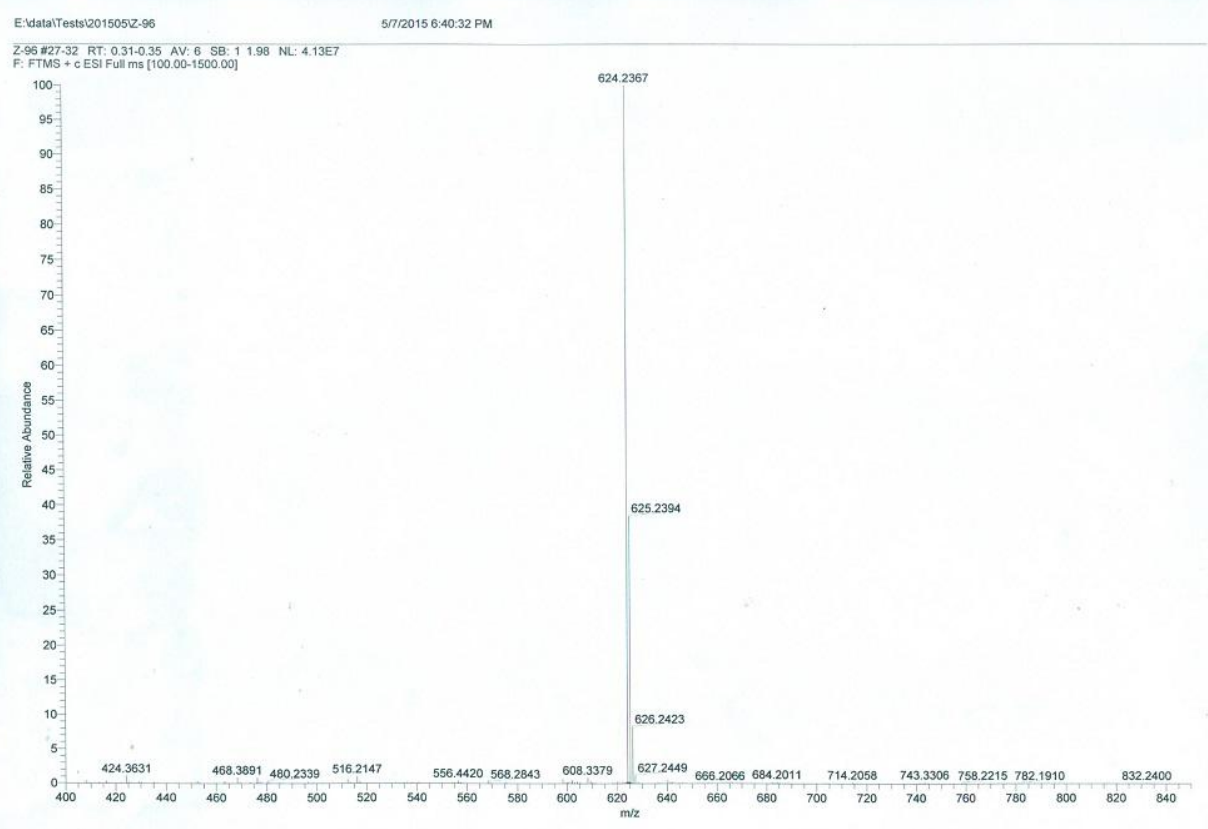

Figure S245. HRESIMS spectrum of $\mathbf{2 1 b}$. 

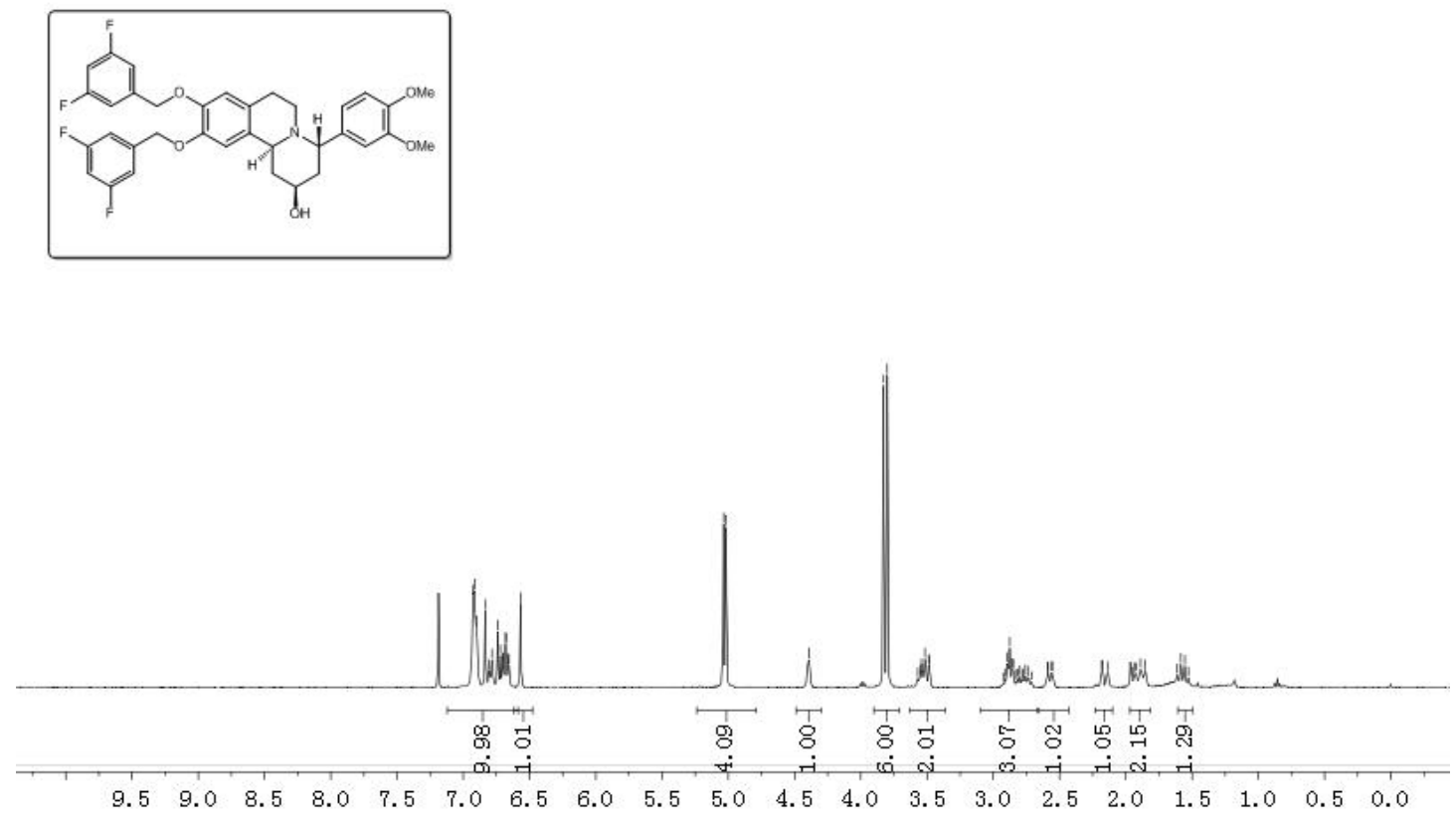

Figure S246. ${ }^{1} \mathrm{H}$ NMR spectrum of $\mathbf{2 1 b}$

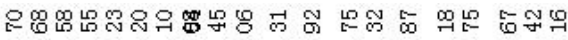

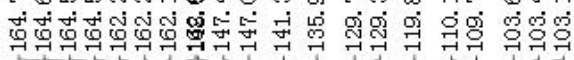

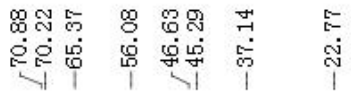
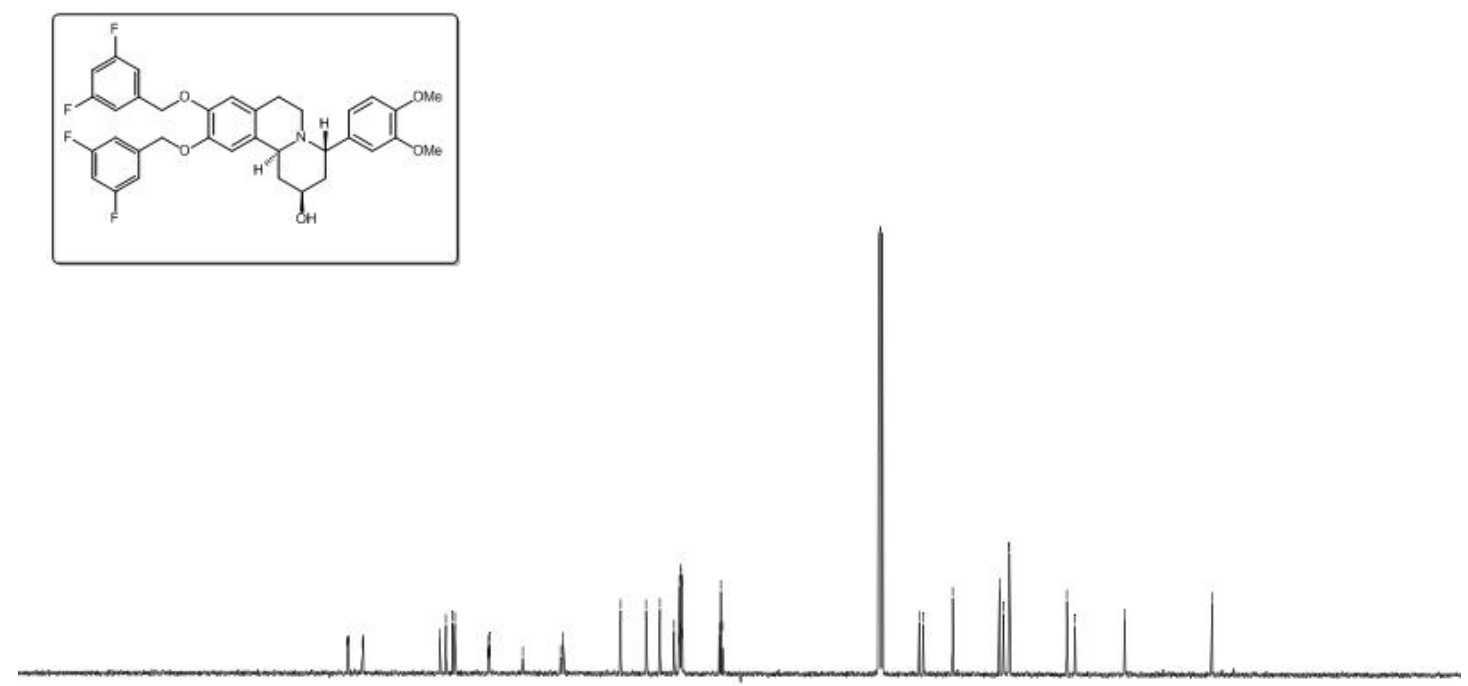

$210 \quad 190 \quad 170 \quad 150$

Figure S247. ${ }^{13} \mathrm{C}$ NMR spectrum of 21b. 


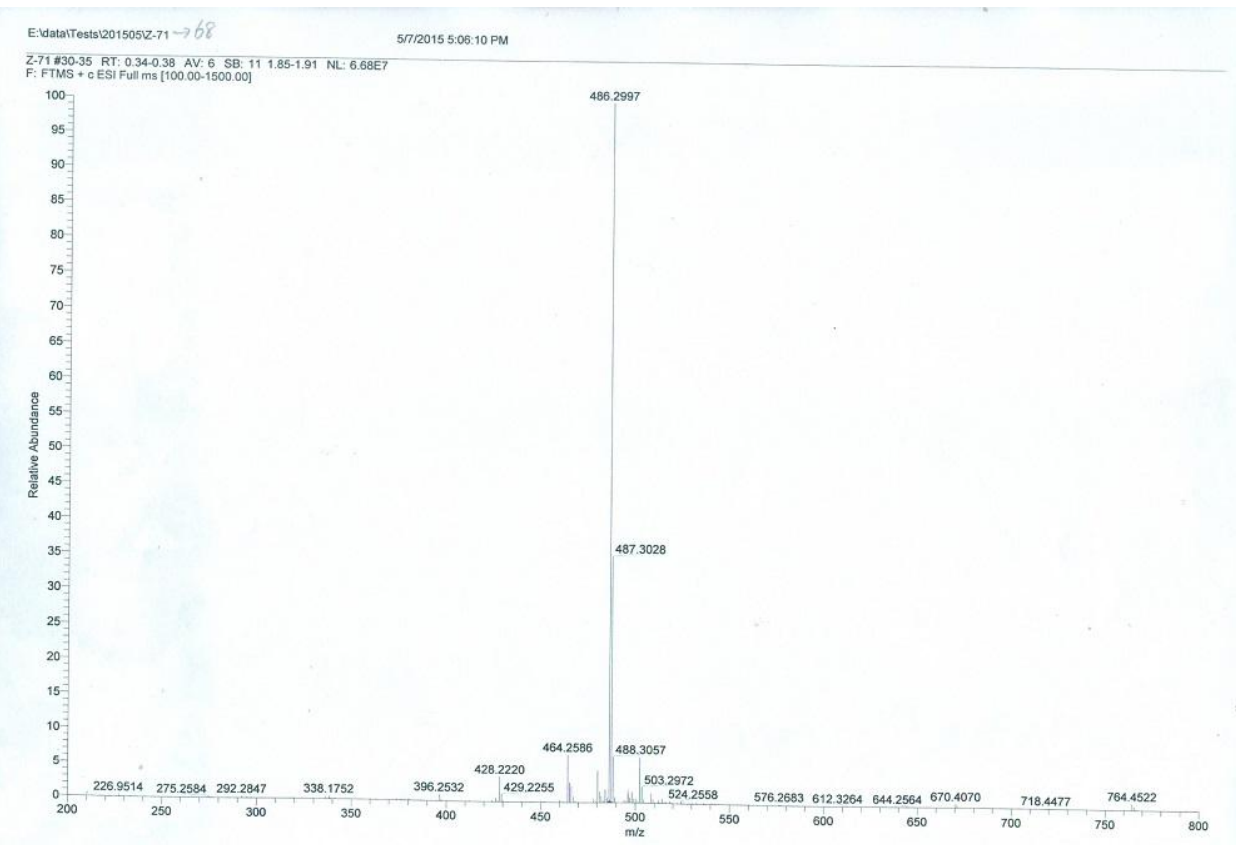

Figure S248. HRESIMS spectrum of 22a.

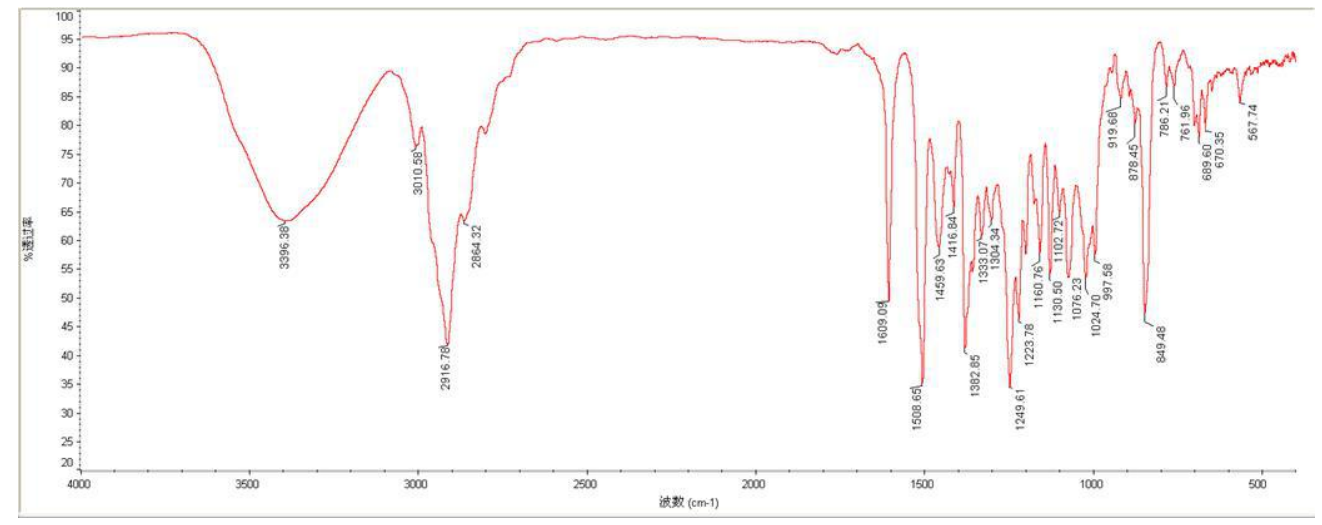

Figure S249. IR spectrum of 22a. 

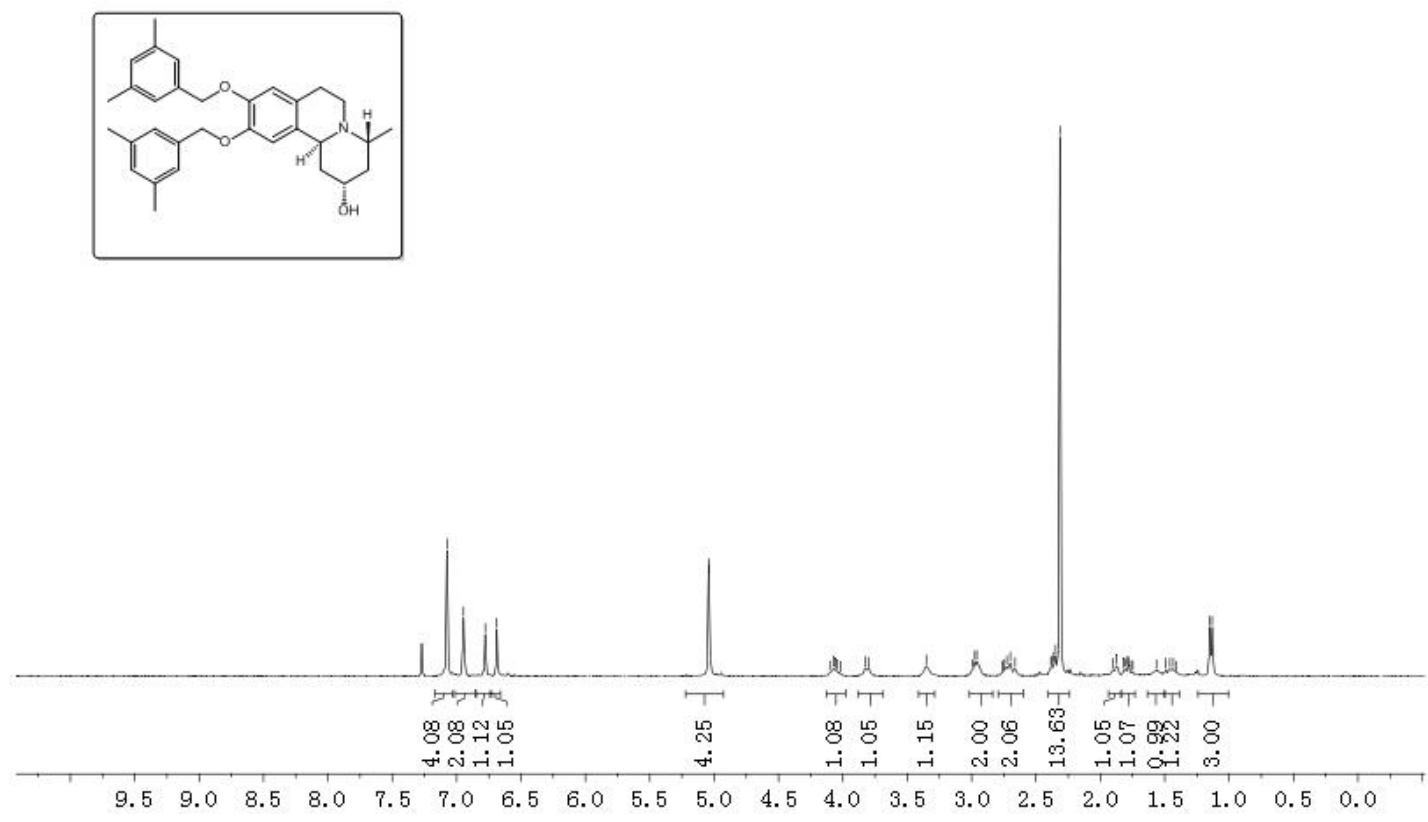

Figure S250. ${ }^{1} \mathrm{H}$ NMR spectrum of 22a.

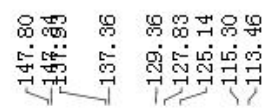

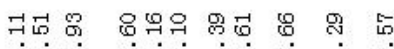

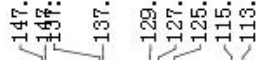

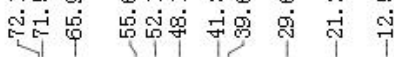
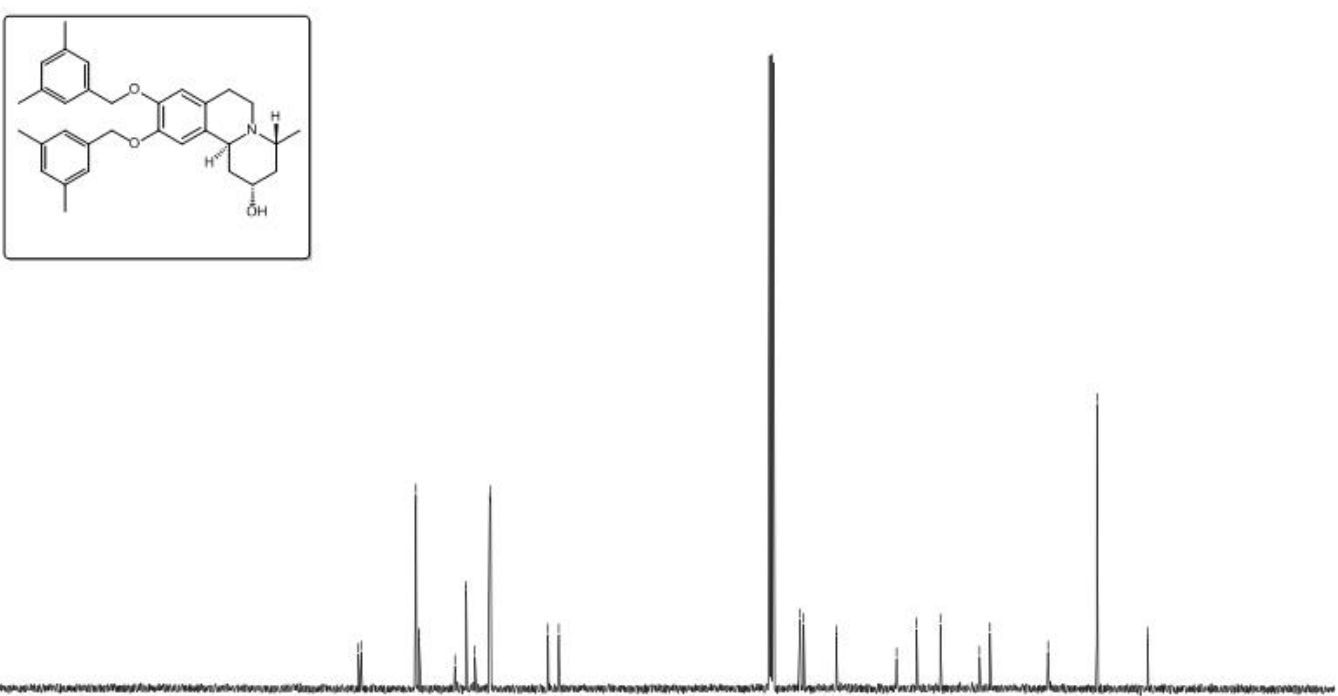

Figure S251. ${ }^{13} \mathrm{C}$ NMR spectrum of 22a. 


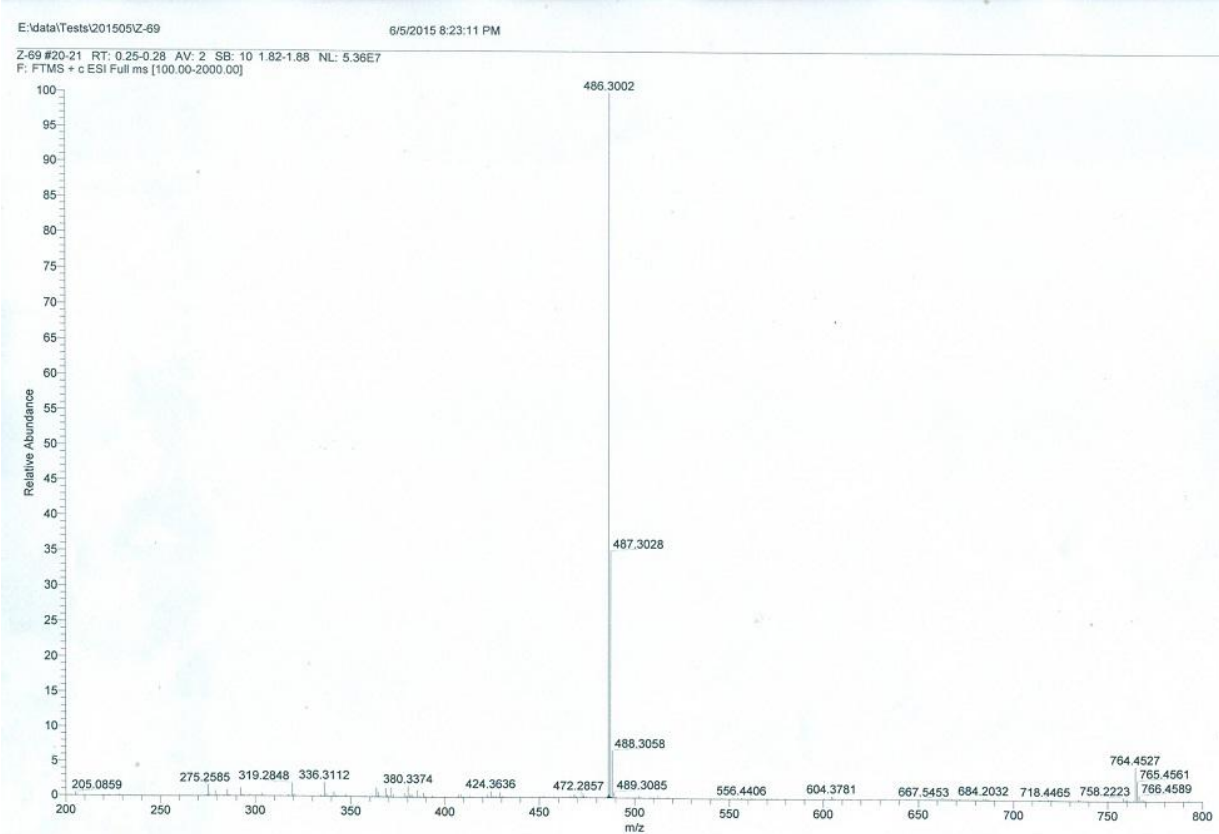

Figure S252. HRESIMS spectrum of 22b.

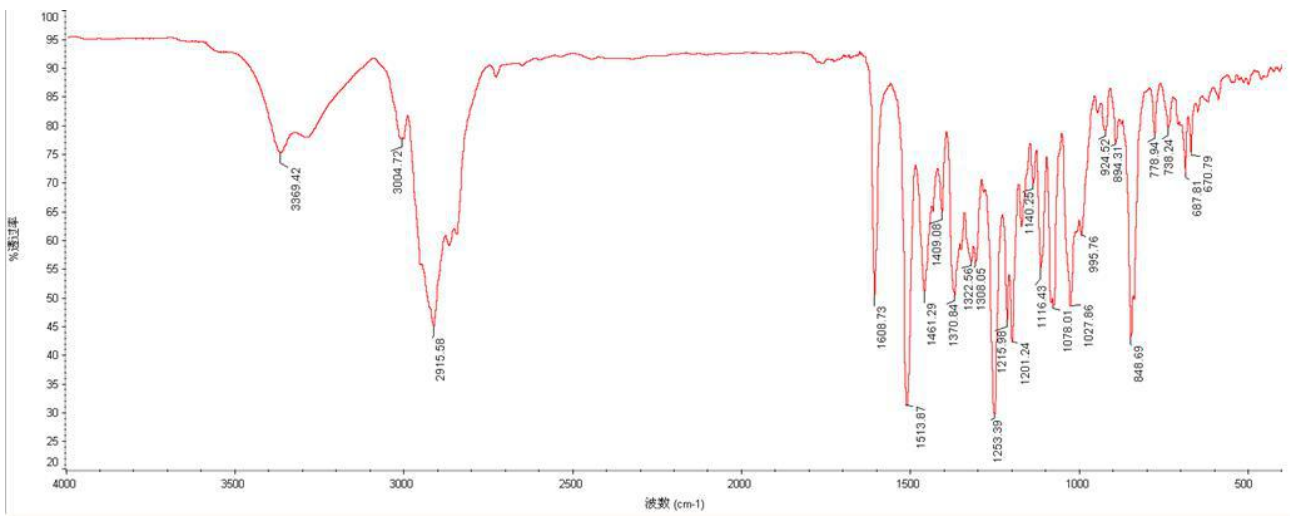

Figure S253. IR spectrum of 22b. 

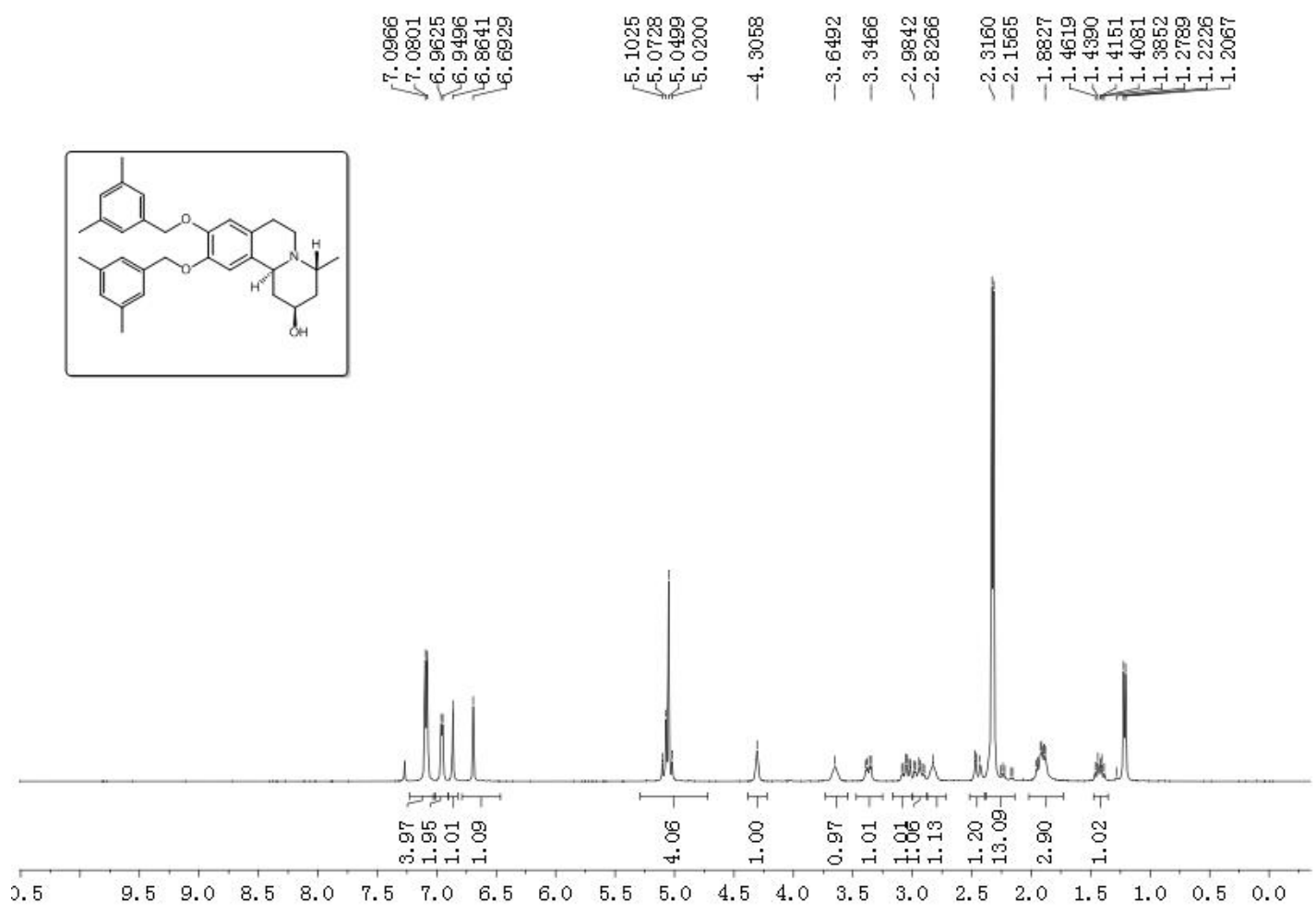

Figure S254. ${ }^{1} \mathrm{H}$ NMR spectrum of $\mathbf{2 2 b}$.

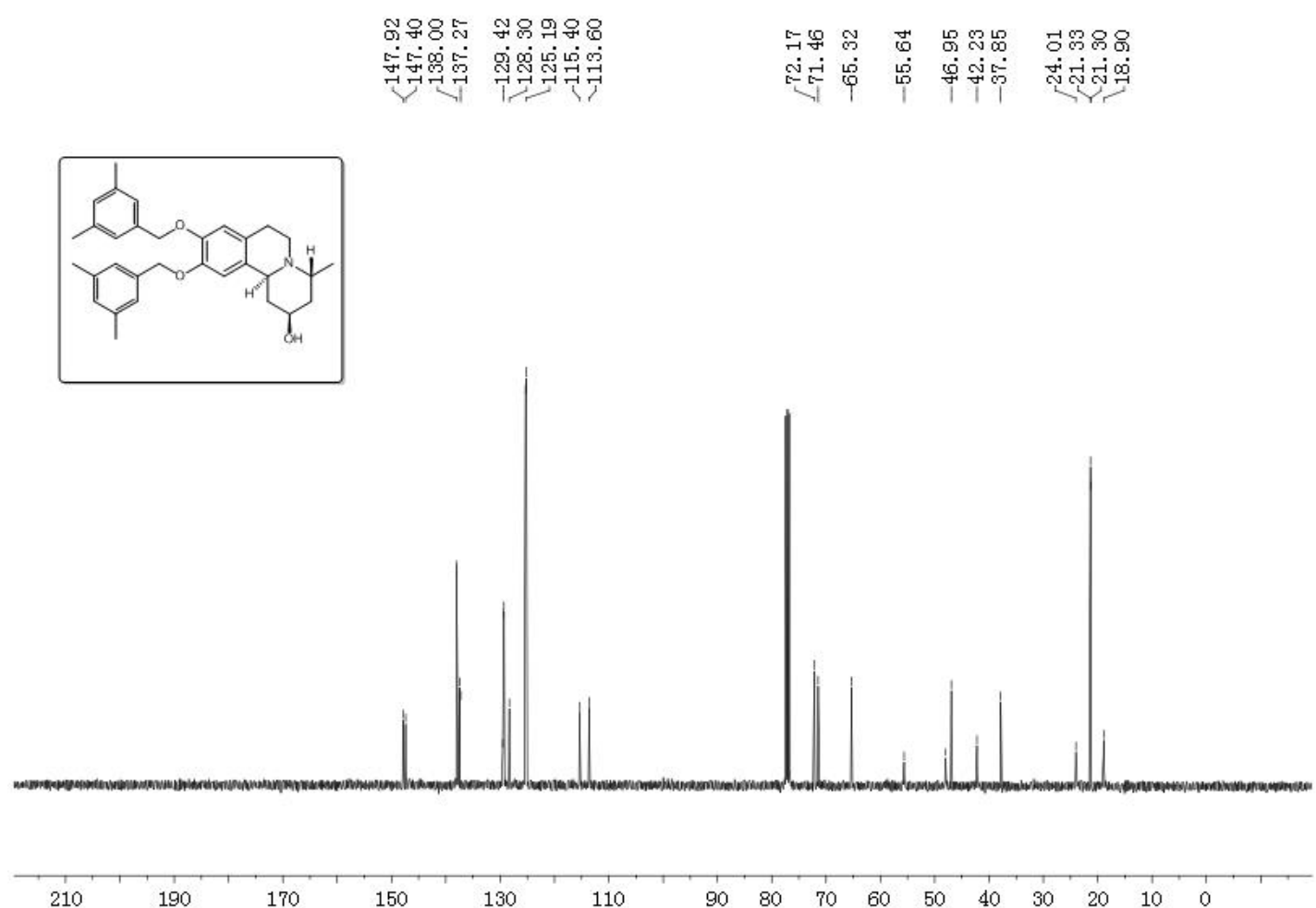

Figure S255. ${ }^{13} \mathrm{C}$ NMR spectrum of $\mathbf{2 2 b}$. 

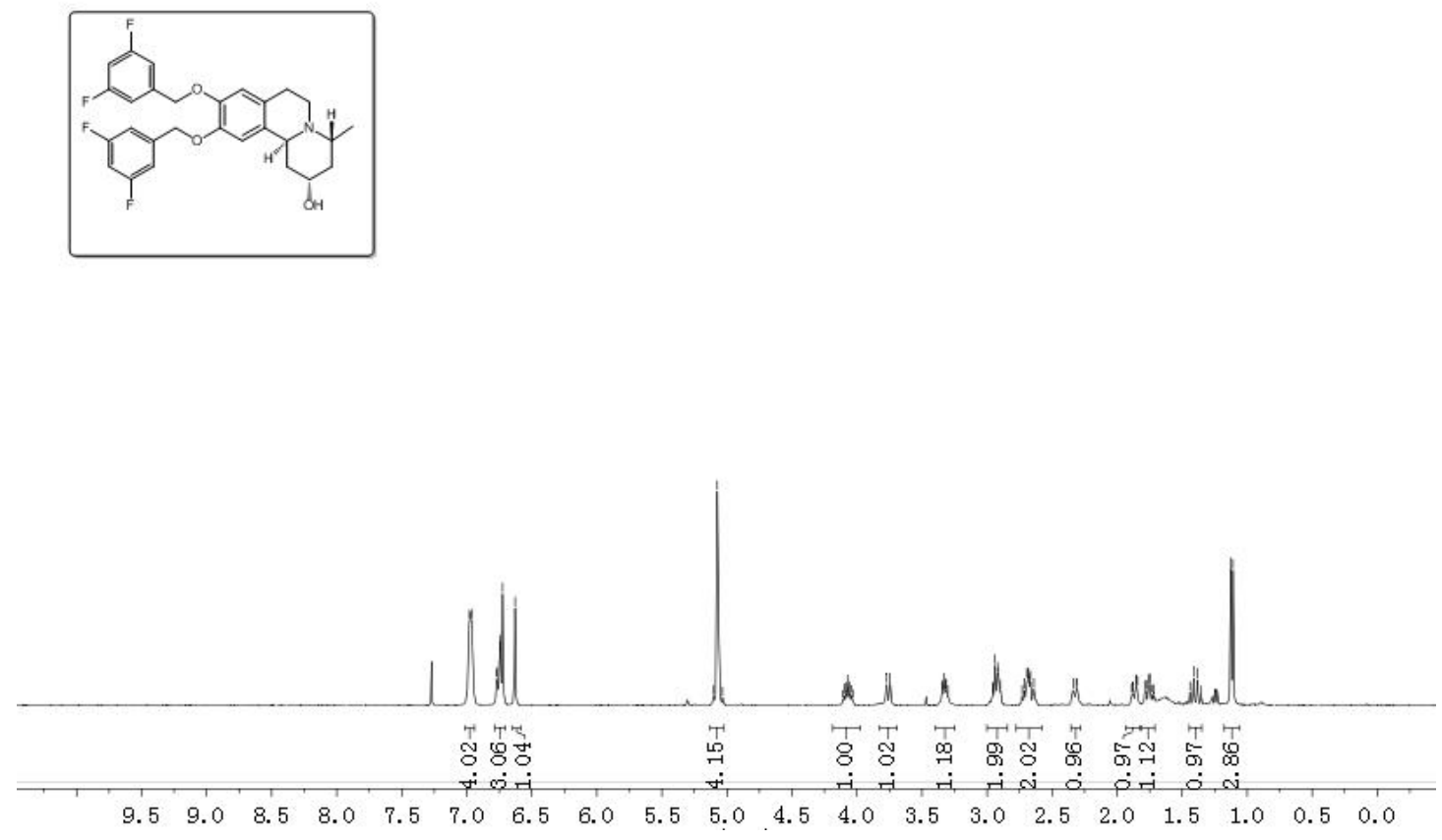

Figure S256. ${ }^{1} \mathrm{H}$ NMR spectrum of 23a.

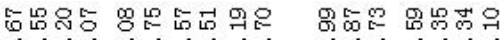

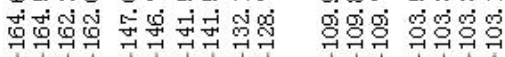

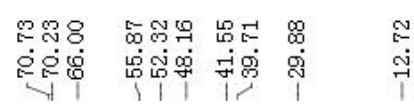
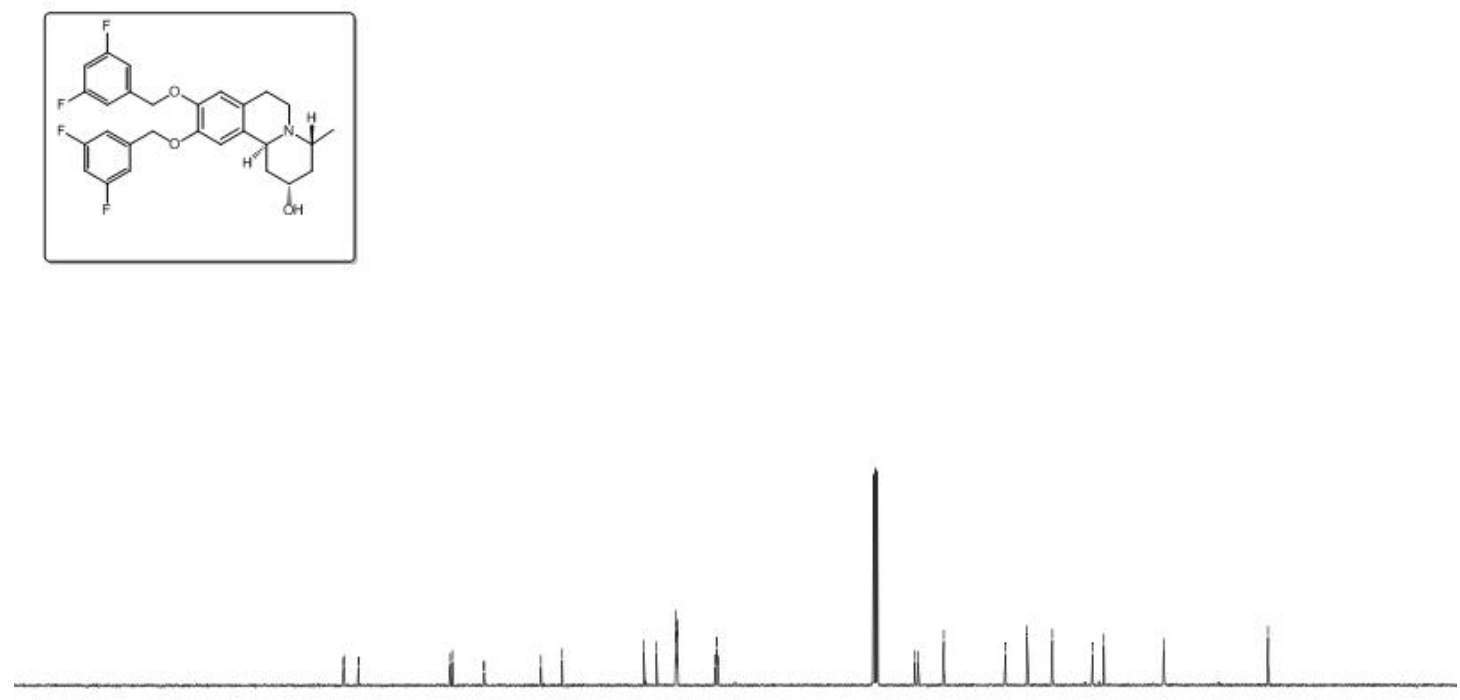

21

190

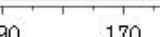

150

Figure S257. ${ }^{13} \mathrm{C}$ NMR spectrum of 23a 


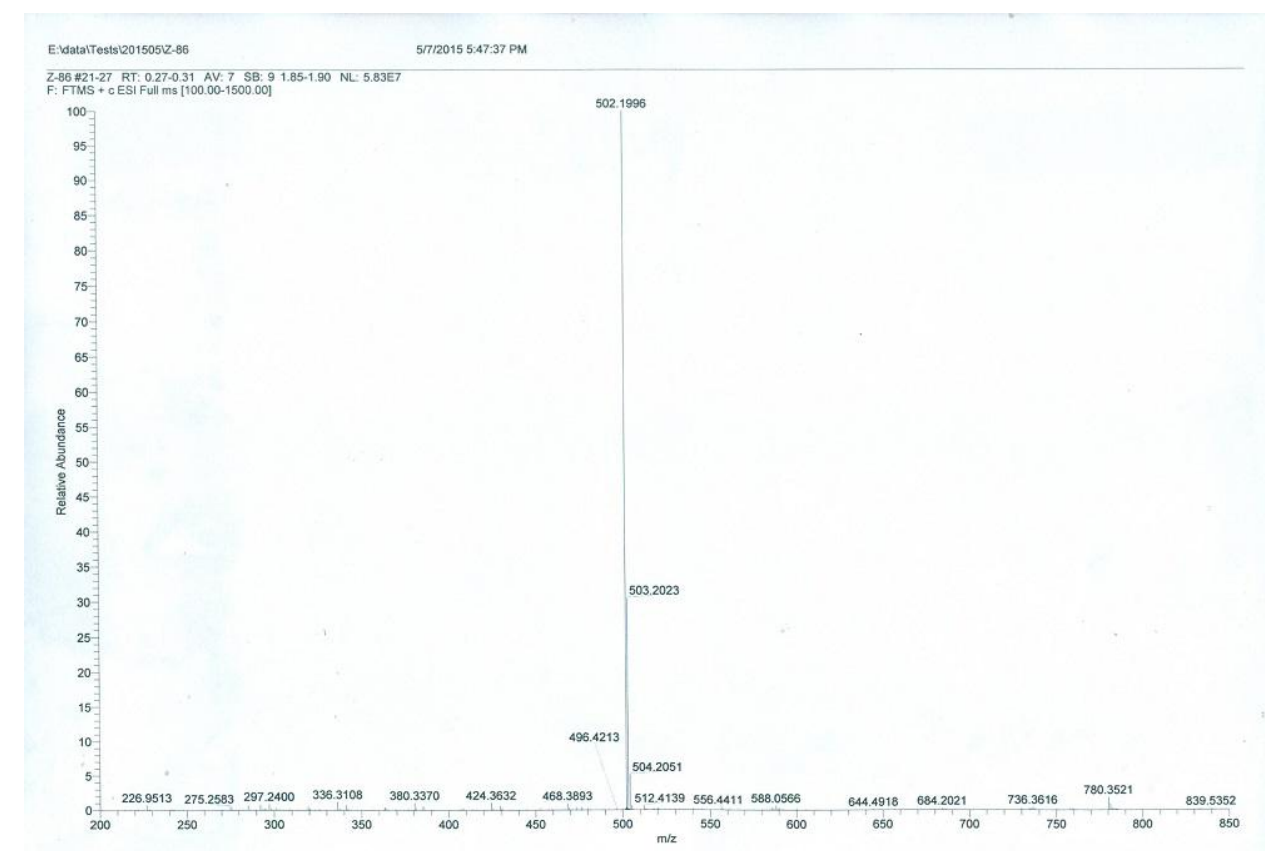

Figure S258. HRESIMS spectrum of $\mathbf{2 3 b}$.
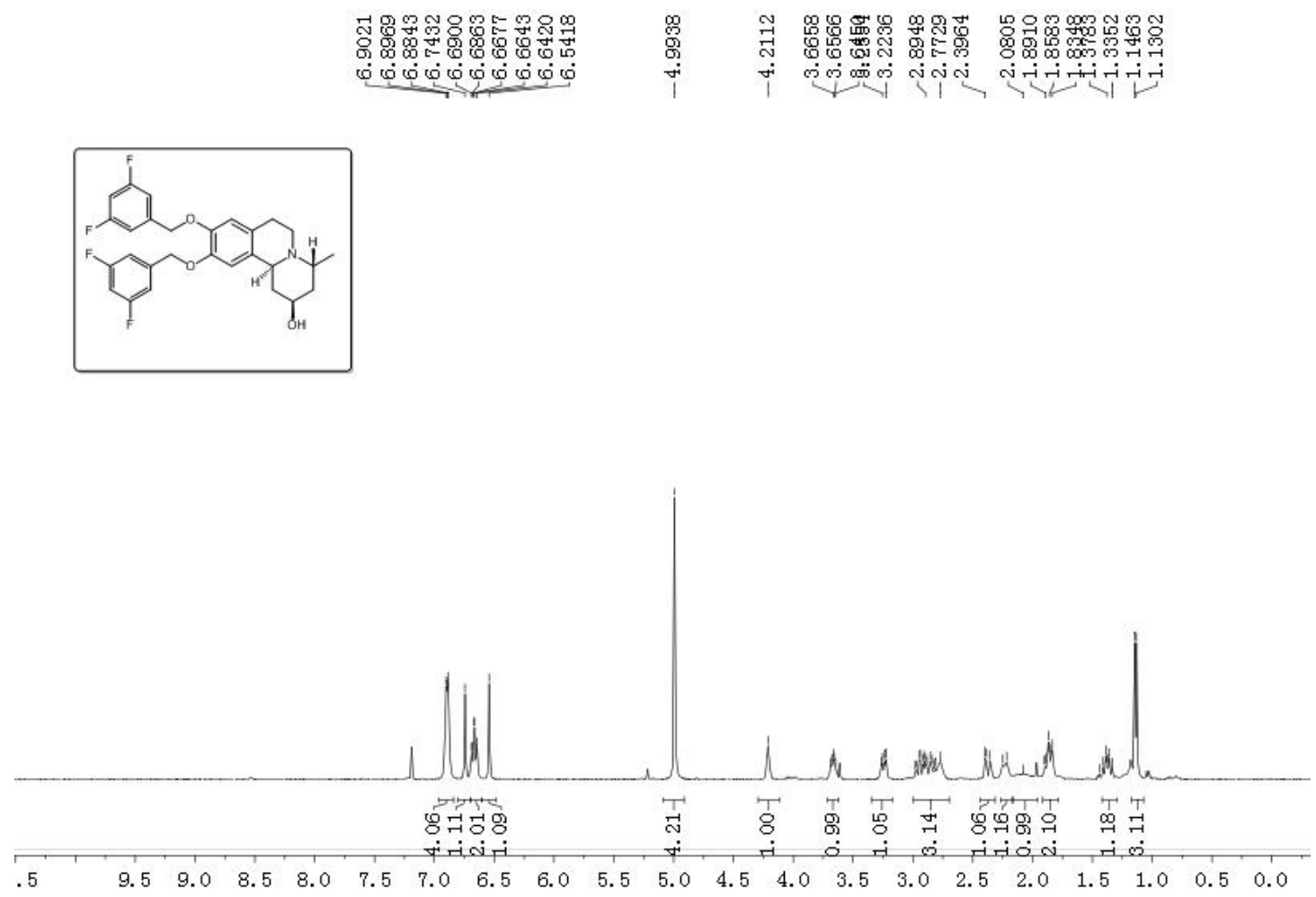

Figure S259. ${ }^{1} \mathrm{H}$ NMR spectrum of 23b. 


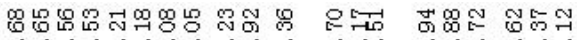

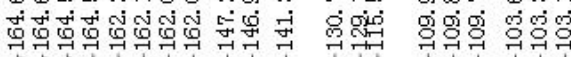

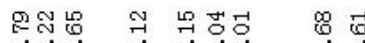

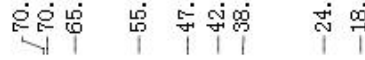
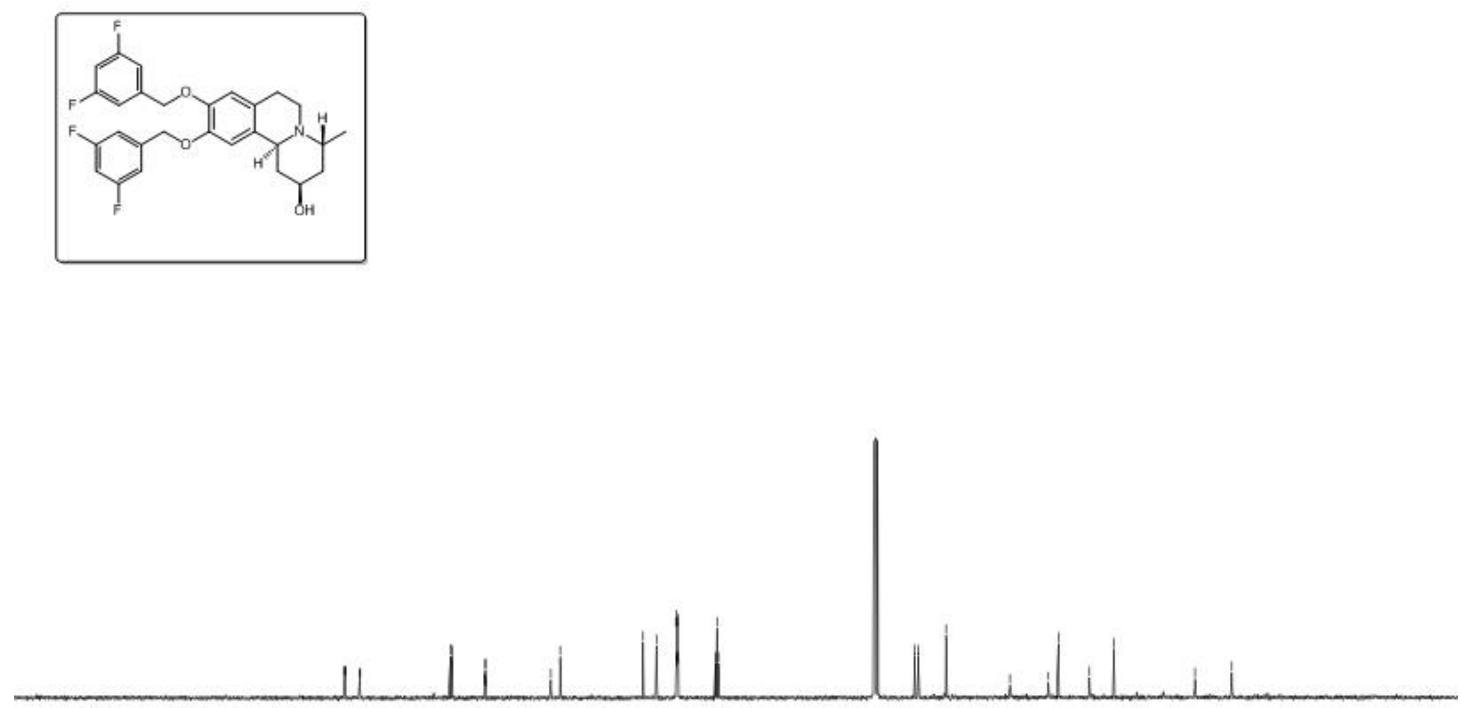

210

190

150

30

110

$90 \quad 80 \quad 70$

Figure S260. ${ }^{13} \mathrm{C}$ NMR spectrum of $\mathbf{2 3 b}$.

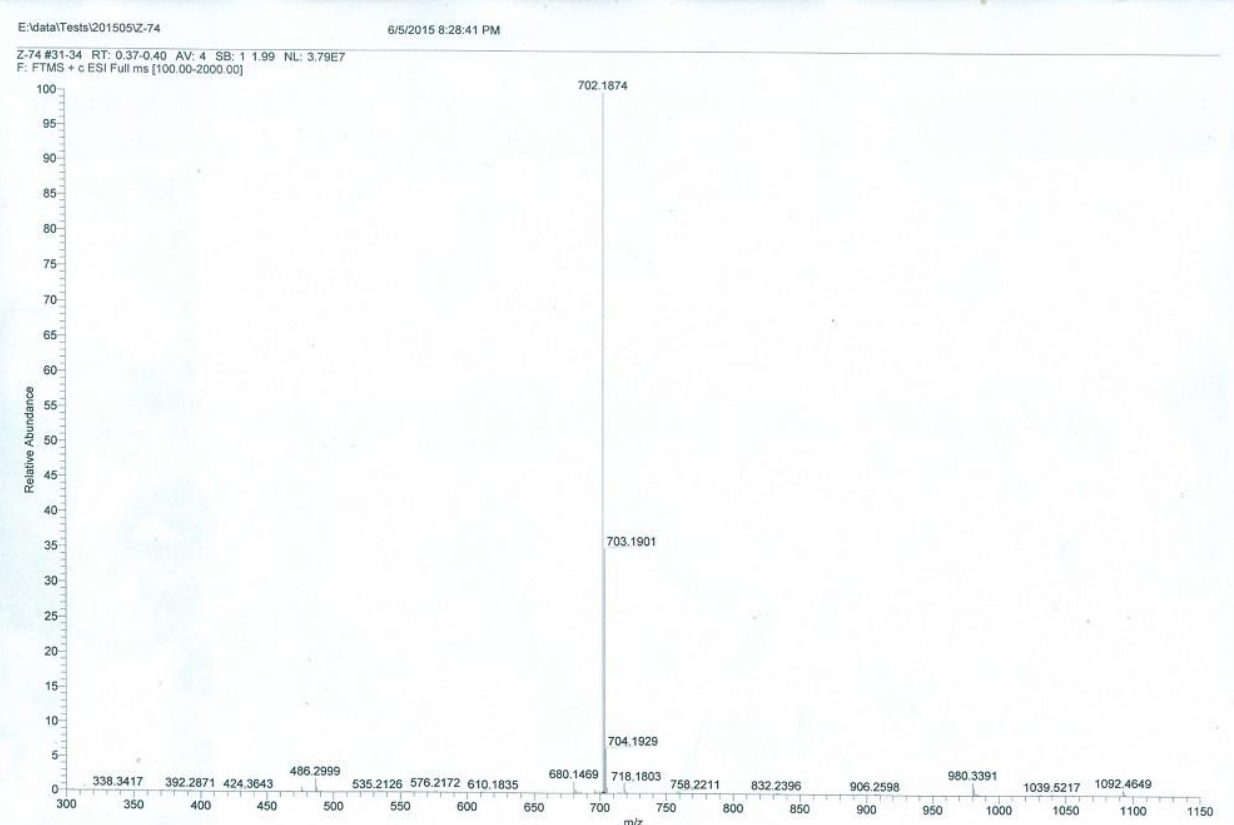

Figure S261. HRESIMS spectrum of 24a. 


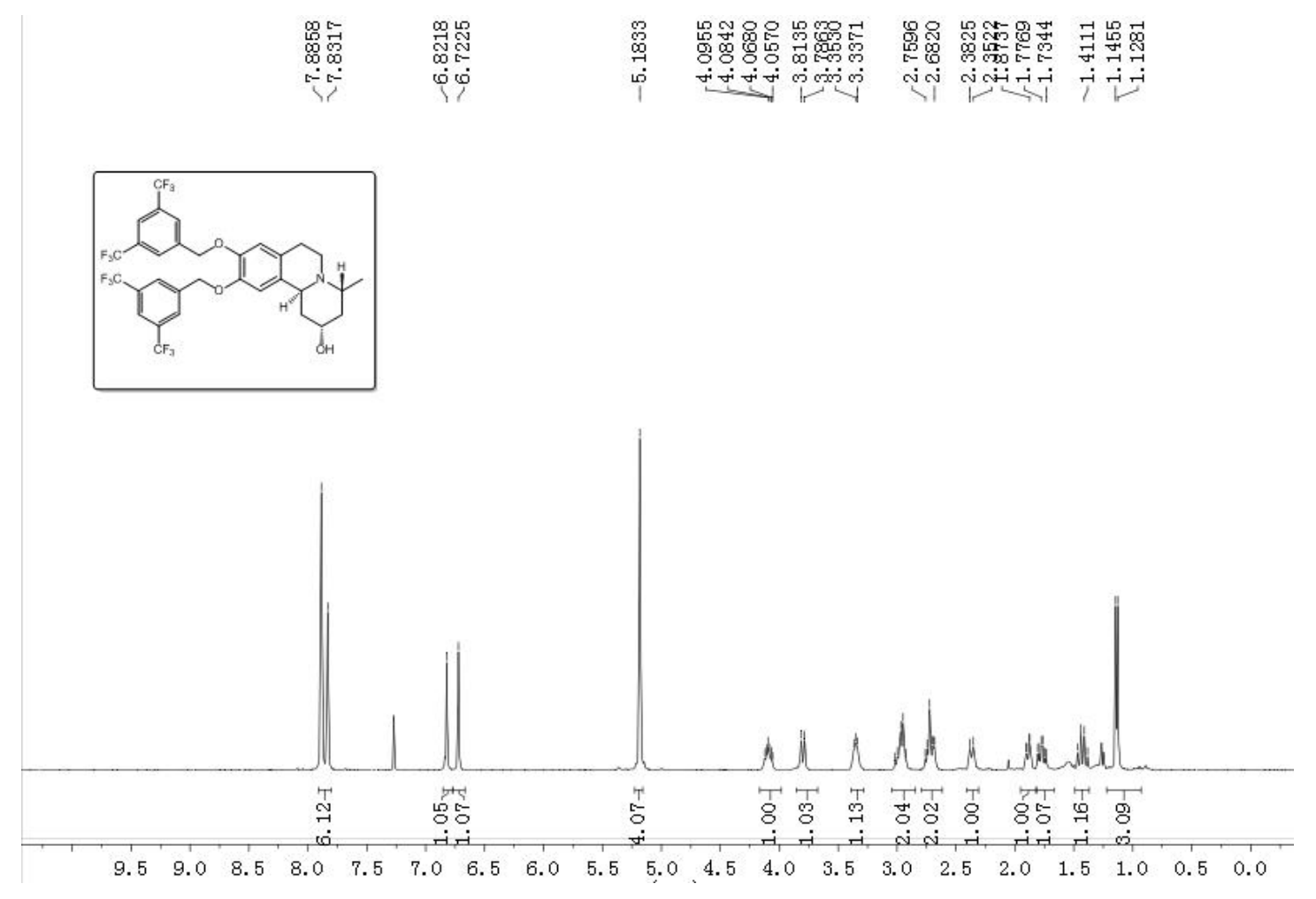

Figure S262. ${ }^{1} \mathrm{H}$ NMR spectrum of 24a.

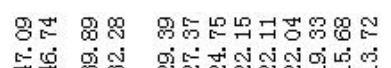

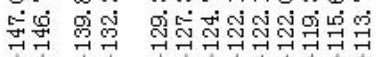

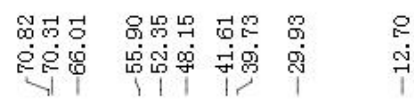
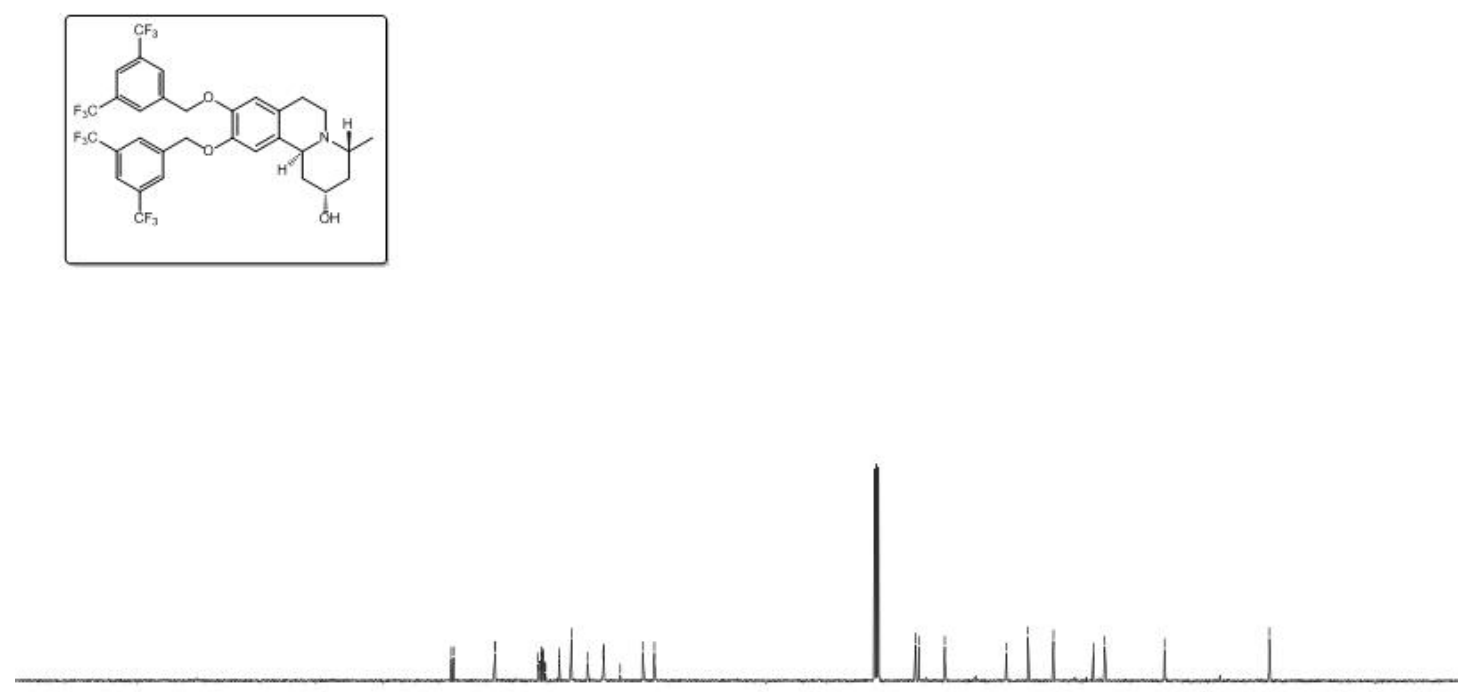

Figure S263. ${ }^{13} \mathrm{C}$ NMR spectrum of 24a. 


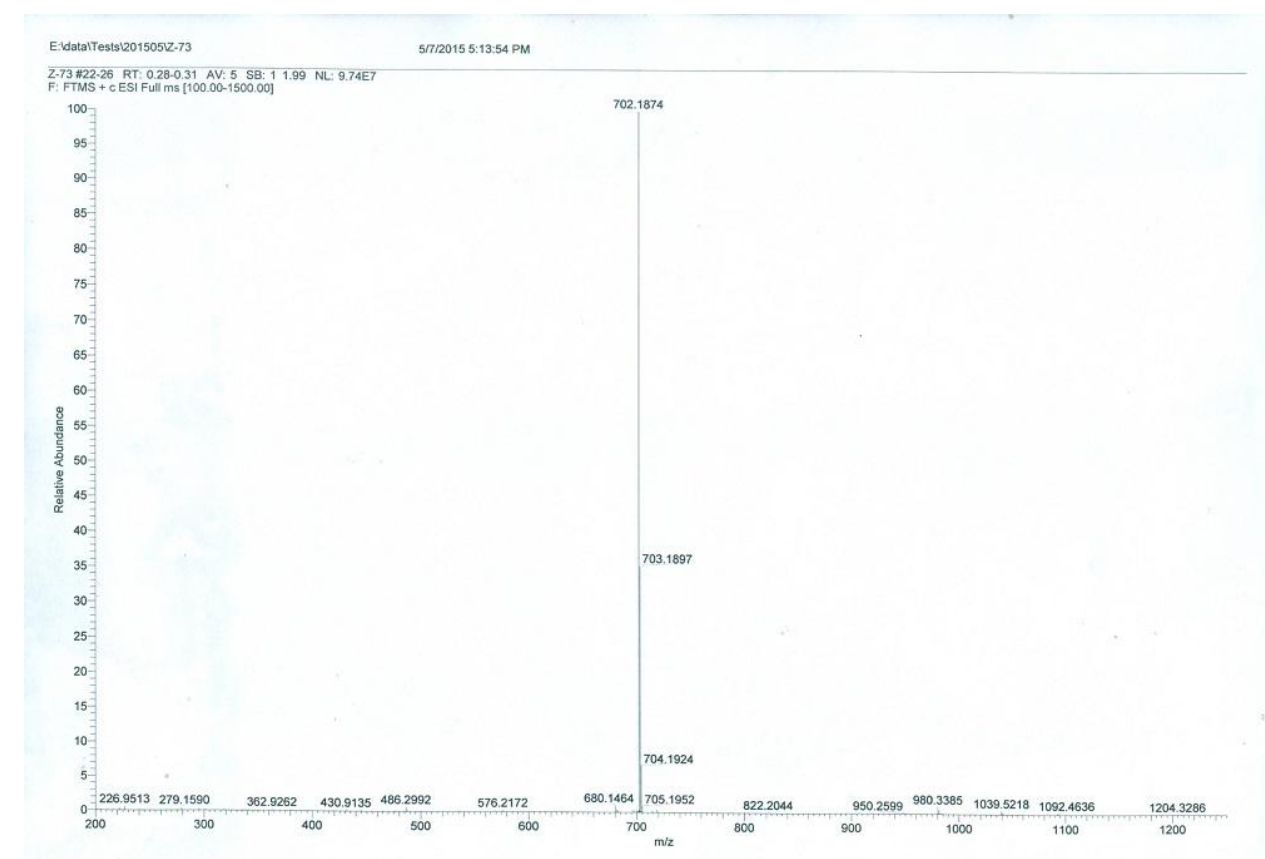

Figure S264. HRESIMS spectrum of 24b.
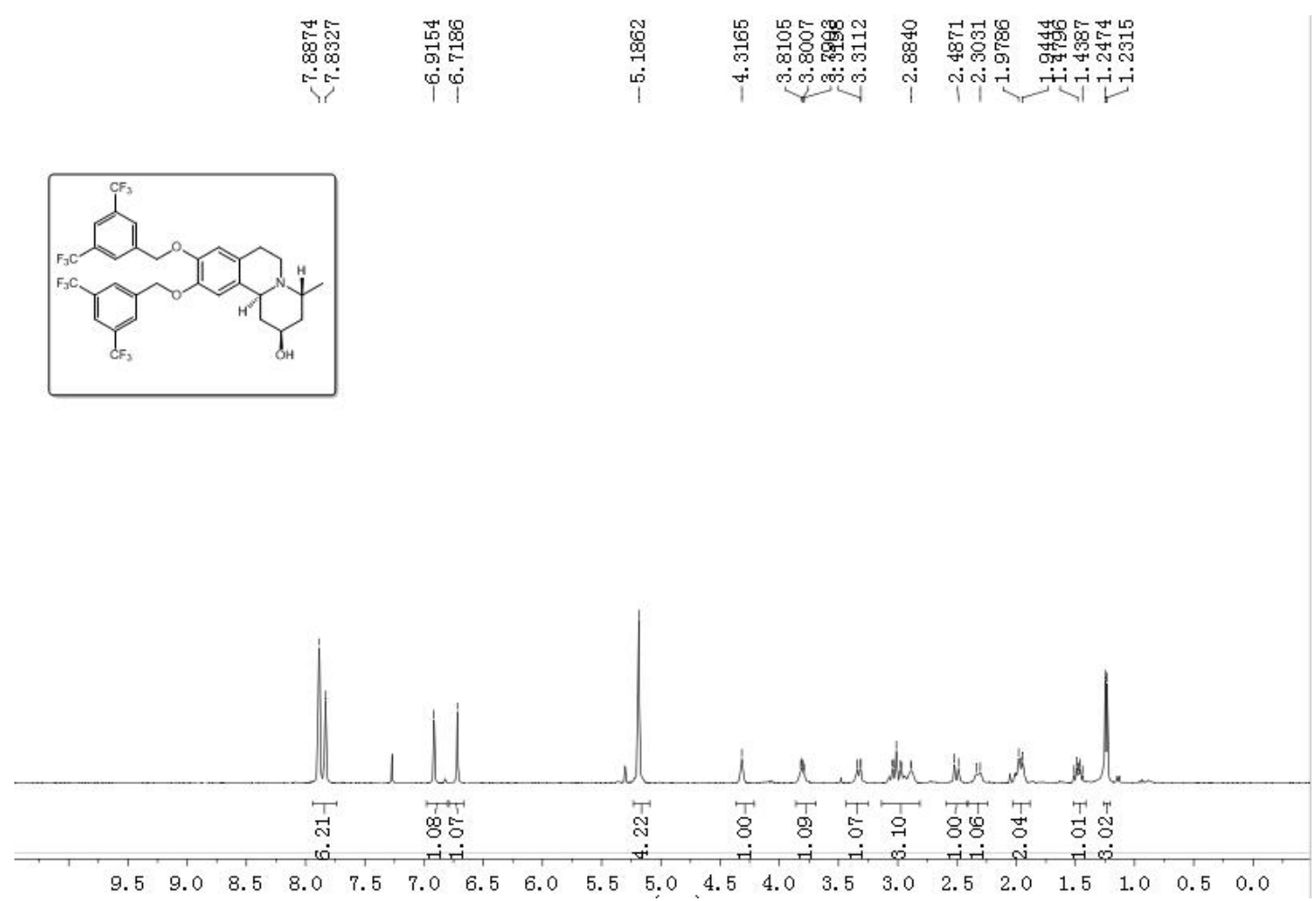

Figure S265. ${ }^{1} \mathrm{H}$ NMR spectrum of 24b. 

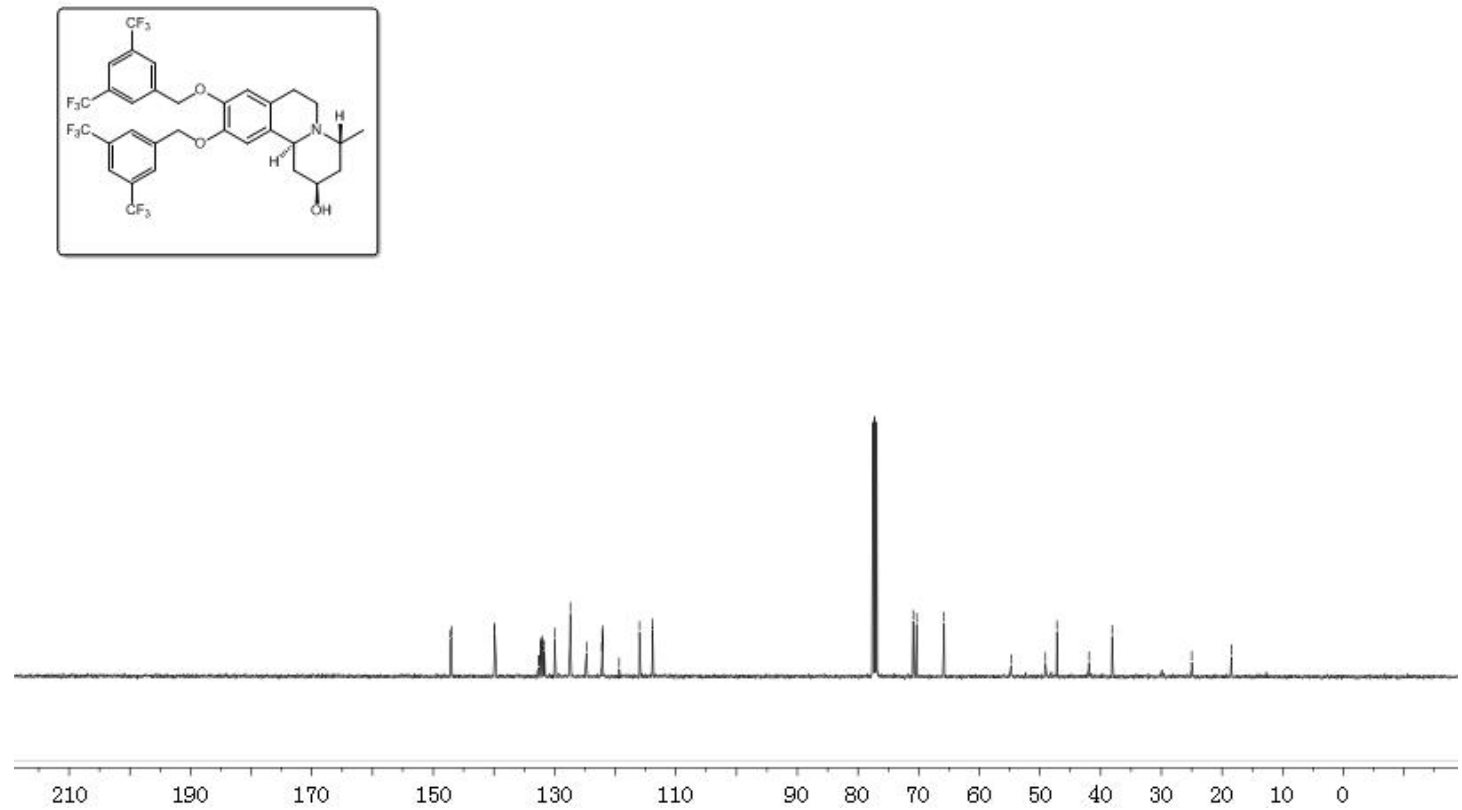

Figure S266. ${ }^{13} \mathrm{C}$ NMR spectrum of $\mathbf{2 4 b}$.
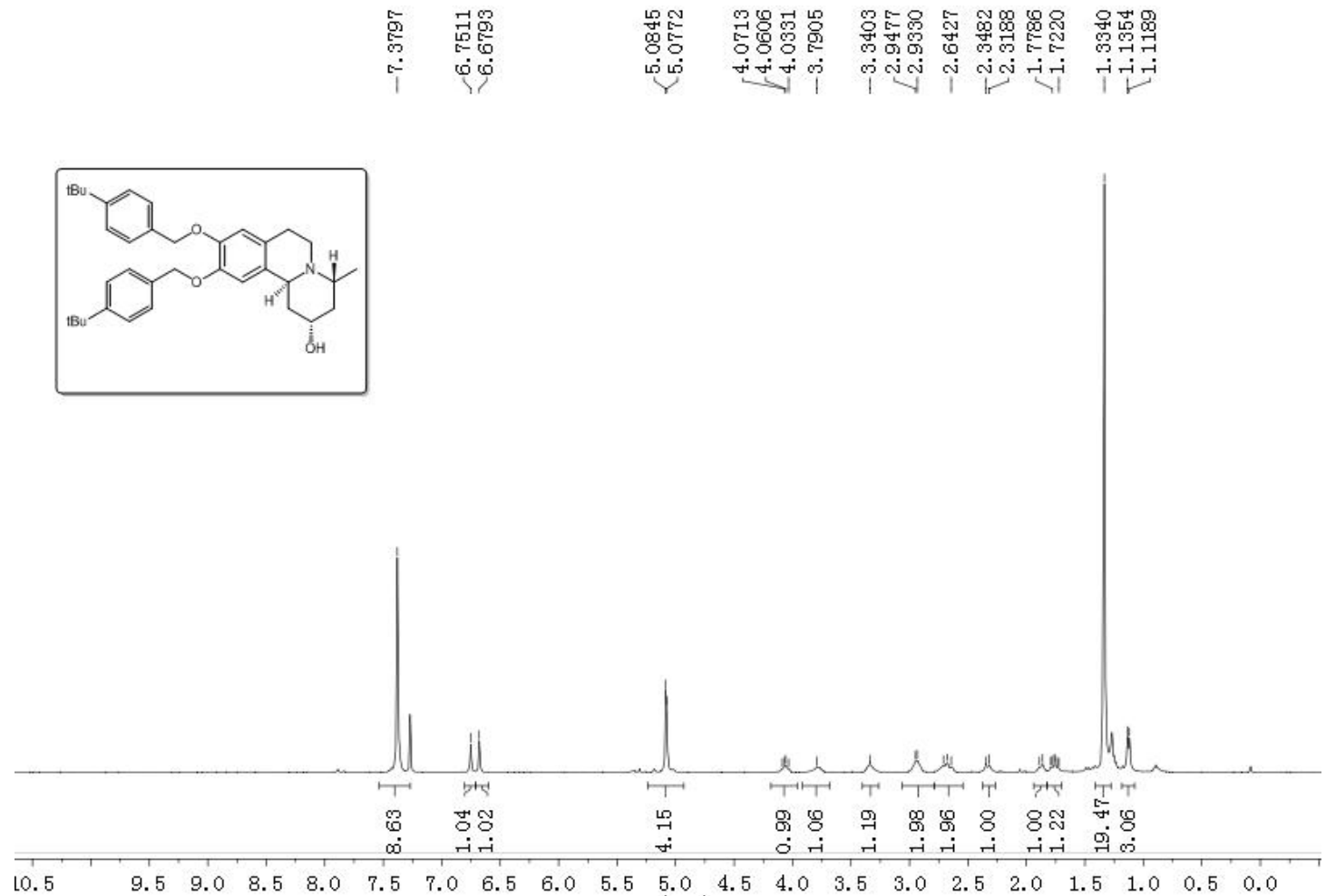

Figure S267. ${ }^{1} \mathrm{H}$ NMR spectrum of 25a. 

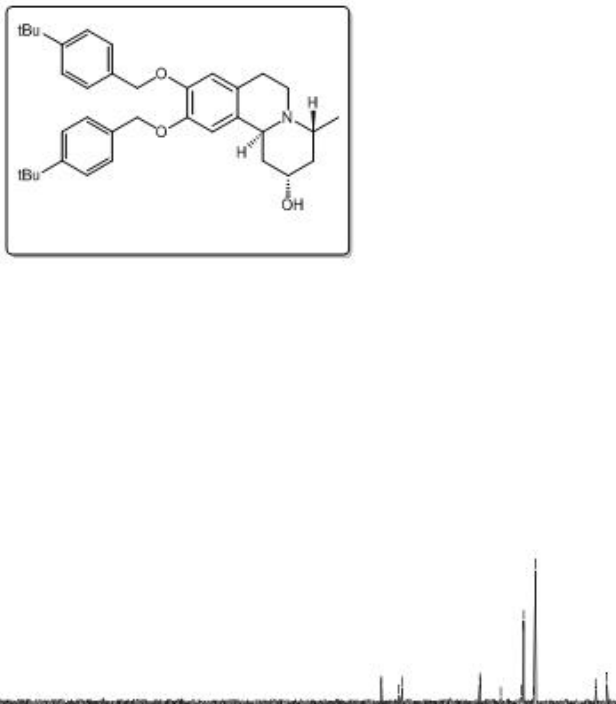

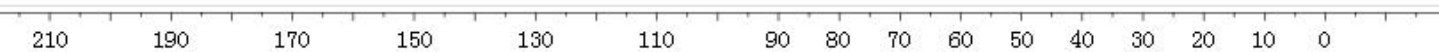

Figure S268. ${ }^{13} \mathrm{C}$ NMR spectrum of $\mathbf{2 5 a}$.

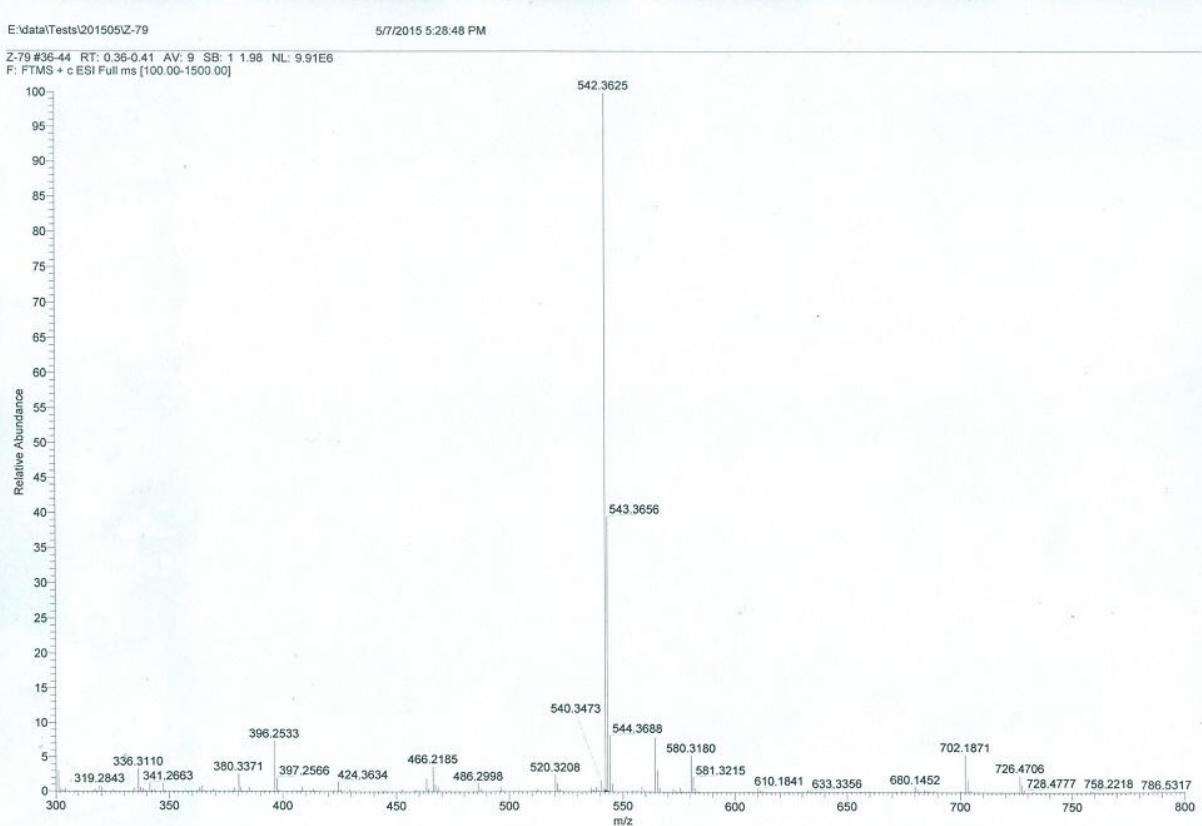

Figure S269. HRESIMS spectrum of $\mathbf{2 5 b}$. 

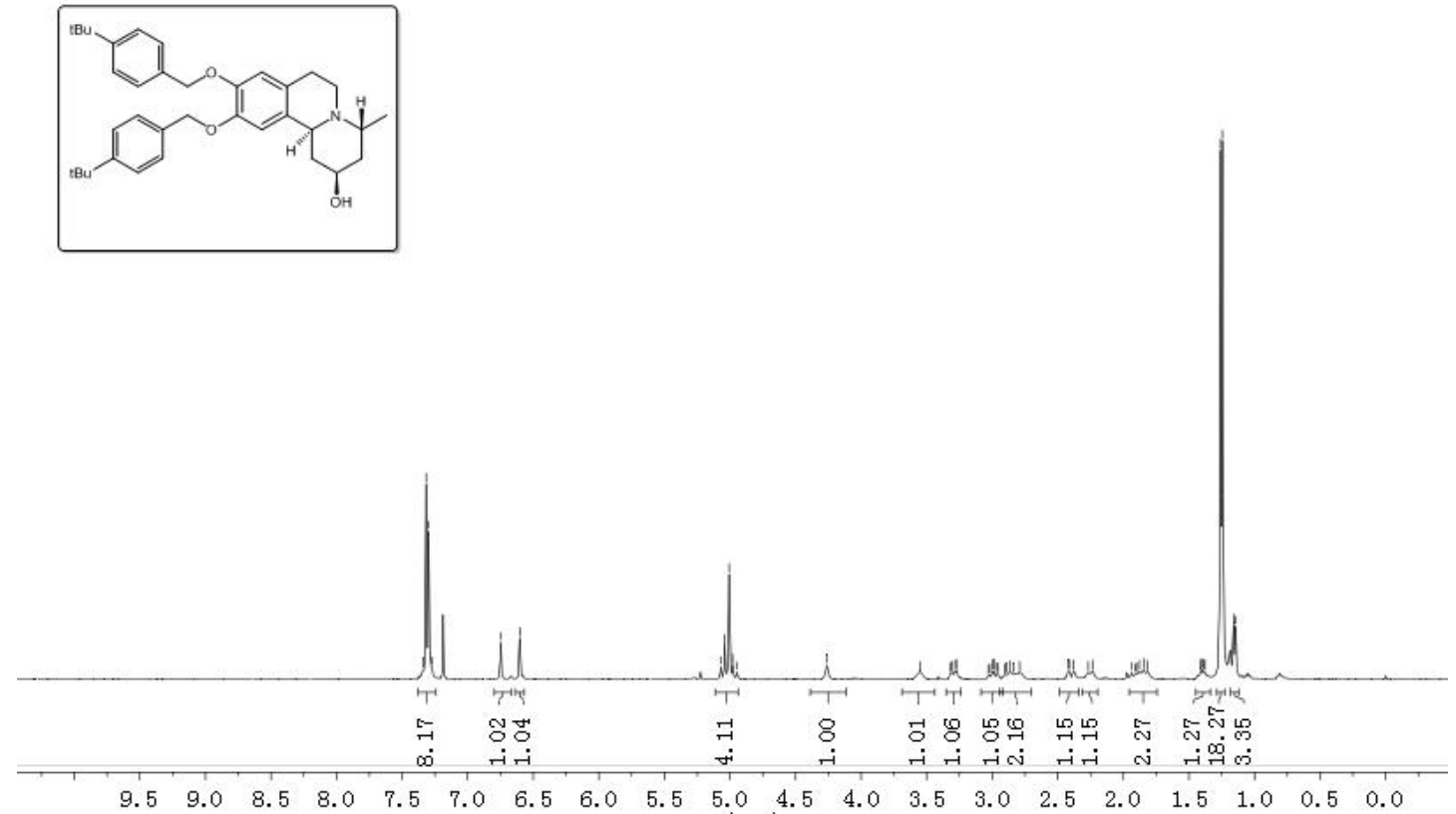

Figure S270. ${ }^{1} \mathrm{H}$ NMR spectrum of $\mathbf{2 5 b}$.

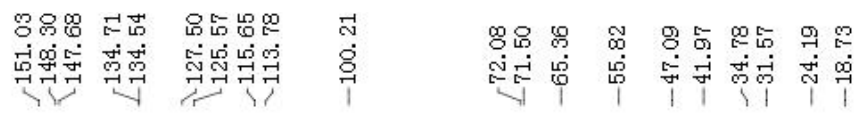
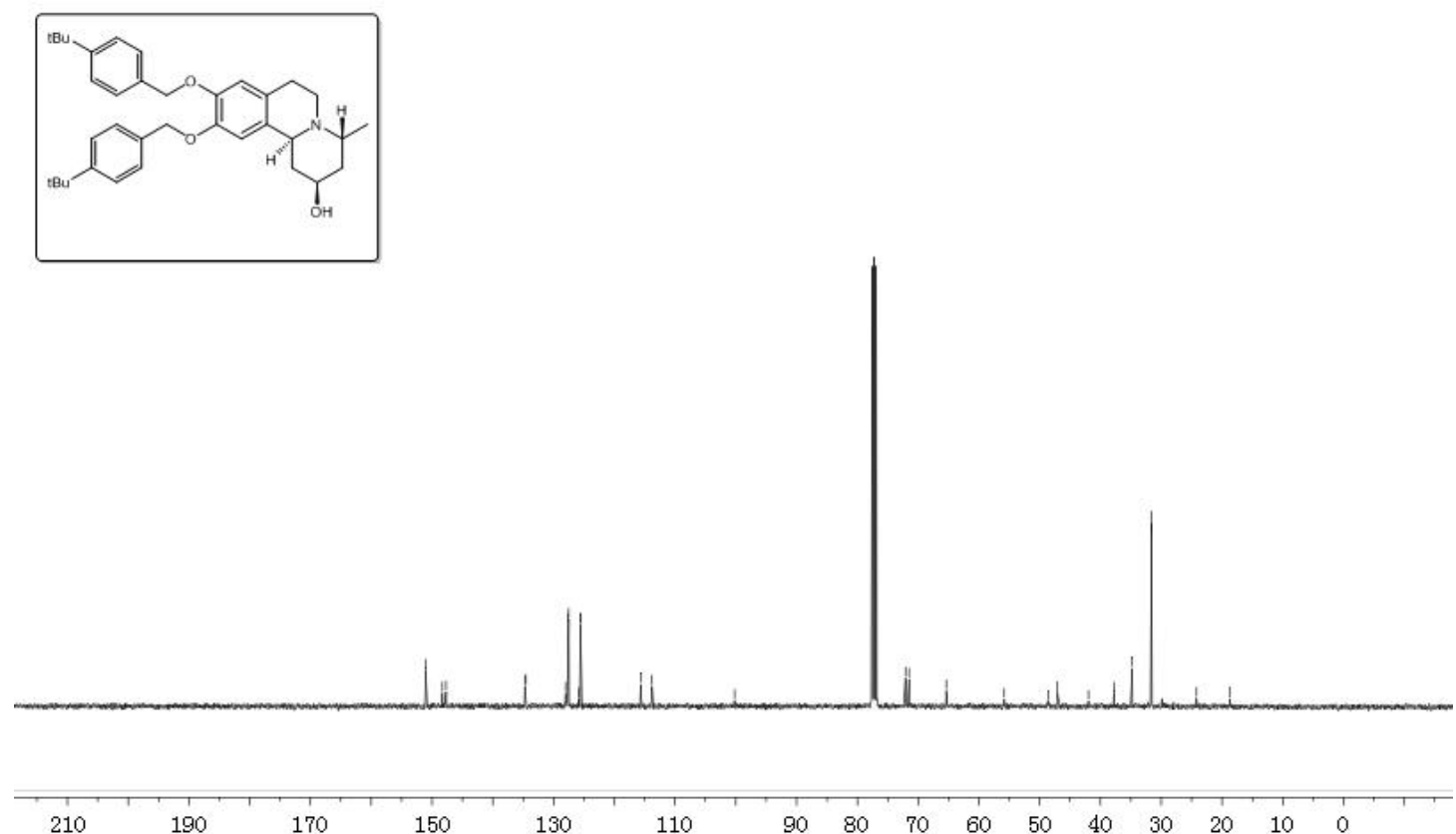

Figure S271. ${ }^{13} \mathrm{C}$ NMR spectrum of $\mathbf{2 5 b}$. 

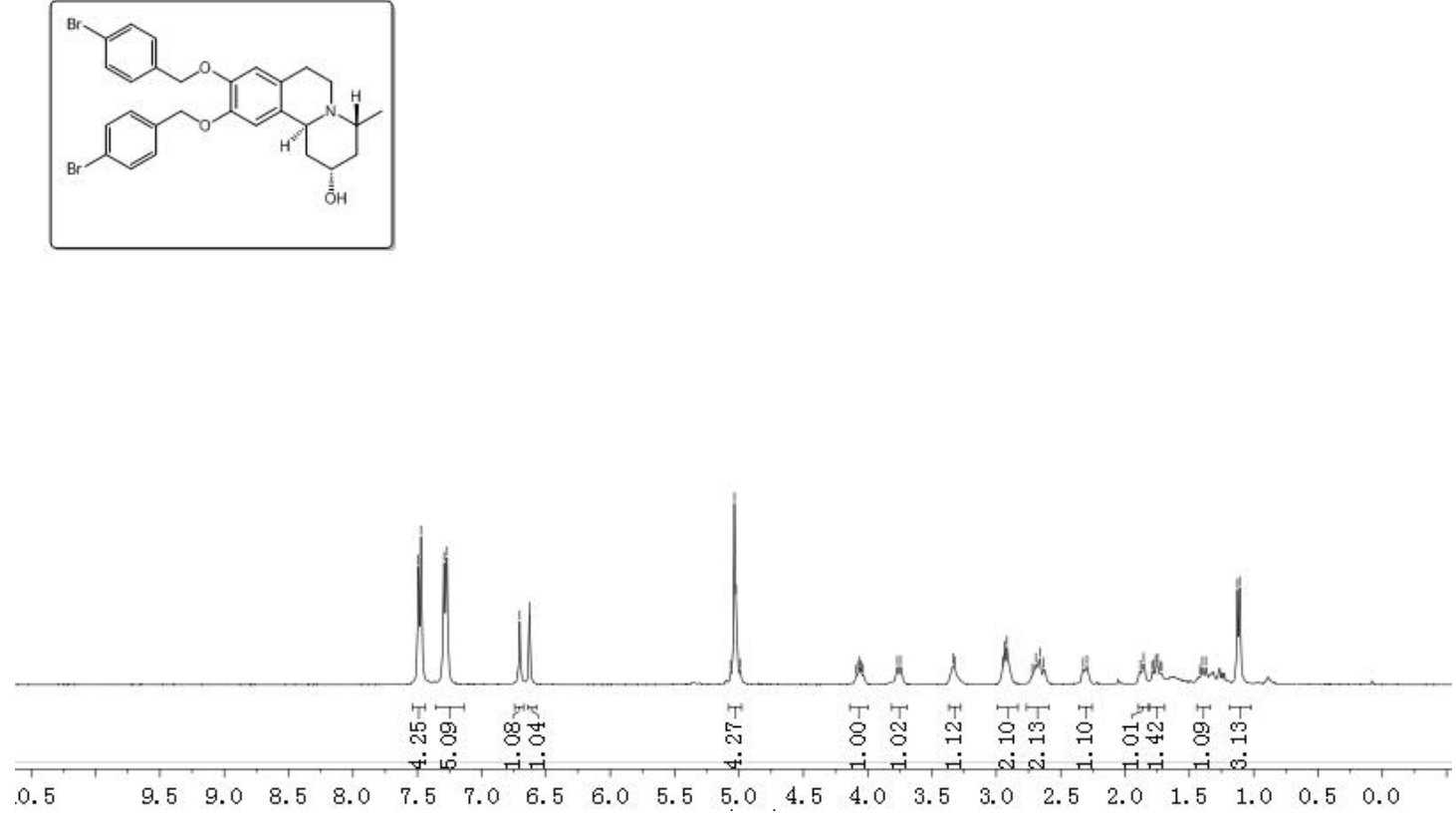

Figure S272. ${ }^{1} \mathrm{H}$ NMR spectrum of 26 a.
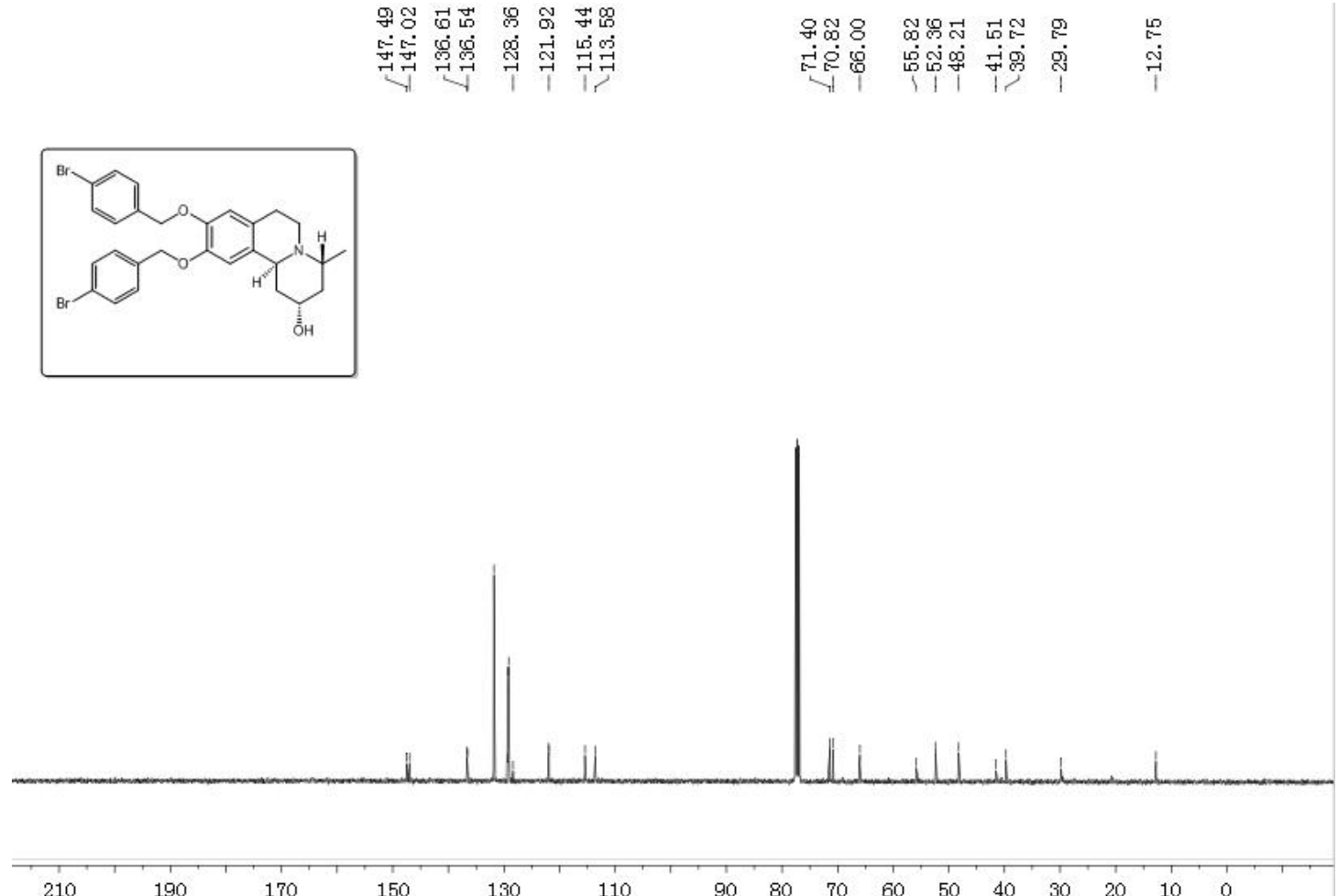

Figure S273. ${ }^{13} \mathrm{C}$ NMR spectrum of 26a. 


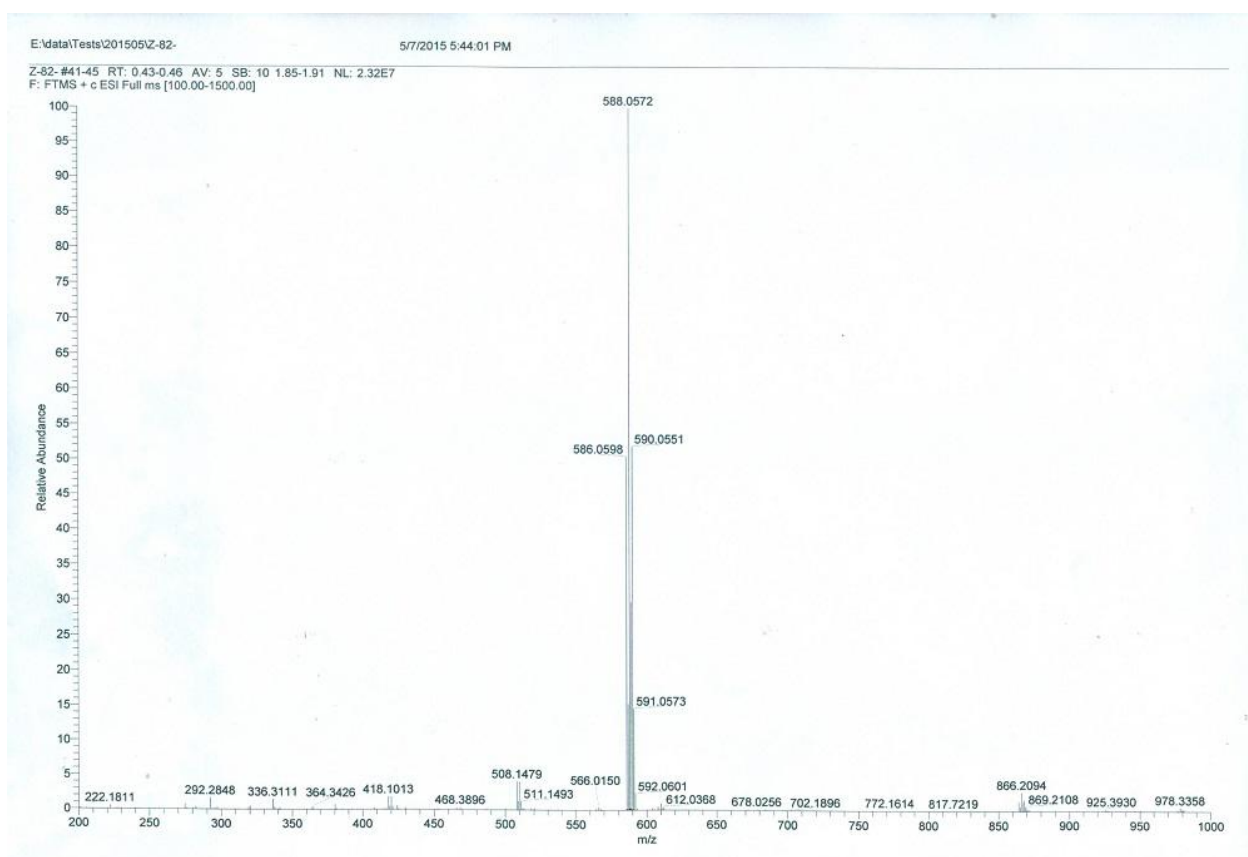

Figure S274. HRESIMS spectrum of $\mathbf{2 6 \mathbf { b }}$.

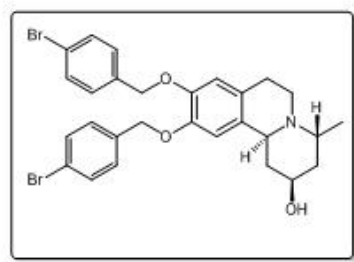

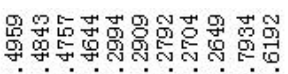

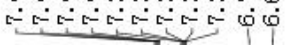
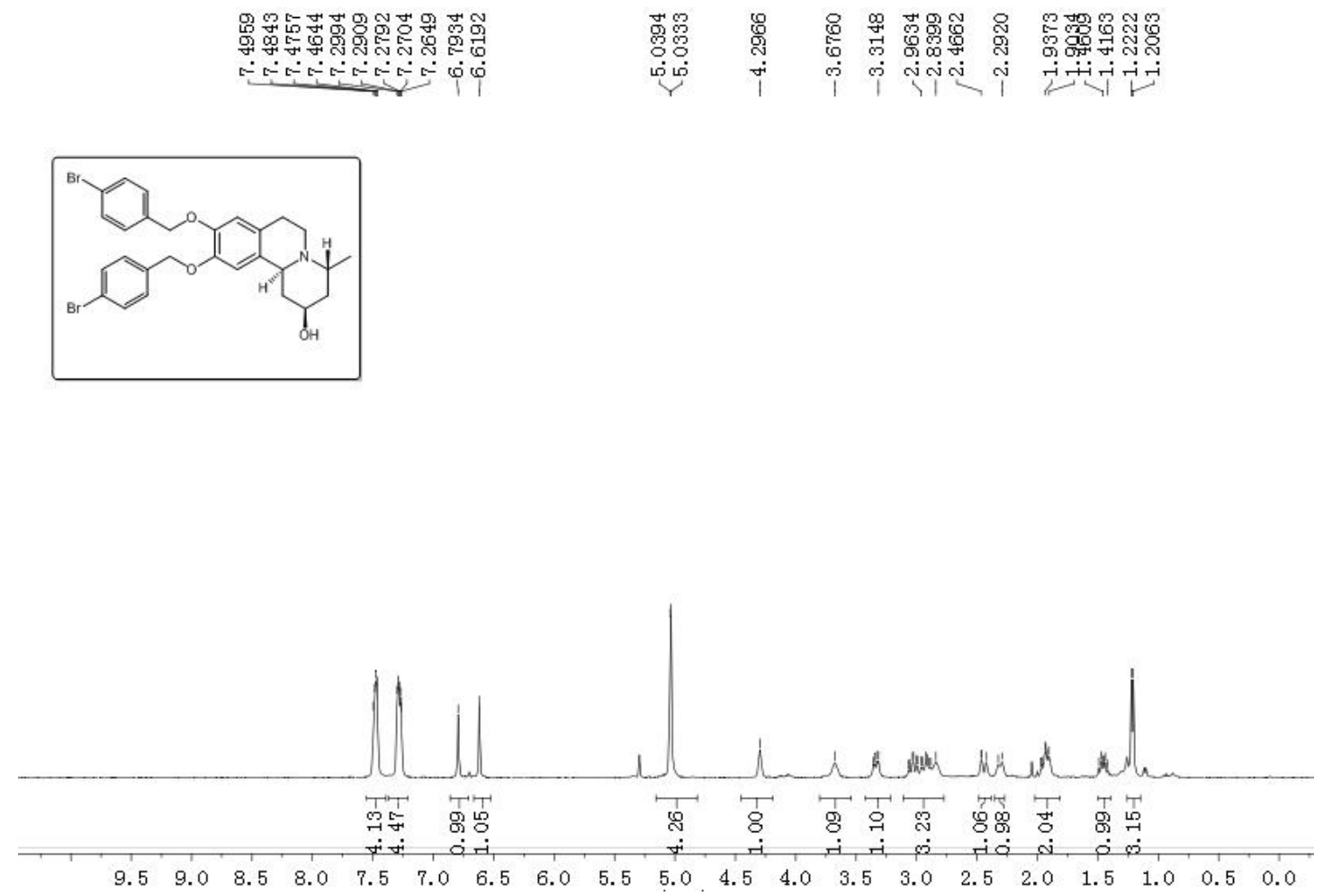

Figure S275. ${ }^{1} \mathrm{H}$ NMR spectrum of $\mathbf{2 6 b}$. 

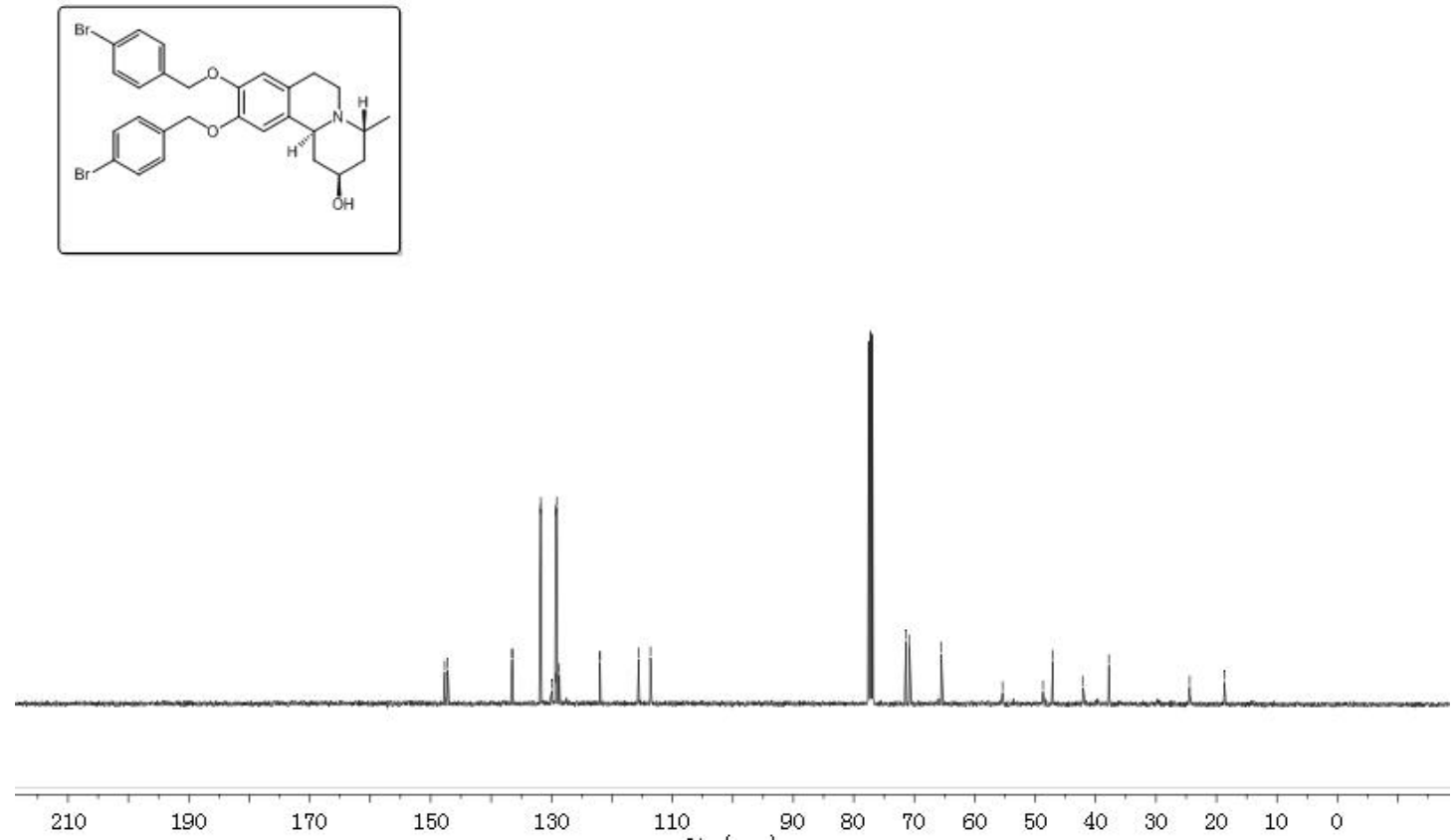

Figure S276. ${ }^{13} \mathrm{C}$ NMR spectrum of $\mathbf{2 6 \mathbf { b }}$.

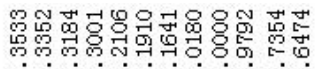

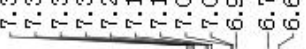

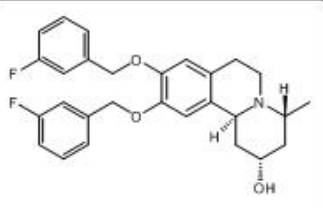

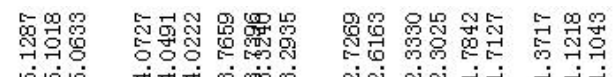

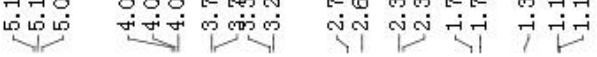

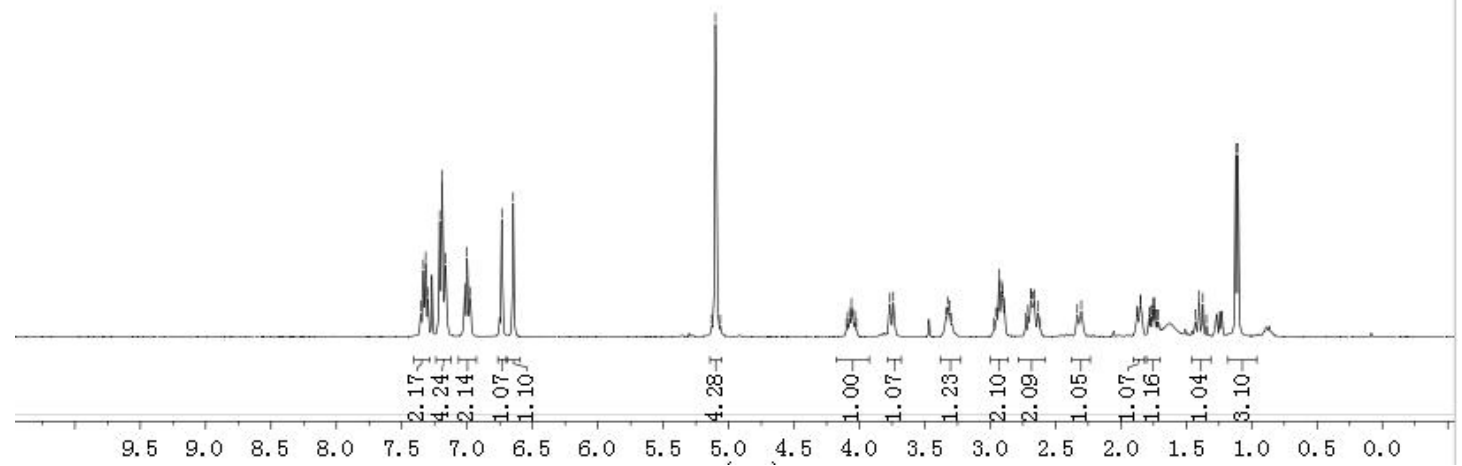

Figure S277. ${ }^{1} \mathrm{H}$ NMR spectrum of $\mathbf{2 7 a}$. 

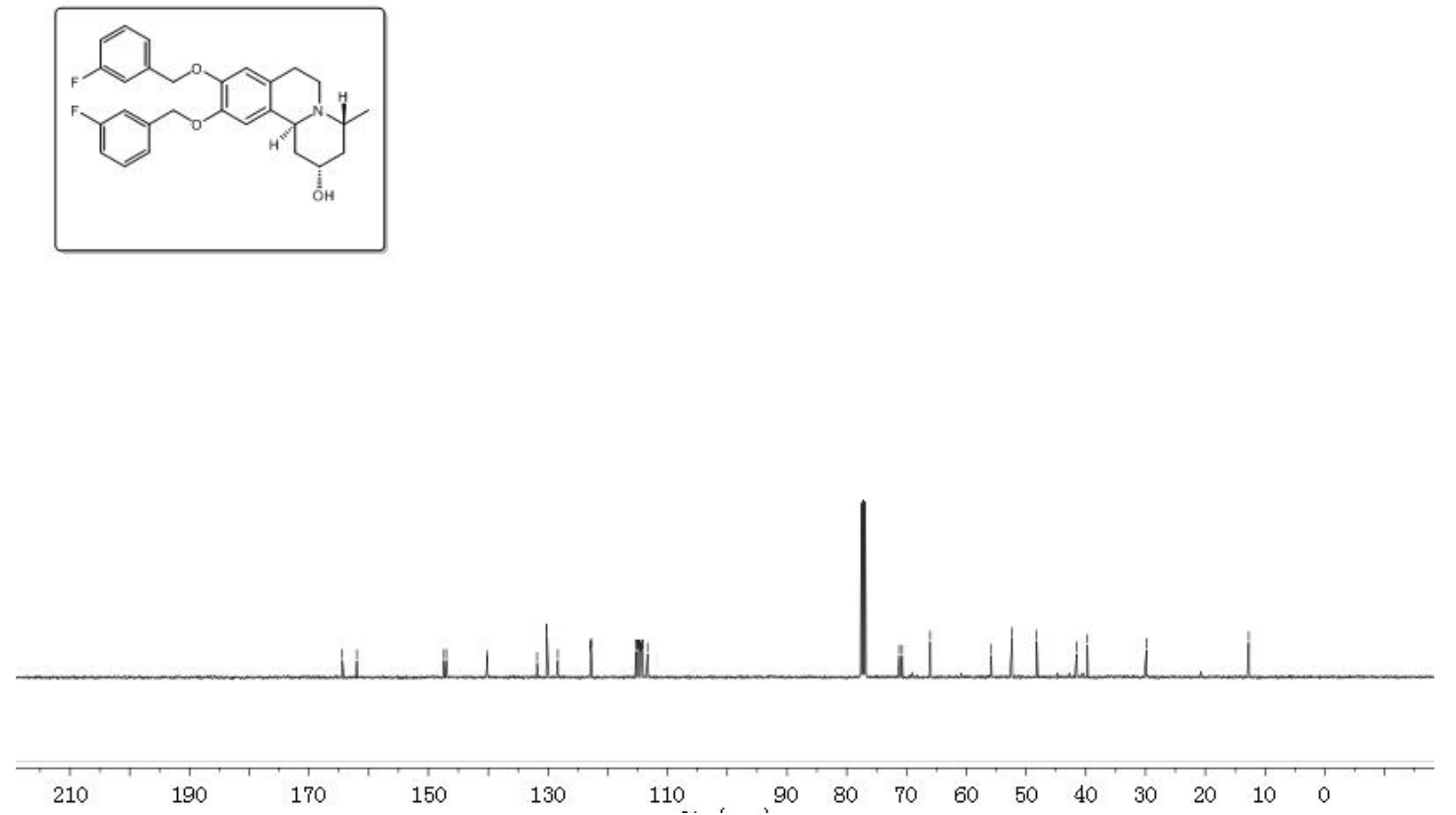

Figure S278. ${ }^{13} \mathrm{C}$ NMR spectrum of $27 \mathbf{a}$.

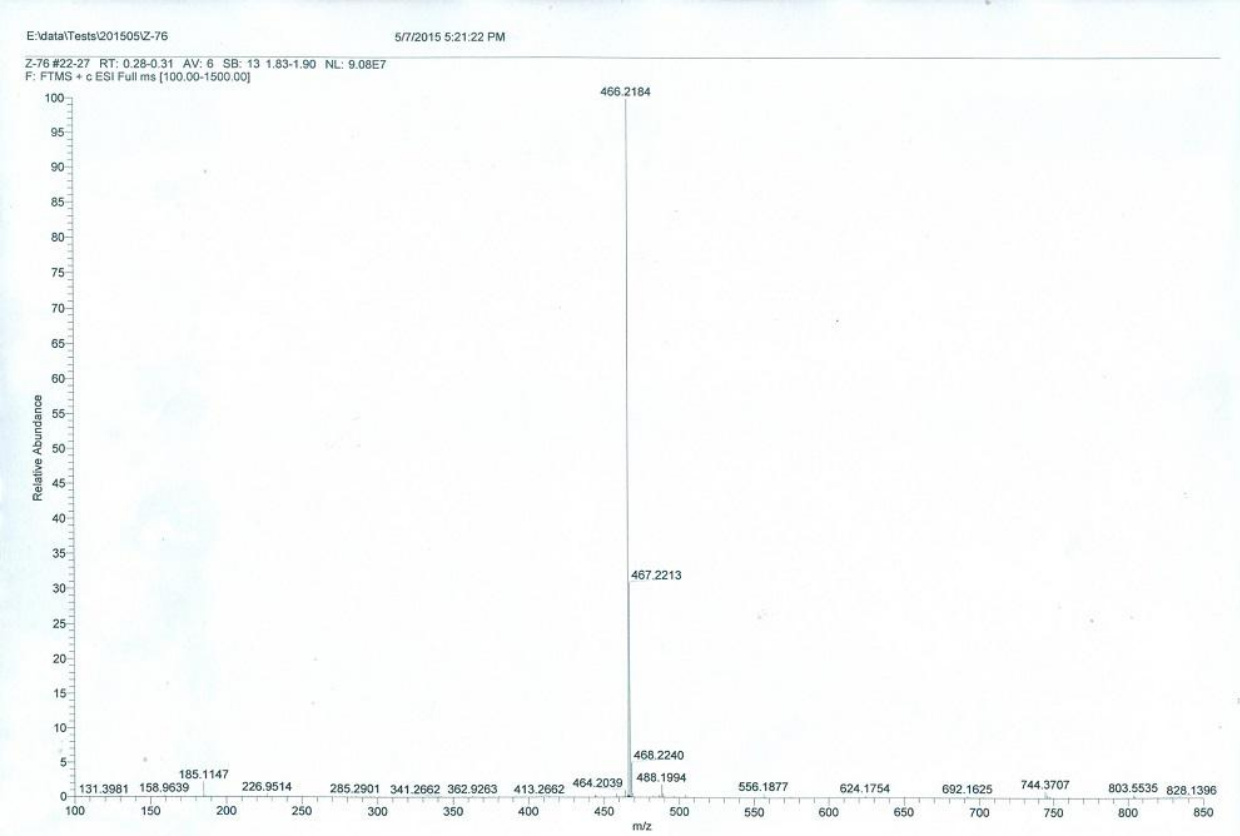

Figure S279. HRESIMS spectrum of 27b. 

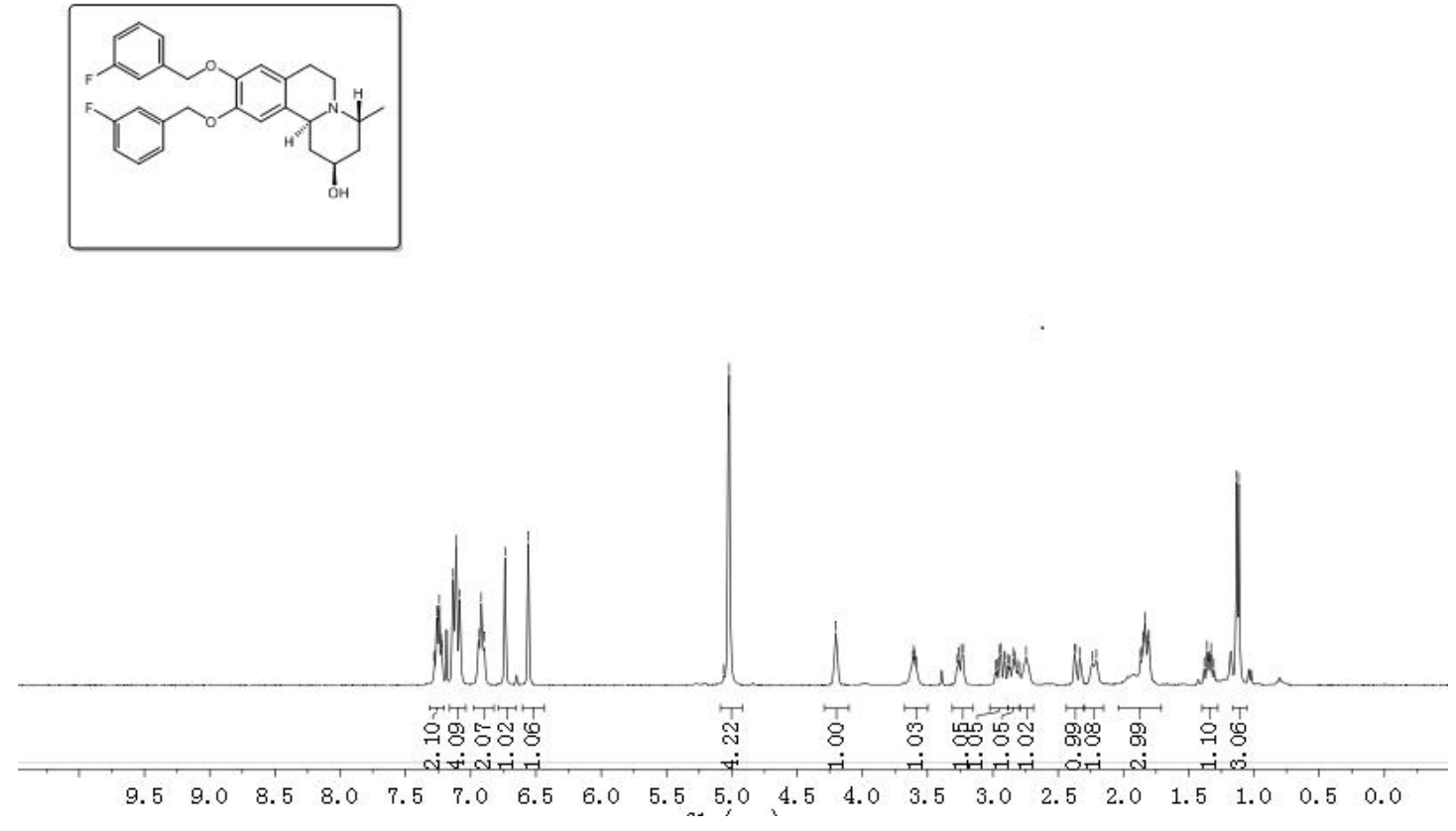

Figure S280. ${ }^{1} \mathrm{H}$ NMR spectrum of $\mathbf{2 7 b}$
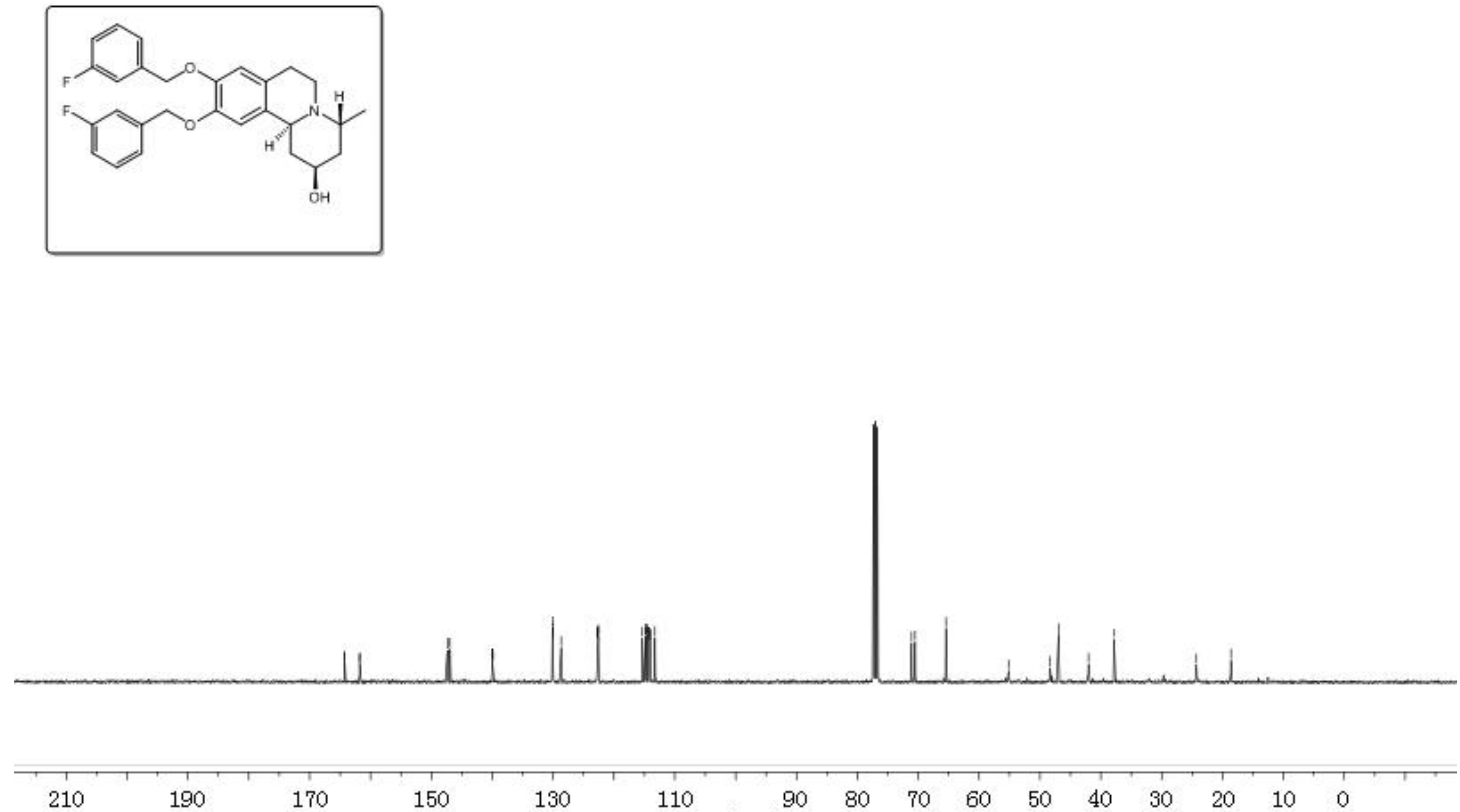

Figure S281. ${ }^{13} \mathrm{C}$ NMR spectrum of $\mathbf{2 7 b}$. 


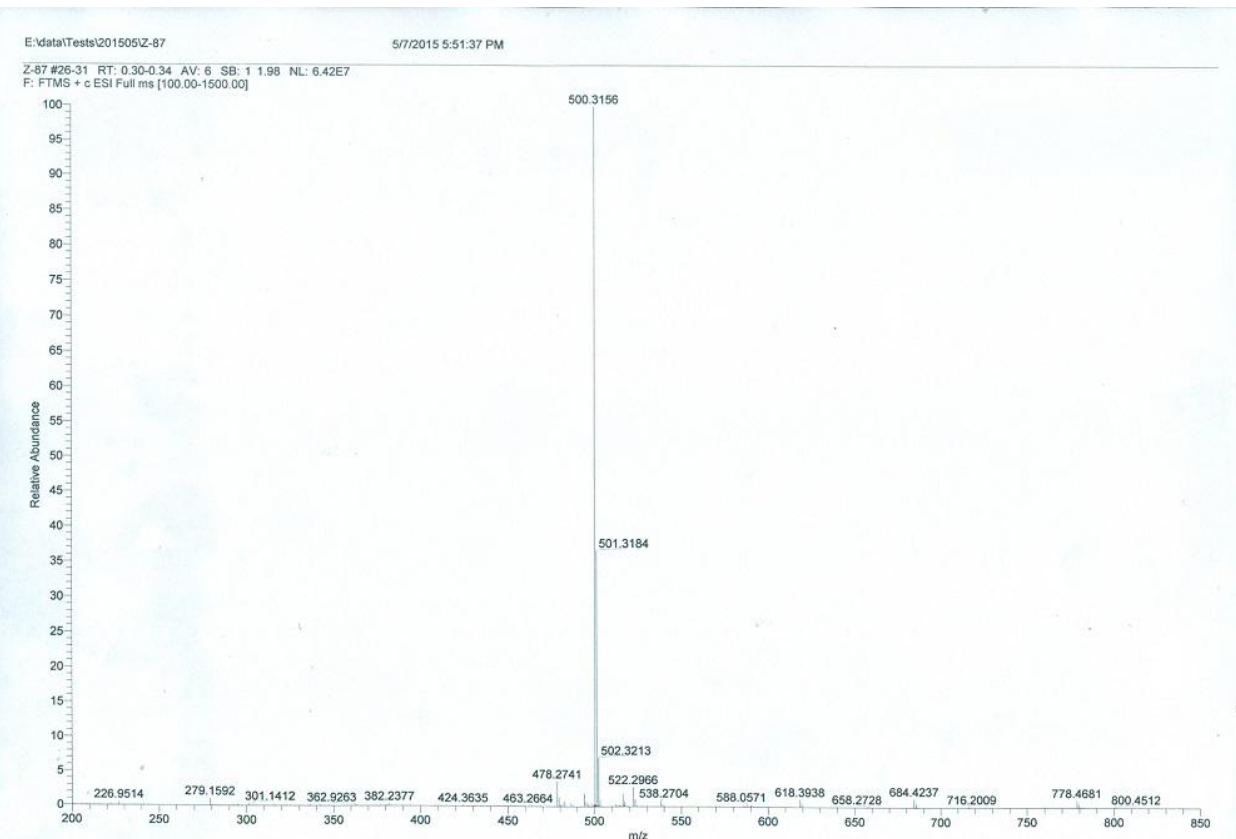

Figure S282. HRESIMS spectrum of $\mathbf{2 8}$.

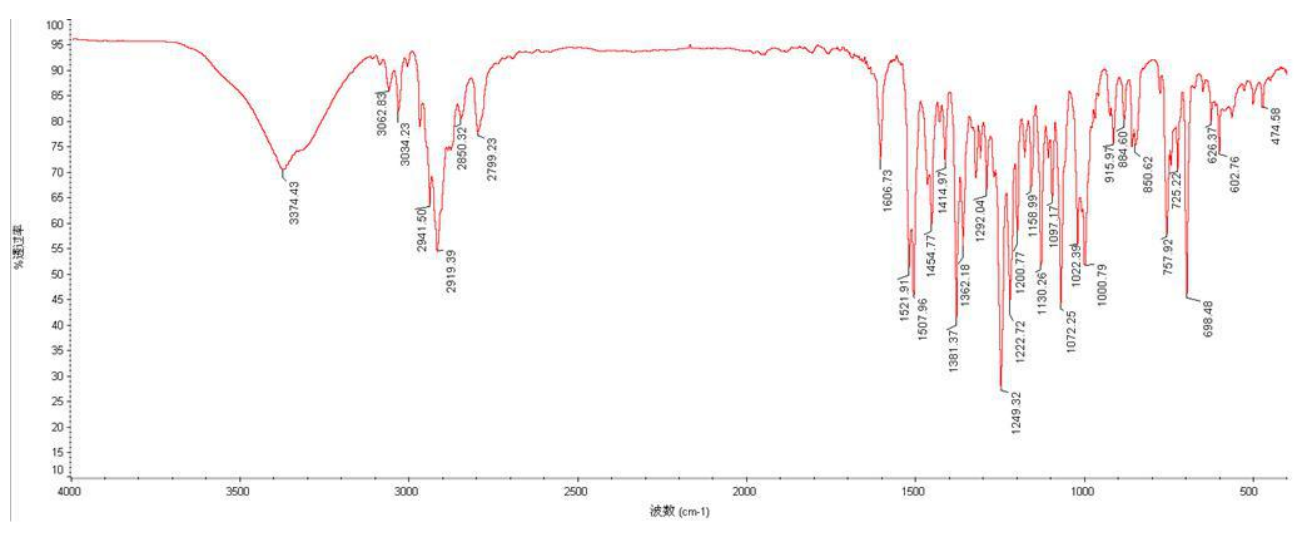

Figure S283. IR spectrum of 28. 

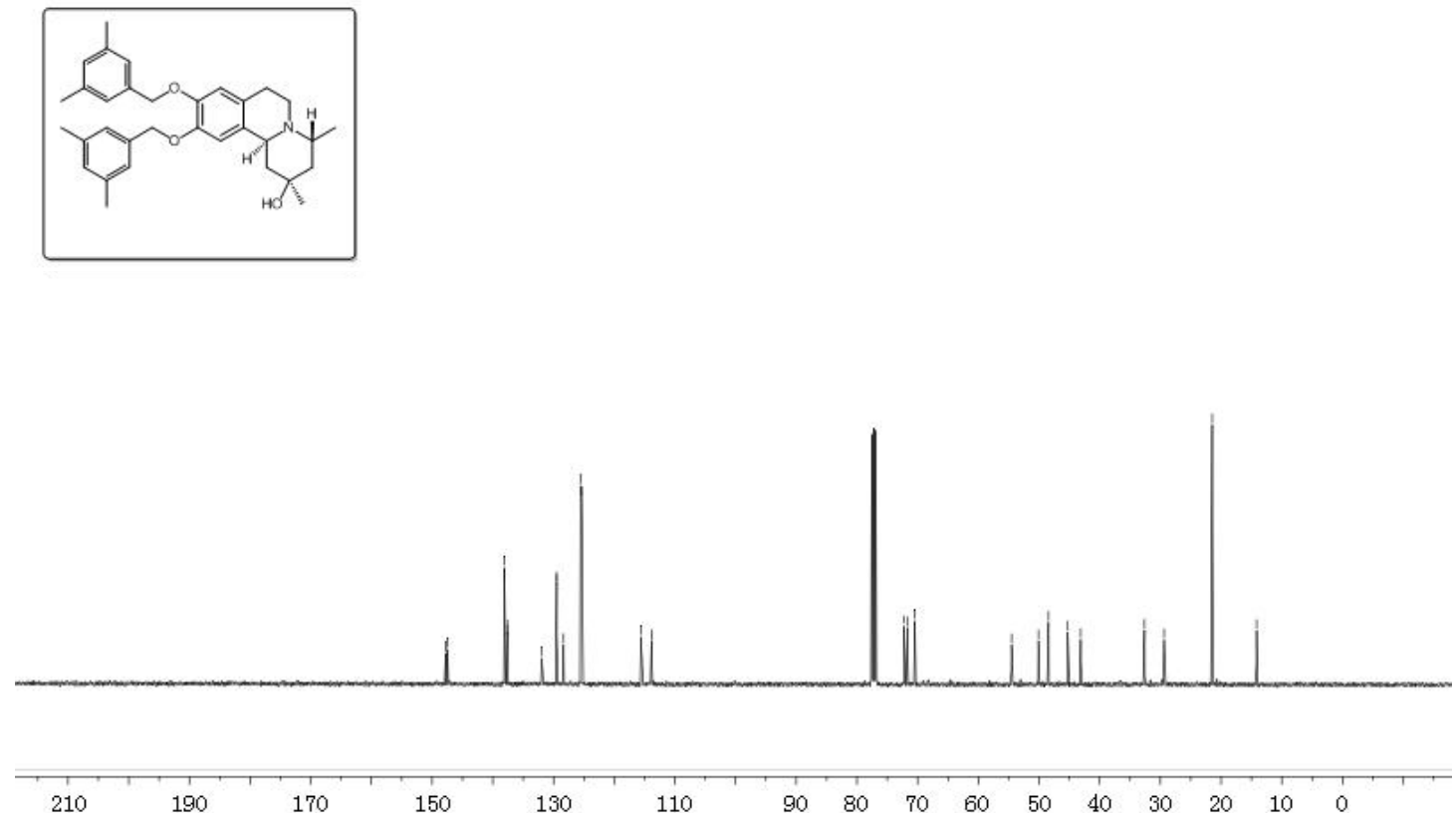

Figure S284. ${ }^{1} \mathrm{H}$ NMR spectrum of 28.

品경

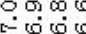

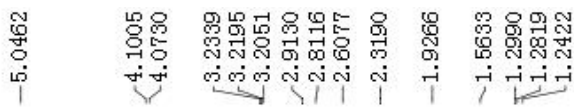

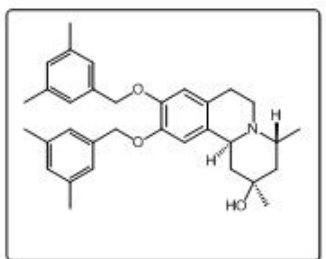




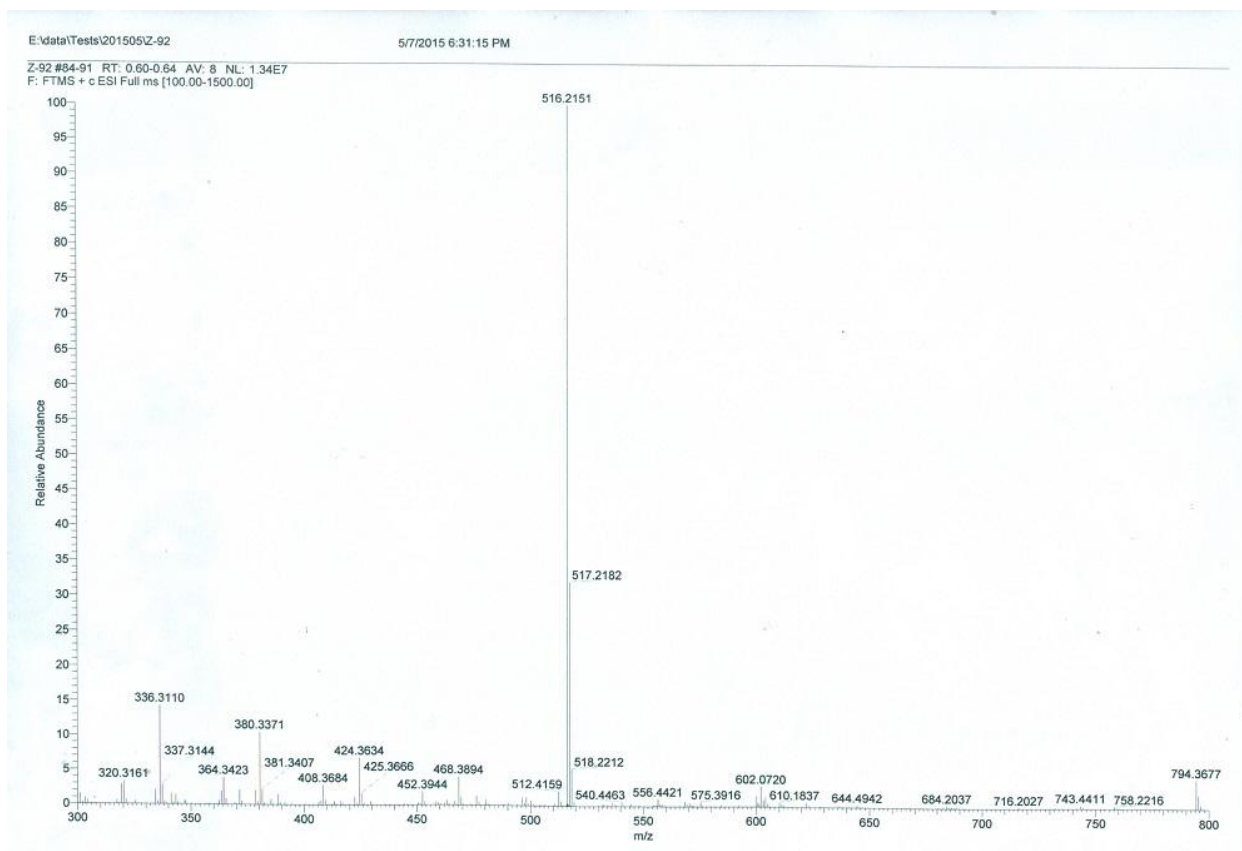

Figure S286. HRESIMS spectrum of 29.
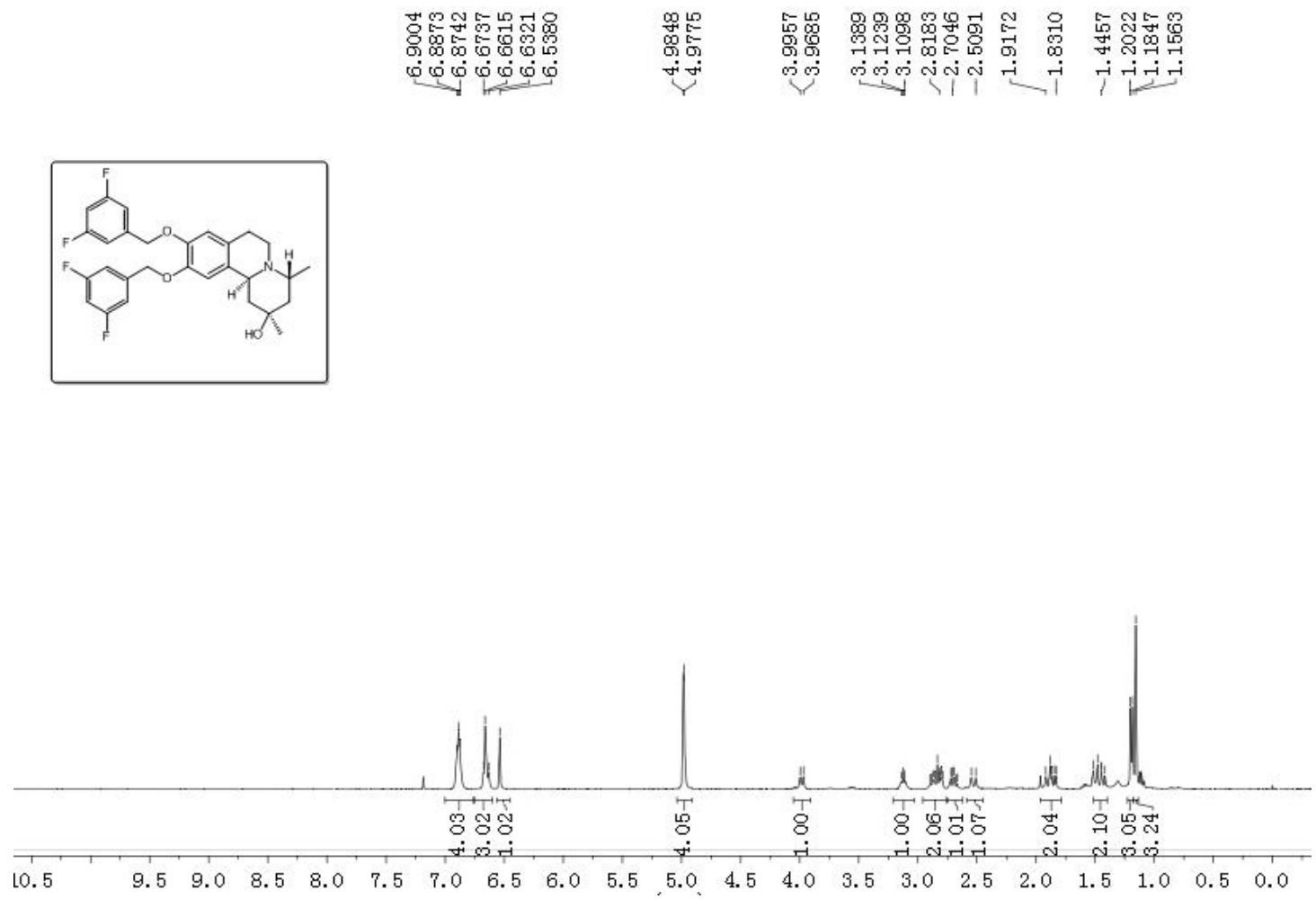

Figure S287. ${ }^{1} \mathrm{H}$ NMR spectrum of 29. 

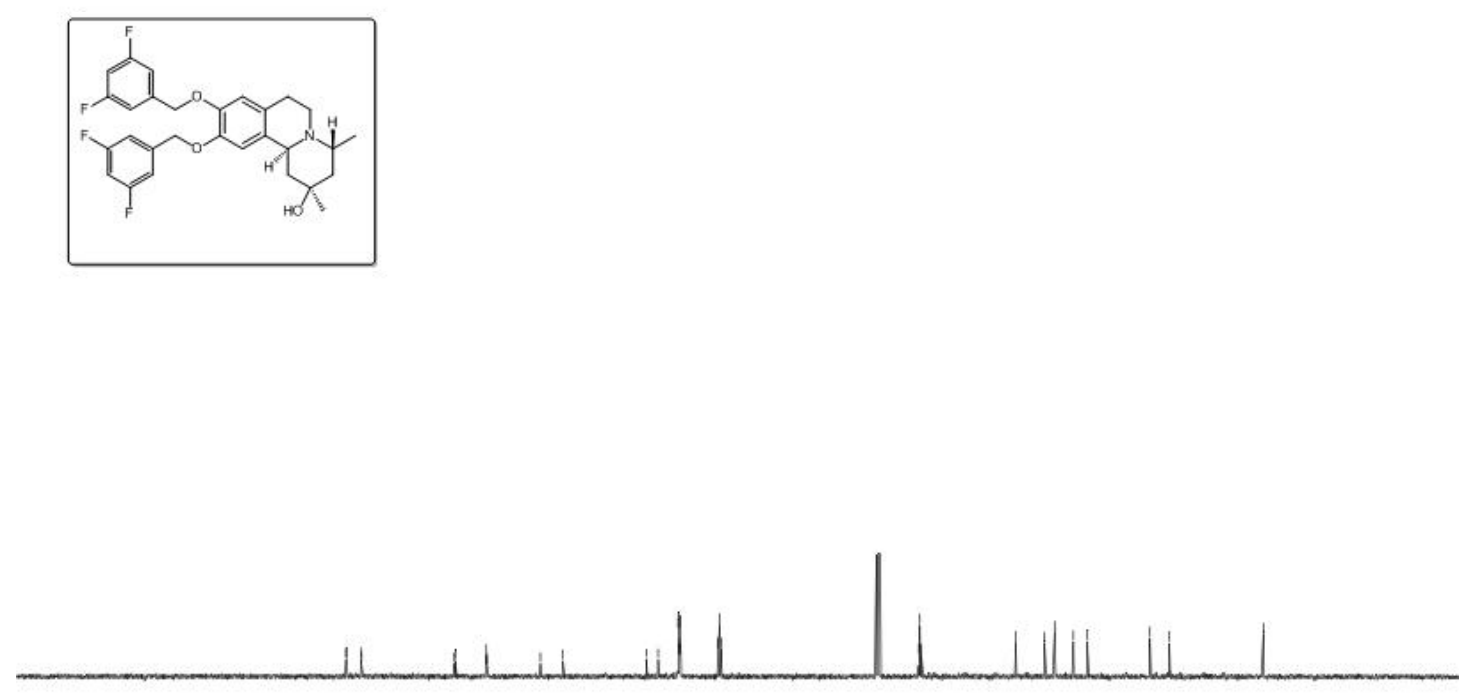

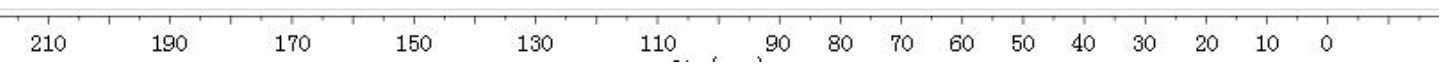

Figure S288. ${ }^{13} \mathrm{C}$ NMR spectrum of 29.

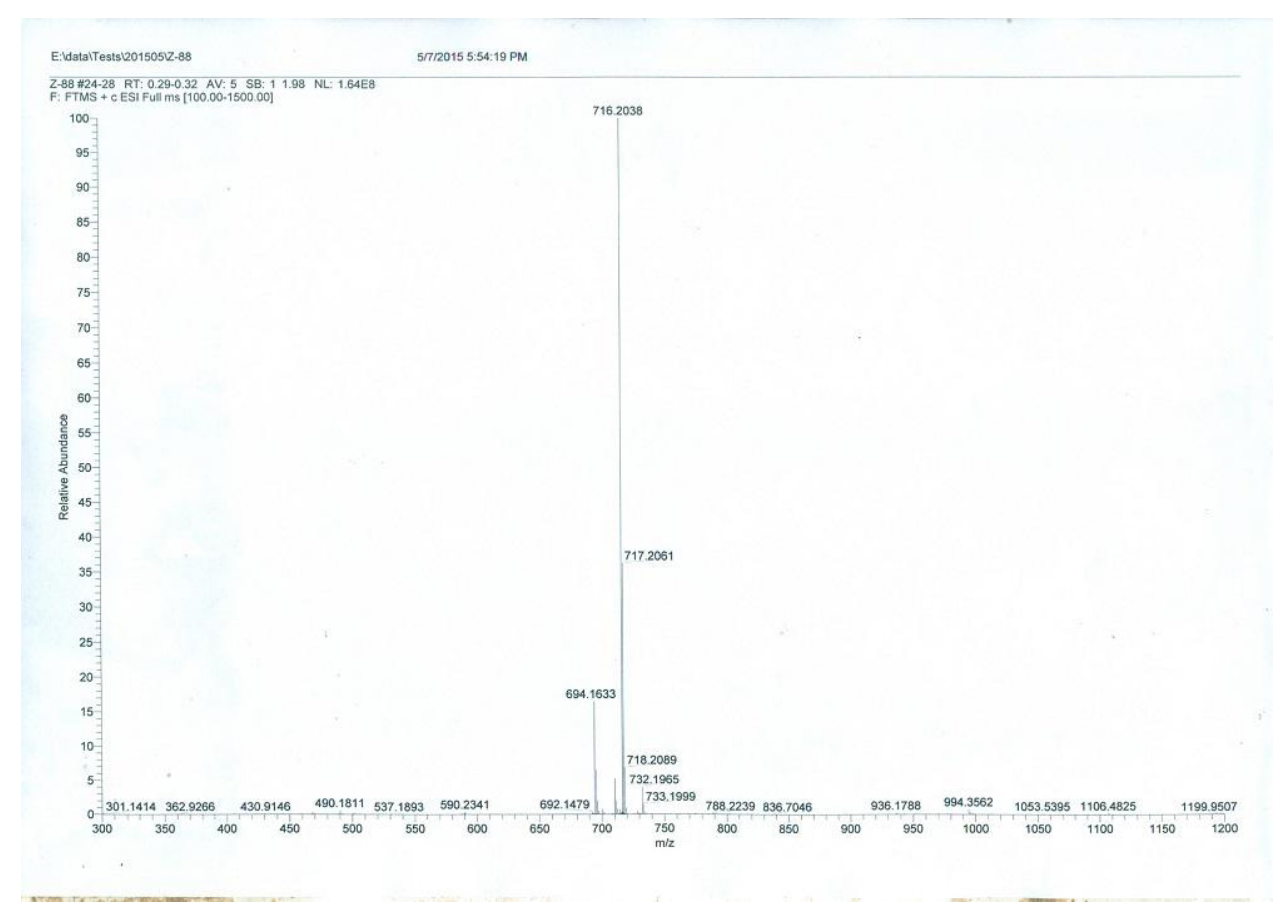

Figure S289. HRESIMS spectrum of $\mathbf{3 0}$. 

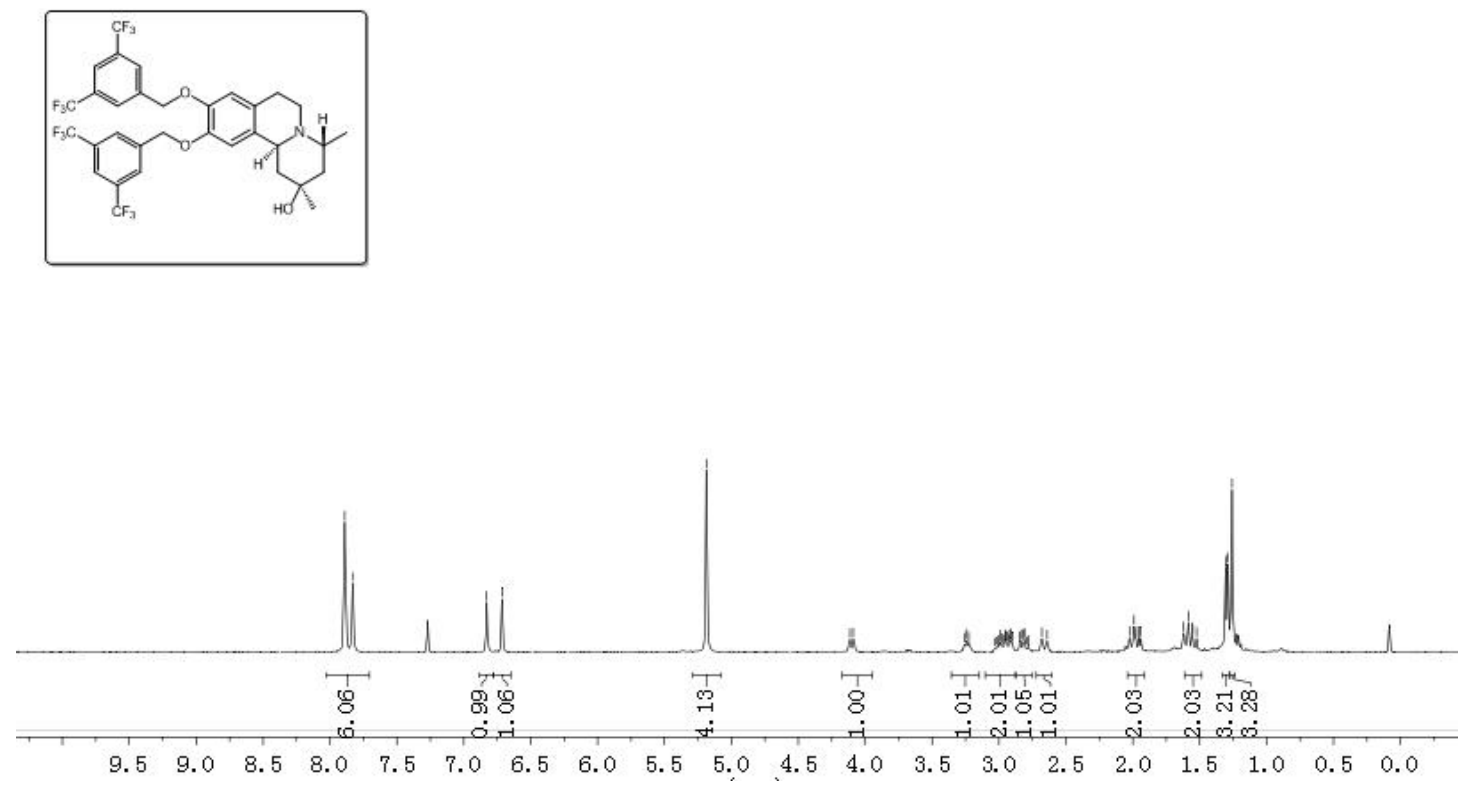

Figure S290. ${ }^{1} \mathrm{H}$ NMR spectrum of $\mathbf{3 0}$.
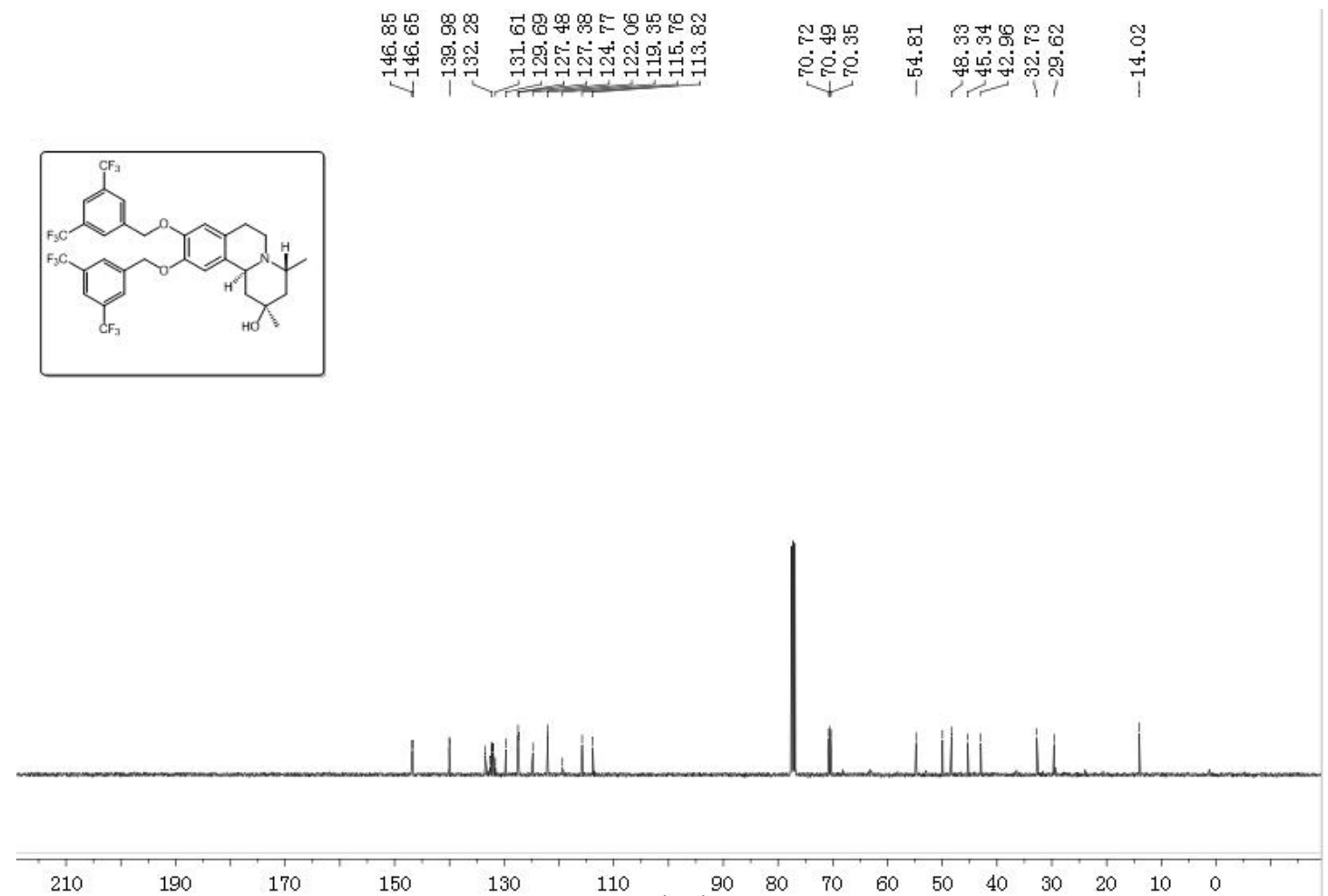

Figure S291. ${ }^{13} \mathrm{C}$ NMR spectrum of $\mathbf{3 0}$. 


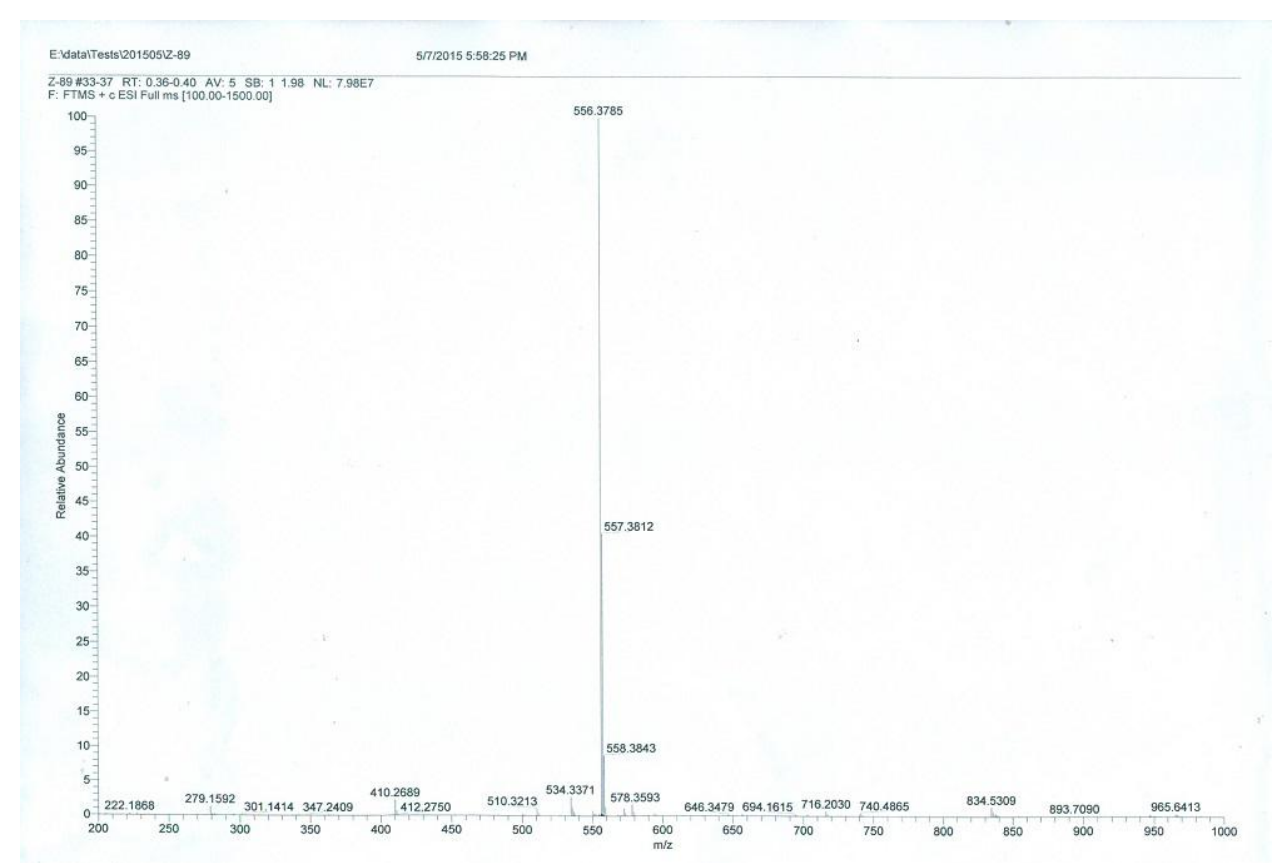

Figure S292. HRESIMS spectrum of $\mathbf{3 1}$.
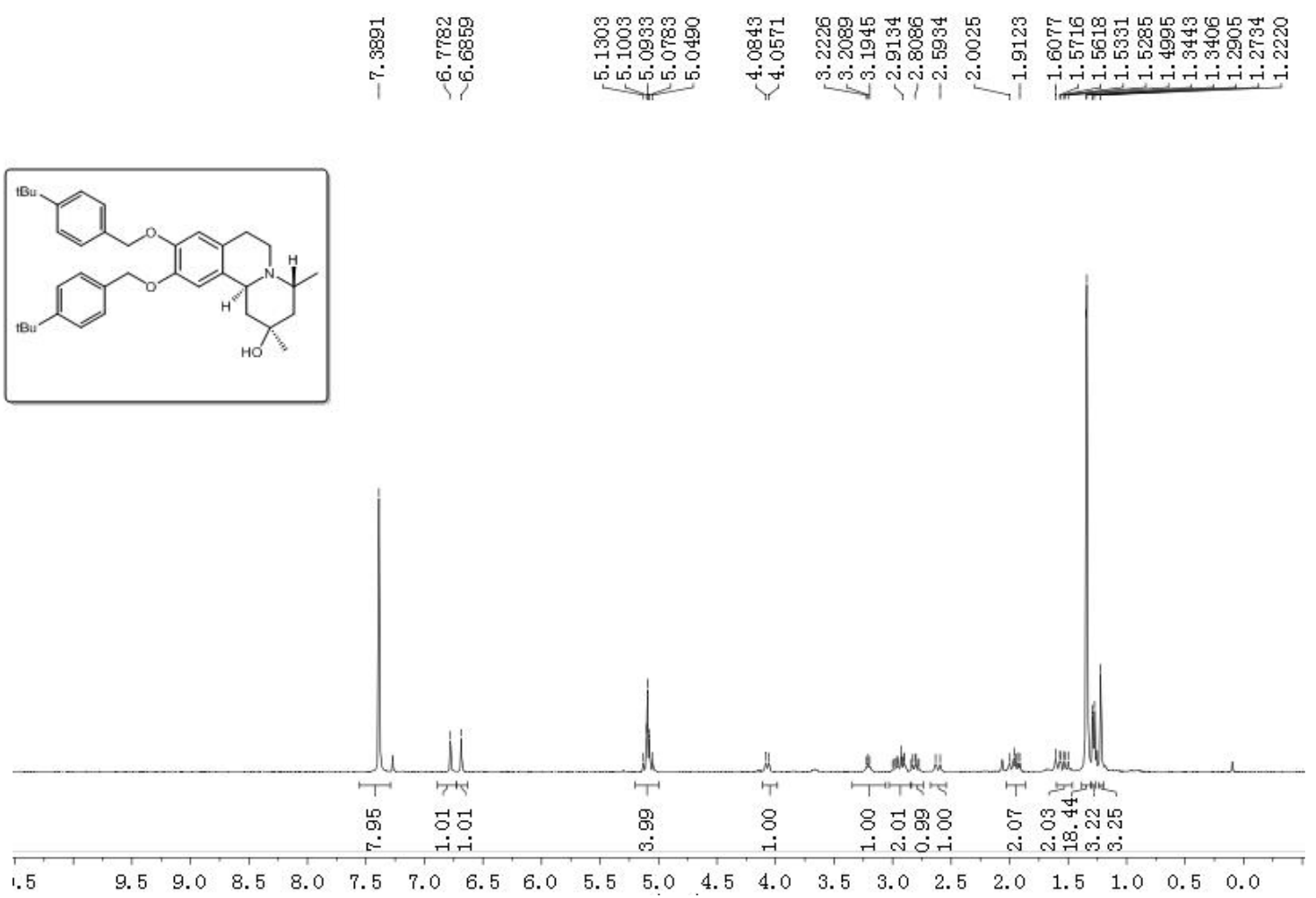

Figure S293. ${ }^{1} \mathrm{H}$ NMR spectrum of $\mathbf{3 1}$. 

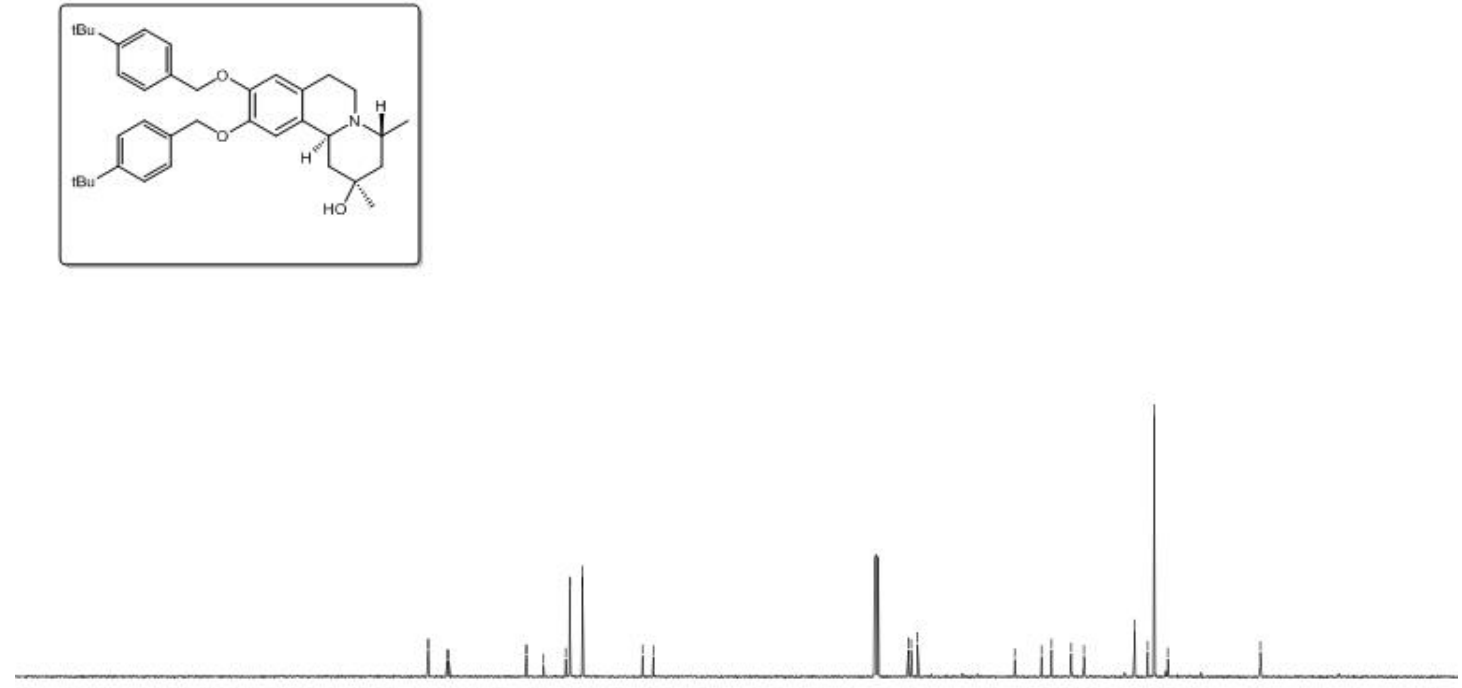

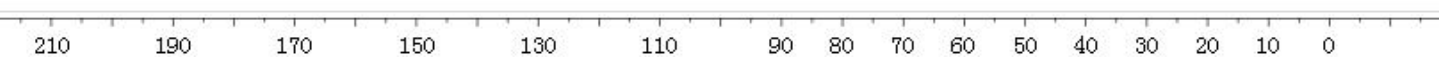

Figure S294. ${ }^{13} \mathrm{C}$ NMR spectrum of 31.

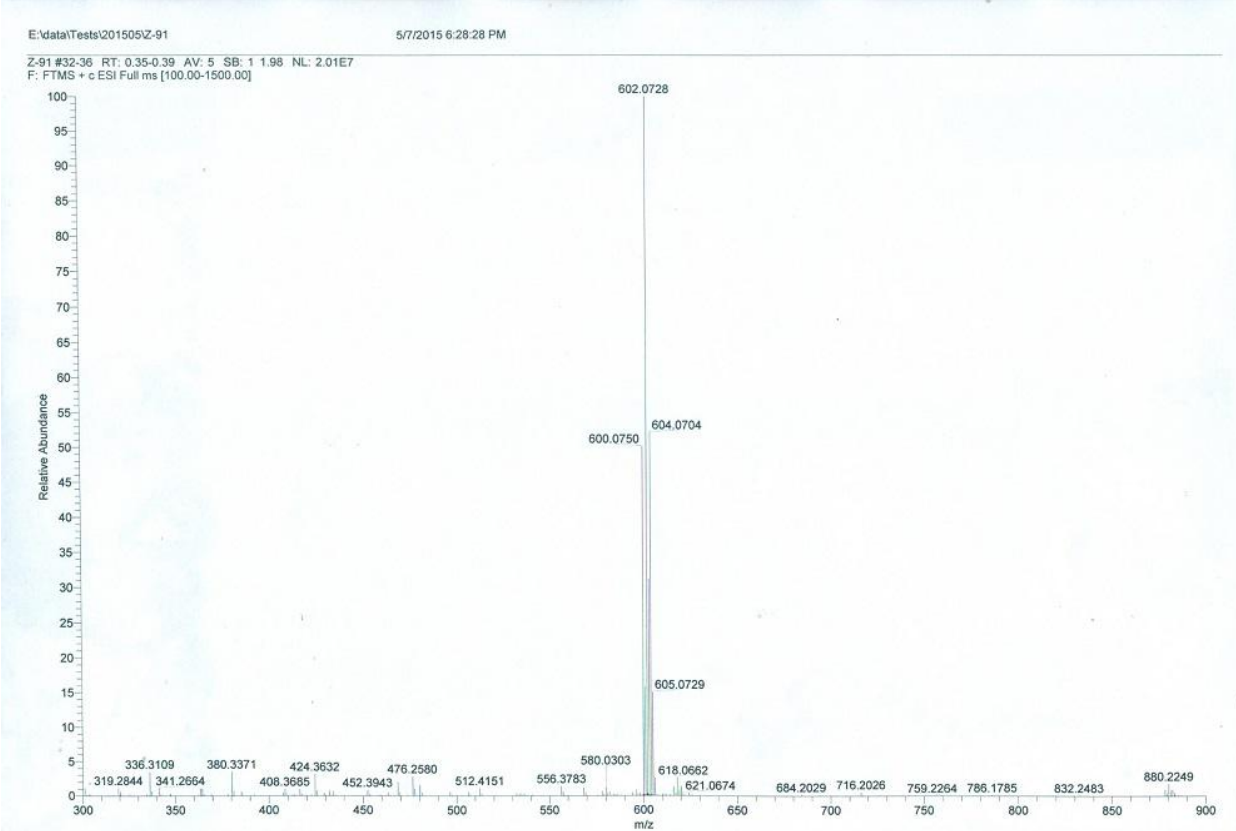

Figure S295. HRESIMS spectrum of 32. 

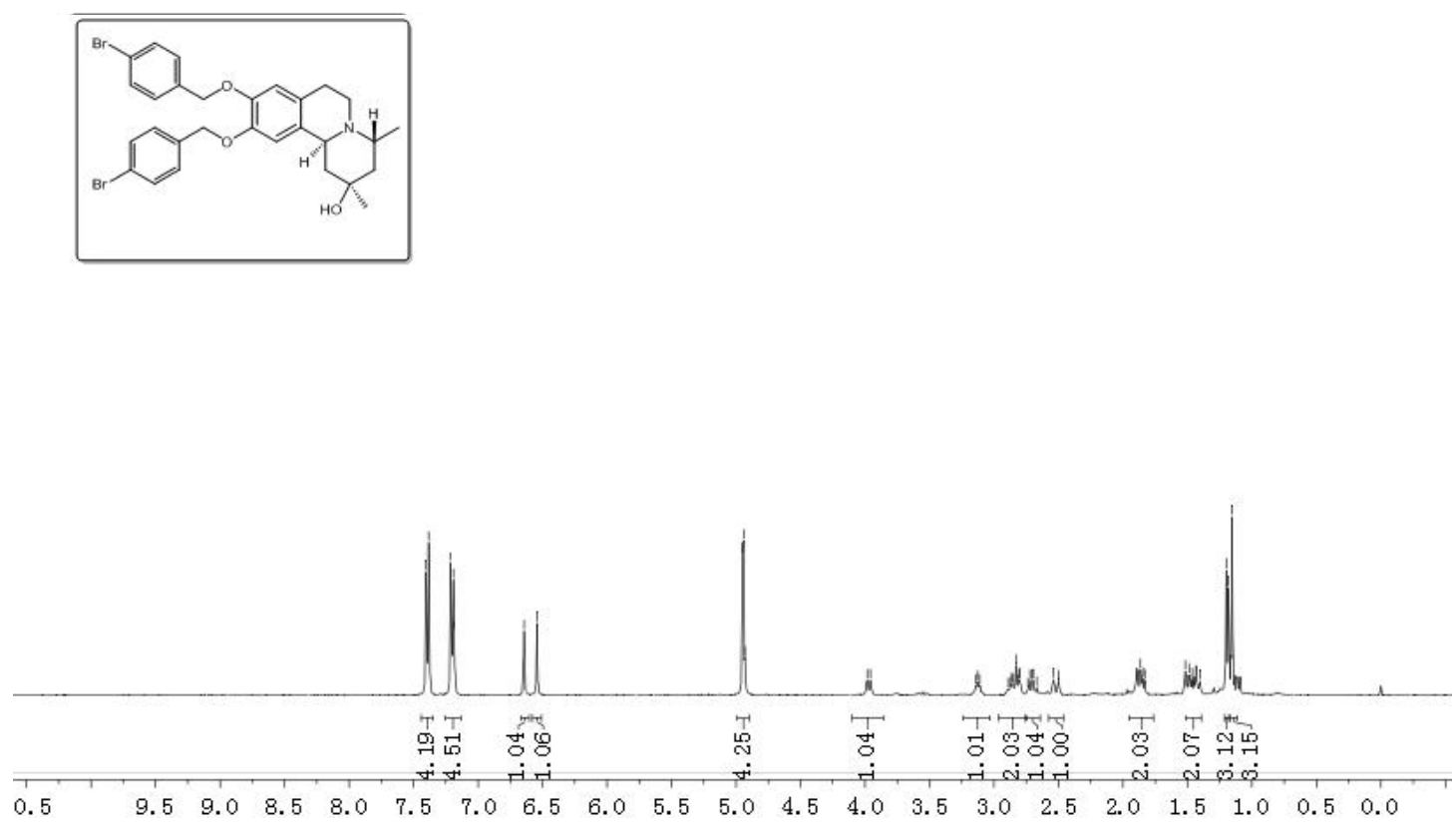

Figure S296. ${ }^{1} \mathrm{H}$ NMR spectrum of $\mathbf{3 2}$.

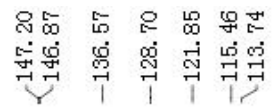

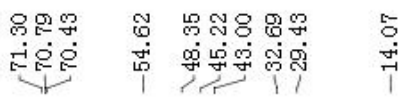
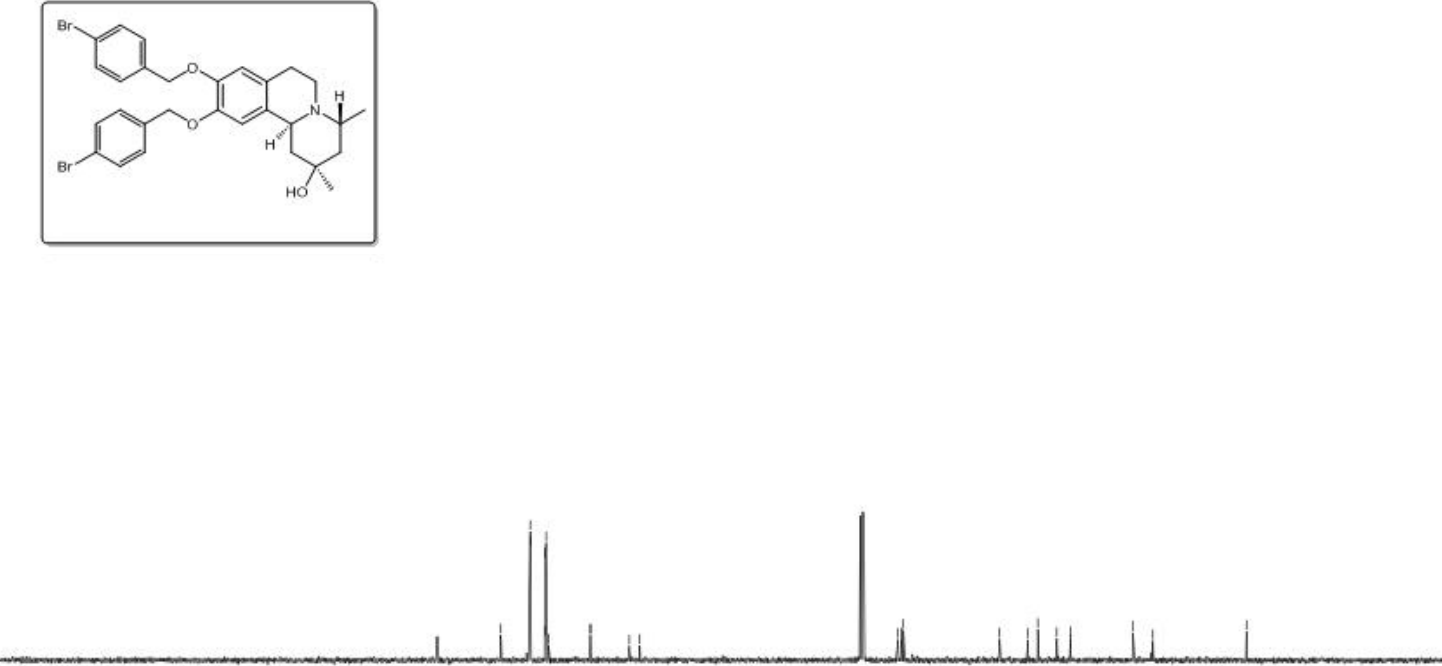

$210 \quad 190 \quad 170$

Figure S297. ${ }^{13} \mathrm{C}$ NMR spectrum of 32 . 


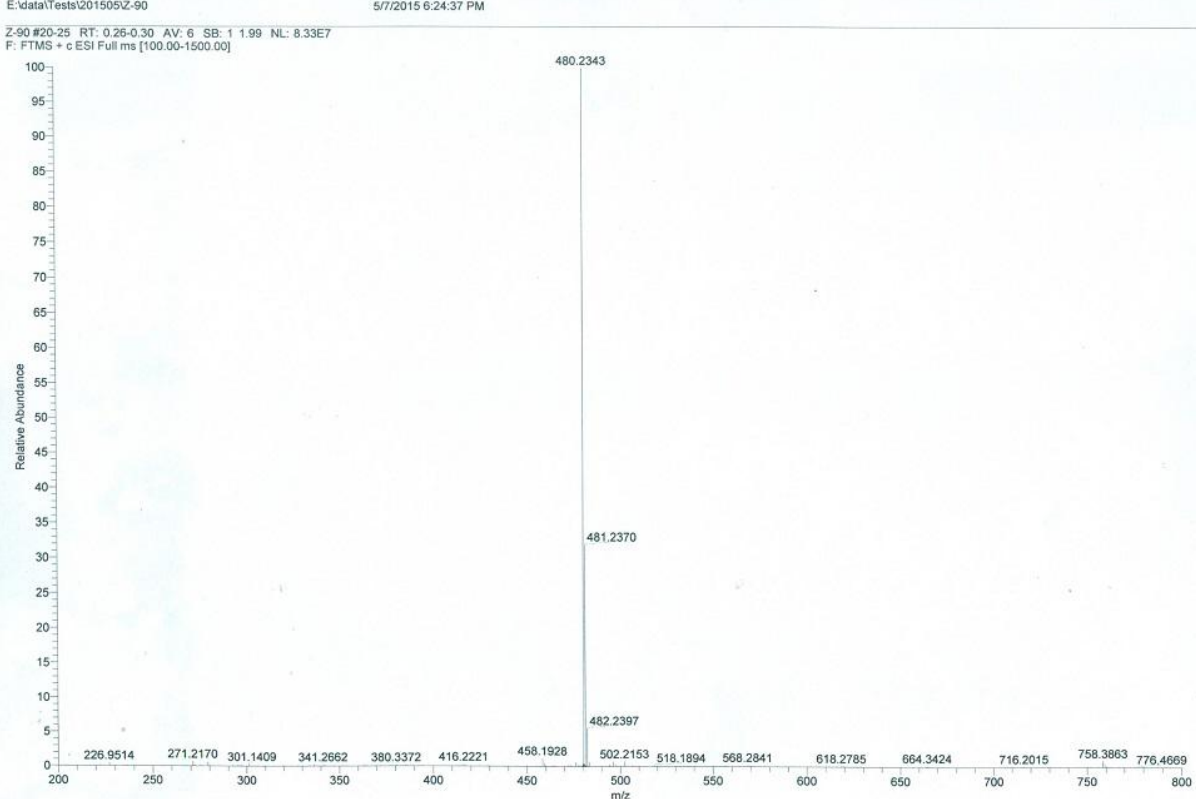

Figure S298. HRESIMS spectrum of $\mathbf{3 3}$.
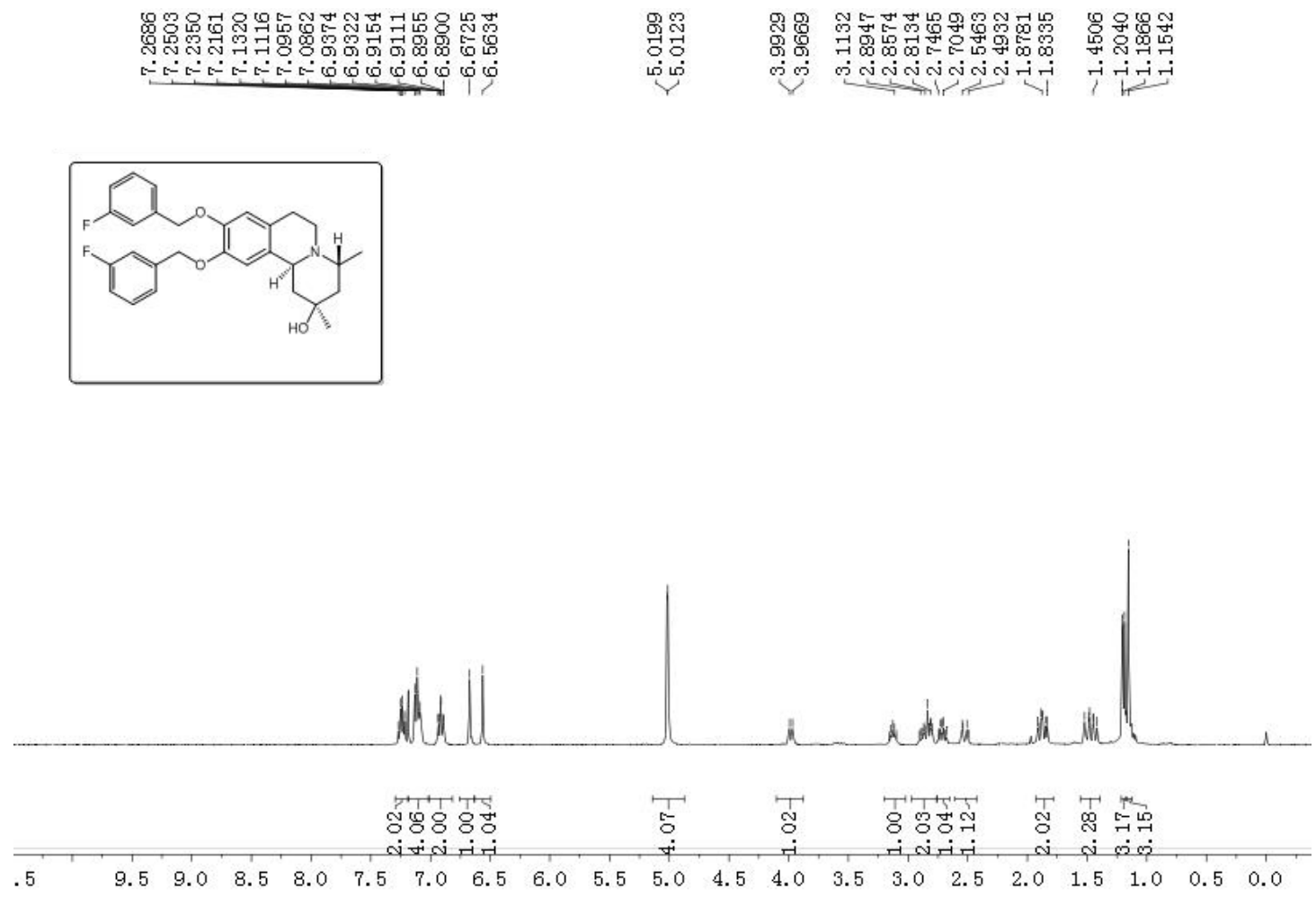

Figure S299. ${ }^{1} \mathrm{H}$ NMR spectrum of $\mathbf{3 3}$. 

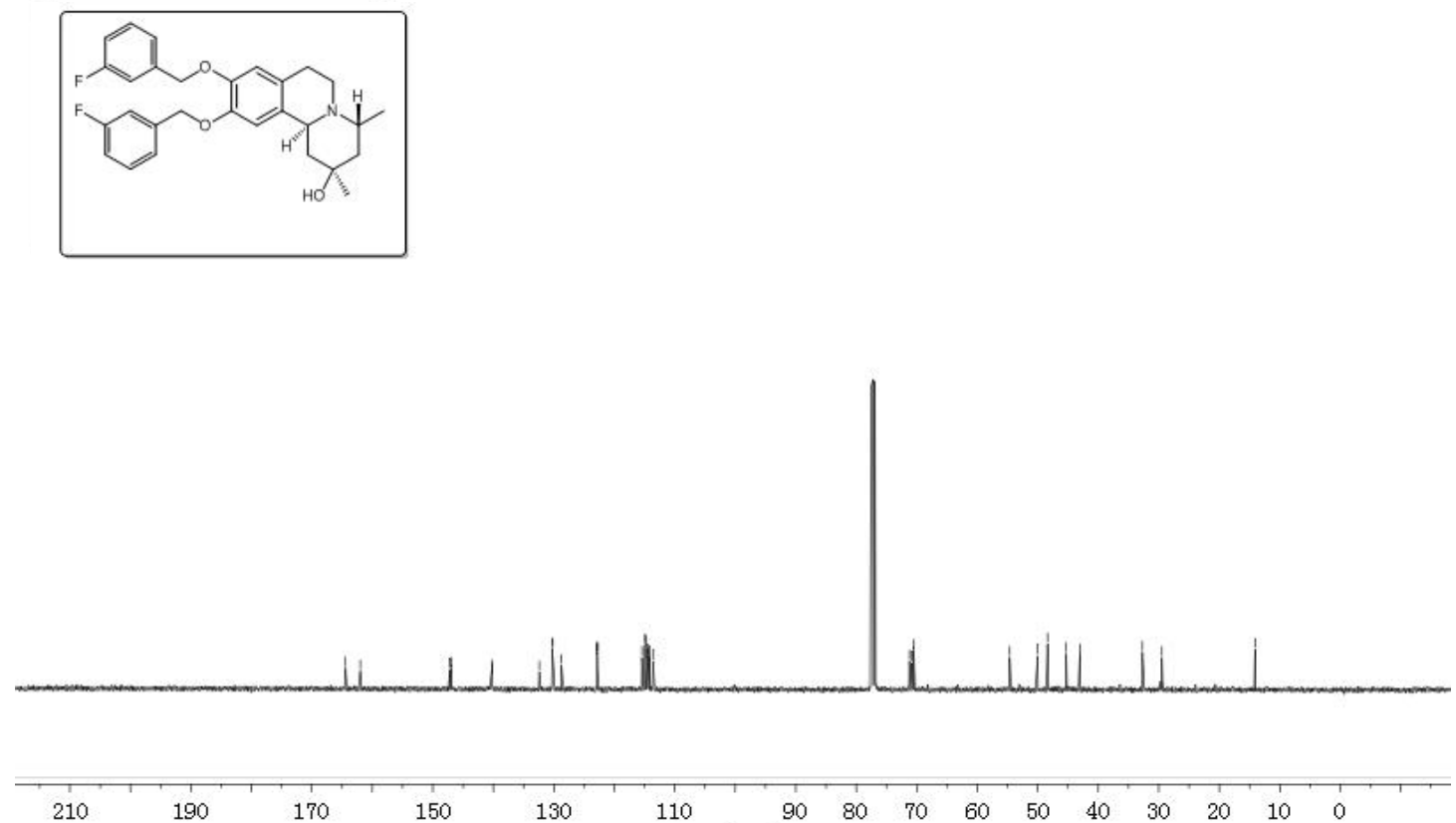

Figure S300. ${ }^{13} \mathrm{C}$ NMR spectrum of 33 .

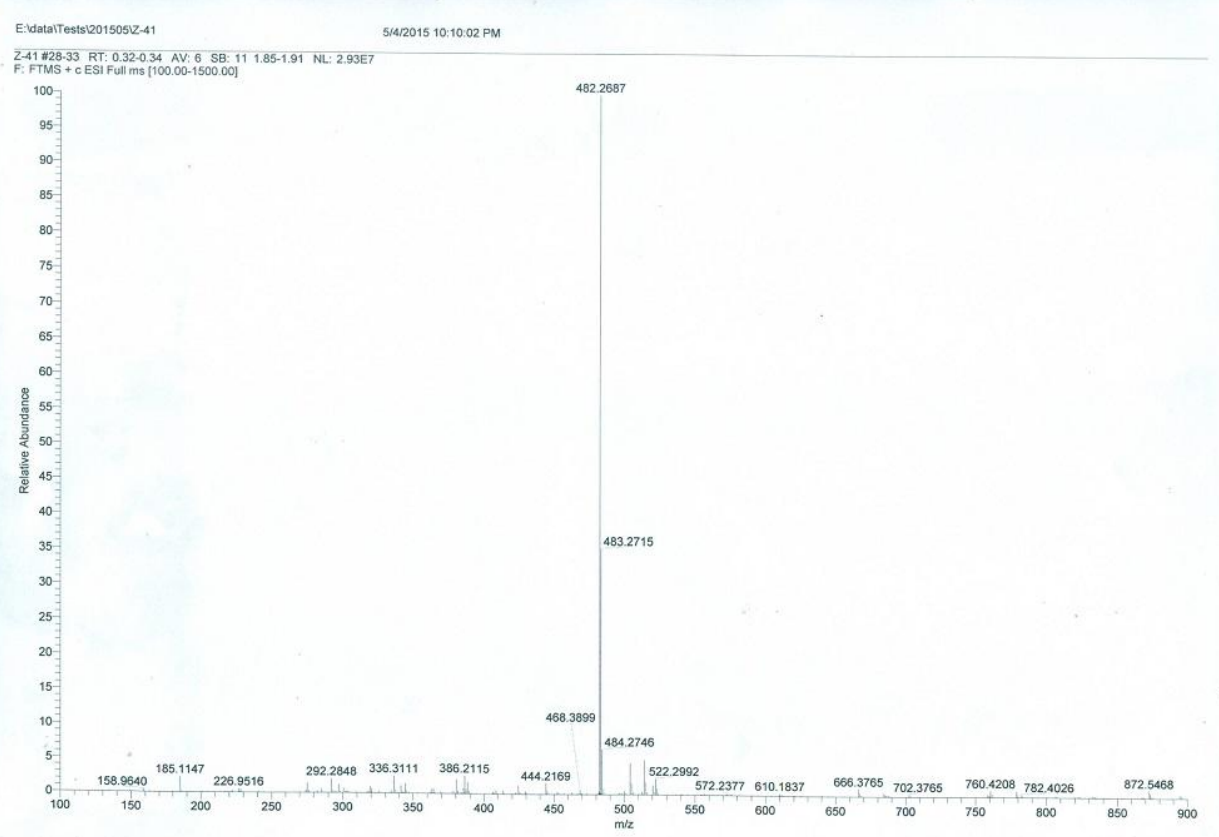

Figure S301. HRESIMS spectrum of SH1. 

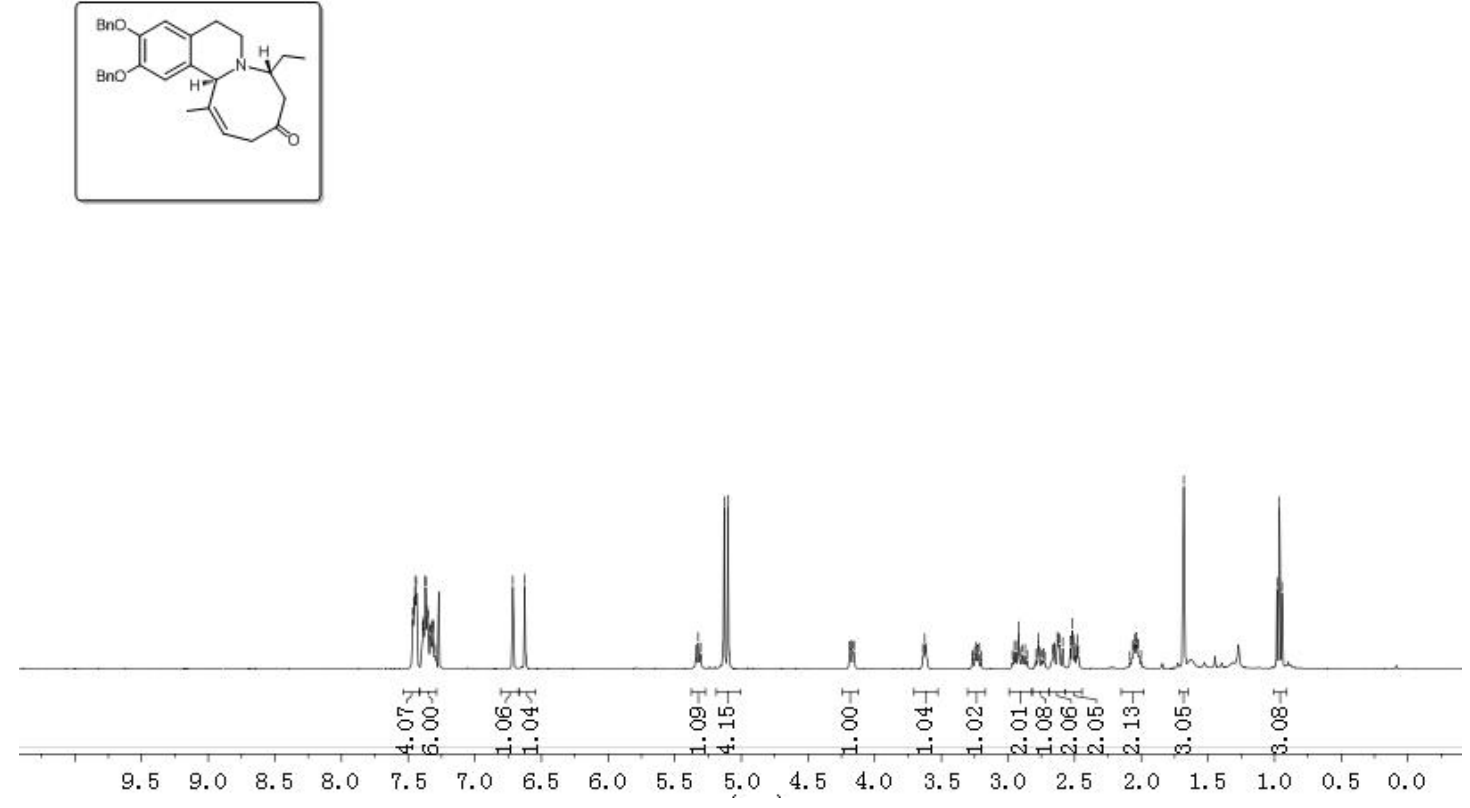

Figure S302. ${ }^{1} \mathrm{H}$ NMR spectrum of SH1.
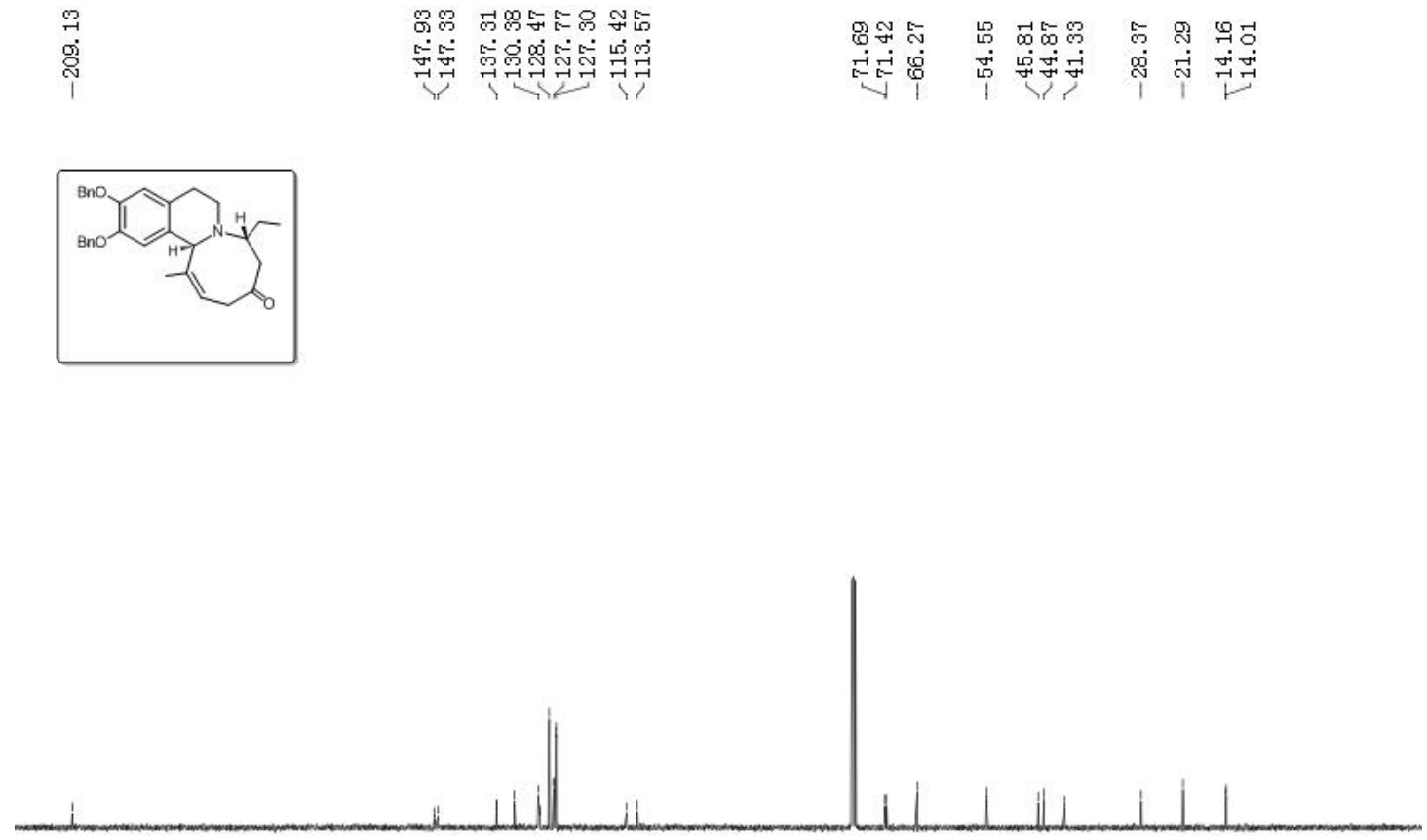

210

190

170

150

130

110

90

Figure S303. ${ }^{13} \mathrm{C}$ NMR spectrum of SH1. 


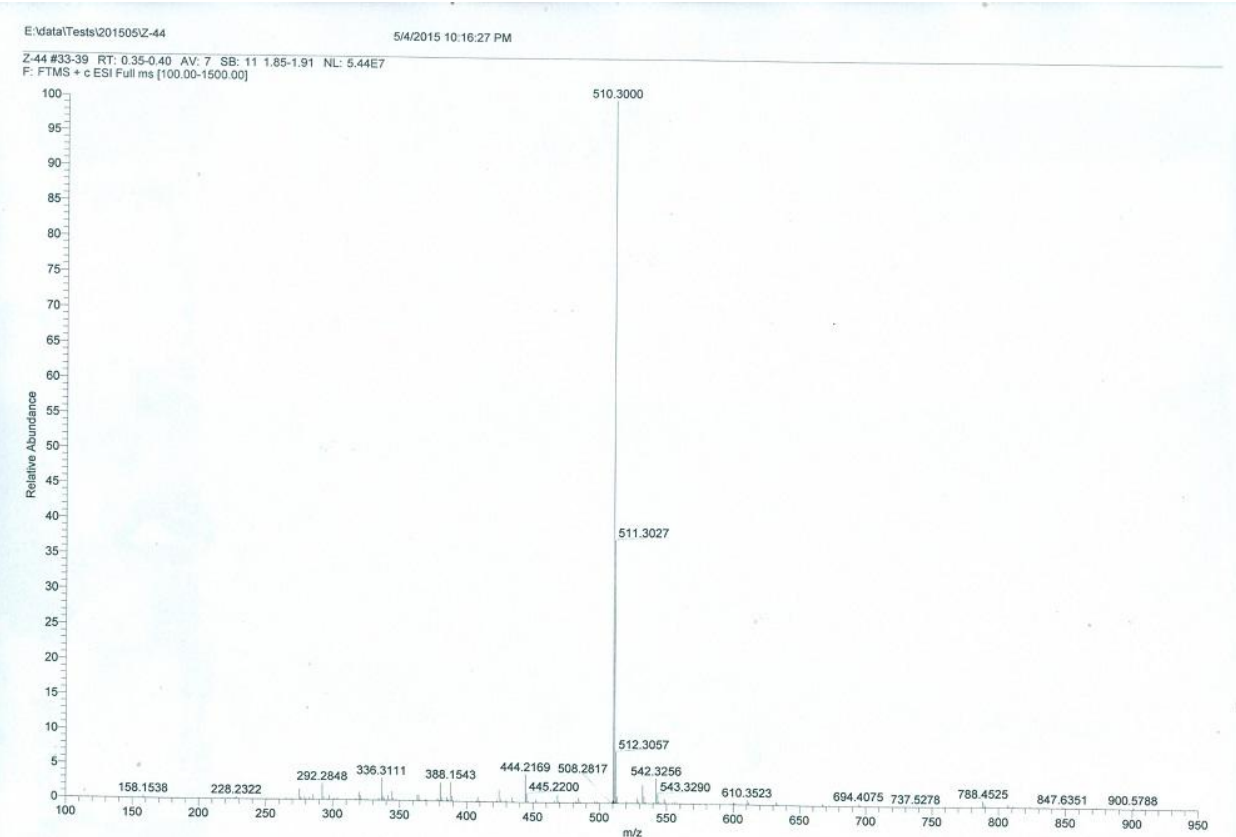

Figure S304. HRESIMS spectrum of SH2.
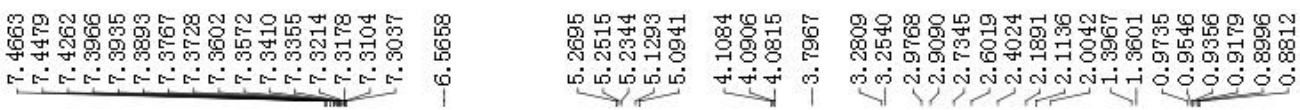

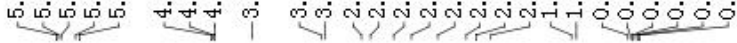
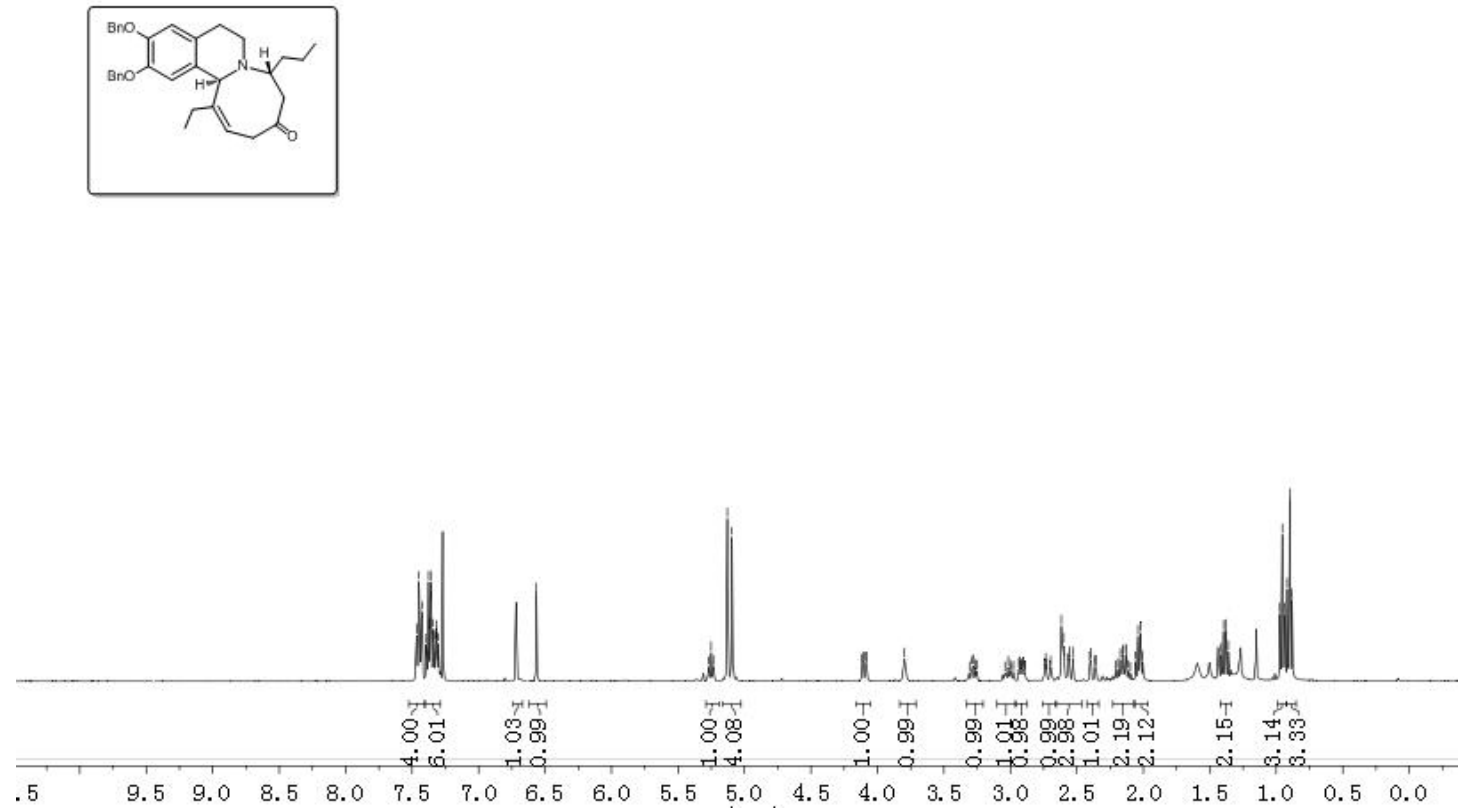

Figure S305. ${ }^{1} \mathrm{H}$ NMR spectrum of $\mathbf{S H 2}$. 

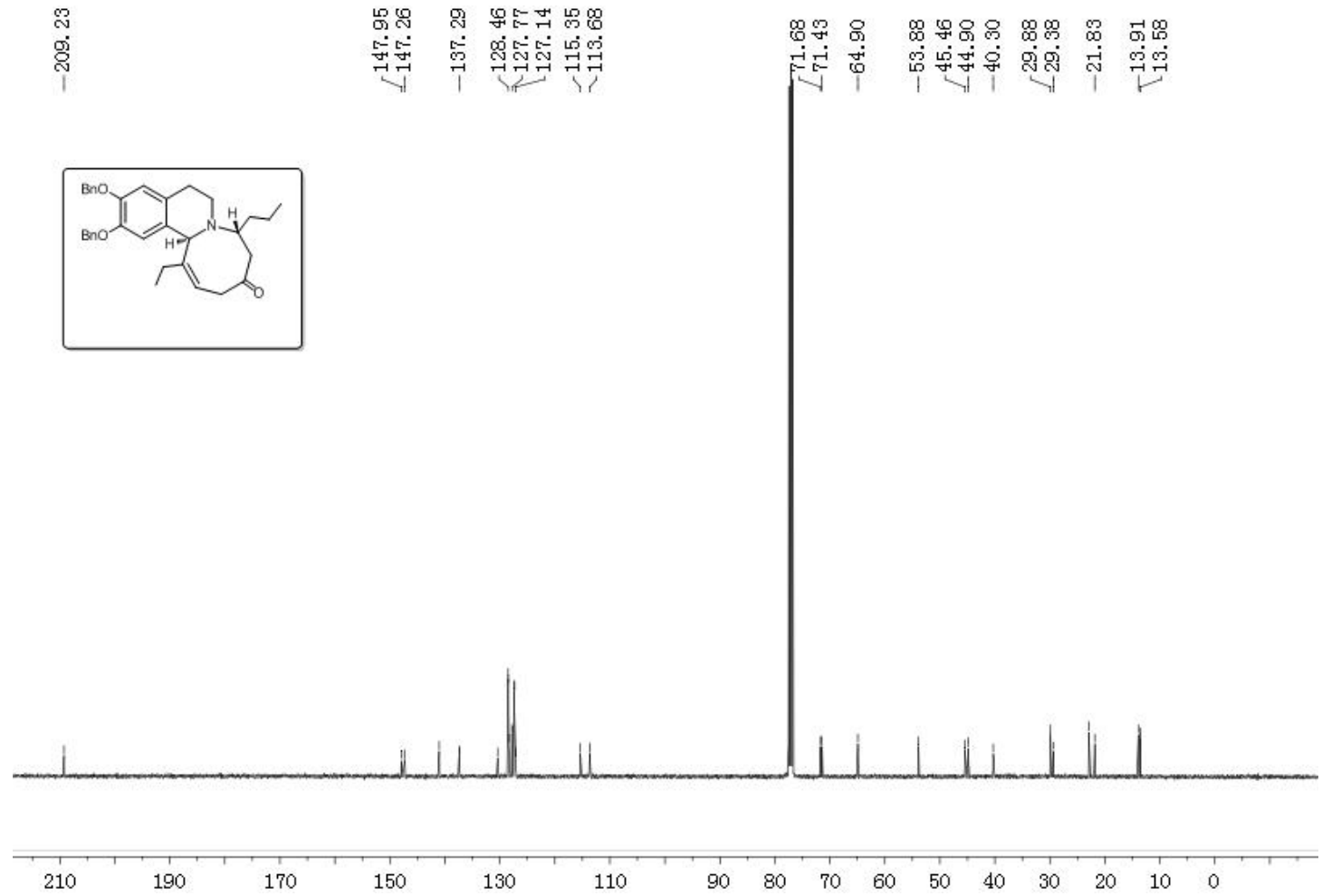

Figure S306. ${ }^{13} \mathrm{C}$ NMR spectrum of SH2.
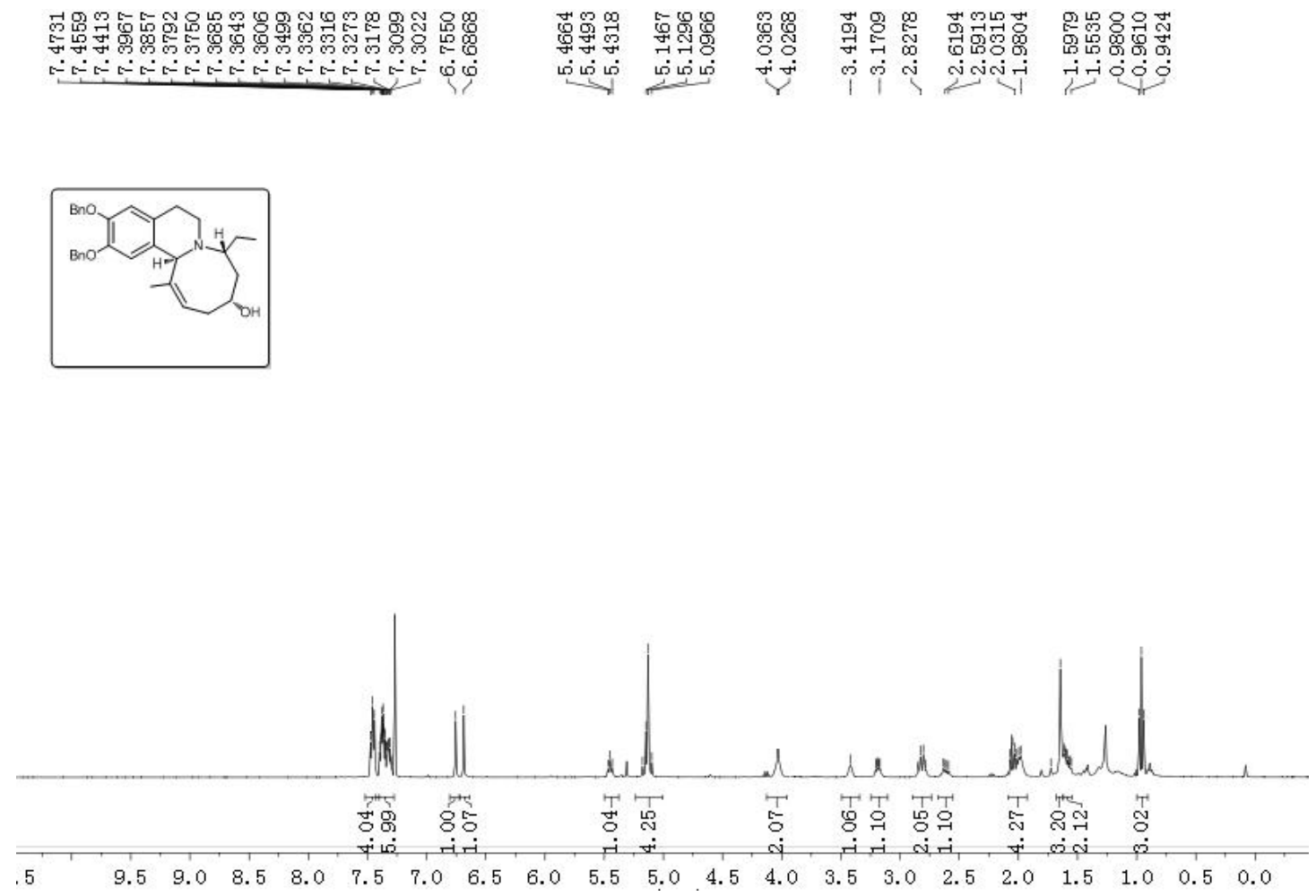

Figure S307. ${ }^{1} \mathrm{H}$ NMR spectrum of $\mathbf{3 7 a}$. 


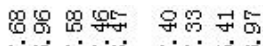

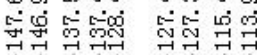

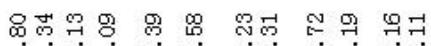

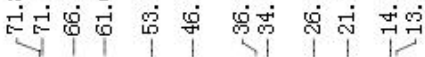
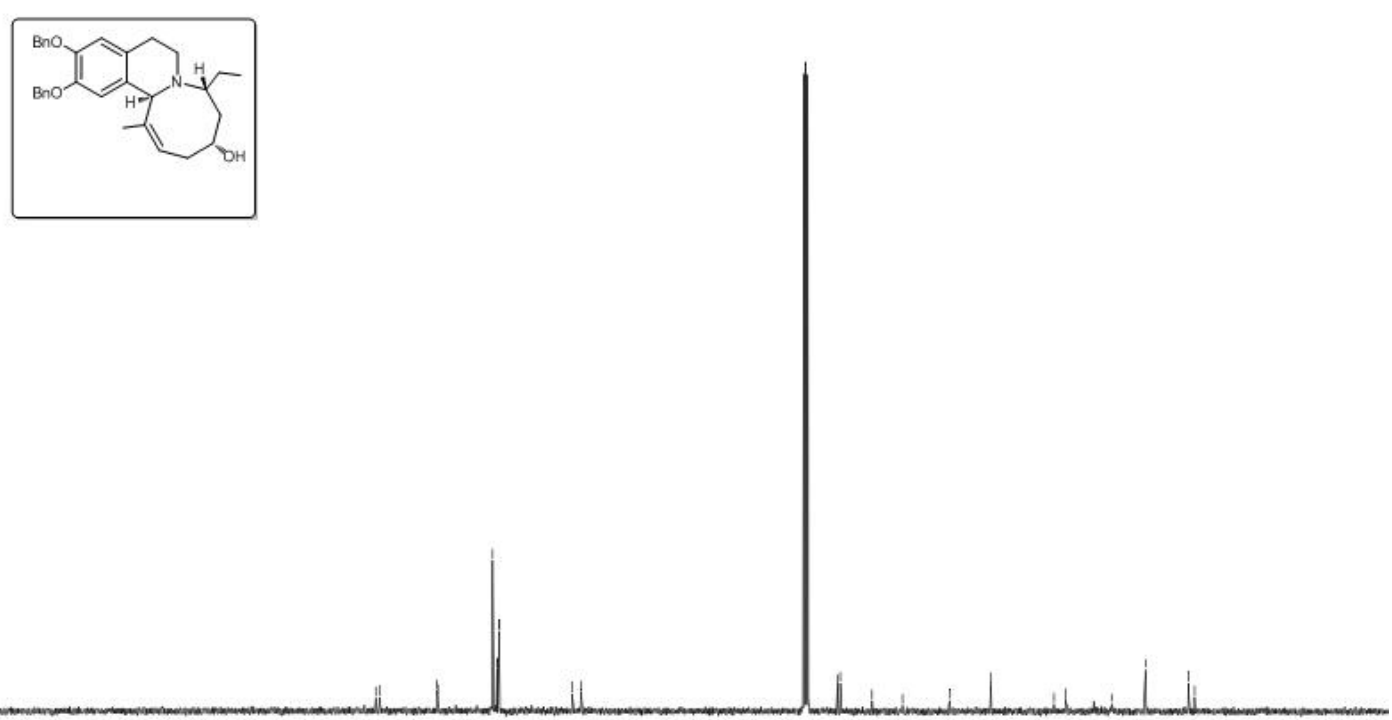

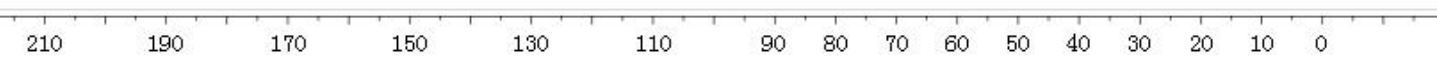

Figure S308. ${ }^{13} \mathrm{C}$ NMR spectrum of $\mathbf{3 7 a}$.

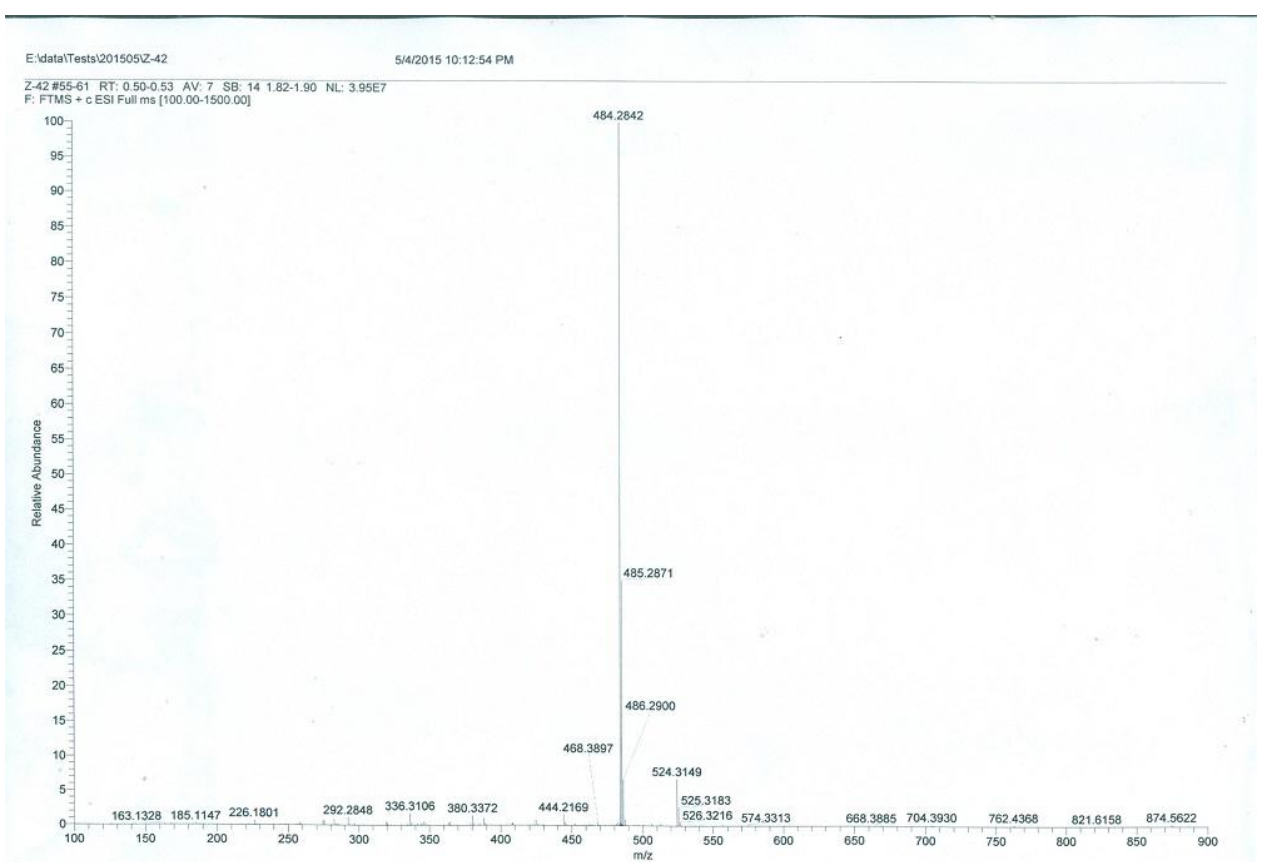

Figure S309. HRESIMS spectrum of $\mathbf{3 7 b}$. 

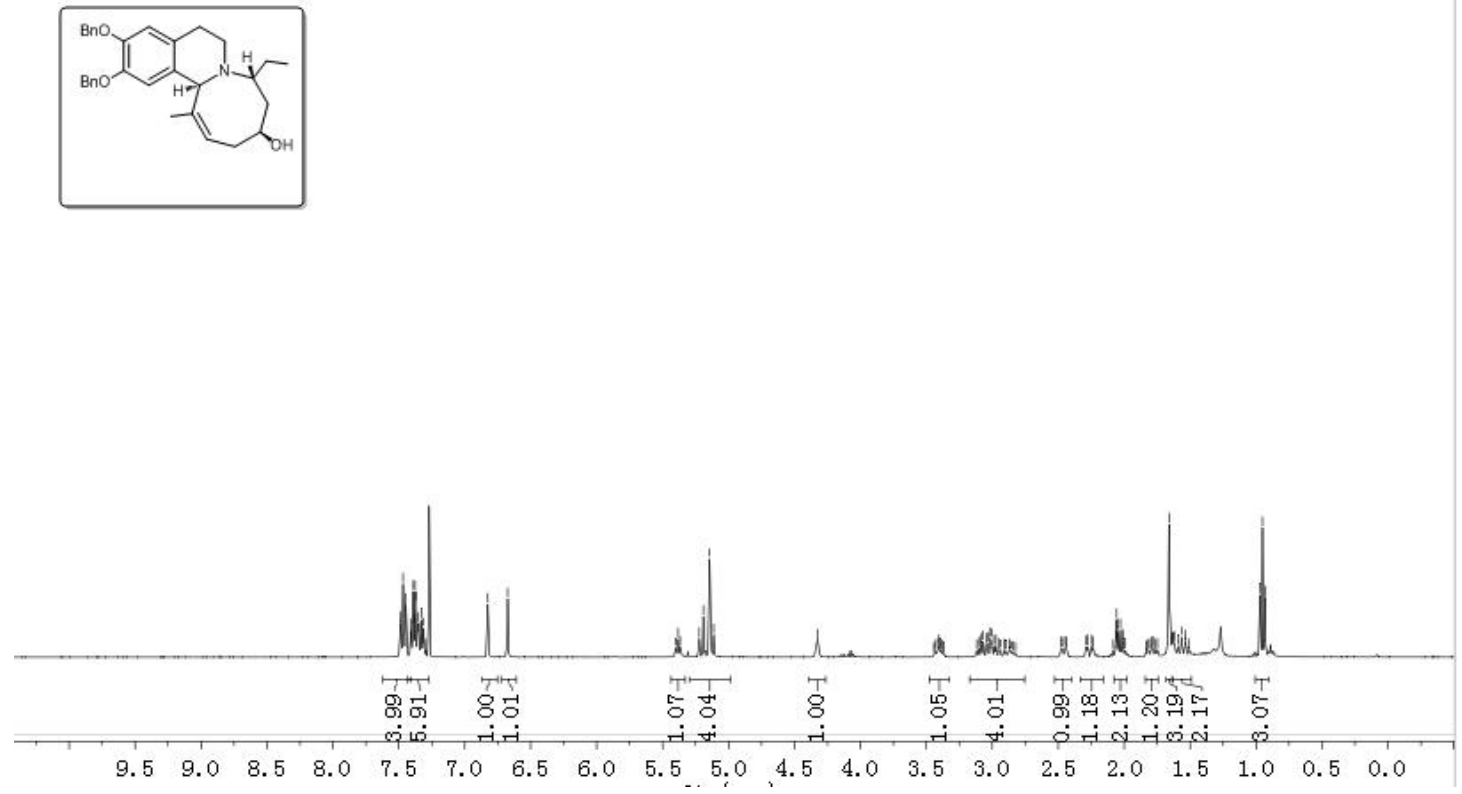

Figure S310. ${ }^{1} \mathrm{H}$ NMR spectrum of $\mathbf{3 7 b}$

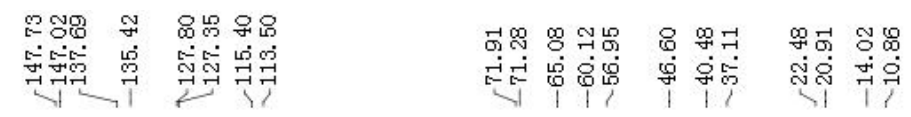

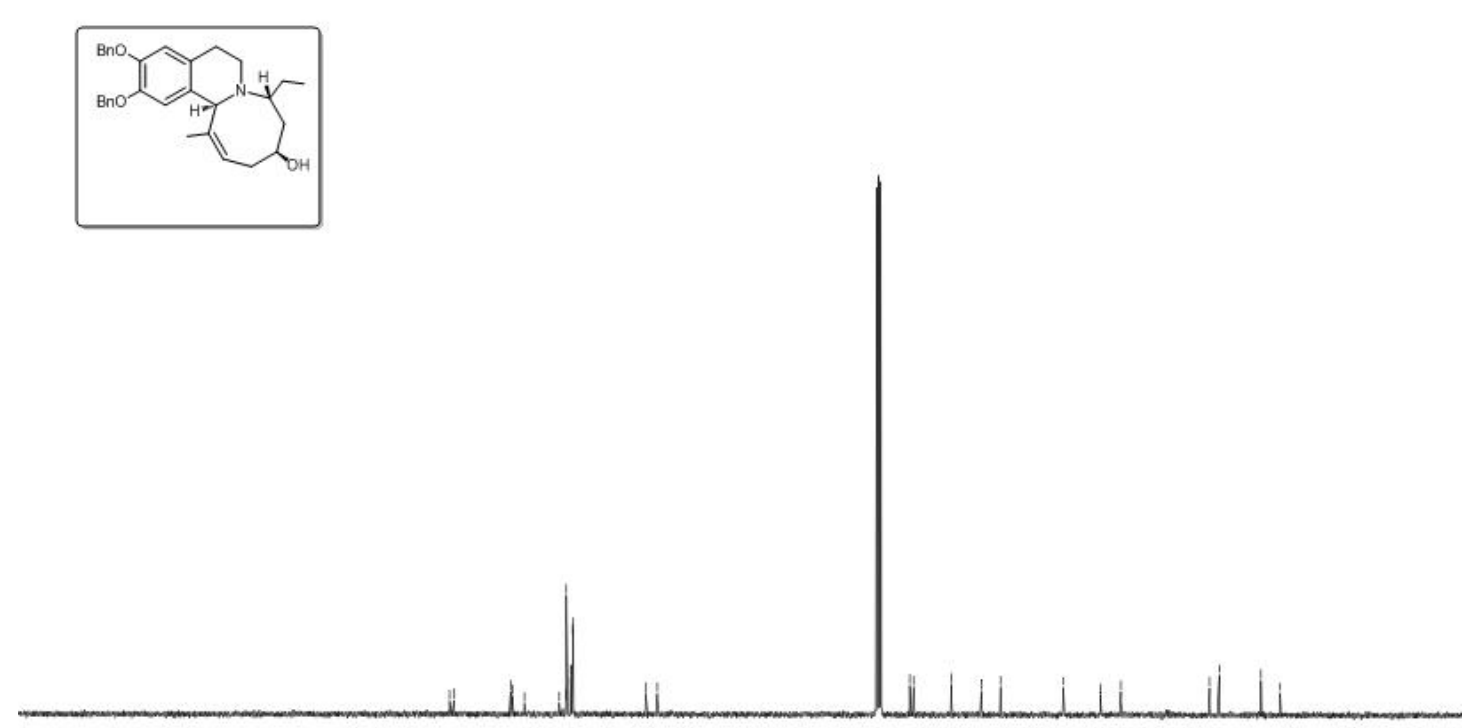

Figure S311. ${ }^{13} \mathrm{C}$ NMR spectrum of $\mathbf{3 7 b}$. 

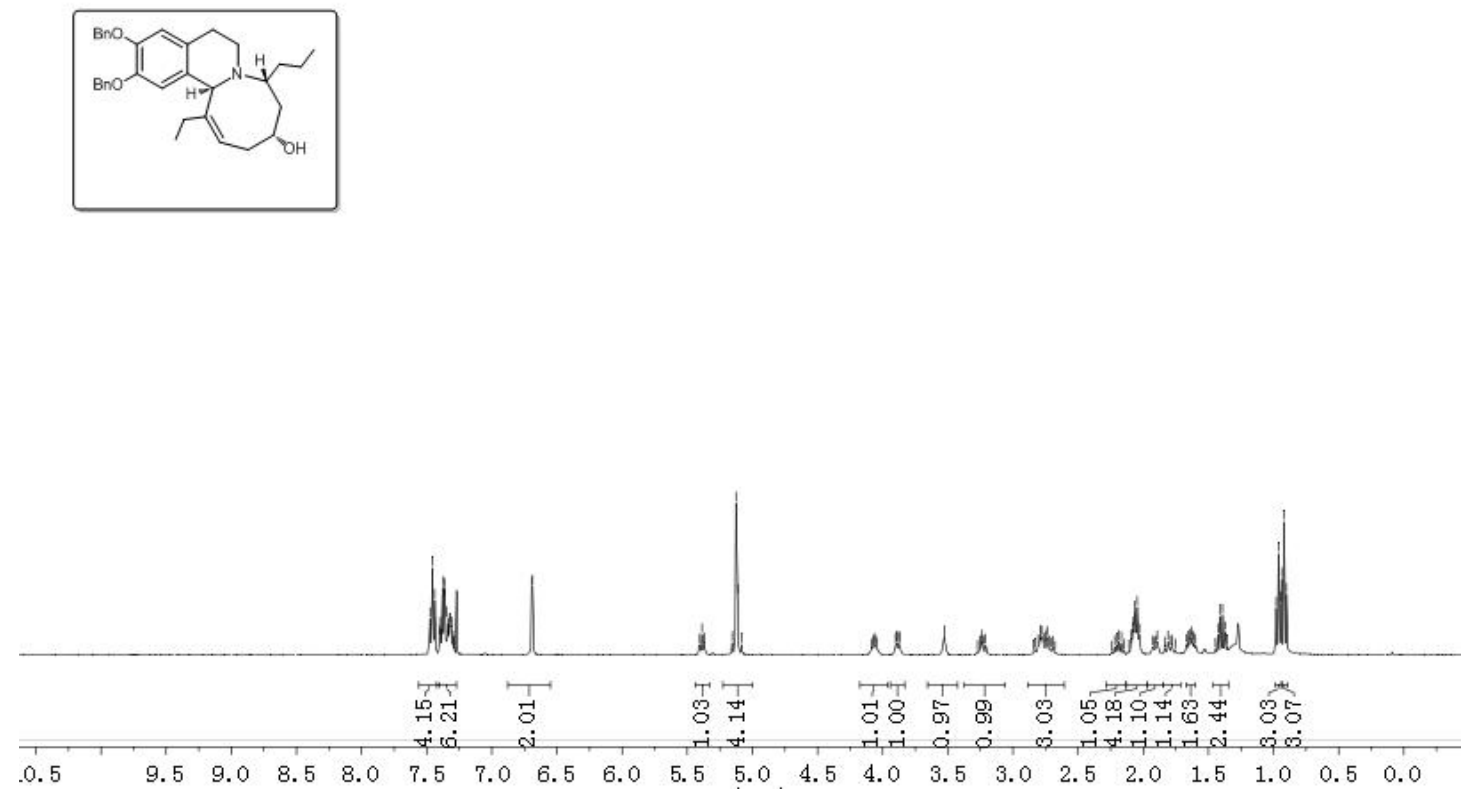

Figure S312. ${ }^{1} \mathrm{H}$ NMR spectrum of $\mathbf{3 8 a}$.

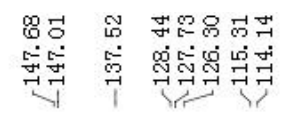

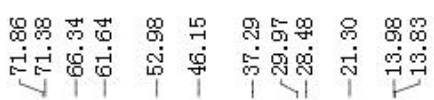
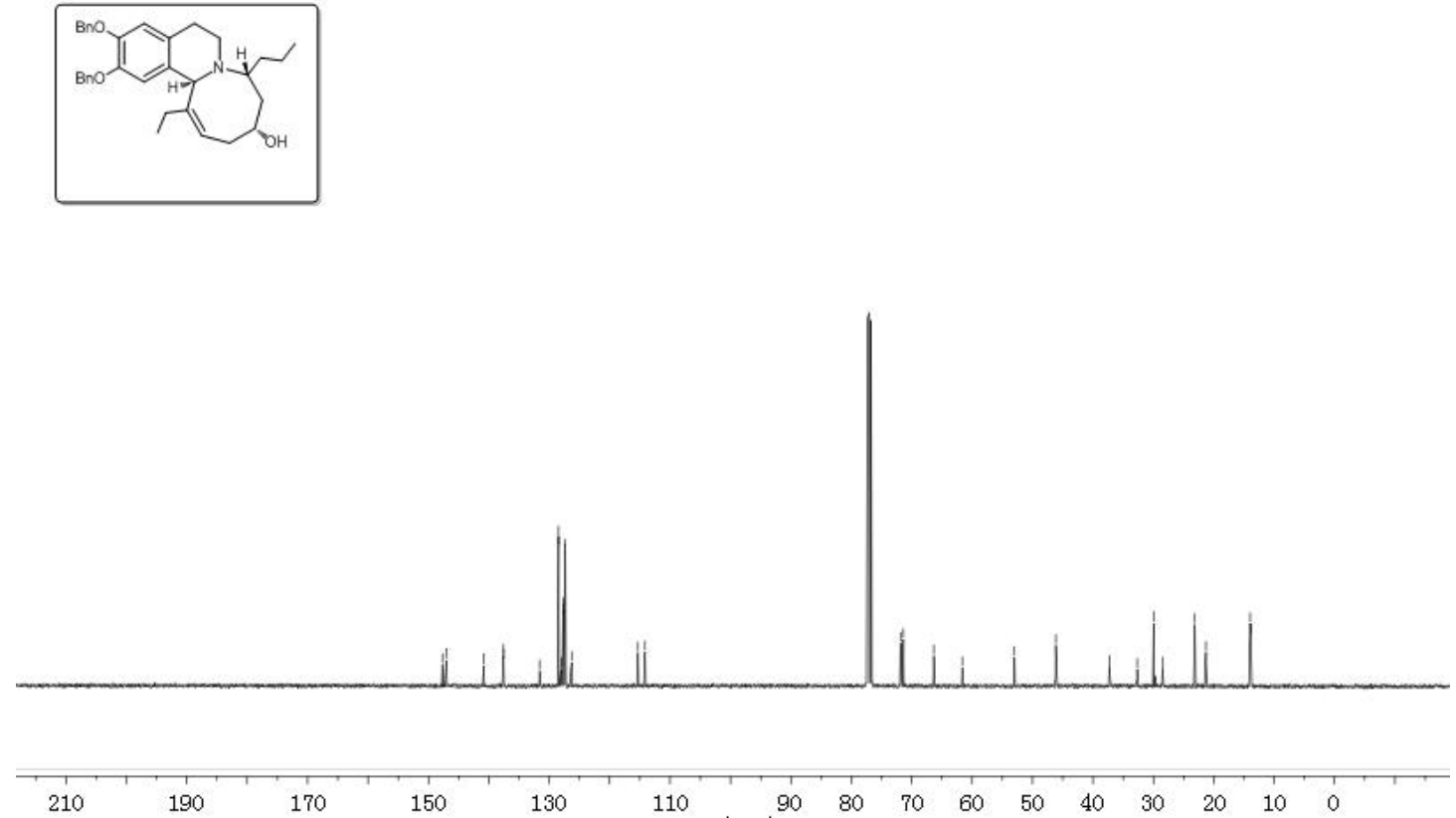

Figure S313. ${ }^{13} \mathrm{C}$ NMR spectrum of $\mathbf{3 8 a}$. 


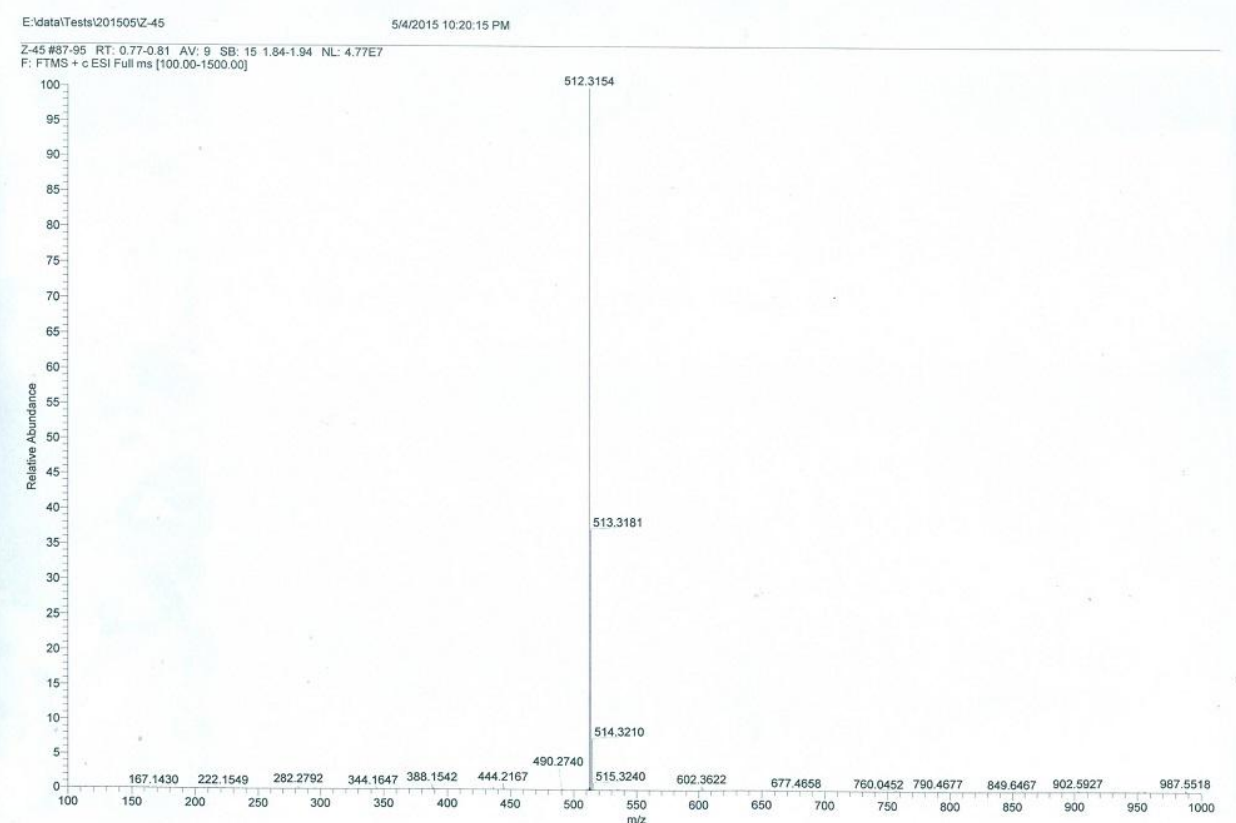

Figure S314. HRESIMS spectrum of $\mathbf{3 8 b}$.

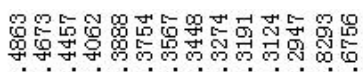

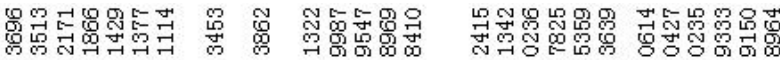

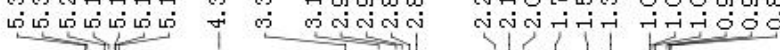

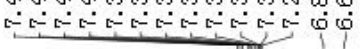
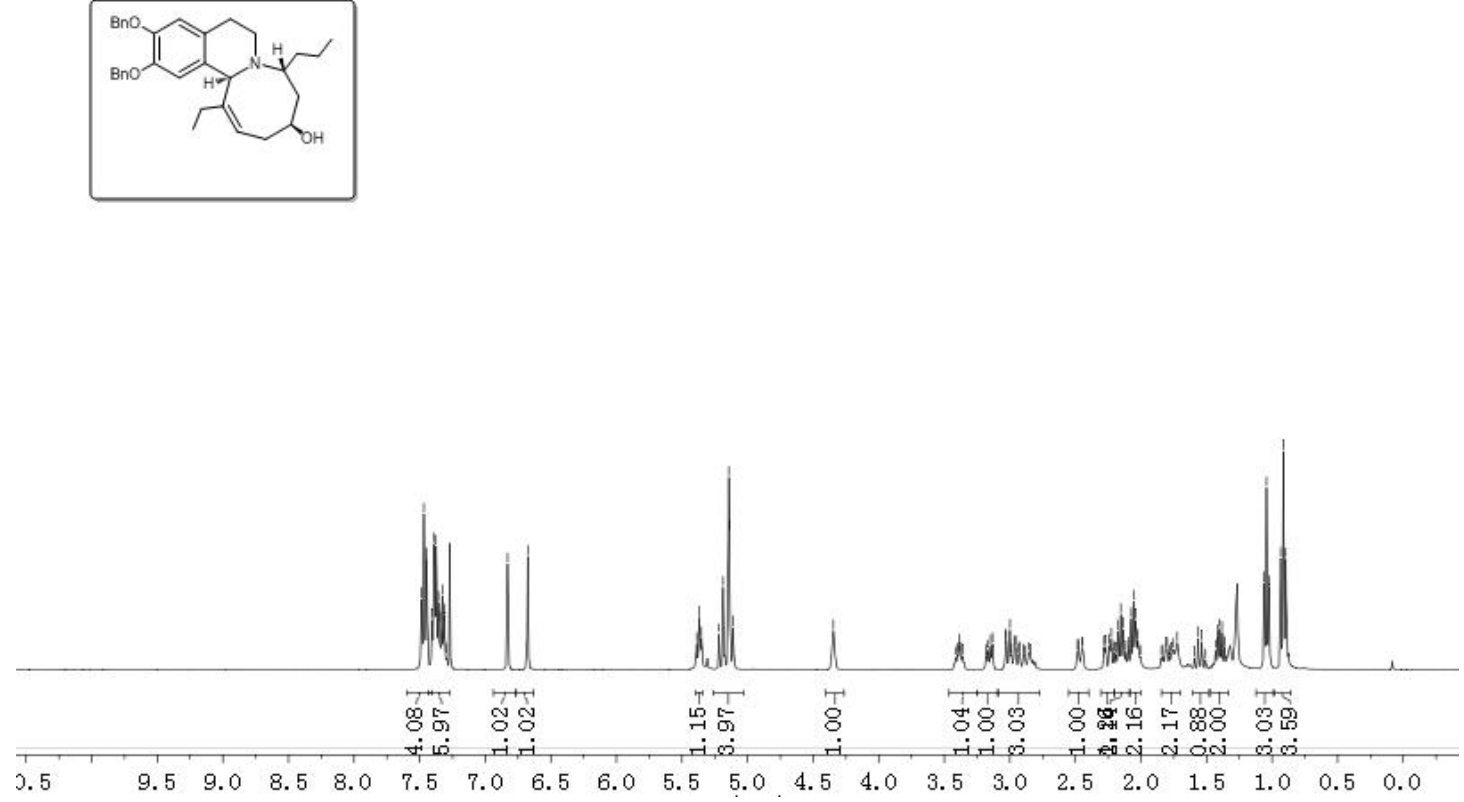

Figure S315. ${ }^{1} \mathrm{H}$ NMR spectrum of $\mathbf{3 8 b}$. 

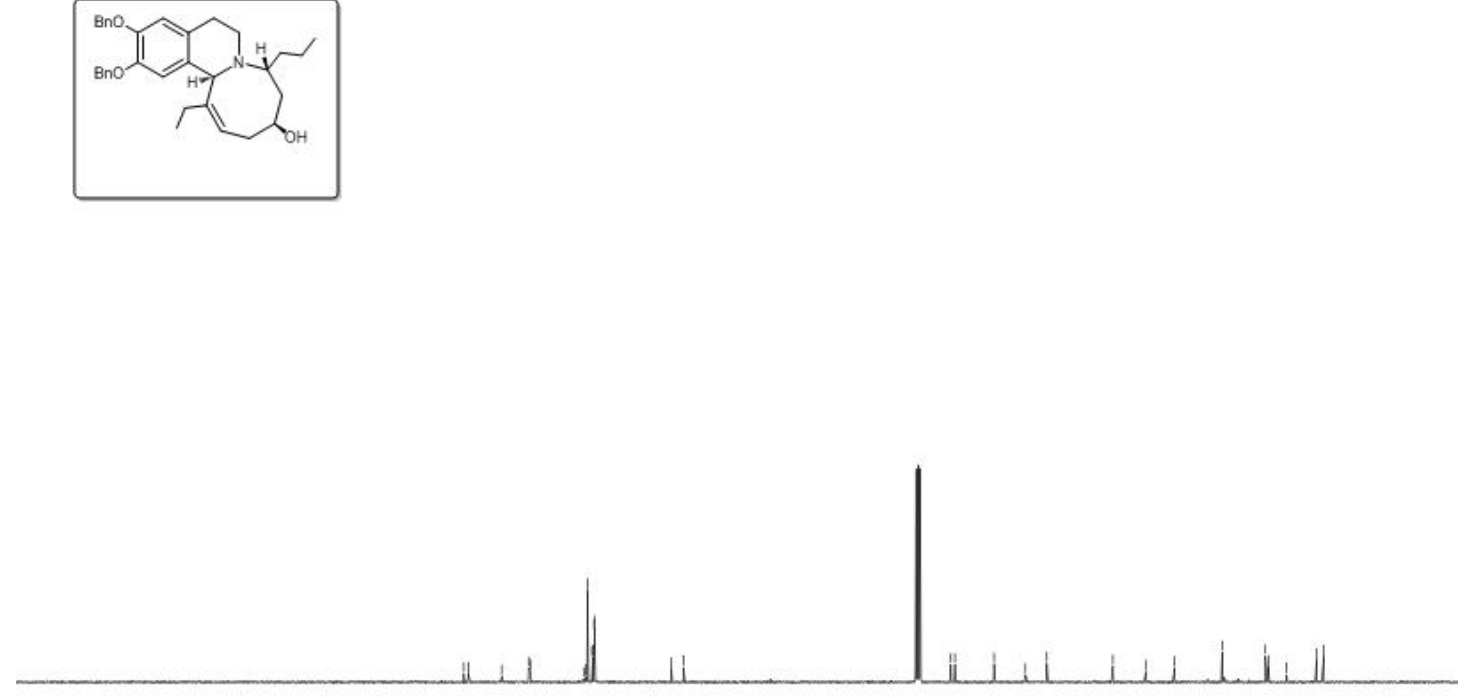

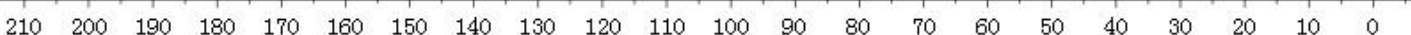

Figure S316. ${ }^{13} \mathrm{C}$ NMR spectrum of $\mathbf{3 8 b}$.

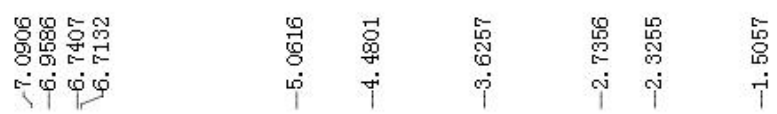
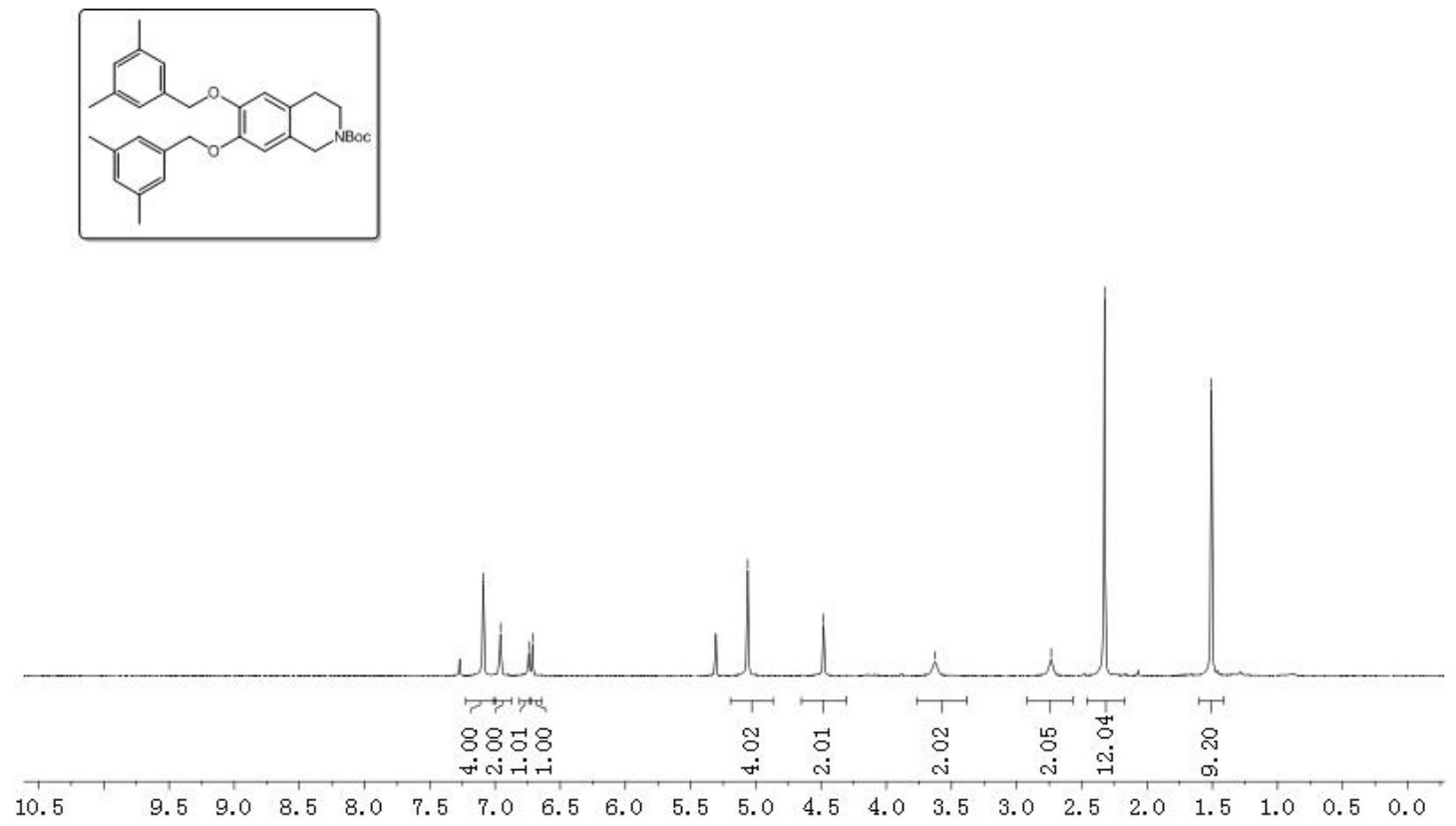

Figure S317. ${ }^{1} \mathrm{H}$ NMR spectrum of IV-22b. 

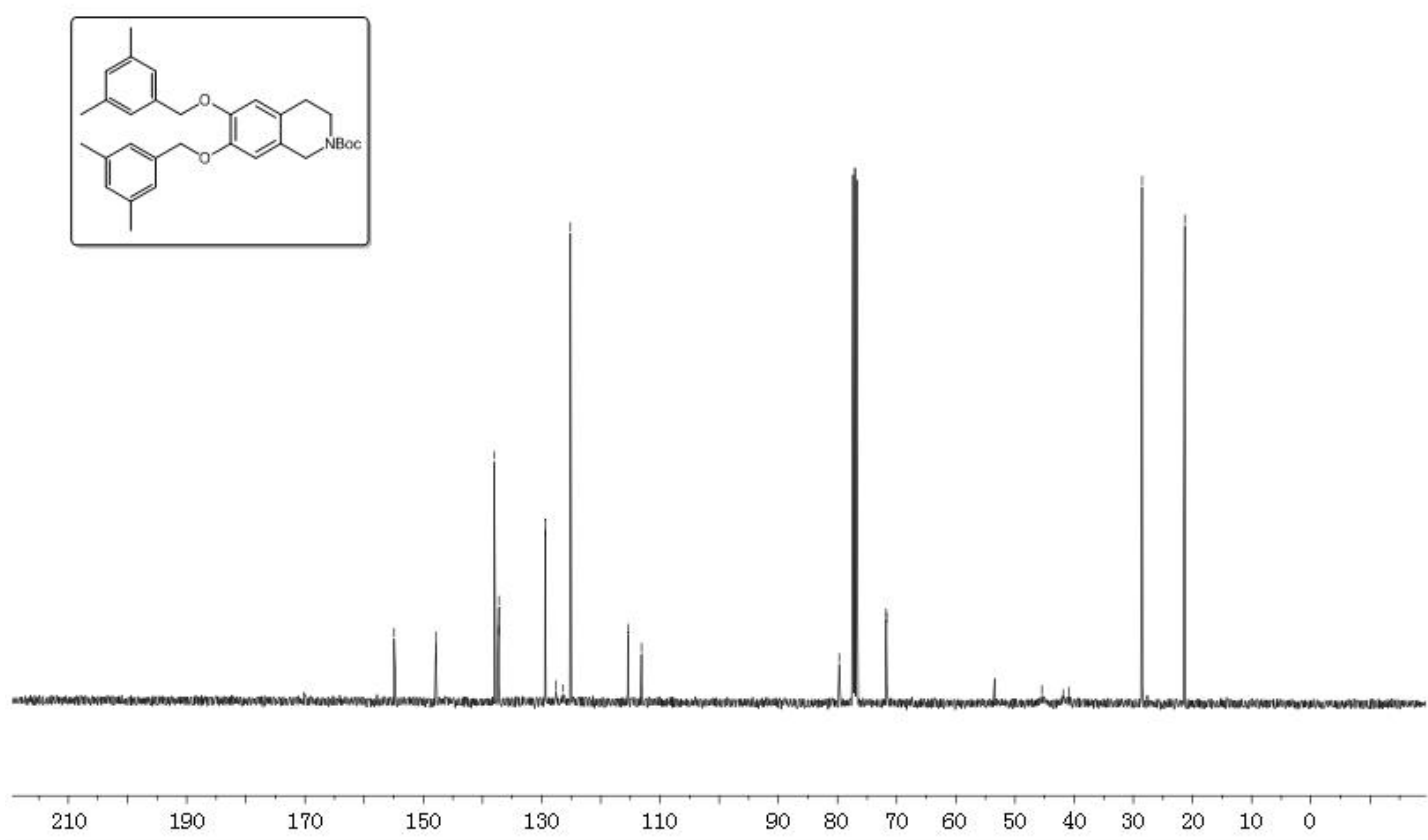

Figure S318. ${ }^{13} \mathrm{C}$ NMR spectrum of IV-22b.
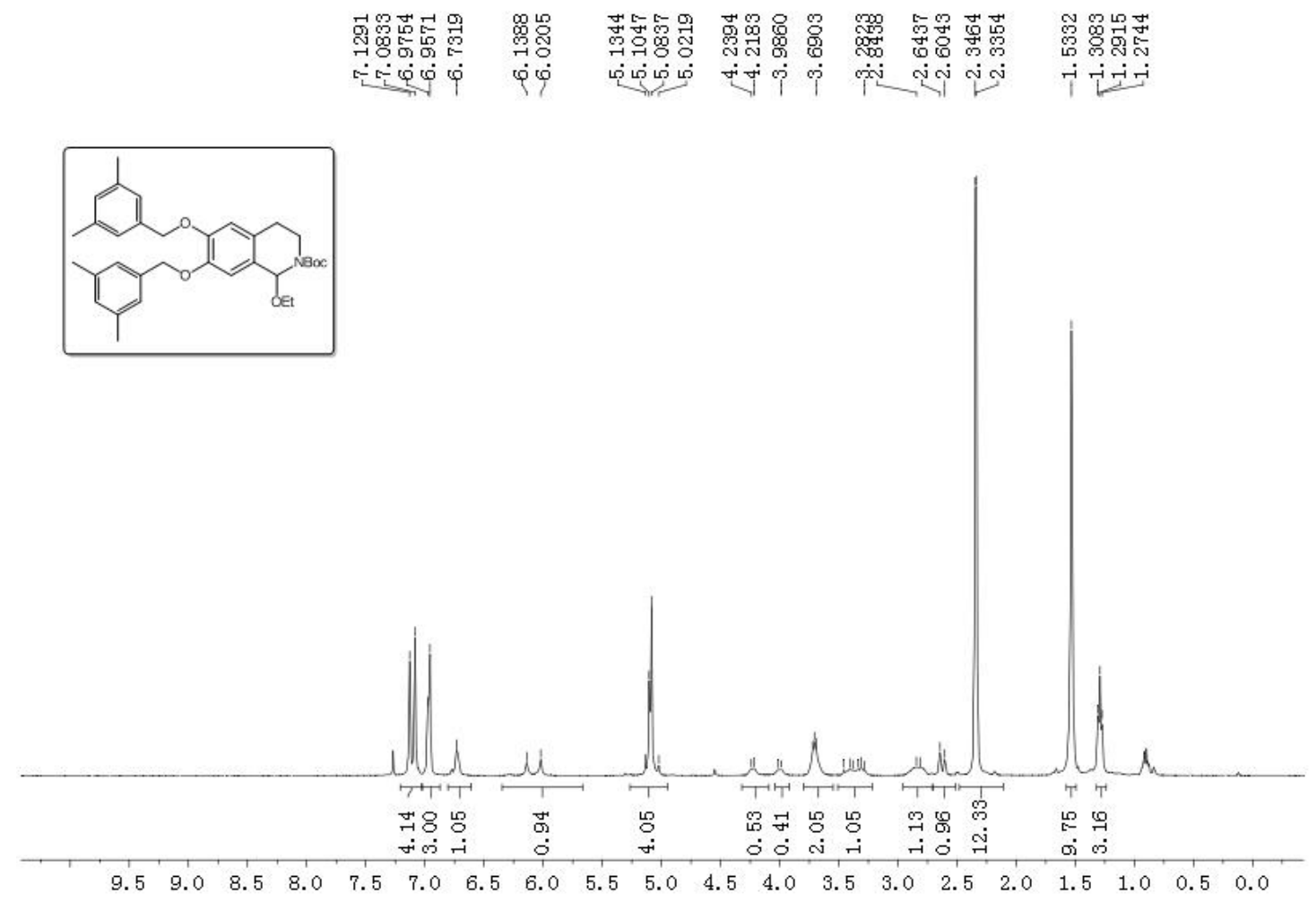

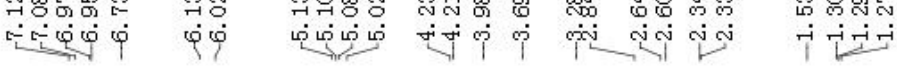

Figure S319. ${ }^{1} \mathrm{H}$ NMR spectrum of V-22b. 

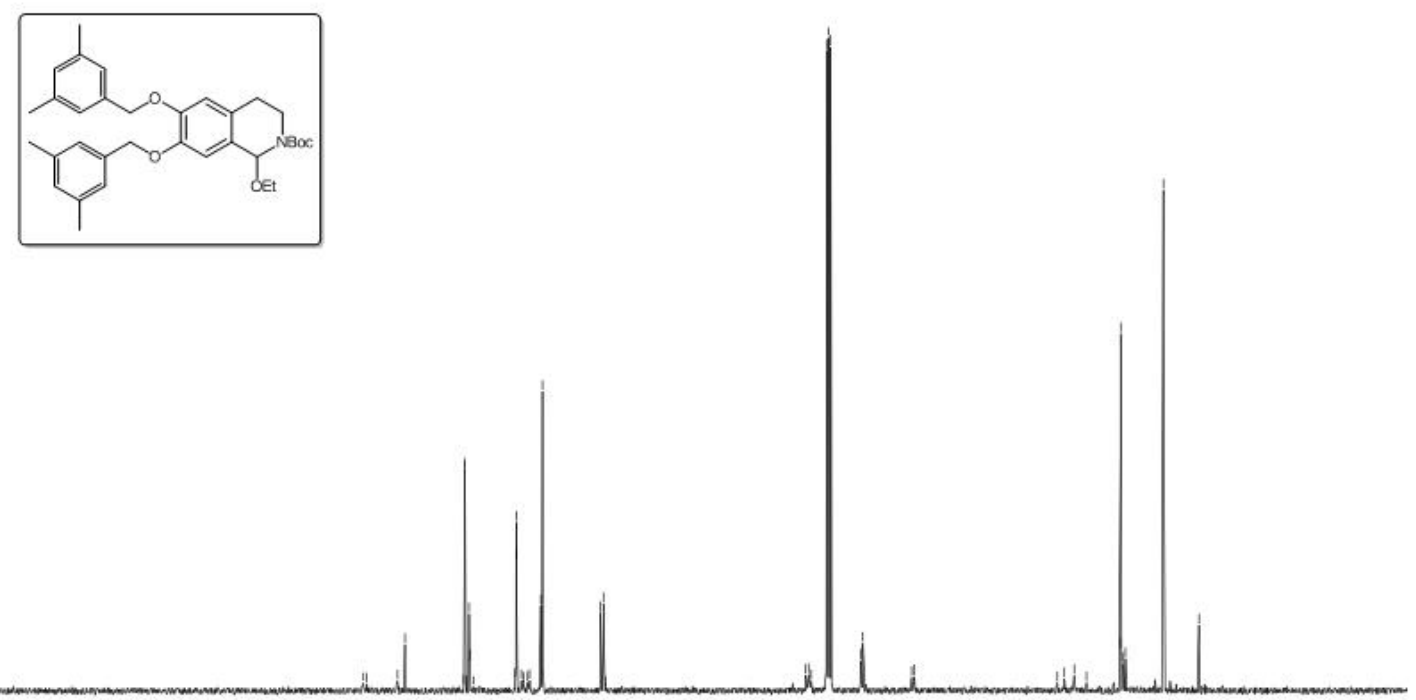

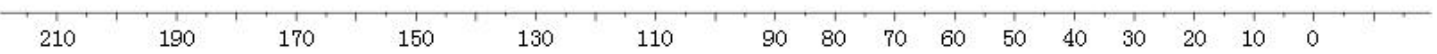

Figure S320. ${ }^{13} \mathrm{C}$ NMR spectrum of $\mathbf{V}-\mathbf{2 2 b}$

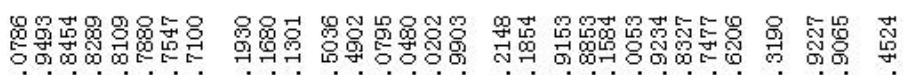

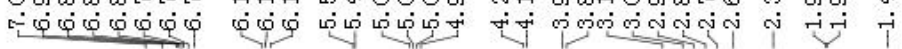
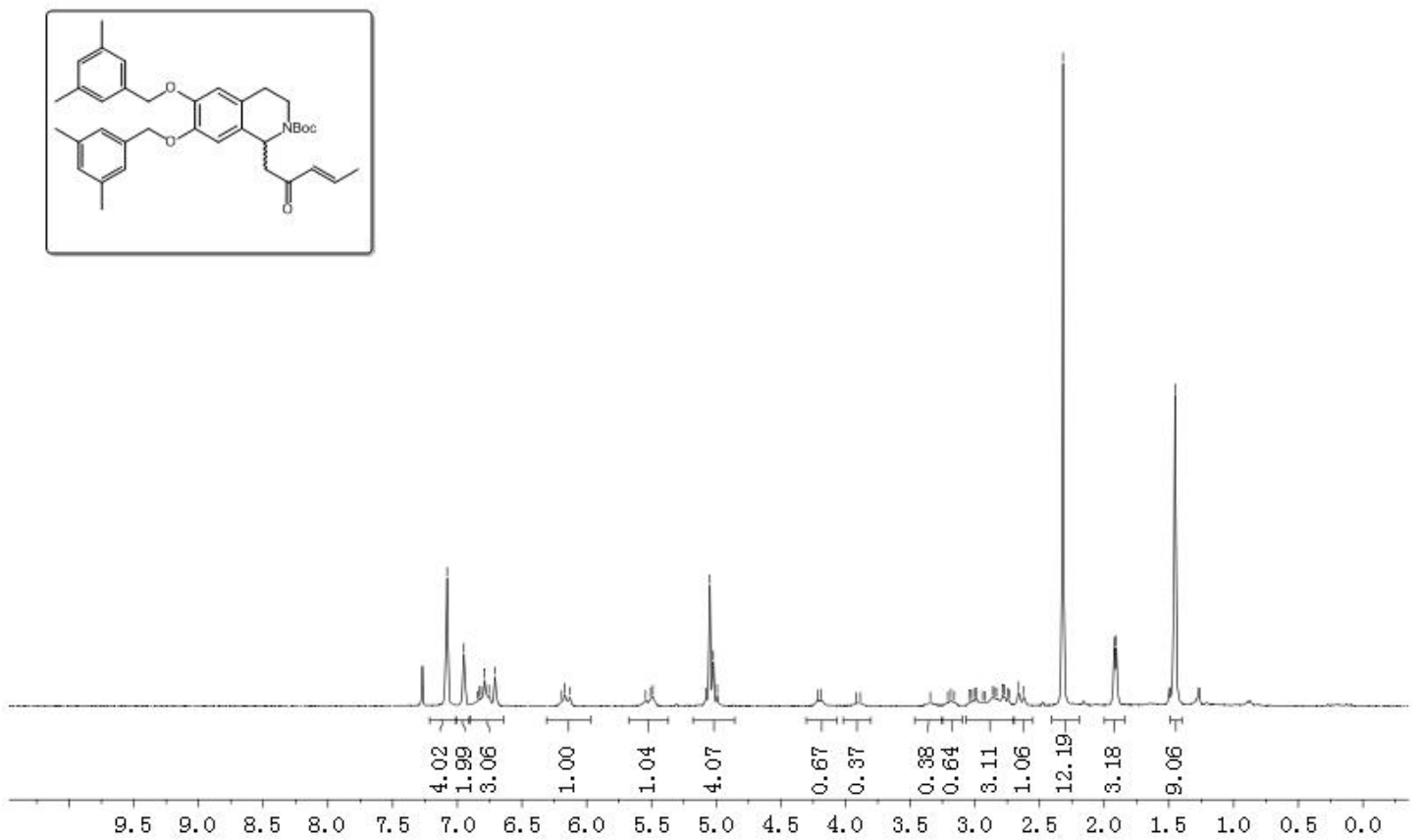

Figure S321. ${ }^{1} \mathrm{H}$ NMR spectrum of VII-22b. 


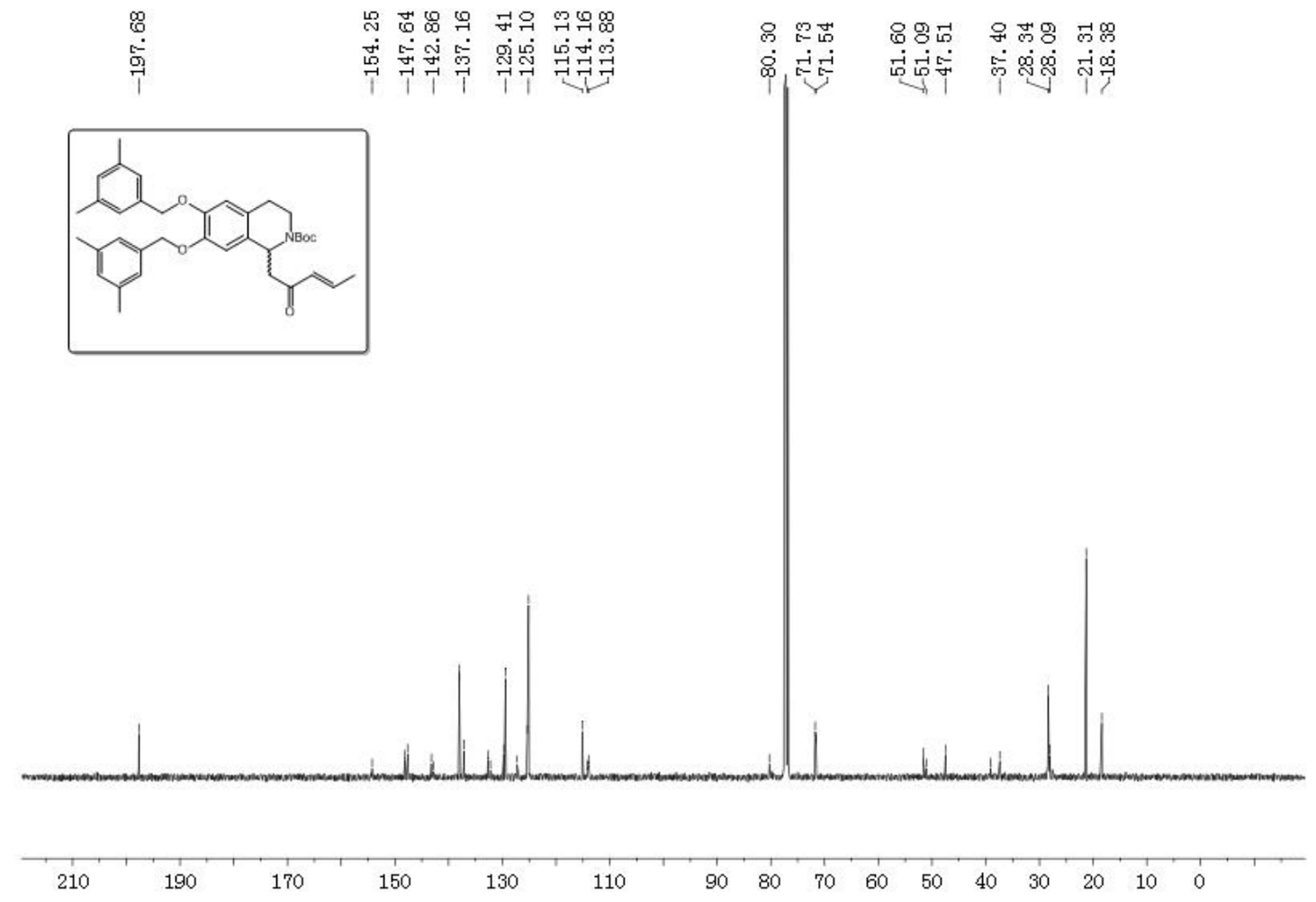

Figure S322. ${ }^{13} \mathrm{C}$ NMR spectrum of VII-22b. 